\title{
Radioactive Waste Management Information for 1993 and Record-To-Date
}

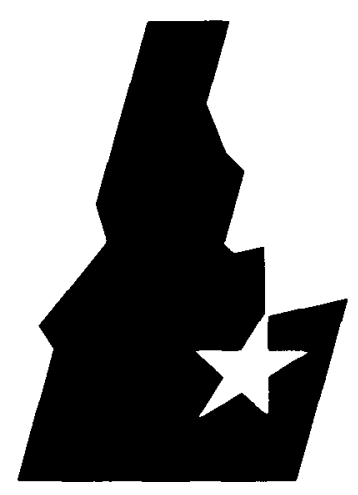

Idaho National Engineering Laboratory

U.S. Department of Energy $\cdot$ Idaho Operations Office 

DOE/ID-10054(93)

Distribution Category

\title{
RADIOACTIVE WASTE MANAGEMENT INFORMATION FOR 1993 AND RECORD-TO-DATE
}

\author{
K. A. Taylor
}

Configuration, Document Control, and Services Unit

Published July 1994

Idaho National Engineering Laboratory

EG\&G Idaho, Inc.

Idaho Falls, Idaho 83415 



\section{DISCLAIMER}

This report was prepared as an account of work sponsored by an agency of the United States Government. Neither the United States Government nor any agency thereof, nor any of their employees, make any warranty, express or implied, or assumes any legal liability or responsibility for the accuracy, completeness, or usefulness of any information, apparatus, product, or process disclosed, or represents that its use would not infringe privately owned rights. Reference herein to any specific commercial product, process, or service by trade name, trademark, manufacturer, or otherwise does not necessarily constitute or imply its endorsement, recommendation, or favoring by the United States Government or any agency thereof. The views and opinions of authors expressed herein do not necessarily state or reflect those of the United States Government or any agency thereof. 



\section{DISCLAIMER}

Portions of this document may be illegible in electronic image products. Images are produced from the best available original document. 



\begin{abstract}
This document presents detailed data, bar graphs, and pie charts on volume, radioactivity, isotopic identity, origin, and decay status of radioactive waste for the calendar year 1993. It also summarizes the radioactive waste data records compiled from 1952 to present for the Idaho National Engineering Laboratory (INEL). The data presented are from the INEL Radioactive Waste Management Information System.
\end{abstract}





\section{EXECUTIVE SUMMARY}

This document, Radioactive Waste Management Information for 1993 and Record-to-Date, contains computerized radioactive waste data records from the Idaho National Engineering Laboratory (INEL). Data are compiled from information supplied by the U.S. Department of Energy (DOE) contractors. Data listed are on airborne and liquid radioactive effluents and solid radioactive waste that is stored, disposed, and sent to the INEL for reduction. Data are summarized for the years 1952 through 1992. Data are detailed for the calendar year 1993.

Airborne and liquid waste was released to the environment through engineered release points identified on Table 1 located in the Engineered Release Points section. Monitoring and effluent sampling systems are an integral part of each engineered release point. Releases to the environment may also occur at locations other than normal release points.

Airborne releases of radioactivity for 1993 were 2,719 curies; noble gases accounted for 2,590 curies or $95 \%$ of the radioactivity. The curie amount compares to 24,179 curies in 1992 and 63,879 curies on 1991 .

Prior to CY1993 data on the actual release of krypton from the Idaho Chemical Processing Plant (ICPP) were classified. Therefore, krypton releases were reported in multiples of 10,000 curies to meet classification requirements. As a result of ICPP's mission change, $\mathrm{Kr}-85$ will no longer be reported in multiples of 10,000 curies. Actual values will be reported, which have resulted in a decrease in total radioactivity being released at ICPP.

A total of 2.7E+09 liters of liquid radioactive waste containing 130 curies was released to the environment at the INEL during 1993. Tritium accounted for $95 \%$ of the radioactivity. As a result of radioactive decay, 122 curies or $93 \%$ of the curies for identified radionuclides remained on January 1, 1994, and 97 curies or $74 \%$ will remain after 5 years. The radioactivity discharged as liquid waste in 1993 was $30 \%$ less than the ac- tivity discharged in 1992 , and $23 \%$ less than the activity discharged in 1991 .

During 1993, a total of $852 \mathrm{~m}^{3}$ of solid radioactive waste containing 429,655 curies was shipped to the Radioactive Waste Management Complex (RWMC) for disposal. This compares to $844 \mathrm{~m}^{3}$ with 143,951 curies disposed in 1992, and $1,272 \mathrm{~m}^{3}$ with 187,569 curies in 1991 . By January 1,1994 , the radionuclides identified in 1993 had decayed to 404,169 curies $(94 \%)$, and in 5 years they will have decayed to 299,811 curies $(70 \%)$.

The majority of the nuclides disposed in solid waste at the RWMC in 1993 consisted of H-3, $\mathrm{Co}-60$, and $\mathrm{Ni}-63$. These nuclides accounted for $92 \%$ of total curies.

No transuranic waste was shipped to the Transuranic Storage Area in 1993. The Waste Calcining Facility at ICPP accounts for $97 \%$ of the volume and $99 \%$ of the radioactivity placed in storage.

The Waste Experimental Reduction Facility (WERF) received $446 \mathrm{~m}^{3}$ and 2 curies of solid radioactive waste for size reduction in 1993. The waste received by WERF for reduction in 1993 compared to $1,139 \mathrm{~m}^{3}$ and 19 curies in 1992 and $2,066 \mathrm{~m}^{3}$ and 17 curies in 1991. Because of reevaluation of the shipments to WERF the 1991 and 1992 totals will be different.

Radioactive effluent discharges at the INEL in 1993 met all applicable DOE requirements (DOE 5400.5, 5820.2) and State of Idaho regulations and standards.

The impact of this waste on the environment, both onsite and offsite, is constantly monitored by the Radiological and Environmental Sciences Laboratory/ Idaho Operations. The results of this program for 1993 are published in the annual report, The Idaho National Engineering Laboratory Site Environmental Report for Calendar Year 1993, DOE/ID-12082(93), published July 1994. 
The effect of radioactive decay reduces quantities of activity at varying rates among the radionuclides. The effect of decay on airborne, liquid, and solid radioactive waste disposed on the INEL in 1993 is in INEL 1993 Summaries section. The decay summary data for the INEL for the period of 1952-1993 is found in the Area Decay Summaries section. Only curies of identified radionuclides have decayed. Although all radioactivity decays, decay schemes cannot be applied to the curies of unidentified radionuclides such as mixed fission or activation products. 


\section{CONTENTS}

ABSTRACT $\ldots \ldots \ldots \ldots \ldots \ldots \ldots \ldots \ldots \ldots \ldots \ldots \ldots \ldots \ldots \ldots \ldots \ldots \ldots \ldots \ldots \ldots \ldots \ldots \ldots$

EXECUTIVE SUMMARY $\ldots \ldots \ldots \ldots \ldots \ldots \ldots \ldots \ldots \ldots \ldots \ldots \ldots \ldots \ldots \ldots$

ACRONYMS AND ABBREVIATIONS $\ldots \ldots \ldots \ldots \ldots \ldots \ldots \ldots \ldots \ldots \ldots \ldots \ldots \ldots \ldots$

CONVERSION FACTORS $\quad \ldots \ldots \ldots \ldots \ldots \ldots \ldots \ldots \ldots \ldots \ldots \ldots \ldots \ldots \ldots \ldots \ldots \ldots$

DEFINITIONS $\ldots \ldots \ldots \ldots \ldots \ldots \ldots \ldots \ldots \ldots \ldots \ldots \ldots \ldots \ldots \ldots \ldots \ldots \ldots \ldots \ldots \ldots \ldots$

INTRODUCTION $\ldots \ldots \ldots \ldots \ldots \ldots \ldots \ldots \ldots \ldots \ldots \ldots \ldots \ldots \ldots \ldots \ldots \ldots \ldots \ldots \ldots$

\section{TAB SECTIONS}

INEL Record-to-Date Summary

INEL Record-to-Date Discharges and Solid Waste

INEL Record-to-Date Decay Summaries

Area Record-to-Date Decay Summaries

INEL 1993 Year-to-Date Summary

INEL Disposed Waste Decay Summary in Curies for 1993

Nuclide Summary in Curies for Airborne, Liquid, and Solid Waste

Argonne National Laboratory-West 1993 Detail Data

Central Facilities Area 1993 Detail Data

Chemical Processing Plant 1993 Detail Data

Decontamination and Decommissioning 1993 Detail Data

Naval Reactor Facility 1993 Detail Data

Power Burst Facility 1993 Detail Data

Radioactive Waste Management Complex 1993 Detail Data

Special Manufacturing Capability 1993 Detail Data

Special Power Excursion Reactor 1993 Detail Data

Test Area North 1993 Detail Data

Test Reactor Area 1993 Detail Data

Waste Experimental Reduction Facility 1993 Detail Data

Engineered Release Points 



\section{ACRONYMS AND ABBREVIATIONS}

\begin{tabular}{|c|c|c|c|}
\hline AlW & Large Ship Reactor at NRF & INEL & $\begin{array}{l}\text { Idaho National Engineering } \\
\text { Laboratory }\end{array}$ \\
\hline ALE & Argonne National Laboratory-East & & \\
\hline ANL & Argonne National Laboratory-West & L\&O & Lab and Office Building \\
\hline ARA & Auxiliary Reactor Area & LOF & Loss-of-Fluid Test \\
\hline ATR & Advanced Test Reactor & LOFT & Loss-of-Fluid Test \\
\hline & Battelle Columbus Laboratories & MAP & Mixed Activation Products \\
\hline & Bettis Atomic Power Laboratory & MFP & Mixed Fission Products \\
\hline & Brookhaven National Laboratory & MTR & Materials Test Reactor \\
\hline & $\begin{array}{l}\text { Brooknaven National Laooralory } \\
\text { Combustion Engineering General }\end{array}$ & MWSF & Mixed Waste Storage Facility \\
\hline & $\begin{array}{l}\text { Combustion Engineering General } \\
\text { Atomics }\end{array}$ & NRF & Naval Reactors Facility \\
\hline CFA & Central Facilities Area & NSA & North Storage Area \\
\hline CG & Concentration Guides (same as DCG) & NWCF & New Waste Calcining Facility \\
\hline CPP & $\begin{array}{l}\text { Chemical Processing Plant (also } \\
\text { referred to as ICPP) }\end{array}$ & $\begin{array}{l}\text { OFF } \\
\text { OTH }\end{array}$ & $\begin{array}{l}\text { Off-site } \\
\text { Other }\end{array}$ \\
\hline CTF & $\begin{array}{l}\text { Containment Test Facility } \\
\text { (LOFT became CTF on 10/1/86) } \\
\text { Calendar Year }\end{array}$ & $\begin{array}{l}\text { PBF } \\
\text { PER }\end{array}$ & $\begin{array}{l}\text { Power Burst Facility } \\
\text { Special Power Excursion Reactor } \\
\text { Test (also referred to as SPERT) }\end{array}$ \\
\hline $\mathrm{D} \& \mathrm{D}$ & $\begin{array}{l}\text { Decontamination and } \\
\text { Decommissioning }\end{array}$ & PREPP & $\begin{array}{l}\text { Process Reduction Experimental Pilot } \\
\text { Plant }\end{array}$ \\
\hline DCG & Derived Concentration Guides & $\mathrm{R} \& \mathrm{D}$ & Research and Development \\
\hline DOE & U.S. Department of Energy & RAL & Radiation Analysis Laboratory \\
\hline $\begin{array}{l}\text { EBR-II } \\
\text { ECF }\end{array}$ & $\begin{array}{l}\text { Experimental Breeder Reactor II } \\
\text { Expended Core Facility }\end{array}$ & RESL/IL & $\begin{array}{l}\text { Radiological and Environmental } \\
\text { Sciences Laboratory/Idaho } \\
\text { Operations }\end{array}$ \\
\hline EG\&G & EG\&G Idaho, Incorporated & RFO & $\begin{array}{l}\text { Rocky Flats Office (EG\&G Rocky } \\
\text { Flats, Colorado) }\end{array}$ \\
\hline $\begin{array}{l}\text { ETR } \\
\text { FASB }\end{array}$ & $\begin{array}{l}\text { Engineering Test Reactor } \\
\text { Fuel Assembly and Storage Building }\end{array}$ & RLWTF & $\begin{array}{l}\text { Radioactive Liquid Waste Treatment } \\
\text { Facility }\end{array}$ \\
\hline FAST & Fluorinel and Storage Facility & RWDS & Radioactive Waste Disposal System \\
\hline $\begin{array}{l}\text { FCF } \\
\text { FMF }\end{array}$ & $\begin{array}{l}\text { Fuel Cycle Facility } \\
\text { Fuel Manufacturing Facility }\end{array}$ & RWMC & $\begin{array}{l}\text { Radioactive Waste Management } \\
\text { Complex }\end{array}$ \\
\hline HFEF & Hot Fuels Examination Facility & RWMIS & $\begin{array}{l}\text { Radioactive Waste Management } \\
\text { Information System }\end{array}$ \\
\hline HFEF-N & $\begin{array}{l}\text { Hot Fuels Examination } \\
\text { Facility-North }\end{array}$ & SiW & Submarine Prototype at NRF \\
\hline HFEF-S & $\begin{array}{l}\text { Hot Fuels Examination } \\
\text { Facility-South }\end{array}$ & $\begin{array}{l}\text { S5G } \\
\text { SDA }\end{array}$ & $\begin{array}{l}\text { Submarine Prototype at NRF } \\
\text { Subsurface Disposal Area }\end{array}$ \\
\hline ICPP & $\begin{array}{l}\text { Idaho Chemical Processing Plant } \\
\text { (also referred to as CPP) }\end{array}$ & $\begin{array}{l}\text { SL-1 } \\
\text { SMC }\end{array}$ & $\begin{array}{l}\text { Stationary Low Power Reactor No. } 1 \\
\text { Specific Manufacturing Capability }\end{array}$ \\
\hline ILTSF & $\begin{array}{l}\text { Intermediate-Level Transuranic } \\
\text { Storage Facility }\end{array}$ & SPERT & $\begin{array}{l}\text { Special Power Excursion } \\
\text { Reactor Test (also referred to as PER) }\end{array}$ \\
\hline
\end{tabular}


SPF Sodium Process Facility

SWEPP Stored Waste Examination Pilot Plant

TAN Test Area North

TRA Test Reactor Area

TREAT Transient Reactor Test Facility

TSA Transuranic Storage Area

TSF Test Support Facility

WCF Waste Calcining Facility
WER Waste Experimental Reduction Facility (also referred to as WERF)

WERF Waste Experimental Reduction Facility (also referred to as WER)

WINCO Westinghouse Idaho Nuclear Company

WMC Waste Management Complex (also referred to as RWMC)

WVRF Waste Volume Reduction Facility

ZPPR Zero Power Plutonium Reactor 


\section{CONVERSION FACTORS}

\begin{tabular}{llr}
\multicolumn{1}{c}{ To Convert } & \multicolumn{1}{c}{ Into } & Multiply by \\
\cline { 2 - 3 } Cubic yards & Cubic meters & 0.7646 \\
Cubic meters & Cubic feet & 35.3140 \\
Liters & Gallons & 0.2642 \\
Kilograms & (U.S. liquid) & \\
& Pounds & 2.2046 \\
& DEFINITIONS &
\end{tabular}

Curie-A unit of radioactivity, defined as that quantity of any radioactive nuclide which has 3.7E+10 disintegrations per second. (Abbreviated $\mathrm{Ci}$.)

Exponential notation-Numbers in this document are expressed as either whole numbers or in exponential notation form. For example: $1,000,000=1 \times 10^{6}=1 \mathrm{E}+06$. 



\section{RADIOACTIVE WASTE MANAGEMENT INFORMATION FOR 1993 AND RECORD-TO-DATE}

\section{INTRODUCTION}

This document summarizes radioactive waste data records for the Idaho National Engineering Laboratory (INEL) compiled since 1952, and provides detailed data for calendar year (CY) 1993. A computerized Radioactive Waste Management Information System (RWMIS) has been used at the INEL since January 1971.

Several improvements to the data base system and data reporting have taken place since data collection was computerized. These improvements include:

- In 1982, the data base language was converted from COBOL to NOMAD, a fourthgeneration data base language. NOMAD has many statistical analysis features that can be applied to the data, and allows for modifying data reporting requirements with minimal impact on the data base.

- In January 1986 , solid waste data reporting requirements changed from a per-shipment basis to a per-container basis. A shipment could consist of 1 to 70 containers. By changing to a per-container basis, solid waste on the INEL is tracked more accurately. It is now possible to pinpoint the exact location of a container and to determine its waste classification.

- In February 1988, waste generators were given the ability to provide the analytical uncertainty, at one sigma, for the reported nuclides of air and liquid effluent releases.

- In May 1988, an RWMIS Graph Download System was made available to waste generators who provide data directly into the data base. This system serves two purposes: (a) it provides an easy way for waste generators to copy their data from the mainframe to a personal computer data base file, and (b) it provides a menu-driven selection process for personal computer data bases with graphing capabilities for trending and other analyses.

U.S. Department of Energy (DOE) contractors routinely report information on airborne and liquid radioactive effluents and solid radioactive waste that is stored, disposed, and sent to the INEL for reduction. Types of information include volume, radioactivity, isotopic identity, origin, and decay status. This system serves as the official repository for this data and provides reports for all types of radioactive effluents and solid waste disposed, sent for reduction, or stored at the INEL.

Graphics were added to the report in 1987 to improve the data presentation. In the Decay Summaries section, those areas having no identified nuclides, or only $<1$ curie of identified nuclides, are not presented with graphics. The area-specific solid low-level waste graphs in this report depict waste in four categories:

1. Direct Disposal-waste that is sent directly to the Radioactive Waste Management Complex for disposal with no volume reduction efforts applied.

2. Compaction-compactible waste that is sent to Waste Experimental Reduction Facility (WERF) to be compacted.

3. Metal Sizing-metallic waste that is sent to WERF for volume reduction.

4. Incineration-combustible waste sent to WERF for incineration.

In 1990, additional reports and graphics were added to better represent previously summarized data. These reports and graphics included a detailed list of generators who ship waste to WERF and details on stored waste by generator.

The RWMIS is continually undergoing review and enhancement. Comments on the system are 
encouraged. This report is updated annually to incorporate waste management data for the current year and to reflect changes from previous annual reports. These changes are made to more accurately reflect the current status of waste operations at the INEL.

Annual and special RWMIS reports have been useful to various levels of management in appraising their radioactive waste programs. Annual reports provide a summary by type and producer of waste. Special reports include radioactive decay from time of disposal to specified future dates. The volume, weight, and curie content in all RWMIS reports are expressed in exponential notation.

A comparison of the annual mean average released nuclides concentrations and the Derived Concentration Guides (DCG) limits are included in the detailed RWMIS. The concentration of liquid releases is compared directly to the reference DCG limits for drinking water. It must be noted that the DCG liquid release limits are for protecting the public from ingesting radiationcontaminated water. INEL liquid releases flow to areas inaccessible to the general public. The calculated concentration of airborne releases as dispersed to the INEL boundary is compared to the reference airborne DCG limits. The reference DCG values are given in DOE Order 5400.5, "Radiation Protection of the Public and the Environment," February 1990. The right-hand column of many RWMIS reports indicate total and average values. Total values include the total content in curies for each radionuclide released during the year. Average values are the annual mean concentrations of radionuclides.

The RWMIS provides readily available information that permits ongoing evaluation of INEL waste management activities, including compliance with DOE regulations and those of other Federal and State agencies. The RWMIS continues to be a valuable asset to the overall management effort at the INEL and continues to alert the DOE Idaho Operations Office (DOE-ID) of major trends and potential problem areas.

\section{Reports Included in Document}

Eight report categories are included in this document. A brief description of each are below.

INEL Record-to-Date Summary-This report summarizes the volume and curies of all effluents and solid waste on the INEL by CY for the period 1984 to 1993 and a cumulative total for 1952 to 1983.

INEL Record-to-Date Discharges and Solid Waste-These reports summarize the volume and curies of all discharges and solid waste on the INEL by discharge/waste type for each area generating waste by $\mathrm{CY}$ for the period 1984 to 1993 and a cumulative total for 1952 to 1983.

INEL Record-to-Date Decay Summaries-These reports summarize both identified and unidentified curies by waste type on the INEL by CY for the period 1984 to 1993 and a cumulative total for 1962 to 1983 . The curie amount is adjusted to allow for radioactive decay through the last day of the current year.

Area Record-to-Date Decay Summaries- These reports summarize the identified and unidentified curies by area and waste type by CY for the period 1984 to 1993 and a cumulative total for 1962 to 1983 . The curie amount is adjusted to allow for radioactive decay through the last day of the current year and then 10 years later.

INEL 1993 Year-to-Date Summary-This report summarizes, by facility, the amounts of radioactivity generated at INEL facilities or at offsite facilities and shipped to the INEL. Liquid and airborne waste that is released is identified, and quantities of solid wastes stored, disposed, or received for reduction are given. The volume of the media containing the radioactivity is also included.

INEL Disposed Waste Decay Summary in Curies for 1993-This report summarizes the total curies of airborne, liquid, and solid waste on the INEL for the current year and then corrects the amount of the total curies for radioactive decay, by year, for 7 years. 
Nuclide Summary in Curies for Airborne, Liquid, and Solid Waste-These reports summarize the annual curie values, by nuclide, for each area reporting effluents or solid waste on the INEL.
Area 1993 Detail Data-These reports provide monthly data on the volumes and radionuclides in the airborne, liquid, and solid waste generated by INEL areas and by non-INEL areas shipping solid waste to the INEL. The year-to-date totals are also shown. 

INEL Record-to-Date Summary

INEL Record-to-Date Summary $\ldots \ldots \ldots \ldots \ldots \ldots \ldots \ldots \ldots \ldots \ldots \ldots \ldots \ldots \ldots \ldots \ldots$ INEL-3

INEL Record-to-Date Summary Bar Graphs $\ldots \ldots \ldots \ldots \ldots \ldots \ldots \ldots$ INEL-4 



\begin{tabular}{|c|c|c|c|c|c|c|c|c|c|c|c|c|}
\hline TYPENYEAR & $1952-1983$ & 1984 & 1985 & 1986 & 1987 & 1988 & 1989 & 1990 & 1991 & 1992 & 1993 & TOTAL \\
\hline \multicolumn{13}{|l|}{ ATRBOWWE } \\
\hline $\begin{array}{l}\text { VOLUME } \\
\text { CURTES }\end{array}$ & $\begin{array}{r}128622 \\
13045298\end{array}$ & $\begin{array}{r}12043 \\
4837\end{array}$ & $\begin{array}{r}9636 \\
77262\end{array}$ & $\begin{array}{l}12289 \\
14518\end{array}$ & $\begin{array}{r}12220 \\
254948\end{array}$ & $\begin{array}{r}13388 \\
173935\end{array}$ & $\begin{array}{l}13514 \\
22370\end{array}$ & $\begin{array}{l}13450 \\
24459\end{array}$ & $\begin{array}{l}12963 \\
63879\end{array}$ & $\begin{array}{l}12715 \\
24179\end{array}$ & $\begin{array}{r}13150 \\
2719\end{array}$ & $\begin{array}{r}253990 \\
13708402\end{array}$ \\
\hline \multicolumn{13}{|l|}{ LIQUID } \\
\hline $\begin{array}{l}\text { VOLLME } \\
\text { CURIES }\end{array}$ & $\begin{array}{l}69494 \\
73747\end{array}$ & $\begin{array}{r}2749 \\
191\end{array}$ & $\begin{array}{r}2744 \\
667\end{array}$ & $\begin{array}{r}2529 \\
346\end{array}$ & $\begin{array}{r}2536 \\
357\end{array}$ & $\begin{array}{r}2357 \\
270\end{array}$ & $\begin{array}{r}1922 \\
137\end{array}$ & $\begin{array}{r}2642 \\
189\end{array}$ & $\begin{array}{r}2490 \\
170\end{array}$ & $\begin{array}{r}2799 \\
187\end{array}$ & $\begin{array}{r}2659 \\
130\end{array}$ & $\begin{array}{l}94920 \\
76391\end{array}$ \\
\hline \multicolumn{13}{|c|}{ SOLID DISPOSED } \\
\hline $\begin{array}{l}\text { VOLUME } \\
\text { CURIES }\end{array}$ & $\begin{array}{r}187602 \\
9713010\end{array}$ & $\begin{array}{r}3906 \\
486043\end{array}$ & $\begin{array}{r}3140 \\
271435\end{array}$ & $\begin{array}{r}3437 \\
95084\end{array}$ & $\begin{array}{r}2969 \\
247521\end{array}$ & $\begin{array}{r}2045 \\
149742\end{array}$ & $\begin{array}{r}1364 \\
590066\end{array}$ & $\begin{array}{r}1762 \\
207543\end{array}$ & $\begin{array}{r}1272 \\
187569\end{array}$ & $\begin{array}{r}844 \\
143951\end{array}$ & $\begin{array}{r}852 \\
429655\end{array}$ & $\begin{array}{r}209193 \\
12521618\end{array}$ \\
\hline $\begin{array}{c}\text { SOLID STORED } \\
\text { VOLUHE } \\
\text { CURIES }\end{array}$ & $\begin{array}{r}53804 \\
82423504\end{array}$ & $\begin{array}{r}3444 \\
2817475\end{array}$ & $\begin{array}{r}3376 \\
55521\end{array}$ & $\begin{array}{r}3128 \\
55109\end{array}$ & $\begin{array}{r}2217 \\
3400798\end{array}$ & $\begin{array}{r}1395 \\
860 \times 486\end{array}$ & $\begin{array}{r}880 \\
51218\end{array}$ & $\begin{array}{r}41 \\
4607\end{array}$ & $\begin{array}{r}181 \\
1603039\end{array}$ & 39741 & $\begin{array}{r}203 \\
1142363\end{array}$ & $\begin{array}{r}68668 \\
98202761\end{array}$ \\
\hline $\begin{array}{l}\text { SOLID TO WER } \\
\text { VOLUME } \\
\text { CURIES }\end{array}$ & F FOR RED & $<1^{78}$ & $\begin{array}{r}1275 \\
1\end{array}$ & $\begin{array}{r}2458 \\
2\end{array}$ & $\begin{array}{r}2529 \\
5\end{array}$ & $\begin{array}{r}2967 \\
6\end{array}$ & $\begin{array}{r}2663 \\
6\end{array}$ & $\begin{array}{r}3179 \\
13\end{array}$ & $\begin{array}{r}2066 \\
17\end{array}$ & $\begin{array}{r}1142 \\
19\end{array}$ & $\begin{array}{r}446 \\
2\end{array}$ & $\begin{array}{r}18802 \\
71\end{array}$ \\
\hline
\end{tabular}




\section{INEL Record-toDate Summary CY 1993}
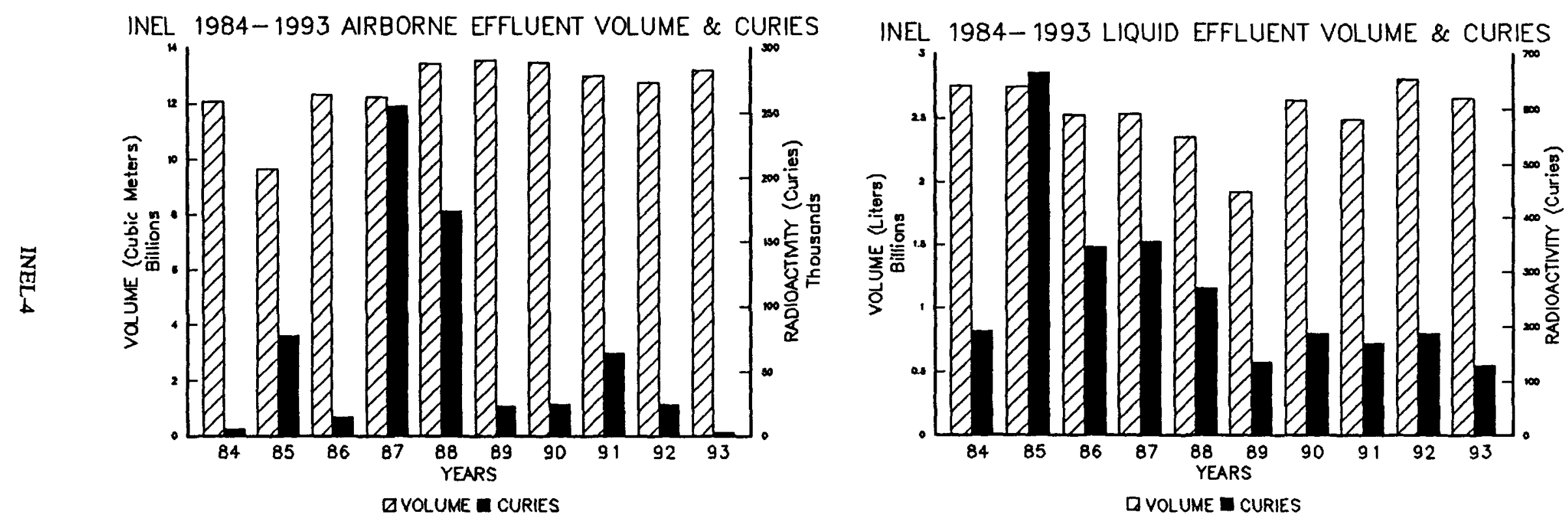


\section{INEL Record-to-Date Summary CY-1993}

INEL 1984-1993 SOLID DISPOSED WASTE VOLUME \& CURIES
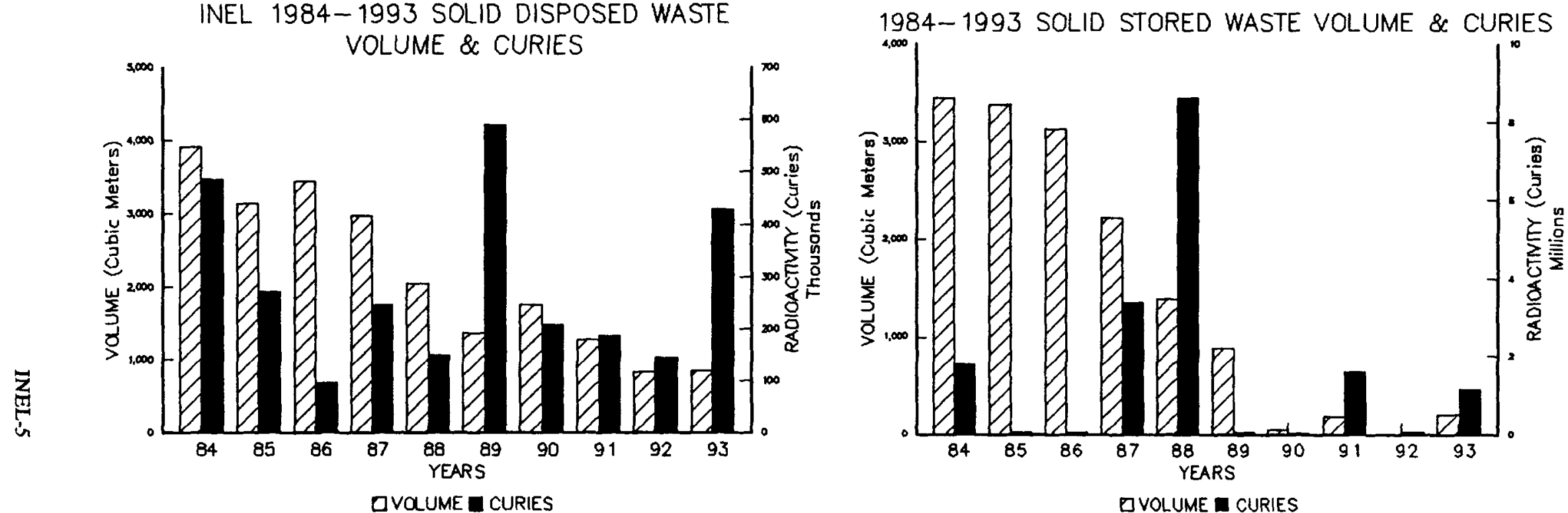

INEL 1984-1993 SOLID WASTE SENT TO WERF FOR VOLUME REDUCTION*

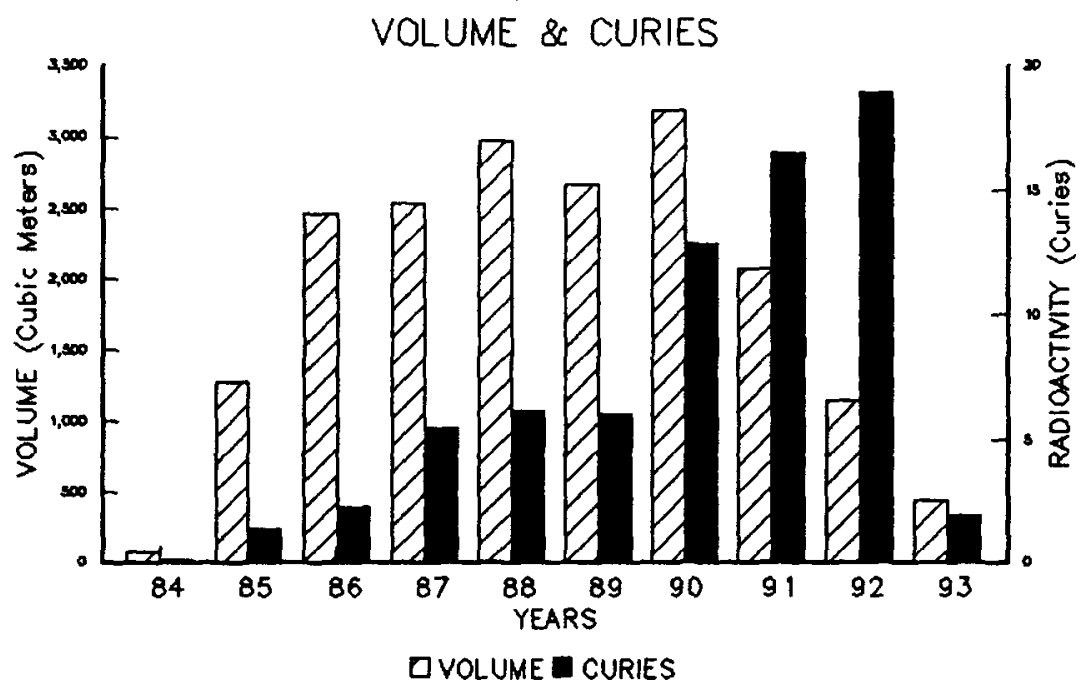





\section{INEL Record-to-Date Discharges and Solid Waste}

INEL Radioactive Airborne Waste Discharges, Record-to-Date $\ldots \ldots \ldots \ldots \ldots$ INEL-9 INEL Radioactive Airborne Waste Discharges Record-to-Date Bar Graphs .. INEL-11

INEL Radioactive Liquid Waste Discharges, Record-to-Date .............. INEL-15 INEL Radioactive Liquid Waste Discharge Record-to-Date Bar Graphs ..... INEL-17 INEL Solid Record-to-Date Summary, Disposed Waste .................. INEL-20 INEL Solid Record-to-Date Summary Disposed Waste Bar Graphs ........ INEL-23 INEL Solid Record-to-Date Summary, Stored Waste $\ldots \ldots \ldots \ldots \ldots \ldots \ldots \ldots$ INEL-28

INEL Solid Record-to-Date Summary Stored Waste Bar Graphs .......... INEL-29 INEL Solid Record-to-Date Summary, ANL Storage Waste $\ldots \ldots \ldots \ldots \ldots \ldots$ INEL-30 INEL Solid Record-to-Date Summary ANL Storage Waste Bar Graph ....... INEL-31 INEL Solid Record-to-Date Summary, ILTSF Waste $\ldots \ldots \ldots \ldots \ldots \ldots \ldots \ldots$ INEL-32

INEL Solid Record-to-Date Summary ILTSF Waste Bar Graphs $\ldots \ldots \ldots \ldots$ INEL-33 INEL Solid Record-to-Date Summary, TSA Waste $\ldots \ldots \ldots \ldots \ldots \ldots \ldots \ldots$ INEL-34

INEL Solid Record-to-Date Summary TSA Waste Bar Graphs ........... INEL-36 CPP High-Level Liquid/Solid Waste Storage, Record-to-Date .............. INEL-39

CPP High-Level Liquid/Solid Waste Storage Record-to-Date Bar Graphs ... INEL-40 INEL Solid Record-to-Date Summary, WERF Waste ................... INEL-41

INEL Solid Record-to-Date Summary WERF Waste Bar Graphs ......... INEL-43 

RECORD-TO-DATE

\begin{tabular}{|c|c|c|c|c|c|c|c|c|c|c|c|c|c|}
\hline AREA & NEAR & $1952-1983$ & 1984 & 1985 & 1986 & 1987 & 1988 & 1989 & 1990 & 1991 & 1992 & 1993 & TOTAL \\
\hline \multicolumn{14}{|l|}{ ANL } \\
\hline & $\begin{array}{l}\text { Volune } \\
\text { curIES }\end{array}$ & $\begin{array}{l}29739 \\
45275\end{array}$ & $\begin{array}{r}2409 \\
250\end{array}$ & $\begin{array}{r}2445 \\
916\end{array}$ & $\begin{array}{r}2406 \\
932\end{array}$ & $\begin{array}{r}2532 \\
889\end{array}$ & $\begin{array}{r}2554 \\
618\end{array}$ & $\begin{array}{r}2540 \\
686\end{array}$ & $\begin{array}{r}2535 \\
716\end{array}$ & $\begin{array}{r}2542 \\
551\end{array}$ & $\begin{array}{r}2542 \\
739\end{array}$ & $\begin{array}{l}2522 \\
1160\end{array}$ & $\begin{array}{l}54766 \\
52733\end{array}$ \\
\hline \multicolumn{14}{|l|}{ ARA } \\
\hline & $\begin{array}{l}\text { VOLUNE } \\
\text { CURIES }\end{array}$ & $\begin{array}{r}352 \\
<1\end{array}$ & $\begin{array}{l}34 \\
<1\end{array}$ & $\begin{array}{l}47 \\
<1\end{array}$ & $\begin{array}{l}47 \\
<1\end{array}$ & $\begin{array}{l}47 \\
<1\end{array}$ & $\begin{array}{l}32 \\
<1\end{array}$ & & & & & & $\begin{array}{r}560 \\
<1\end{array}$ \\
\hline \multicolumn{14}{|l|}{ CFA } \\
\hline & Volune & 427 & 393 & 240 & 217 & 77 & 59 & 58 & 58 & 58 & 58 & 34 & 1481 \\
\hline & CURIES & $<1$ & $<1$ & $<1$ & $<1$ & $<1$ & $<1$ & $<1$ & $<1$ & $<1$ & $<1$ & $<1$ & $<1$ \\
\hline \multicolumn{14}{|l|}{ CPP } \\
\hline & $\begin{array}{l}\text { VOLUNE } \\
\text { CURIES }\end{array}$ & $\begin{array}{r}35538 \\
7524492\end{array}$ & $\begin{array}{r}5201 \\
398\end{array}$ & $\begin{array}{r}3332 \\
64279\end{array}$ & $\begin{array}{r}3923 \\
10845\end{array}$ & $\begin{array}{r}3911 \\
250918\end{array}$ & $\begin{array}{r}3893 \\
170708\end{array}$ & $\begin{array}{r}3794 \\
20002\end{array}$ & $\begin{array}{r}3827 \\
20002\end{array}$ & $\begin{array}{r}3916 \\
60043\end{array}$ & $\begin{array}{r}3895 \\
20000\end{array}$ & $\begin{array}{r}4244 \\
62\end{array}$ & $\begin{array}{r}75476 \\
8141749\end{array}$ \\
\hline \multicolumn{14}{|l|}{ CTF } \\
\hline & $\begin{array}{l}\text { Volune } \\
\text { CurIES }\end{array}$ & & & & $\begin{array}{r}155 \\
<1\end{array}$ & 63 & & & & & & & $\begin{array}{r}218 \\
<1\end{array}$ \\
\hline \multicolumn{14}{|l|}{ LOF } \\
\hline & $\begin{array}{l}\text { VOLUnE } \\
\text { CURIES }\end{array}$ & $\begin{array}{r}528 \\
4\end{array}$ & $\begin{array}{r}129 \\
27\end{array}$ & $\begin{array}{r}165 \\
8780\end{array}$ & & & & & & & & & $\begin{array}{r}822 \\
8810\end{array}$ \\
\hline \multicolumn{14}{|c|}{ NRF } \\
\hline & Volune & 25407 & 2114 & 2187 & 2722 & 1630 & 1745 & 2377 & 2151 & 1795 & 1654 & 2646 & 45428 \\
\hline & cuRres & 35 & $<1$ & $<1$ & $<1$ & $<1$ & $<1$ & $<1$ & $<1$ & $<1$ & $<1$ & 1 & 39 \\
\hline \multicolumn{14}{|l|}{ Paf } \\
\hline & Volune & 845 & 61 & 62 & 57 & 58 & 60 & 60 & 62 & 63 & 58 & 52 & 1436 \\
\hline & CURIES & $<1$ & $<1$ & $<1$ & $<1$ & $<1$ & $<1$ & $<1$ & $<1$ & $<1$ & $<1$ & $<1$ & $<2$ \\
\hline \multicolumn{14}{|l|}{ PER } \\
\hline & $\begin{array}{l}\text { VOLUME } \\
\text { CURIES }\end{array}$ & $\begin{array}{r}* * \\
6\end{array}$ & & & & & & & & & & & $x$ \\
\hline \multicolumn{14}{|l|}{ six } \\
\hline & volune & & & 8 & 1424 & 1962 & 3046 & 2812 & 3031 & 3122 & 3047 & 3141 & 21593 \\
\hline & CURIES & & & $<1$ & $<1$ & $<1$ & $<1$ & $<1$ & $<1$ & $<1$ & $<1$ & $<1$ & $<1$ \\
\hline \multirow[t]{3}{*}{ TAN } & & . & & & & & & & & & & & \\
\hline & volure & 14331 & 651 & 173 & 332 & 660 & 645 & 632 & 492 & 191 & 182 & 191 & 18480 \\
\hline & CURIES & 53725 & $<1$ & $<1$ & $<1$ & $<1$ & $<1$ & $<1$ & $<1$ & $<1$ & $<1$ & $<1$ & 53725 \\
\hline \multirow[t]{2}{*}{ TRA } & & & & & & & & & & & & & \\
\hline & $\begin{array}{l}\text { VOLUYE } \\
\text { CURIES }\end{array}$ & $\begin{array}{r}21455 \\
5421763\end{array}$ & $\begin{array}{l}1051 \\
4162\end{array}$ & $\begin{array}{l}1076 \\
3288\end{array}$ & $\begin{array}{l}1089 \\
2741\end{array}$ & $\begin{array}{l}1199 \\
3140\end{array}$ & $\begin{array}{l}1190 \\
2609\end{array}$ & $\begin{array}{l}1069 \\
1682\end{array}$ & $\begin{array}{l}1130 \\
3741\end{array}$ & $\begin{array}{l}1140 \\
3284\end{array}$ & $\begin{array}{l}1130 \\
3439\end{array}$ & $\begin{array}{l}1155 \\
1495\end{array}$ & $\begin{array}{r}32684 \\
5451343\end{array}$ \\
\hline
\end{tabular}


RECORD-TO-DATE

\begin{tabular}{|c|c|c|c|c|c|c|c|c|c|c|c|c|}
\hline ARENYEAR & $1952-1983$ & 1984 & 1985 & 1986 & 1987 & 1988 & 1989 & 1990 & 1991 & 1992 & 1993 & TOTAL \\
\hline MER & & & & & & & & & & & & \\
\hline $\begin{array}{l}\text { VOLUME } \\
\text { CURIES }\end{array}$ & & & & $\begin{array}{l}16 \\
<1\end{array}$ & $\begin{array}{l}72 \\
<1\end{array}$ & $\begin{array}{r}161 \\
<1\end{array}$ & $\begin{array}{r}156 \\
<1\end{array}$ & $\begin{array}{r}164 \\
<1\end{array}$ & $\begin{array}{r}137 \\
<1\end{array}$ & $\begin{array}{r}135 \\
<1\end{array}$ & 155 & $\begin{array}{r}996 \\
<1\end{array}$ \\
\hline nexc & & & & & & & & & & & & \\
\hline $\begin{array}{l}\text { VOLUNE } \\
\text { CURIES }\end{array}$ & & & & & $<1$ & $\begin{array}{r}4 \\
<1\end{array}$ & $\begin{array}{l}15 \\
<1\end{array}$ & & & $\begin{array}{l}14 \\
<1\end{array}$ & $\begin{array}{l}10 \\
<1\end{array}$ & $\begin{array}{l}52 \\
<1\end{array}$ \\
\hline TOTAL & & & & & & & & & & & & \\
\hline $\begin{array}{l}\text { VOLURE } \\
\text { CURTES }\end{array}$ & $\begin{array}{r}128622 \\
13045298\end{array}$ & $\begin{array}{r}12043 \\
4837\end{array}$ & $\begin{array}{r}9636 \\
77262\end{array}$ & $\begin{array}{l}12289 \\
14518\end{array}$ & $\begin{array}{r}12220 \\
254948\end{array}$ & $\begin{array}{r}13388 \\
173935\end{array}$ & $\begin{array}{l}13514 \\
22370\end{array}$ & $\begin{array}{l}13450 \\
24459\end{array}$ & $\begin{array}{l}12963 \\
63879\end{array}$ & $\begin{array}{l}12715 \\
24179\end{array}$ & $\begin{array}{r}13150 \\
2719\end{array}$ & $\begin{array}{r}253990 \\
13708402\end{array}$ \\
\hline
\end{tabular}

VOLURE IN MLLLIONS OF CUBIC METERS

** NOT AVAILABLE

DETAILS MAY NOT ADD UP tO TOTALS BECAUSE OF RONDING 
INEL Radioactive Airborne Waste Discharges Record-to-Date
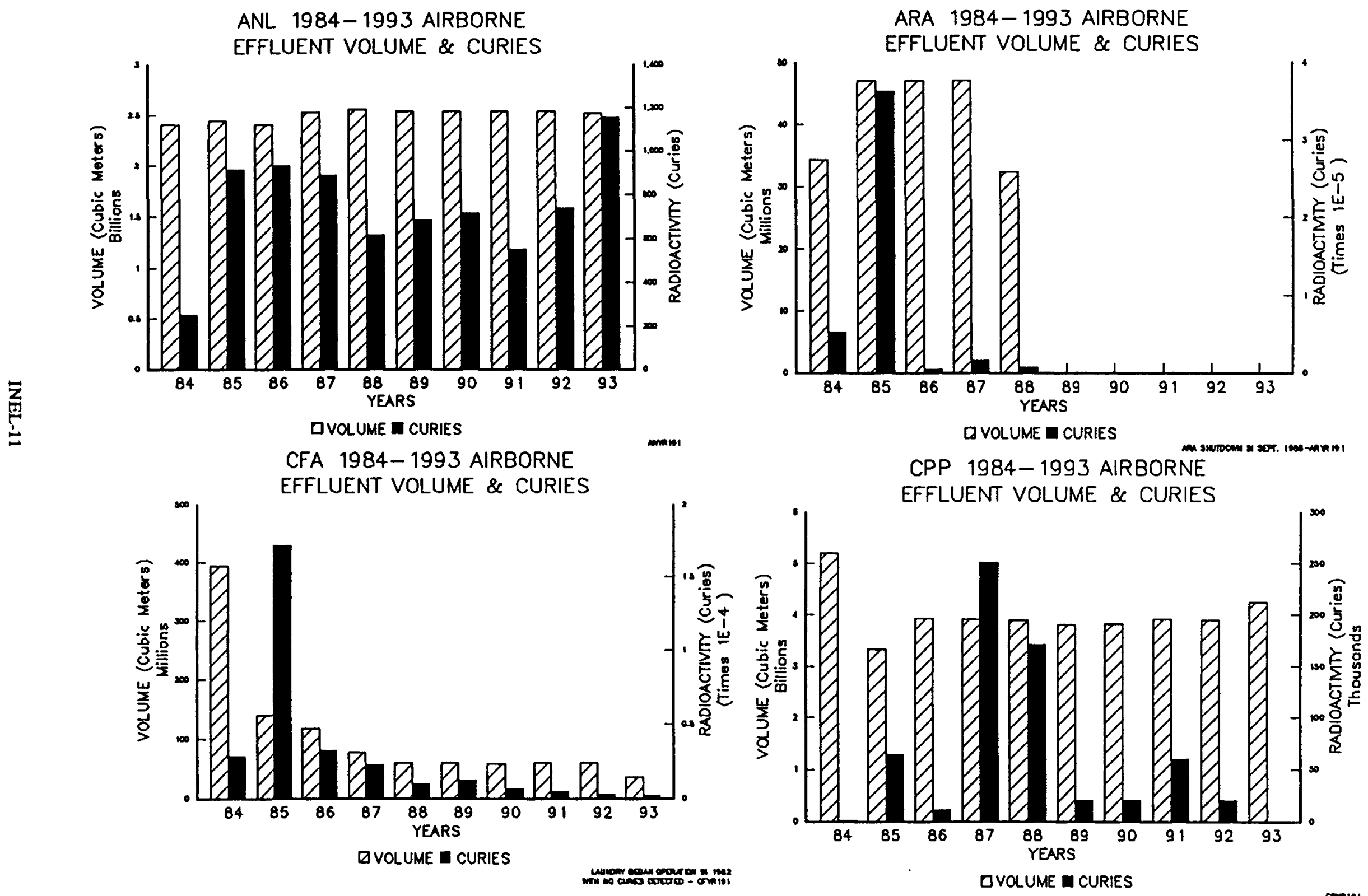
INEL Radioactive Aiborne Waste Discharges Record-to-Date
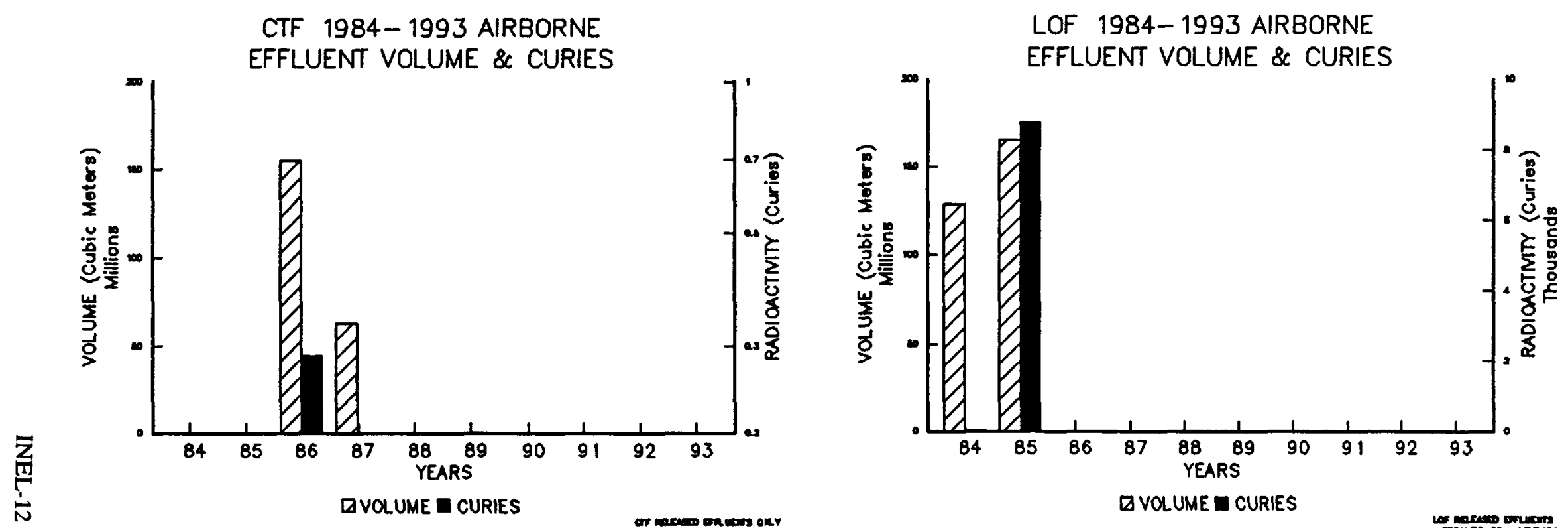

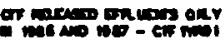

NRF 1984-1993 AIRBORNE

EFFLUENT VOLUME \& CURIES

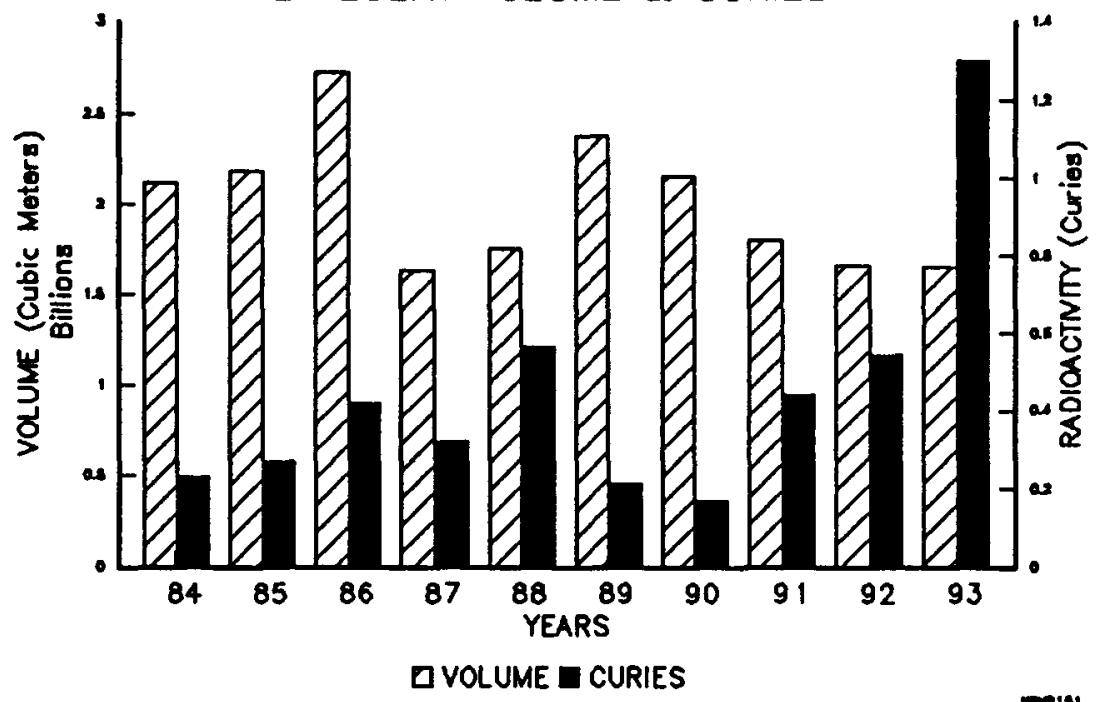


INEL Radioactive Airborne Waste Discharges Record-to-Date
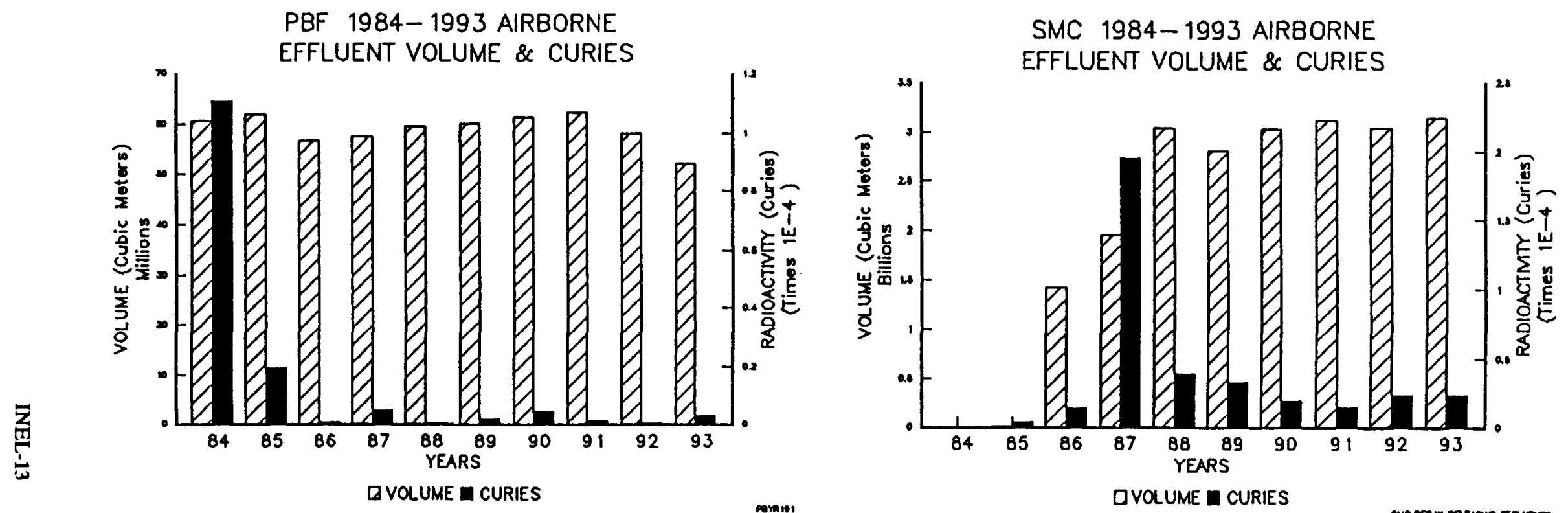

TAN 1984-1993 AIRBORNE

DVOLUME CURIES

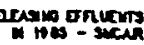
EFFLUENT VOLUME \& CURIES

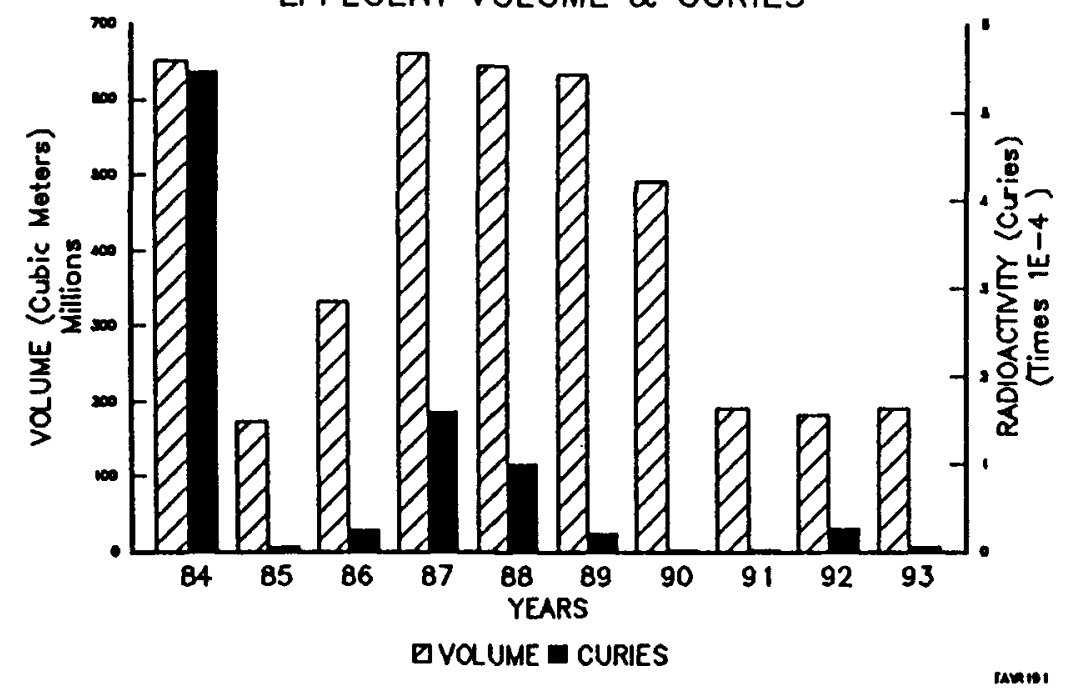


INEL Radioactive Airborne Waste Discharges Record-to-Date
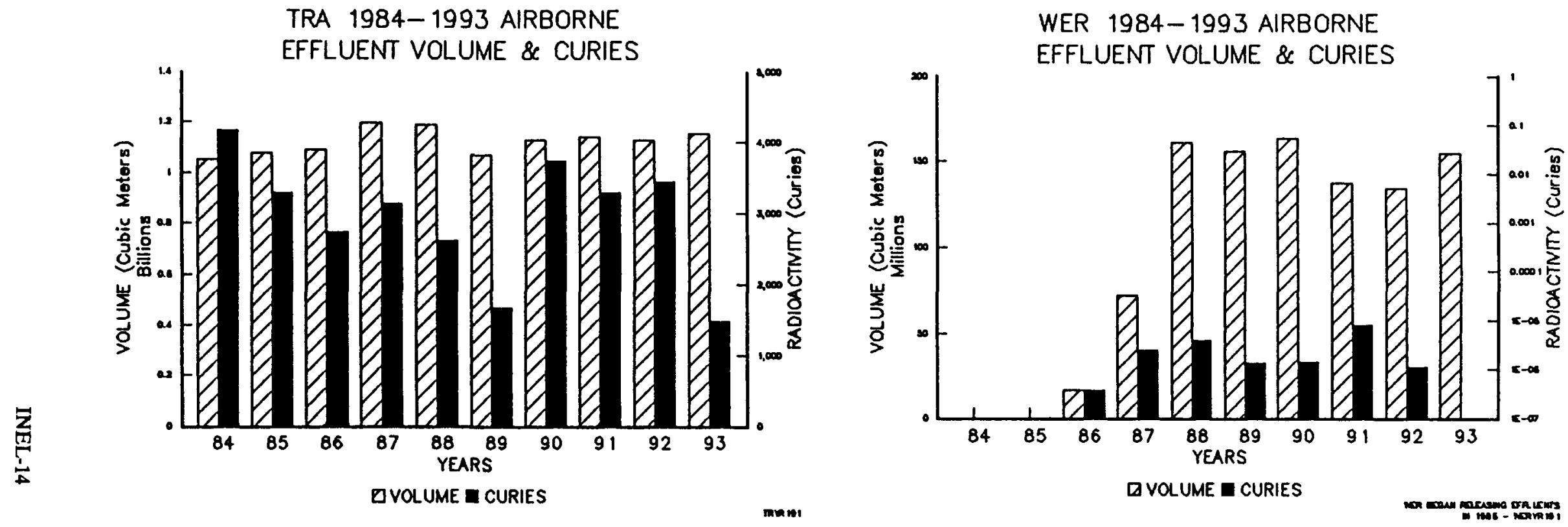

WMC 1984-1993 AIRBORNE

EFFLUENT VOLUME \& CURIES

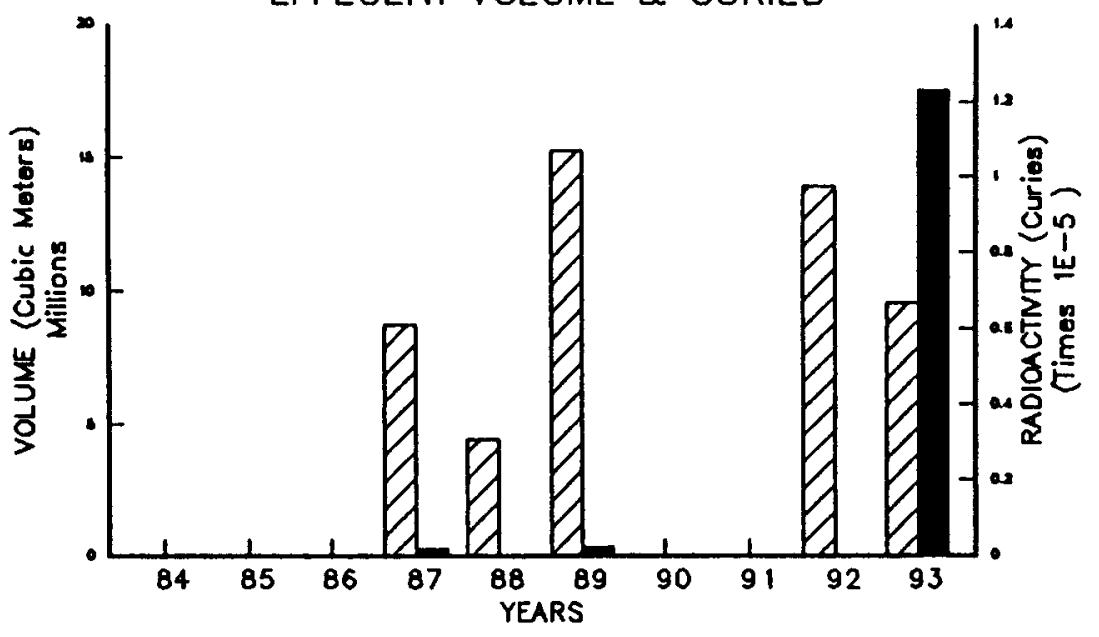

DVOLUME — CURIES 
RECORD-TO-DATE

\begin{tabular}{|c|c|c|c|c|c|c|c|c|c|c|c|c|c|}
\hline AREN & MEAR & $1952-1983$ & 1984 & 1985 & 1986 & 1987 & 1988 & 1989 & 1990 & 1991 & 1992 & 1993 & TOTAL \\
\hline \multicolumn{14}{|l|}{ MNL } \\
\hline & $\begin{array}{l}\text { VOLUME } \\
\text { CURIES }\end{array}$ & $\begin{array}{l}64 \\
21\end{array}$ & $\begin{array}{r}2 \\
<1\end{array}$ & $\begin{array}{r}2 \\
<1\end{array}$ & $\begin{array}{l}2 \\
<1\end{array}$ & $\begin{array}{r}2 \\
<1\end{array}$ & $\begin{array}{r}2 \\
<1\end{array}$ & $\begin{array}{l}1 \\
<1\end{array}$ & $\begin{array}{r}2 \\
<1\end{array}$ & $\begin{array}{l}2 \\
<2\end{array}$ & $\begin{array}{l}1 \\
<1\end{array}$ & $\begin{array}{r}5 \\
<1\end{array}$ & $\begin{array}{l}86 \\
23\end{array}$ \\
\hline \multicolumn{14}{|l|}{ ARA } \\
\hline & $\begin{array}{l}\text { VOLUME } \\
\text { CURTES }\end{array}$ & 48 & 13 & & $<1$ & 2 & 1 & & & & & & 65 \\
\hline \multicolumn{14}{|l|}{ CFA } \\
\hline & $\begin{array}{l}\text { VOLUAE } \\
\text { CURIES }\end{array}$ & $\begin{array}{r}4402 \\
39\end{array}$ & $\begin{array}{r}176 \\
5\end{array}$ & $\begin{array}{r}166 \\
4\end{array}$ & $\begin{array}{r}104 \\
3\end{array}$ & $\begin{array}{r}89 \\
2\end{array}$ & $\begin{array}{r}62 \\
1\end{array}$ & $\begin{array}{r}117 \\
2\end{array}$ & $\begin{array}{r}154 \\
2\end{array}$ & $\begin{array}{r}192 \\
3\end{array}$ & $\begin{array}{r}160 \\
3\end{array}$ & $\begin{array}{l}44 \\
<1\end{array}$ & $\begin{array}{r}5668 \\
65\end{array}$ \\
\hline \multicolumn{14}{|l|}{ CPP } \\
\hline & $\begin{array}{l}\text { VOLUME } \\
\text { CURIES }\end{array}$ & $\begin{array}{l}40551 \\
21285\end{array}$ & $\begin{array}{r}2092 \\
13\end{array}$ & $\begin{array}{r}2020 \\
393\end{array}$ & $\begin{array}{r}2183 \\
251\end{array}$ & $\begin{array}{r}2268 \\
216\end{array}$ & $\begin{array}{r}2140 \\
89\end{array}$ & $\begin{array}{r}1664 \\
<1\end{array}$ & $\begin{array}{r}2357 \\
<1\end{array}$ & $\begin{array}{r}2107 \\
2\end{array}$ & $\begin{array}{r}2433 \\
<1\end{array}$ & $\begin{array}{r}2515 \\
<1\end{array}$ & $\begin{array}{l}62331 \\
22250\end{array}$ \\
\hline \multicolumn{14}{|l|}{ CTF } \\
\hline & $\begin{array}{l}\text { VOLUNE } \\
\text { CURIES }\end{array}$ & & & & $\begin{array}{l}71 \\
<1\end{array}$ & 22 & & & & & & & $\begin{array}{l}93 \\
<1\end{array}$ \\
\hline \multicolumn{14}{|l|}{ LOF } \\
\hline & VOLUME & 2061 & 325 & 427 & & & & & & & & & 2813 \\
\hline & CURIES & $<1$ & $<1$ & $<1$ & & & & & & & & & $<1$ \\
\hline \multicolumn{14}{|l|}{ NRF } \\
\hline & VOLUAE & 1569 & & & & & & & & & $<1$ & & 1569 \\
\hline & CURIES & 349 & & & & & & & & & $<1$ & & 349 \\
\hline \multicolumn{14}{|l|}{ PBF } \\
\hline & VOLLME & 9 & & & & & & & & & & & 9 \\
\hline & CURIES & $<1$ & & & & & & & & & & & $<1$ \\
\hline \multicolumn{14}{|l|}{ PER } \\
\hline & $\begin{array}{l}\text { VOLUAE } \\
\text { CURIES }\end{array}$ & $\begin{array}{r}76 \\
155\end{array}$ & & & & & & & & & & & $\begin{array}{r}76 \\
255\end{array}$ \\
\hline \multirow[t]{3}{*}{ TAN } & & & & & & & & & & & & & \\
\hline & VOLUME & 1991 & 79 & 51 & 73 & 82 & 81 & 67 & 58 & 90 & 126 & 24 & 2712 \\
\hline & CURIES & 58 & $<1$ & $<1$ & $<1$ & $<1$ & $<1$ & $<1$ & $<1$ & $<1$ & $<1$ & 2 & 61 \\
\hline \multicolumn{14}{|l|}{ TRA } \\
\hline & $\begin{array}{l}\text { VOLUME } \\
\text { CURIES }\end{array}$ & $\begin{array}{l}28724 \\
51838\end{array}$ & $\begin{array}{r}61 \\
173\end{array}$ & $\begin{array}{r}78 \\
269\end{array}$ & $\begin{array}{l}94 \\
91\end{array}$ & $\begin{array}{r}70 \\
139\end{array}$ & $\begin{array}{r}70 \\
179\end{array}$ & $\begin{array}{r}73 \\
134\end{array}$ & $\begin{array}{r}71 \\
185\end{array}$ & $\begin{array}{r}99 \\
165\end{array}$ & $\begin{array}{r}88 \\
184\end{array}$ & $\begin{array}{r}71 \\
128\end{array}$ & $\begin{array}{l}19498 \\
53485\end{array}$ \\
\hline \multicolumn{14}{|l|}{$\operatorname{mic}$} \\
\hline & $\begin{array}{l}\text { VOLUNE } \\
\text { CURIES }\end{array}$ & $<1$ & & & & & & & & & & & $<1$ \\
\hline
\end{tabular}


RECORO-TO-DATE

\begin{tabular}{|c|c|c|c|c|c|c|c|c|c|c|c|c|}
\hline AREA NEAR & $1952-1983$ & 1984 & 1985 & 1986 & 1987 & 1988 & 1989 & 1990 & 1991 & 1992 & 1993 & TOTAL \\
\hline JTAL & & & & & & & & & & & & \\
\hline $\begin{array}{l}\text { VOLUHE } \\
\text { CURIES }\end{array}$ & $\begin{array}{l}69494 \\
73745\end{array}$ & $\begin{array}{r}2749 \\
191\end{array}$ & $\begin{array}{r}2744 \\
667\end{array}$ & $\begin{array}{r}2529 \\
346\end{array}$ & $\begin{array}{r}2536 \\
357\end{array}$ & $\begin{array}{r}2357 \\
270\end{array}$ & $\begin{array}{r}1922 \\
137\end{array}$ & $\begin{array}{r}2642 \\
189\end{array}$ & $\begin{array}{r}2490 \\
170\end{array}$ & $\begin{array}{r}2799 \\
187\end{array}$ & $\begin{array}{r}2659 \\
130\end{array}$ & $\begin{array}{l}94920 \\
76389\end{array}$ \\
\hline
\end{tabular}

* VOLLHE IN MILLION LITERS

* FOR THE YEARS 1962-70 THE SHORT-LIVED RADIOACTIVITY (LESS THAN 2-3 DAY HALF LIFE) MERE NOT REPORTED

DETAILS MAY NOT ADD UP TO TOTALS BECAUSE OF ROUNDING 
INEL Radioactive Liquid Waste Discharge Record-to-Date
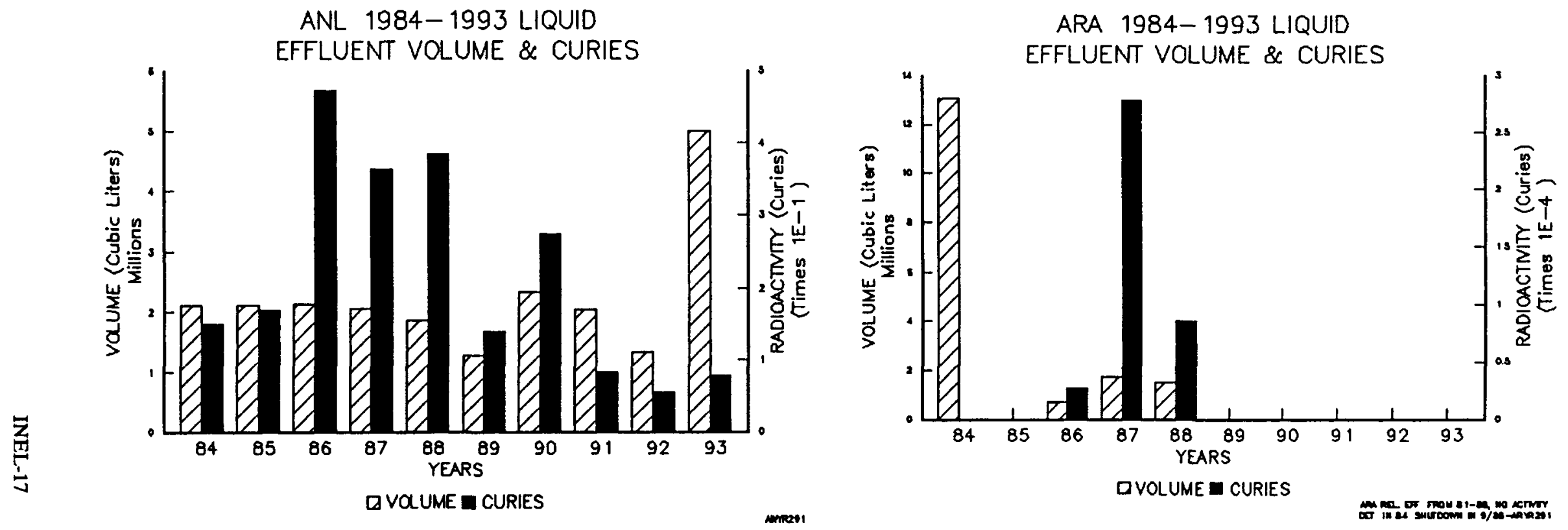

CFA $1984-1993$ LIQUID

EFFLUENT VOLUME \&C CURIES

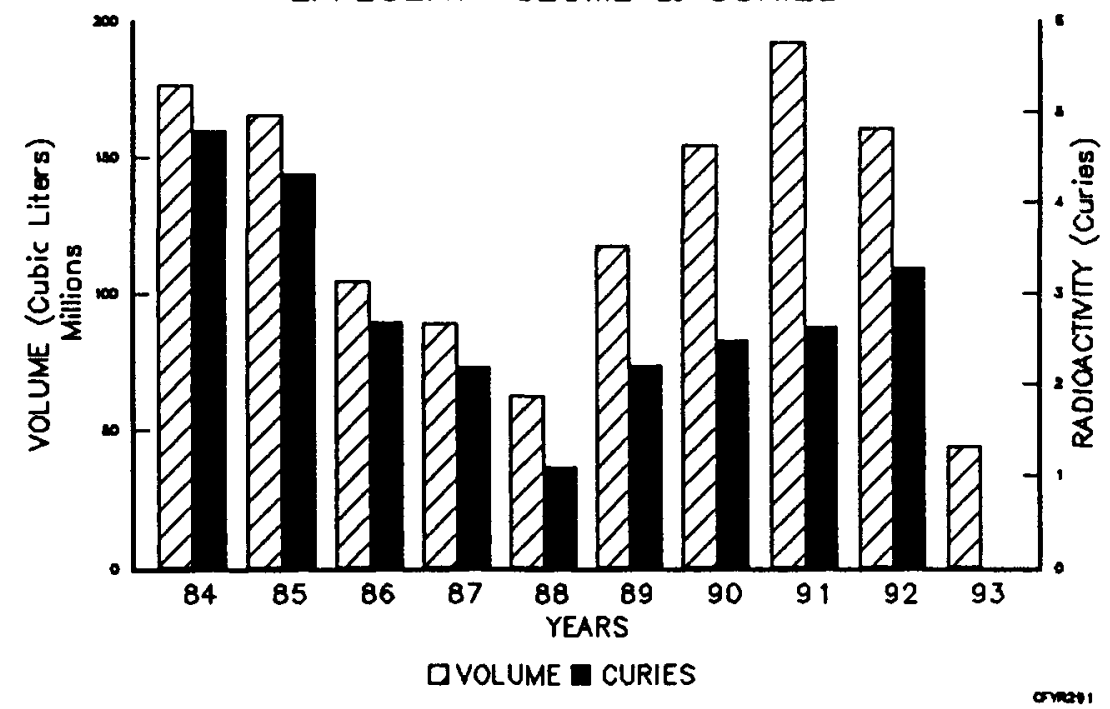


INEL Radioactive Liquid Waste Discharge Record-to-Date

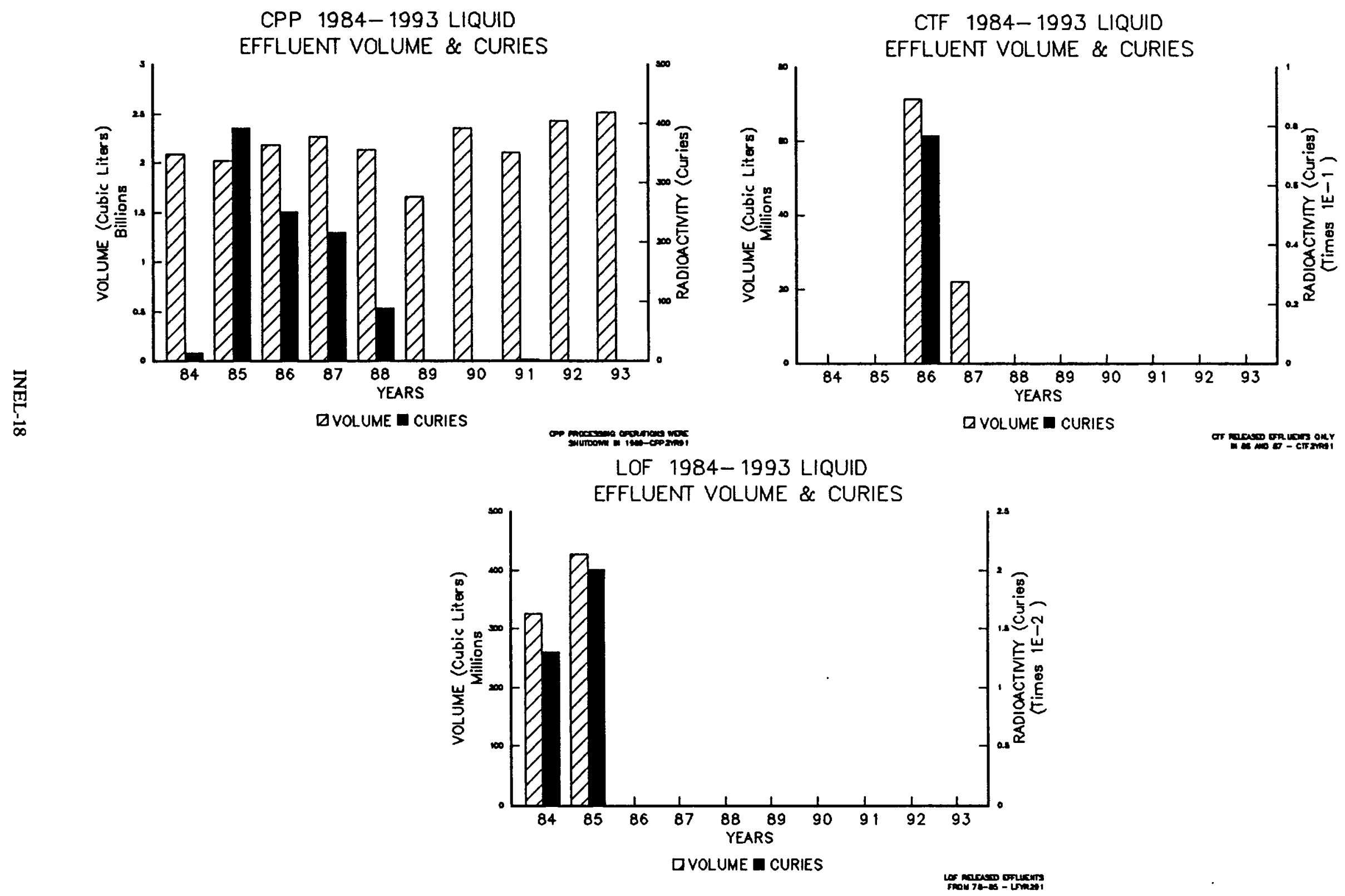


<smiles>C#C[Si]1([Si]2(#C)C=CC=C2)C=CC=C1</smiles> 
SCHEDULE NO. P6LPMO22-A

INEL SOLID RECORD-TO-DATE SUMYARY

RUN DATE: 06/20/94

dISPOSEd MUSTE

\begin{tabular}{|c|c|c|c|c|c|c|c|c|c|c|c|c|c|}
\hline AREA & NEAR & $1952-1983$ & 1984 & 1985 & 1986 & 1987 & 1988 & 1989 & 1990 & 1991 & 1992 & 1993 & TOTAL \\
\hline \multicolumn{14}{|l|}{ ALE } \\
\hline & $\begin{array}{l}\text { VOLLUNE } \\
\text { CURIES }\end{array}$ & $\begin{array}{r}3544 \\
473\end{array}$ & $\begin{array}{r}442 \\
37\end{array}$ & $\begin{array}{r}592 \\
71\end{array}$ & $\begin{array}{r}306 \\
10\end{array}$ & $\begin{array}{l}309 \\
840\end{array}$ & $\begin{array}{r}204 \\
50\end{array}$ & & & & & & $\begin{array}{l}5398 \\
1461\end{array}$ \\
\hline \multicolumn{14}{|l|}{ ANL } \\
\hline & $\begin{array}{l}\text { VOLUME } \\
\text { CURIES }\end{array}$ & $\begin{array}{r}7112 \\
1112839\end{array}$ & $\begin{array}{r}153 \\
119289\end{array}$ & $\begin{array}{r}121 \\
126651\end{array}$ & $\begin{array}{r}135 \\
57382\end{array}$ & $\begin{array}{r}109 \\
204682\end{array}$ & $\begin{array}{r}473 \\
142614\end{array}$ & $\begin{array}{r}172 \\
463320\end{array}$ & $\begin{array}{r}65 \\
130511\end{array}$ & $\begin{array}{r}30 \\
82261\end{array}$ & $\begin{array}{r}56 \\
92896\end{array}$ & $\begin{array}{r}77 \\
87058\end{array}$ & $\begin{array}{r}8503 \\
2618502\end{array}$ \\
\hline \multicolumn{14}{|l|}{ ARA } \\
\hline & $\begin{array}{l}\text { VOLUNE } \\
\text { CURIES }\end{array}$ & $\begin{array}{r}381 \\
4017\end{array}$ & $\begin{array}{r}16 \\
2\end{array}$ & $\begin{array}{r}17 \\
5\end{array}$ & $\begin{array}{r}1 \\
<1\end{array}$ & $\begin{array}{l}20 \\
<1\end{array}$ & & & & & & & $\begin{array}{r}435 \\
4025\end{array}$ \\
\hline \multicolumn{14}{|c|}{ BEN } \\
\hline & $\begin{array}{l}\text { VOLUANE } \\
\text { CURIES }\end{array}$ & & & & $\begin{array}{l}<1 \\
<1\end{array}$ & & & & & & & & $\begin{array}{l}<1 \\
<1\end{array}$ \\
\hline \multicolumn{14}{|l|}{ BNL } \\
\hline & $\begin{array}{l}\text { VOLUME } \\
\text { CURIES }\end{array}$ & $\begin{array}{r}5 \\
<1\end{array}$ & & & & & & & & & & & $\begin{array}{r}5 \\
<1\end{array}$ \\
\hline \multicolumn{14}{|l|}{ CEG } \\
\hline & $\begin{array}{l}\text { VOLUME } \\
\text { CURIES }\end{array}$ & & & & & & & & & & & $\begin{array}{r}22 \\
1967\end{array}$ & $\begin{array}{r}22 \\
1967\end{array}$ \\
\hline \multicolumn{14}{|l|}{ CFA } \\
\hline & VOLUNE & 2429 & 37 & 9 & 16 & 4 & 5 & 4 & 45 & & & 84 & 2633 \\
\hline & CURIES & 381 & $<1$ & $<1$ & $<1$ & 4 & $<1$ & $<1$ & $<1$ & & & $<1$ & 385 \\
\hline \multicolumn{14}{|l|}{ CPP } \\
\hline & $\begin{array}{l}\text { VOLUNE } \\
\text { CURIES }\end{array}$ & $\begin{array}{r}25941 \\
613408\end{array}$ & $\begin{array}{r}1817 \\
301\end{array}$ & $\begin{array}{l}807 \\
112\end{array}$ & $\begin{array}{l}948 \\
214\end{array}$ & $\begin{array}{r}1151 \\
218\end{array}$ & $\begin{array}{l}578 \\
192\end{array}$ & $\begin{array}{l}513 \\
115\end{array}$ & $\begin{array}{l}752 \\
211\end{array}$ & $\begin{array}{r}544 \\
24\end{array}$ & $\begin{array}{r}129 \\
2\end{array}$ & $\begin{array}{r}7 \\
<1\end{array}$ & $\begin{array}{r}33187 \\
614798\end{array}$ \\
\hline \multicolumn{14}{|l|}{ CTF } \\
\hline & $\begin{array}{l}\text { VOLUWE } \\
\text { CURIES }\end{array}$ & & & & $\begin{array}{l}805 \\
277\end{array}$ & $\begin{array}{r}251 \\
25\end{array}$ & $\begin{array}{l}27 \\
<1\end{array}$ & & & & & $\begin{array}{l}54 \\
<1\end{array}$ & $\begin{array}{r}1138 \\
303\end{array}$ \\
\hline \multicolumn{14}{|l|}{$D+D$} \\
\hline & $\begin{array}{l}\text { VOLURE } \\
\text { CURTES }\end{array}$ & $\begin{array}{l}2023 \\
5689\end{array}$ & $\begin{array}{r}293 \\
3\end{array}$ & $\begin{array}{l}34 \\
<1\end{array}$ & $\begin{array}{l}53 \\
<1\end{array}$ & $\begin{array}{r}8 \\
<1\end{array}$ & $\begin{array}{l}95 \\
<1\end{array}$ & $\begin{array}{l}24 \\
<1\end{array}$ & $\begin{array}{l}21 \\
<1\end{array}$ & $\begin{array}{l}58 \\
<1\end{array}$ & $\begin{array}{r}185 \\
<1\end{array}$ & $\begin{array}{r}344 \\
2\end{array}$ & $\begin{array}{l}3137 \\
5695\end{array}$ \\
\hline \multicolumn{14}{|l|}{ LOF } \\
\hline & $\begin{array}{l}\text { VOLUHE } \\
\text { CURIES }\end{array}$ & $\begin{array}{l}13 \\
<1\end{array}$ & $\begin{array}{r}11 \\
2\end{array}$ & $\begin{array}{l}49 \\
<1\end{array}$ & & & & & & & & & $\begin{array}{r}73 \\
3\end{array}$ \\
\hline \multicolumn{14}{|l|}{ NRF } \\
\hline & $\begin{array}{l}\text { VOLUME } \\
\text { CURIES }\end{array}$ & $\begin{array}{r}19778 \\
3650802\end{array}$ & $\begin{array}{r}416 \\
365125\end{array}$ & $\begin{array}{r}347 \\
141753\end{array}$ & $\begin{array}{r}270 \\
35930\end{array}$ & $\begin{array}{r}171 \\
29665\end{array}$ & $\begin{array}{r}111 \\
6732\end{array}$ & $\begin{array}{r}134 \\
126486\end{array}$ & $\begin{array}{r}419 \\
74087\end{array}$ & $\begin{array}{r}270 \\
102849\end{array}$ & $\begin{array}{r}159 \\
49795\end{array}$ & $\begin{array}{r}146 \\
42259\end{array}$ & $\begin{array}{r}22222 \\
4625484\end{array}$ \\
\hline
\end{tabular}


DISPOSED MUSTE

\begin{tabular}{|c|c|c|c|c|c|c|c|c|c|c|c|c|c|}
\hline AREA & NEAR & $1952-1983$ & 1984 & 2985 & 1986 & 1987 & 1988 & 1989 & 1990 & 1991 & 1992 & 1993 & TOTAL \\
\hline \multicolumn{14}{|l|}{ PBF } \\
\hline & $\begin{array}{l}\text { VOLUME } \\
\text { CURIES }\end{array}$ & $\begin{array}{r}513 \\
7\end{array}$ & $\begin{array}{l}239 \\
181\end{array}$ & $\begin{array}{l}121 \\
371\end{array}$ & $\begin{array}{r}4 \\
<1\end{array}$ & $\begin{array}{l}45 \\
11\end{array}$ & $\begin{array}{r}29 \\
3\end{array}$ & & & & & & $\begin{array}{l}950 \\
573\end{array}$ \\
\hline \multicolumn{14}{|l|}{ PER } \\
\hline & $\begin{array}{l}\text { VOLUNE } \\
\text { CURIES }\end{array}$ & $\begin{array}{r}499 \\
24\end{array}$ & & & & $\begin{array}{r}4 \\
<1\end{array}$ & & $\begin{array}{r}6 \\
<1\end{array}$ & & $\begin{array}{r}2 \\
<1\end{array}$ & & & $\begin{array}{r}510 \\
24\end{array}$ \\
\hline \multicolumn{14}{|l|}{ RFO } \\
\hline & $\begin{array}{l}\text { VOLUAE } \\
\text { CURIES }\end{array}$ & $\begin{array}{r}73124 \\
256010\end{array}$ & & & & & & & & & & & $\begin{array}{r}73124 \\
256010\end{array}$ \\
\hline \multicolumn{14}{|l|}{$\sin x$} \\
\hline & VOLUAE & & & & 43 & 7 & 53 & 21 & & 24 & 50 & & 198 \\
\hline & CURIES & & & & $<1$ & $<1$ & $<1$ & $<1$ & & $<1$ & $<1$ & & 1 \\
\hline \multicolumn{14}{|l|}{ TAN } \\
\hline & $\begin{array}{l}\text { VOLLAEE } \\
\text { CURIES }\end{array}$ & $\begin{array}{r}7993 \\
101441\end{array}$ & $\begin{array}{r}219 \\
2\end{array}$ & $\begin{array}{r}237 \\
17\end{array}$ & $\begin{array}{r}235 \\
17\end{array}$ & $\begin{array}{l}224 \\
359\end{array}$ & $\begin{array}{r}56 \\
1\end{array}$ & $\begin{array}{l}76 \\
67\end{array}$ & $\begin{array}{r}43 \\
2658\end{array}$ & $\begin{array}{l}24 \\
<1\end{array}$ & $\begin{array}{r}87 \\
347\end{array}$ & $\begin{array}{r}82 \\
216\end{array}$ & $\begin{array}{r}9275 \\
105124\end{array}$ \\
\hline \multicolumn{14}{|l|}{ TRA } \\
\hline & $\begin{array}{l}\text { VOLUME } \\
\text { CURIES }\end{array}$ & $\begin{array}{r}14281 \\
3866659\end{array}$ & $\begin{array}{r}261 \\
1098\end{array}$ & $\begin{array}{r}613 \\
2452\end{array}$ & $\begin{array}{r}387 \\
1253\end{array}$ & $\begin{array}{r}219 \\
11631\end{array}$ & $\begin{array}{l}158 \\
147\end{array}$ & $\begin{array}{l}62 \\
77\end{array}$ & $\begin{array}{l}37 \\
68\end{array}$ & $\begin{array}{r}72 \\
2430\end{array}$ & $\begin{array}{l}179 \\
909\end{array}$ & $\begin{array}{r}28 \\
298152\end{array}$ & $\begin{array}{r}16298 \\
4184878\end{array}$ \\
\hline \multicolumn{14}{|l|}{ WER } \\
\hline & $\begin{array}{l}\text { VOLLAME } \\
\text { CURIES }\end{array}$ & & & $\begin{array}{r}192 \\
<1\end{array}$ & $\begin{array}{r}199 \\
<1\end{array}$ & $\begin{array}{r}273 \\
<1\end{array}$ & $\begin{array}{r}254 \\
<1\end{array}$ & $\begin{array}{r}328 \\
<1\end{array}$ & $\begin{array}{r}359 \\
6\end{array}$ & $\begin{array}{r}248 \\
3\end{array}$ & & $\begin{array}{l}7 \\
<1\end{array}$ & $\begin{array}{r}1861 \\
10\end{array}$ \\
\hline \multicolumn{14}{|l|}{$\operatorname{manc}$} \\
\hline & $\begin{array}{l}\text { VOLUME } \\
\text { CURIES }\end{array}$ & $\begin{array}{l}19778 \\
60926\end{array}$ & $\begin{array}{l}<1 \\
<1\end{array}$ & & $\begin{array}{l}35 \\
<1\end{array}$ & $\begin{array}{r}177 \\
83\end{array}$ & & $\begin{array}{l}23 \\
<1\end{array}$ & $\begin{array}{l}22 \\
<1\end{array}$ & & & & $\begin{array}{l}20036 \\
61009\end{array}$ \\
\hline \multicolumn{14}{|c|}{ OTHER* } \\
\hline & $\begin{array}{l}\text { VOLUME } \\
\text { CURIES }\end{array}$ & $\begin{array}{l}1365 \\
2434\end{array}$ & & & & & & & & & & & $\begin{array}{l}1365 \\
2434\end{array}$ \\
\hline \multicolumn{14}{|c|}{ OFFSITE } \\
\hline & $\begin{array}{l}\text { VOLUNE } \\
\text { CURIES }\end{array}$ & $\begin{array}{r}6501 \\
37734\end{array}$ & & & & & & & & & & & $\begin{array}{r}6501 \\
37734\end{array}$ \\
\hline \multicolumn{14}{|c|}{$S L-1$} \\
\hline & $\begin{array}{l}\text { VOLLME } \\
\text { CURIES }\end{array}$ & $\begin{array}{r}2319 \\
599\end{array}$ & & & & & & & & & & & $\begin{array}{r}2319 \\
599\end{array}$ \\
\hline
\end{tabular}




\section{DISPOSED MASTE}

\begin{tabular}{|c|c|c|c|c|c|c|c|c|c|c|c|c|}
\hline AREAMEAR & $1952-1983$ & 1984 & 1985 & 1986 & 1987 & 2988 & 1989 & 1990 & 1991 & 1992 & 1993 & TOTAL \\
\hline TOTAL & & & & & & & & & & & & \\
\hline $\begin{array}{l}\text { VOLUNE: } \\
\text { CURIESE }\end{array}$ & $\begin{array}{r}187599 \\
9712441\end{array}$ & $\begin{array}{r}3906 \\
486041\end{array}$ & $\begin{array}{r}3140 \\
271433\end{array}$ & $\begin{array}{r}3437 \\
95084\end{array}$ & $\begin{array}{r}2969 \\
247519\end{array}$ & $\begin{array}{r}2045 \\
249740\end{array}$ & $\begin{array}{r}1364 \\
590065\end{array}$ & $\begin{array}{r}1762 \\
207541\end{array}$ & $\begin{array}{r}1272 \\
187566\end{array}$ & $\begin{array}{r}844 \\
143948\end{array}$ & $\begin{array}{r}852 \\
429654\end{array}$ & $\begin{array}{r}209190 \\
12521033\end{array}$ \\
\hline
\end{tabular}

DETAILS MAY NOT ADD UP TO TOTALS BECAUSE OF ROUNDING 


\section{INEL Solid Record-to-Date Summary Disposed Waste}

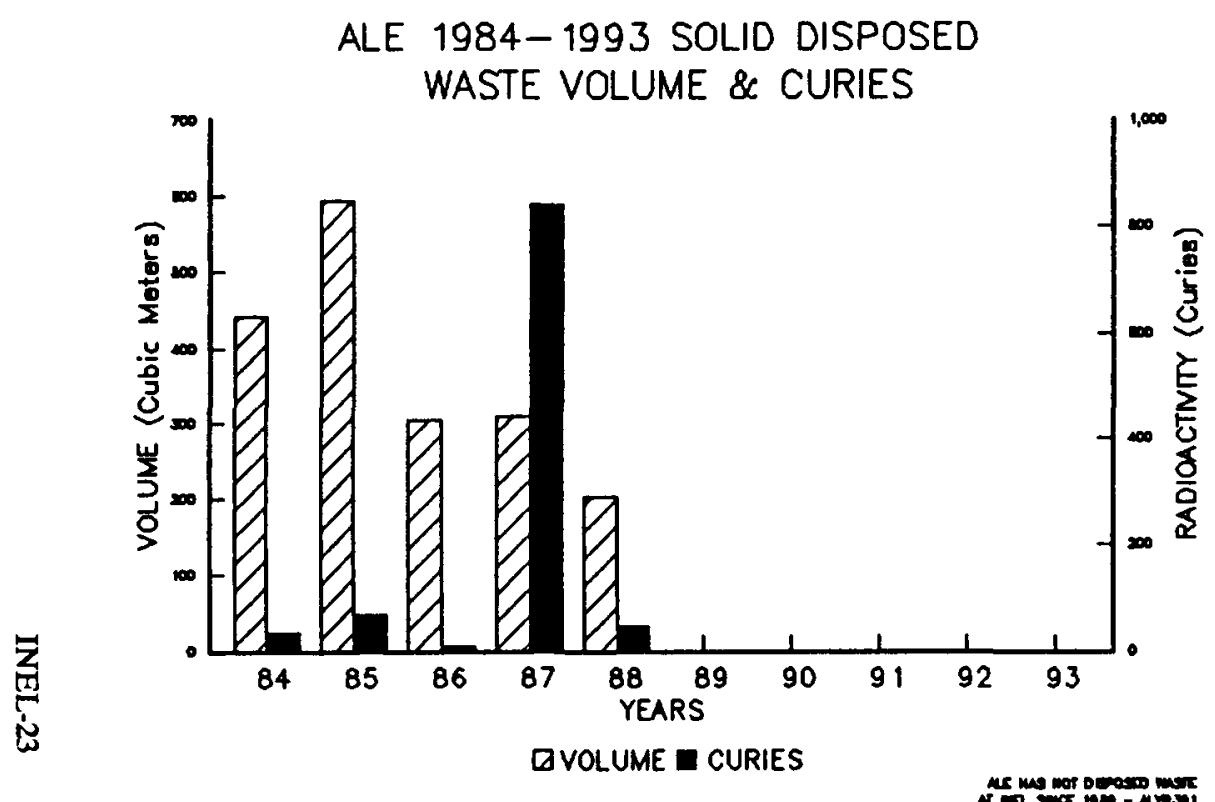

ARA 1984-1993 SOLID DISPOSED WASTE VOLUME \& CURIES

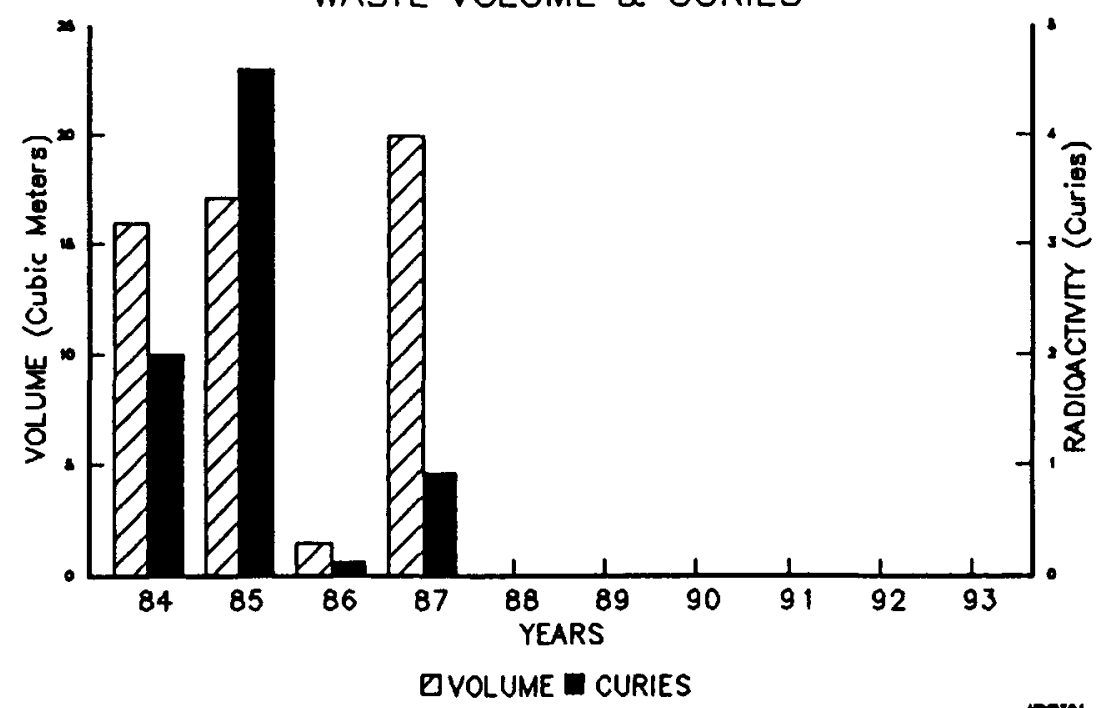

ANL 1984-1993 SOLID DISPOSED WASTE VOLUME \& CURIES

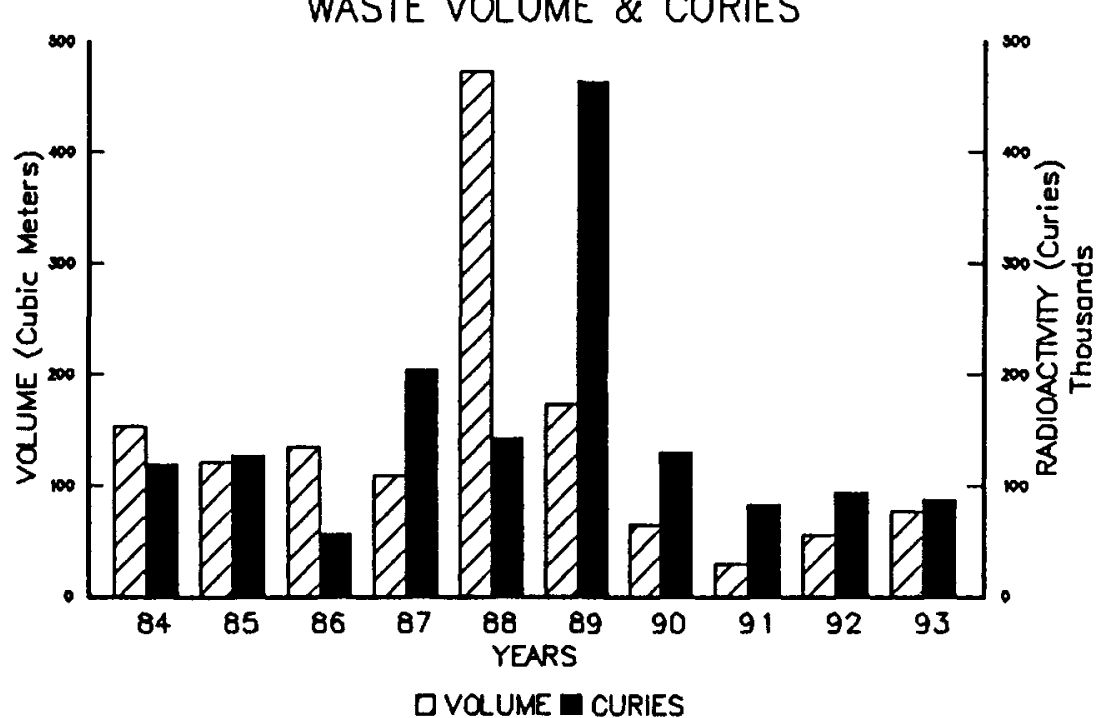

BEN 1984- 1993 SOLID DISPOSED WASTE VOLUME \& CURIES

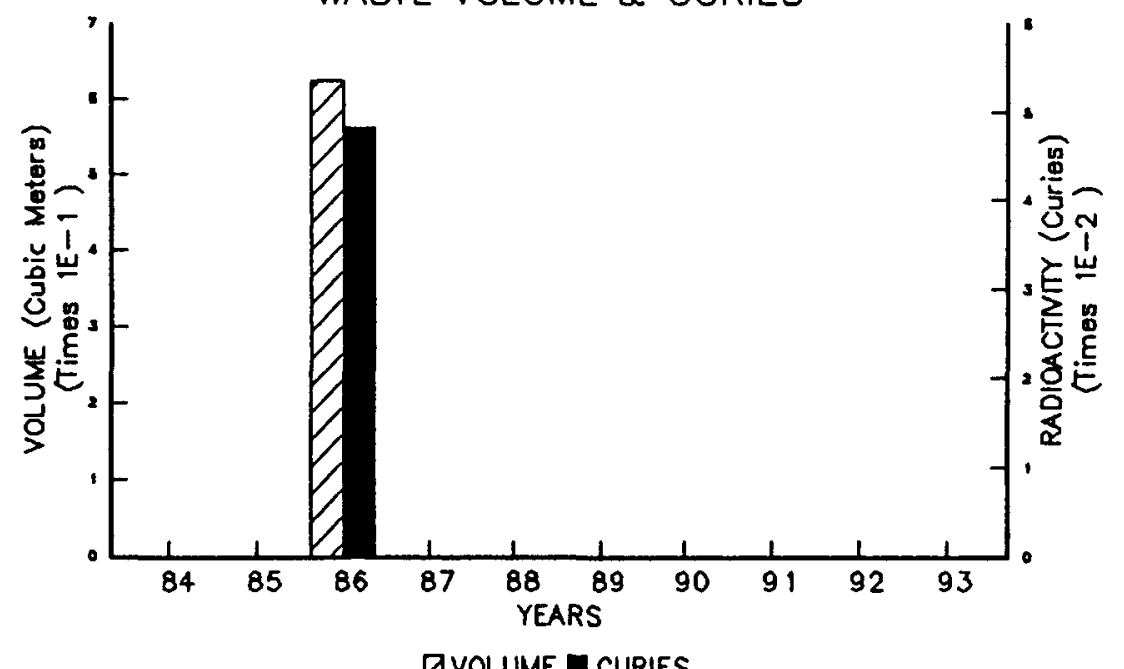

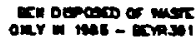




$$
\begin{aligned}
& 1 \text { A } \\
& \text { hillow }
\end{aligned}
$$




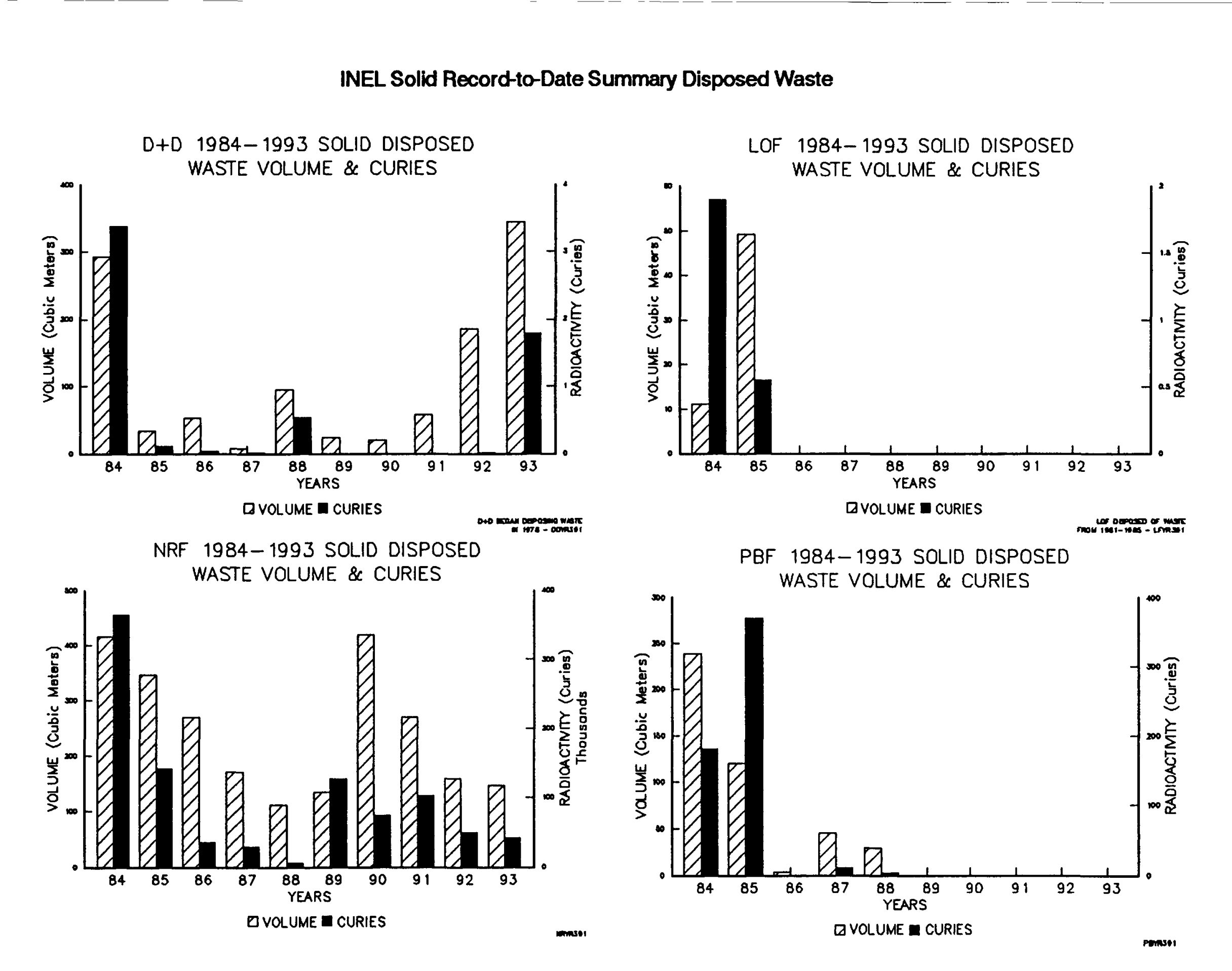




\section{INEL Solid Record-to-Date Summary Disposed Waste}
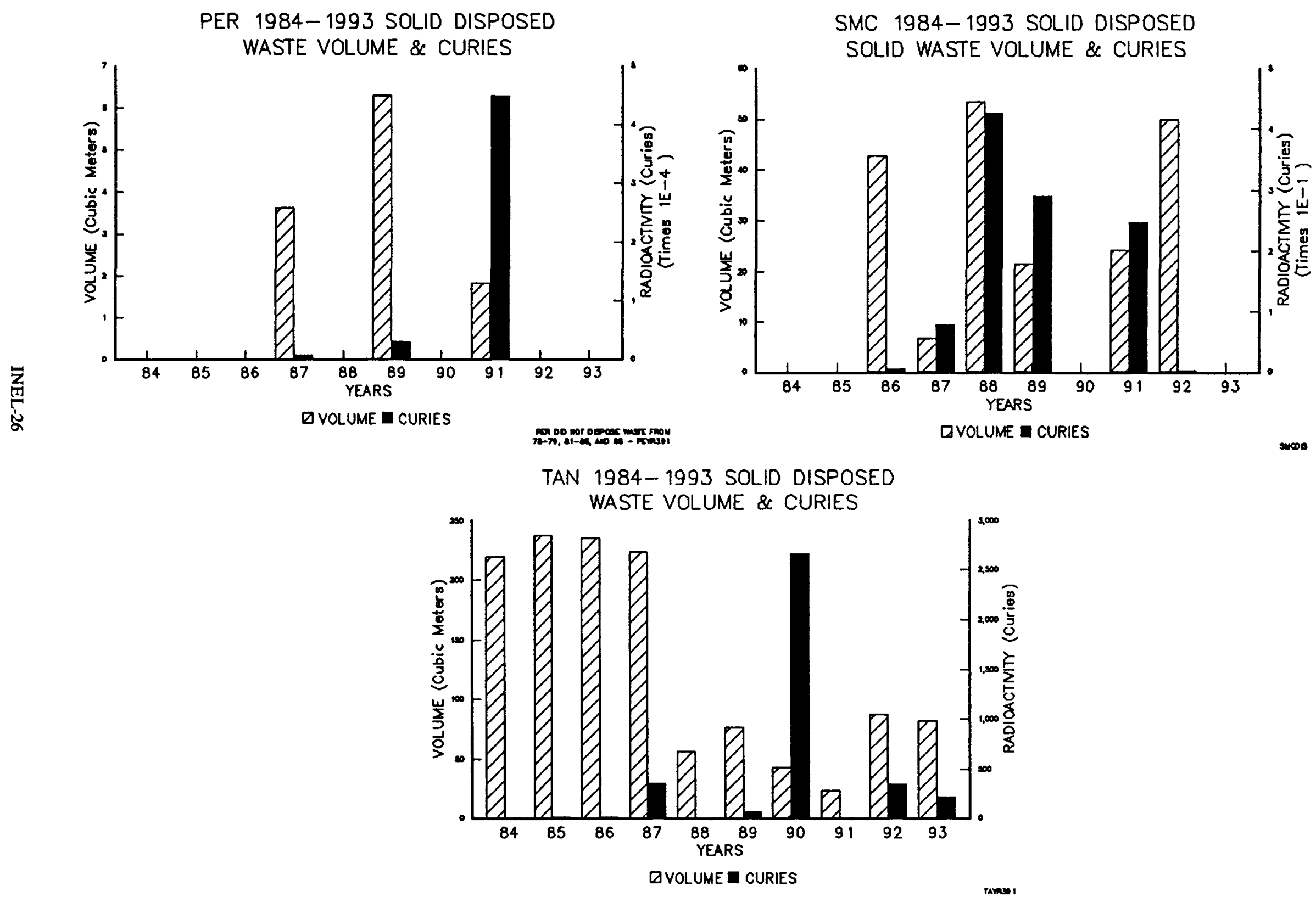


\section{INEL Solid Record-to-Date Summary Disposed Waste}
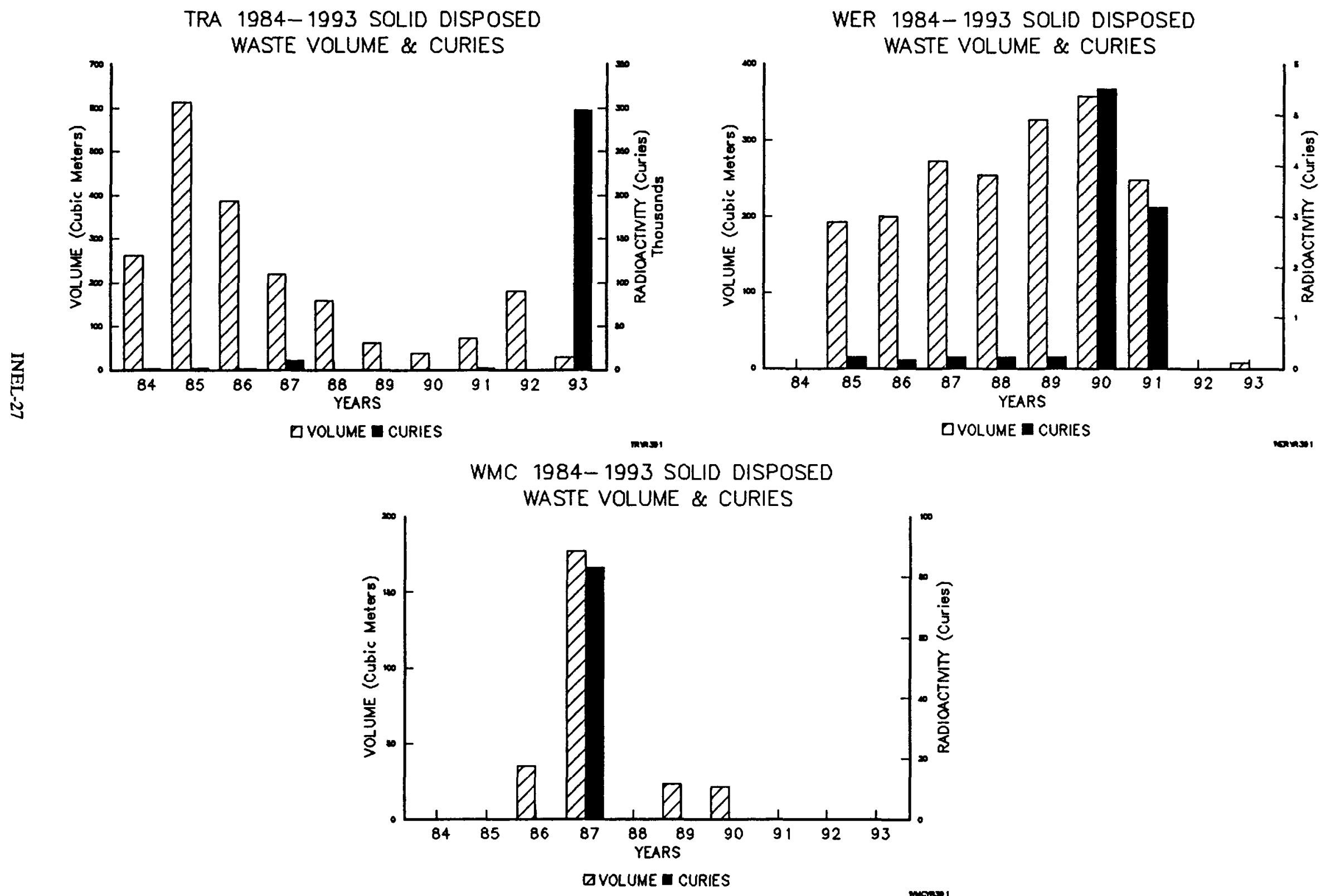


\section{STORED MASTE}

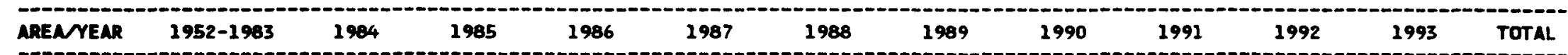

NL STORAGE

vouve

Volure

9921761

$<1$

$\begin{array}{rl}<1 & 15468\end{array}$

1201

$<1<1$

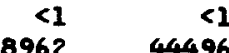

$\begin{array}{rr}<1 & 1 \\ 4996 & 2774\end{array}$

$<1$
39742

$\begin{array}{rr}<1 & 93 \\ 1165 & 10087630\end{array}$

ILTSF

volurite

$\begin{array}{lr}<1 & <1 \\ 49 & 2258\end{array}$

$\begin{array}{rr}8 & 5 \\ 1524 & 3913\end{array}$

12
120

$\begin{array}{rr}6 & 80 \\ 60 & 10570\end{array}$

TSA

VOLUNE $50871 \quad 3343 \quad 3375$

CURIES $332241 \quad 35070 \quad 37793$

3126
43211

$2091 \quad 1068$

1068
10612

872
6623

(2)

$24265 \quad 10612 \quad 6623$

34.40

WeF

$\begin{array}{lrr}\text { VOLUNE } & 2796 & 100 \\ \text { CURIES } & 71166976 & 1777000\end{array}$

$\begin{array}{rr}118 & 322 \\ 3359000 & 8566000\end{array}$

7

26

177
1603000

$<1$

64752

8566000

TOTAL

VOLUNE $\quad 53803 \quad 3444 \quad 3376$

3376
55520

$\begin{array}{rrr}3128 & 2217 & 1395\end{array}$

880

$\begin{array}{rr}41 & 181 \\ 4607 & 1603039\end{array}$

39741

<1 203

20368668 VOLUNE IN CUBIC METERS

DETAILS MaY NOT ADD UP TO TOTALS BECAUSE OF RONNING 


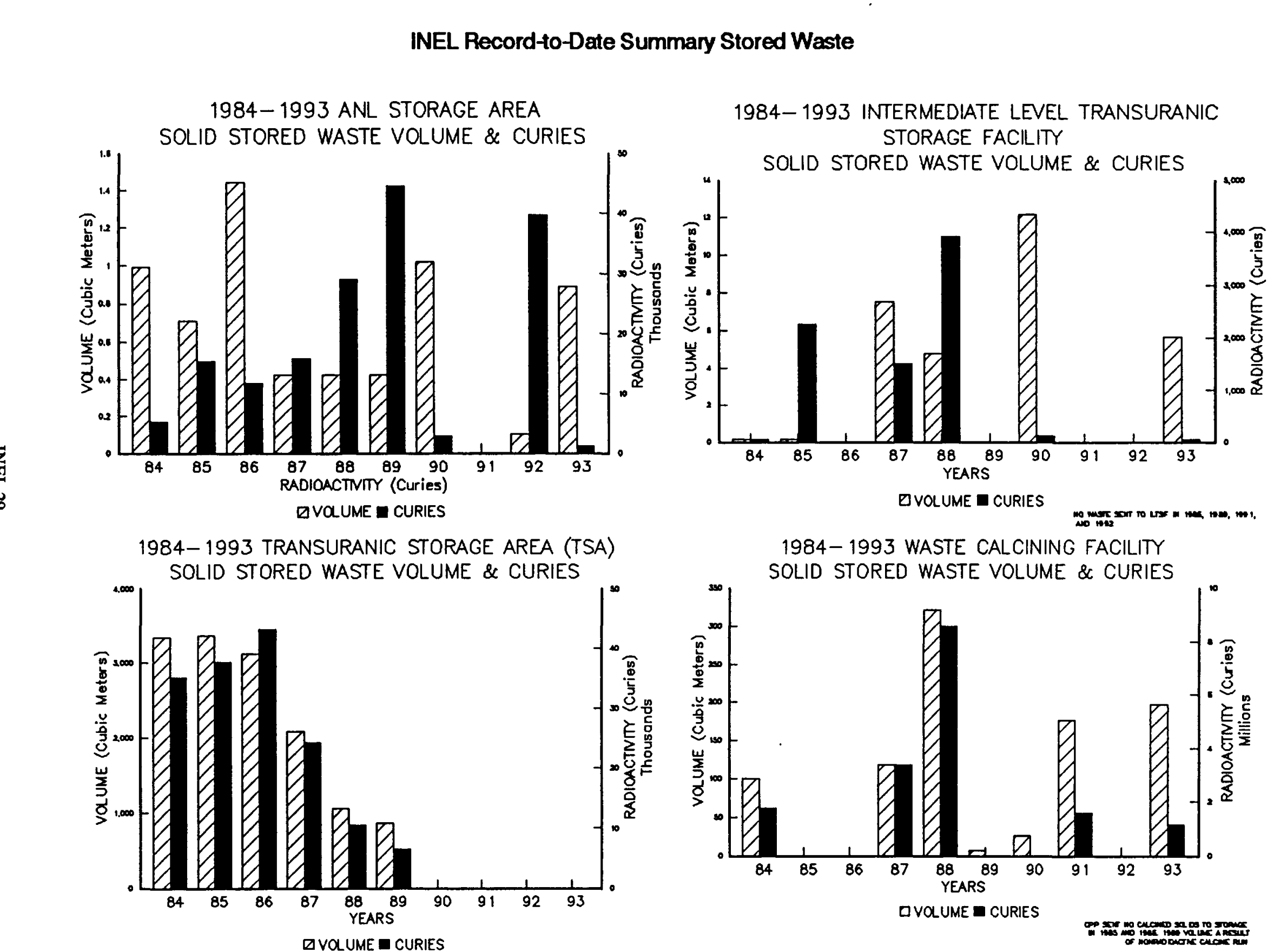


ANL STORAGE WASTE

\begin{tabular}{|c|c|c|c|c|c|c|c|c|c|c|c|c|}
\hline AREAYYAR & $1952-1983$ & 1984 & 1985 & 1986 & 1987 & 1988 & 1989 & 1990 & 1991 & 1992 & 1993 & TOTAL \\
\hline \multicolumn{13}{|l|}{ NL } \\
\hline $\begin{array}{l}\text { VolURE } \\
\text { CURIES }\end{array}$ & $\begin{array}{r}86 \\
9921705\end{array}$ & $\begin{array}{r}<1 \\
5356\end{array}$ & $\begin{array}{r}<1 \\
15468\end{array}$ & 11897 & $\begin{array}{r}<1 \\
16010\end{array}$ & $\begin{array}{r}<1 \\
28962\end{array}$ & $\begin{array}{r}<1 \\
44496\end{array}$ & 2774 & & $\begin{array}{r}<1 \\
39741\end{array}$ & $\begin{array}{r}<1 \\
1165\end{array}$ & $\begin{array}{r}93 \\
10087574\end{array}$ \\
\hline \multicolumn{13}{|l|}{ TRA } \\
\hline Volure & $<1$ & & & & & & & & & & & $<1$ \\
\hline CURTES & $<2$ & & & & & & & & & & & $<1$ \\
\hline \multicolumn{13}{|l|}{ TOTAL } \\
\hline $\begin{array}{l}\text { VOLUAE } \\
\text { CURIES }\end{array}$ & $\begin{array}{r}87 \\
9922705\end{array}$ & $\begin{array}{r}<1 \\
5356\end{array}$ & $\begin{array}{r}<1 \\
15468\end{array}$ & $\begin{array}{r}1 \\
11897\end{array}$ & $\begin{array}{r}<1 \\
16010\end{array}$ & $\begin{array}{r}<1 \\
28962\end{array}$ & $\begin{array}{r}<1 \\
4496\end{array}$ & $\begin{array}{r}1 \\
2774\end{array}$ & & $\begin{array}{r}<1 \\
39741\end{array}$ & $\begin{array}{r}<1 \\
1165\end{array}$ & $\begin{array}{r}93 \\
10087574\end{array}$ \\
\hline
\end{tabular}

DETAILS MAY NOT ADD UP TO TOTALS BECAUSE OF ROUNDING 


\section{INEL Solid Record-to-Date Summary ANL Storage Waste}

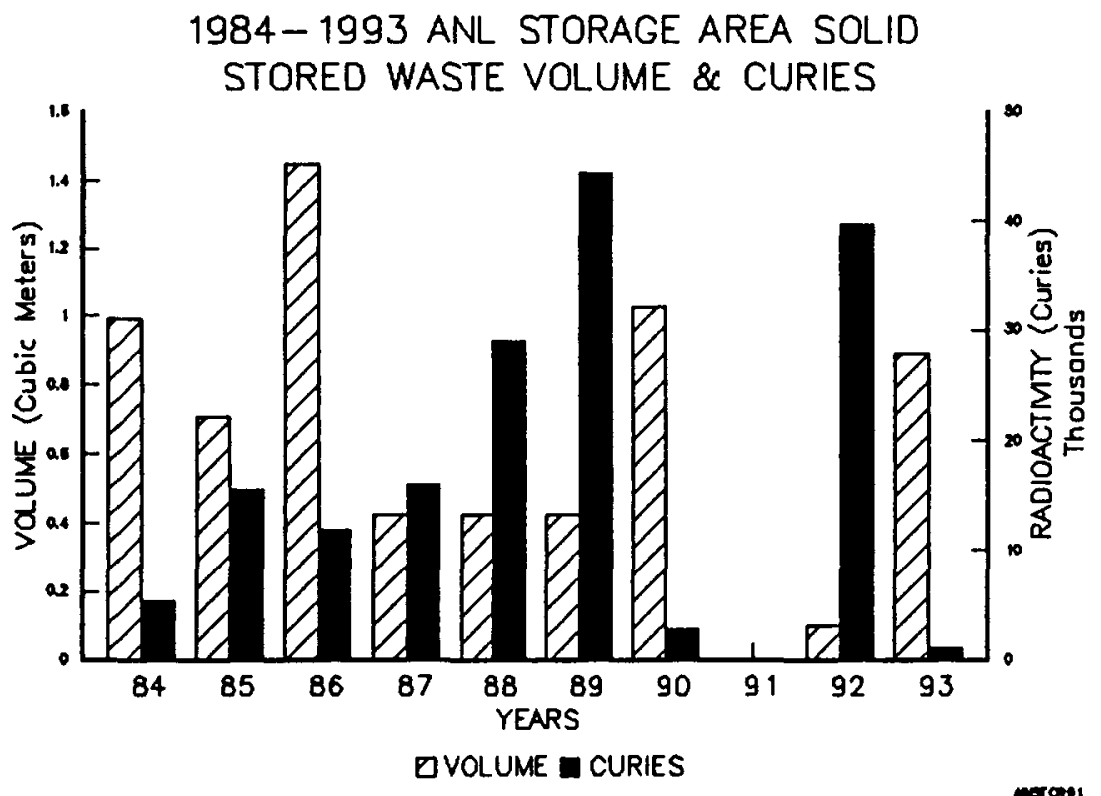


ILTSF WASTE

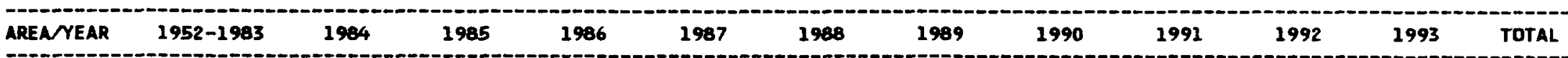

ALE

$\begin{array}{lr}\text { VOLLME } & 38 \\ \text { CURIES } & 233\end{array}$

ANL

VOLUME

CURIES 1485

BET

VOLUME

CURIES

CPP

$\begin{array}{lr}\text { VOLLUE } & 6 \\ \text { CURIES } & 847\end{array}$

NRF

VOLUME 3

CURIES 81

Z TOTAL

䍐

$\begin{array}{lr}\text { VOLUME } & 50 \\ \text { CURIES } & 2646\end{array}$

$\begin{array}{rlr}50 & <1 \quad<1\end{array}$

VOLUME IN CUBIC METERS

$\begin{array}{rrr}7 & 3 & 12\end{array}$

120

$\begin{array}{rr}<1 & 1 \\ 1429 & 3268\end{array}$

$<1$

604

604

847

3

$\begin{array}{rrr}8 & 5 & 12 \\ 1524 & 3913 & 120\end{array}$

12
120

$\begin{array}{rr}6 & 80 \\ 60 & 10570\end{array}$

DETAILS maY NOT ADD UP TO TOTALS BECAUSE OF ROUNDING 


\section{INEL Solid Record-to-Date Summary ILTSF Waste}

ALE 1984- 1993 INTERMEDIATE LEVEL TRANSURANIC STORAGE FACILITY SOLID WASTE VOLUME \& CURIES

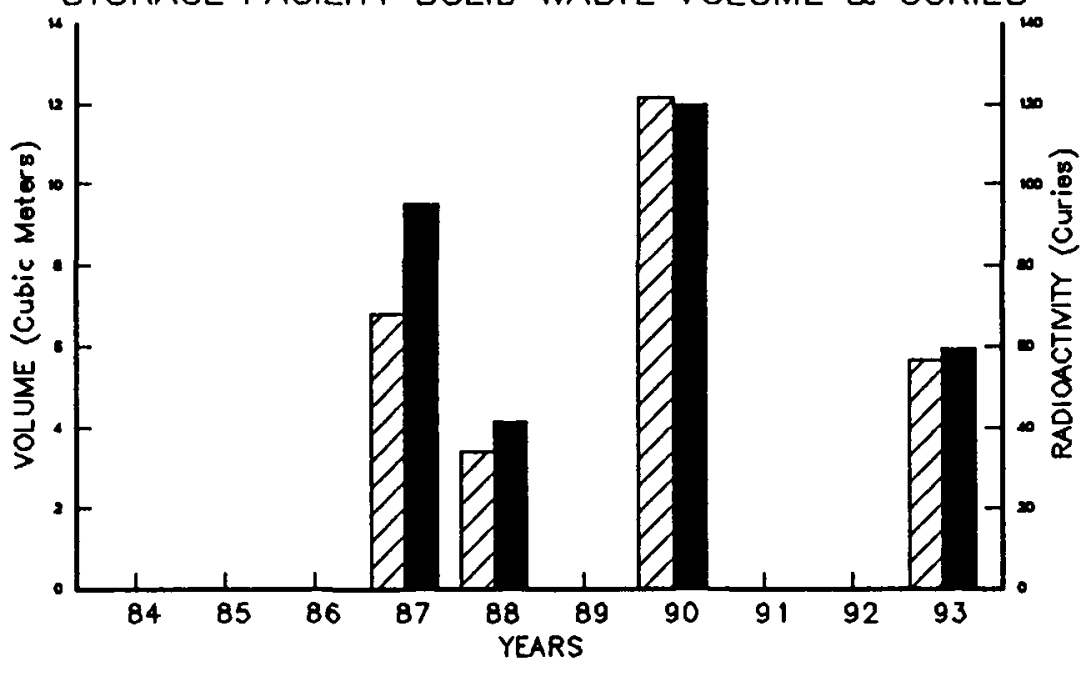

OVOLUME I CURIES

BET 1984- 1993 INTERMEDIATE LEVEL TRANSURANIC STORAGE FACILITY SOLID WASTE VOLUME \& CURIES

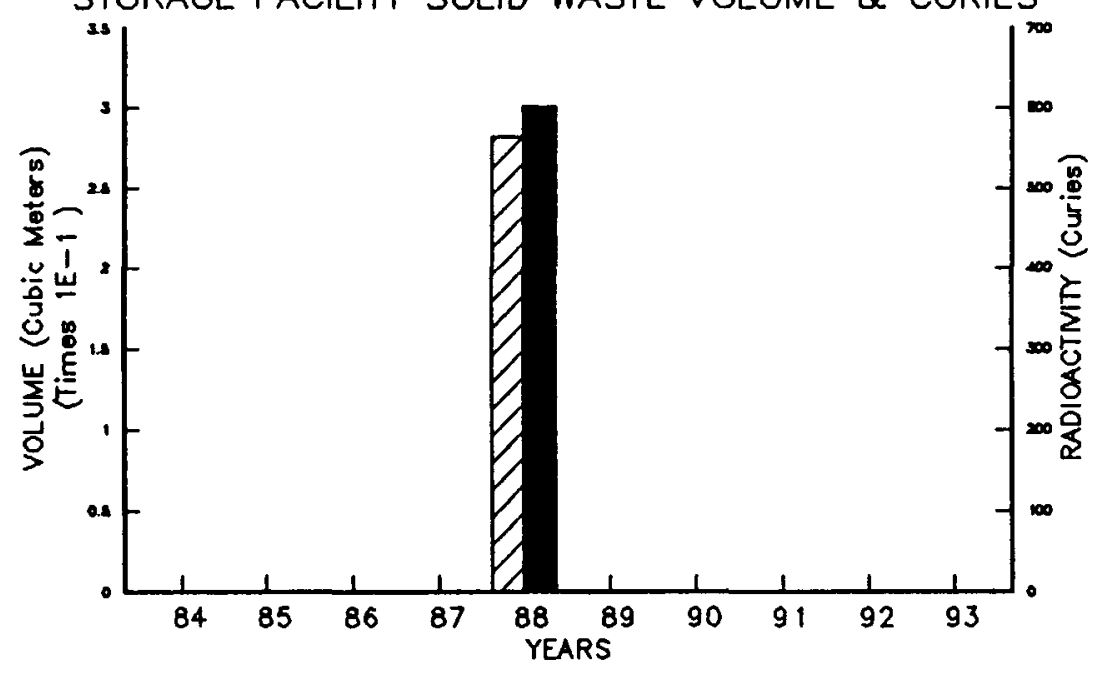

DVOLUME $\square$ CURIES
ANL 1984-1993 INTERMEDIATE LEVEL TRANSURANIC STORAGE FACILITY SOLID WASTE VOLUME \& CURIES

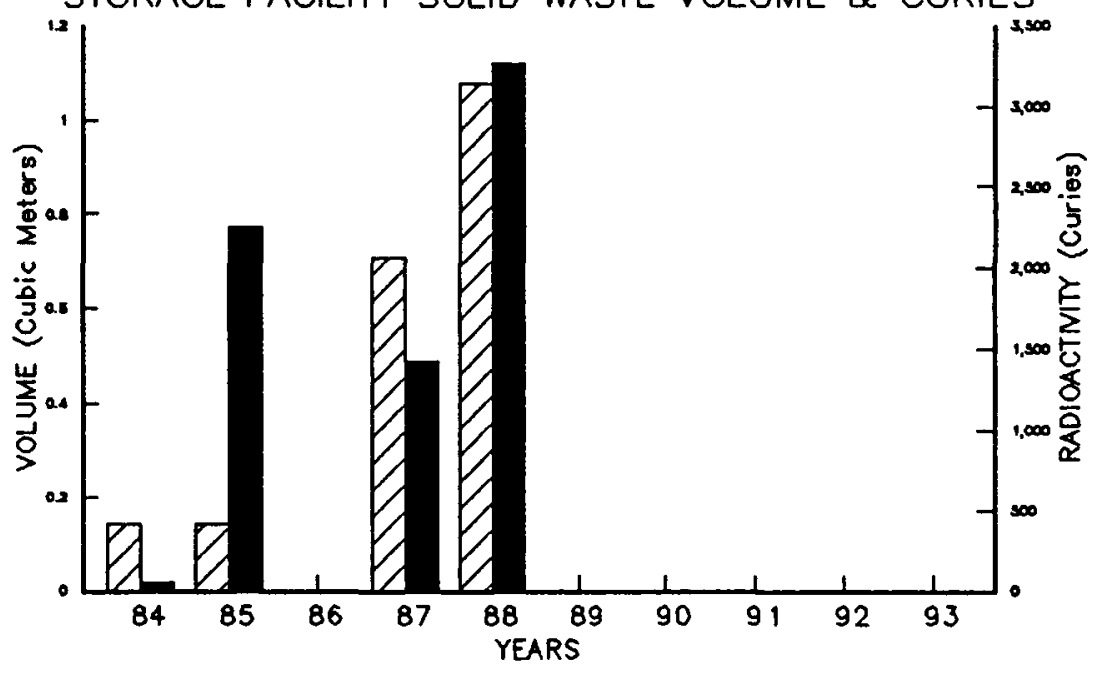

QVOLUME I CURIES

TRA 1984- 1993 INTERMEDIATE LEVEL TRANSURANIC STORAGE FACILITY SOLID WASTE VOLUME \& CURIES

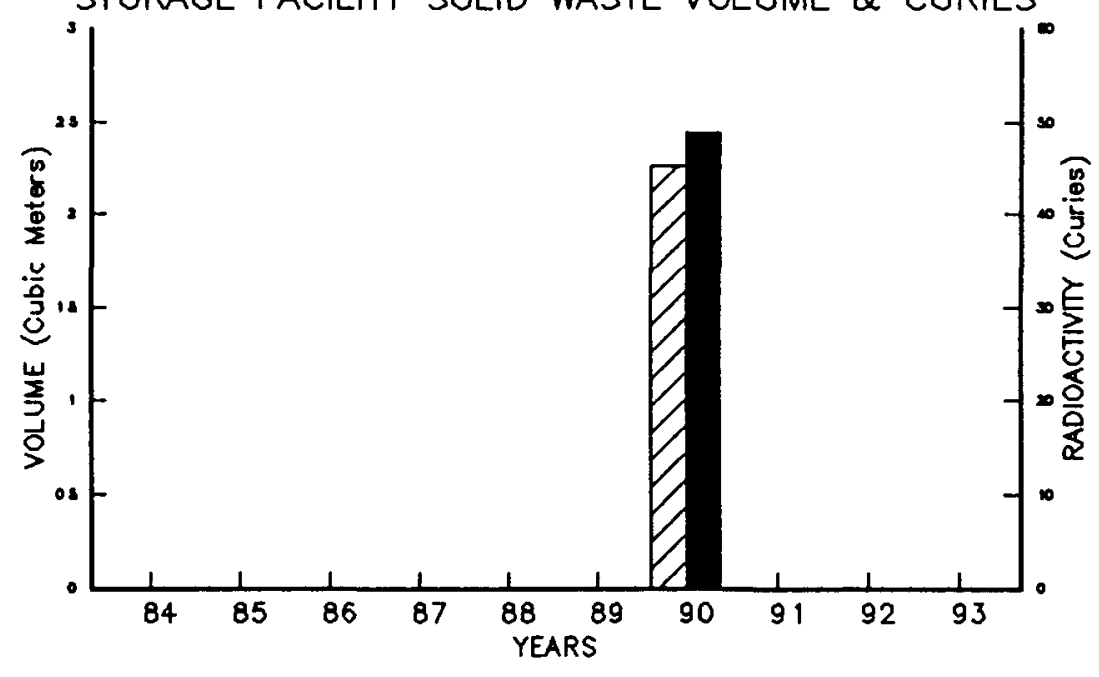

DVOLUME I CURIES 
TSA WASTE

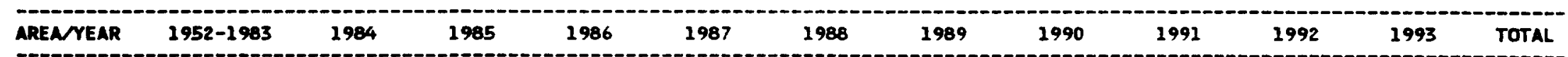

ALE

$\begin{array}{rrrrrr}\text { VOLLTE } & 1372 & 37 & 20 & 36 & \\ \text { CURIES } & 6932 & 772 & 28 & 508 & 12\end{array}$

5
12

1471

ANL

$\begin{array}{rrrrrr}\text { VOLLEE } & 7 & <1 & 5 & 6\end{array}$

9

$\begin{array}{rr}4 & 6 \\ 59 & 126\end{array}$

23

$<1$

$<1$

39
832

ARA

VOLUAE $\quad 10$

CURIES 21

BCL

VOLUME $\quad 214$

180

BEN

volune

CURIES

$<1$

$\begin{array}{lr}<1 & 4 \\ 62 & 26\end{array}$

$\begin{array}{ll}\text { VOLUNE } & 429 \\ \text { CURIES } & 895\end{array}$

$\begin{array}{ll}\text { VOLUNE } & <1 \\ \text { CURIES } & <1\end{array}$

cpp

$\begin{array}{lrr}\text { VOLUNE } & 41 & 2 \\ \text { CURIES } & 148 & 195\end{array}$

CTF

volure

CURIES

$<1$
1

$\mathbf{0}+\mathbf{0}$

VOLUME

$\begin{array}{rrrrrr}\text { VOLUNE } & 3532 & 10 & 76 & 126 & 87 \\ \text { CURIES } & 66826 & 882 & 55 & 104 & 1527\end{array}$

76
55

$126 \quad 87$

3831

984

342

$<1$

10

1

214
180

<1

984

$<1$
$<1$

c1

42

339 
TSA MASTE

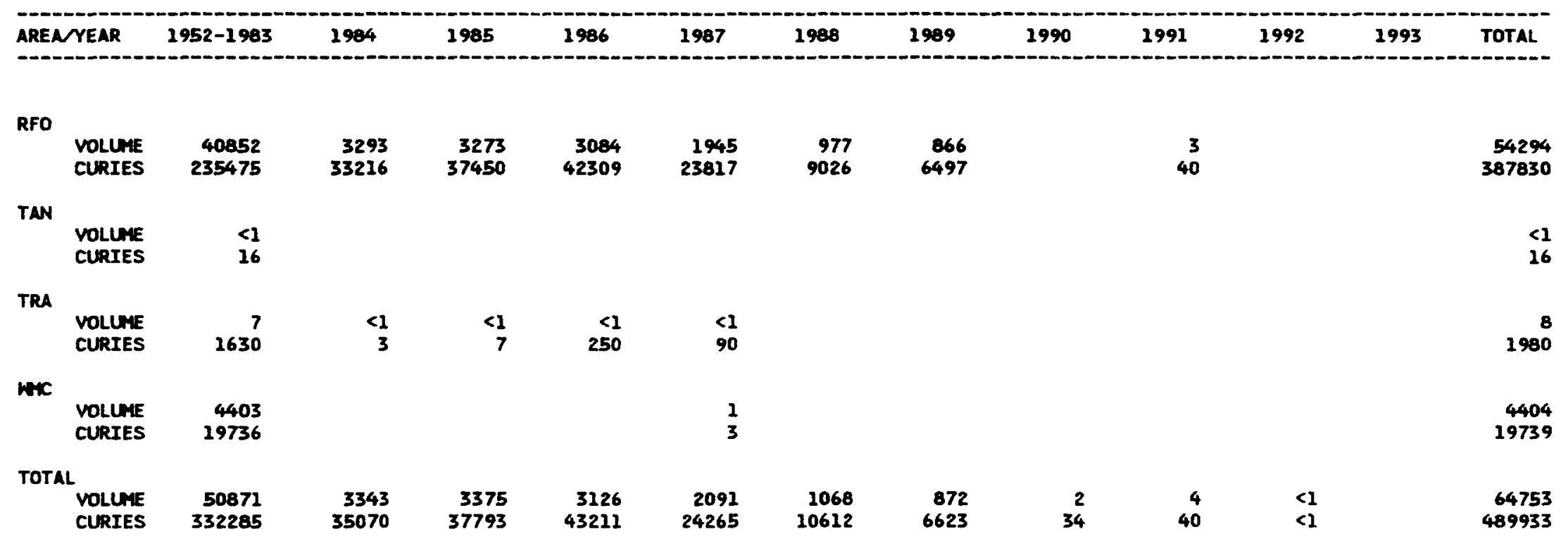




\section{INEL Solid Record-to-Date Summary TSA Waste}

ALE 1984- 1993 TRANSURANIC STORAGE AREA SOLID WASTE VOLUME \& CURIES

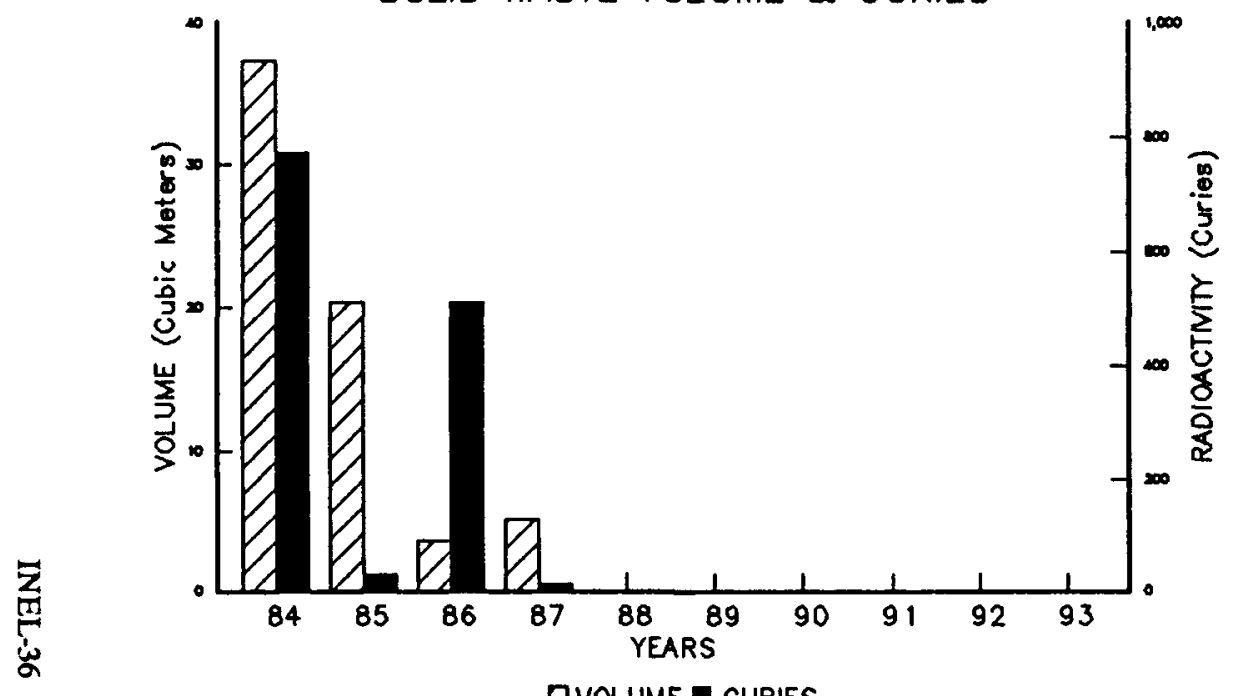

BEN 1984-1993 TRANSURANIC STORAGE AREA SOLID WASTE VOLUME \& CURIES

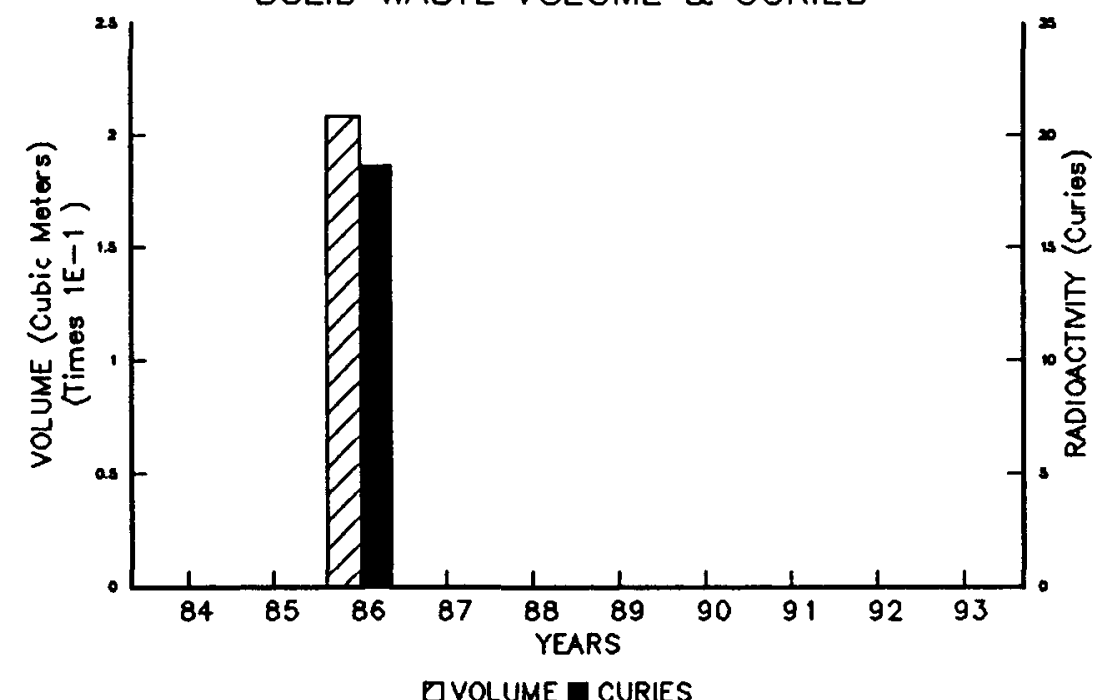

ANL 1984-1993 TRANSURANIC STORAGE AREA SOLID WASTE VOLUME \& CURIES

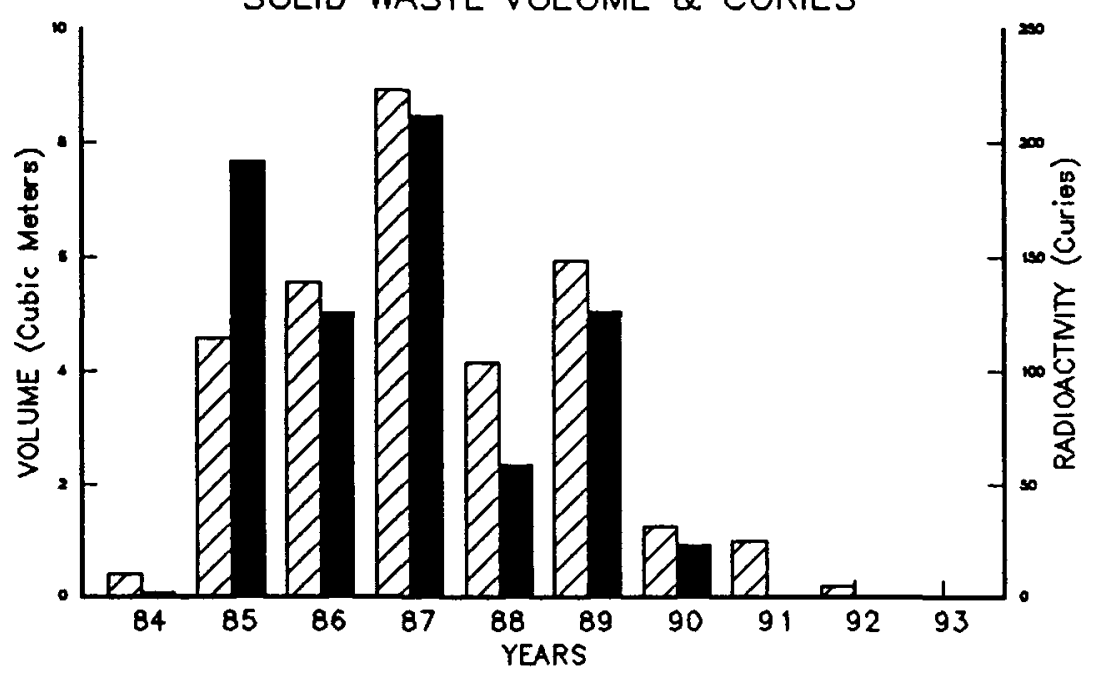

BET 1984.-1993 TROLUNEUERARIES STORAGE AREA SOLID WASTE VOLUME \& CURIES

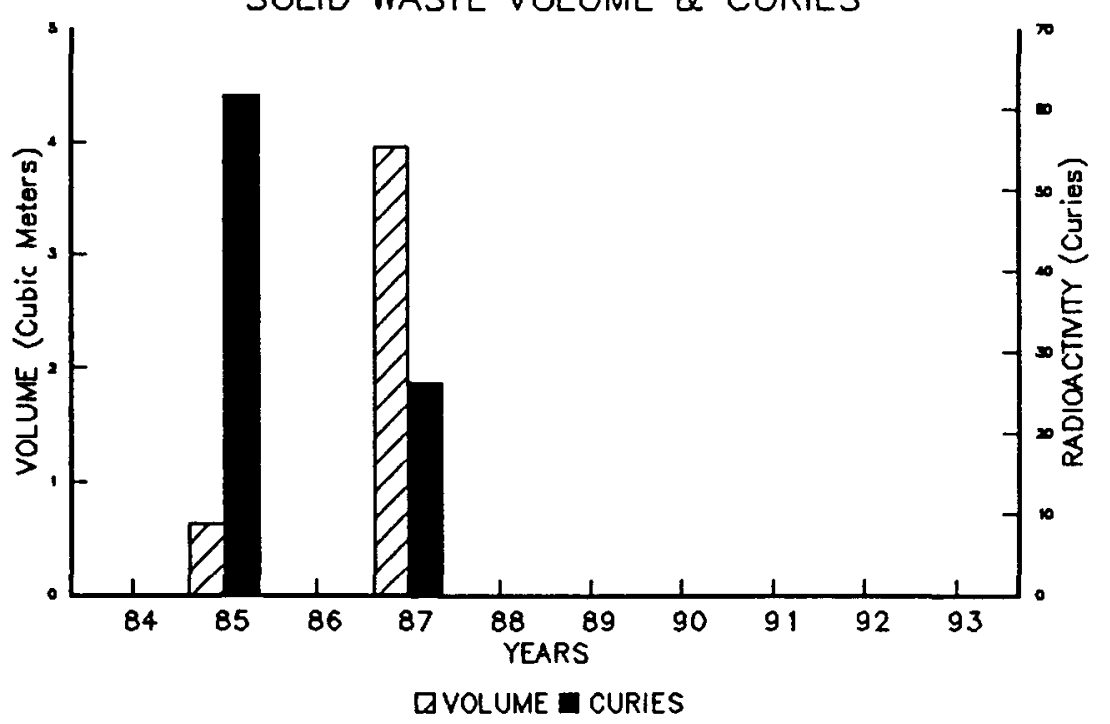




\section{INEL Solid Record-to-Date Summary TSA Waste}

CPP 1984- 1993 TRANSURANIC STORAGE AREA

SOLID WASTE VOLUME \& CURIES

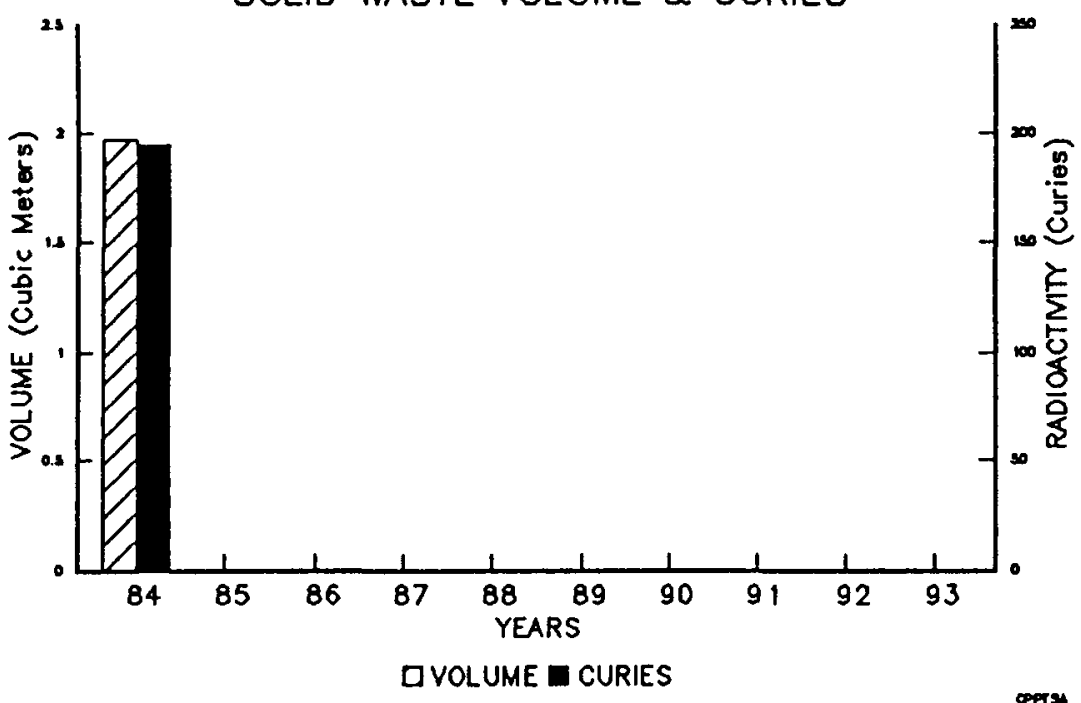

D\&D 1984-1993 TRANSURANIC STORAGE AREA SOLID WASTE VOLUME \& CURIES

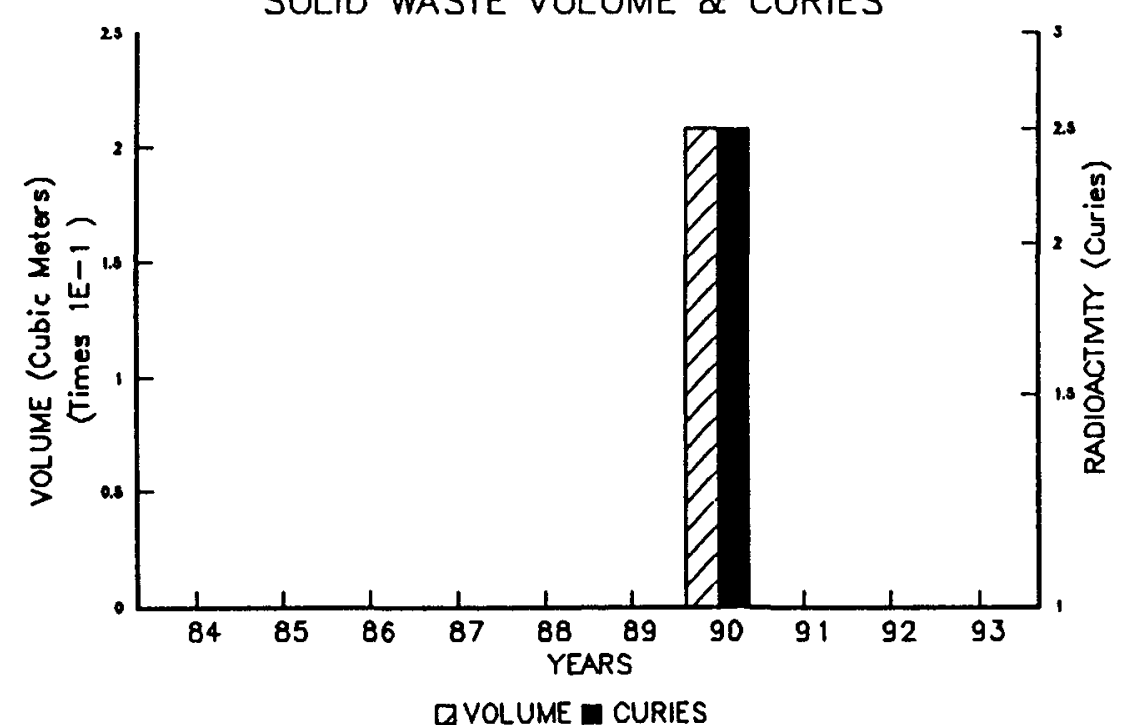

CTF 1984-1993 TRANSURANIC STORAGE AREA SOLID WASTE VOLUME \& CURIES

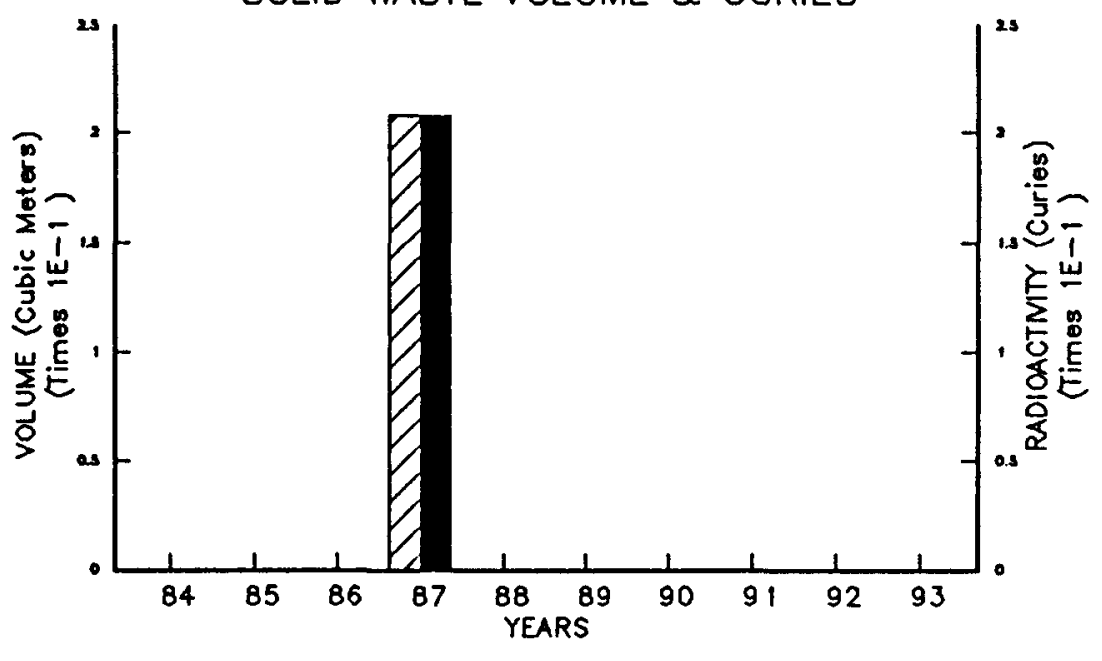

a VOLUME Q CURIES

arrs

MRC 1984-1993 TRANSURANIC STORAGE AREA SOLID WASTE VOLUME \& CURIES

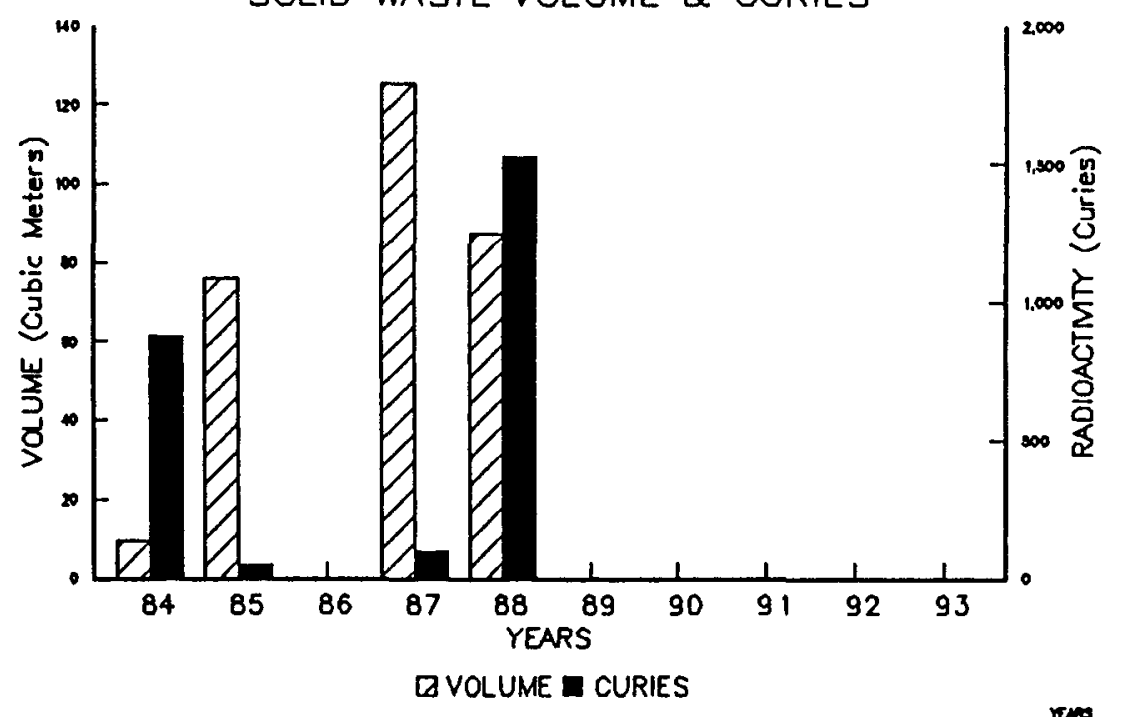




\section{INEL Solid Record-to-Date Summary TSA Waste}
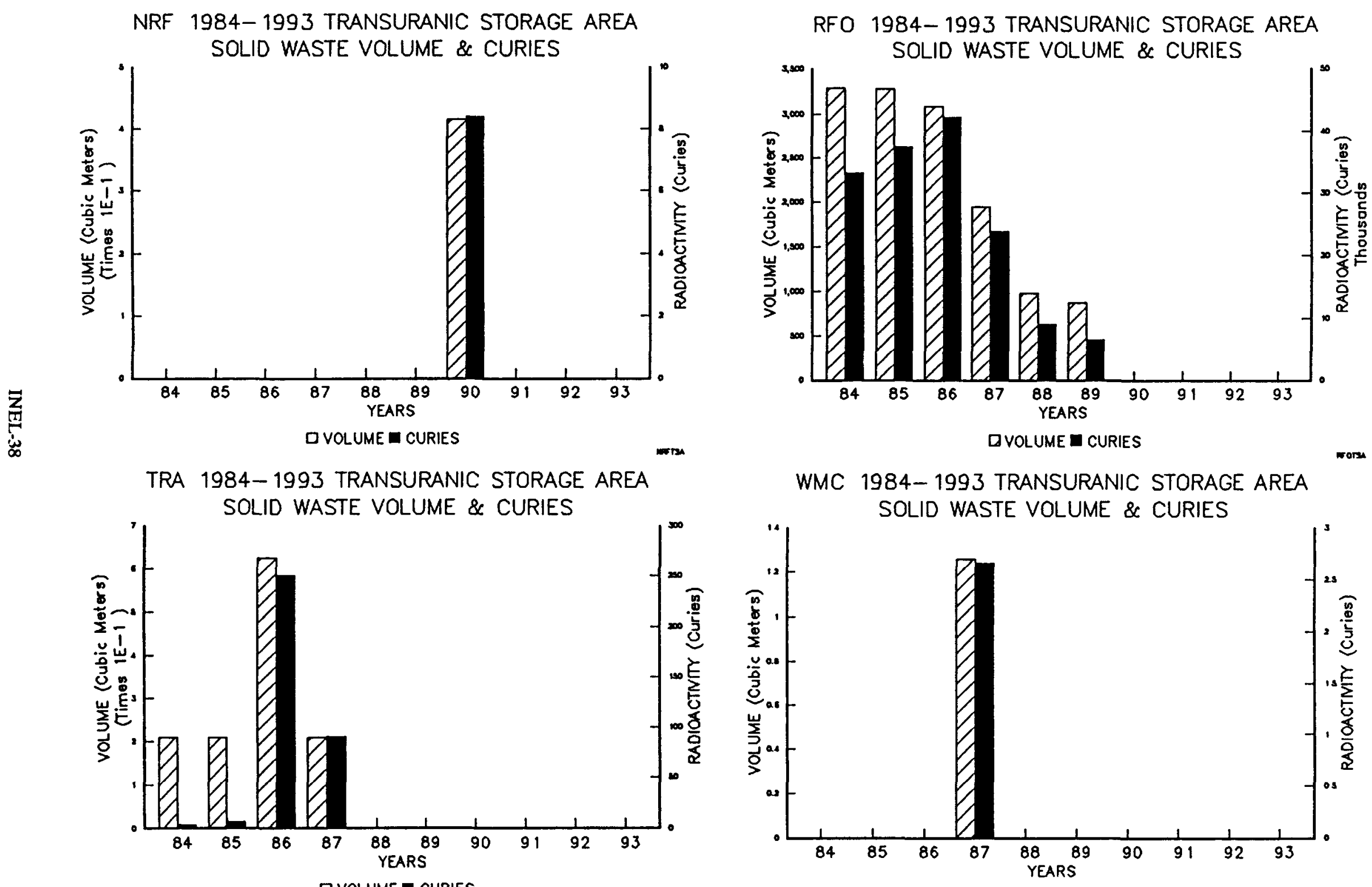

WMC 1984-1993 TRANSURANIC STORAGE AREA

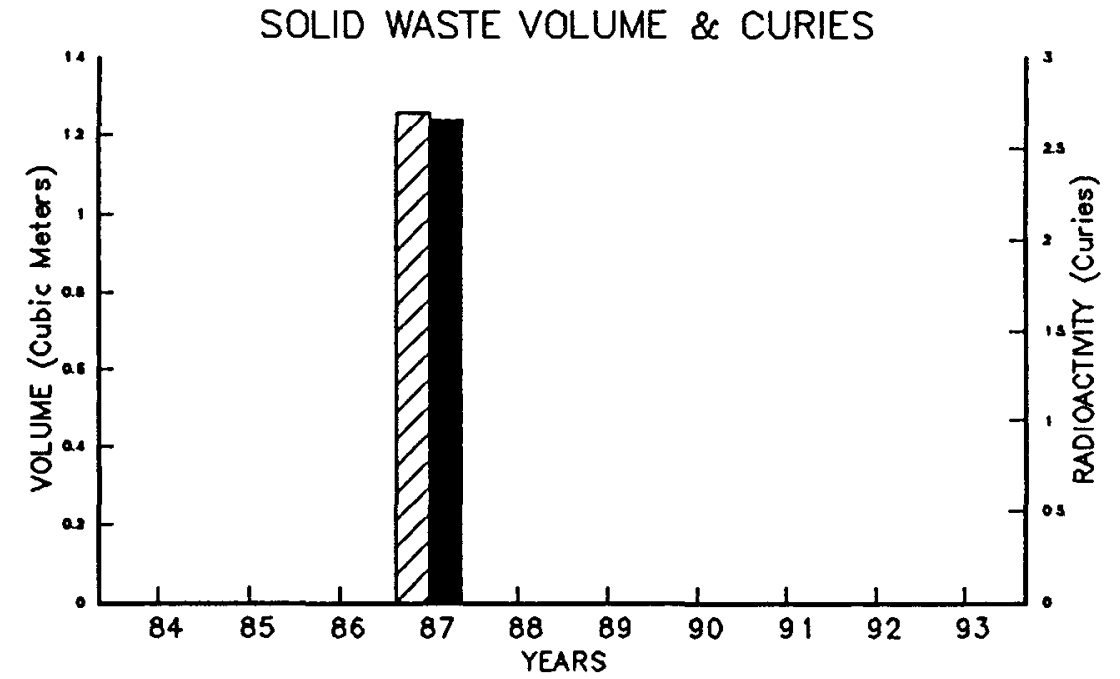

mans

DVOLUME I CURIES 
FILE: RPT22F NOMAD B1 INEL VMESA CMS7 at PUT 9301.

SCHEDULE NO. P6IPYO22-F

CPP HIGH-LEVEL LIQUID/SOLID WUSTE STORAGE

RECORD-TO-DATE

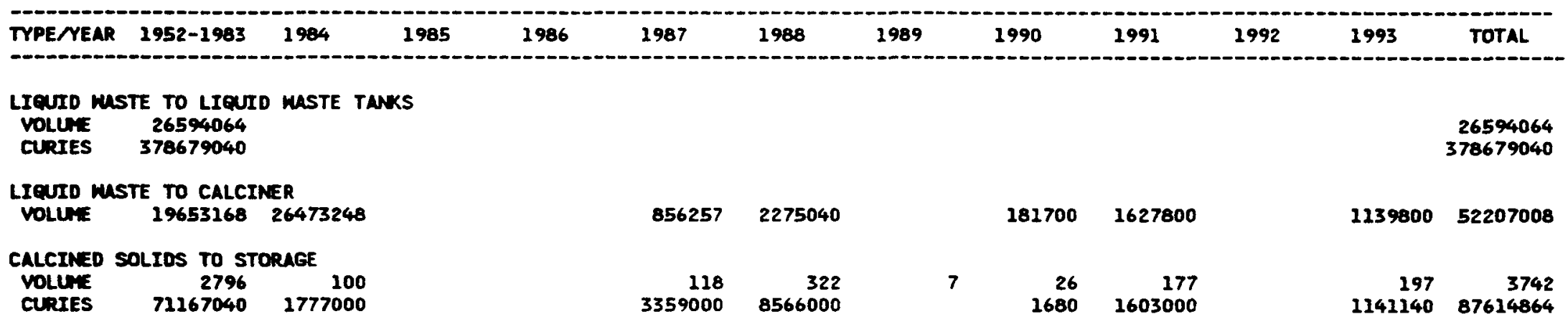

LIEUID VOLUNE IN LITERS

SOLID VOLUNE IN CUBIC METERS

LIQUID MUSTES KERE GENERATED BEGINNING IN 1953;

HONEVER, THE WUSTE CALCINUTION PROCESS DID NOT BEGIN UNTIL 1963.

DETAILS MAY NOT ADD UP TO TOTALS BECAUSE OF RONNDING 
CPP High-Level Liquid/Solid Waste Storage Record-to-Date
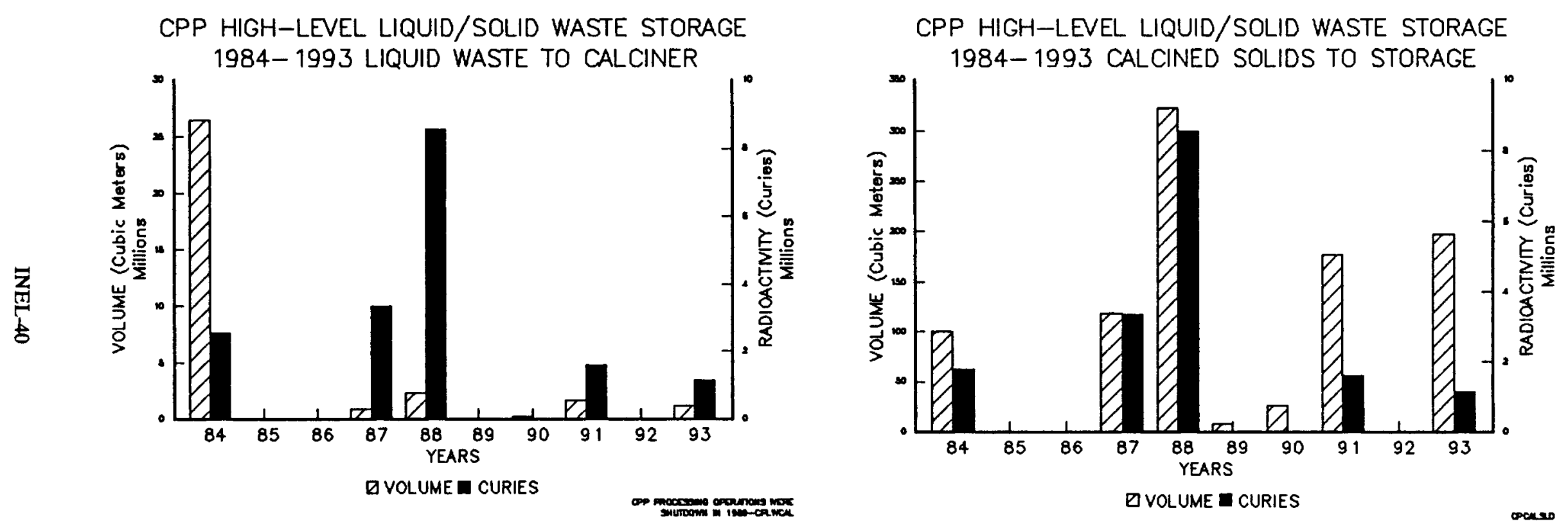
MERF MASTE

\begin{tabular}{|c|c|c|c|c|c|c|c|c|c|c|c|c|c|}
\hline AREA & NEAR & $1952-1983$ & 1984 & 1985 & 1986 & 1987 & 1988 & 2989 & 1990 & 1991 & 1992 & 1993 & TOTAL \\
\hline \multicolumn{14}{|l|}{ ANL } \\
\hline & $\begin{array}{l}\text { VOLUME } \\
\text { CURIES }\end{array}$ & & & $\begin{array}{l}37 \\
<1\end{array}$ & $\begin{array}{l}84 \\
<1\end{array}$ & $\begin{array}{r}115 \\
<1\end{array}$ & $\begin{array}{r}231 \\
<1\end{array}$ & $\begin{array}{r}221 \\
<1\end{array}$ & $\begin{array}{r}150 \\
<1\end{array}$ & $\begin{array}{r}106 \\
<1\end{array}$ & $\begin{array}{l}81 \\
<1\end{array}$ & & $\begin{array}{r}1026 \\
2\end{array}$ \\
\hline \multicolumn{14}{|l|}{ ARA } \\
\hline & VOLUANE & & & $<1$ & 6 & & 27 & & & & & & 34 \\
\hline & CURIES & & & $<1$ & $<1$ & & $<1$ & & & & & & $<1$ \\
\hline \multicolumn{14}{|l|}{ CFA } \\
\hline & VOLUAE & & 10 & 90 & 78 & 104 & 66 & 37 & 74 & $<1$ & 3 & 20 & 483 \\
\hline & CURIES & & $<1$ & $<1$ & $<1$ & $<1$ & $<1$ & $<1$ & $<1$ & $<1$ & $<1$ & $<1$ & $<1$ \\
\hline \multicolumn{14}{|l|}{ CPP } \\
\hline & VOLUME & & & 245 & 898 & 1095 & 1005 & 1094 & 1439 & 892 & 77 & 55 & 6800 \\
\hline & CURIES & & & $<1$ & 2 & 4 & 4 & 4 & 7 & 3 & $<1$ & $<1$ & 24 \\
\hline \multicolumn{14}{|l|}{ CTF } \\
\hline & VOLLAME & & & & 91 & & & & & & & & 91 \\
\hline & CURIES & & & & $<1$ & & & & & & & & $<1$ \\
\hline \multicolumn{14}{|l|}{$D+D$} \\
\hline & Volune & & 57 & 3 & 342 & & & 2 & 4 & 103 & 5 & 53 & 571 \\
\hline & CURIES & & $<1$ & $<1$ & $<1$ & & & $<1$ & $<1$ & $<1$ & $<1$ & $<1$ & $<1$ \\
\hline \multicolumn{14}{|l|}{ LOF } \\
\hline & VOLUME & & & 77 & & & & & & & & & 77 \\
\hline & CURIES & & & $<1$ & & & & & & & & & $<1$ \\
\hline \multicolumn{14}{|l|}{ NRF } \\
\hline & VOLUME & & & 173 & 367 & 572 & 1119 & 674 & 1002 & 556 & 389 & 291 & 5143 \\
\hline & CURIES & & & $<1$ & $<1$ & $<1$ & 2 & 2 & 4 & 7 & 2 & 2 & 19 \\
\hline \multicolumn{14}{|l|}{ PBF } \\
\hline & VOLUAE & & & & 9 & 30 & 6 & $<1$ & 12 & & & & 58 \\
\hline & CURIES & & & & $<1$ & $<1$ & $<1$ & $<\overline{1}$ & $<1$ & & & & $<1$ \\
\hline \multicolumn{14}{|l|}{ PER } \\
\hline & VOLUME & & & $<1$ & 7 & & & 16 & 3 & & 1 & 26 & 53 \\
\hline & CURIES & & & $<1$ & $<1$ & & & $<1$ & $<1$ & & $<1$ & $<1$ & $<1$ \\
\hline \multicolumn{14}{|c|}{$\sin C$} \\
\hline & VOLLAE & & & & 38 & 130 & 178 & 225 & 186 & 181 & & & 938 \\
\hline \multirow{2}{*}{\multicolumn{14}{|c|}{ TAN }} \\
\hline & & & & & & & & & & & & & \\
\hline & VOLUME & & & 308 & 278 & 192 & 92 & 104 & 57 & 37 & & & 1067 \\
\hline & CURIES & & & $<1$ & $<1$ & $<1$ & $<1$ & $<1$ & $<1$ & $<1$ & & & $<1$ \\
\hline
\end{tabular}




\section{MERF MASTE}

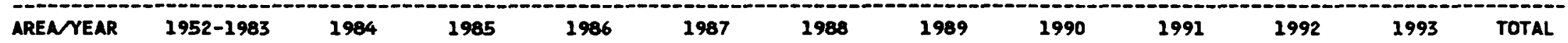

1985

1986

1987

1988

1989

1990

1991

1992

TOTAL

TRA

VOLUME
CURTES

$\begin{array}{rr}11 & 340 \\ <1 & <1\end{array}$

257
$<1$

286
$<1$

244

290

246

184

586
17

2441

$\operatorname{rac}$

VOLUME

CURIES

TOTAL

VOLUNE
CURIES

$\begin{array}{rr}2 & 4 \\ <1 & <1\end{array}$

4
$<1$

78
$<1$

1275
1

2458
2

2529
5

2967
6

2663
6

$<1 \quad 6$

19

VOLUME IN CUBIC METERS

DETAILS MAY NOT ADD UP TO TOTALS bECAUSE OF ROUNDING 


\section{INEL Solid Record-to-Date Summary WERF Waste}
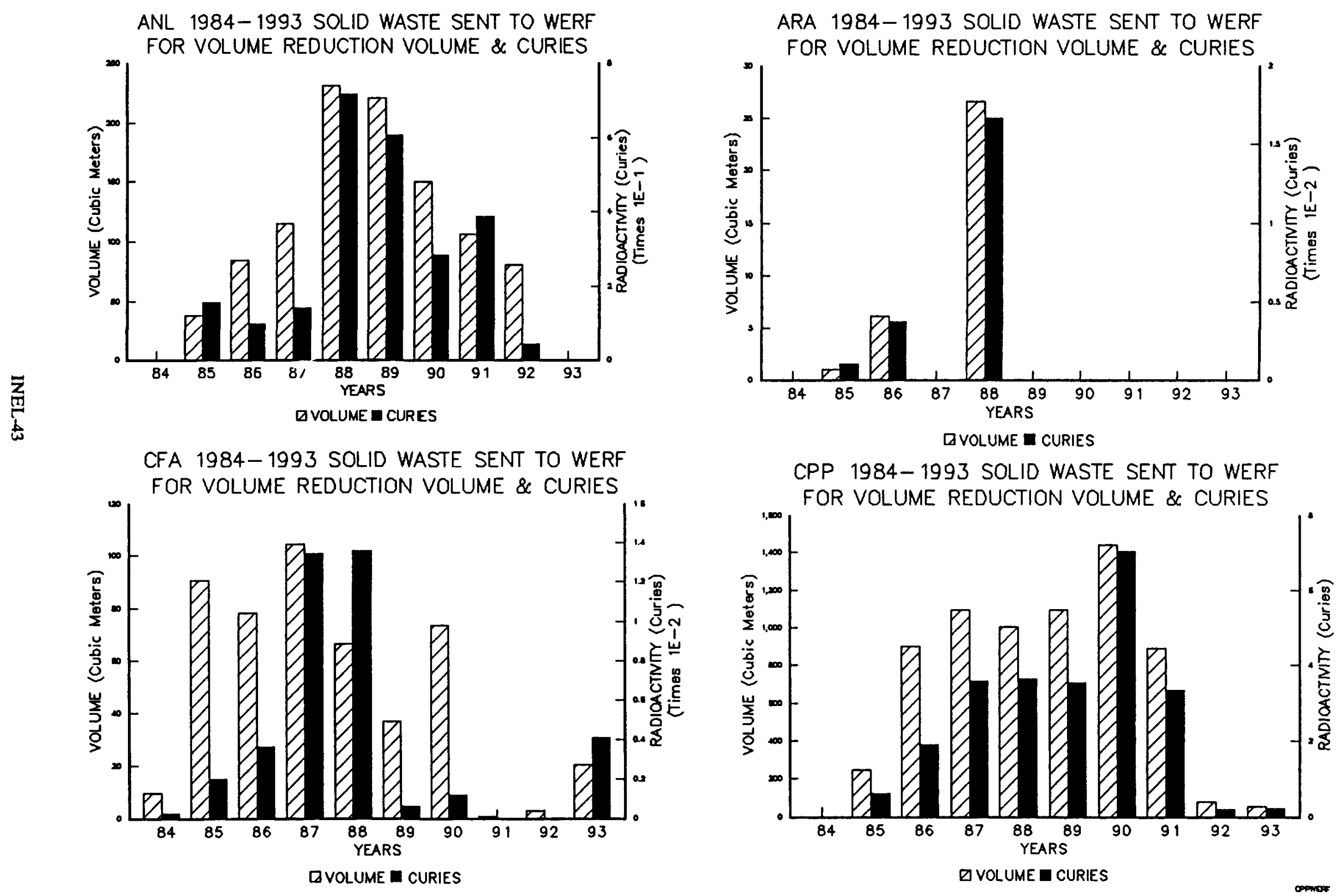


\section{INEL Solid Record-to-Date Summary WERF Waste}
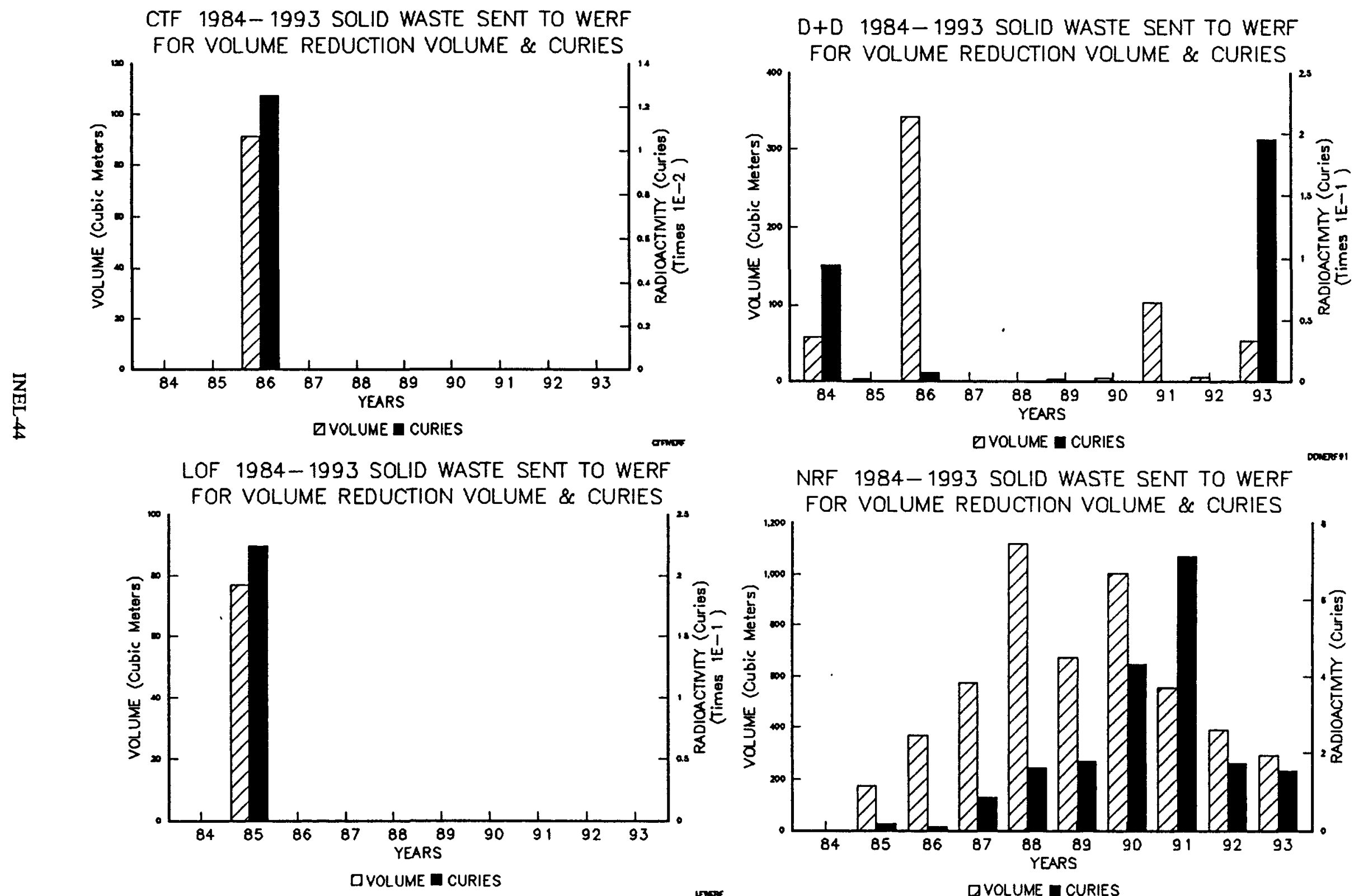

NRF 1984-1993 SOLID WASTE SENT TO WERF

mansen

FOR VOLUME REDUCTION VOLUME \& CURIES

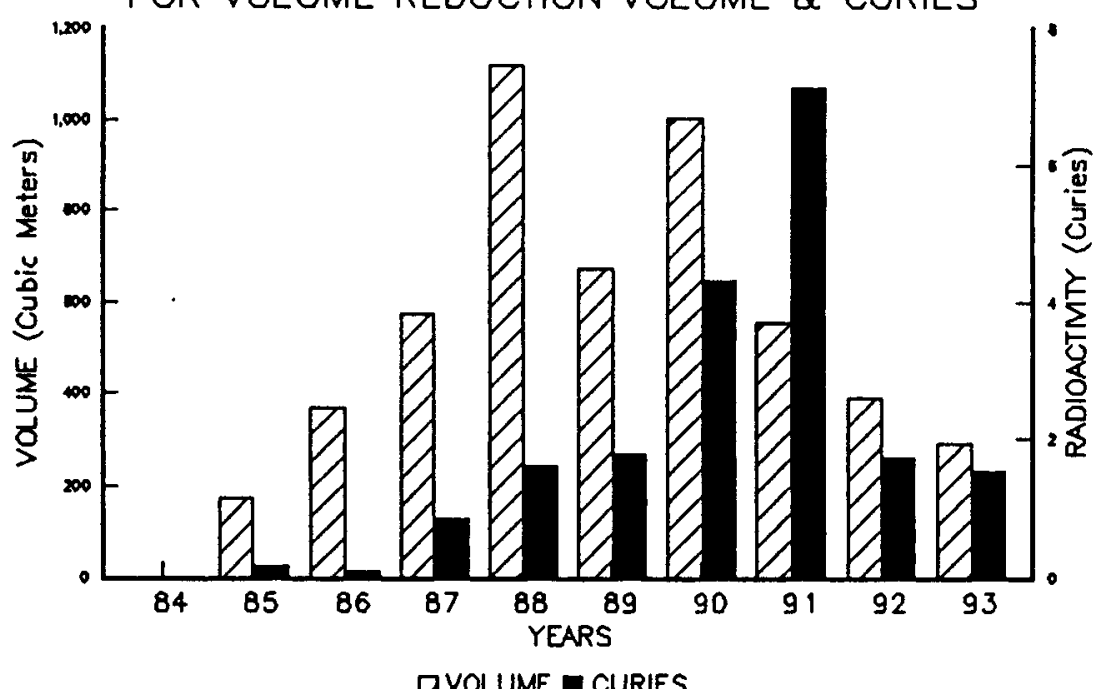


INEL Solid Record-to-Date Summary WERF Waste

PBF 1984- 1993 SOLID WASTE SENT TO WERF WASTE VOLUME \& CURIES
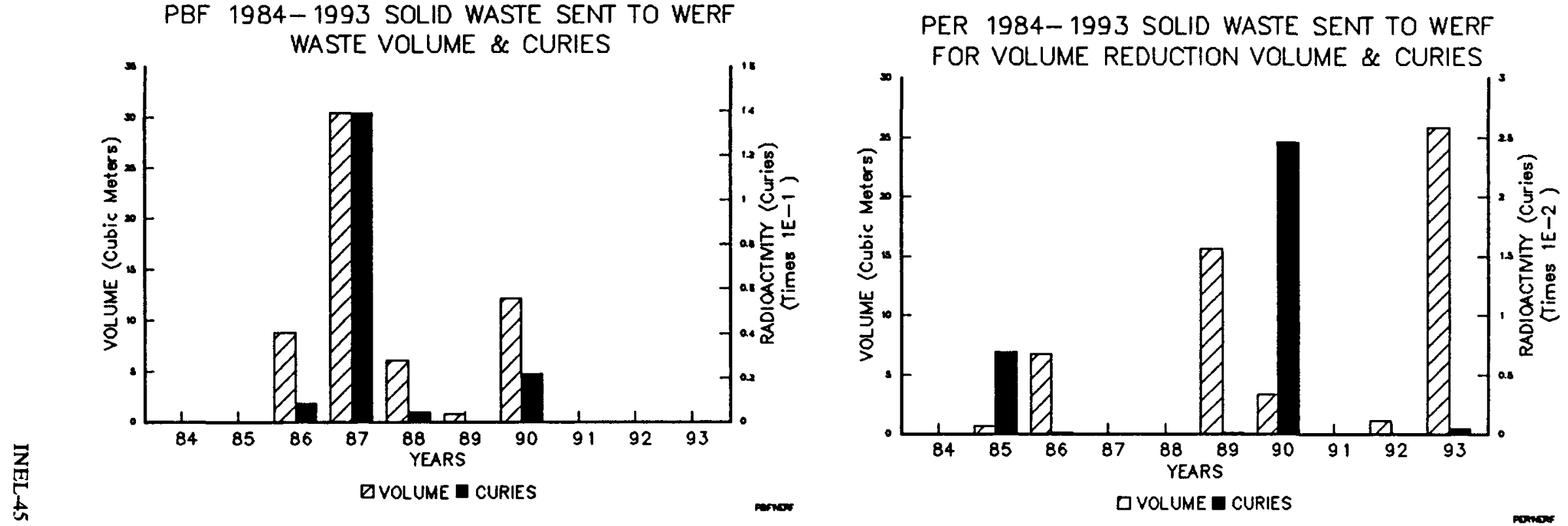

SMC 1984-1993 SOLID WASTE SENT TO WERF

FOR VOLUME REDUCTION VOLUME \& CURIES

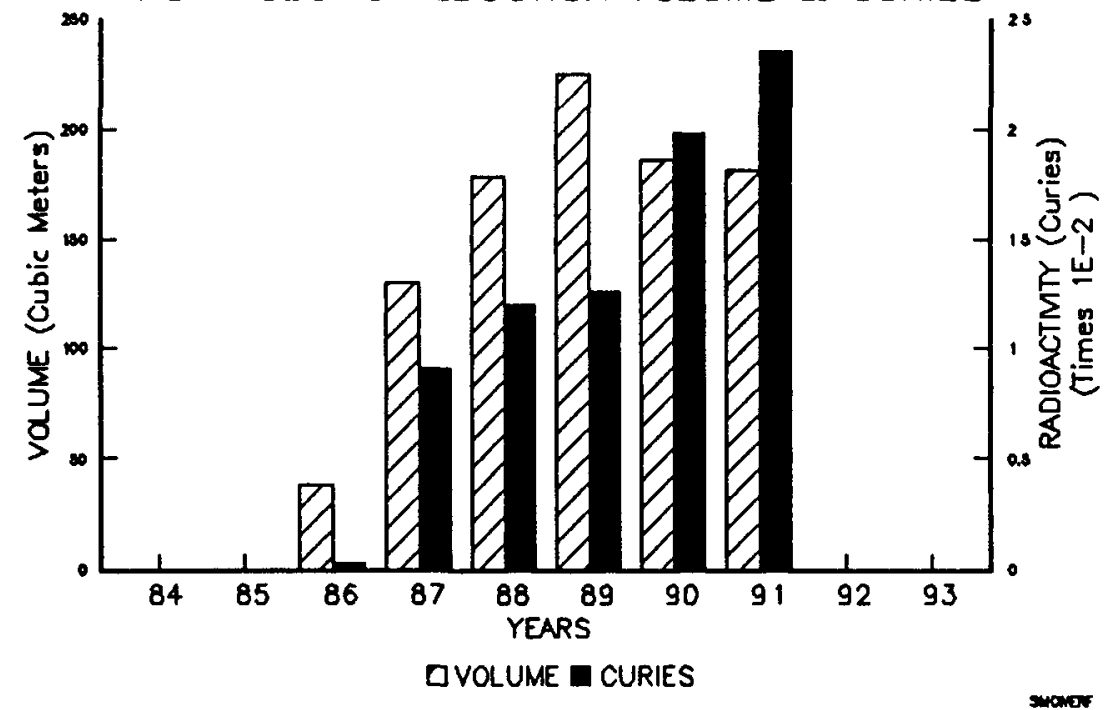


INEL Solid Record-to-Date Summary WERF Waste

TAN 1984-1993 SOLID WASTE SENT TO WERF FOR VOLUME REDUCTION VOLUME \& CURIES

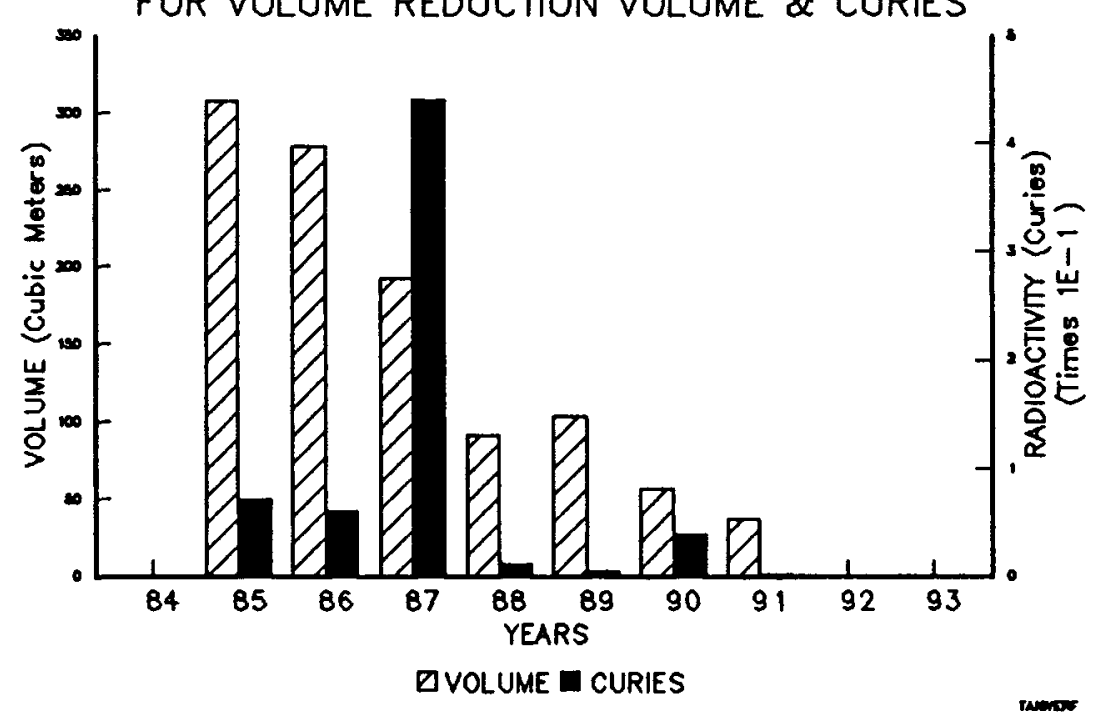

TRA 1984-1993 SOLID WASTE SENT TO WERF FOR VOLUME REDUCTION VOLUME \& CURIES

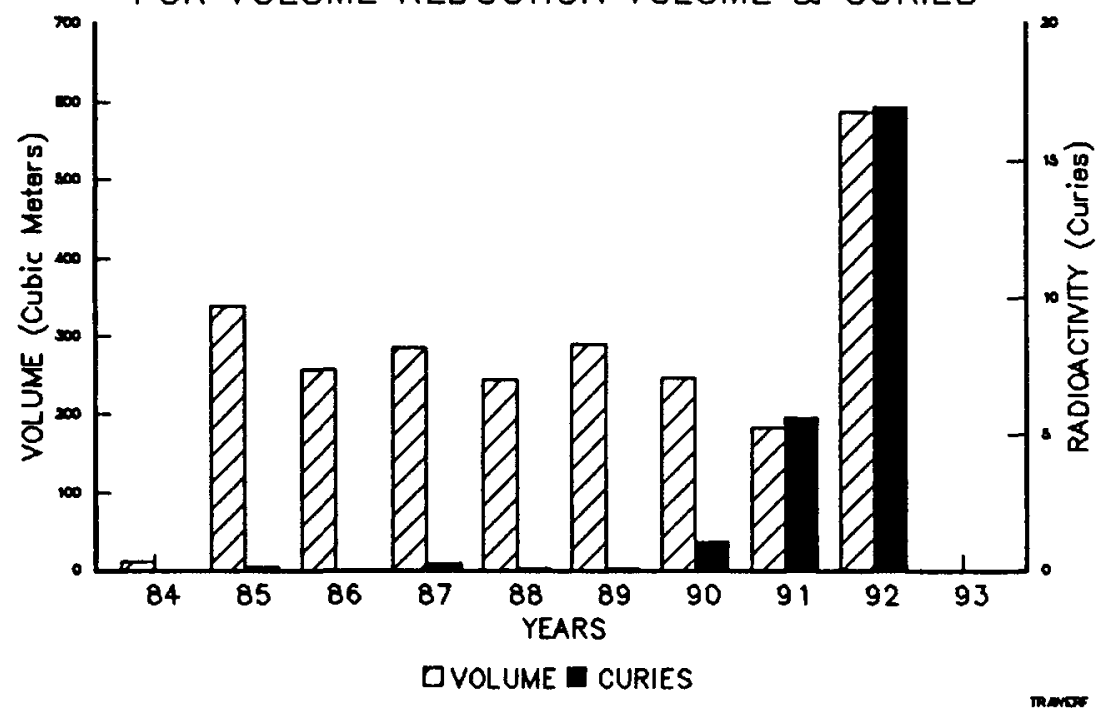

WMC 1984-1993 SOLID WASTE SENT TO WERF FOR VOLUME REDUCTION VOLUME \& CURIES

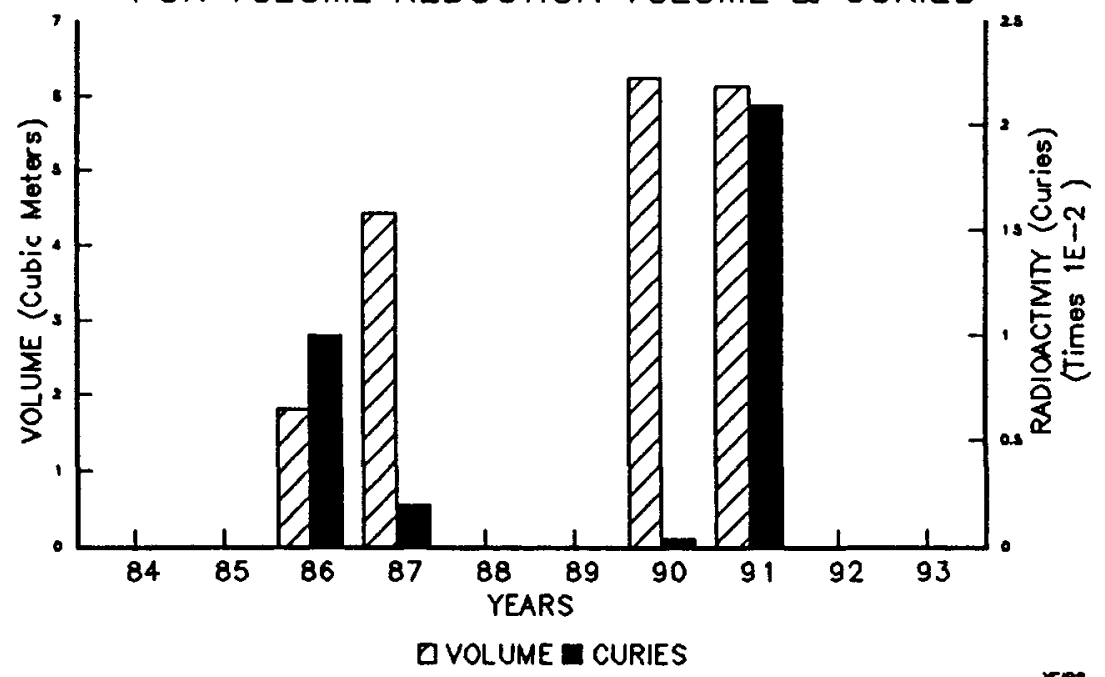

Q VOLUME $\square$ CURIES 


\section{INEL Record-to-Date Decay Summaries}

INEL Airborne Waste Decay Data and Bar Graph .................... INEL-49

INEL Liquid Waste Decay Data and Bar Graph

INEL-50

INEL Solid Disposed Waste Decay Data and Bar Graph

INEL-51 

AIRBORNE MASTE DECAY DATA

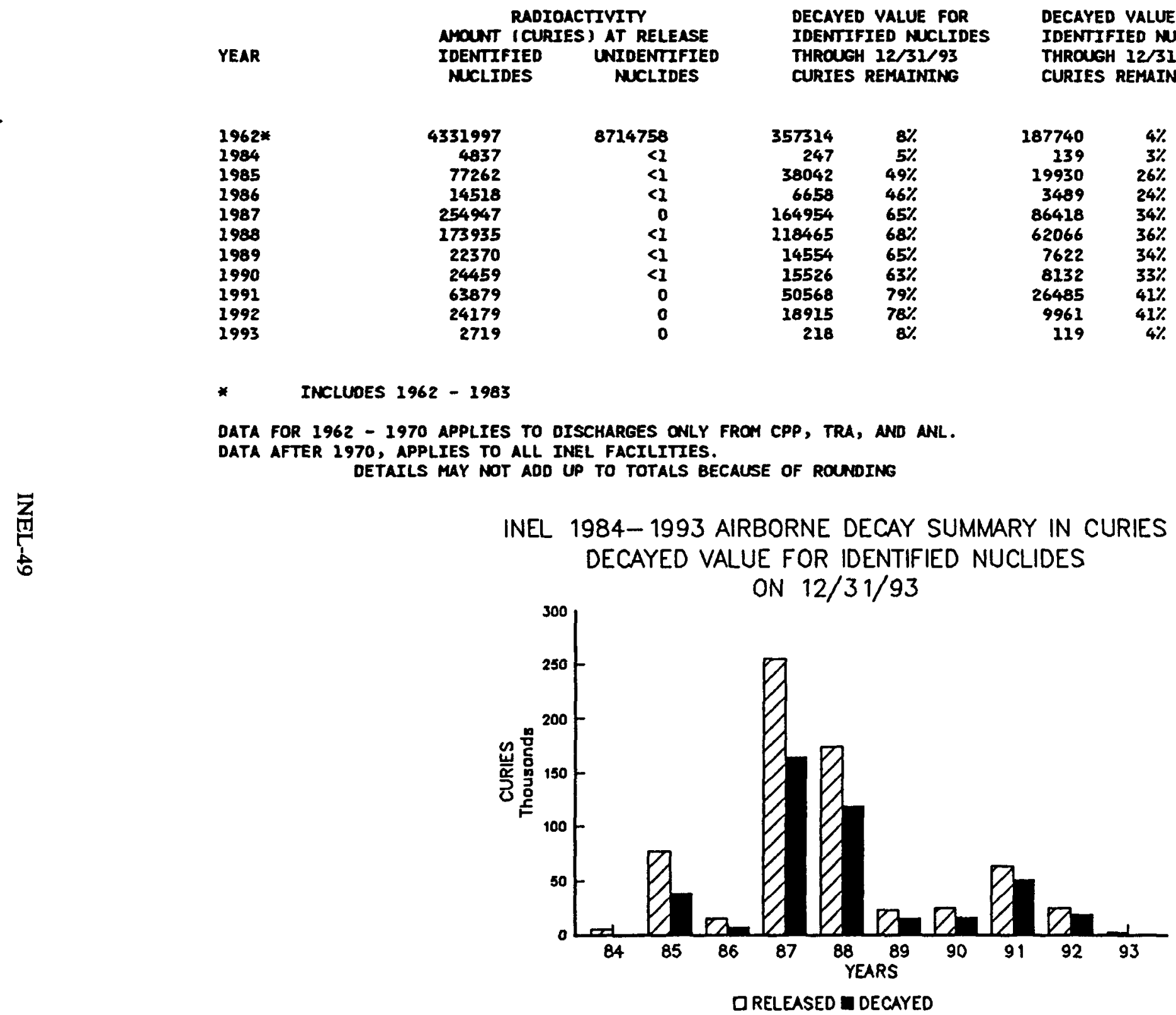


LIQUID MUSTE DECAY DATA
RADIOACTIVITY

AHOUNT (CURIES) AT RELEASE

IDENTIFIED UNIDENTIFIED

NUCLIDES

NUCLIDES

4494
191
667
346
357
270
137
189
170
187
130

29310
$<1$
$<1$
$<1$
0
0
0
0
0
0
0

DECAYED VALUE FOR IDENTIFIED MUCLIDES

THROUGH 12/31/93

CURIES REMUINING
DECAYED VALUE FOR

IDENTIFIED MUCLIDES

THROUEH 12/31/03

CURIES REMAINING

$\begin{array}{rr}3601 & 8 \% \\ 60 & 32 \% \\ 236 & 35 \% \\ 123 & 35 \% \\ 137 & 38 \% \\ 110 & 41 \% \\ 57 & 42 \% \\ 86 & 46 \% \\ 83 & 49 \% \\ 95 & 51 \% \\ 69 & 53 \%\end{array}$

* INCLUDES $1962-1983$

DATA FOR 1962 - 1970 APPLIES TO DISCHARGES ONLY FROM CPP, TRA, AND NRF. DATA AFTER 1970, APPLIES TO ALL INEL FACILITIES.

INEL 1984- 1993 LIQUID DECAY SUMMARY

IN CURIES, DECAYED VALUE FOR IDENTIFIED

NUCLIDES ON $12 / 31 / 93$

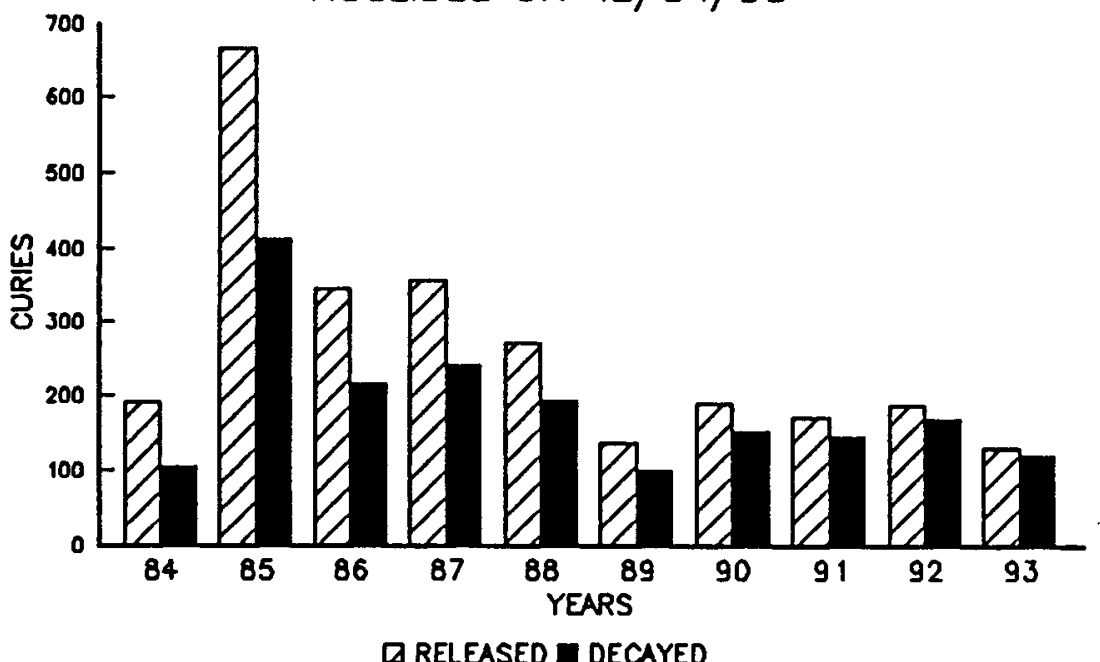


YEAR

$1962 *$
1984
1985
1986
1987
1988
1989
1990
1991
1992
1993

*

\begin{tabular}{ll}
\multicolumn{2}{c}{ RADIOACTIVITY } \\
AHOUNT & (CURIES) \\
IDENTIFIED & UNIDENTIFIED \\
MUCLIDES & NUCLIDES
\end{tabular}

\section{3}

485687

271119

94919

241678

149587

590003

207530

187565

143949

429654

5180572
355
316
165
5841
155
64
13
4
0
0

Decayed VALUE for IDENTIFIED MUCLIDES THROUGH 12/31/93 CURIES REMUINING
DECAYED VALUE FoR

IDENTIFIED NUCLIDES

THROUEH $12 / 31 / 03$ CURIES REMAINING

$\begin{array}{rr}96251 & 2 \% \\ 167154 & 34 \% \\ 67705 & 25 \% \\ 18382 & 19 \% \\ 18906 & 8 \% \\ 5180 & 3 \% \\ 124411 & 21 \% \\ 50738 & 24 \% \\ 46172 & 25 \% \\ 42647 & 30 \% \\ 207769 & 48 \%\end{array}$

INCLUDES $1962-1983$

DETAILS MAY NOT ADD UP TO TOTALS BECAUSE OF ROUNDING

INEL 1984- 1993 SOLID DISPOSED DECAY SUMMARY IN CURIES, DECAYED VALUE FOR IDENTIFIED

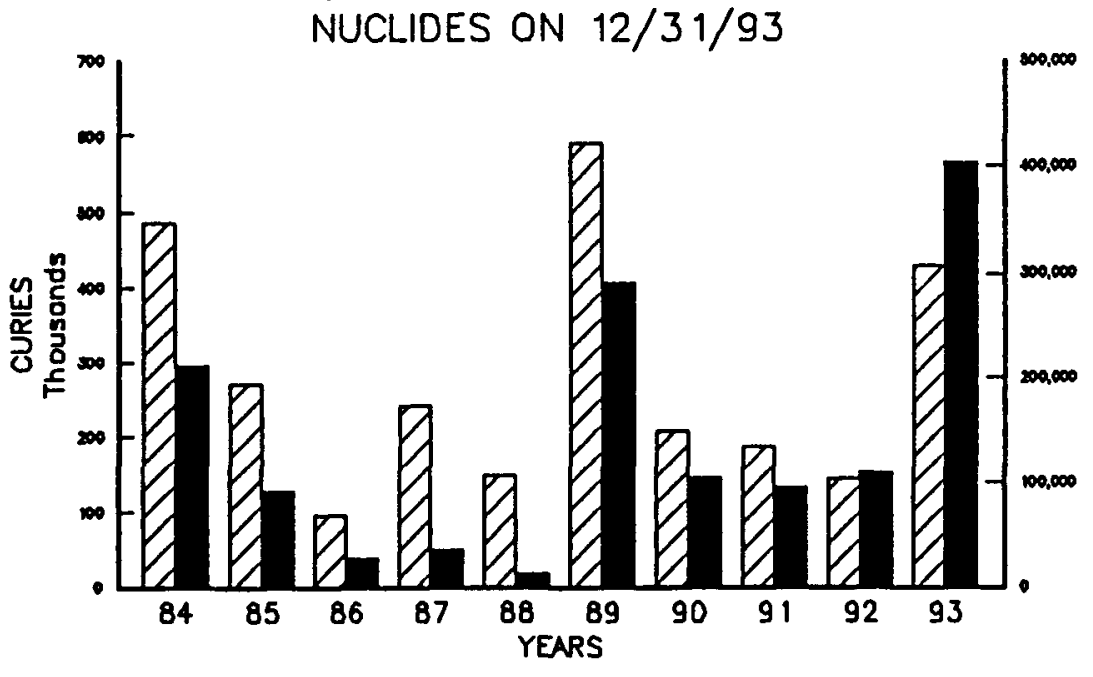

$\square$ DISPOSED $\square$ DECAYED 



\section{Area Record-to-Date Decay Summaries}

ANL-East Solid Disposed Waste Decay Data and Bar Graph $\ldots \ldots \ldots \ldots \ldots \ldots$ INEL-55

ANL-West Airborne Effluent Decay Data and Bar Graph . . . . . . . . . . . . . . INEL-56

ANL-West Liquid Effluent Data ............................ INEL-57

ANL-West Solid Disposed Waste Decay Data and Bar Graph . . . . . . . . . . INEL-58

ARA Airborne Effluent Decay Data $\ldots \ldots \ldots \ldots \ldots \ldots \ldots \ldots \ldots \ldots \ldots \ldots$ INEL-59

ARA Liquid Effluent Data $\ldots \ldots \ldots \ldots \ldots \ldots \ldots \ldots \ldots \ldots \ldots \ldots \ldots \ldots$ INEL-60

ARA Solid Disposed Waste Decay Data and Bar Graph . . . . . . . . . . . INEL-61

Bendix Solid Disposed Waste Decay Data $\ldots \ldots \ldots \ldots \ldots \ldots \ldots \ldots \ldots \ldots$ INEL-62

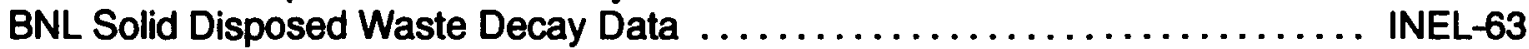

CFA Airborne Effluent Decay Data $\ldots \ldots \ldots \ldots \ldots \ldots \ldots \ldots \ldots \ldots \ldots \ldots$ INEL-64

CFA Liquid Effluent Data and Bar Graph $\ldots \ldots \ldots \ldots \ldots \ldots \ldots \ldots \ldots \ldots$ INEL-65

CFA Solid Disposed Waste Decay Data ....................... INEL-66

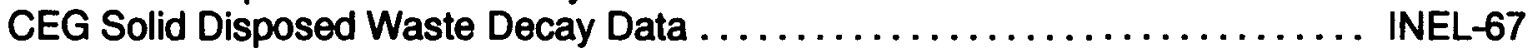

CPP Airborne Effluent Decay Data and Bar Graph . . . . . . . . . . . . . INEL-68

CPP Liquid Effluent Data and Bar Graph ..................... INEL-69

CPP Solid Disposed Waste Decay Data and Bar Graph ............... INEL-70

CTF Airborne Effluent Decay Data ........................ INEL-71

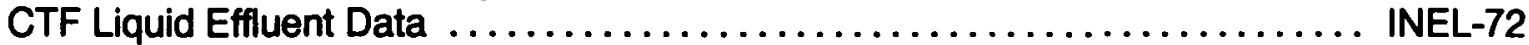

CTF Solid Disposed Waste Decay Data and Bar Graph ............... INEL-73

D\&D Solid Disposed Waste Decay Data and Bar Graph $\ldots . \ldots \ldots \ldots \ldots \ldots$. . . . . . . .

LOFT Facility Airborne Effluent Decay Data and Bar Graph .............. INEL-75

LOFT Facility Liquid Effluent Data ............................... INEL-76

LOFT Facility Solid Disposed Waste Decay Data and Bar Graph . . . . . . . . . . . INEL-77

NRF Airborne Effluent Decay Data $\ldots \ldots \ldots \ldots \ldots \ldots \ldots \ldots \ldots \ldots \ldots \ldots \ldots \ldots \ldots \ldots \ldots$ INEL -78

NRF Liquid Effluent Data $\ldots \ldots \ldots \ldots \ldots \ldots \ldots \ldots \ldots \ldots \ldots \ldots \ldots \ldots \ldots \ldots \ldots \ldots$ INEL-79

NRF Solid Disposed Waste Decay Data and Bar Graph .................. INEL-80

PBF Airborne Effluent Decay Data $\ldots \ldots \ldots \ldots \ldots \ldots \ldots \ldots \ldots \ldots \ldots \ldots \ldots \ldots \ldots \ldots$ INEL-81

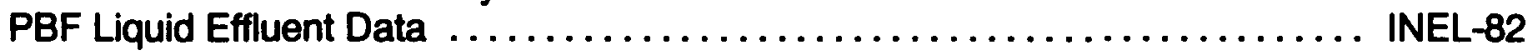

PBF Solid Disposed Waste Decay Data and Bar Graph ............... INEL-83

RFO Solid Disposed Waste Decay Data $\ldots \ldots \ldots \ldots \ldots \ldots \ldots \ldots \ldots \ldots$ INEL-84

SMC Airborne Effluent Decay Data ............................ INEL-85

SMC Solid Disposed Waste Decay Data $\ldots \ldots \ldots \ldots \ldots \ldots \ldots \ldots \ldots \ldots \ldots$ INEL-86

SPERT Airbome Effluent Decay Data $\ldots \ldots \ldots \ldots \ldots \ldots \ldots \ldots \ldots \ldots \ldots$ INEL-87

SPERT Liquid Effluent Data ............................. INEL-88

SPERT Solid Disposed Waste Decay Data $\ldots \ldots \ldots \ldots \ldots \ldots \ldots \ldots \ldots \ldots \ldots \ldots$. . . . . . . . . . .

TAN Airborne Effluent Decay Data $\ldots \ldots \ldots \ldots \ldots \ldots \ldots \ldots \ldots \ldots \ldots \ldots \ldots$ INEL-90

TAN Liquid Effluent Data ................................. INEL-91

TAN Solid Disposed Waste Decay Data and Bar Graph . . . . . . . . . . . . INEL-92

TRA Airborne Effluent Decay Data and Bar Graph ................. INEL-93

TRA Liquid Effluent Data and Bar Graph ......................... INEL-94

TRA Solid Disposed Waste Decay Data and Bar Graph ................. INEL-95

WERF Airbome Effluent Decay Data .............................. INEL-96

WERF Solid Disposed Waste Decay Data $\ldots \ldots \ldots \ldots \ldots \ldots \ldots \ldots \ldots \ldots$. . . . . . . . . . . $\ldots 7$

WMC Airborne Effluent Decay Data ............................ INEL-98

WMC Liquid Effluent Data $\ldots . \ldots \ldots \ldots \ldots \ldots \ldots \ldots \ldots \ldots \ldots \ldots \ldots \ldots \ldots$. . . . . . . . . . . . . .

WMC Solid Disposed Waste Decay Data and Bar Graph .............. INEL-100 

SOLID DISPOSED MASTE DECAY DATA

\begin{tabular}{|c|c|c|c|}
\hline \multirow[b]{3}{*}{$\mathrm{ACC}$} & \multicolumn{2}{|c|}{ RADIOACTIVITY } & DECAYEd VALUE FOR \\
\hline & Nmon: & (CURIES) & IDENTIFIED MUCLIOES \\
\hline & $\begin{array}{l}\text { IDENTIFIED } \\
\text { NUCLIDES }\end{array}$ & $\begin{array}{l}\text { UNIDENTIFIED } \\
\text { MUCLIDES }\end{array}$ & $\begin{array}{l}\text { THROUGH } 12 / 31 / 93 \\
\text { CURIES REMUINING }\end{array}$ \\
\hline
\end{tabular}

$\begin{array}{lrr}1962 * & 185 & 288 \\ 1984 & <1 & 36 \\ 1985 & 52 & 19 \\ 1986 & 4 & 6 \\ 1987 & 837 & 3 \\ 1988 & 50 & <1 \\ & 1128 & 353\end{array}$

TOTALS

INCLUDES $1962-1983$
DECAYED VALUE FOR

IDENTIFIED MUCLIDES

THROUGH 12/31/03

CURIES REMAINING

$\begin{array}{rr}8 & 5 \% \\ 21 & 57 \% \\ 22 & 42 \% \\ 2 & 55 \% \\ 331 & 39 \% \\ 22 & 44 \% \\ 385 & 34 \%\end{array}$

DETAILS MAY NOT ADD UP TO TOTALS BECAUSE OF ROUNDINE

ALE 1984- 1993 SOLID DISPOSED WASTE SUMMARY IN CURIES, DECAYED VALUE FOR IDENTIFIED NUCLIDES

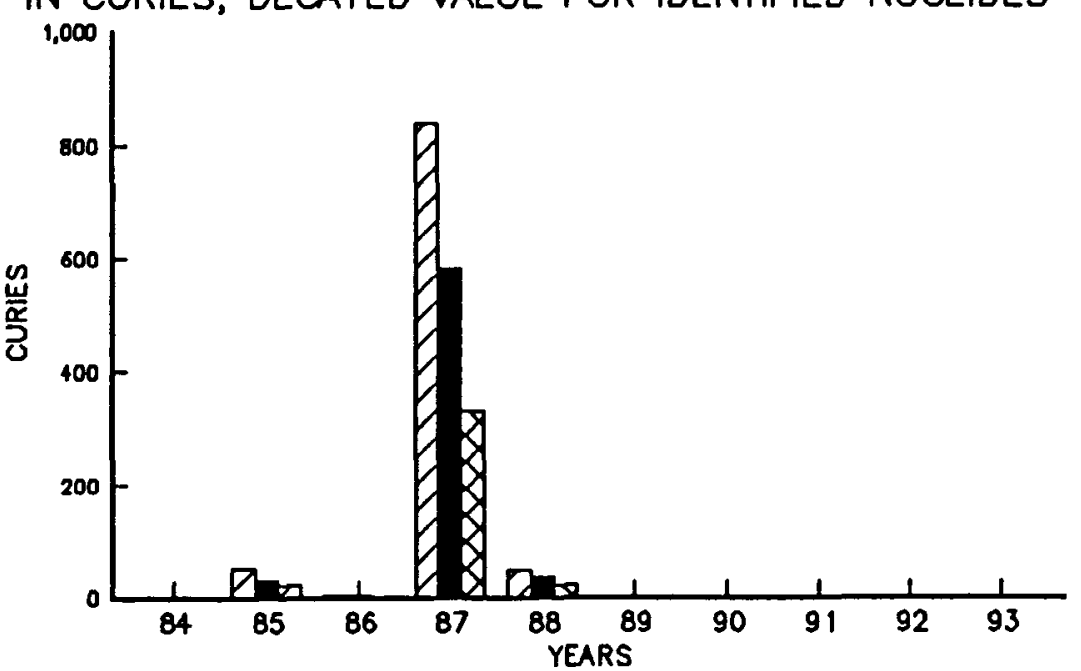

DDISPOSED $=$ DECAYED ON $12 / 31 / 93$ DECAYED ON $12 / 31 / 03$ 
AIRBORNE EFFLUENT DECAY DATA

\begin{tabular}{|c|c|c|c|c|c|c|}
\hline ACCUNDLATED IN & $\begin{array}{l}\text { RAD } \\
\text { AMOUNT ICU } \\
\text { IOENTIFIED } \\
\text { MUCLIDES }\end{array}$ & $\begin{array}{l}\text { TIVITY } \\
\text { J AT RELEASE } \\
\text { UNIDENTIFIED } \\
\text { NUCLIDES }\end{array}$ & \multicolumn{2}{|c|}{$\begin{array}{l}\text { DECAYED VALUE FOR } \\
\text { IDENTIFIED NUCLIDES } \\
\text { THROUGH } 12 / 31 / 93 \\
\text { CURIES REMAINING }\end{array}$} & \multicolumn{2}{|c|}{$\begin{array}{l}\text { DECAYED YALUE F } \\
\text { IDENTIFIED NUCL: } \\
\text { THROUEH } 12 / 31 / 0 \\
\text { CURIES REMAININ }\end{array}$} \\
\hline $\begin{array}{l}1962 * \\
1984 \\
1985 \\
1986 \\
1987 \\
1988 \\
1989 \\
1990 \\
1991 \\
1992 \\
1993\end{array}$ & $\begin{array}{r}32615 \\
250 \\
916 \\
932 \\
889 \\
618 \\
686 \\
716 \\
551 \\
739 \\
1160\end{array}$ & $\begin{array}{r}12662 \\
0 \\
0 \\
<1 \\
0 \\
<1 \\
<1 \\
<1 \\
0 \\
0 \\
0\end{array}$ & $\begin{array}{r}640 \\
18 \\
5 \\
5 \\
6 \\
3 \\
1 \\
2 \\
2 \\
170 \\
157\end{array}$ & $\begin{array}{l}2 \% \\
7 \% \\
<1 \% \\
<1 \% \\
<1 \% \\
<1 \% \\
<1 \% \\
<1 \% \\
<1 \% \\
23 \% \\
14 \%\end{array}$ & $\begin{array}{r}336 \\
10 \\
3 \\
3 \\
3 \\
1 \\
<1 \\
1 \\
<1 \\
143 \\
84\end{array}$ & $\begin{array}{l}1 \% \\
4 \% \\
<1 \% \\
<1 \% \\
<1 \% \\
<1 \% \\
<1 \% \\
<1 \% \\
<1 \% \\
19 \% \\
7 \%\end{array}$ \\
\hline TOTALS & 40073 & 12662 & 1009 & $3 \%$ & 586 & $1 \%$ \\
\hline
\end{tabular}

* INCLUdes $1962-1983$

DETAILS MAY NOT ADD UP TO TOTALS BECAUSE OF ROUNOING

ANL 1984- 1993 AIRBORNE DECAY SUMMARY IN CURIES, DECAYED VALUE FOR IDENTIFIED NUCLIDES

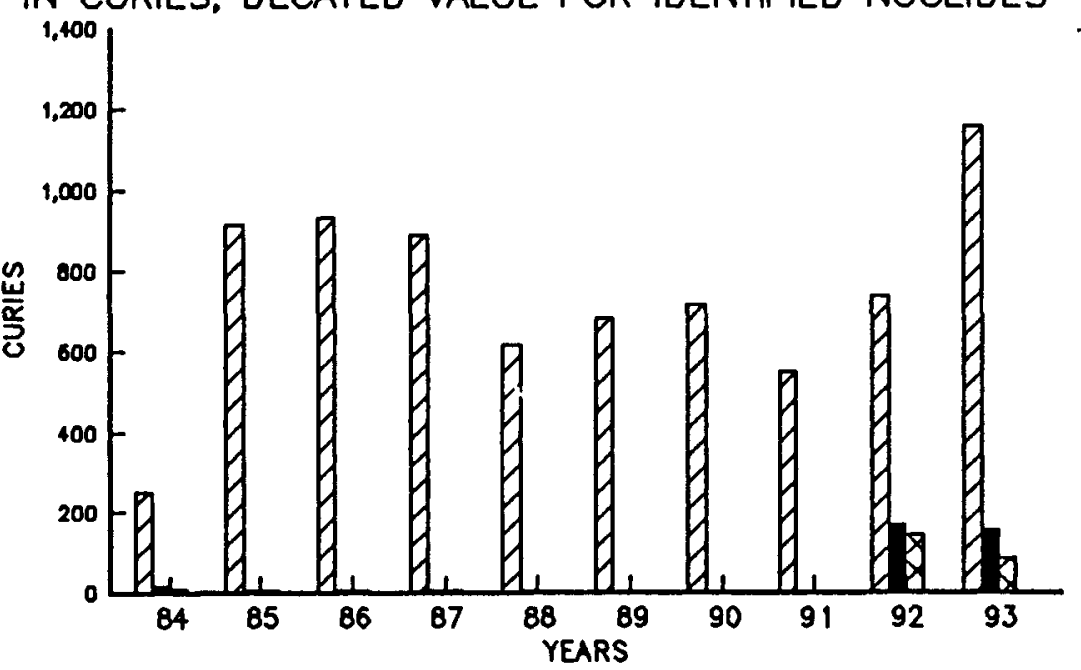

DRELEASED $\square$ DECAYED ON $12 / 31 / 93 \square$ DECAYED ON $12 / 31 / 03$ 


\section{LIQUID EFFLUENT DATA}

RADIOACTIVITY

AMONT (CURIES) AT RELEASE

IDENTIFIED UNIDENTIFIED

NUCLIDES

DECAYED VALUE FOR

IDENTIFIED NUCLIDES

THROUGH 12/31/93

CURIES REMUINING

DECAYEd VALUE FOR

IDENTIFIED NUCLIDES

THROUEH 12/31/03

CURIES REMUINING

$$
\begin{aligned}
& 1962 * \\
& 1984 \\
& 1985 \\
& 1986 \\
& 1987 \\
& 1988 \\
& 1989 \\
& 1990 \\
& 1991 \\
& 1992 \\
& 1993
\end{aligned}
$$

TOTALS

IMCLUDES $1962-1983$

2
$<1$
$<1$
$<1$
$<1$
$<1$
$<1$
$<1$
$<1$
$<1$
$<1$

4

19
0
0
0
0
0
0
0
0
0
0

19 $\begin{array}{ll}<1 & 47 \% \\ <1 & 59 \% \\ <1 & 63 \% \\ <1 & 66 \% \\ <1 & 70 \% \\ <1 & 74 \% \\ <1 & 76 \% \\ <1 & 83 \% \\ <1 & 87 \% \\ <1 & 93 \% \\ <1 & 98 \%\end{array}$

$261 \%$

$\begin{array}{ll}<1 & 28 \% \\ <1 & 33 \% \\ <1 & 36 \% \\ <1 & 38 \% \\ <1 & 40 \% \\ <1 & 42 \% \\ <1 & 43 \% \\ <1 & 47 \% \\ <1 & 49 \% \\ <1 & 53 \% \\ <1 & 56 \%\end{array}$

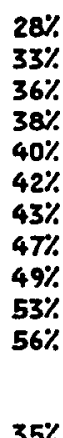

$35 \%$

DETAILS MAY NOT ADD UP TO TOTALS BECAUSE OF ROUNDING 
SOLID DISPOSED MUSTE DECAY DATA

\begin{tabular}{|c|c|c|c|c|c|c|}
\hline ACCUNLLATEO IN & $\begin{array}{r}\text { RADIO } \\
\text { NOWNT } \\
\text { IDENTIFIED } \\
\text { NUCLIDES }\end{array}$ & $\begin{array}{l}\text { CTIVITY } \\
\text { (CURIES) } \\
\text { UNIDENTIFIED } \\
\text { MCLIDES }\end{array}$ & \multicolumn{2}{|c|}{$\begin{array}{l}\text { DECAYED VALUE FOR } \\
\text { IDENTIFIED NUCLIDES } \\
\text { THROUEH } 12 / 31 / 93 \\
\text { CURIES REMAINING }\end{array}$} & \multicolumn{2}{|c|}{$\begin{array}{l}\text { DECAYED VALUE F } \\
\text { IDENTIFIED NUCL } \\
\text { THROUGH } 12 / 31 / 0 \\
\text { CURIES REMAININ }\end{array}$} \\
\hline $\begin{array}{l}1962 * \\
1984 \\
1985 \\
1986 \\
1987 \\
1988 \\
1989 \\
1990 \\
1991 \\
1992 \\
1993\end{array}$ & $\begin{array}{r}1061535 \\
119270 \\
126476 \\
57325 \\
204634 \\
142488 \\
463273 \\
130505 \\
82261 \\
92898 \\
87058\end{array}$ & $\begin{array}{r}50388 \\
20 \\
177 \\
57 \\
50 \\
128 \\
48 \\
7 \\
<1 \\
0 \\
0\end{array}$ & $\begin{array}{r}8585 \\
5346 \\
7952 \\
5246 \\
13868 \\
7506 \\
195552 \\
49734 \\
36632 \\
70663 \\
74327\end{array}$ & $\begin{array}{r}<1 \% \\
4 \% \\
6 \% \\
9 \% \\
7 \% \\
5 \% \\
42 \% \\
38 \% \\
45 \% \\
76 \% \\
85 \%\end{array}$ & $\begin{array}{r}3117 \\
1562 \\
2137 \\
1466 \\
3633 \\
1870 \\
52202 \\
13076 \\
9623 \\
18346 \\
16879\end{array}$ & $\begin{array}{r}<1 \% \\
1 \% \\
2 \% \\
3 \% \\
2 \% \\
1 \% \\
11 \% \\
10 \% \\
12 \% \\
20 \% \\
19 \%\end{array}$ \\
\hline TOTALS & 2567722 & 50875 & 475410 & $19 \%$ & 123913 & 5\%. \\
\hline
\end{tabular}

DETAILS MAY NOT ADD UP TO TOTALS BECAUSE OF ROUNDING

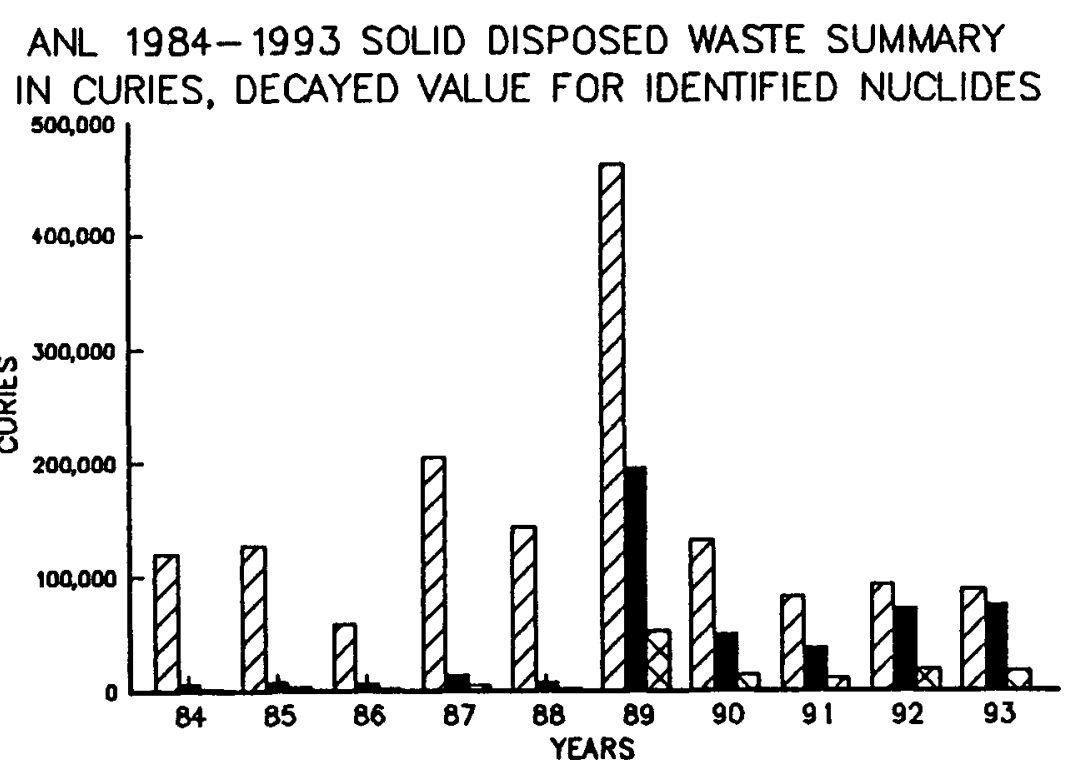

$\square$ DISPOSED $\square$ DECAYED ON $12 / 31 / 93$ DDECAYED ON $12 / 31 / 03$ 


\section{AIRBORNE EFFLUENT DECAY DATA}

\begin{tabular}{|c|c|c|c|}
\hline ACCUMULATED IN & $\begin{array}{l}\text { RONT I } \\
\text { IDENTIFI } \\
\text { MCLIDE }\end{array}$ & $\begin{array}{l}\text { RADIOACTIVITY } \\
\text { (CURIES) AT RELEASE } \\
\text { IED WITOENTIFIED } \\
\text { ES } \\
\text { MUCLIDES }\end{array}$ & $\begin{array}{l}\text { DECAYED VALUE FOR } \\
\text { IDENTIFIED MULLIDES } \\
\text { THROUSH } 12 / 31 / 93 \\
\text { CURIES REMAINING }\end{array}$ \\
\hline
\end{tabular}

decayed VALUE For

IDENTIFIED MULIDES

THROUGH 12/31/03

\begin{tabular}{|c|c|c|c|c|c|c|}
\hline \multirow{7}{*}{$\begin{array}{l}1962 \% \\
1984 \\
1985 \\
1986 \\
1987 \\
1988\end{array}$} & $<1$ & $<1$ & $<1$ & $<1 \%$ & $<1$ & $<1 \%$ \\
\hline & $<1$ & $<1$ & <1 & $<1 \%$ & $<1$ & $<1 \%$ \\
\hline & $<1$ & $<1$ & $<1$ & $4 \%$ & $<1$ & $4 \%$ \\
\hline & $<1$ & $<1$ & $<1$ & $65 \%$ & $<1$ & $52 \%$ \\
\hline & $<1$ & 0 & $<1$ & $2 \%$ & $<1$ & $<1 \%$ \\
\hline & <1 & 0 & $<1$ & $3 \%$ & $<1$ & $2 \%$ \\
\hline & $<1$ & $<1$ & $<1$ & $<1 \%$ & $<1$ & $<1 \%$ \\
\hline
\end{tabular}

\section{TOTALS}

INCLUDES 1962 - 1983

DETAILS MAY NOT ADD UP TO TOTALS BECAUSE OF ROUNDING 
LIQUTD EFFLUENT DATA

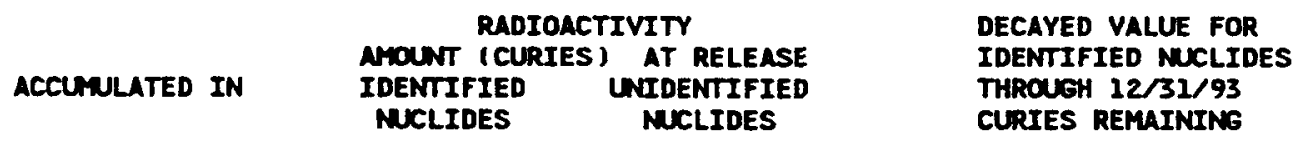

$\begin{array}{lr}1962 * & <1 \\ 1984 & 0 \\ 1986 & <1 \\ 1987 & <1 \\ 1988 & <1\end{array}$

TOTALS

$<1$

$<1$
0
0
0
0

* INCLUDES $1962-1983$

$\begin{array}{rr}0 & 0 \% \\ 0 & 0 \% \\ <1 & 12 \% \\ 0 & 0 \% \\ 0 & 0 \%\end{array}$

$<1<1 \%$
DeCAYED VALUE FOR

IDENTIFIED NUCLIDES

THROUGH 12/31/03

CURIES REMAINING

$\begin{array}{rr}0 & 0 \% \\ 0 & 0 \% \\ <1 & 10 \% \\ 0 & 0 \% \\ 0 & 0 \%\end{array}$

$<1<1 \%$ 
SOLID DISPOSED WUSTE DECAY DATA

\begin{tabular}{|c|c|c|c|c|c|c|}
\hline \multirow[b]{2}{*}{ ACCUMULATED IN } & \multicolumn{2}{|c|}{ RADIOACTIVITY } & \multicolumn{2}{|c|}{$\begin{array}{l}\text { DECAYED VALUE FOR } \\
\text { IDENTIFIED MUCLIDES }\end{array}$} & \multicolumn{2}{|c|}{$\begin{array}{l}\text { DECAYED VALUE FO } \\
\text { IDENTIFIED MUCLI }\end{array}$} \\
\hline & $\begin{array}{l}\text { IDENTIFIED } \\
\text { NUCLIDES }\end{array}$ & $\begin{array}{l}\text { UNIDENTIFIED } \\
\text { NUCLIDES }\end{array}$ & $\begin{array}{l}\text { THRO } \\
\text { CURI }\end{array}$ & $\begin{array}{l}2 / 31 / 93 \\
\text { UAINING }\end{array}$ & $\begin{array}{l}\text { THRO } \\
\text { CURI }\end{array}$ & $\begin{array}{l}\text { 2/31/03 } \\
\text { MAINING }\end{array}$ \\
\hline $\begin{array}{l}1962 * \\
1984 \\
1985 \\
1986 \\
1987\end{array}$ & $\begin{array}{r}541 \\
<1 \\
4 \\
<1 \\
0\end{array}$ & $\begin{array}{r}3477 \\
2 \\
<1 \\
<1 \\
<1\end{array}$ & $\begin{array}{r}168 \\
<1 \\
2 \\
<1 \\
0\end{array}$ & $\begin{array}{r}31 \% \\
62 \% \\
48 \% \\
49 \% \\
0 \%\end{array}$ & $\begin{array}{r}154 \\
<1 \\
2 \\
<1 \\
0\end{array}$ & $\begin{array}{r}29 \% \\
\mathbf{5 3 \%} \\
\mathbf{3} \% \% \\
\mathbf{3 7 \%} \\
0 \%\end{array}$ \\
\hline TOTALS & 545 & 3480 & 170 & $31 \%$ & 156 & $29 \%$ \\
\hline
\end{tabular}

ARA 1984-1993 SOLID DISPOSED WASTE SUMMARY

IN CURIES, DECAYED VALUE FOR IDENTIFIED NUCLIDES

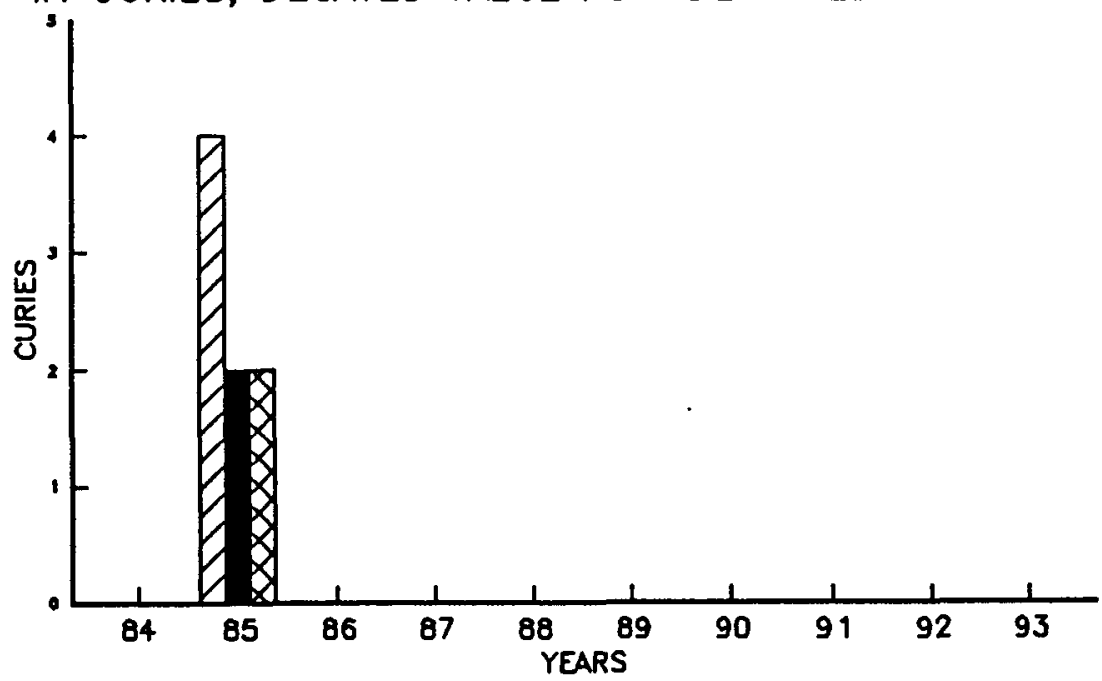

$\square$ DISPOSED $\square$ DECAYED ON $12 / 31 / 93$ D DECAYED ON $12 / 31 / 03$ 


\section{SOLIO DISPOSEO WASTE DECAY DATA}

\section{ACCUMULATED IN}

1986

TOTALS

\section{RADIOACTIVITY \\ NHOUNT (CURIES) \\ IDENTIFIED UNIDENTIFIED \\ NUCLIDES \\ MUCLIDES}

\section{$<1$}

$<1$ o

$\mathbf{0}$
DECAYEd VALUE FOR IDENTIFIED NUCLIDES THROUEH 12/31/93

CURIES REMAINING

$<1 \quad 80 \%$

$<1 \quad 80 \%$
DECAYED VALUE FOR IDENTIFIED MULIDES

THROUEH 12/31/03

CURIES REMUINTM

* INCLUdes $1962-1983$

DETAILS MAY NOT ADD UP TO TOTALS BECAUSE OF ROUNDING 
SOLID DISPOSED MUSTE DECAY DATA

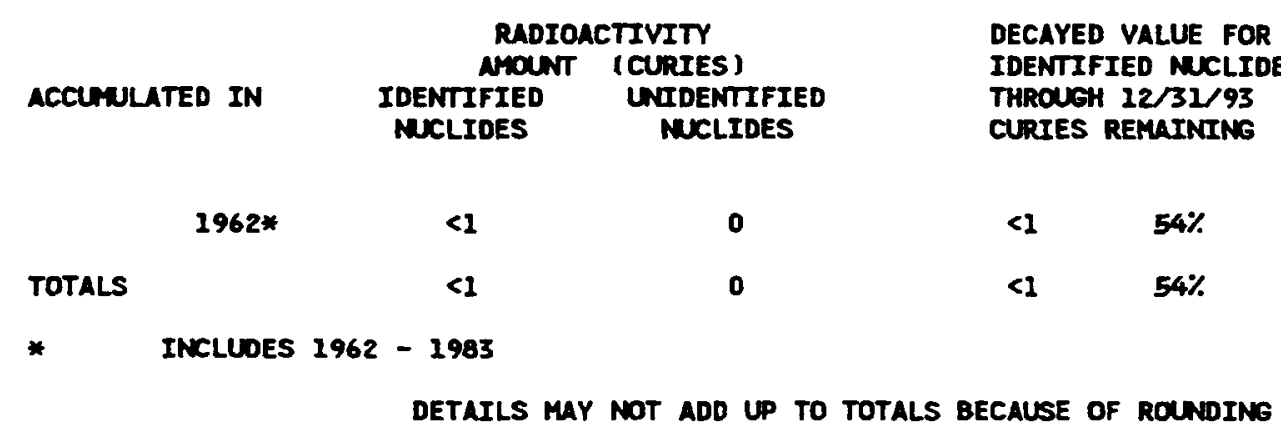

DECAYED VALUE FOR

IDENTIFIED MUCLIDES

THROUGH 12/31/03

CURIES REMAINING

$\begin{array}{ll}<1 & 30 \% \\ <1 & 30 \%\end{array}$


AIRBORNE EFFLUENT DECAY DATA

ACCUNULATED IN

RADIOACTIVITY
NOUNT (CURIES ) AT RELEASE
IDENTIFIED CNIDENTIFIED
NUCLIDES NUCLIDES

DECAYED VALUE FOR IDENTIFIED NUCLIDES THROLEH 12/31/93 CURIES REMAINING

$$
\begin{aligned}
& <1 \\
& <1 \\
& <1 \\
& <1 \\
& <1 \\
& <1 \\
& <1 \\
& <1 \\
& <1 \\
& <1 \\
& <1
\end{aligned}
$$

$<1$
$<1$
$<1$
$<1$
0
0
0
0
0
0
0

$<1$

TOTALS

* INCLUDES $1962-1983$

$\begin{array}{rr}<1 & 35 \% \\ <1 & 35 \% \\ <1 & 42 \% \\ <1 & 29 \% \\ <1 & 10 \% \\ <1 & 18 \% \\ <1 & 20 \% \\ <1 & 17 \% \\ <1 & 14 \% \\ <1 & 5 \% \\ <1 & 2 \%\end{array}$

<1 $20 \%$
DECAYEd VALUe for IDENTIFIED NUCLIDES THROUGH 12/31/03 CURIES REMAINING

$\begin{array}{rr}<1 & 24 \% \\ <1 & 20 \% \\ <1 & 23 \% \\ <1 & 17 \% \\ <1 & 4 \% \\ <1 & 7 \% \\ <1 & 8 \% \\ <1 & 7 \% \\ <1 & 7 \% \\ <1 & 3 \% \\ <1 & 2 \%\end{array}$

DETAILS MAY NOT ADD UP TO TOTALS BECAUSE OF ROUNDING 


\section{LIEUID EFFLUENT DATA}

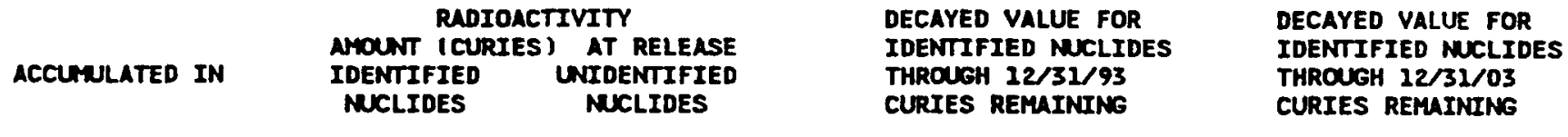

$1962 *$
1984
1985
1986
1987
1988
1989
2990
1991
1992

$\begin{array}{rr}19 & 20 \\ 5 & 0 \\ 4 & 0 \\ 3 & 0 \\ 2 & 0 \\ 1 & 0 \\ 2 & 0 \\ 2 & 0 \\ 3 & 0 \\ 3 & 0 \\ <1 & 0\end{array}$

$\begin{array}{rl}10 & 51 \% \\ 3 & 58 \% \\ 3 & 62 \% \\ 2 & 66 \% \\ 2 & 69 \% \\ <1 & 73 \% \\ 2 & 78 \% \\ 2 & 82 \% \\ 2 & 87 \% \\ 3 & 91 \% \\ <1 & 97 \%\end{array}$

$\begin{array}{ll}6 & 29 \% \\ 2 & 33 \% \\ 2 & 35 \% \\ 1 & 37 \% \\ <1 & 39 \% \\ <1 & 42 \% \\ <1 & 44 \% \\ 1 & 47 \% \\ 1 & 50 \% \\ 2 & 52 \% \\ <1 & 55 \%\end{array}$

TOTALS

45

20

28

$63 \%$

16

$36 \%$

* INCLUDES $1962-1983$

DETAILS MAY NOT ADD UP TO TOTALS BECAUSE OF ROUNDING

CFA 1984-1993 LIQUID DECAY SUMMARY

IN CURIES, DECAYED VALUE FOR IDENTIFIED NUCLIDES

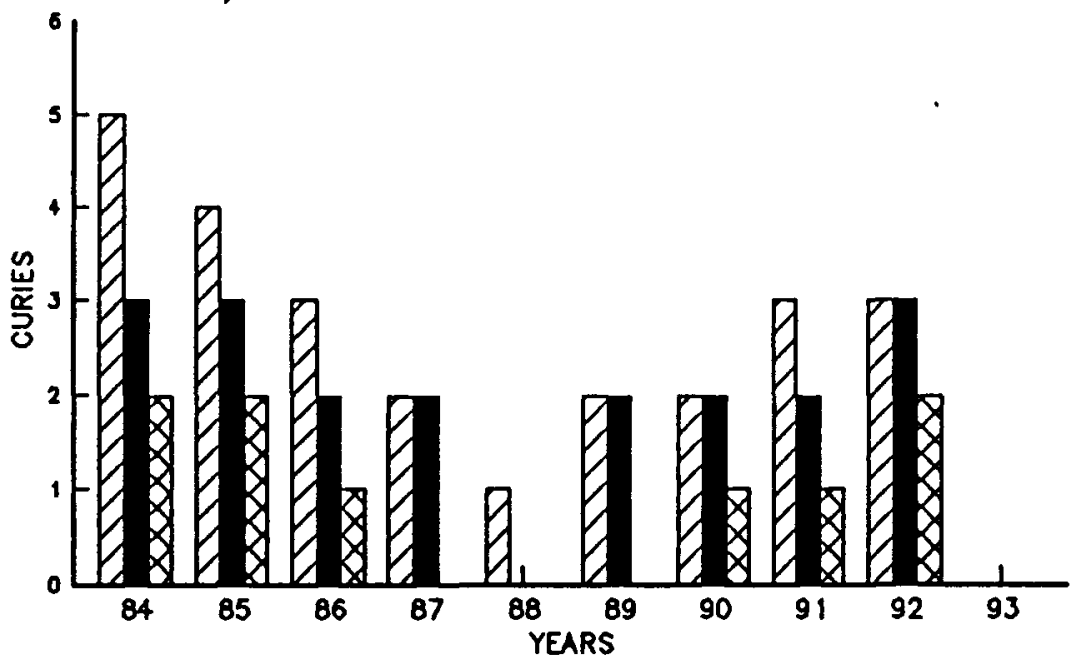

DDISPOSED $\square$ DECAYED ON 12/31/91 $\triangle$ DECAYED ON 12/31/01 
SOLID DISPOSED MASTE DECAY DATA

\begin{tabular}{|c|c|c|c|c|}
\hline ACCUMLATED IN & $\begin{array}{r}\text { RADI } \\
\text { AMOUN } \\
\text { IDENTIFIED } \\
\text { MUCLIDES }\end{array}$ & $\begin{array}{l}\text { TIVITY } \\
\text { (CURIES) } \\
\text { UNIDENIIFIED } \\
\text { MCLIDES }\end{array}$ & $\begin{array}{l}\text { DECAYED VALUE FOR } \\
\text { IDENTIFIED NUCLIDES } \\
\text { THROUEH } 12 / 31 / 93 \\
\text { CURIES REMAINING }\end{array}$ & $\begin{array}{l}\text { DECAYED VALUE FOR } \\
\text { IDENTIFIED NUCLIDES } \\
\text { THROUGH } 22 / 31 / 03 \\
\text { CURIES REMAINING }\end{array}$ \\
\hline
\end{tabular}

\begin{tabular}{|c|c|c|c|c|c|c|}
\hline 1962* & 186 & 196 & 40 & $22 \%$ & 18 & $10 \%$ \\
\hline 1984 & 0 & $<1$ & 0 & $0 \%$ & 0 & $0 \%$ \\
\hline 1985 & $<1$ & $<1$ & $<1$ & $99 \%$ & $<1$ & $98 \%$ \\
\hline 1986 & 0 & $<1$ & 0 & $0 \%$ & 0 & $0 \%$ \\
\hline 1987 & 0 & 4 & 0 & $0 \%$ & 0 & $0 \%$ \\
\hline 1988 & 0 & $<1$ & 0 & $0 \%$ & 0 & $0 \%$ \\
\hline 1989 & 0 & $<1$ & 0 & $0 \%$ & 0 & $0 \%$ \\
\hline 1990 & $<1$ & 0 & $<1$ & $77 \%$ & $<1$ & $40 \%$ \\
\hline 1992 & $<1$ & 0 & $<1$ & $96 \%$ & $<1$ & $68 \%$ \\
\hline 1993 & $<1$ & 0 & $<1$ & $99 \%$ & $<1$ & $65 \%$ \\
\hline & 186 & 200 & 40 & $22 \%$ & 18 & $10 \%$ \\
\hline
\end{tabular}

DETAILS MAY NOT ADD UP TO TOTALS BECAUSE OF ROUNDING 
Combustion Engineering - General Atomics

SOLID DISPOSED MASTE DECAY DATA

\section{ACCUNULATED IN}

1993

TOTALS

\section{RAOIOACTIVITY \\ MONT (CURIES) \\ IDENTIFIED UNIDENTIFIED \\ MCLIDES}

$\begin{array}{ll}1967 & 0 \\ 1967 & 0\end{array}$

\section{0}

0
DECAYED VALUE FOR IDENTIFIED NUCLIDES THROUGH 12/31/93 CURIES REMAINING

* INClUDES $1962-1983$

$\begin{array}{ll}1892 & 96 \% \\ 1892 & 96 \%\end{array}$

1892
DECAYEd VALUE FOR IDENTIFIED NUCLIDES THROUGH $12 / 31 / 03$

CURIES REMUINING
DETAILS MAY NOT ADd UP TO TOTALS BECAUSE OF ROUNDING 


\section{AIRBORNE EFFLUENT DECAY DATA}

RADIOACTIVITY (CURIES) AT RELEASE IDENTIFIED UNIDENTIFIED IDENTIFIED MUCLIDES

THROUGH $12 / 31 / 93$

CURIES REMAINING

decAyed VALUE For

IDENTIFIED MUCLIDES

THROUGH 12/31/03

CURIES REMUINING

$\begin{array}{lrrrrrr}1962 * & 1239535 & 6285051 & 356668 & 29 \% & 187400 & 15 \% \\ 1984 & 398 & 0 & 229 & 57 \% & 129 & 33 \% \\ 1985 & 64279 & 0 & 38030 & 59 \% & 19924 & 31 \% \\ 1986 & 10845 & 0 & 6652 & 61 \% & 3486 & 32 \% \\ 1987 & 250918 & 0 & 164945 & 66 \% & 86413 & 34 \% \\ 1988 & 170708 & 0 & 118462 & 69 \% & 62064 & 36 \% \\ 1989 & 20002 & 0 & 14552 & 73 \% & 7621 & 38 \% \\ 1990 & 20002 & 0 & 15524 & 78 \% & 8130 & 41 \% \\ 1991 & 60043 & 0 & 50566 & 84 \% & 26484 & 44 \% \\ 1992 & 20000 & 0 & 18745 & 94 \% & 9817 & 49 \% \\ 1993 & 62 & 0 & 60 & 97 \% & 34 & 55 \% \\ & 1856787 & 6285051 & 784434 & 42 \% & 411502 & 22 \%\end{array}$

DETAILS maY NOT ADD UP TO TOTALS BECAUSE OF RONDING

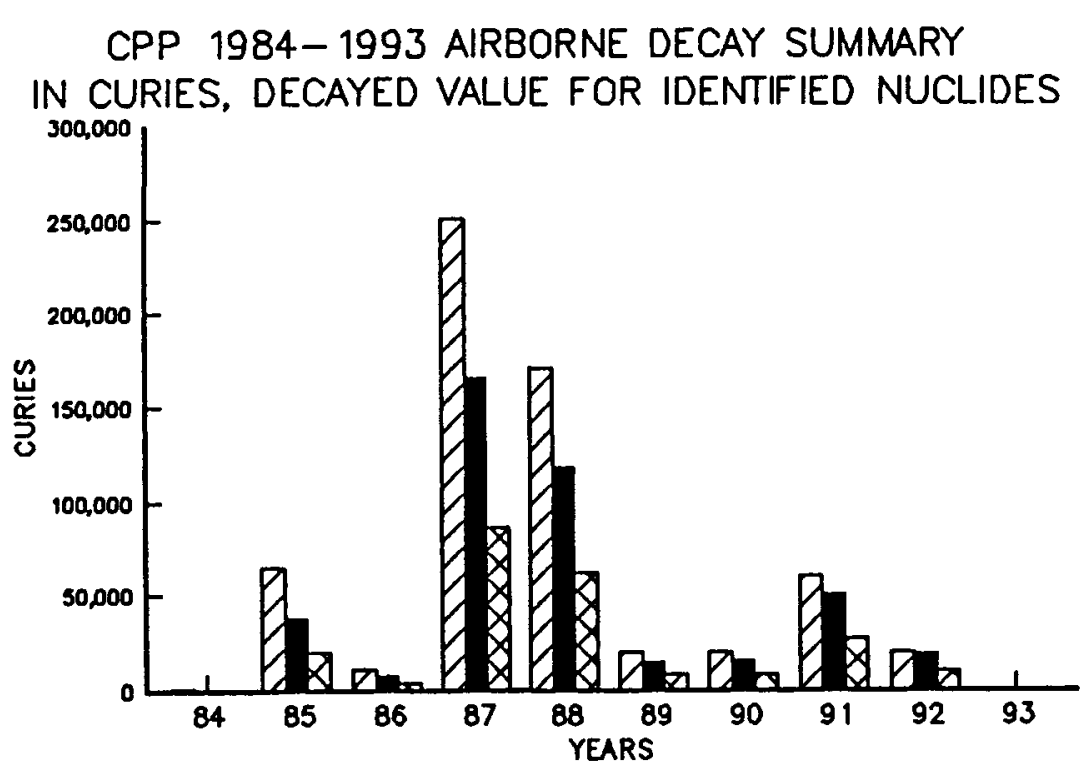

$\square$ RELEASED DECAYED ON $12 / 31 / 93 \otimes$ DECAYED ON $12 / 31 / 03$ 
LIQUID EFFLUENT DATA

\begin{tabular}{|c|c|c|c|c|c|c|}
\hline ACCUMULATED IN & \multicolumn{2}{|c|}{$\begin{array}{l}\text { RADIOACTIVITY } \\
\text { AMOUNT ICURIES ) AT RELEASE } \\
\text { IDENTIFIED } \\
\text { MUCLIDES } \\
\text { NUENTIFIED } \\
\text { NUCLIDES }\end{array}$} & \multicolumn{2}{|c|}{$\begin{array}{l}\text { DECAYED VALUE FOR } \\
\text { IDENTIFIED MUCLIDES } \\
\text { THROUGH } 12 / 31 / 93 \\
\text { CURIES REMINING }\end{array}$} & \multicolumn{2}{|c|}{$\begin{array}{l}\text { DECAYED VALUE F } \\
\text { IDENTIFIED MUCL } \\
\text { THROUGH } 12 / 31 / 0 \\
\text { CURIES REMAININ }\end{array}$} \\
\hline $\begin{array}{l}1962 * \\
1984 \\
1985 \\
1986 \\
1987 \\
1988 \\
1989 \\
1990 \\
1991 \\
1992 \\
1993\end{array}$ & $\begin{array}{r}19803 \\
13 \\
393 \\
251 \\
216 \\
89 \\
<1 \\
<1 \\
2 \\
<1 \\
<1\end{array}$ & $\begin{array}{r}2484 \\
0 \\
0 \\
0 \\
0 \\
0 \\
0 \\
0 \\
0 \\
0 \\
0\end{array}$ & $\begin{array}{r}3984 \\
7 \\
249 \\
162 \\
149 \\
65 \\
<1 \\
<1 \\
2 \\
<1 \\
<1\end{array}$ & $\begin{array}{l}20 \% \\
58 \% \\
63 \% \\
65 \% \\
69 \% \\
73 \% \\
44 \% \\
78 \% \\
86 \% \\
89 \% \\
49 \%\end{array}$ & $\begin{array}{r}2280 \\
4 \\
142 \\
92 \\
85 \\
37 \\
<1 \\
<1 \\
1 \\
<1 \\
<1\end{array}$ & $\begin{array}{l}12 \% \\
33 \% \\
36 \% \\
37 \% \\
39 \% \\
42 \% \\
31 \% \\
47 \% \\
49 \% \\
51 \% \\
35 \%\end{array}$ \\
\hline TOTALS & 20769 & 2484 & 4620 & $22 \%$ & 2642 & $13 \%$ \\
\hline
\end{tabular}

* INCLUdes $1962-1983$

DETAILS MAY NOT ADD UP TO TOTALS BECAUSE OF ROUNDING

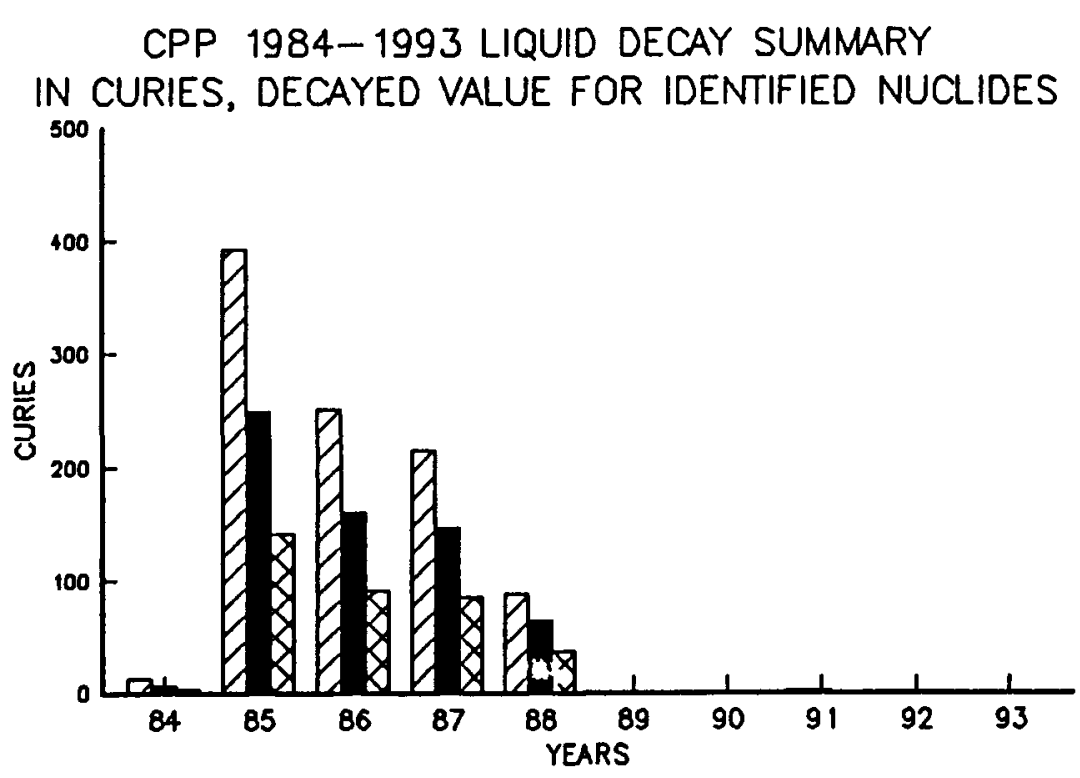

DDISPOSED D DECAYED ON $12 / 31 / 93$ Q DECAYED ON 12/31/03 
SOLID DISPOSED WUSTE DECAY DATA

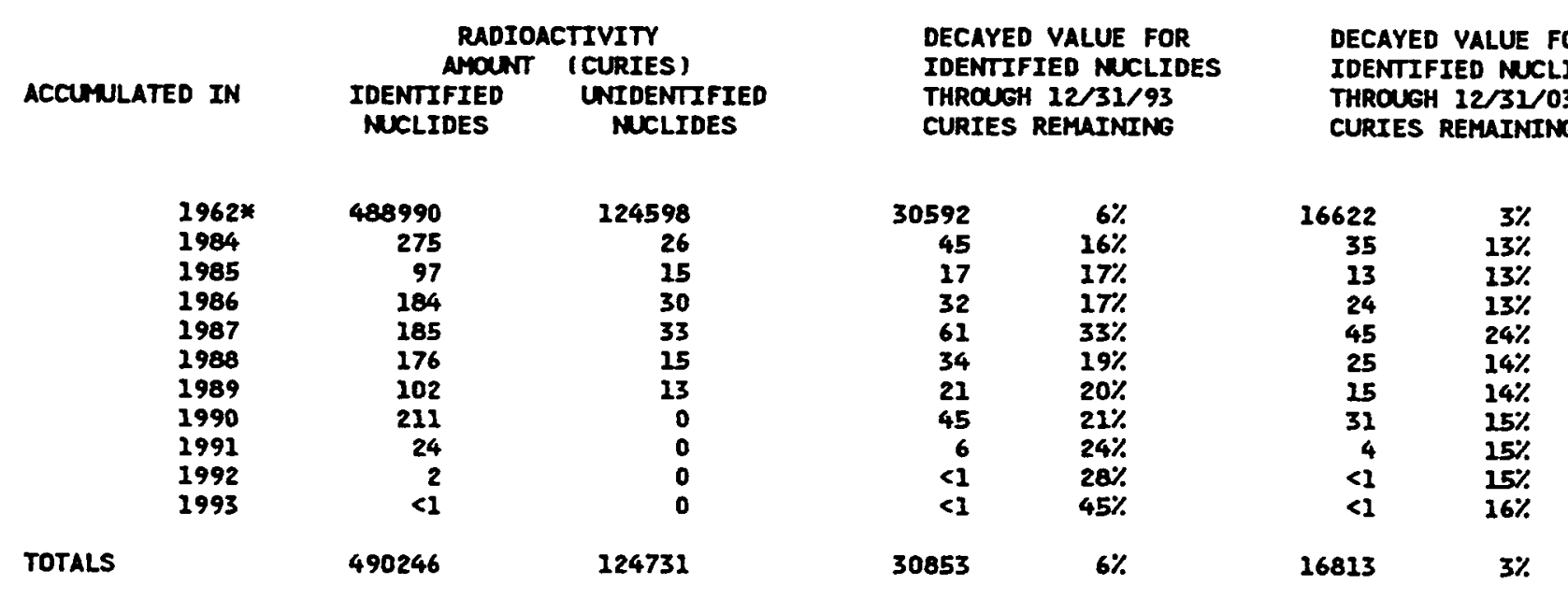

* INClUdes 1962 - 1983

DETAILS MAY NOT ADD UP TO TOTALS BECAUSE OF ROUNDING

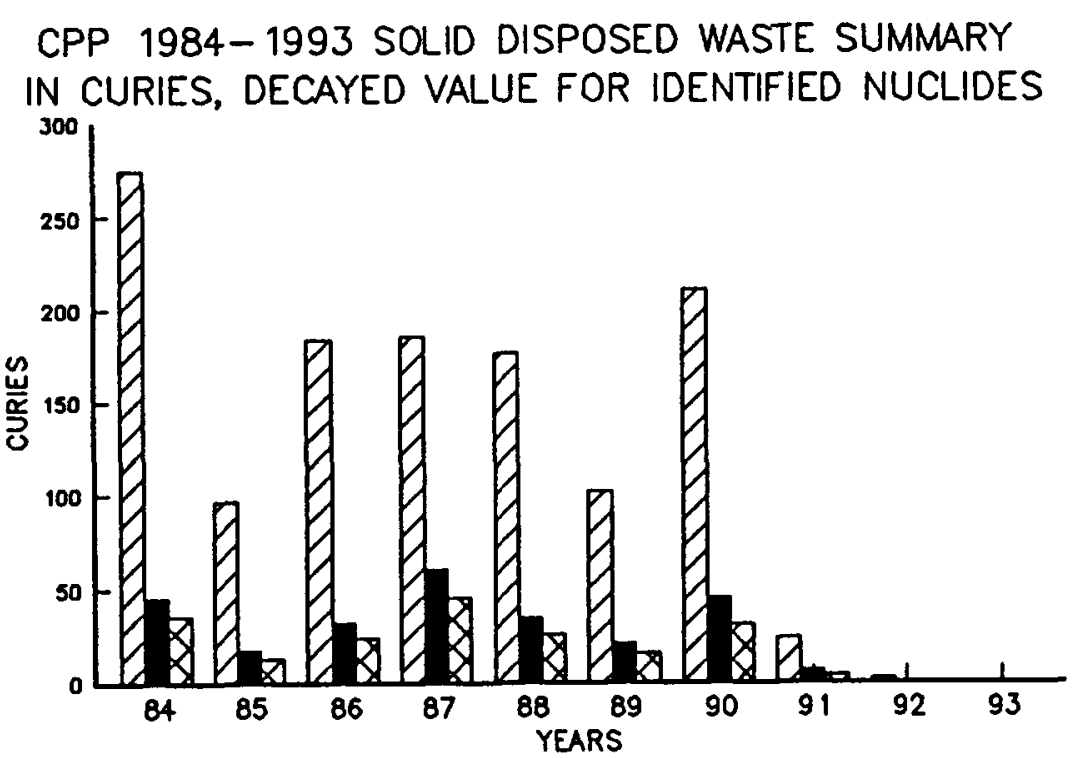

$\square$ DISPOSED $\square$ DECAYED ON 12/31/93 $\square$ DECAYED ON $12 / 31 / 03$ 
AIRBOPAE EFFLUENT DECAY DATA

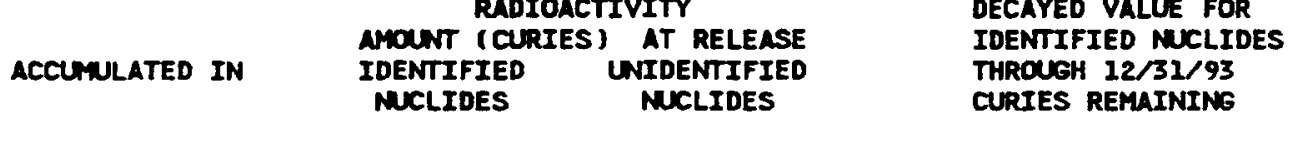

DECAYED VALUE FOR

IDENTIFIED NUCLIDES

THROUGH $12 / 31 / 03$

CURIES REMAINING

\begin{tabular}{|c|c|c|c|c|c|c|}
\hline $\begin{array}{l}1986 \\
1987\end{array}$ & $\begin{array}{r}<1 \\
0\end{array}$ & $\begin{array}{l}0 \\
0\end{array}$ & $\begin{array}{r}<1 \\
0\end{array}$ & $\begin{array}{r}60 \% \\
0 \%\end{array}$ & $\begin{array}{r}<1 \\
0\end{array}$ & $\begin{array}{r}31 \% \\
0 \%\end{array}$ \\
\hline OTALS & $<1$ & 0 & $<1$ & $60 \%$ & $<1$ & $31 \%$ \\
\hline
\end{tabular}

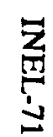

DETAILS MAY NOT ADD UP TO TOTALS BECAUSE OF ROUNDING 
LIQUID EFFLUENT DATA

\begin{tabular}{|c|c|c|}
\hline ACCUMLATED IN & $\begin{array}{l}\text { RADIOACTIVITY } \\
\text { AMONT ICURIES ) AT RELEASE } \\
\text { IDENTIFIED } \\
\text { MNIDENTIFIED } \\
\text { MCLIDES }\end{array}$ & $\begin{array}{l}\text { DECAYED VALUE FOR } \\
\text { IDENTIFIED NUCLIDES } \\
\text { THROUEH } 12 / 31 / 93 \\
\text { CURIES REMAINING }\end{array}$ \\
\hline
\end{tabular}

1986

1986

$<1$
0

$\mathbf{0}$

TOTALS

$<1$
$<1$

$84 \%$

$<1$

$84 \%$

decayed value for

IDENTIFIED NUCLIDES

THROUEH 12/31/03

CURIES REMUINING

* INCLUDES 1962 - 1983

DETAILS MAY NOT ADD UP TO TOTALS BECAUSE OF ROUNDING

$<1 \quad 66 \%$

$<166 \%$ 
SOLID DISPOSED MASTE DECAY DATA

\begin{tabular}{|c|c|c|c|}
\hline \multicolumn{3}{|c|}{ RADIOACTIVITY } & DECAYED VALUE FOR \\
\hline ACCUMULATED IN & $\begin{array}{l}\text { IDENTIFIED } \\
\text { NUCLIDES }\end{array}$ & $\begin{array}{l}\text { UNIDENTIFIED } \\
\text { MUCLIDES }\end{array}$ & $\begin{array}{l}\text { THROUGH } 12 / 31 / 93 \\
\text { CURTES REMATNTHE }\end{array}$ \\
\hline
\end{tabular}

$\begin{array}{rrrrr}1986 & 242 & 35 & 66 & 27 \% \\ 1987 & 20 & 6 & 8 & 40 \% \\ 1988 & 0 & <1 & 0 & 0 \% \\ 1993 & <1 & 0 & <1 & 96 \% \\ & 262 & 41 & 74 & 28 \%\end{array}$

TOTALS

INCLUDES $1962-2983$

DETAILS MAY NOT ADD UP TO TOTALS BECAUSE OF ROUNDING

CTF 1984-1993 SOLID DISPOSED WASTE SUMMARY IN CURIES, DECAYED VALUE FOR IDENTIFIED NUCLIDES

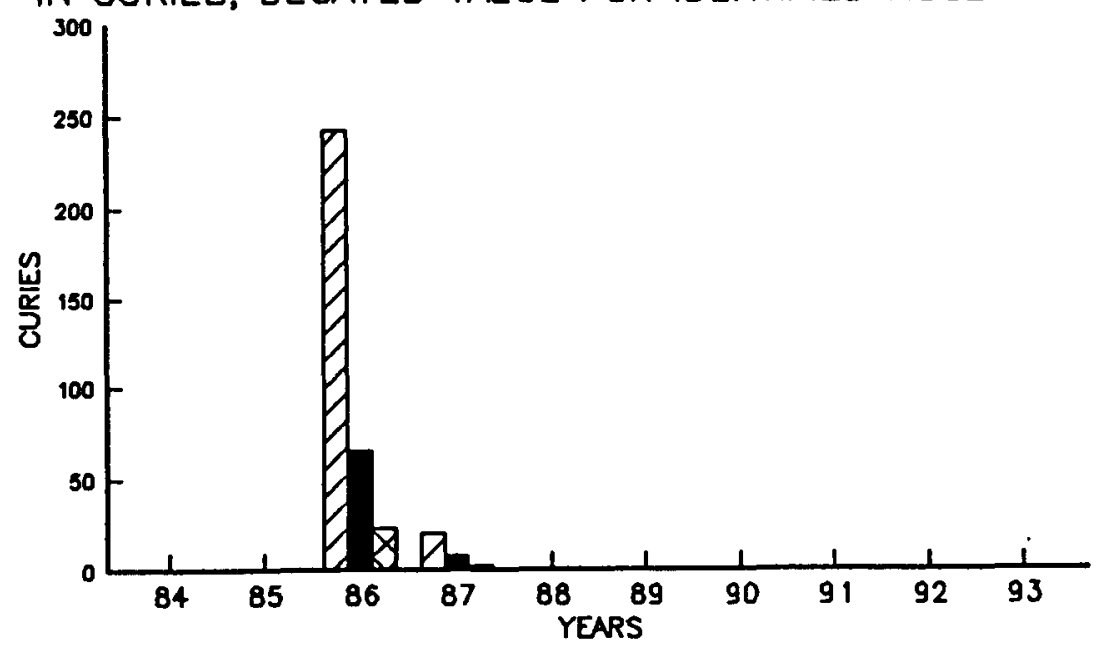

D DISPOSED DECAYED ON 12/31/93 DDECAYED ON 12/31/03 CTF DISPOSED WASTE IN 86, 87 NNO 88
DECAYED VALUE FOR IDENTIFIED MUCLIDES THROUGH 12/31/03 CURIES REMUINING

$\begin{array}{rr}23 & 10 \% \\ 2 & 11 \% \\ 0 & 0 \% \\ <1 & 60 \% \\ 25 & 20 \%\end{array}$


SOLID DISPOSED WASTE DECAY DATA

\begin{tabular}{|c|c|c|c|c|c|c|}
\hline ACCUMULATED IN & \multicolumn{2}{|c|}{$\begin{array}{l}\text { RADIOACTIVITY } \\
\text { AMOUNT (CURIES) }\end{array}$} & \multicolumn{2}{|c|}{$\begin{array}{l}\text { DECAYED VALUE FOR } \\
\text { IOENTIFIED MUCLIDES }\end{array}$} & $\begin{array}{l}\text { DECA } \\
\text { IDEA } \\
\text { THRO } \\
\text { CURI }\end{array}$ & $\begin{array}{l}\text { VALUE } \\
\text { ED NUC } \\
12 / 31 / \\
\text { EMAINI }\end{array}$ \\
\hline $\begin{array}{l}1962 * \\
1984 \\
1985 \\
1986 \\
1987 \\
1988 \\
1989 \\
1990 \\
1991 \\
1992 \\
1993\end{array}$ & $\begin{array}{r}5631 \\
1 \\
<1 \\
<1 \\
<1 \\
<1 \\
<1 \\
<1 \\
<1 \\
<1 \\
2\end{array}$ & $\begin{array}{r}58 \\
2 \\
<1 \\
<1 \\
<1 \\
0 \\
0 \\
0 \\
0 \\
0 \\
0\end{array}$ & $\begin{array}{r}2910 \\
<1 \\
<1 \\
<1 \\
<1 \\
<1 \\
<1 \\
<1 \\
<1 \\
<1 \\
2\end{array}$ & $\begin{array}{r}52 \% \\
56 \% \\
33 \% \\
100 \% \\
70 \% \\
46 \% \\
74 \% \\
88 \% \\
92 \% \\
97 \% \\
98 \%\end{array}$ & $\begin{array}{r}1775 \\
<1 \\
<1 \\
<1 \\
<1 \\
<1 \\
<1 \\
<1 \\
<1 \\
<1 \\
1\end{array}$ & $\begin{array}{r}32 \% \\
37 \% \\
9 \% \\
100 \% \\
52 \% \\
12 \% \\
44 \% \\
69 \% \\
70 \% \\
77 \% \\
64 \%\end{array}$ \\
\hline TOTALS & 5635 & 60 & 2913 & $52 \%$ & 1776 & $32 \%$ \\
\hline
\end{tabular}

* INCLUdeS $1962-1983$

DETAILS MAY NOT ADD UP TO TOTALS BECAUSE OF ROUNDING

D+D 1984- 1993 SOLID DISPOSED WASTE SUMMARY IN CURIES, DECAYED VALUE FOR IDENTIFIED NUCLIDES

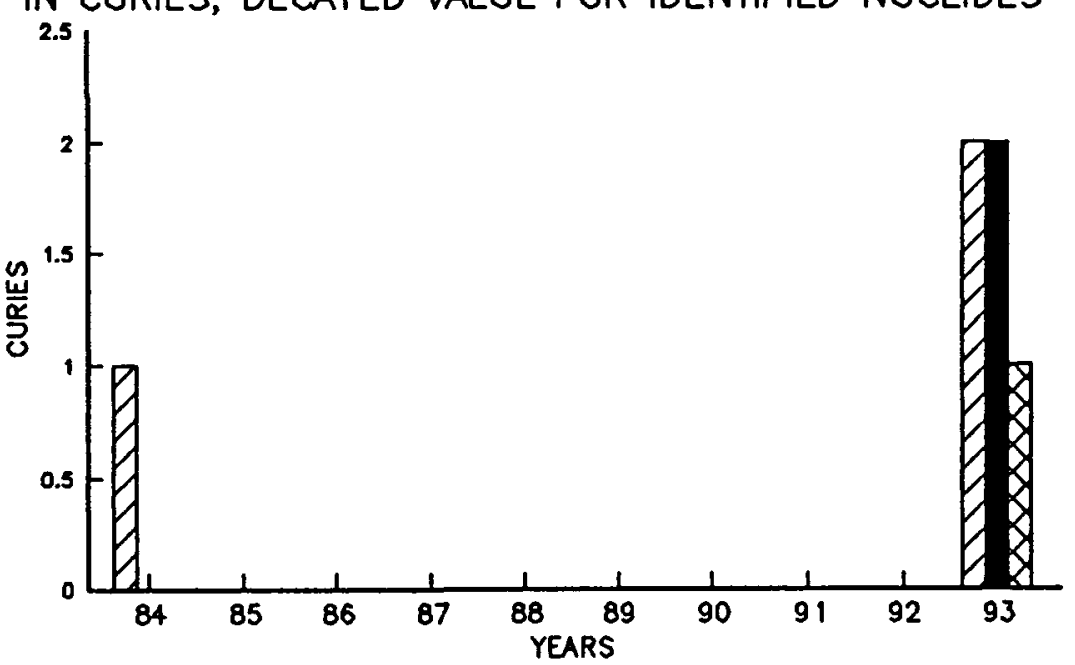

$\square$ DISPOSED $\square$ DECAYED ON $12 / 31 / 93$ QDECAYED ON $12 / 31 / 03$ 
AIRBORNE EFFLUENT DECAY DATA

RADIOACTIVITY
AMOUNT (CURIES) AECAYED VALUE FOR

AMOUNT (CURIES) AT RELEASE IDENTIFIED NUCLIDES

IDENIIFIED UNIDENTIFIED

MUCLIDES

THROUGH 12/31/93

CURIES REMAININE

$\begin{array}{rrr}<1 & <1 & <1 \% \\ 0 & <1 & <1 \% \\ 0 & 6 & <1 \% \\ <1 & 6 & <1 \%\end{array}$

TOTALS

$\begin{array}{lr}1962 * & 4 \\ 2984 & 27 \\ 1985 & 8780\end{array}$

8810

* Includes 1962 - 1983
DECAYED VALUE FOR

IDENTIFIED NUCLIDES

THROUGH 12/31/03

CURIES REMAINING

$\begin{array}{ll}<1 & <1 \% \\ <1 & <1 \% \\ 3 & <1 \% \\ 3 & <1 \%\end{array}$

DETAILS MAY NOT ADD UP TO TOTALS BECAUSE OF ROUNDING

LOF 1984-1993 AIRBORNE DECAY SUMMARY IN CURIES, DECAYED VALUE FOR IDENTIFIED NUCLIDES

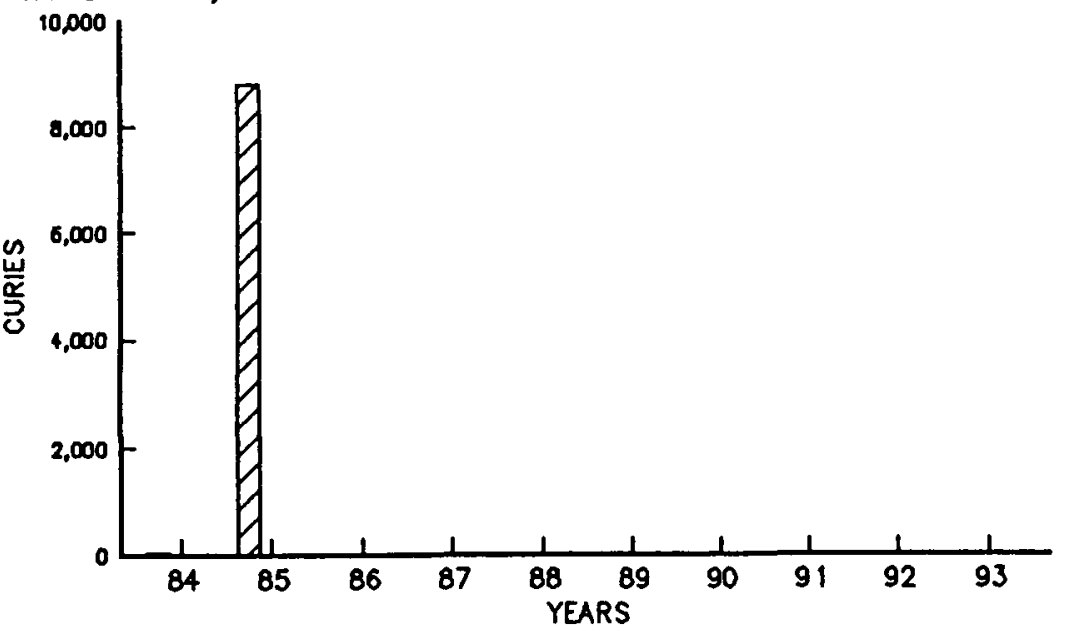

DRELEASED D DECAYED ON 12/31/93 D DECAYED ON 12/31/03

LOF RELEASED EFTUUATS FROM 78-85 
LIQUTD EFFLUENT DATA

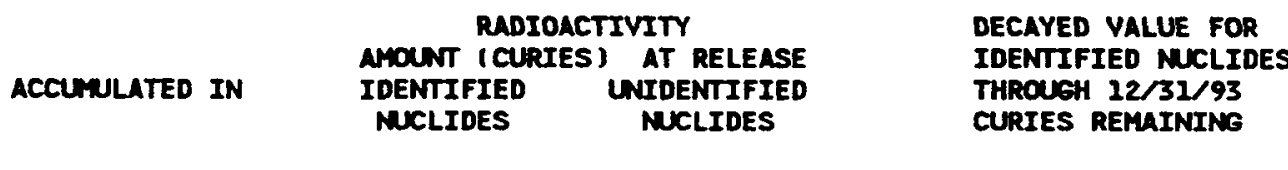

$\begin{array}{llrllll}1962 \% & <1 & <1 & <1 & 39 \% & <1 & 31 \% \\ 1984 & <1 & 0 & <1 & 16 \% & <1 & 4 \% \\ 1985 & <1 & <1 & <1 & 54 \% & <1 & 38 \% \\ & <1 & <1 & <1 & 39 \% & <1 & 26 \%\end{array}$

TOTALS

* INCLUDES $1962-1983$

DETAILS MAY NOT ADD UP TO TOTALS BECAUSE OF ROUNDING

DECAYEd VALUE FOR

IDENTIFIED NUCLIDES

THROUGH 12/31/03

CURIES REMAINING 
LOSS-OF-FLUTD TEST FACILITY

SOLID DISPOSED MASTE DECAY DATA

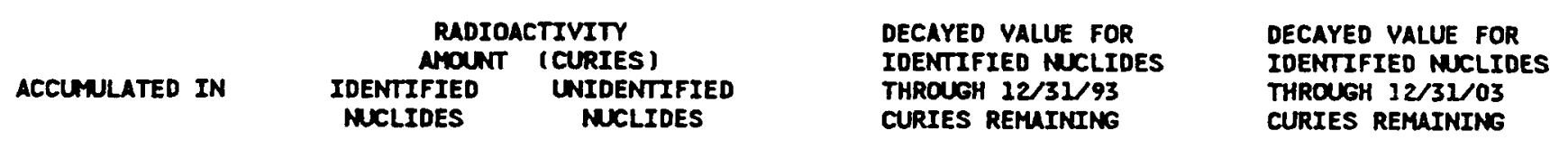

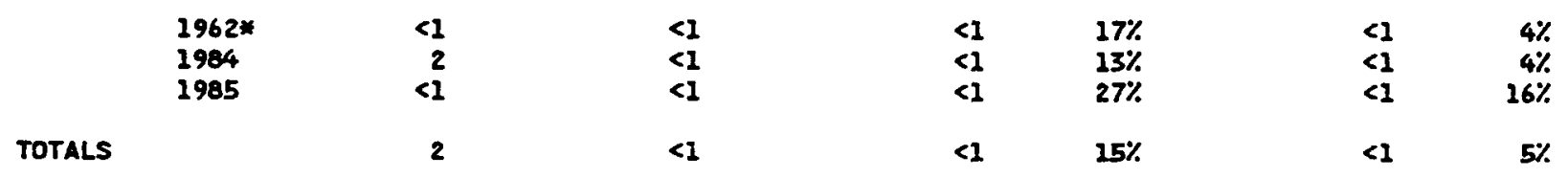

* INCLUDes $2962-1983$

DETAILS MAY NOT ADD UP TO TOTALS BECAUSE OF ROUNDING IN CURIES, DECAYED VALUE FOR IDENTIFIED NUCLIDES

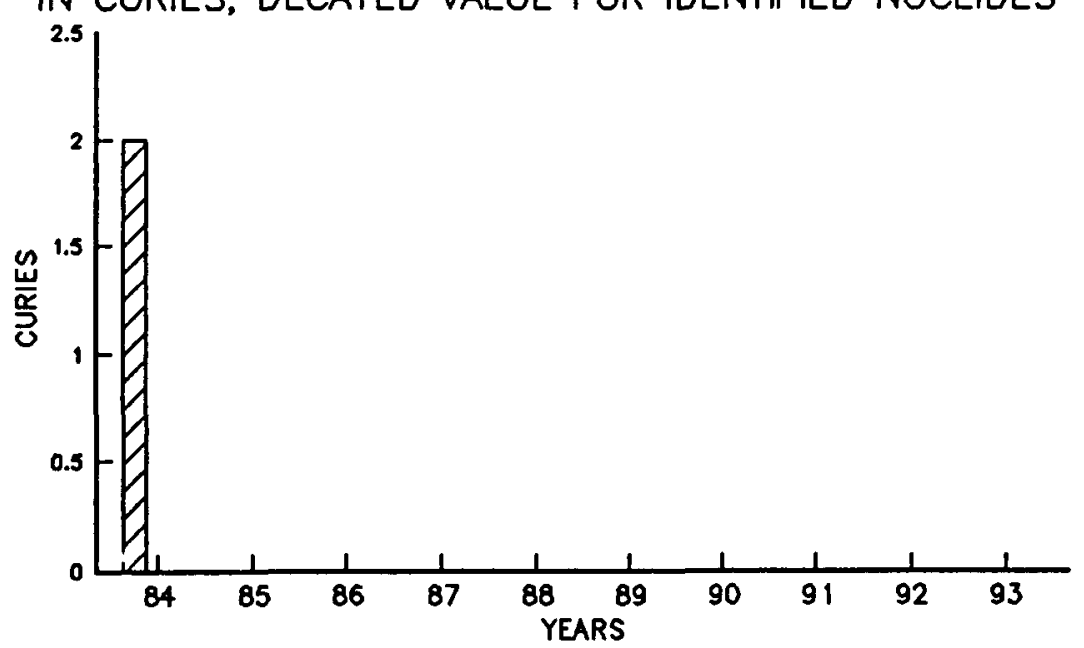

DOISPOSED DECAYED ON 12/31/93 $\triangle$ DECAYED ON $12 / 31 / 03$

LOF DISPOSED OF WASE FROM B 1-85 
SCHEDULE NO. P61PM021-B

MAVAL REACTORS FACILITY

\section{AIRBORNE EFFLUENT DECAY DATA}

RADIOACTIVITY

AHOWT (CURIES) AT RELEASE

IDENTIFIED UNIDENTIFIED

NUCLIDES

$$
\text { MUCLIDES }
$$

IDENTIFIED MUCLIDES

THROUEH 12/31/93

CURIES REMAINING

$$
\begin{aligned}
& 1962 * \\
& 1984 \\
& 1985 \\
& 1986 \\
& 1987 \\
& 1988 \\
& 1989 \\
& 1990 \\
& 1991 \\
& 1992 \\
& 1993
\end{aligned}
$$

TOTALS

$$
\begin{aligned}
& <1 \\
& <1 \\
& <1 \\
& 0 \\
& 0 \\
& 0 \\
& 0 \\
& 0 \\
& 0 \\
& 0 \\
& 0
\end{aligned}
$$

$\begin{array}{ll}5 & 13 \% \\ <1 & 94 \% \\ <1 & 95 \% \\ <1 & 88 \% \\ <1 & 86 \% \\ <1 & 82 \% \\ <1 & 96 \% \\ <1 & 95 \% \\ <1 & 89 \% \\ <1 & 44 \% \\ <1 & 75 \% \\ & \\ 8 & 21 \%\end{array}$

DECAYED VALUE FoR

RUN DATE: 05/17/94

DECAYED VALUE FOR

IDENTIFIED NUCLIDES

THROUGH 12/31/03

CURIES REMAINING

$\begin{array}{rr}3 & 9 \% \\ <1 & 90 \% \\ <1 & 91 \% \\ <1 & 84 \% \\ <1 & 73 \% \\ <1 & 59 \% \\ <1 & 89 \% \\ <1 & 86 \% \\ <1 & 58 \% \\ <1 & 36 \% \\ <1 & 73 \% \\ & \\ 6 & 16 \%\end{array}$

DETAILS MAY NOT ADD UP TO TOTALS BECALSE OF ROUNDING 
LIEUTO EFFLUENT DATA

$$
\text { RADIOACTIVITY }
$$

AMONT (CURIES) AT RELEASE

IDENTIFIED UNIDENTIFIED

MUCLIDES

DECAYEd VALUE FOR

IDENTIFIED NUCLIDES

THROUCH 22/31/93

CURIES REMAINING

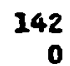

29

$14 \%$

$<1$

0

142

TOTALS

208

INCLUDES $1962-1983$
DECAYED VALUE FOR

IDENTIFIED NUCLIDES

THROUGH 12/31/03

CURIES REMAINING

$18 \quad 9 \%$

$189 \%$

DETAILS MAY NOT ADD UP TO TOTALS BECAUSE OF RONNOING 
SOLID DISPOSED WASTE DECAY DATA

\begin{tabular}{|c|c|c|c|c|c|c|}
\hline ACCUMLLATED IN & $\begin{array}{r}\text { RAD } \\
\text { AMOU } \\
\text { IDENTIFIED } \\
\text { MUCLIDES }\end{array}$ & $\begin{array}{l}\text { TIVITY } \\
\text { (CURIES) } \\
\text { UNIDENIIFIED } \\
\text { NUCLIDES }\end{array}$ & \multicolumn{2}{|c|}{$\begin{array}{l}\text { DECAYED VALUE FOR } \\
\text { IDENTIFIED MUCLIOES } \\
\text { THROUGH } 12 / 31 / 93 \\
\text { CURIES REMAINING }\end{array}$} & \multicolumn{2}{|c|}{$\begin{array}{l}\text { DECAYED VALUE } \\
\text { IDENTIFIED NUCI } \\
\text { THROUGH } 12 / 31 / \\
\text { CURIES REMAINII }\end{array}$} \\
\hline $\begin{array}{l}1962 * \\
1984 \\
1985 \\
1986 \\
1987 \\
1988 \\
1989 \\
1990 \\
1991 \\
1992 \\
1993\end{array}$ & $\begin{array}{r}555513 \\
365127 \\
141754 \\
35930 \\
29665 \\
6732 \\
126487 \\
74089 \\
102852 \\
49795 \\
42260\end{array}$ & $\begin{array}{r}3095387 \\
0 \\
0 \\
0 \\
0 \\
0 \\
<1 \\
0 \\
0 \\
0 \\
0\end{array}$ & $\begin{array}{r}65529 \\
205489 \\
82776 \\
21878 \\
18780 \\
4481 \\
95157 \\
52260 \\
57316 \\
37545 \\
38752\end{array}$ & $\begin{array}{l}12 \% \\
56 \% \\
58 \% \\
61 \% \\
63 \% \\
67 \% \\
75 \% \\
71 \% \\
56 \% \\
75 \% \\
92 \%\end{array}$ & $\begin{array}{r}41779 \\
165499 \\
65245 \\
16819 \\
14097 \\
3262 \\
72150 \\
35708 \\
36327 \\
23999 \\
24652\end{array}$ & $\begin{array}{l}8 \% \\
45 \% \\
46 \% \\
47 \% \\
48 \% \\
48 \% \\
57 \% \\
48 \% \\
35 \% \\
48 \% \\
58 \%\end{array}$ \\
\hline TOTALS & 2530299 & 3095387 & 679964 & $44 \%$ & 499537 & $33 \%$ \\
\hline
\end{tabular}

DETAILS MAY NOT ADD UP TO TOTALS BECAUSE OF ROUNDING

NRF 1984-1993 SOLID DISPOSED WASTE SUMMARY

IN CURIES, DECAYED VALUE FOR IDENTIFIED NUCLIDES

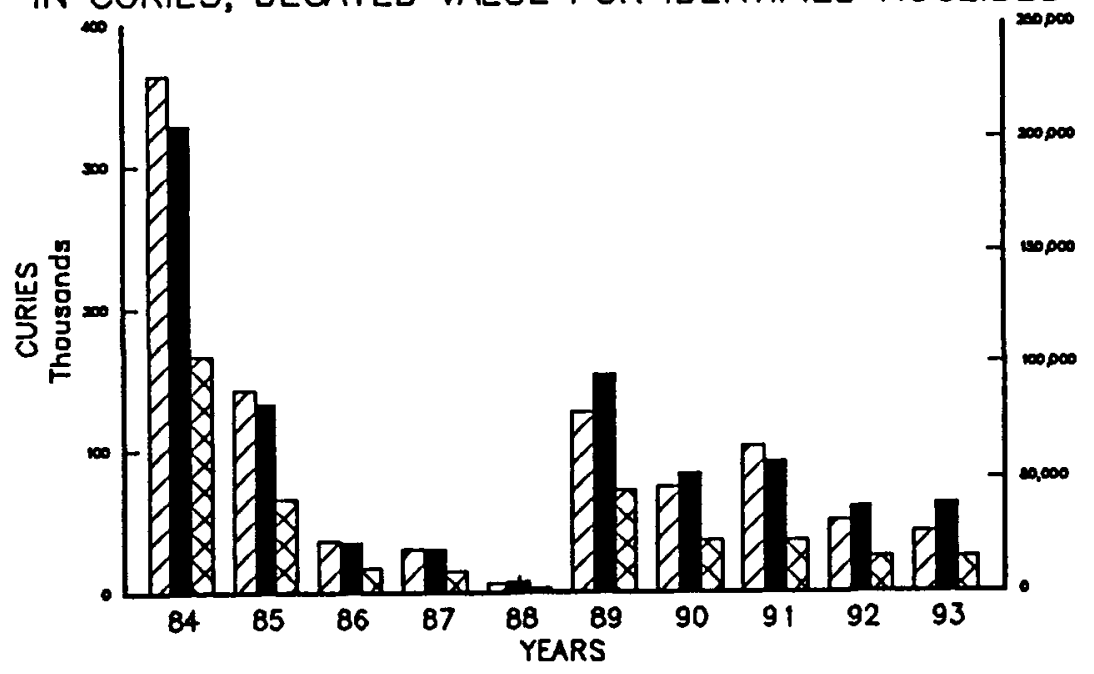

$\square D I S P O S E D=D E C A Y E D$ ON $12 / 31 / 93 \Delta$ DECAYED ON $12 / 31 / 03$ 
RADIOACTIVITY

AMOUNT (CURIES) AT RELEASE IDENTIFIED UNIDENTIFIED

NUCLIDES

1962*

1984

1985

1986

1987

1988

1989

1989

1992
1993

MUCLIDES

DECAYEd VALUE FoR IDENTIFIED MUCLIDES THROUEH 12/31/93

CURIES REMAINING

decAYed VALUE FoR

IDENTIFIED MUCLIDES

THROUGH $12 / 31 / 03$

CURIES REMATNTHG

TOTALS

$\begin{array}{ll}<1 & <1 \\ <1 & <1 \\ <1 & <1 \\ <1 & <1 \\ <1 & 0 \\ <1 & 0 \\ <1 & 0 \\ <1 & 0 \\ <1 & 0 \\ <1 & 0 \\ <1 & 0 \\ <1 & <1\end{array}$

* INCLUdes $1962-1983$

DETATLS MAY NOT ADD UP TO TOTALS BECALSE OF ROUNDING

$\begin{array}{rr}<1 & 22 \% \\ <1 & 45 \% \\ <1 & 24 \% \\ <1 & 38 \% \\ 0 & 0 \% \\ <1 & 4 \% \\ <1 & 40 \% \\ <1 & 4 \% \\ <1 & 36 \% \\ <1 & 22 \% \\ <1 & <1 \% \\ & <1 \\ & 22 \%\end{array}$


SCHEDULE NO. P61PMO21-C

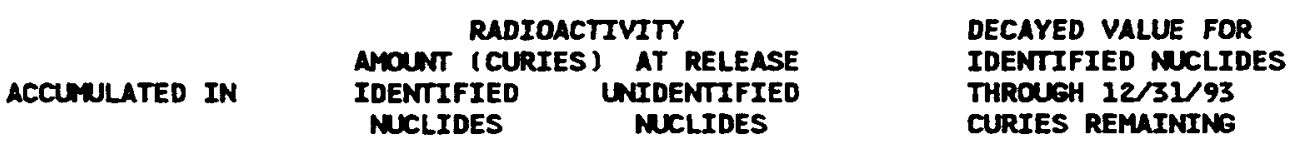

1962*

$<1$

$<1$

$<1$

TOTALS

$<1$

INCLUDES 1962 - 1983

DETAILS MAY NOT ADD UP TO TOTALS BECAUSE OF ROUNDING
RUN DATE: 05/17/94

DECAYED YALUE TOR

IDENTIFIED NUCLIDES

THROUGH 12/31/03

CURIES REMAINING

$<1$

$39 \%$

$<1$

$39 \%$ 


\section{SOLID DISPOSED MUSTE DECAY DATA}

ACCUMLATED IN

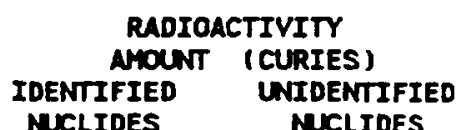

MCLIDES NUCLIDES

$<1$
0
367
0
8
$<1$

$$
\begin{array}{r}
7 \\
181 \\
4 \\
<1 \\
3 \\
2
\end{array}
$$

376

197

TOTALS

INCLUDES $1962-1983$

DETAILS MAY NOT ADD UP TO TOTALS BECAUSE OF ROUNDING

PBF 1984- 1993 SOLID DISPOSED WASTE SUMMARY IN CURIES, DECAYED VALUE FOR IDENTIFIED NUCLIDES

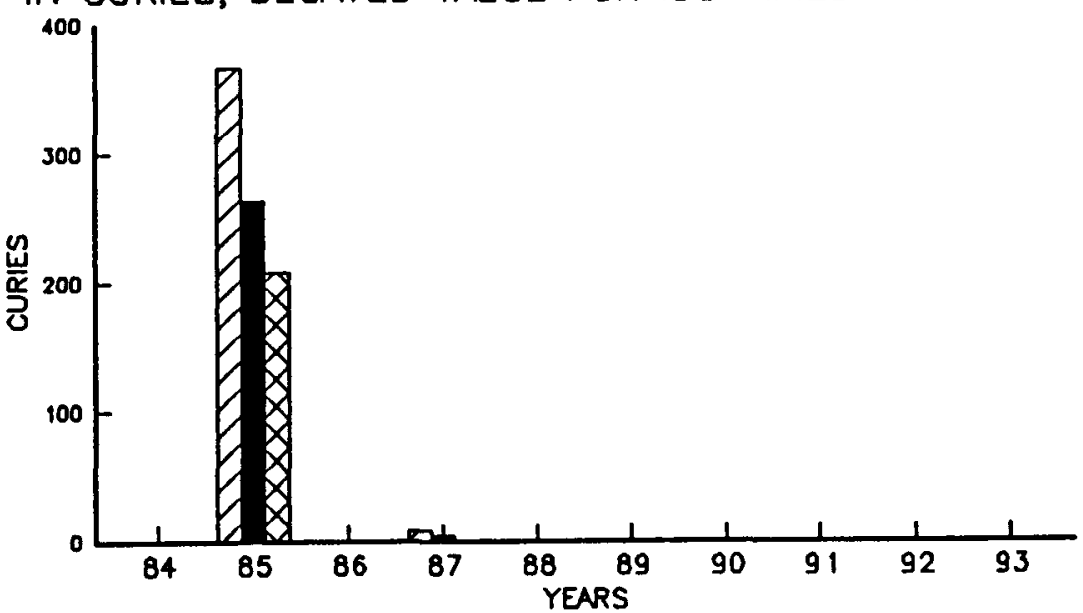

D DISPOSED DECAYED ON 12/31/93 DECCAYED ON 12/31/03

PQF HWD NO IOENTIFIED NUCUDES IN 81,83,
84, 86 OR 88 THRU 93 
SOLID DISPOSED MASTE DECAY DATA

\begin{tabular}{|c|c|c|c|c|c|c|}
\hline ACCUMULATED IN & $\begin{array}{r}\text { RADIC } \\
\text { AMOUN } \\
\text { IDENTIFIED } \\
\text { MCLIDES }\end{array}$ & $\begin{array}{l}\text { TIVITY } \\
\text { (CURIES) } \\
\text { UNIDENTIFIED } \\
\text { MUCLIDES }\end{array}$ & \multicolumn{2}{|c|}{$\begin{array}{l}\text { DECAYED VALUE FOR } \\
\text { IDENTIFIED NUCLIDES } \\
\text { THROUEH } 12 / 31 / 93 \\
\text { CURIES REMAINING }\end{array}$} & \multicolumn{2}{|c|}{$\begin{array}{l}\text { DECAYED VALUE FOR } \\
\text { IDENTIFIED MUCLIDES } \\
\text { THROUEH } 12 / 31 / 03 \\
\text { CURIES REMAINING }\end{array}$} \\
\hline 1962* & 4870 & 251240 & 4791 & $98 \%$ & 4784 & $98 \%$ \\
\hline TOTALS & 4870 & 251140 & 4791 & $98 \%$ & 4784 & $98 \%$ \\
\hline
\end{tabular}

DETAILS MAY NOT ADD UP TO TOTALS BECAUSE OF ROUNDING 


\section{AIRBORNE EFFLUENT DECAY DATA}

\section{RADIOACTIVITY}

AMOUNT (CURIES) AT RELEASE

IDENTIFIED UNIDENTIFIED

MUCLIDES

DECAYED VALUE FOR

IDENTIFIED NUCLIDES

THROUEH $12 / 31 / 93$
CURIES REMINING

$$
\begin{aligned}
& 1985 \\
& 1986 \\
& 1987 \\
& 1988 \\
& 1989 \\
& 1990 \\
& 1991 \\
& 1992 \\
& 1993
\end{aligned}
$$

TOTALS

INCLUDES $1962-1983$

$\begin{array}{lll}0 & <1 & 43 \% \\ 0 & <1 & 43 \% \\ 0 & <1 & 22 \% \\ 0 & <1 & 43 \% \\ 0 & <1 & 36 \% \\ 0 & <1 & 36 \% \\ 0 & <1 & 36 \% \\ 0 & <1 & 36 \% \\ 0 & <1 & 40 \% \\ 0 & <1 & 30 \%\end{array}$

DECAYED VALUE FOR

IDENTIFIED NUCLIDES

THROUGH 12/31/03

CURIES REMAINING

DETAILS MAY NOT ADD UP TO TOTALS BECAUSE OF ROUNDING 
SOLID DISPOSED MASTE DECAY DATA

ACCUNULATED IN

1986
1987
1988
2989
1991
1992

RADIOACTIVITY
AMOWT
(CURIES)
IDENTIFIED
NUCLIDEENTIFIED
NUCLIDES
DECAYED VALUE FOR
IDENTIFIED MUCLIDES
THROUGH $22 / 31 / 93$
CURIES REMAINING

TOTALS

$<1$
$<1$
$<1$
$<1$
$<1$
$<1$
1

* INCLUDES 1962 - 1983

DETAILS MAY NOT ADD UP TO TOTALS BECALSE OF ROUNDING

DECAYED VALUE FOR

IDENTIFIED NUCLIDES

THROUEH 12/31/03

CURIES REMAINING

$\begin{array}{ll}<1 & 100 \% \\ <1 & 100 \% \\ <1 & 100 \% \\ <1 & 100 \% \\ <1 & 100 \% \\ <1 & 100 \% \\ 1 & 100 \%\end{array}$




\section{AIRBORNE EFFLUENT DECAY DATA}

\section{RADIOACTIVITY}

ANOUNT (CURIES) AT RELEASE

IDENTIFIED UNIDENTIFIED

MUCLIDES

NUCLIOES

2962*

o

TOTALS

06

INCLUDES $1962-1983$

DECAYED VALUE FOR

IDENTIFIED MUCLIOES

THROUSH $22 / 31 / 93$

CURIES REMUINING

\section{6}

$0 \quad 0 \%$

o $\%$

DECAYED VALUE FOR

IDENTIFIED MUCLIDES

THROUEH 12/31/03

CURIES REMAINING

DETAILS MAY NOT ADD UP TO TOTALS BECALSE OF ROUNDING 
LIEUTD EFFLUENT DATA

\section{RAOIOACTIVITY}

AHOUNT (CURIES) AT RELEASE

IDENTIFIED UNIDENTIFIED

MXLIDES

DECAYEd VALUE FOR

IDENTIFIED NUCLIDES

THROUEH 12/31/93

DECAYEd VALUE FOR

IDENTIFIED NUCLIDES

THROUGH $12 / 31 / 03$

MCLIDES

155

28\%

$<1 \quad 23 \%$

TOTALS

$<1$

155

$<$

$28 \%$

C1 $23 \%$

* INCLUDES 1962 - 1983

DETAILS MAY NOT ADO UP TO TOTALS BECAUSE OF RONDING 
SPECIAL POMER EXCURSION REACTOR TEST

SOLID DISPOSED MUSTE DECAY DATA

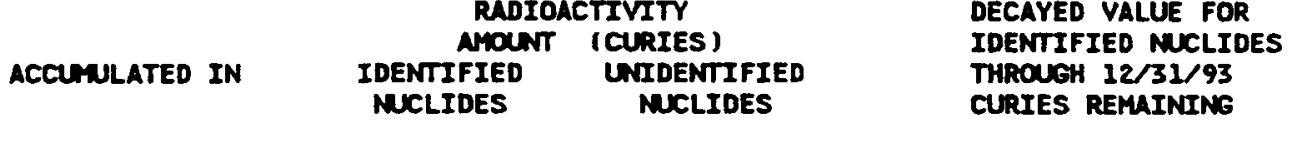

DECAYED VALUE FOR

IDENTIFIED NUCLIDES

THROUGH 12/31/03

CURIES REMAINING

$\begin{array}{lrrrrrr}1962 * & 5 & 19 & <1 & 10 \% & <1 & 6 \% \\ 1987 & <1 & <1 & <1 & 100 \% & <1 & 100 \% \\ 1989 & 01 & 0 & 0 \% & 0 & 0 \% \\ 1991 & <1 & 0 & <1 & 82 \% & <1 & 48 \% \\ 1993 & <1 & 0 & <1 & 97 \% & <1 & 71 \% \\ & 5 & 19 & <1 & 10 \% & <1 & 6 \%\end{array}$

TOTALS

INCLUDES $1962-1983$

DETAILS MAY NOT ADD UP TO TOTALS BECALSE OF ROUNDING 
AIRBORNE EFFLLENT DECAY DATA

\begin{tabular}{|c|c|c|c|}
\hline ACCLNLLATED IN & $\begin{array}{l}\text { RADIOACTIVITY } \\
\text { ANOUNT (CURIES) AT RELEASE } \\
\text { IDENTIFIED } \\
\text { NUCLIDES } \\
\text { NUENTIFIED } \\
\text { NUCLIDES }\end{array}$ & $\begin{array}{l}\text { DECAYED VALUE FOR } \\
\text { IDENTIFIED NUCLIDES } \\
\text { THROUGH } 12 / 31 / 93 \\
\text { CURIES REMUINING }\end{array}$ & $\begin{array}{l}\text { DECAYED VALUE FOR } \\
\text { IDENTIFIED NUCLIDES } \\
\text { THROUGH I2/31/03 } \\
\text { CURIES REMAINING }\end{array}$ \\
\hline
\end{tabular}

$\begin{array}{lrrrrrr}1962 * & 51 & 53724 & <1 & 48 \% & <1 & 25 \% \\ 1984 & <1 & <1 & <1 & 71 \% & <1 & 56 \% \\ 1985 & <1 & <1 & <1 & 42 \% & <1 & 33 \% \\ 1986 & <1 & <1 & 01 & 10 \% & <1 & 8 \% \\ 1987 & <1 & 01 & <1 & 56 \% & <1 & 32 \% \\ 1988 & <1 & 0 & <1 & 45 \% & <1 & 25 \% \\ 1989 & <1 & 0 & <1 & 18 \% & <1 & 36 \% \\ 1990 & <1 & 0 & <1 & 18 \% & <1 & 14 \% \\ 1991 & <1 & 0 & <1 & <1 \% & <1 & 14 \% \\ 1992 & <1 & 0 & <1 & 32 \% & <1 & <1 \% \\ 1993 & <1 & 0 & <1 & 48 \% & <1 & 23 \% \\ & <1 & 53724 & & & <1 & 25 \%\end{array}$

* INCludes 1962 - 1983

DETAILS MAY NOT ADD UP TO TOTALS BECAUSE OF ROUNDING 


\section{LIEUID EFFLUENT DATA}

\begin{tabular}{|c|c|c|c|}
\hline ACCLMLATED IN & $\begin{array}{l}\text { RADIOACTIVITY } \\
\text { NOWN (CURIES) AT RELEASE } \\
\text { IDENTIFIED } \\
\text { MUCLIDIDENTIFIED } \\
\text { MUCLIDES }\end{array}$ & $\begin{array}{l}\text { DECAYED VALUE FOR } \\
\text { IDENTIFIED NUCLIDES } \\
\text { THROUGH } 12 / 31 / 93 \\
\text { CURIES REMAINING }\end{array}$ & $\begin{array}{l}\text { DECAYED VALUE FOR } \\
\text { IDENTIFIED NUCLIDES } \\
\text { THROUGH } 12 / 31 / 03 \\
\text { CURIES REMAINING }\end{array}$ \\
\hline
\end{tabular}

$$
\begin{aligned}
& 1962 * \\
& 1984 \\
& 1985 \\
& 1986 \\
& 1987 \\
& 1988 \\
& 1989 \\
& 1990 \\
& 1991 \\
& 1992 \\
& 1993
\end{aligned}
$$

$\begin{array}{lr}19 & 39 \\ <1 & <1 \\ <1 & <1 \\ <1 & 0 \\ <1 & 0 \\ <1 & 0 \\ <1 & 0 \\ <1 & 0 \\ <1 & 0 \\ <1 & 0 \\ 2 & 0\end{array}$

39
$<1$
$<1$
0
0
0
0
0
0
0
0

$\begin{array}{rr}6 & 31 \% \\ <1 & 59 \% \\ <1 & 60 \% \\ <1 & 63 \% \\ <1 & <1 \% \\ <1 & 9 \% \\ <1 & 70 \% \\ <1 & 68 \% \\ <1 & 84 \% \\ <1 & 87 \% \\ 2 & 97 \%\end{array}$

$\begin{array}{ll}3 & 18 \% \\ <1 & 33 \% \\ <1 & 34 \% \\ <1 & 37 \% \\ <1 & <1 \% \\ <1 & 5 \% \\ <1 & 40 \% \\ <1 & 39 \% \\ <1 & 48 \% \\ <1 & 50 \% \\ 1 & 55 \%\end{array}$

TOTALS

22

39

8

$38 \%$

$5 \quad 22 \%$ 
SOLIO DISPOSED MASTE DECAY DATA

\begin{tabular}{|c|c|c|c|c|c|c|}
\hline ACCUMLLATED IN & $\begin{array}{r}\text { RADIO } \\
\text { AMOUNT } \\
\text { IDENTIFIED } \\
\text { MUCLIDES }\end{array}$ & $\begin{array}{l}\text { TIVITY } \\
\text { (CURIES) } \\
\text { UNIDENTIFIED } \\
\text { NUCLIDES }\end{array}$ & \multicolumn{2}{|c|}{$\begin{array}{l}\text { DECAYED VALUE FOR } \\
\text { IDENTIFIED MUCLIDES } \\
\text { THROUGH } 12 / 31 / 93 \\
\text { CURIES REMAINING }\end{array}$} & \multicolumn{2}{|c|}{$\begin{array}{l}\text { DECAYED VALUE } \\
\text { IDENTIFIED NUC } \\
\text { THROUEH } 12 / 31 / \\
\text { CURIES REMAINI }\end{array}$} \\
\hline $\begin{array}{l}1962 * \\
1984 \\
1985 \\
1986 \\
1987 \\
1988 \\
1989 \\
1990 \\
1991 \\
1992 \\
1993\end{array}$ & $\begin{array}{r}18690 \\
0 \\
0 \\
1 \\
11 \\
<1 \\
66 \\
2658 \\
<1 \\
347 \\
216\end{array}$ & $\begin{array}{r}82753 \\
2 \\
17 \\
16 \\
347 \\
1 \\
<1 \\
0 \\
0 \\
0 \\
0\end{array}$ & $\begin{array}{r}6802 \\
0 \\
0 \\
<1 \\
7 \\
<1 \\
56 \\
2425 \\
<1 \\
327 \\
208\end{array}$ & $\begin{array}{r}36 \% \\
0 \% \\
0 \% \\
85 \% \\
64 \% \\
52 \% \\
85 \% \\
91 \% \\
91 \% \\
94 \% \\
96 \%\end{array}$ & $\begin{array}{r}6458 \\
0 \\
0 \\
<1 \\
4 \\
<1 \\
44 \\
1921 \\
<1 \\
254 \\
157\end{array}$ & $\begin{array}{l}35 \% \\
0 \% \\
0 \% \\
67 \% \\
38 \% \\
19 \% \\
66 \% \\
72 \% \\
67 \% \\
73 \% \\
73 \%\end{array}$ \\
\hline TOTALS & 21989 & 83137 & 9826 & $45 \%$ & 8839 & $40 \%$ \\
\hline
\end{tabular}

* INCludes 1962 - 1983

DETAILS MAY NOT ADD UP TO TOTALS BECAUSE OF ROUNDING

TAN 1984- 1993 SOLID DISPOSED WASTE SUMMARY IN CURIES, DECAYED VALUE FOR IDENTIFIED NUCLIDES

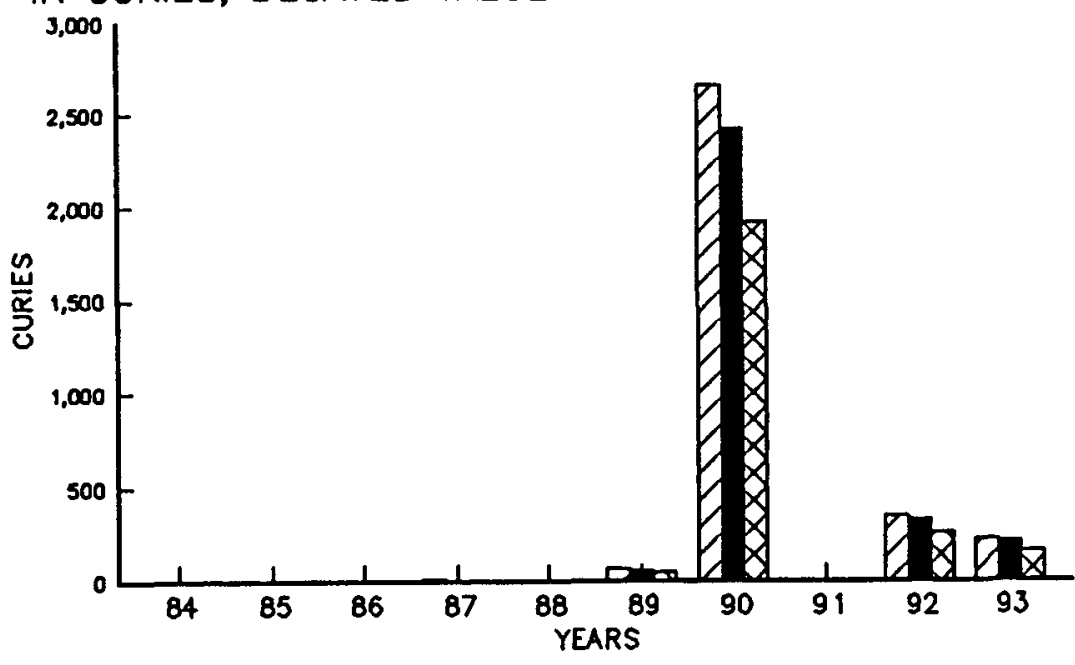

DDISPOSED $\square$ DECAYED ON $12 / 31 / 93 \square$ DECAYED ON $12 / 31 / 03$ 
AIRBORNE EFFLUENT DECAY DATA

\section{RADIOACTIVITY}

AMOUNT (CURIES) AT RELEASE

IDENTIFIED UNIDENTIFIED

MUCLIDES

NUCLIDES

$1962 *$
1984
1985
1986
1987
1988
1989
1990
1991
1992
1993

TOTALS

$\begin{array}{rr}3059811 & 2363315 \\ 4162 & <1 \\ 3288 & <1 \\ 2741 & <1 \\ 3140 & 0 \\ 2609 & 0 \\ 1682 & 0 \\ 3741 & 0 \\ 3284 & 0 \\ 3439 & 0 \\ 1495 & 0\end{array}$

2363315
DECAYED VALUE FOR IDENTIFIED NUCLIDES THROUGH 12/31/93 CURIES REMUINTNG

$\begin{array}{ll}2 & <1 \% \\ <1 & <1 \% \\ <1 & <1 \% \\ <1 & <1 \% \\ 2 & <1 \% \\ <1 & <1 \% \\ <1 & <1 \% \\ <1 & <1 \% \\ <1 & <1 \% \\ <1 & <1 \% \\ <1 & <1 \%\end{array}$

$<I \%$
DECAYED VALUE FOR IDENTIFIED NUCLIDES THROUGH $12 / 31 / 03$ CURIES REMUININE

DETAILS MAY NOT ADD UP TO TOTALS BECAUSE OF RONNDING

TRA 1984-1993 AIRBORNE DECAY SUMMARY IN CURIES, DECAYED VALUE FOR IDENTIFIED NUCLIDES

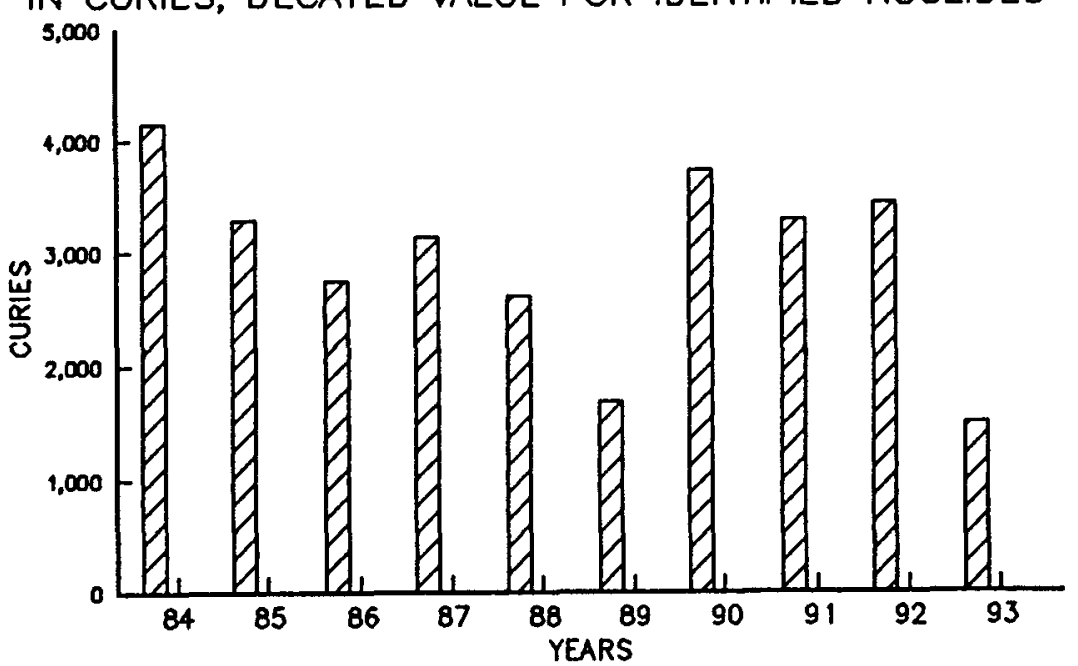

DRELEASED D DECAYED ON $12 / 31 / 93$ DECAYED ON $12 / 31 / 03$ 
LIQUID EFFLUENT DATA

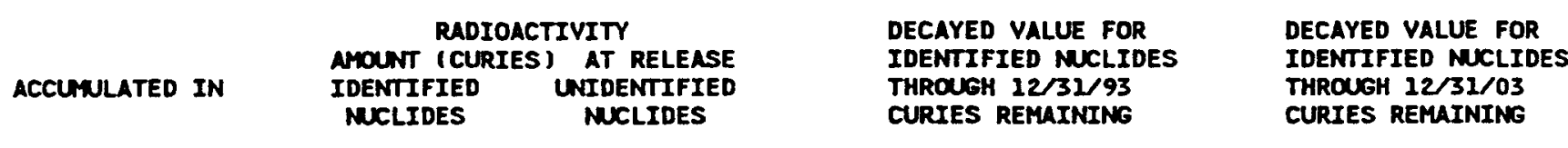

\begin{tabular}{|c|c|c|c|c|c|c|}
\hline $\begin{array}{l}1962 * \\
1984 \\
1985 \\
1986 \\
1987 \\
1988 \\
1989 \\
1990 \\
1991 \\
1992 \\
1993\end{array}$ & $\begin{array}{r}24394 \\
173 \\
269 \\
91 \\
139 \\
179 \\
134 \\
185 \\
165 \\
184 \\
128\end{array}$ & $\begin{array}{r}27451 \\
<1 \\
0 \\
<1 \\
0 \\
0 \\
0 \\
0 \\
0 \\
0 \\
0\end{array}$ & $\begin{array}{r}2231 \\
95 \\
162 \\
51 \\
89 \\
127 \\
98 \\
149 \\
141 \\
165 \\
120\end{array}$ & $\begin{array}{r}9 \% \\
55 \% \\
60 \% \\
56 \% \\
64 \% \\
71 \% \\
73 \% \\
80 \% \\
85 \% \\
90 \% \\
94 \%\end{array}$ & $\begin{array}{r}1293 \\
54 \\
92 \\
29 \\
51 \\
72 \\
56 \\
85 \\
80 \\
94 \\
68\end{array}$ & $\begin{array}{r}5 \% \\
31 \% \\
34 \% \\
32 \% \\
37 \% \\
40 \% \\
42 \% \\
46 \% \\
49 \% \\
51 \% \\
53 \%\end{array}$ \\
\hline TOTALS & 26041 & 27451 & 3430 & $13 \%$ & 1974 & $8 \%$ \\
\hline
\end{tabular}

* INCLUDES $1962-1983$

DETAILS MAY NOT ADD UP TO TOTALS BECAUSE OF ROUNDING

TRA 1984-1993 LIQUID DECAY SUMMARY IN CURIES, DECAYED VALUE FOR IDENTIFIED NUCLIDES

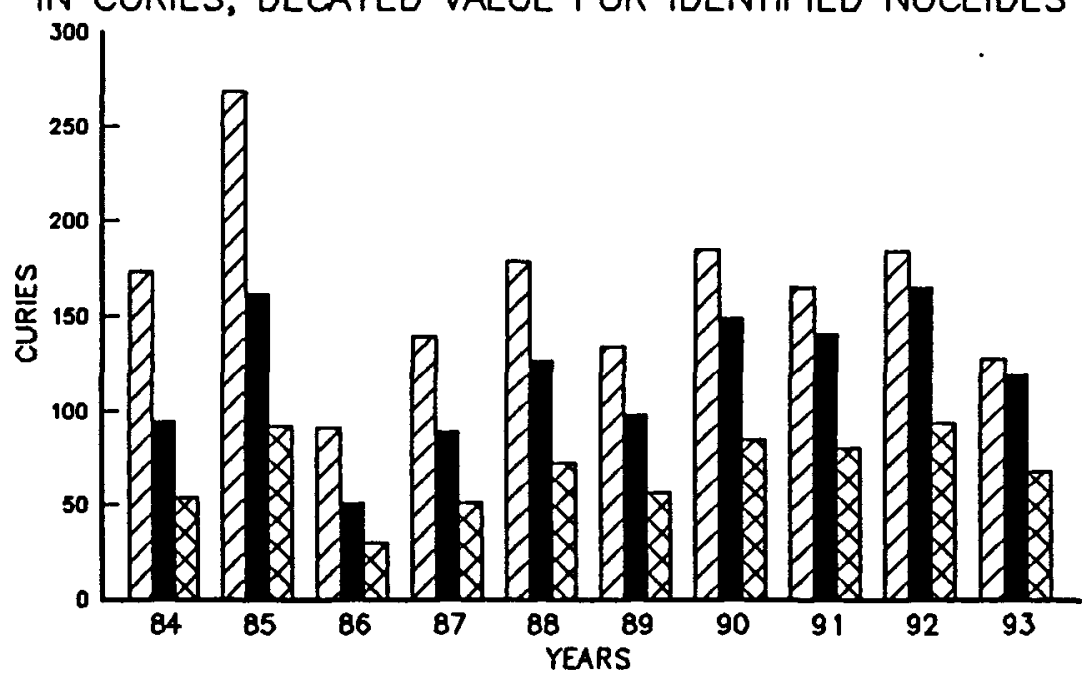

DRELEASED $\square$ DECAYED ON $12 / 31 / 93$ DECAYED ON $12 / 31 / 03$ 
TEST REACTOR AREA

SOLID DISPOSED WUSTE DECAY DATA

\begin{tabular}{|c|c|c|c|c|c|c|}
\hline ACCUMULATED IN & $\begin{array}{r}\text { RADI } \\
\text { AMOU } \\
\text { IDENTIFIED } \\
\text { MCLIDES }\end{array}$ & $\begin{array}{l}\text { TIVITY } \\
\text { (CURIES) } \\
\text { UNIDENTIFIED } \\
\text { MUCLIDES }\end{array}$ & \multicolumn{2}{|c|}{$\begin{array}{l}\text { DECAYED VALUE FOR } \\
\text { IDENTIFIED NUCLIDES } \\
\text { THROUEH } 12 / 31 / 93 \\
\text { CURIES REMAINING }\end{array}$} & \multicolumn{2}{|c|}{$\begin{array}{l}\text { DECAYED VALUE F } \\
\text { IDENTIFIED MULL } \\
\text { THROUGH } 12 / 31 / 0 \\
\text { CURIES REMUININ }\end{array}$} \\
\hline $\begin{array}{l}1962 * \\
1984 \\
1985 \\
1986 \\
1987 \\
1988 \\
1989 \\
1990 \\
1991 \\
1992 \\
1993\end{array}$ & $\begin{array}{r}2396315 \\
1013 \\
2369 \\
1233 \\
6237 \\
139 \\
75 \\
68 \\
2430 \\
909 \\
298152\end{array}$ & $\begin{array}{r}1470582 \\
85 \\
83 \\
20 \\
5394 \\
8 \\
2 \\
<1 \\
0 \\
0 \\
0\end{array}$ & $\begin{array}{r}59524 \\
105 \\
138 \\
87 \\
2576 \\
1 \\
1 \\
5 \\
914 \\
283 \\
289111\end{array}$ & $\begin{array}{r}2 \% \\
10 \% \\
6 \% \\
7 \% \\
41 \% \\
2 \% \\
2 \% \\
7 \% \\
38 \% \\
31 \% \\
97 \%\end{array}$ & $\begin{array}{r}21532 \\
57 \\
80 \\
46 \\
713 \\
<1 \\
<1 \\
1 \\
218 \\
47 \\
165004\end{array}$ & $\begin{array}{r}<1 \% \\
6 \% \\
3 \% \\
4 \% \\
11 \% \\
<1 \% \\
<1 \% \\
2 \% \\
9 \% \\
5 \% \\
55 \%\end{array}$ \\
\hline TOTALS & 2708936 & 1476172 & 352746 & $13 \%$ & 187699 & $7 \%$ \\
\hline
\end{tabular}

DETAILS MAY NOT ADD UP TO TOTALS BECAUSE OF ROUNDING

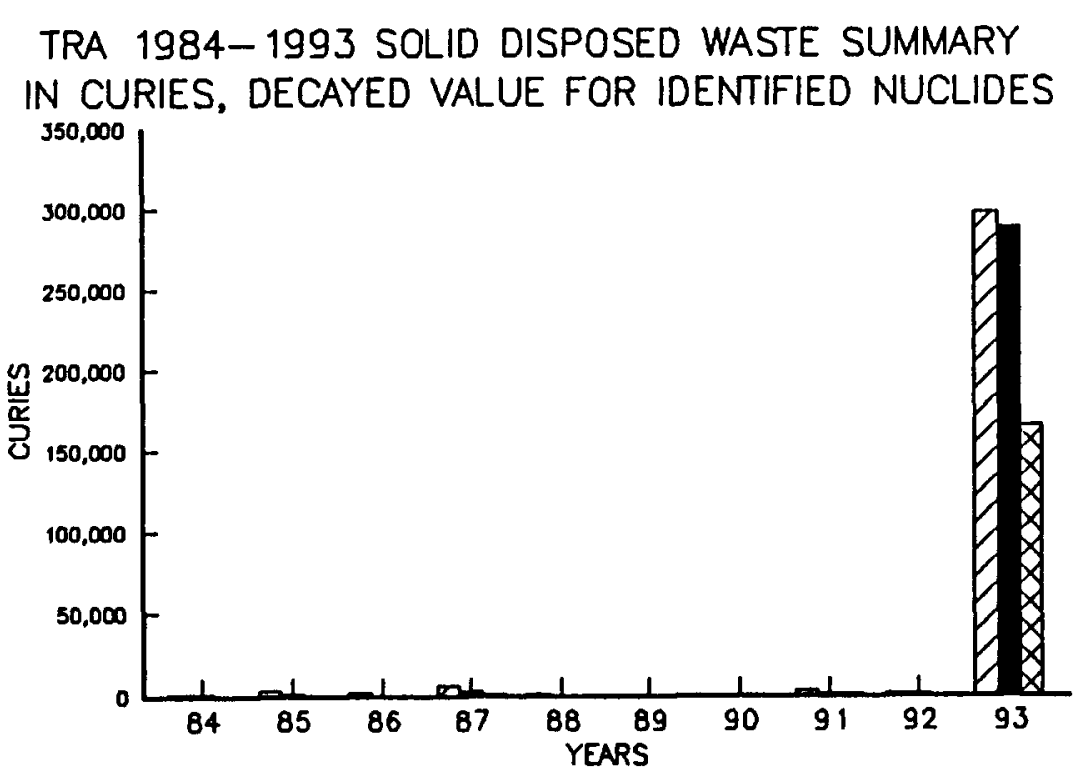

DDISPOSED DECAYED ON 12/31/93 D DECAYED ON 12/31/03 
AIRBORNE EFFLUENT DECAY DATA
RADIOACTIVITY

AMOUNT (CURIES) AT RELEASE IDENTIFIED UNIDENTIFIED NUCLIDES MUCLIDES

ACCUMLATED IN

$$
\begin{aligned}
& 1986 \\
& 1987 \\
& 1988 \\
& 1989 \\
& 1990 \\
& 1991 \\
& 1992 \\
& 1993
\end{aligned}
$$

TOTALS

$$
\begin{aligned}
& <1 \\
& <1 \\
& <1 \\
& <1 \\
& <1 \\
& <1 \\
& <1 \\
& 0 \\
& <1
\end{aligned}
$$

$<1$
0
0
0
0
0
0
0
$<1$

* INCLUDES $1962-1983$

DETAILS MAY NOT ADD UP TO TOTALS BECAUSE OF ROUNDINE

IDENTIFIED NUCLIDES
DECAYED VALUE FOR

IDENTIFIED MUCLIDES

THROUGH 12/31/03

CURIES REMAINING

THROUGH 12/31/93

$\begin{array}{rr}<1 & 21 \% \\ <1 & 26 \% \\ <1 & 25 \% \\ <1 & 5 \% \\ <1 & 7 \% \\ <1 & 29 \% \\ <1 & 6 \% \\ 0 & 39 \% \\ <1 & 23 \%\end{array}$




\section{SOLID DISPOSED MUSTE DECAY DATA}

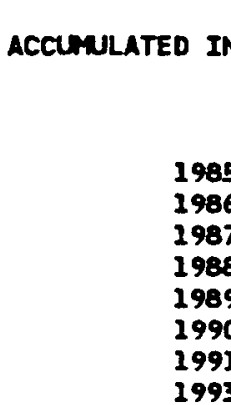

RADIOACTIVITY
MOWN (CURIES)
IDENIIFIED
MUCLIOES

DECAYED VALUE FOR IDENTIFIED MUCLIDES

THROUEH $12 / 31 / 93$

DECAYEd VALUE FOR

IDENTIFIED NUCLIDES

THROUEH 12/31/03

CURIES REMAINING

TOTALS

$\begin{array}{rr}0 & <1 \\ 0 & <1 \\ 0 & <1 \\ 0 & <1 \\ 0 & <1 \\ 0 & 6 \\ 0 & 3 \\ <1 & 0 \\ <1 & 10\end{array}$

* INCLUDES $1962-1983$

DETAILS MAY NOT ADD UP TO TOTALS BECAUSE OF ROUNDING

$\begin{array}{rrrr}0 & 0 \% & 0 & 0 \% \\ 0 & 0 \% & 0 & 0 \% \\ 0 & 0 \% & 0 & 0 \% \\ 0 & 0 \% & 0 & 0 \% \\ 0 & 0 \% & 0 & 0 \% \\ 0 & 0 \% & 0 & 0 \% \\ 0 & 0 \% & 0 & 0 \% \\ <1 & 94 \% & <1 & 53 \% \\ <1 & 94 \% & <1 & 53 \%\end{array}$


AIRBORNE EFFLUENT DECAY DATA

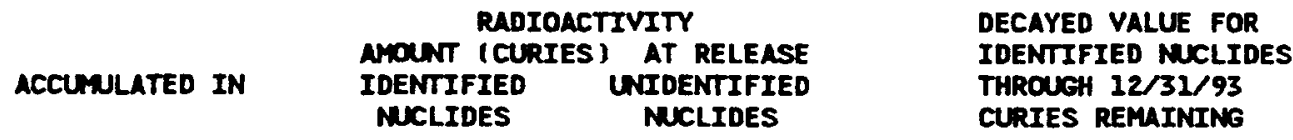

\begin{tabular}{|c|c|c|c|c|c|}
\hline $\begin{array}{l}1987 \\
1988 \\
1989 \\
1992 \\
1993\end{array}$ & $\begin{array}{l}<1 \\
<1 \\
<1 \\
<1 \\
<1\end{array}$ & $\begin{array}{l}0 \\
0 \\
0 \\
0 \\
0\end{array}$ & $\begin{array}{r}<1 \\
0 \\
0 \\
0 \\
0\end{array}$ & $\begin{array}{l}3 \% \\
0 \% \\
0 \% \\
0 \% \\
0 \%\end{array}$ & $\begin{array}{r}<1 \\
0 \\
0 \\
0 \\
0\end{array}$ \\
\hline OTALS & $<1$ & 0 & $<1$ & $<1 \%$ & $<1$ \\
\hline
\end{tabular}

* INCLUDES $1962-1983$

DETAILS MAY NOT ADD UP TO TOTALS BECALSE OF ROUNDING

DECAYED VALUE FOR

IOENTIFIED NUCLIDES

THROUGH $12 / 31 / 03$

CURIES REMAINING 


\section{LIQUTD EFFLUENT DATA}

RADIOACTIVITY

NHONT (CURIES) AT RELEASE

IDENTIFIED UNIDENTIFIED

NUCLIDES

DeCAYEd VALUE FOR

IDENTIFIED NUCLIDES

THROUGH 12/31/93

CURIES REMUINING

TOTALS
0

0\%
$\%$

DECAYED VALUE FOR

IDENTIFIED NUCLIDES

THROUEH 12/31/03

CURIES REMAINING

o\%

* INCLUDES $1962-1983$

DETAILS MAY NOT ADD UP TO TOTALS BECAUSE OF ROUNDING 
SOLID DISPOSEO MASTE DECAY DATA

\begin{tabular}{|c|c|c|c|c|c|c|}
\hline ACCUNULATED IN & $\begin{array}{r}\text { RADI } \\
\text { AMOUN } \\
\text { IOENTIFIED } \\
\text { NUCLIDES }\end{array}$ & $\begin{array}{l}\text { TIVITY } \\
\text { (CURIES) } \\
\text { UNIDENTIFIED } \\
\text { MUCLIDES }\end{array}$ & \multicolumn{2}{|c|}{$\begin{array}{l}\text { DECAYED VALUE FOR } \\
\text { IDENTIFIED MULIDES } \\
\text { THROUEH } 12 / 31 / 93 \\
\text { CURIES REMAINING }\end{array}$} & \multicolumn{2}{|c|}{$\begin{array}{l}\text { DECAYED VALUE FO } \\
\text { IDENTIFIED MUCLI } \\
\text { THROUGH } 12 / 31 / 03 \\
\text { CURIES REMAININE }\end{array}$} \\
\hline $\begin{array}{l}1962 * \\
1984 \\
1986 \\
1987 \\
1989 \\
1990\end{array}$ & $\begin{array}{l}6 \\
<1 \\
<1 \\
83 \\
<1 \\
<1\end{array}$ & $\begin{array}{r}60920 \\
0 \\
0 \\
<1 \\
0 \\
0\end{array}$ & $\begin{array}{l}4 \\
<1 \\
<1 \\
82 \\
<1 \\
<1\end{array}$ & $\begin{array}{r}69 \% \\
100 \% \\
99 \% \\
99 \% \\
100 \% \\
100 \%\end{array}$ & $\begin{array}{l}3 \\
<1 \\
<1 \\
81 \\
<1 \\
<1\end{array}$ & $\begin{array}{r}55 \% \\
100 \% \\
97 \% \\
97 \% \\
100 \% \\
100 \%\end{array}$ \\
\hline TOTALS & 89 & 60920 & 86 & 97\% & 84 & $94 \%$ \\
\hline
\end{tabular}

* Includes $1962-1983$

DETAILS MAY NOT ADD UP TO TOTALS BECAUSE OF ROUNDING

WMC 1984-1993 SOLID DISPOSED WASTE SUMMARY IN CURIES, DECAYED VALUE FOR IDENTIFIED NUCLIDES

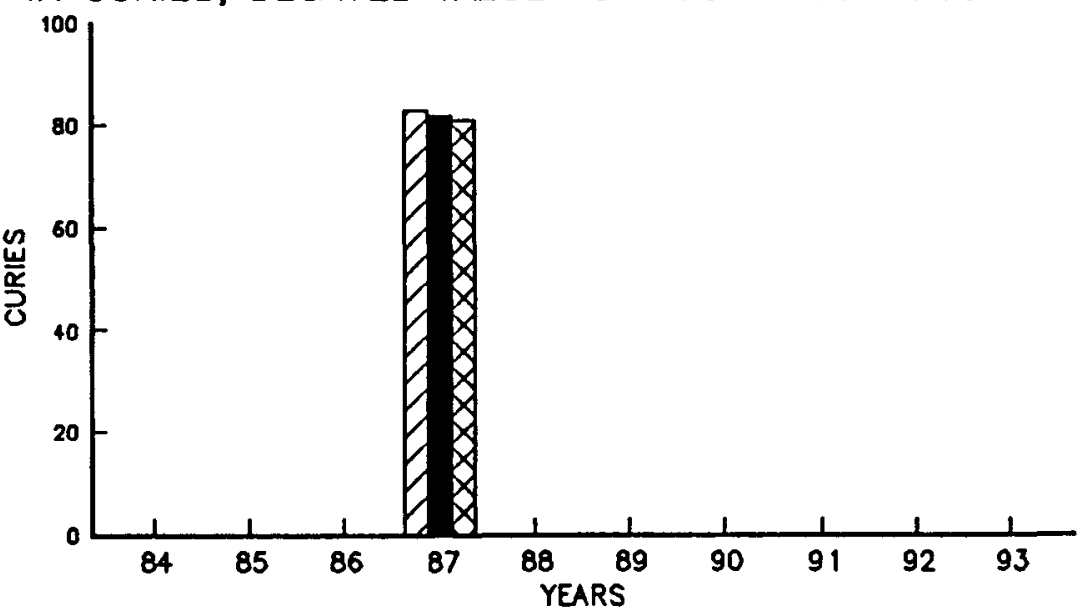

DDISPOSED $\square$ DECAYED ON 12/31/93 D DECAYED ON $12 / 31 / 03$ 


\section{INEL 1993 Year-to-Date Summary}

INEL Year To Date Summary, $1993 \ldots \ldots \ldots \ldots \ldots \ldots \ldots \ldots \ldots \ldots \ldots$ INEL-103

INEL Year-To-Date CY1993 Pie Charts

INEL-106

INEL Year-To-Date Summary CY1992 Bar Graphs

INEL-107 

INEL YEAR TO OATE SUMYARY

FOR JANUARY THROUGH DECEMBER 1993

PG 1 RPT. 2

AIRBORNE

$\begin{array}{cc}\text { VOLLME } & \text { RADIO- } \\ \text { (CU. METERS) } & \text { ACTIVITY } \\ \text { (CURIES ) }\end{array}$

LICUID

\begin{tabular}{cc} 
VOLUNE & $\begin{array}{c}\text { RADIO- } \\
\text { ACTIVITY } \\
\text { (LITERS) } \\
\text { (CURIES) }\end{array}$ \\
\hline
\end{tabular}

5.009E+06 7.881E-02

$2.522 E+09 \quad 2.260 E+03$

$3.439 E+07 \quad 1.541 E-06$

4.244E+09 $6.214 E+01$

4.385E+07 1.226E-04

$2.515 E+09 \quad 1.167 E-02$

$1.646 E+09 \quad 1.298 E+00$

5.225E+07 3.099E-06

$3.141 E+09 \quad 2.400 E-05$

$1.907 E+08 \quad 5.996 E-06$

$1.155 E+09 \quad 1.495 E+03$

$1.547 E+08-1.432 E-07$

$9.565 E+06 \quad 1.231 E-05$
$2.428 E+07 \quad 1.863 E+00$

$\begin{array}{ll}2.428 E+07 & 1.863 E+00 \\ 7.068 E+07 & 1.282 E+02\end{array}$
VOLURE

SOLID

to-

ACTIVITY
(CURIES )

(CU. METERS)

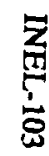

wance

$1.315 E+10 \quad 2.719 E+03$

$2.659 E+09 \quad 1.301 E+02$

8.519E+02

$4.297 E+05$

* solio values are for maste disposed at ruAc and are end products of werf reduction processes. 
INEL WASTE MANAGEMENT INFORMATION SYSTEM

INEL YEAR TO DATE SUMMARY

FOR JANUARY THROUGH OCTOBER 1993

FACILITY

STOTED WASTES

$\begin{array}{cc}\text { RADIO- } \\ \text { VOLLE } & \begin{array}{c}\text { ACTIVITY } \\ \text { (CU. METERS ) } \\ \text { (CURIES ) }\end{array}\end{array}$

RADIO-
VOLUME
(LITERS)
ACTIVITY
(CURIES )

NL STORMGE AREA

NL

\section{$0.000 E+00 \quad 0.000 E+00$}

$0.000 E+00$

$0.000 E+00$

ILTSF MSTE FROH:

ALE

$0.000 E+00 \quad 0.000 E+00$

$0.000 E+00 \quad 0.000 E+00$

LIEUTD MSTE TAKKS

cPp

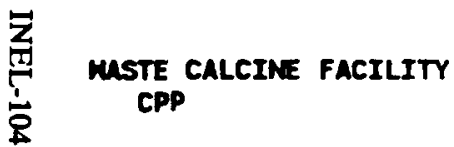

\section{$1.140 E+06 \quad 1.141 E+06$}

$1.140 E+06 \quad 1.141 E+06$

8.900E-01 1.165E+03

8. 900E-01 1.165E+03

$5.680 E+00 \quad 5.985 E+01$

$5.680 E+00 \quad 5.985 E+01$

PG 2 RPT. 2

RADIO-

CTIVITY

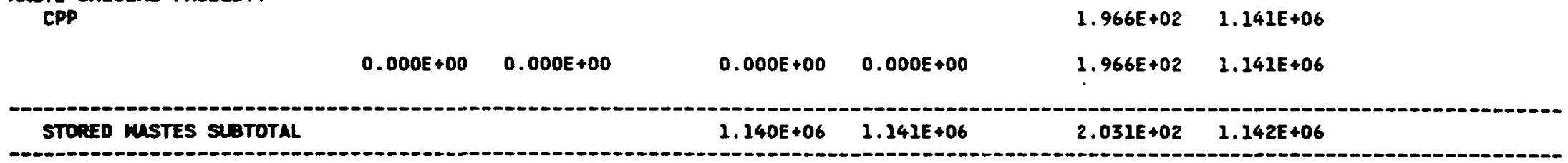


INEL WASTE MANAGEMENT INFORMATION SYSTEM
TNEL YEAR TO DATE SUMARY

FOR JANUARY THROUGH OECEMBER 1993

AIRBORNE

RADIO- LIOUID

\section{LIQUID}

\section{FACILITY}

VOLUEE RADIO-

(CU. METERS) (CURIES

\begin{tabular}{cc}
$\begin{array}{c}\text { RADIO- } \\
\text { (LITERS) }\end{array}$ & $\begin{array}{c}\text { ACTIVITY } \\
\text { (CURIES) }\end{array}$ \\
\hline
\end{tabular}

SOLID

\section{USTTES TO BE PROCESSED}

AT neRF FROM:
CFA
CPP
MRF
PER

$\begin{array}{ll}2.020 E+01 & 4.103 E-03 \\ 5.505 E+01 & 2.100 E-01 \\ 5.332 E+01 & 1.951 E-01 \\ 2.912 E+02 & 1.533 E+00 \\ 2.582 E+01 & 4.635 E-04\end{array}$


INEL Year-to-Date Summary CY 1993

INEL AIRBORNE EFFLUENT

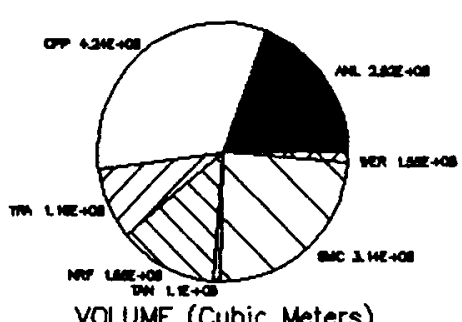

VOLUME (Cubic Meters)

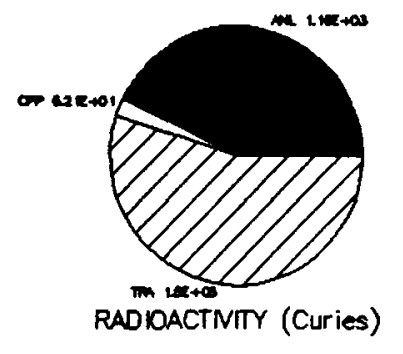

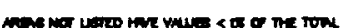

INEL LIQUID EFFLUENT

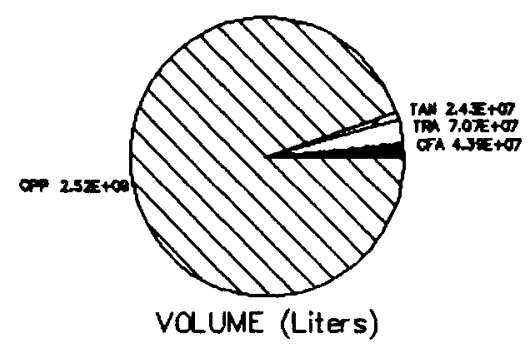

INEL SOLID WASTE VOLUME (Cubic Meters) DISPOSED AT RWMC AND SENT FOR REDUCTION EFFORTS

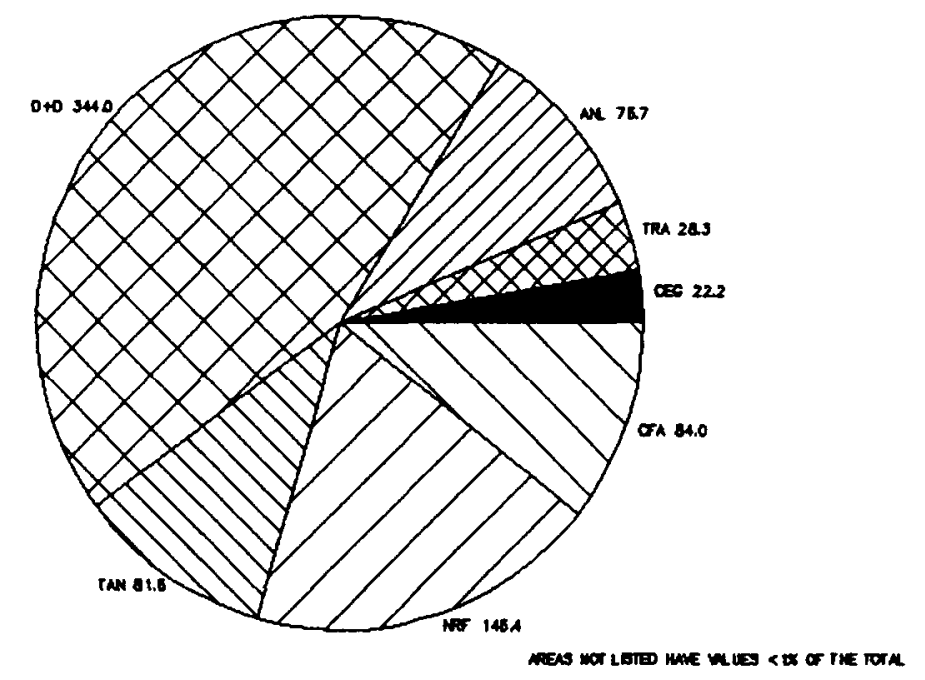


INEL Year-to-Date Summary CY 1993
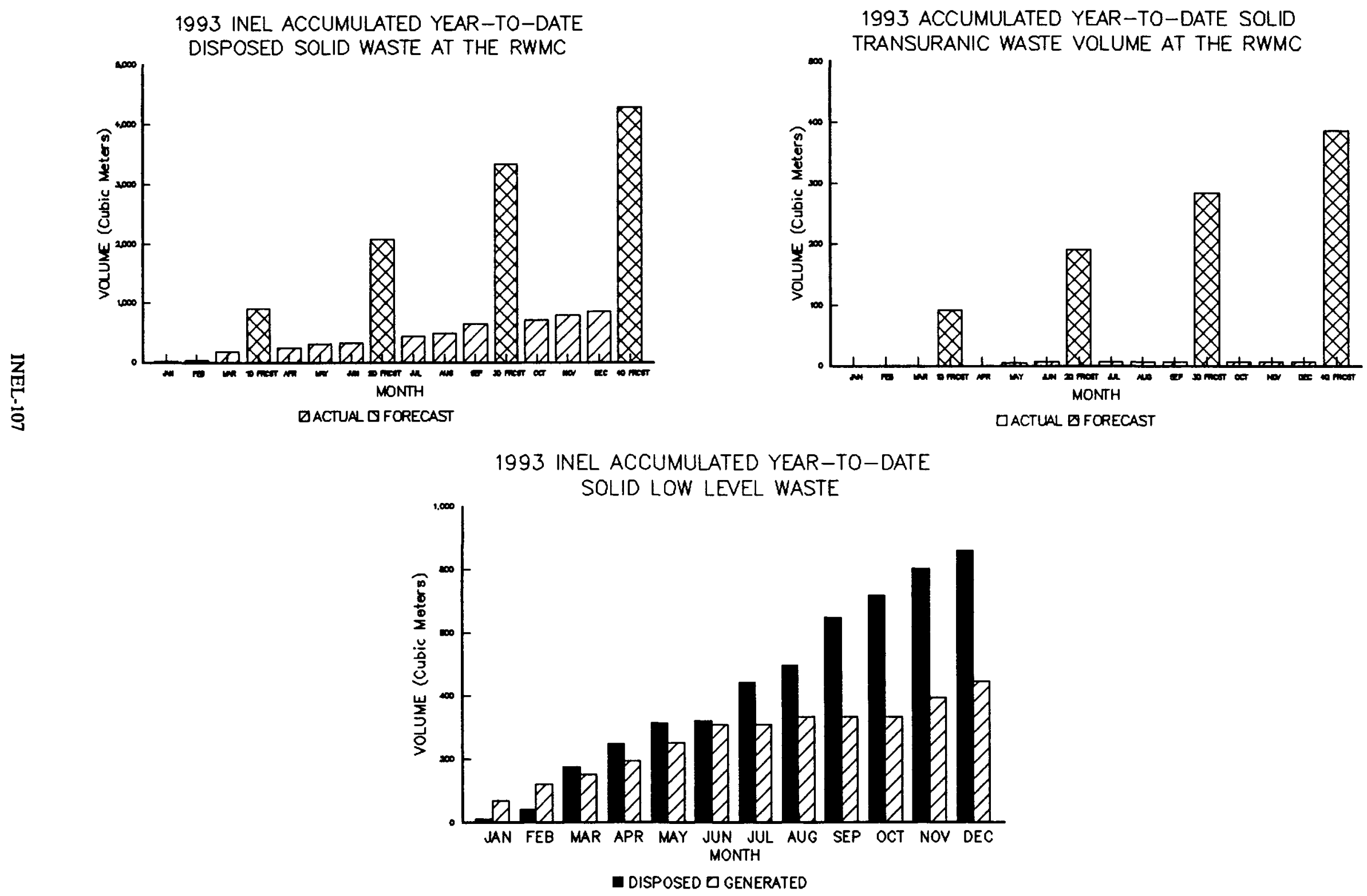

INEL Disposed Waste Decay Summary in Curies for 1993

INEL Disposed Waste Decay Summary In Curies For $1993 \ldots \ldots \ldots \ldots \ldots$ INEL-111 



\section{DISPOSED MASTE DECAY SUMMARY IN CURIES FOR 1993}

$\begin{array}{cc}\text { TOTAL } & \text { TOTAL } \\ \text { IDENTIFIED } & \text { UNIDENTIFIED } \\ \text { NMCLIDES } & \text { MUCLIDES } \\ \text { AT TIME OF } & \text { AT TIME OF } \\ \text { DISPOSAL } & \text { DISPOSAL }\end{array}$

$\begin{array}{lrr}\text { AIRBORNE } & 2719 & 0 \\ \text { LIEUID } & 130 & 0 \\ \text { SOLID } & 429654 & 0\end{array}$

210

DECAYED VALUE FOR IDENTIFIED NUCLIDES THROUGH

$01 / 01 / 9401 / 01 / 9501 / 01 / 96$ 01/01/97 01/01/98 01/01/99 01/01/04

429654

122

183

174

166

158

150

119

DETAILS MAY NOT ADD UP TO TOTALS BECAUSE OF ROUNDING

$\stackrel{\text { 离 }}{\stackrel{5}{\Xi}}$ 

Nuclide Summary in Curies for Airborne, Liquid, and Solid Waste

Airborne Nuclide Summary In Curies, 1993

INEL-115

Liquid Nuclide Summary In Curies, 1993

INEL-117

Disposed Solid Waste Nuclide Summary In Curies, 1993

INEL-118

ANL Storage Area Nuclide Summary in Curies, 1993

INEL-120

WERF Processed Nuclide Summary In Curies, 1993

INEL-121 

INEL WUSTE MANAGEMENT INFORYATION SYSTEM

$$
\text { AIRBORNE }
$$

MCLIDE SUMMARY IN CURIES

FOR JANUARY THROUEH DECEMBER 1993

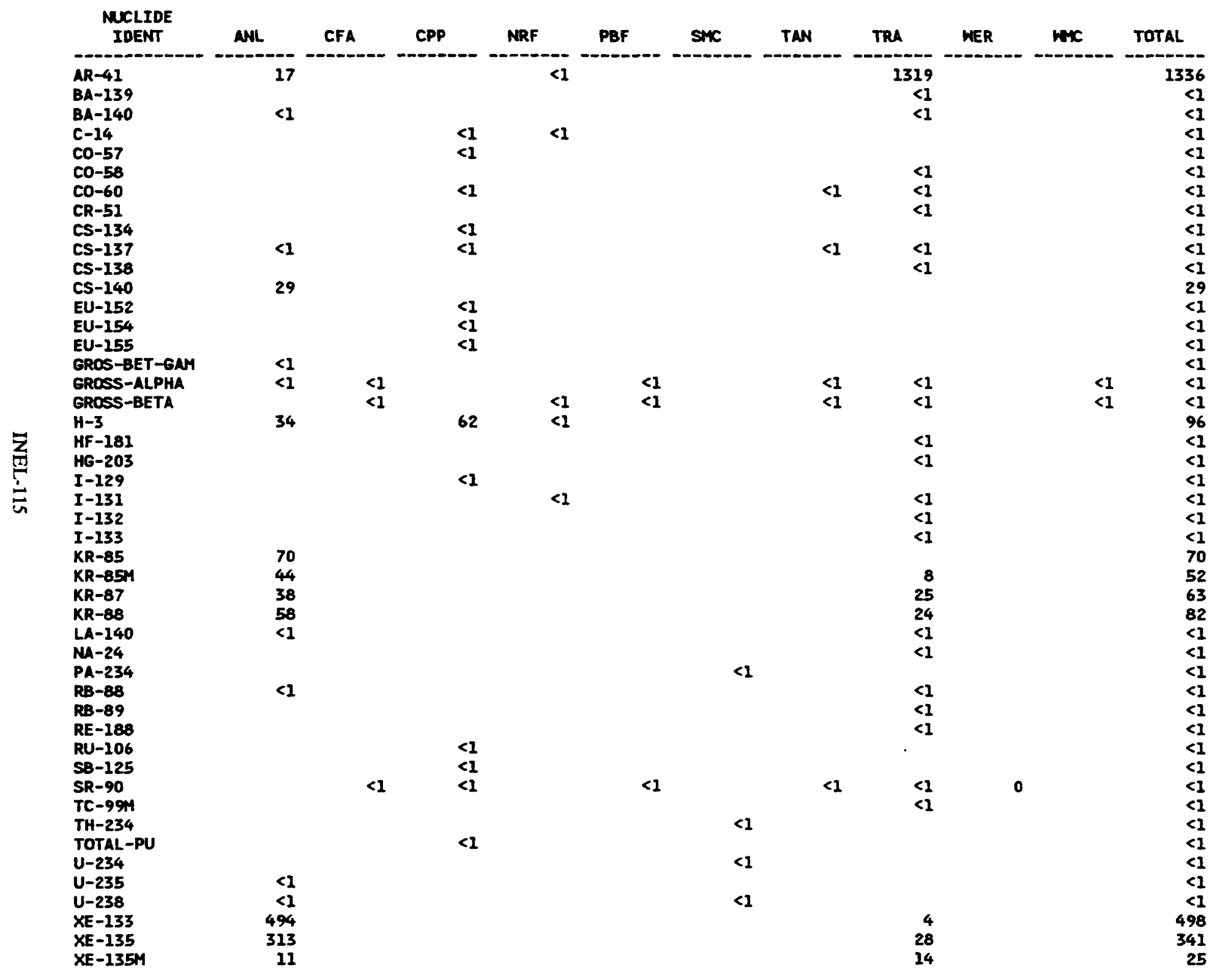


INEL WASTE MANAGEMENT INFORMATION SYSTEM

\section{AIRBORNE}

NUCLIOE SUMARY IN CURIES

FOR JANUARY THROUEH DECEMBER 1993

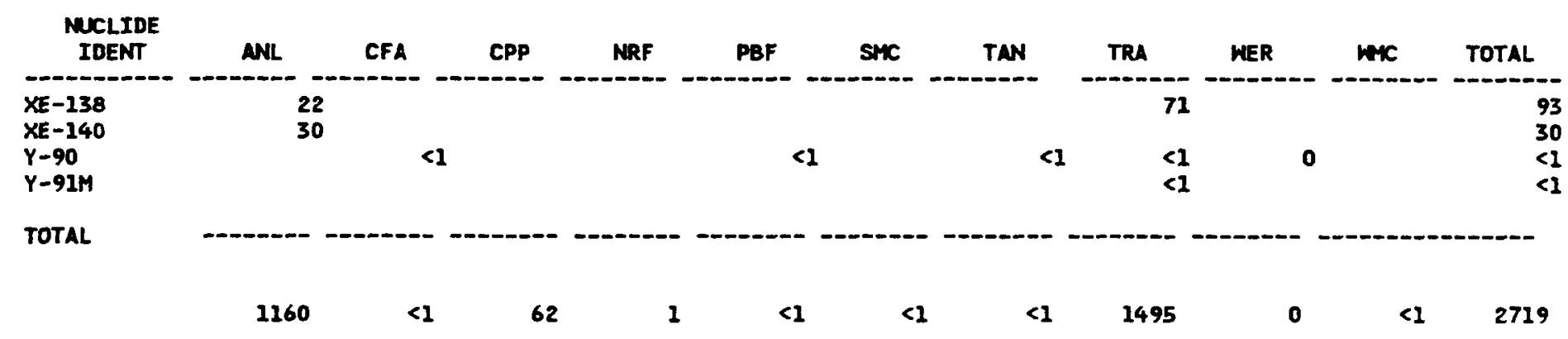


INEL WASTE MANAGEMENT INFORMATION SYSTEM

\section{LIQUTD}

MCLIDE SUMUARY IN CURIES

FOR JANUARY THROUGH DECEMBER 1993

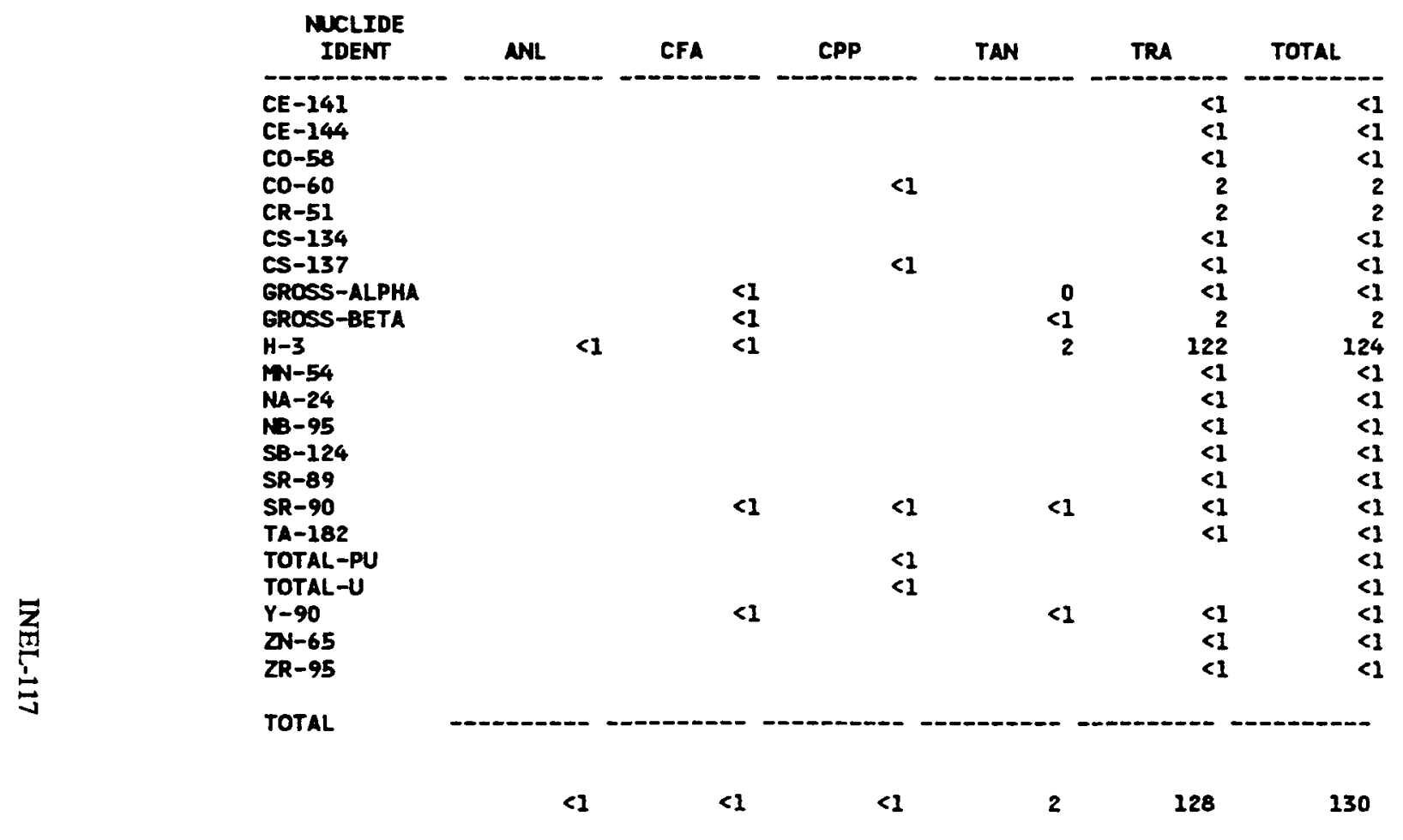


NCLIDE SUMMARY IN CURTES

FOR JANUARY THROUGH DECEKBER 1993

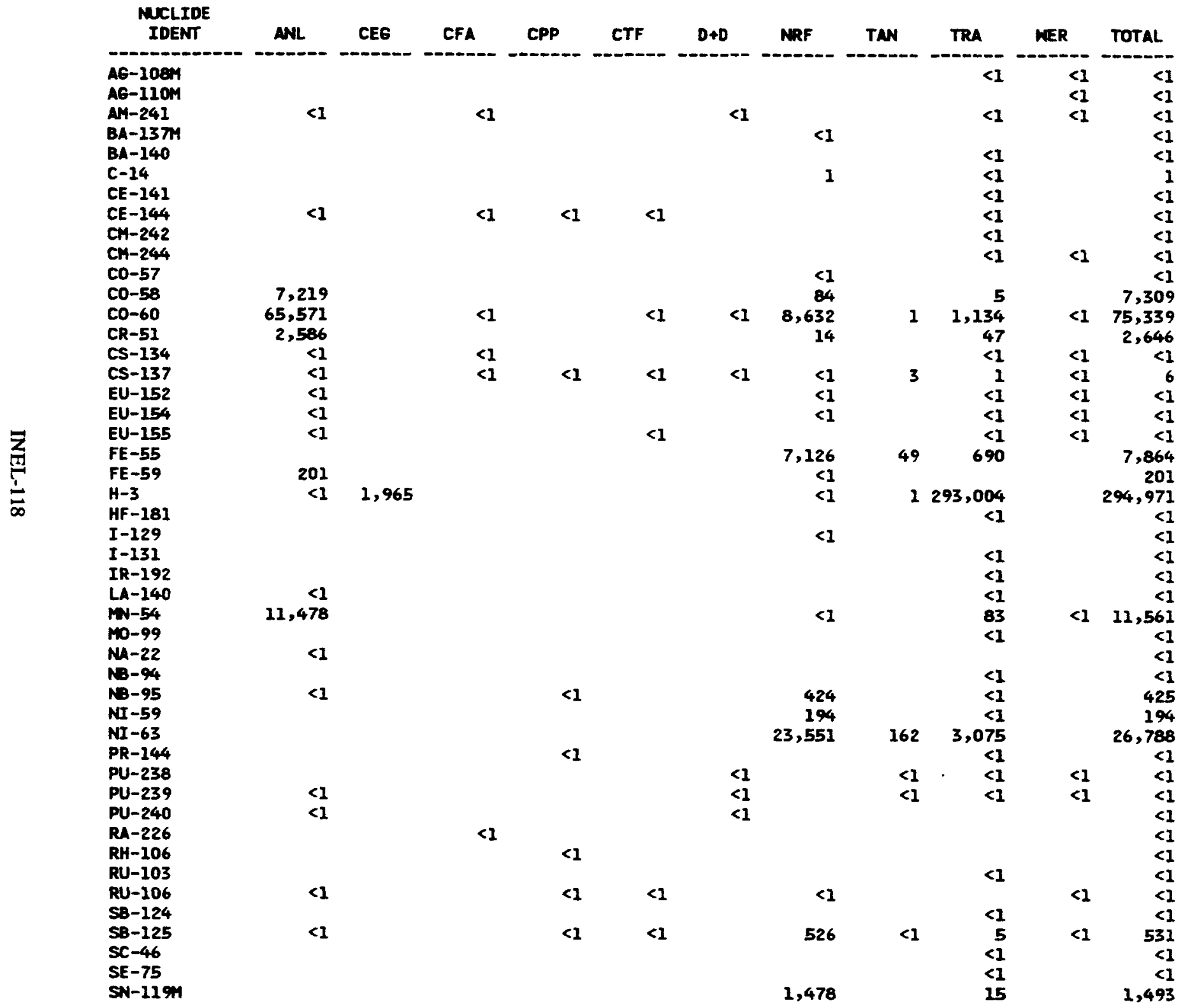


INEL MASTE MUUGEMENT INFORMUTION SYSTEM

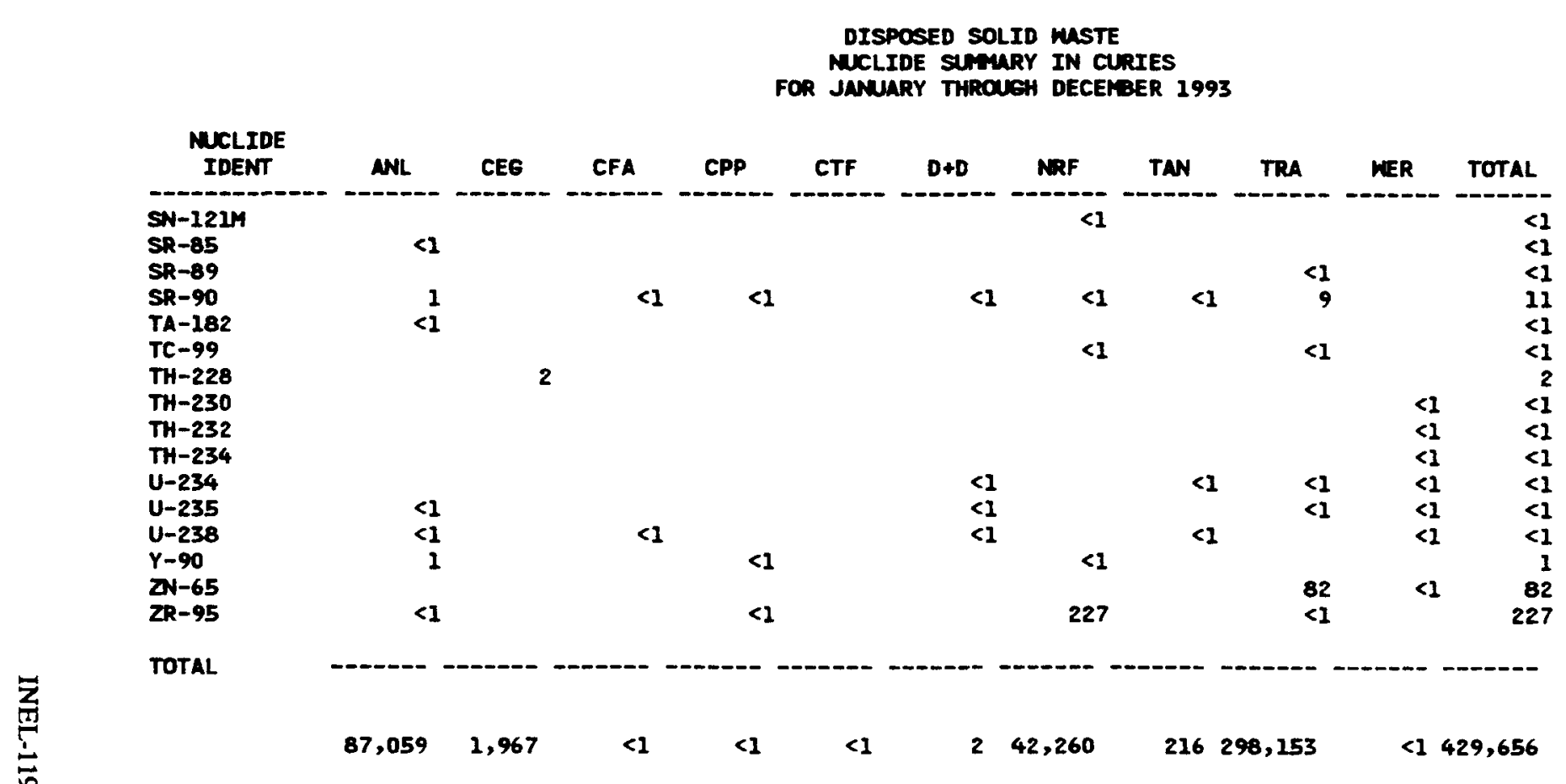


IDAHO OPERATIONS OFFICE

RUN DATE: 05/26/94

INEL WUSTE MANAGEMENT INFORMATION SYSTEM

ANL STORAGE AREA

CURIES

PG 1

FOR JANUARY THROUGH DECEBBER 1993

MULIDE

IDENT

ANL

TOTAL

BA-13T

CE-141

CE-144

Co-57

Co-58

CO-60

CS- 134

CS -137

EU-154

EU-155

$\mathrm{H}-3$

KR-85

$M N-5$

NA-22

$\mathrm{NB}-95$

No-95

N-95M

NP-237

PM-147

PR-144

PU-239

PU-240

RH-103M

RH-106

RH-106

RU-106

SB-125

SM-151

SN-123

SR-85

SR-89

SR-90

TE-125M

TE-127M

TH-234

U-233

U -235

$U-238$

$Y-90$

$Y-91$
$Z R-95$

TOTAL

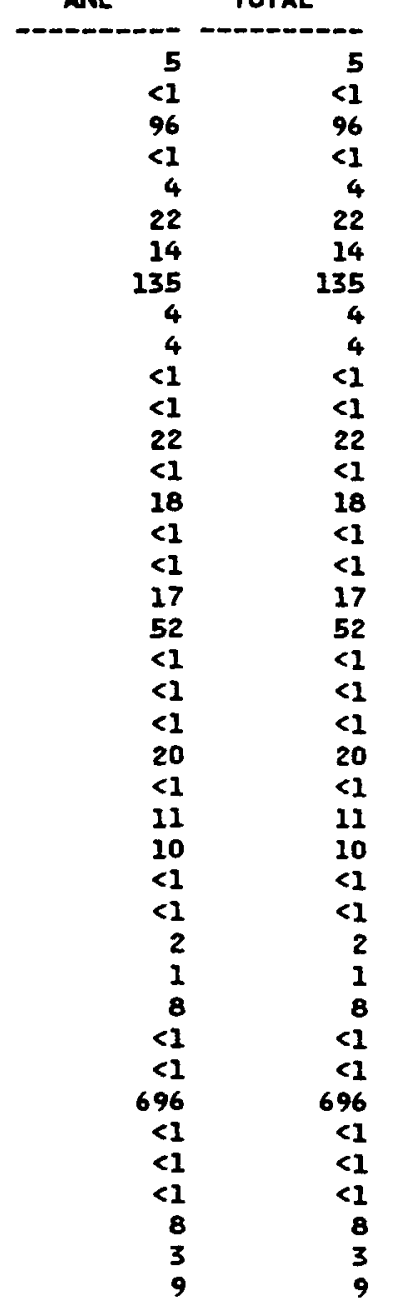


INEL WASTE MANAGEMENT INFORMATION SYSTEM

WERF PROCESSED

MUCLIDE SUMAARY IN CURIES

FOR JANAARY THROUGH DECEMBER 1993

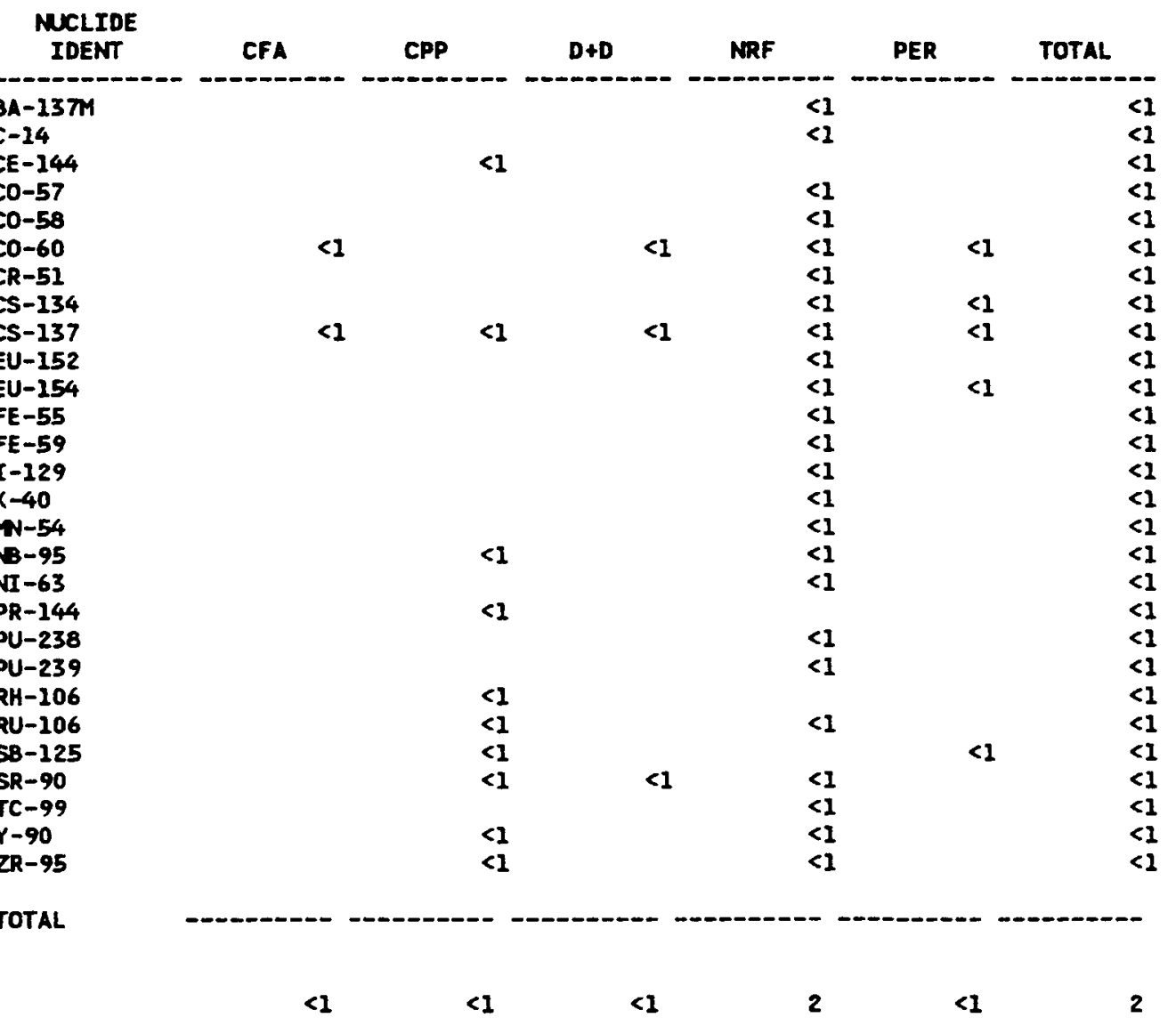





\section{Argonne National Laboratory-West 1993 Detail Data}

Argonne National Laboratory-West (ANL) Bar Graphs of Annual Data

by Month

CY1993 Bar Graphs

ANL-5

ANL Detail Data for Months July to December,

Annual Average Concentration and Total Curies for January to December

Report 401-ANL Airborne Waste Summary . . . . . . . . . . . . . . . ANL-9

Report 402-ANL Liquid Waste Summary .................... ANL-12

Report 403-ANL Disposed Solid Waste Summary ................ ANL-13

Report 404-ANL Solid Waste Summary, ANL Stored Wastes ......... ANL-16

ANL Detail Data for Months January to June,

Annual Average Concentration and Total Curies for January to June

ANL-21

Report 401-ANL Airborne Waste Summary . .................. ANL-23

Report 402-ANL Liquid Waste Summary $\ldots \ldots \ldots \ldots \ldots \ldots \ldots \ldots \ldots$ ANL-26

Report 403-ANL Disposed Solid Waste Summary ............... ANL-27

ANL Facility Detail Data for Months July to December,

Annual Average Concentration and Total Curies for January to December ...... ANL-29

Report 440-EBR-II Airborne Waste Summary $\ldots \ldots \ldots \ldots \ldots \ldots \ldots \ldots$ ANL-31

Report 441-EBR-II Liquid Waste Summary $\ldots \ldots \ldots \ldots \ldots \ldots \ldots \ldots$ ANL-33

Report 470-FASB Airborne Waste Summary $\ldots \ldots \ldots \ldots \ldots \ldots \ldots \ldots$ ANL-34

Report 475-FMF Airborne Waste Summary $\ldots \ldots \ldots \ldots \ldots \ldots \ldots \ldots$ ANL-35

Report 460-HFEF-N Airborne Waste Summary $\ldots \ldots \ldots \ldots \ldots \ldots \ldots$ ANL-36

Report 430-Fuel Cycle FAC Airborne Waste Summary ............. ANL-37

Report 420_L\&O Main Stack Airborne Waste Summary $\ldots \ldots \ldots \ldots \ldots$ ANL-38

Report 422-NDA Laboratory Airborne Waste Summary $\ldots . \ldots \ldots \ldots .$. ANL-39

Report 490-RLWTF Airborne Waste Summary $\ldots \ldots \ldots \ldots \ldots \ldots \ldots$ ANL-40

Report 480-SCMS Airborne Waste Summary $\ldots \ldots \ldots \ldots \ldots \ldots \ldots$. ANL-41

Report 410_TREAT Airborne Waste Summary $\ldots \ldots \ldots \ldots \ldots \ldots \ldots$ ANL-42

Report 450-ZPPR Airborne Waste Summary $\ldots \ldots \ldots \ldots \ldots \ldots \ldots$ ANL-45

ANL Facility Detail Data for Months January to June,

Annual Average Concentration and Total Curies for January to June .......... ANL-47

Report 440-EBR-II Airborne Waste Summary . ................. ANL-49

Report 441-EBR-II Liquid Waste Summary $\ldots \ldots \ldots \ldots \ldots \ldots \ldots \ldots$ ANL-51

Report 470-FASB Airborne Waste Summary $\ldots \ldots \ldots \ldots \ldots \ldots \ldots$ ANL-52

Report 475-FMF Airborne Waste Summary $\ldots \ldots \ldots \ldots \ldots \ldots \ldots$. ANL-53

Report 460-HFEF-N Airborne Waste Summary $\ldots \ldots \ldots \ldots \ldots \ldots \ldots$ ANL-54

Report 430-Fuel Cycle FAC Airborne Waste Summary ............. ANL-55

Report 420-L\&O Main Stack Airborne Waste Summary $\ldots \ldots \ldots \ldots \ldots$ ANL-56

Report 422-NDA Laboratory Airborne Waste Summary ............. ANL-57

Report 490-RLWTF Airborne Waste Summary $\ldots \ldots \ldots \ldots \ldots \ldots \ldots$ ANL-58

Report 480-SCMS Airborne Waste Summary $\ldots \ldots \ldots \ldots \ldots \ldots \ldots$ ANL-59

Report 410 -TREAT Airborne Waste Summary $\ldots \ldots \ldots \ldots \ldots \ldots \ldots \ldots$ ANL-60

Report 450_ZPPR Airborne Waste Summary $\ldots \ldots \ldots \ldots \ldots \ldots \ldots \ldots$ ANL-63 
Argonne National Laboratory-West (ANL) Bar Graphs of Annual Data by Month 

Argonne National Laboratory-West (ANL) Monthly Details - CY 1993
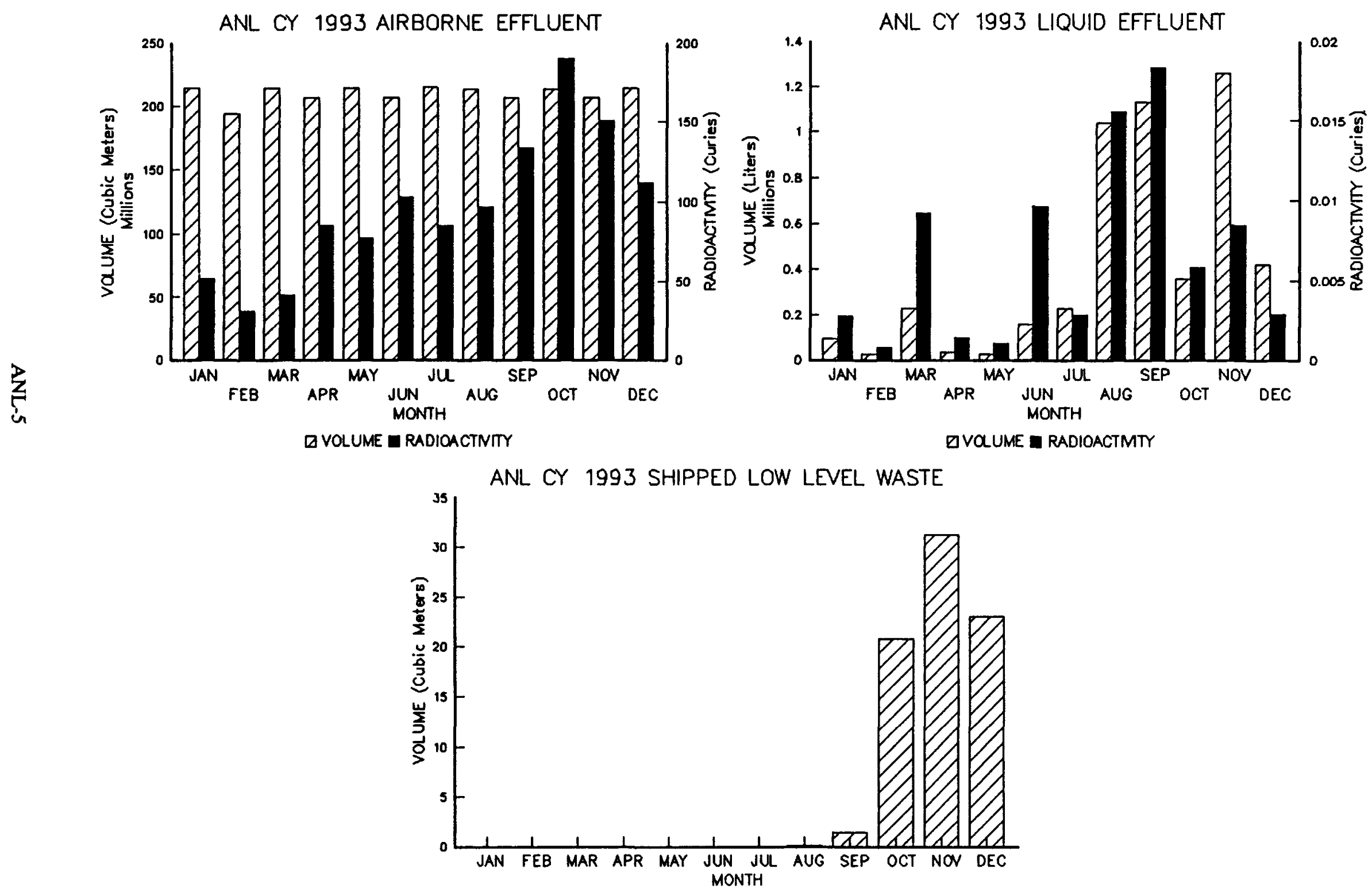

口INCINERATION $\square$ METAL SIZING I COMPACTION DDIRECT DISPOSAL 
Argonne National Laboratory-West (ANL) Monthly Details - CY 1993

疍

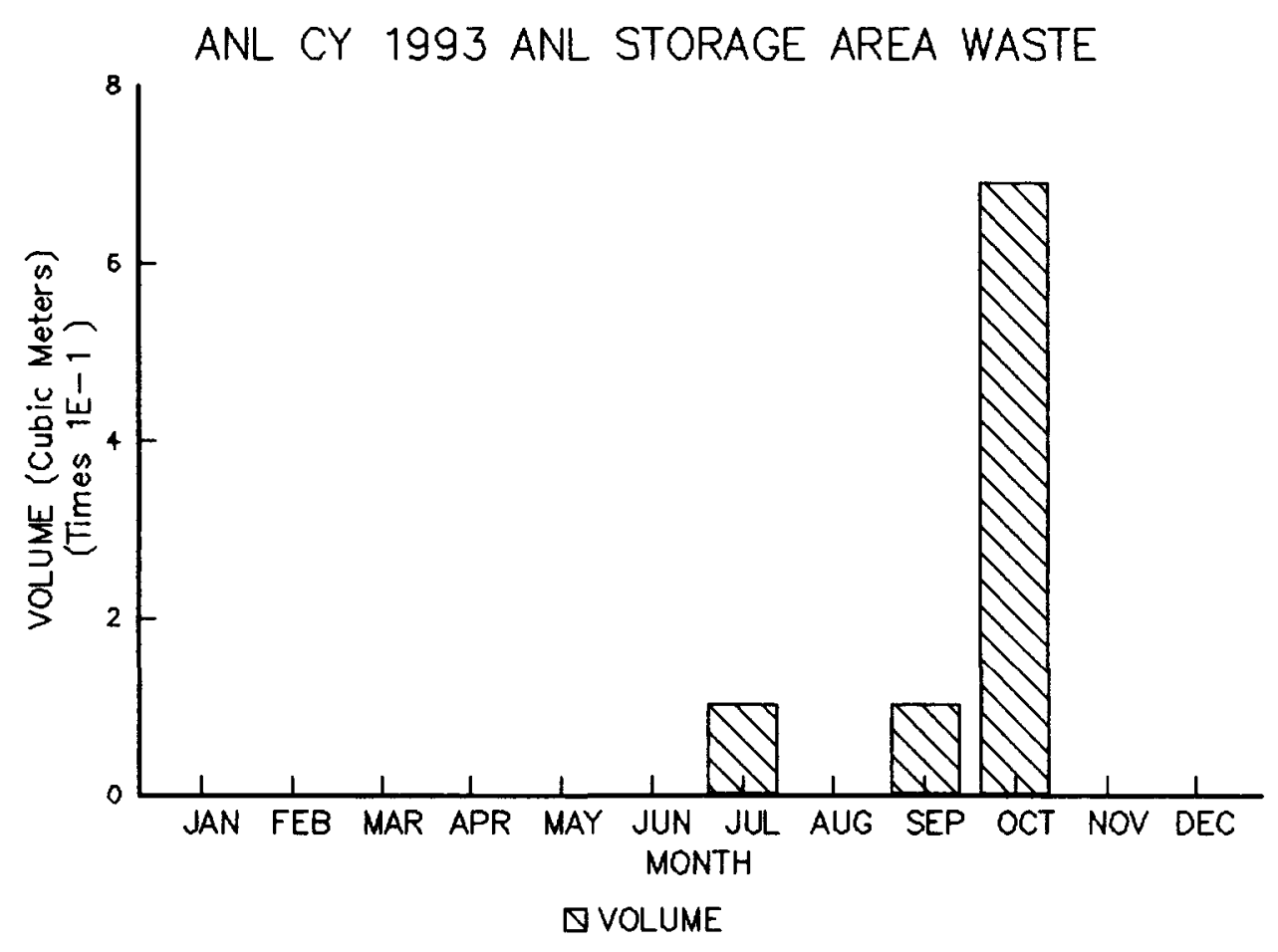


ANL Detail Data for Months July to December Annual Average Concentration and Total Curies for January to December 

NNL AIRBORNE MUSTE SUMARY FOR JAN THROUGH DEC 1993

MUCLIDES
AR-A1
CONCEN (UCML)
CURIES
$\%$ OF TOTAL
BA-140
CONCEN (UCML)
ONRES
$\%$ OF TOTAL
CS-137
CONCEN (UCML)
CURIES
$\%$ OF TOTAL
CS-140
CONCEN (UCML)
CURIES
$\%$ OF TOTAL
GROS-BET-GNM
CONCEN (UCML)
CURIES
$\%$ OF TOTAL

\section{cross-ALPHA}

CONCEN (UCMM)

curres

$\%$ OF TOTAL

${ }_{\text {CONCEN (UCML) }}^{H-3.490 E+03}$ CORIES $\%$ of TOTAL

$1.101 E+04$

$7.708 E-17$

$1.657 \mathrm{E}-08$

$1.260 E+09$

$1.054 E-08$
$2.266 E+00$ 2.662

August

$\begin{array}{rrr}4.643 E-09 & 1.449 E-10 & 8.145 E-09 \\ 9.900 E-01 & 3.100 E-02 & 1.688 E+00\end{array}$

$\begin{array}{rrr}900 E-01 & 3.100 E-02 & 1.688 E+00 \\ 1.173 & 0.032 & 1.262\end{array}$

$1.878 E-13$

$1.882 E-13$

3. $900 E-05$

OCTOBER

NOVERBER
PAGE I RPT. 401
aNeuL
ANL $-A$

$\begin{array}{lll}\text { AVG CONCEN YEARLY } & \text { YEARLY } \\ \text { DECEIBER TOT CURIES RELEASE* CG* } & \% \text { OF CSE }\end{array}$
$\begin{array}{lllll}4.858 E-09 & 9.805 E-09 & 6.641 E-09 & 5.377 E-15 & 1.00 E-08\end{array}$
$1.007 E+00 \quad 2.101 E+00 \quad 1.675 E+01$
$\begin{array}{rrr}0.666 & 1.871 & 1.444\end{array}$

$2.884 E-13 \quad 2.063 E-13 \quad 1.670 E-19 \quad 3.00 E-09$

$6.180 E-05 \quad 5.202 E-04$

6.570E-18 $5.320 E-24 \quad 4.00 E-10$

$1.657 E-08$

$1.062 E-08$
$2.200 E+00$

1.645

$\begin{array}{llll}1.179 E-12 & 1.831 E-14 & 2.574 E-14 & 4.788 E-15\end{array}$

$2.534 E-04 \quad 3.918 E-06 \quad 5.333 E-06 \quad 1.024 E-06$

$\begin{array}{llll}957 E-14 & 6.062 E-14 & 2.830 E-13 & 2.291 E-19\end{array}$

$1.619 E-08 \quad 1.258 E-08 \quad 9.380 E-15$

$3.470 E+00 \quad 2.922 E+01$

$\begin{array}{lr}3.091 & 2.519\end{array}$

4.340E-16 $9.299 E-16 \quad 8.091 E-17 \quad 2.227 E-16 \quad 8.437 E-16 \quad 1.591 E-15 \quad 6.250 E-16 \quad 5.060 E-22$

$\begin{array}{llllllll}9.329 E-08 & 1.990 E-07 & 1.676 E-08 & 4.552 E-08 & 1.748 E-07 & 3.410 E-07 & 1.576 E-06\end{array}$

$\begin{array}{llllllllll}2.557 E-08 & 2.593 E-08 & 3.383 E-08 & 6.725 E-09 & 3.364 E-08 & 3.350 E-08 & 1.350 E-08 & 1.094 E-14 & 1.00 E-07\end{array}$ $5.497 E+00 \quad 5.5496+007.010 E+00 \quad 1.439 E+00$

$6.970 E+00 \quad 7.179 E+00 \quad 3.406 E+01$

$$
\begin{array}{rrrrrrr}
6.459 & 5.744 & 5.241 & 0.756 & 4.612 & 6.395 & 2.936
\end{array}
$$

KR-A5
CONCEN (UCML)

CURTES

$3.913 E+03$

$\%$ of rotAl

3.280E-08 1.292E-08

$\begin{array}{rr}.050 E+00 & 2.550 E+00 \\ 8.283 & 2.640\end{array}$

4.392E-09 2.000E-08

$1.231 E-09$

7.186E-09

$100 E-01 \quad 4.280 E+00$

$2.550 E+00$
1.687

$1.540 E+00$

$2.792 E-08 \quad 2.261 E-14 \quad 3.00 E-06$

KR-8BM

$1.867 E-01$

CONCEN IUCAR

curres

1.883E-08 2.777E-08 $1.377 E-08 \quad 1.616 E-08$

$3.031 E-08$

1.372

$042 E+01$
6.070

$\%$ OF Total
2.134
$6.281 E+00$

3. $943 E-08$

$1.746 E-08 \quad 1.413 E-14 \quad 1.00 E-07$ 6.153
$450 E+00 \quad 4.403 E+01$ 
RADIOACTIVE WASTE MANAGEMENT INFORMATION SYSTEM

ANL AIRBORNE MASTE SIMMARY

FOR JAN THROUEH DEC 1993

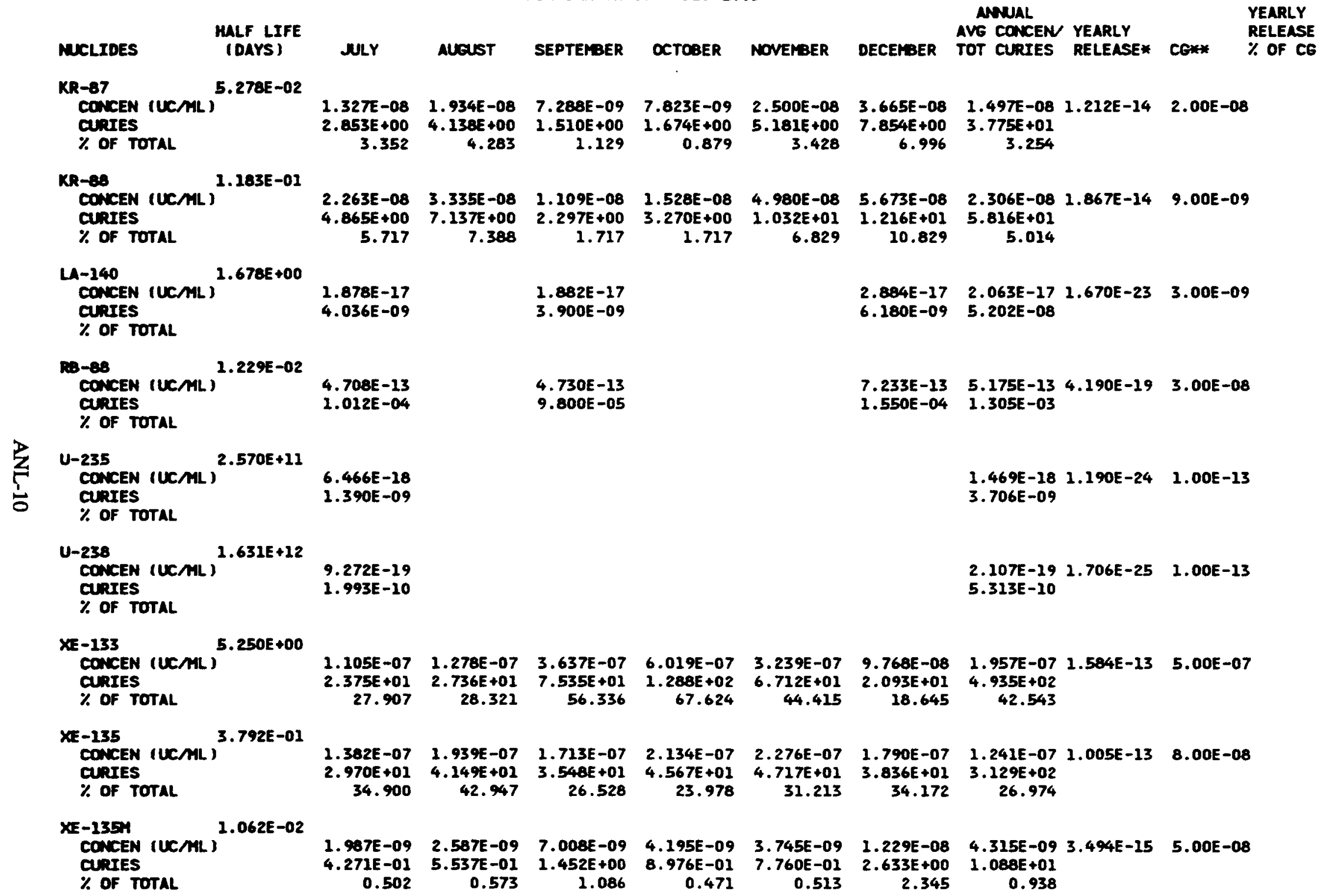


RADIOACTIVE WUSTE MUNAGEMENT INFORMATION SYSTEM

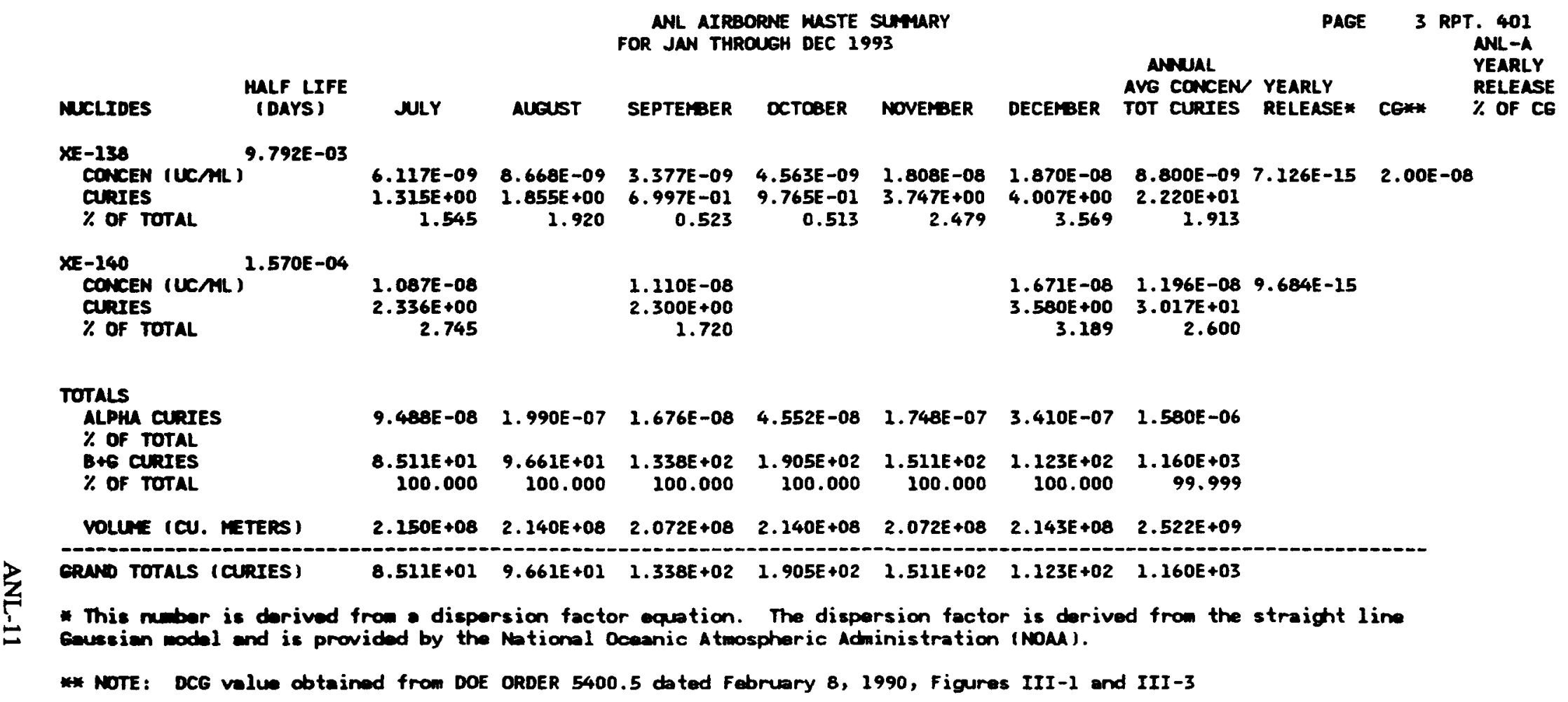


SCHED. NO. P61PYOO9-E

IDAHO OPERATIONS OFFICE

NL LIQUTD WASTE SUMARY

FOR JAN THROUEH DEC 1993

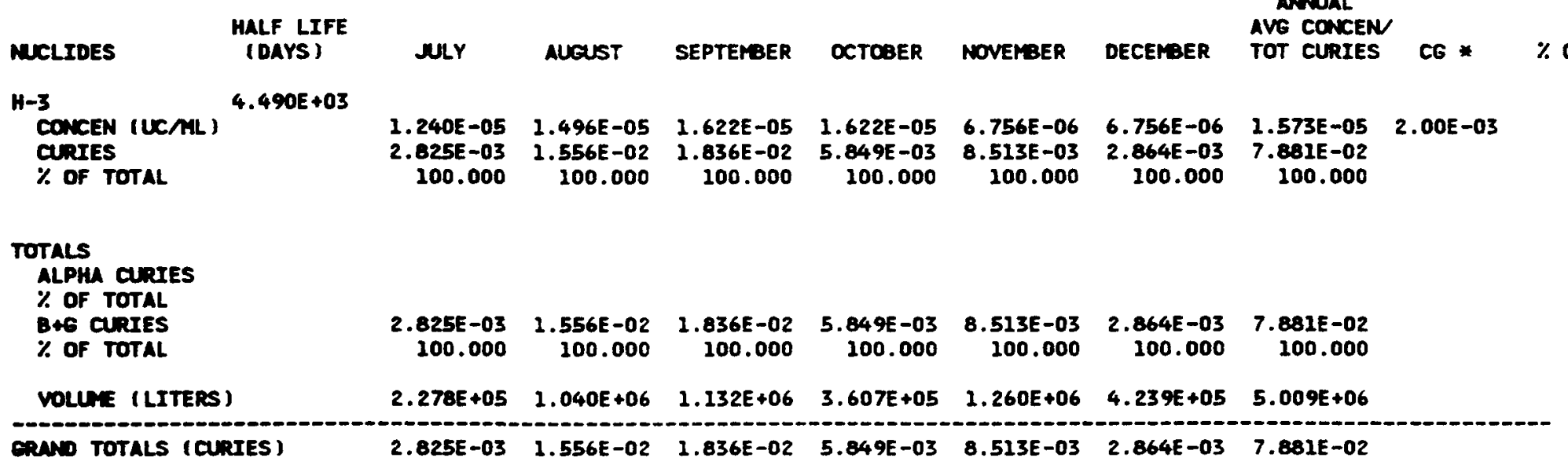

* NOTE: DCG value abtained from DOE ORDER 5400.5 dated February8, 1990, Figures III-1 and III-3

RUN DATE : 05/11/94

PAgE 1 RPT. 402 $A N L-L$ 

RADIOACTIVE WASTE MANAGEMENT INFORMUTION SYSTEM

ANL DISPOSED SOLID WLSTE SUMMARY FOR JAN THROUGH DEC 1993

\section{MULIDES}

HALF LIFE

(DAYS)

JULY

AUGUST

SEPTERER
AM-241

CURIEs

$\%$ of total

erans

CE-144

$\%$ OF TOTAL

\section{CO-58}

CURIES

$\%$ of total

\section{CO-60}

CURIES

CR-5I

CURIES

$\%$ of total

$\sum_{\omega}$ CS-134
CURIES
$\%$ OF TOTAL

CS-137

CURIES

\% OF total

EU-252

CURIES

\% OF TOTAL

EU-254
CURIES

\% Of total

EU-255

CURIES

$\%$ of total

FE-59

curres

\% OF TOTAL

H-3

curres

$\%$ of total.

\section{$1.577 E+05$}

$2.844 E+02$

$7.091 E+01$

$1.924 E+03$

$2.770 E+01$

$7.537 E+02$

$1.101 E+04$

$4.891 E+03$

$3.103 E+03$

$1.727 E+03$

$4.451 E+01$

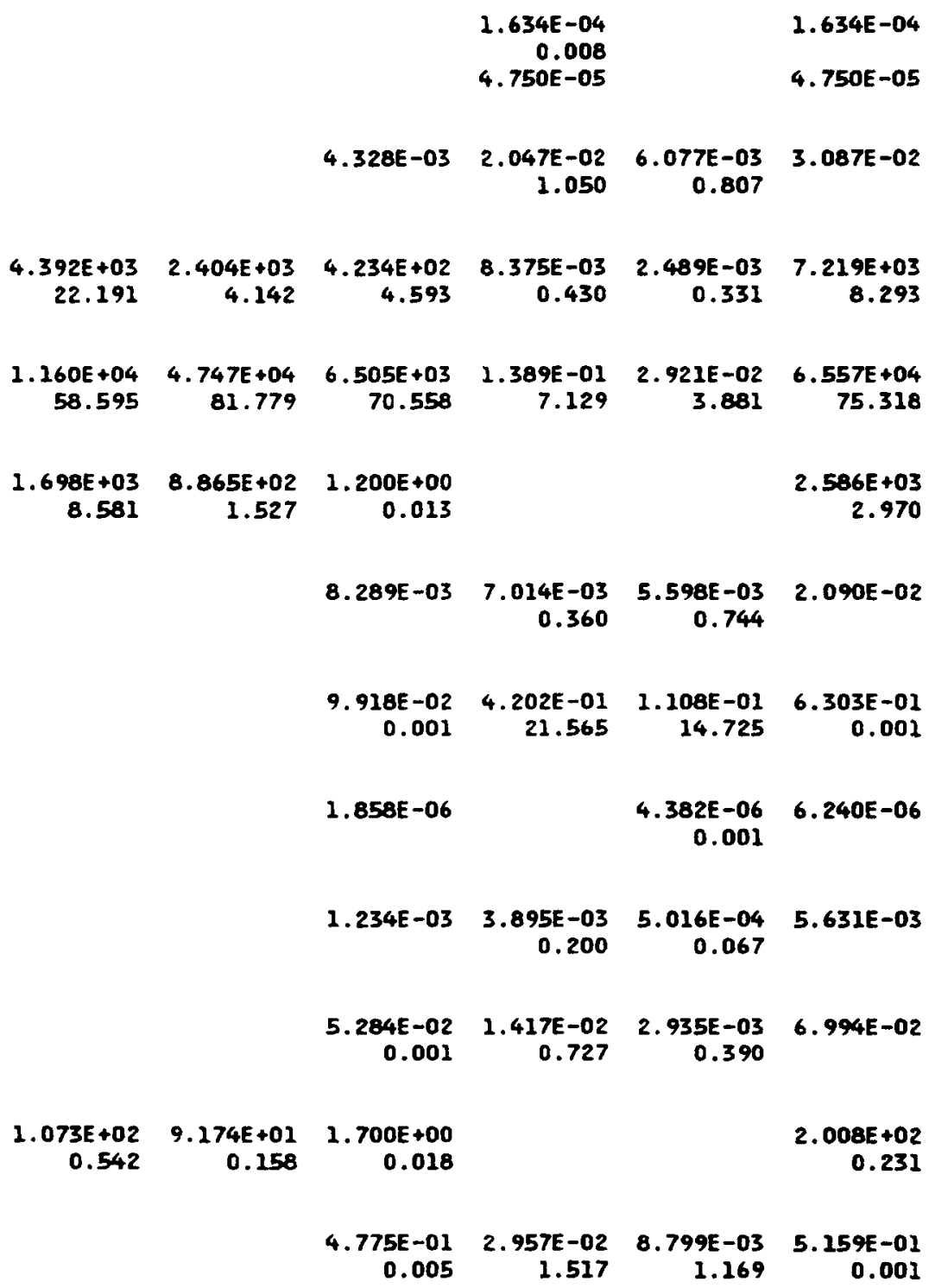

1.634E-04$$
\begin{array}{r}
0.008 \\
.750 E-05
\end{array}
$$

1.634E-04

4. 750E-05

$4.328 E-03 \quad 2.047 E-02 \quad 6.077 E-03 \quad 3.087 E-02$ $\begin{array}{rr}1.050 & 0.807\end{array}$

$\begin{array}{llllll}4.392 E+03 & 2.404 E+03 & 4.234 E+02 & 8.375 E-03 & 2.489 E-03 & 7.219 E+03\end{array}$ $\begin{array}{rrrrrr}22.191 & 4.142 & 4.593 & 0.430 & 0.331 & 8.293\end{array}$ $\begin{array}{rrrrrr}1.160 E+04 & 4.747 E+04 & 6.505 E+03 & 1.389 E-01 & 2.921 E-02 & 6.557 E+04 \\ 58.595 & 81.779 & 70.558 & 7.129 & 3.881 & 75.318\end{array}$

$\begin{array}{llll}1.698 E+03 & 8.865 E+02 & 1.200 E+00 & 2.586 E+03\end{array}$ $\begin{array}{rrrr}8.581 & 1.527 & 0.013 & 2.586 E+03 \\ & & & 2.970\end{array}$

DECEMBER ANUAL

PAGE

2 RPT 403

TOTAL 
PADIOACTIVE WASTE MANAGEMENT INFORMATION SYSTEM

ANL DISPOSED SOLID WASTE SIMMARY

FOR JAN THROUGH DEC 1993

\begin{tabular}{|c|c|}
\hline MULIDES & $\begin{array}{l}\text { HALF LIFE } \\
\text { (DAYS) }\end{array}$ \\
\hline $\begin{array}{l}\text { LA-140 } \\
\text { CURIES } \\
\% \text { OF TOTAL }\end{array}$ & $1.678 E+00$ \\
\hline $\begin{array}{l}\text { M-54 } \\
\text { CURIES } \\
\% \text { OF TOTAL }\end{array}$ & $3.122 E+02$ \\
\hline $\begin{array}{l}\text { MA-22 } \\
\text { CURIES } \\
\text { \% OF TOTAL }\end{array}$ & $9.508 E+02$ \\
\hline $\begin{array}{l}\text { N3-95 } \\
\text { CURIES } \\
\% \text { OF TOTAL }\end{array}$ & $3.498 E+01$ \\
\hline $\begin{array}{l}\text { PU-239 } \\
\text { cunIES } \\
\% \text { OF TOTAL } \\
\text { cRAMS }\end{array}$ & $8.800 E+06$ \\
\hline $\begin{array}{l}\text { PU-240 } \\
\text { CURIES } \\
\% \text { OF TOTAL } \\
\text { GRAMS }\end{array}$ & $2.394 E+06$ \\
\hline $\begin{array}{l}\text { RU-106 } \\
\text { CURIES } \\
\% \text { OF TOTAL }\end{array}$ & $3.726 E+02$ \\
\hline $\begin{array}{l}\text { SB-125 } \\
\text { CURIES } \\
\% \text { OF TOTAL }\end{array}$ & $1.007 E+03$ \\
\hline $\begin{array}{l}\text { SR-85 } \\
\text { CURIES } \\
\text { \% OF TOTAL }\end{array}$ & $6.480 E+01$ \\
\hline $\begin{array}{l}\text { SA-90 } \\
\text { CURIES } \\
\% \text { OF TOTAL }\end{array}$ & $1.059 E+04$ \\
\hline $\begin{array}{l}\text { TA-182 } \\
\text { CURIES } \\
\% \text { OF TOTAL }\end{array}$ & $1.145 E+02$ \\
\hline $\begin{array}{l}\text { U-235 } \\
\text { CURIES } \\
\text { \% OF TOTAL }\end{array}$ & $2.570 E+11$ \\
\hline
\end{tabular}

JULY

AUEUST
$2.917 E-05$

5.000E-06

\section{PAGE 2 RPT 403}

ANUAL

TOTAL
8. 256E-04
8. 256E-04 10.091
2.287 12.393
$287 E+03$
24.806

$1.523 E-02$
0.782

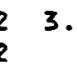

$333 E-03$
0.443

$1.248 E+04$
1.997E+03 7.1

$2.164 E-03 \quad 1.535 E-03 \quad 1.513 E-04 \quad 3.850 E-03$

$$
0.079 \quad 0.020
$$

$3.491 E-04 \quad 1.751 E-03 \quad 5$
0.090

$$
\begin{array}{r}
5.207 E-04 \\
0.069
\end{array}
$$

$\begin{array}{rrrrrr}3.614 E-06 & 1.830 E-06 & 2.845 E-04 & 1.857 E-03 & 4.248 E-04 & 2.572 E-03 \\ & & & 0.095 & 0.056 & \\ 5.820 E-05 & 2.947 E-05 & 4.581 E-03 & 2.990 E-02 & 6.841 E-03 & 4.141 E-02\end{array}$

$\begin{array}{cccc}6.810 E-08 & 2.951 E-08 & 4.540 E-09 & 9.268 E-06\end{array}$

$9.370 E-06$

$\begin{array}{llll}3.000 E-07 & 1.300 E-07 & 2.000 E-08 & 4.083 E-05\end{array}$

4. $128 E-05$

1. 793E-04

2. 917E-05

5.000E-06

$$
\begin{array}{rrrr}
2.629 E-01 & 6.361 E-01 & 2.886 E-01 & 1.188 E+00 \\
0.003 & 32.643 & 38.343 & 0.001
\end{array}
$$
$\begin{array}{rrrrr}3.000 E-01 & 1.474 E-03 & 7.396 E-03 & 2.201 E-03 & 3.111 E-01 \\ 0.001 & & 0.380 & 0.292 & \end{array}$

7.594E-04 1.242E-05 9.192E-06 6.100E-04 $\quad 1.391 E-03$ 
ANL DISPOSED SOLID MUSTE SLMMARY

FOR JAN THROUGH DEC 1993

\begin{tabular}{|c|c|}
\hline MCLIDES & $\begin{array}{l}\text { HALF LIFE } \\
\text { (DAYS) }\end{array}$ \\
\hline \multicolumn{2}{|l|}{ GRurs } \\
\hline $\begin{array}{l}\text { U-238 } \\
\text { CURIES } \\
\% \text { OF TOTAL } \\
\text { GRANS }\end{array}$ & $1.631 E+12$ \\
\hline $\begin{array}{l}Y-90 \\
\text { CURIES } \\
\% \text { OF TOTAL }\end{array}$ & $2.667 E+00$ \\
\hline $2 R-95$ & $6.403 E+01$ \\
\hline
\end{tabular}

CURIES

\% OF TOTAL

TOTALS
ALPHA CURIES
$\%$ OF TOTAL
B+G CURIES
$\%$ OF TOTAL
$\frac{2}{n}$ TOTAL ERAHS OF AM

\section{TOTAL ERAMS OF NH}

TOTAL ERANS OF PU

TOTAL ERANS OF PU $-239+241$

TOTAL ERATS OF $u$

TOTAL ERNAS OF TH

TOTAL ERNAS OF NP

VOLUNE (CU. METERS)

VEIEAT (KILOERAMS)
vLY

AUEUST
PAge

3 RPT 403

NIL -5

ERND TOTALS (CURIES)

$\begin{array}{llll}3.516 E+0 z & 5.751 E+00 \quad 4.255 E+00 \quad 2.824 E+02 \quad 6.440 E+02\end{array}$

5.365E-05 $\quad 9.924 E-07 \quad 1.319 E-06 \quad 3.905 E-05 \quad 9.501 E-05$ 0.005

$\begin{array}{lllll}1.597 E+02 & 2.953 E+00 & 3.925 E+00 & 1.162 E+02 & 2.828 E+02\end{array}$

$$
\begin{array}{llll}
1.597 E-01 & 6.361 E-01 & 2.886 E-01 & 1.084 E+00
\end{array}
$$$$
\begin{array}{lrrr}
0.002 & 32.643 & 38.343 & 0.001
\end{array}
$$

$1.179 E-03 \quad 5.917 E-03 \quad 1.760 E-03 \quad 8.856 E-03$ $0.304 \quad 0.234$
3.682E-06
8.149E-04 2.979E-04
$.040 E-03$
$1.074 E-03$
$1.979 E+04$
$5.804 E+04$
$.219 E+03$
0.105
0.143
100.000
100.000
100.000
99.896
99.857
$706 E+04$
99.999

$\begin{array}{lllllll}5.850 E-05 & 2.960 E-05 & 4.581 E-03 & 2.750 E-05 & & 4.750 E-05 \\ & 2.994 E-02 & 6.841 E-03 & 4.145 E-02\end{array}$

$\begin{array}{lllllll}5.850 E-05 & 2.960 E-05 & 4.581 E-03 & 2.994 E-02 & 6.841 E-03 & 4.145 E-02 \\ 5.820 E-05 & 2.947 E-05 & 4.581 E-03 & 2.990 E-02 & 6.841 E-03 & 4.141 E-02\end{array}$

$\begin{array}{llllll}5.820 E-05 & 2.947 E-05 & 4.581 E-03 & 2.990 E-02 & 6.841 E-03 & 4.141 E-02 \\ & 5.113 E+02 & 8.705 E+00 & 8.181 E+00 & 3.986 E+02 & 9.268 E+02\end{array}$

$\begin{array}{llllll}1.000 E-01 & 1.450 E+00 & 2.079 E+01 & 3.124 E+01 & 2.305 E+01 & 7.666 E+01\end{array}$

$2.660 E+02 \quad 2.022 E+03 \quad 1.085 E+04 \quad 2.786 E+04 \quad 1.184 E+04 \quad 5.285 E+04$

$1.979 E+04 \quad 5.804 E+04 \quad 9.219 E+03 \quad 1.949 E+00 \quad 7.528 E-01 \quad 8.706 E+04$ 
RADIOACTIVE WASTE MAUAGEMENT INFORMATION SYSTEM

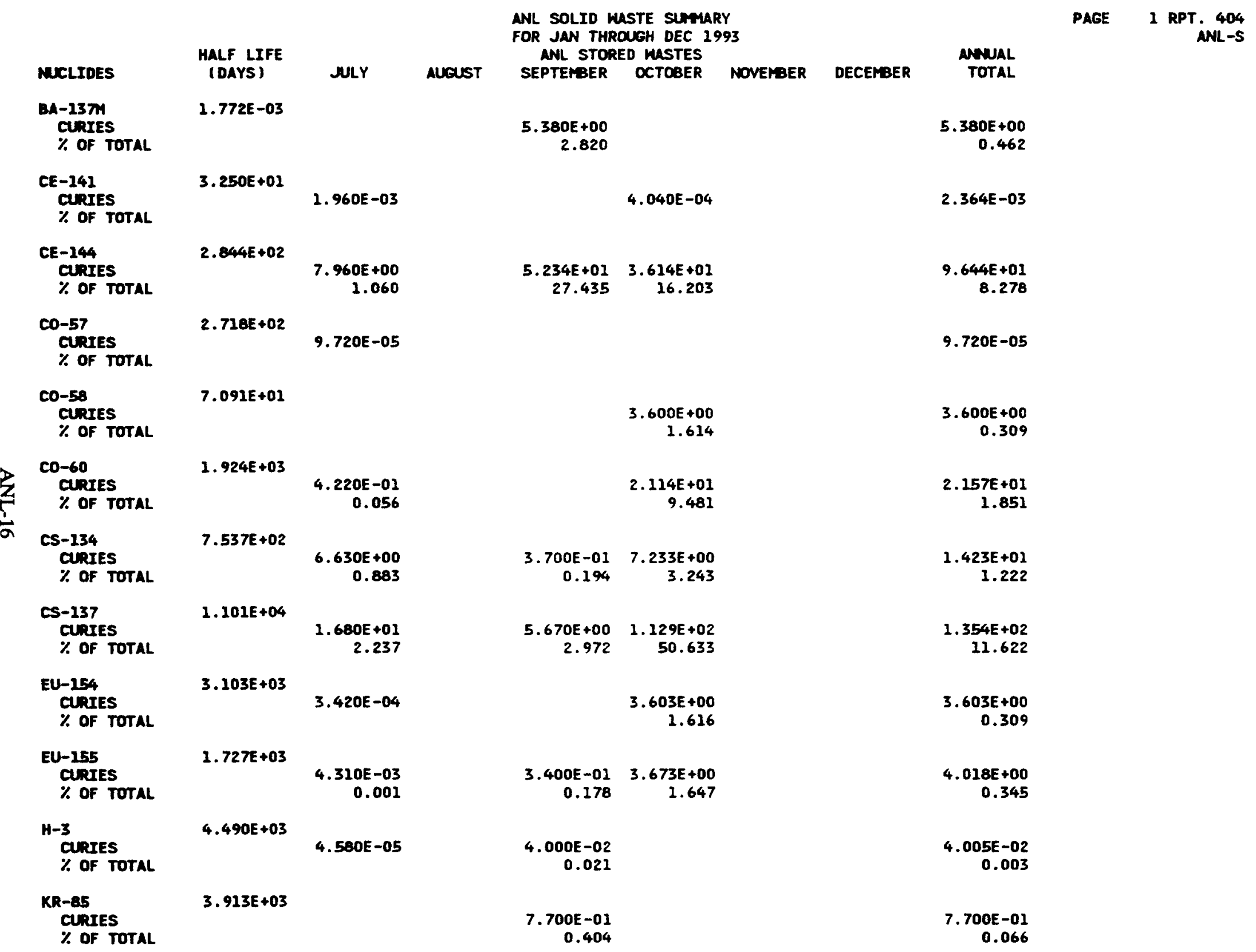


FOR JNN THROUGH DEC 1993

ANL STORED MASTES

\begin{tabular}{lcr} 
MUCLIDES & $\begin{array}{c}\text { MALF LIFE } \\
\text { (DAYS) }\end{array}$ & \multicolumn{1}{c}{ NULY } \\
MN-54 & $3.122 E+02$ & \\
CURIES & & $2.260 E+01$ \\
$\%$ OF TOTAL & & 1.677 \\
NM-22 & &
\end{tabular}

AUEUST

8.000E-02

$\%$ of TOTAL

\section{N-95}

CURIES

$\%$ of total

\section{N-95N}

CURIES

$\%$ of total

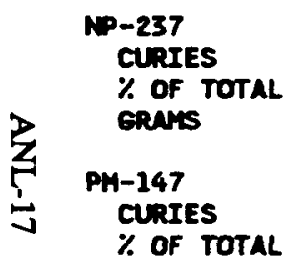

$$
\begin{aligned}
& \text { PR-144 } \\
& \text { CURIES } \\
& \% \text { OF TOTAL }
\end{aligned}
$$

pu-239

CURIES
\% OF TOTAL

ERurs

\section{PU-240 \\ CURIES \\ $\%$ of total}

epurs

RH-103M

Cunies

RH-106

CURIES

$\%$ of total
$3.498 E+01$

$3.620 E+00$

7.8IIE+08

7.050E-06

1.000E-02

$9.575 E+02$

1. 200E-02

8.800E+06

$$
\begin{array}{r}
\text { 4. } 931 E-01 \\
0.066
\end{array}
$$$$
\begin{array}{r}
0.066 \\
\times 40 E+00
\end{array}
$$

$2.394 E+06$

\section{$1.589 E-01$}

0.021
$.000 E-01$

3.897E-02
8. $420 E+00$
$1.410 E-05 \quad 2.115 E-05$

2.000E-02 3.000E-02

$1.678 E+01$

8. 796

5. $222 E+01$

27.372

$\begin{array}{rr}2.422 E-01 & 2.441 E-01 \\ 0.127 & 0.109\end{array}$

$3.900 E+00 \quad 3.931 E+00$

4.540E-03 8.172E-02

$\begin{array}{rr}0.002 & 0.037 \\ .000 E-02 & 3.600 E-01\end{array}$

2.300E-01

$1.071 E+01 \quad 4.732 E-01$

$\begin{array}{rr}5.614 & 0.212\end{array}$
ANL SOLID WUSTE SUMARY

SEPTEMBER OCTOBER

NOVErBE

$$
\begin{array}{r}
9.301 E+00 \\
4.170
\end{array}
$$

$\begin{array}{rr}1.214 E+01 & 5.400 E+00 \\ 6.363 & 2.421\end{array}$

4. $000 E-02$

0.021

PAGE

2 RPT. 404

ANL-S

TOTAL

$2.190 E+01$

1.880

8.000E-02

$1.754 E+01$

1.506

$4.000 E-02$

0.003

4.230E-05

6.000E-02

$1.678 E+01$

1.440

5.222E+01

4.483

$9.794 \mathrm{E}-01$

0.084
$577 E+03$

$2.452 E-01$

0.021

$1.080 E+00$

$2.300 E-01$

0.020

$1.960 E+01$ 
RADIOACTIVE WASTE MANAGEMENT INFORMATION SYSTEM

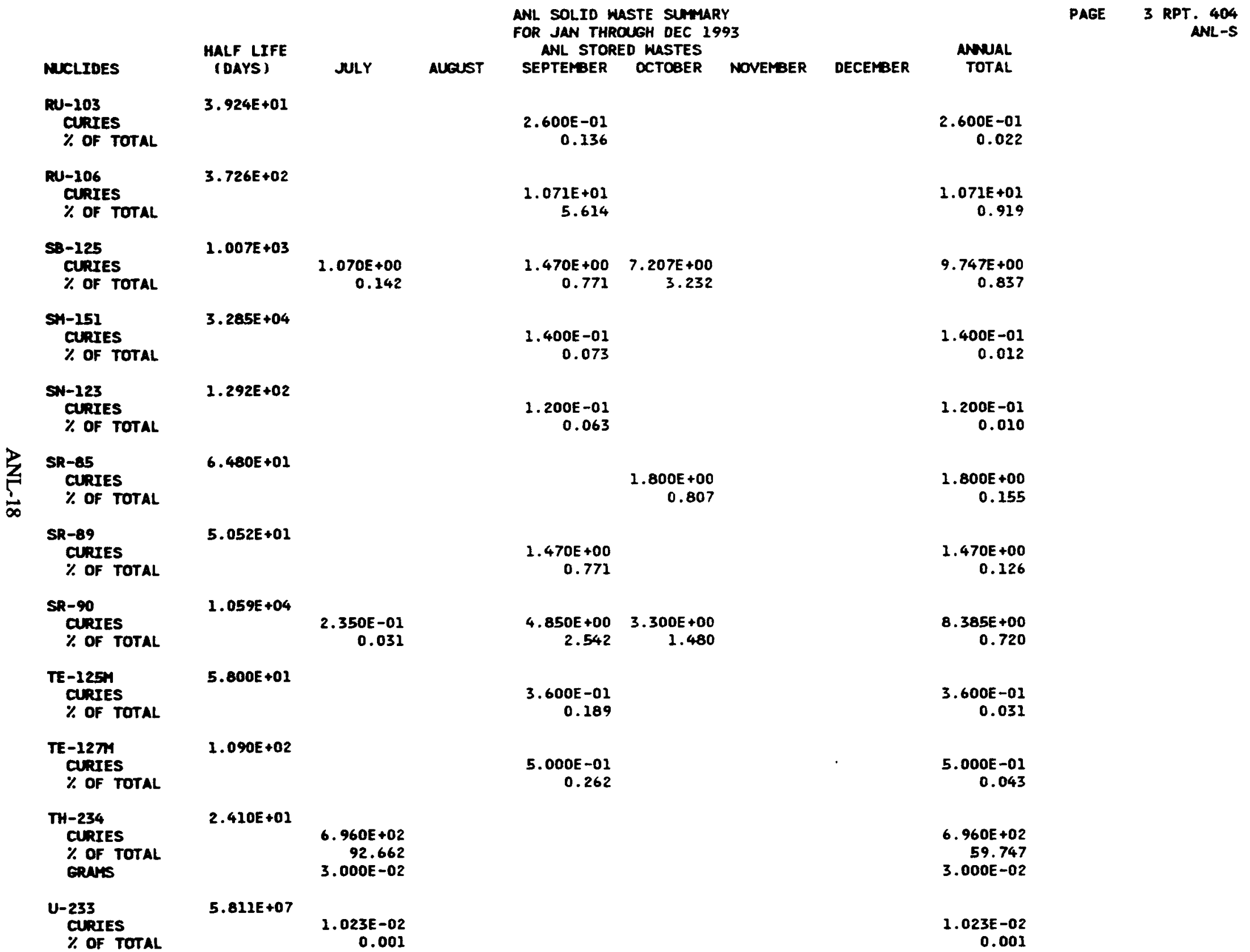


SCHED. NO. P61PHOO9-K

IDAHO OPERATIONS OFFICE

IDAHO OPERATIONS OFFICE

RUN DATE: 05/11/94

RADIOACTIVE WASTE MANAGEMENT INFORMATION SYSTEM

ANL SOLID WASTE SUMARY

FOR JAN THROUGH DEC 1993

ANL STORED WASTES

HALF LIF

JULY

AUQUST

SEPTEMER D WASTES

$1.060 E+00$

eRNis

$2.570 E+11$

U-235

CURIES

epars

$1.135 E-04$

$5.255 E+01$

$1.631 E+12$

U-238

CURIES

OF TOTAL

erents

1.820E-05

$5.417 E+01$

Y-90

$\%$ OF TOTAL

$2.667 E+00$

$2.350 E-01$

0.031

Y-91

CURIES

$6.403 E+01$

CURIES

\% OF TOTAL

TOTALS

ALPHA CURIES

$\%$ OF TOTAL

B+6 Curies

$\%$ OF TOTAL

6.624E-01

0.088

$7.505 E+02$

99.912

TOTAL ERAMS OF AM

TOTAL ERNAS OF PU $8.640 E+00$

$\begin{array}{ll}\text { TOTAL ERNS } & 8.640 E+00 \\ \text { TOTAL ERANS OF PU-239+241 } & 7.940 E+00\end{array}$

TOTAL ERAMS OF $U$ 1.078E +02

TOTAL GRAYS OF TH

$1.078 E+02$
3. $000 E-02$

$1.000 E-02$

DOUNE (CU. METERS

NEIEHT (KILOERAMS)
7.007E-05 1.883E-05

$3.244 E+01 \quad 8.716 E+00$

1.253E-05 5.154E-06

$3.729 E+01 \quad 1.534 E+01$

$4.850 E+00 \quad 3.300 E+00$

2.542

1.480

$3.190 E+00$

1.672

$5.580 E+00 \quad 3.600 E+00$

$\begin{array}{rr}2.468 E-01 & 3.259 E-01 \\ 0.129 & 0.146\end{array}$

$\begin{array}{rr}1.905 E+02 & 2.227 E+02 \\ 99.871 & 99.854\end{array}$

$3.920 E+00 \quad 4.291 E+00$

$3.900 E+00 \quad 3.931 E+00$

$6.973 E+01 \quad 2.406 E+01$

2.000E-02 3.000E-02

$1.000 E-01 \quad 6.900 E-01$

2.599E+02 $1.283 E+03$

1. $908 E+02 \quad 2.230 E+02$ $\begin{array}{rr}2.925 & 1.614\end{array}$

\section{ANNUAL}

PAGE 4 RPT. 404

TOTAL

$1.060 E+00$

2.024E-04

$9.370 E+01$

$3.588 E-05$

$1.068 E+02$

$8.385 E+00$

$$
0.720
$$

$3.190 E+00$ 0.274

$9.180 E+00$

0.788

1. 235E+00 0.106

$1.164 E+03$

$1.685 E+01$

$1.577 E+01$

$2.026 E+02$

3.000E-02

6.000E-02

$8.900 E-01$

$1.816 E+03$

ERAD TOTALS (CURIES) $7.511 E+02$

$2.265 E+03$



ANL Detail Data for Months January to June 



\section{NL ATRBORNE MASTE SUMYRY}

FOR JAN THROUEH JUN 1993

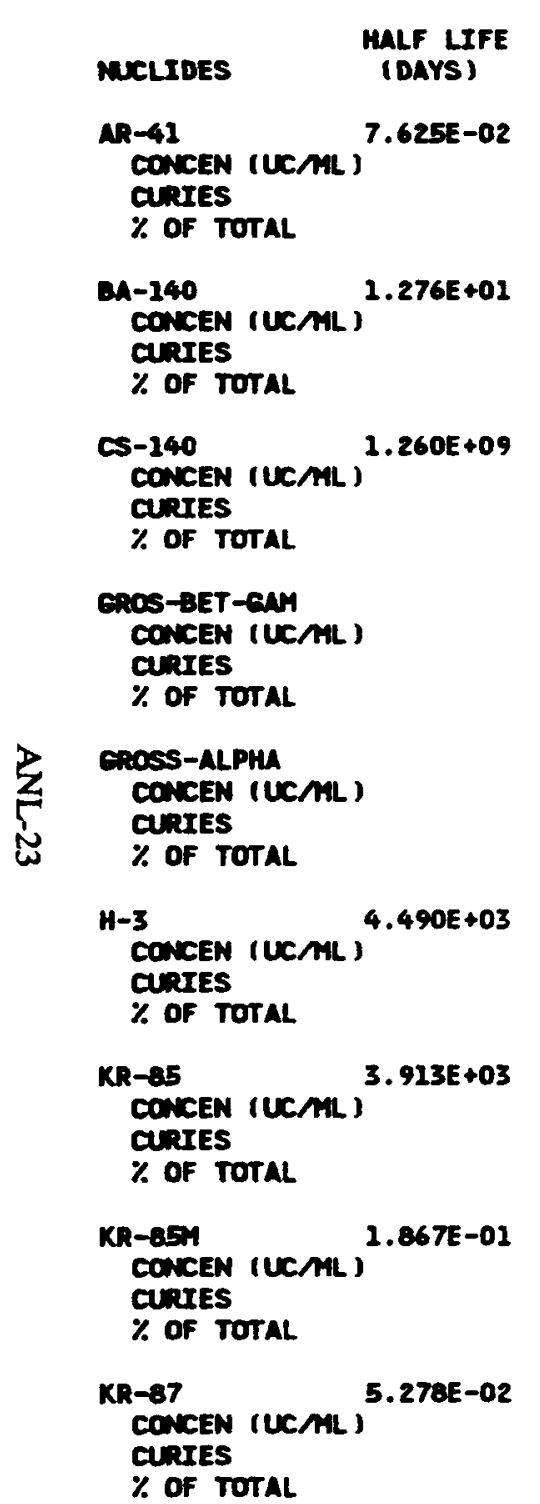

5. 903E-09 $1.929 E-08$

$1.264 E+00 \quad 3.736 E+00$ 2.438
SANUARY FEBRUARY
MARCH

$9705-13 \quad 0.422 E-13$ $4.218 E-05 \quad 1.631 E-04$ 0.001

$\begin{array}{lllllll}1.086 E-08 & 9.287 E-09 & 5.379 E-09 & 2.626 E-09 & 8.737 E-09 & 7.074 E-15 & 1.00 E-08\end{array}$ $2.325 E+00 \quad 1.904 E+00 \quad 1.152 E+00 \quad 5.439 E-01 \quad 1.093 E+01$

$\begin{array}{lllllllll}1.106 E-08 & 4.729 E-08 & 1.578 E-08 & 1.044 E-08 & 1.367 E-08 & 6.208 E-09 & 1.702 E-08 & 1.378 E-14\end{array}$

$\begin{array}{lllllll}2.368 E+00 & 9.158 E+00 & 3.379 E+00 & 2.163 E+00 & 2.928 E+00 & 1.286 E+00 & 2.128 E+01\end{array}$ $\begin{array}{lrrrrrr}4.567 & 29.193 & 8.098 & 2.526 & 3.786 & 1.251 & 5.447\end{array}$

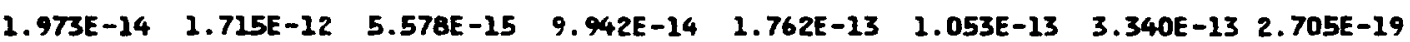

$\begin{array}{lllllll}4.225 E-06 & 3.321 E-04 & 1.194 E-06 & 2.060 E-05 & 3.773 E-05 & 2.181 E-05 & 4.177 E-04\end{array}$

$\begin{array}{lllllllll}2.105 E-15 & 5.552 E-16 & 2.6 \% 4 E-17 & 2.968 E-16 & 5.124 E-17 & 3.346 E-16 & 5.645 E-16 & 4.571 E-22\end{array}$

$\begin{array}{lllllll}4.508 E-07 & 1.075 E-07 & 5.768 E-09 & 6.251 E-08 & 1.097 E-08 & 6.932 E-08 & 7.059 E-07\end{array}$

$\begin{array}{llllllllll}4.773 E-10 & 5.420 E-11 & 7.578 E-10 & 2.618 E-11 & 2.030 E-11 & 6.394 E-10 & 3.336 E-10 & 2.701 E-16 & 1.00 E-07\end{array}$ $\begin{array}{llllllll}1.022 E-01 & 1.050 E-02 & 1.622 E-01 & 5.426 E-03 & 4.347 E-03 & 1.325 E-01 & 4.172 E-01\end{array}$

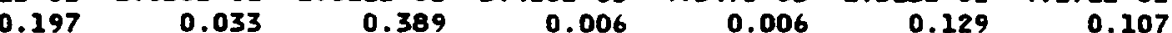

$\begin{array}{lllllllll}1.952 E-08 & 9.140 E-09 & 1.845 E-09 & 1.410 E-08 & 1.339 E-07 & 6.560 E-08 & 4.121 E-08 & 3.337 E-14 & 3.00 E-06\end{array}$ $\begin{array}{llllllll}4.180 E+00 & 1.770 E+00 & 3.950 E-01 & 2.922 E+00 & 2.868 E+01 & 1.359 E+01 & 5.154 E+01\end{array}$

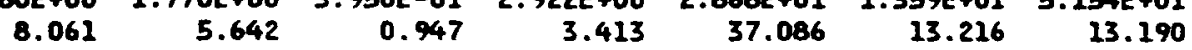

$\begin{array}{llllllllll}9.573 E-09 & 8.764 E-11 & 1.501 E-08 & 2.051 E-09 & 5.681 E-09 & 2.930 E-08 & 1.039 E-08 & 8.413 E-15 & 1.00 E-07\end{array}$ $\begin{array}{lllllll}2.050 E+00 & 1.697 E-02 & 3.214 E+00 & 4.251 E-01 & 1.217 E+00 & 6.070 E+00 & 1.299 E+01\end{array}$

$$
\begin{array}{rrrrrrr}
.050 E+00 & 1.697 E-02 & 3.214 E+00 & 4.251 E-01 & 1.217 E+00 & 6.070 E+00 & 1.299 E+01 \\
3.953 & 0.054 & 7.703 & 0.497 & 1.573 & 5.902 & 3.325
\end{array}
$$

$\begin{array}{ccccccccc}6.584 E-09 & 3.992 E-10 & 1.946 E-08 & 4.768 E-09 & 1.638 E-08 & 2.118 E-08 & 1.163 E-08 & 9.413 E-15 & 2.00 E-08\end{array}$ $\begin{array}{llllllll}1.410 E+00 & 7.730 E-02 & 4.167 E+00 & 9.882 E-01 & 3.508 E+00 & 4.387 E+00 & 1.454 E+01\end{array}$ 
RADIOACTIVE WUSTE MUMAGEMENT INFORMATION SYSTEM

AVL AIPBODANE WASTE SUMMRY

FOR JAN THROUGH JUN 1993

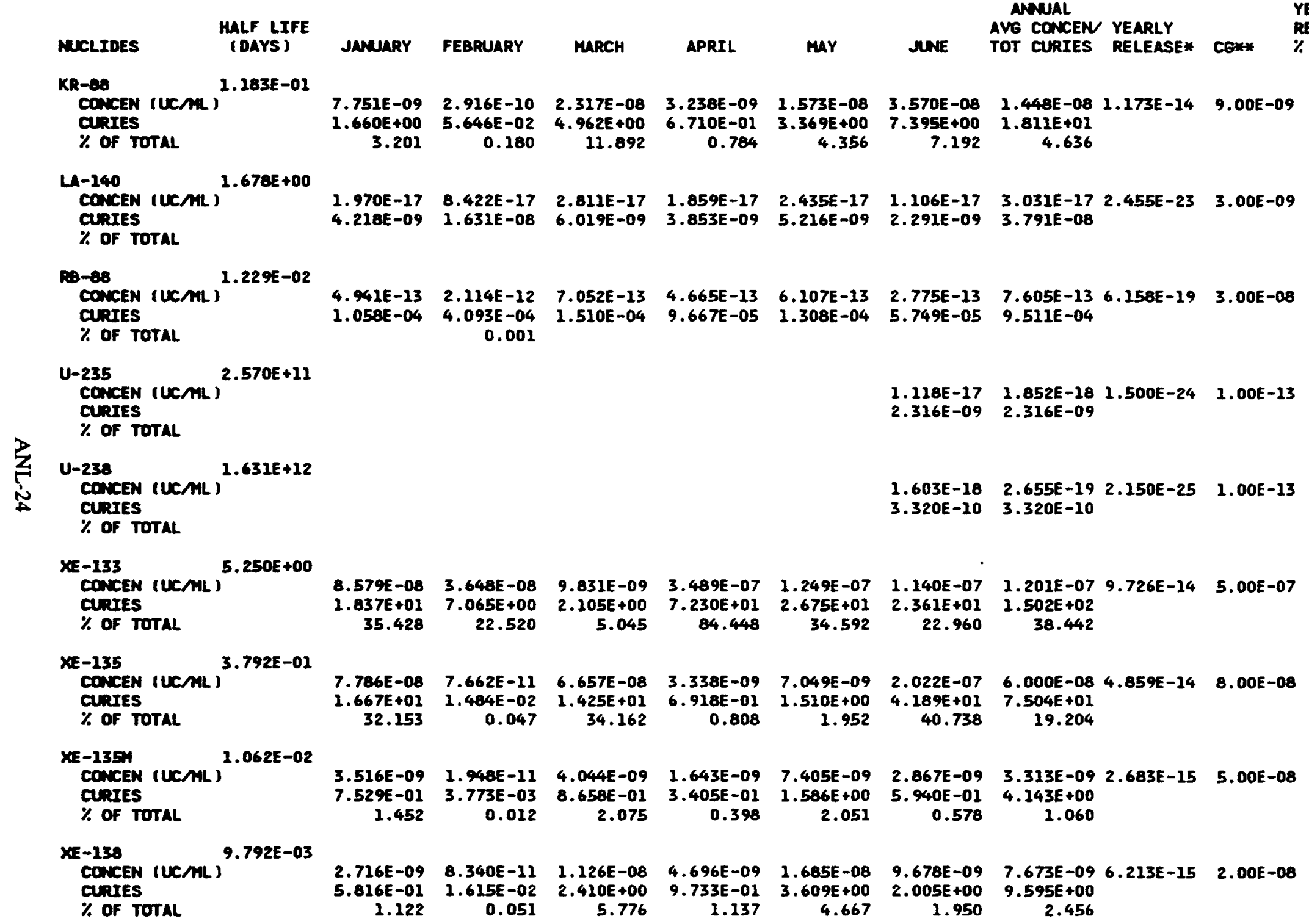

\section{of Toral}$$
1.122
$$$$
0.051
$$$$
5.776
$$

1.137

1.950

2.456
40

ANL-A

YEARLY

$\%$ of CG 
ANL AIRBORE MUSTE SUMPARY

FOR JAN THROUGH JUN 1993

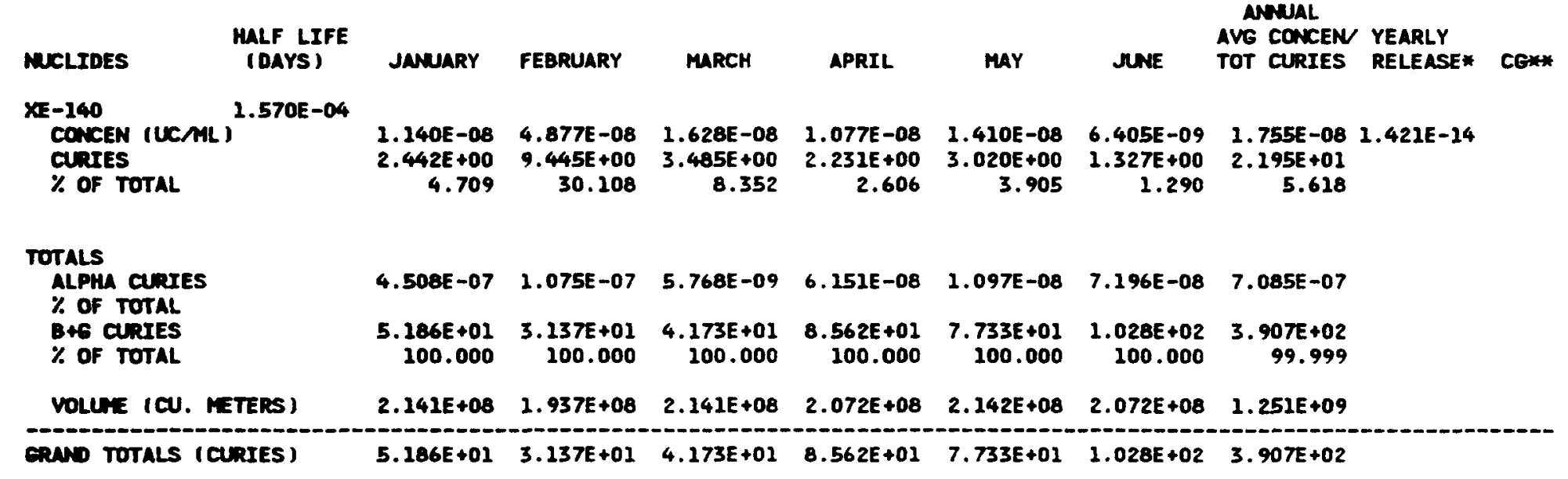

* This nuber is derived from a disparsion factor equation. The dispersion factor is derived from the straight line cusseisn model and is providad by the Mtional Dcoanic Atmospheric Administration INOMA.

* NOTE: DCE value obtained from DOE ORDER 5400.5 detad February 8, 1990, Figures III-I and III-3 
IDAHO OPERATIONS OFFICE

U.S. DEPARTMENT OF ENERGY

RADIOACTIVE MASTE MANAGEMENT INFORMATION SYSTEM

WNL LIQUID WASTE SUMMARY FOR JAN THROUGH JUN 1993

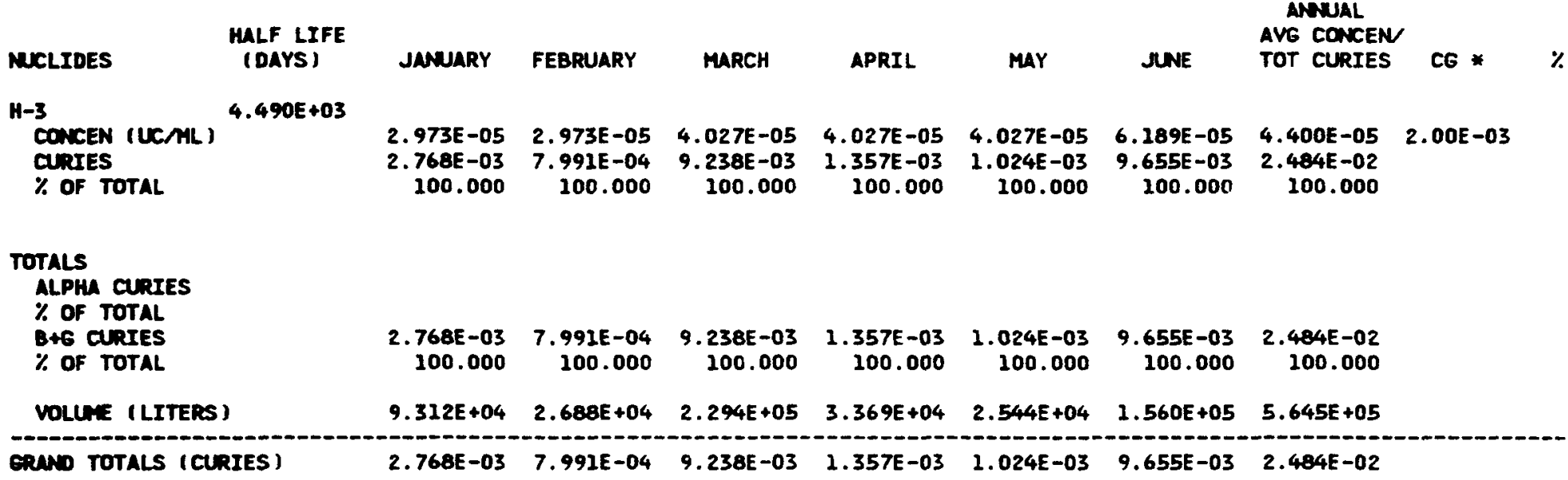

* NOTE: DCG value obtainad from DOE ORDER 5400.5 dated February8, 1990, Figures III-I and III-3
RUN DATE: 05/12/94

AGE 1 RPT. 402

ANL-L 
RADIOACTIVE WASTE MANAGEMENT INFORMATION SYSTEM

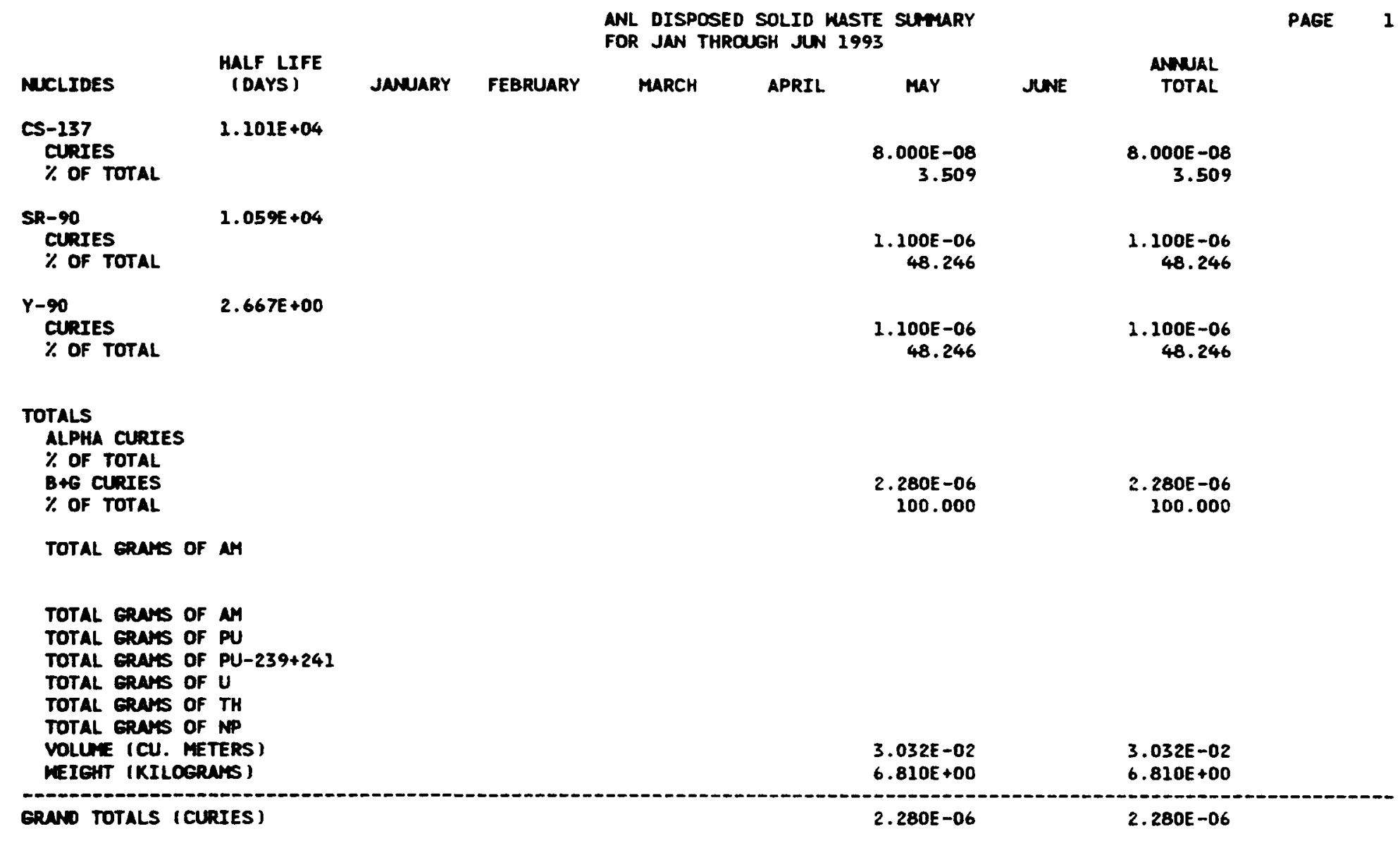


ANL Facility Detail Data for Months July to December Annual Average Concentration and Total Curies for January to December 


RADIOACTIVE WASTE MANAGEMENT INFORMATION SYSTEM

EBR-II AIRBORNE WASTE SUMMARY

FOR JAN THROUGH DEC 1993

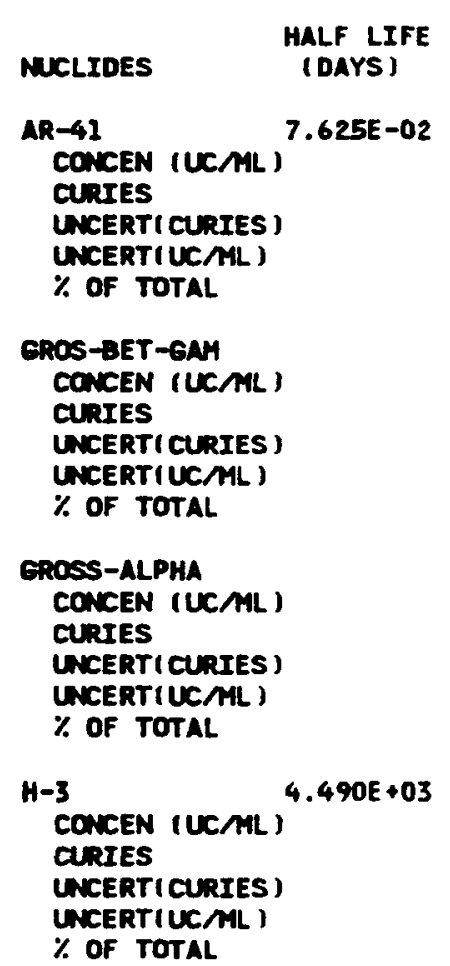

JULY

AUEUST
SEPTEMBER

OCTOBER

\section{$1.033 E-07$}

8. $846 E-01$

0.694
7.590

5.902

5.498

0.773

4.695

\section{KR-85M \\ CONCEN (UCAL) \\ UNCERTICURIES ) \\ UNCERTIUCML ) \\ $\%$ OF TOTAL.}

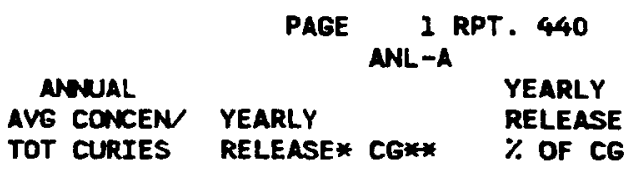

7.008

3.351

$\begin{array}{llllllllll}4.570 E-07 & 6.717 E-07 & 3.329 E-07 & 3.908 E-07 & 7.335 E-07 & 9.545 E-07 & 4.222 E-07 & 3.419 E-13 & 1.00 E-07\end{array}$

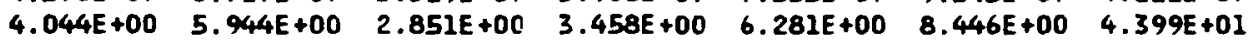
5.584
6.322
2.236
1.857
4.231
8.245
4.330

$\begin{array}{llllllllll}3.206 E-07 & 4.676 E-07 & 1.745 E-07 & 1.892 E-07 & 6.050 E-07 & 8.847 E-07 & 3.603 E-07 & 2.917 E-13 & 2.00 E-08\end{array}$ $\begin{array}{lllllll}2.837 E+00 & 4.138 E+00 & 1.494 E+00 & 1.674 E+00 & 5.181 E+00 & 7.829 E+00 & 3.754 E+01\end{array}$
3.917
4.401
1.172
0.899
3.490
7.643
3.695

$\begin{array}{llllllllll}5.485 E-07 & 8.065 E-07 & 2.670 E-07 & 3.695 E-07 & 1.205 E-06 & 1.372 E-06 & 5.568 E-07 & 4.509 E-13 & 9.00 E-09 & 0.01\end{array}$

CONCEN (UCAL)

CURIES

$\begin{array}{llllllll}4.854 E+00 & 7.137 E+00 & 2.286 E+00 & 3.270 E+00 & 1.032 E+01 & 1.214 E+01 & 5.801 E+01\end{array}$
6.702
7.590
1.793
1,756
6.952
11.851
5.711 
IDAHO OPERATIONS OFFICE

U.S. DEPARTMENT OF ENERGY

RADIOACTIVE WASTE MANAGEMENT INFORMATION SYSTEM

EBR-II AIRBORNE WASTE SUMARY

FOR JAN THROUEH DEC 1993

\begin{tabular}{|c|c|}
\hline CLIDES & $\begin{array}{l}\text { HALF LIFE } \\
\text { (DAYS) }\end{array}$ \\
\hline $\begin{array}{l}\text { XE- } 133 \\
\text { CONCEN (UCML) } \\
\text { CURIES } \\
\text { UNCERT(CURIES) } \\
\text { UNCERTIUCML) } \\
\text { \% OF TOTAL }\end{array}$ & $5.250 E+00$ \\
\hline $\begin{array}{l}\text { XE-135 } \\
\text { CONCEN (UCML) } \\
\text { CURIES } \\
\text { UNCERTI CURIES) } \\
\text { UNCERTI UCML) } \\
\text { \% OF TOTAL }\end{array}$ & $3.792 E-01$ \\
\hline $\begin{array}{l}\text { XE-13SM } \\
\text { CONCEN (UCML) } \\
\text { CURIES } \\
\text { UNCERT(CURIES) } \\
\text { UNCERT (UCAL) } \\
\text { \% OF TOTAL }\end{array}$ & $1.062 E-02$ \\
\hline $\begin{array}{l}\text { XE-138 } \\
\text { CONCEN IUCML) } \\
\text { CURIES } \\
\text { UNCERT(CURIES) } \\
\text { UNCERT(UCAML ) } \\
\text { \% OF TOTAL }\end{array}$ & $9.792 E-03$ \\
\hline
\end{tabular}

NLY

Aueust

$2.684 E-06 \quad 3.092 E-06$ $.375 E+01 \quad 2.736 E+01$

32.793

29.098

59.095

69.178

45.214

$3.356 E-06 \quad 4.689 E-06 \quad 4.143 E-06 \quad 5.161 E-06$

$2.970 E+01 \quad 4.149 E+01$

4. $143 E-06$

$5.161 E-06$
$4.567 E+01$
41.008

44.126

27.826

24.529

31.775
5.509E-06 4.335E-06

$4.717 E+01 \quad 3.836 E+01$

37.447

$\begin{array}{lllllllll}4.827 E-08 & 6.257 E-08 & 1.696 E-07 & 1.014 E-07 & 9.062 E-08 & 2.975 E-07 & 1.045 E-07 & 8.458 E-14 & 5.00 E-08\end{array}$ $\begin{array}{lllllll}4.271 E-01 & 5.537 E-01 & 1.452 E+00 & 8.976 E-01 & 7.760 E-01 & 2.633 E+00 & 1.088 E+01\end{array}$
0.590
0.589
1.139
0.482
0.523
2.570
1.071

$\begin{array}{llllllllll}1.486 E-07 & 2.096 E-07 & 8.171 E-08 & 1.104 E-07 & 4.376 E-07 & 4.528 E-07 & 2.130 E-07 & 1.725 E-13 & 2.00 E-08\end{array}$ $\begin{array}{lllllll}1.315 E+00 & 1.855 E+00 & 6.997 E-01 & 9.765 E-01 & 3.747 E+00 & 4.007 E+00 & 2.220 E+01\end{array}$
1.816
1.973
0.549
0.524
2.524
3.912
2.185

\section{TOTALS}

\begin{tabular}{|c|c|c|c|c|c|c|c|}
\hline $\begin{array}{l}\text { ALPHA CURIES } \\
\% \text { OF TOTAL } \\
\text { B+G CURIES } \\
\% \text { OF TOTAL }\end{array}$ & $\begin{array}{r}7.242 E+01 \\
100.000\end{array}$ & $\begin{array}{r}9.403 E+01 \\
100.000\end{array}$ & $\begin{array}{r}1.275 E+02 \\
100.000\end{array}$ & $\begin{array}{r}1.862 E+02 \\
100.000\end{array}$ & $\begin{array}{r}9.590 E-10 \\
1.484 E+02 \\
100.000\end{array}$ & $\begin{array}{r}1.150 E-08 \\
1.024 E+02 \\
100.000\end{array}$ & $1.246 E-0$ \\
\hline Lune I & 8. $849 E+06$ & $8.849 E+06$ & $8.563 E+06$ & $8.849 E+06$ & $8.563 E+06$ & $8.849 E+06$ & $1.042 E$ \\
\hline
\end{tabular}

* This maber is derived from a disparsion factor equation. The dispersion factor is derived from the straight line onssien model and is proveded by the National Oceanic Atmospheric Administration (NOAA).

* NOTE: DCG valu obtained from DOE ORDER 5400.5 datad Fabruary 8, 1990, figures III-1 and III-3
RUN DATE: 05/11/94

PAGE 2 RPT. 440
ANL-A

YEARLY RELEASE $\%$ OF CG 
WASTE MANAGEMENT INFORMATION SYSTEM

EBR-II LIEUID WUSTE SUMMARY

FOR JAN THROUGH DEC 1993

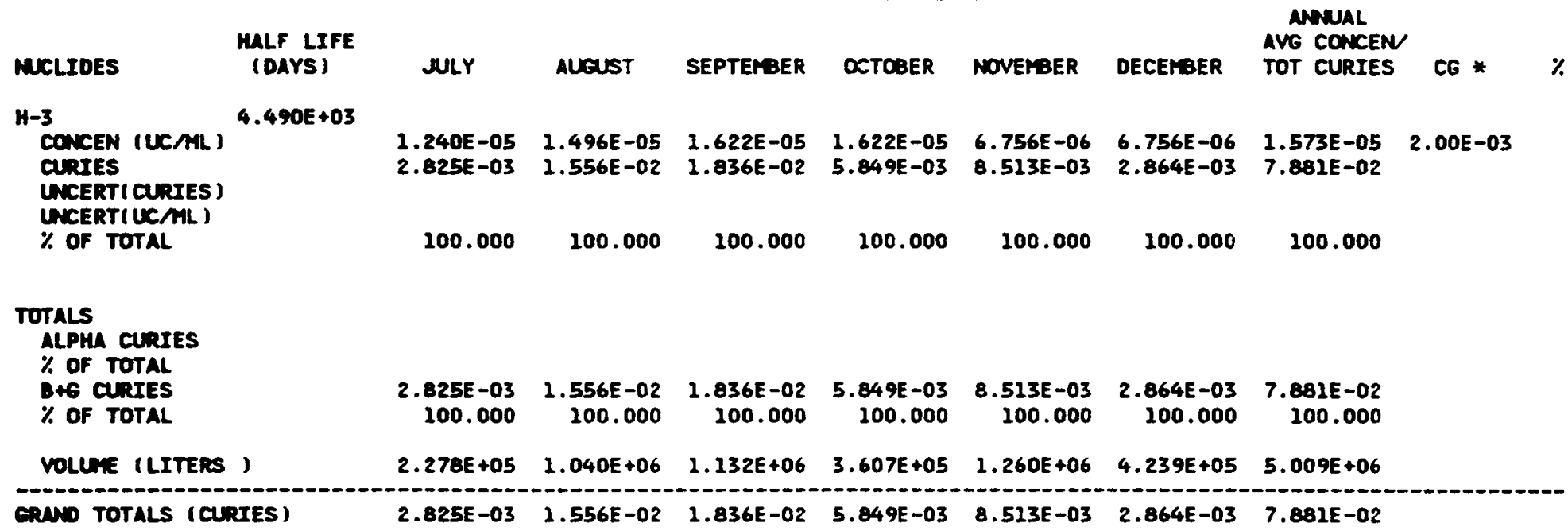

* NOTE: DCE value obtained from DOE ORDER 5400.5 dated february8, 1990, Figures III-1 and III-3 
RADIOACTIVE WASTE MANAGEMENT INFORMATION SYSTEM

\section{FASB AIRBOPNE WASTE SUMARY}

FOR JAN THROUGH DEC 1993

MUCLIDES
GROS-BET-GAM
CONCEN (UCML)
CURIES
UNCERT (CURIES)
UNCERTIUCAL)
\% OF TOTAL

\section{GROSS-ALPHA}

CONCEN (UCAL)

CURIES

UNCERT ( CURIES )

UNCERTIUCAL )

$\%$ of total

\section{MALF LIFE}

DAYS

JULY

SEPTEMER

OCTOBER

NOVEMER

december

NWNAL

AVG CONCEN

TOT CURIES

\section{$1.400 E-15$}

$1.721 E-08$

2. $970 E-14$

$5.196 E-15 \quad 6.900 E-22$

$\begin{array}{ll}7.500 E-15 & 7.420 E-15 \\ 9.525 E-08 & 9.119 E-08\end{array}$

100.000

93.338

89.221

$87.209 \quad 93.286$

$7.360 E-16 \quad 1.100 E-15$

$5.340 E-16$ $9.347 E-09 \quad 1.397 E-08 \quad 6.563 E-09$

100.000

12.791

6.714

\section{$2.120 E-15 \quad 6.277 E-16 \quad 8.337 E-23$}

$2.692 E-08 \quad 9.387 E-08$
6.662
10.779

\section{TOTALS}

ALPHA CURIES

$\%$ OF total

B+G OF TOTAL

$\%$ OF TOTAL

VOLUME (CU. MeTERS)

ERAN TOTALS (CURIES)

$1.397 E-08$

$$
12.791
$$

$6.563 E-09$

6.714

$87.209 \quad 93.286$

\section{PAGE I RPT. 470 ANL $-A$}

YEARLY YEARLY
RELEASE $\%$ OF CE

* This muber is derived from a disparsion factor equation. The dispersion factor is derived from the straight line goussian model and is proveded by the National Dceanic Atmospheric Administration (NOAA).

** NOTE: DCE velue obtained from DOE ORDER 5400.5 dated February 8, 1990 , Figures III-1 and III-3 
FMF AIRBORNE MUSTE SUMYARY
FOR JAN THROUGH DEC 1993

MUCLIDES
GROS-BET-GNM
CONCEN (UCML)
CURIES
UNCERT(CURIES)
UNCERT (UCAML)
\% OF TOTAL

\section{HALF LIFE}

(DAYS)

\section{Auars}

\section{$2.480 E-15$
$2.946 E-08$}

100.000
SEPTEMER

OCTOBER NOVEMBER

$\begin{array}{llll}1.700 E-15 & 1.800 E-16 & 7.300 E-15 & 1.090 E-15\end{array}$

$1.955 E-08$

$2.138 E-09$

8.395E-08
70.892
100.000
100.000

GROSS-ALPHA

CONCEN (UCML)

CURIES

UNCERTICURIES

UNCERT (UCML)

$\%$ OF TOTAL

\section{TOTALS}

ALPHA CURIES

$\%$ of total

B+6 CUNIES

$\%$ of total

1.295E-08

7.176

\section{$6.980 E-16$ \\ 8.027E-09}

29.108 $\begin{array}{lll}1.410 E-14 & 2.424 E-15 & 2.006 E-22 \\ 1.675 E-07 & 3.390 E-07\end{array}$

92.824

9.431 PAGE $\underset{\text { ANL }-A}{1 \text { RPT. } 475}$
ANNAL

AVG CONCEN

YEARLY

RELEASE* CE**

$.327 E-14$ 1. $927 E-21$

$3.256 E-06$

100.000

8.027E-09

29.108
$955 E-08$

$1.955 E-08$
70.892

$2.138 E-09$
100.000

$1.675 E-07$
92.824

100.000

$1.295 E-08$

7.176

$3.390 E-07$ 9.431 $256 E-06$
90.569

VOLUME (CU. METERS )

2.046-08

$1.150 E+07 \quad 1.188 E+07 \quad 1.150 E+07 \quad 1.188 E+07 \quad 1.399 E+08$

GRND TOTALS (CURIES) 2.946E-08

2.758E-08 2.138E-09 8.395E-08 1.805E-07, 3.595E-06

* This number is darived from a disparsion factor equetion. The dispersion factor is darived from the straight line gavesien model and is proveded by the National Oceanic Atmospheric Adninistration (NOAA).

* NOTE: DCG value obtainad from DOE ORDER 5400.5 dated February 8, 1990, Figures III-1 and III-3 


\section{RADIOACTIVE WASTE MANAGEMENT INFORMATION SYSTEM}

HFEF-N AIRBORNE MUSTE SUMAARY FOR JAN THROUGH DEC 1993

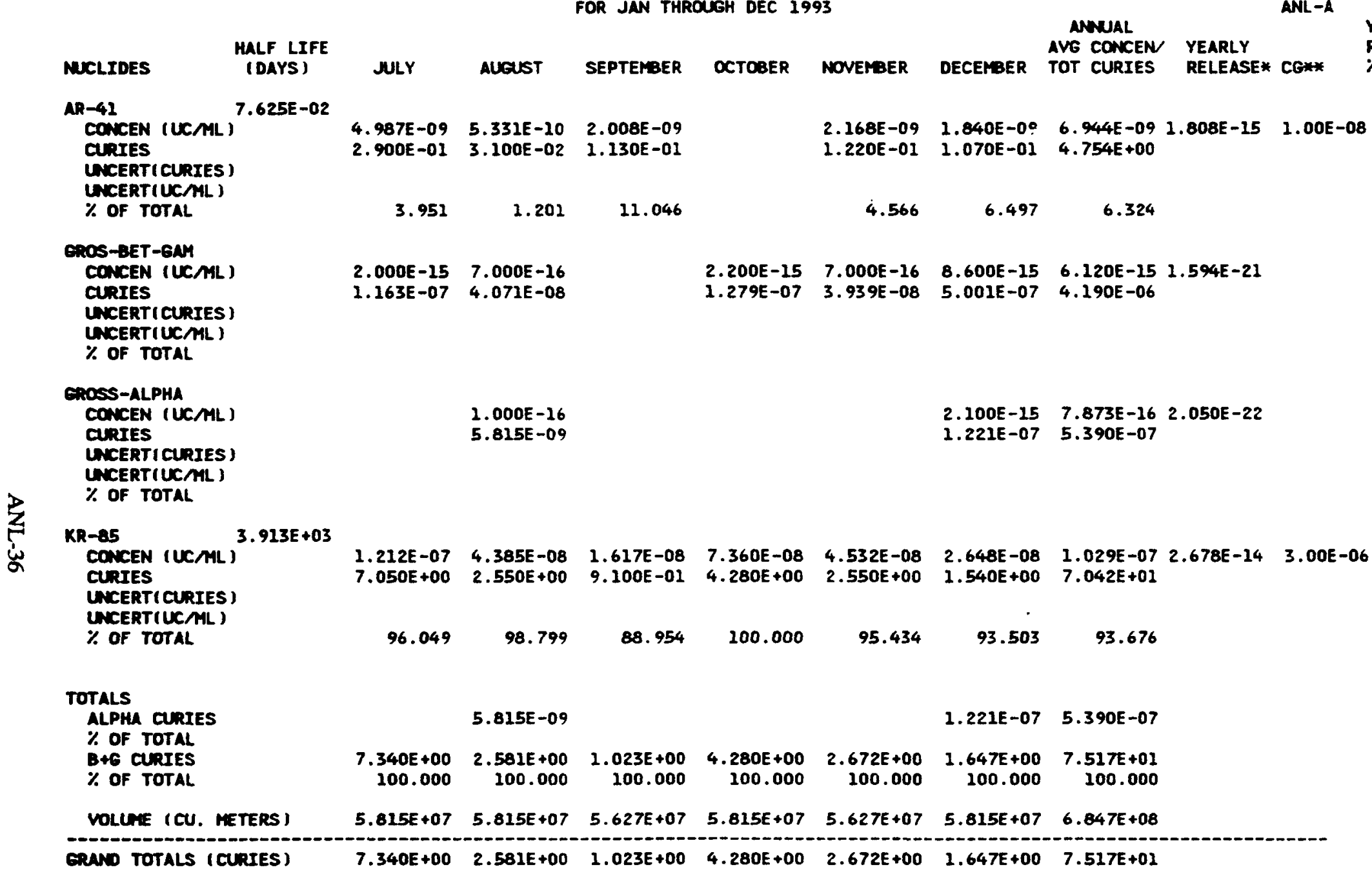

* This muber is derived from a dispersion factor equation. The dispersion factor is derived from the straight line gavesien model and is proveded by the National Dceanic Atmospheric Adwinistration (NOAA).

* NOTE: DCE value obtained from DOE ORDER 5400.5 doted February 8, 1990 , Figures III-1 and III-3 
FUEL CYCLE FAC AIRBORNE WASTE SUMMARY FOR JAN THROUGH DEC 1993

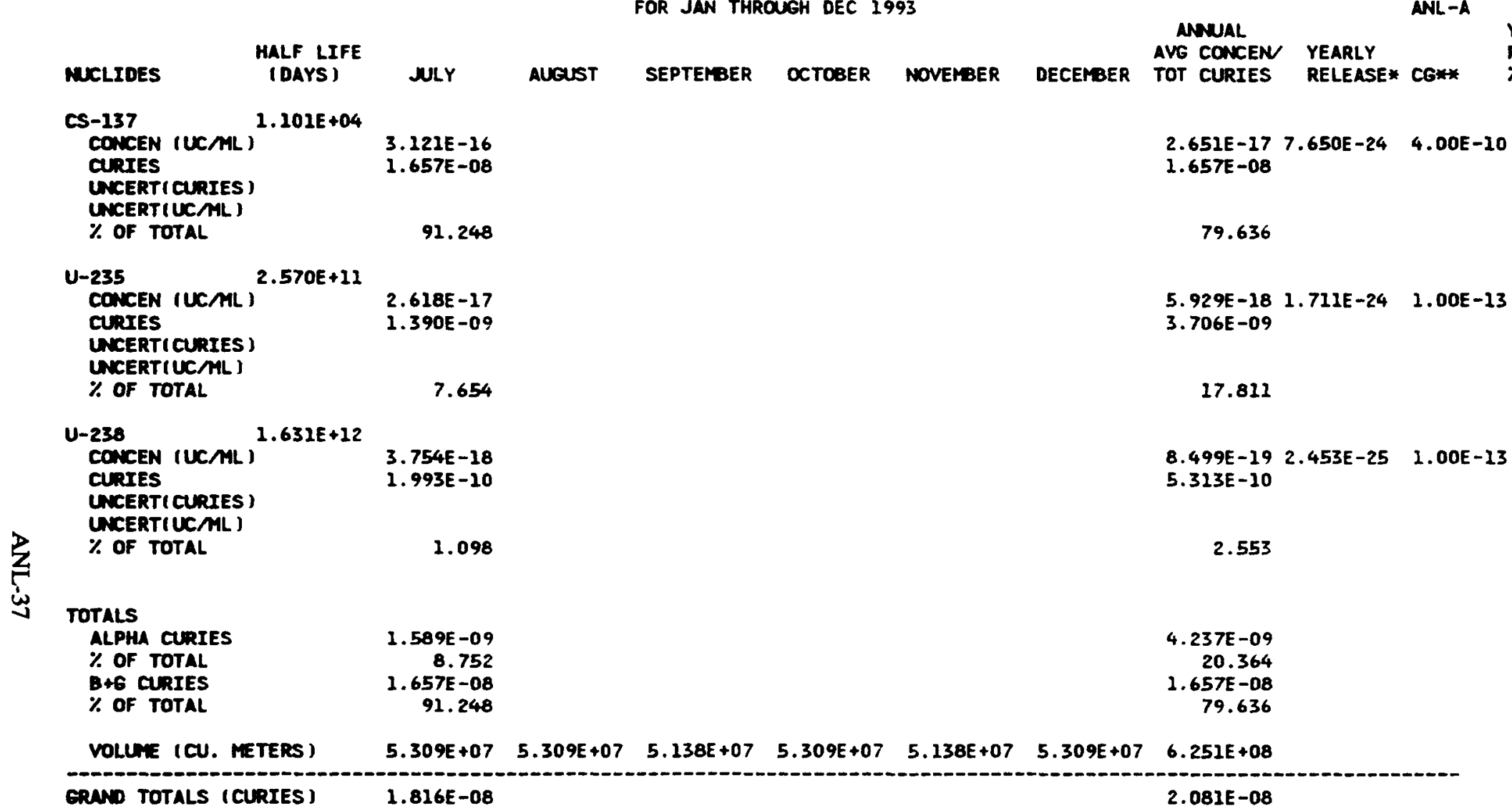

* This nuber is derived from a dispersion factor equation. The dispersion factor is derived from the straight line oussian model and is proveded by the National Oceanic Atmospheric Adninistration (NOMA).

* NOTE: DCG value obtained from DOE ORDER 5400.5 dated February 8, 2990 , Figures III-I and III-3 


\section{RADIOACTIVE WASTE MANAGEMENT INFORMATION SYSTE}

FOR JAN THROUEH DEC 1993

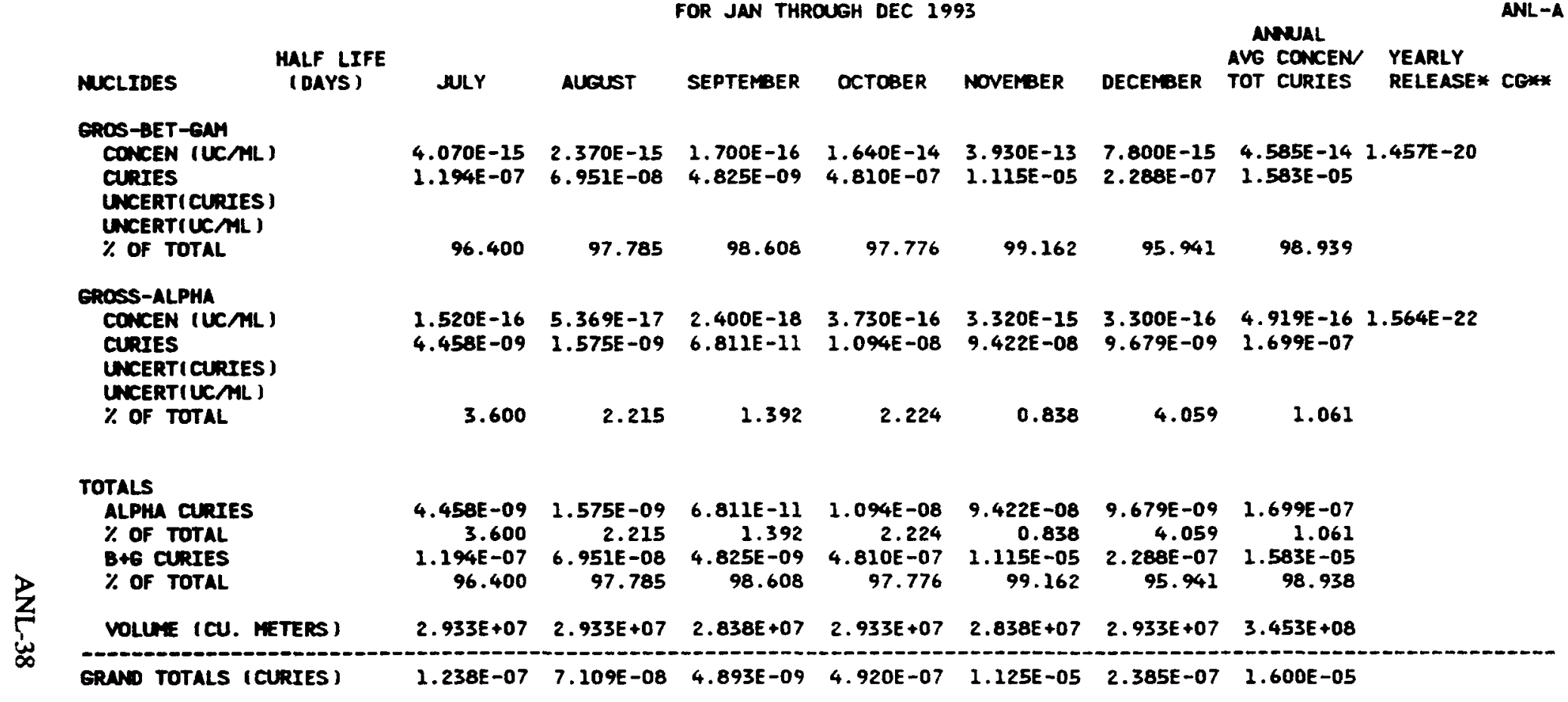

* This nuber is derived from a dispersion factor equation. The dispersion factor is derived from the straight line geussian modal and is proveded by the National Oceanic Atmospheric Adninistration (NOAA).

** NOTE: DCE value obtained from DOE ORDER 5400.5 dated February 8, 1990, Figures III-1 and III-3 
RADIOACTIVE WASTE MANAGEMENT INFORMATION SYSTEM

NDA LABORATORY AIRBORNE MASTE SUMMARY FOR JAN THROUGH DEC 1993

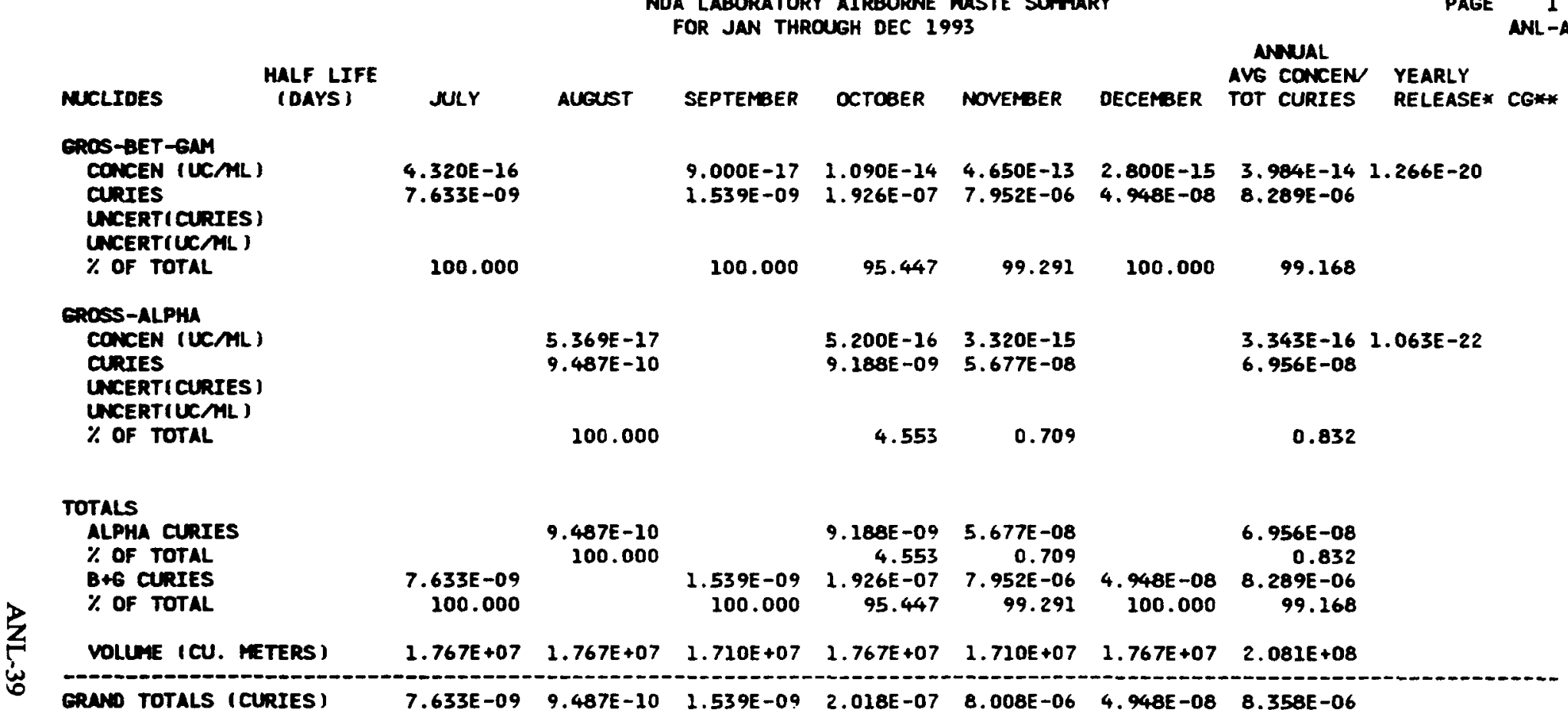

* This number is derived from a disparsion factor aqution. The dispersion factor is derived from the straight line oassian modal and is proveded by the National Oceanic Atmospheric Administration (NOAA).

* NOTE: DCG value obtained from DOE ORDER 5400.5 dated February 8, 1990, Figures III-I and III-3 
RADIOACTIVE WUSTE MANAGEMENT INFORMATION SYSTEM

RLKTF AIRBORNE WUSTE SUMUARY FOR JAN THROUEH DEC 1993

\section{MCLIDES \\ EROS-BET - GNM
CONCEN (UCML) \\ CONCEN \\ UNEERT C CURIES \\ UACERT (UCAL) \\ $\%$ OF TOTAL}

HALF LIFE

(DAYS)

JULY

AUEUST SEPTEMBER

\section{EROSS-ALPHA}

CONCEN (UCAL)

CURTES

UNCERT (CURIES)

UNCERT (UCALI)

$\%$ OF TOTAL

$$
\begin{array}{llll}
8.000 E-16 & 3.860 E-16 & 4.900 E-16 & 1.000 E-15 \\
2.882 E-09 & 1.391 E-09 & 1.708 E-09 & 3.486 E-09
\end{array}
$$

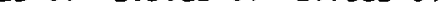

100.000

0.001

100.000

\section{$9.880 E-17$}

$3.444 E-10$

8.992

4. $000 E-17$

$1.441 E-10$

H-3 CONCEN (UCML)

$4.490 E+03$

CURIES

ANG CONAL

PAgE 1 RPT. 490 ANL-A

TOT CURIES RELEASE* CG** \% OF CG

\section{$2.485 E-15 \quad 8.873 E-23$}

$1.054 \mathrm{E}-07$

$$
0.001
$$

$5.302 E-17$ 1.893E-24

2. 249E-09

$3.386 E-11 \quad 3.178 E-10 \quad 1.134 E-17 \quad 1.00 E-07$

5. 246E-11

1.220E-04 1.348E-02

99.999

100.000

99.999

UNCERT (UCALI)

$1.441 E-10$

2.882E-09 $1.890 E-04 \quad 2.708 E-09$ $100.000 \quad 100.000 \quad 100.000$
ALPHA CURIES

$\%$ OF TOTAL

B+6 CURIES

$\%$ OF TOTAL

VOLUNE ICU. METERS I

ERND TOTALS (CURIES)

$3.603 E+06 \quad 3.603 E+06$

$3.486 E+06 \quad 3.603 E+06$

2.882E-09 1.890E-04 1.708E-09

$$
\begin{array}{rrr}
3.444 E-10 & & 2.249 E-09 \\
8.992 & & \\
3.486 E-09 & 1.220 E-04 & 1.348 E-02 \\
91.008 & 100.000 & 100.000
\end{array}
$$
goussien model and is proveded by the Notional Dcemic Atmospheric Acministration (NOMA).

* NOTE: DCE value obtained from DOE ORDER 5400.5 dated February 8, 1990, Figures III-1 and III-3 
SCMS AIRBORNE WASTE SUMARY

FOR JAN THROUGH DEC 1993
NUCLIDES
SROS-BET-GAM
CONCEN IUC

CONCEN (UCAL)

CURIES

UNCERTICURIES

UNCERTIUCAL

$\%$ OF TOTAL

\section{EROSS-ALPHA}

CONCEN (UCAL)

CURIES

UNCERT ( CURIES

UNCERT (UC/ML)

$\%$ OF TOTAL

\section{HALF LIFE}

(DAYS)

JULY

Aucust

SEPTEMBER

5. 271E-08

8.070E-15

3.300E-

OCTOe日R

NOVERBR
98.691

100.00

3.600E-16 1.070E-16

4.550E-09 1.352E-09

$$
7.947
$$

1.309

$\begin{array}{llll} & \text { PAGE } & \text { I RPT. } 480 \\ \text { ANL-A } & \\ \text { ANUAL } & & & \text { YEARLY } \\ \text { AVG CONCEN } & \text { YEARLY } & \text { RELEASE } \\ \text { TOT CURIES } & \text { RELEASE* CG** } & \% \text { OF CG }\end{array}$

\section{TOTALS \\ ALPHA curres \\ $\%$ OF TOTAL \\ $z \quad \begin{aligned} & \text { B+G CURTES } \\ & \text { \% OF TOTAL }\end{aligned}$}
4.550E-09 1.352E-09
5. $271 \mathrm{E}-08$
1.309

92.053

98.691
$4.036 E-06 \quad 5.309 E-08$
100.000
100.000

3.602

$2.720 E-16 \quad 2.600 E-16 \quad 1.393 E-16 \quad 1.244 E-23$

$3.327 E-09 \quad 3.286 E-09 \quad 2.073 E-08$
3.602
3.351
0.435

VOLUNE (CU. METERS)

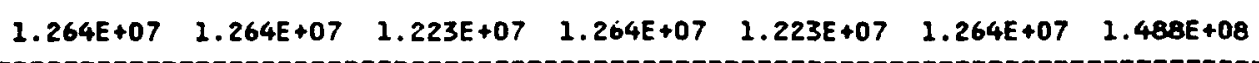

GRAN TOTALS (CURIES )

$5.726 E-08 \quad 2.034 E-07 \quad 4.036 E-06 \quad 5.309 E-08 \quad 9.236 E-08 \quad 9.809 E-08 \quad 4.760 E-06$

* This muber is derived from a dispersion factor equation. The dispersion factor is derived from the straight line gevesian model and is proveded by the National Oceanic Atmospheric Adninistration (NOAA).

** NOTE: DCE value obtained from DOE ORDER 5400.5 dated Fabruary 8, 1990, Figures III-1 and III-3 
RADIOACTIVE WASTE MANAGEMENT INFORMATION SYSTEM

TREAT AIRBORNE WASTE SUMMARY FOR JAN THROUEH DEC 1993

\begin{tabular}{|c|c|}
\hline MCLIDES & $\begin{array}{l}\text { HALF LIFE } \\
\text { (DAYS) }\end{array}$ \\
\hline $\begin{array}{l}\text { AR-A1 } \\
\text { CONCEN (UCML) } \\
\text { CURIES } \\
\text { UNCERT(CURIES) } \\
\text { UNCERTIUCML) } \\
\% \text { OF TOTAL }\end{array}$ & \\
\hline $\begin{array}{l}\text { BA-140 } \\
\text { CONCEN (UCML) } \\
\text { CURIES } \\
\text { UNCERT(CURIES) } \\
\text { UNCERT(UCAML) } \\
\text { \% OF TOTAL }\end{array}$ & $1.276 E+01$ \\
\hline $\begin{array}{l}\text { CS-140 } \\
\text { CONCEN (UCML) } \\
\text { CURIES } \\
\text { UNCERTI CURIES) } \\
\text { UNCERT (UCML) } \\
\text { \% OF TOTAL }\end{array}$ & $1.260 E+09$ \\
\hline $\begin{array}{l}\text { GROS-BET-GNM } \\
\text { CONCEN (UCML) } \\
\text { CURIES } \\
\text { UNCERT(CURIES) } \\
\text { UNCERT(UCAL) } \\
\text { \% OF TOTAL }\end{array}$ & \\
\hline $\begin{array}{l}\text { GROSS-ALPHA } \\
\text { CONCEN (UCML) } \\
\text { CURIES } \\
\text { UNCERT(CURIES) } \\
\text { UNCERT (UCAL) } \\
\text { \% OF TOTAL }\end{array}$ & \\
\hline $\begin{array}{l}\text { KR-85M } \\
\text { CONCEN (UCML) } \\
\text { CURIES } \\
\text { UNCERT(CURIES) } \\
\text { UNCERT(UCML) } \\
\text { \% OF TOTAL }\end{array}$ & , \\
\hline $\begin{array}{l}\text { KR-87 } \\
\text { CONCEN (UCML) } \\
\text { CURIES } \\
\text { UNCERT(CURIES) } \\
\text { UNCERTIUC/ML) }\end{array}$ & , 5.278E-02 \\
\hline
\end{tabular}

JLY

ALGUST SEPTEMBER OCTOBER NOVEMBER

DECEMBER

ANNAL
AVG CONCEN YEARLY

PAGE 1 RPT. 410 ANL $-A$ TOT CURIES RELEASE* CE** \% OF CG

\section{$7.235 E-07$ \\ $7.080 E-01$}

13.246

4.124E-11

4.036E-05

0.001

$2.315 E-06$

$2.266 E+00$

42.395
$5.641 E-06$

$6.900 E-01$

13.208

\section{$3.188 E-10$}

$3.900 E-05$

0.001

\section{$1.798 E-05$}

2. 200E+D0

42.114

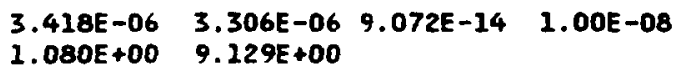

O.00E-08

$.229 E+00$

13.198

9.500E-12

4.700E-11 $2.690 E-13$

$3.609 E-06$

0.005

95.390

86.957

86.886

$1.956 E-10$

$6.180 E-05$

1.884E-10 5.170E-18 3.00E-09

0.001

0.001

1. $098 \mathrm{E}-05$

$3.470 E+00$

$1.058 E-05 \quad 2.904 E-13$

42.404

42.368

$7.600 E-14$

.438E-08 $5.800 E-12$

1. 500E -14

3.700E-11

$2.450 E-10 \quad 6.722 E-18$

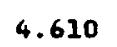

4.610

13.043

13.114

$2.894 E-09$

$2.832 E-03$

0.053

$1.656 E-08$

$1.621 E-02$

0.303

2. 207E-08

2. $700 E-03$

0.052

$1.308 E-07$

$1.600 E-02$
$1.373 E-08$ $4.340 E-03$

1.321E-08 $3.625 E-16 \quad 1.00 E-07$ $3.648 E-02$

0.053

0.053

$\begin{array}{llll}7.848 E-08 & 7.580 E-08 & 2.080 E-15 & 2.00 E-08\end{array}$ $2.480 E-02 \quad 2.093 E-01$

\% OF TOTAL

0.303

0.304

$1.135 E-13 \quad 3.115 E-21$
$3.135 E-07$ 
RADIOACTIVE WASTE MANAGEMENT INFORMATION SYSTEM

TREAT AIRBORNE WASTE SUMMARY FOR JAN THROUGH DEC 1993

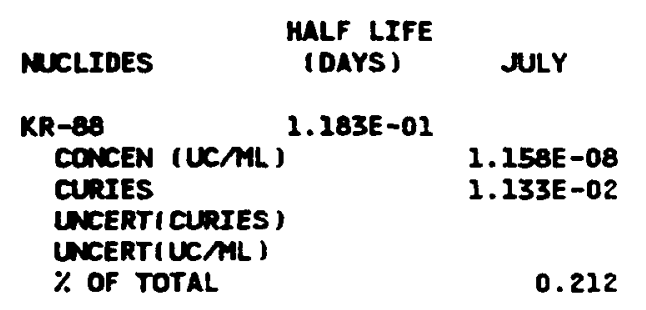

LA-140
CONCEN (UCAL) $1.678 E+00$
CURIES
UNCERT(CURIES)
UNCERT(UCML)
$\%$ OF TOTAL

AUGUST SEPTEMBER

OCTOBER

NOVEMBER
$3.188 E-14$
4.124E-15

4.036E-09

$1.034 E-10$

I.012E-04

0.002

$1.447 E-09$

$1.416 E-03$

0.026

$2.8 \% 4 E-09$

$2.832 E-03$

0.053

2. 387E-06

$2.336 E+00$

43.704

\section{8. $992 E-08$ \\ 8.
1. $100 E-02$}

0.211

$3.900 E-09$

B.011E-10

$9.800 E-05$

0.002

$1.144 E-08$

$1.400 E-03$

0.027

2. 207E-08

$2.700 E-03$

0.052

1. 880E-05

$2.300 E+00$

44.028
PAge 2 RPT. 410
ANL $-A$
YEARLY

MNNAL AVG CONCEN YEARLY $\quad$ RELEASE DECERBER TOT CURIES RELEASE* CG** \% OF CG

5.506E-08 5.292E-08 $1.452 E-15 \quad 9.00 E-09$ $1.740 E-02 \quad 1.462 E-01$

0.213

0.212

$1.956 E-14 \quad 1.884 E-14 \quad 5.170 E-22 \quad 3.00 E-09$ 6.180E-09 5.202E-08

4. $905 E-10 \quad 4.727 E-10 \quad 1.297 E-17 \quad 3.00 E-08$ $1.550 E-04$ 1.305E-03
0.002
0.002

6.867E-09 6.622E-09 $1.817 E-16 \quad 5.00 E-07$ $2.170 E-03 \quad 1.829 E-02$
0.027
0.027

$1.373 E-08 \quad 1.324 E-08 \quad 3.634 E-16 \quad$ B. $00 E-08$ $4.340 E-03$ 3.657E-02

$0.053 \quad 0.053$

$1.133 E-05 \quad 1.092 E-05 \quad 2.998 E-23$ $3.580 E+00 \quad 3.017 E+01$

$43.748 \quad 43.743$ 
SCHED. NO. P61PMOO9-D

\begin{abstract}
RADIOACTIVE WASTE MANAGEMENT INFORMATION SYSTEM
TREAT AIRBORNE WASTE SUMMARY

FOR JAN THROUGH DEC 2993
\end{abstract}

U.S. DEPARTMENT OF ENERGY

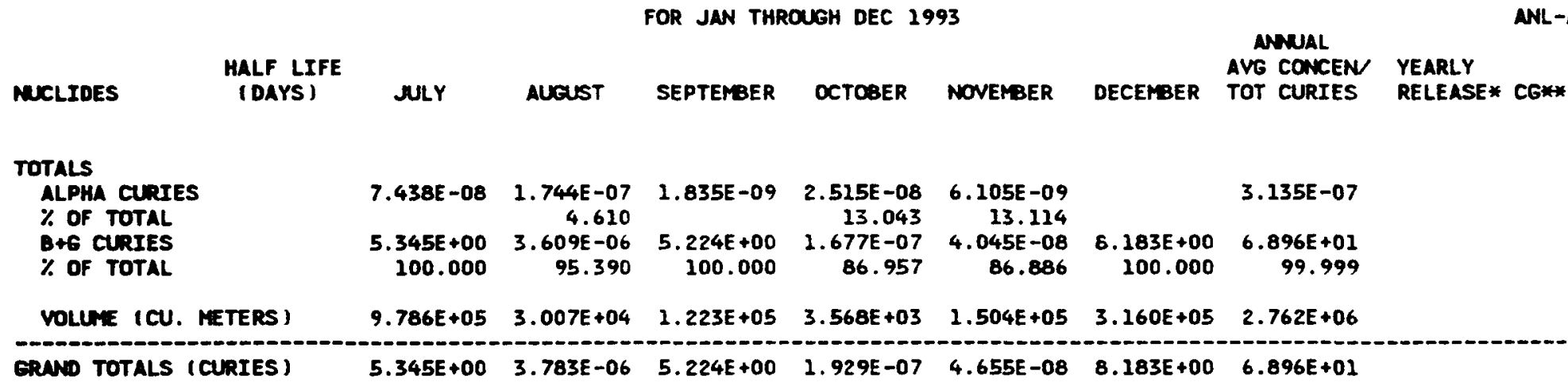

* This nubar is chrived from a dispersion factor equation. The dispersion factor is derived from the straight line gauseian model and is proveded by the National Dceanic Atmospheric Administration (NOAA).

* NOTE: DCG value obtained from DOE ORDER 5400.5 dated February 8, 1990, Figures III-1 and III-3
RUN DATE: 05/11/94

ARE 3 RPT. 410

YEARLY

RELEASE 
RADIOACTIVE WUSTE MUUGEMENT INFORMATION SYSTEM

ZPPR AIRBOPANE WUSTE SUMYARY

FOR JAN THROUGH DEC 1993

NUCLIDES
GROS-BET-GAM
CONCEN (LCML)
CURIES
UNCERT ( CURIES)
UNCERT UCAML)
\% OF TOTAL

GROSS-ALPHA CONCEN (UCML)

CURIES

UNCERTI CURIES I

UNCERT (UCML)

$\because$ Of TOTAL
JULY

$$
\text { Aveust }
$$

SEPTEMBER

october

NOVEMBER

\subsection{E- -25}

$1.644 E-08$

98.373

$\begin{array}{llllll}9.200 E-17 & 1.280 E-16 & 4.630 E-17 & 3.880 E-17 & 2.230 E-15\end{array}$

100.000

100.000

1.627

100.000

100.000
5.583E-10 $\quad 7.767 E-10 \quad 2.719 E-10 \quad 2.354 E-10 \quad 1.309 E-08$

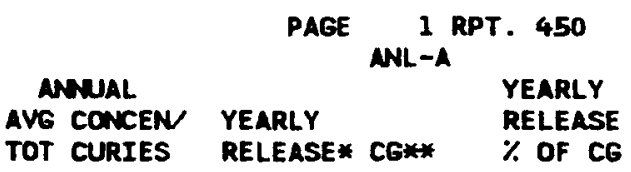

TOT CURIES

RELEASE* CG**

\subsection{E-16}

$3.350 E-09$

100.000

77.624

$2.245 E-16 \quad 7.743 E-24$

1.604E-08

22.376

\section{TOTALS}

ALPHA CURIES

$\%$ OF TOTAL

B+G CURIES

5.583E-10 7.767E-10

100.000

100.000
$2.719 E-10 \quad 2.354 E-20 \quad 1.309 E-08$
1.627
100.000
100.000
$.644 E-08$
98.373
100.000

$\%$ OF TOTAL
$1.604 E-08$
$350 E-09 \quad 5.563 E-08$
$100.000 \quad 77.624$

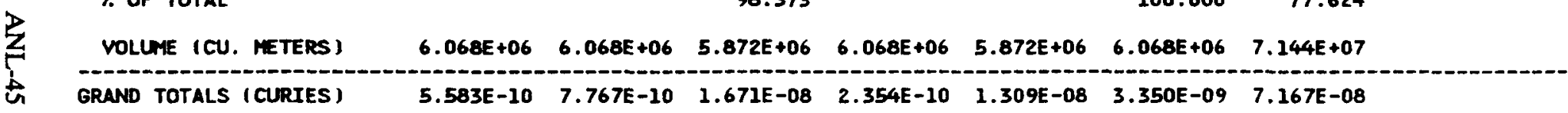

* This number is derived from a dispersion factor equation. The dispersion factor is derived from the straight line gaussian model and is provedad by the National Oceanic Atmospheric Administration (NOAA).

* NOTE: DCG value obtained from DOE ORDER 5400.5 dated February 8, 1990, Figures III-1 and III-3 

ANL Facility Detail Data for Months January to June Annual Average Concentration and Total Curies for January to June 

EBR-II AIRBORNE MASTE SUMHARY FOR JAN THROUGH JUN 1993

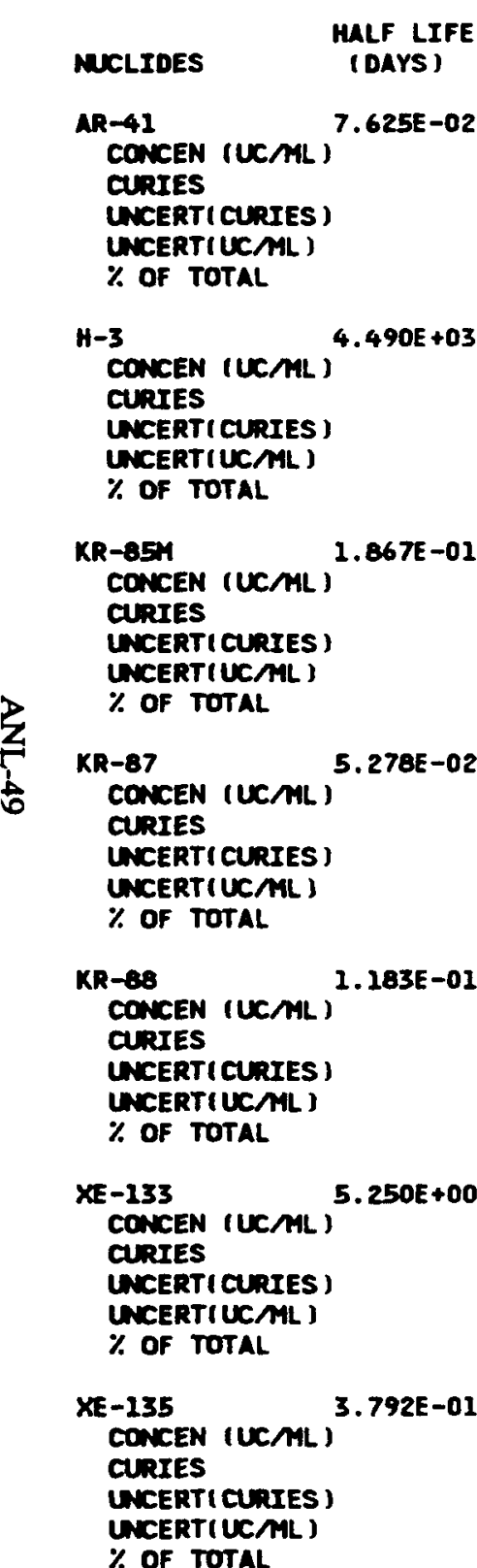

JANUARY FEBRUARY MARCH APRIL MAY

5.667E-09 4.904E-10 8.072E-09 $5.014 E-02 \quad 3.919 E-03 \quad 7.142 E-02$
0.120
0.055
0.222

$1.143 E-08 \quad 1.190 E-09 \quad 1.718 E-08 \quad 6.337 E-10 \quad 4.630 E-10$ $\begin{array}{llllll}1.011 E-01 & 9.508 E-03 & 1.520 E-01 & 5.426 E-03 & 4.097 E-03\end{array}$
0.243

0.135

0.473

0.007

0.020

$\begin{array}{llllll}3.914 E-07 & 6.909 E-10 & 3.628 E-07 & 4.933 E-08 & 1.371 E-07\end{array}$

$2.047 E+00 \quad 5.522 E-03 \quad 3.210 E+00 \quad 4.224 E-01 \quad 1.213 E+00$ 4.919

0.078

9.984

0.553

2.922
0.153

0.242

$\begin{array}{llll}7.086 E-07 & 2.510 E-07 & 2.032 E-13 \quad 1.00 E-07\end{array}$

7.046

4.551

$\begin{array}{llllllllll}1.574 E-07 & 1.471 E-09 & 4.682 E-07 & 1.136 E-07 & 3.941 E-07 & 5.113 E-07 & 2.785 E-07 & 2.255 E-13 & 2.00 E-08\end{array}$ $1.393 E+00 \quad 1.176 E-02 \quad 4.143 E+00 \quad 9.727 E-01 \quad 3.487 E+00 \quad 4.378 E+00 \quad 1.439 E+01$
3.347
0.165
12.886
1.274
8.401
5.083
5.050

$\begin{array}{lllllllll}1.863 E-07 & 1.335 E-09 & 5.589 E-07 & 7.710 E-08 & 3.791 E-07 & 8.629 E-07 & 3.486 E-07 & 2.822 E-13 & 9.00 E-09\end{array}$ $1.648 E+00 \quad 2.067 E-02 \quad 4.945 E+00 \quad 6.602 E-01 \quad 3.354 E+00 \quad 7.389 E+00 \quad 1.801 E+01$
3.960
0.150
15.381
0.865
8.080
8.580
6.321

$\begin{array}{lllllllll}2.076 E-06 & 8.833 E-07 & 2.377 E-07 & 8.443 E-06 & 3.023 E-06 & 2.757 E-06 & 2.907 E-06 & 2.354 E-12 & 5.00 E-07\end{array}$ $\begin{array}{lllllll}1.837 E+01 & 7.059 E+00 & 2.103 E+00 & 7.230 E+01 & 2.675 E+01 & 2.361 E+01 & 1.502 E+02\end{array}$
44.144
99.092
6.541
94.679
64.444
27.414
52.720

$\begin{array}{lllllllll}1.884 E-06 & 4.238 E-10 & 1.611 E-06 & 8.047 E-08 & 1.702 E-07 & 4.892 E-06 & 1.452 E-06 & 1.176 E-12 & 8.00 E-08\end{array}$ $\begin{array}{lllllll}1.667 E+01 & 3.387 E-03 & 1.425 E+01 & 6.891 E-01 & 1.506 E+00 & 4.189 E+01 & 7.501 E+01\end{array}$
40.059
0.048
44.323
0.902
3.628
48.639
26.329

PAGE 2 RPT. 440 YEARLY RELEASE 
RADTOACTIVE WUSTE MANAGEMENT INFORMATION SYSTEM

EBR-II AIRBORNE WUSTE SUMHARY FOR JAN THROUGH JUN 1993

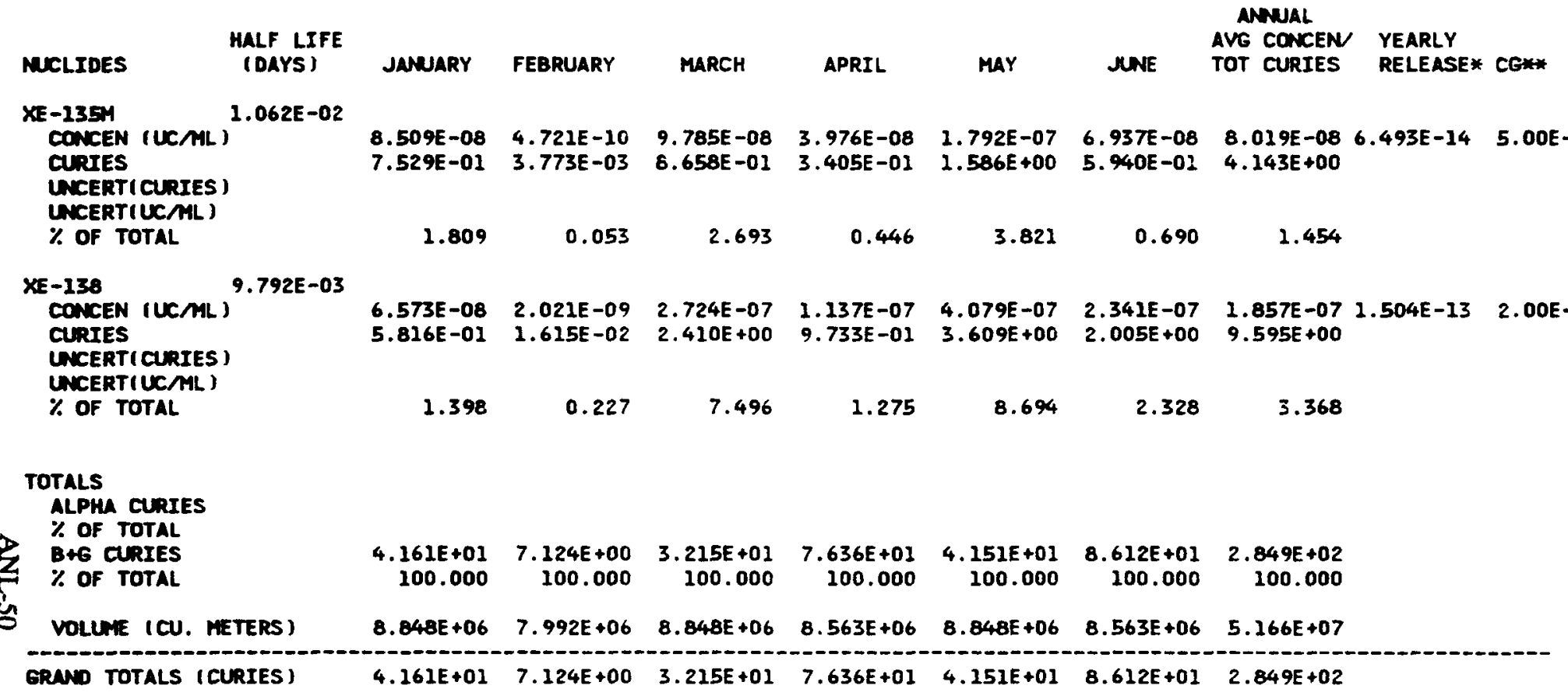

* This nuber is chrived from a dispersion factor equation. The dispersion factor is derived from the straight line ooussian model and is proveded by the National Dceanic Atmospheric Administration (NOAA).

** NOTE: DCE value obtained from DOE ORDER 5400.5 dated February 8, 1990, Figures III-I and III-3 


\section{WASTE MANAGEMENT INFORMATION SYSTEM}

U.S. DEPARTMENT OF ENERGY

EBR-II LIQUID WASTE SUMMRY

FOR JAN THROUGH JUN 1993

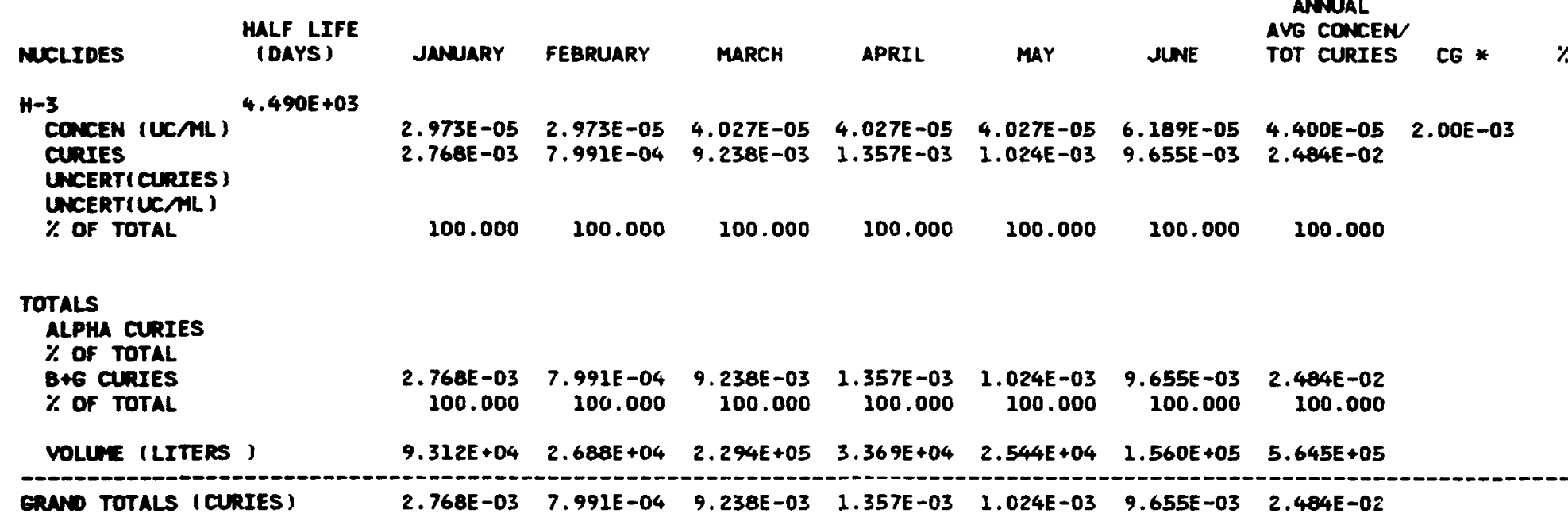

* NOTE: DCE value obtainad from DOE ORDER 5400.5 dated February8, 1990, Figures III-1 and III-3 
RADIOACTIVE WASTE MANAGEMENT INFORMATION SYSTEM

FASB AIRBORNE MASTE SUMAARY FOR JAN THROUGH JUN 1993

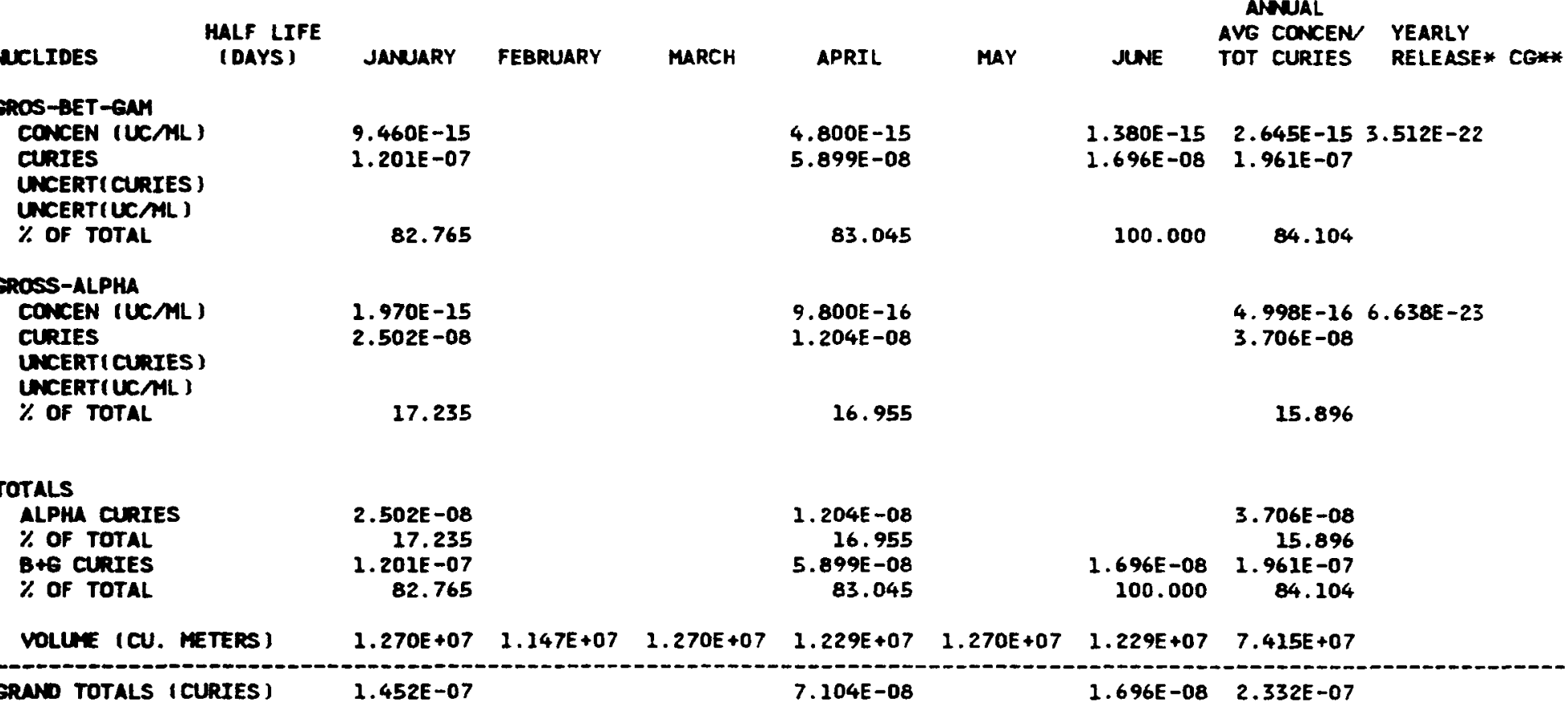

This muber is darived from a dispersion factor eqution. The dispersion factor is derived from the straight line oussian model and is proveded by the National Oceanic Atmospheric Actinistration (NOAA).

* NOTE: DCG value obtainad from DOE ORDER 5400.5 dated Fabruary 8, 1990 , Figures III-1 and III-3 
RADIOACTIVE WASTE MANAGEMENT INFORMATION SYSTEM

FMF AIRBORNE WUSTE SUMMARY FOR JAN THROUGH JUN 1993

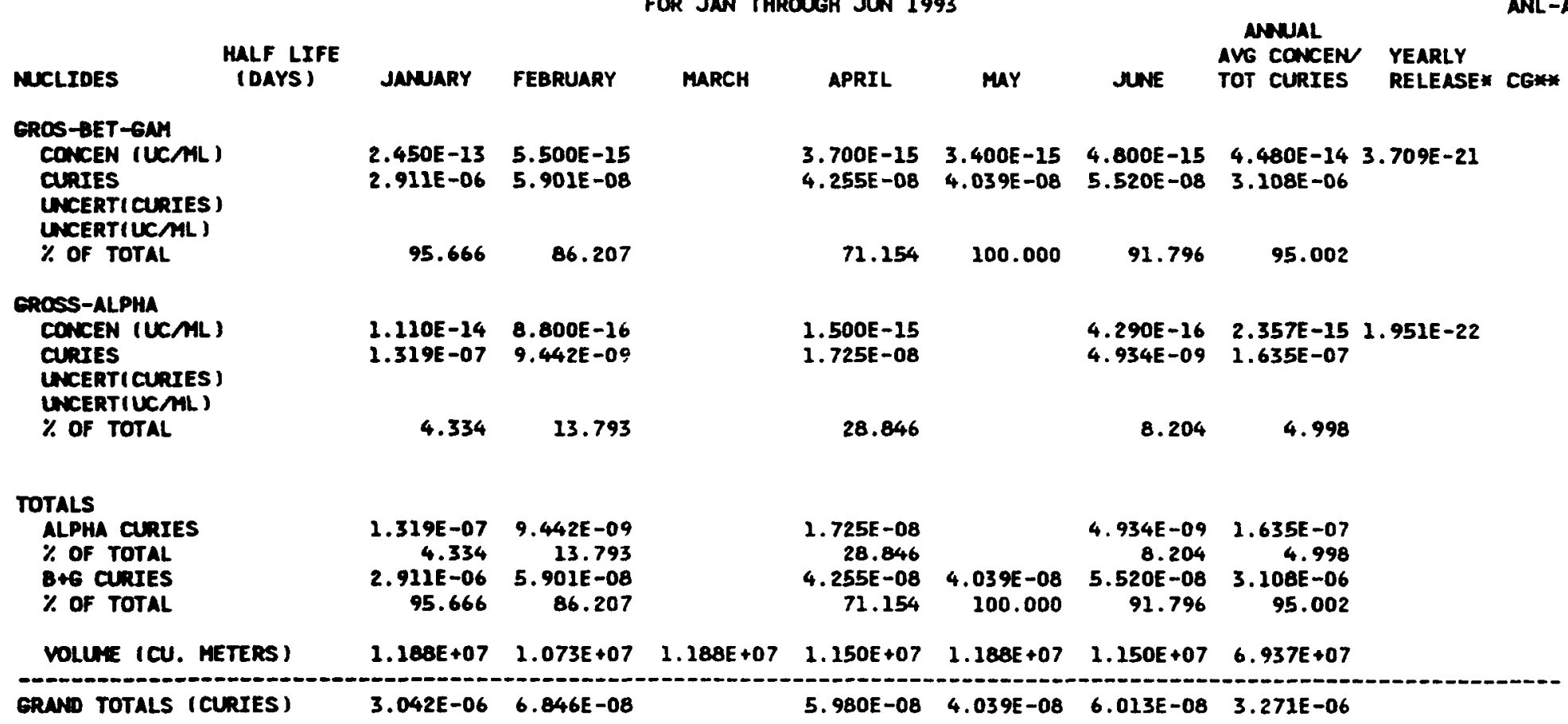

* This nuber is derived from a dispersion factor equation. The dispersion factor is derived from the straight line geussian model and is provedad by the Notional Oceanic Atmospheric Administration (NOAA).

** NOTE: DCG value obtained from DOE ORDER 5400.5 dated Fabruary 6,1990 , figures III-1 and III-3 
RADTOACTIVE WASTE MANAGEMENT INFORMATION SYSTEM

HFEF-N ATPBOONE MUSTE SUMURY

FOR JAN THROUEH JUN 1993

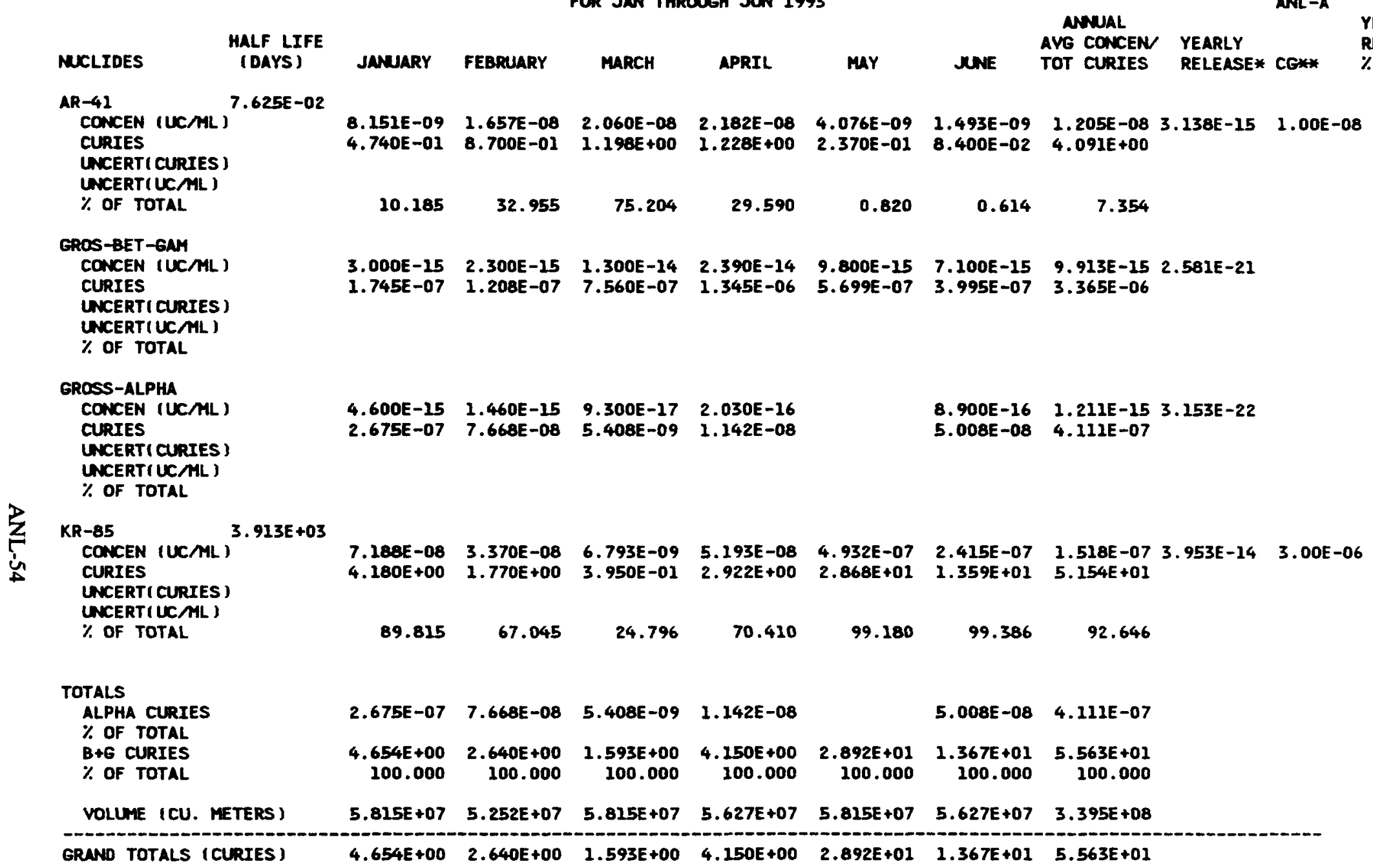

GRAND TOTALS (CURIES) $\quad 4.654 E+00 \quad 2.640 E+00 \quad 1.593 E+00 \quad 4.150 E+00 \quad 2.892 E+01 \quad 1.367 E+01 \quad 5.563 E+01$

* This number is derived from a disparsion factor equetion. The dispersion factor is derived from the straight line goussian model and is proveded by the National Dceanic Atmospheric Administration (NOMA).

** NOTE: DCG value obtained from DOE ORDER 5400.5 datad Fabruary 8, 1990 , Figures III-1 and III-3 
FUEL CYCLE FAC AIRBOANE WASTE SUMMARY FOR JAN THROUGH JN 1993

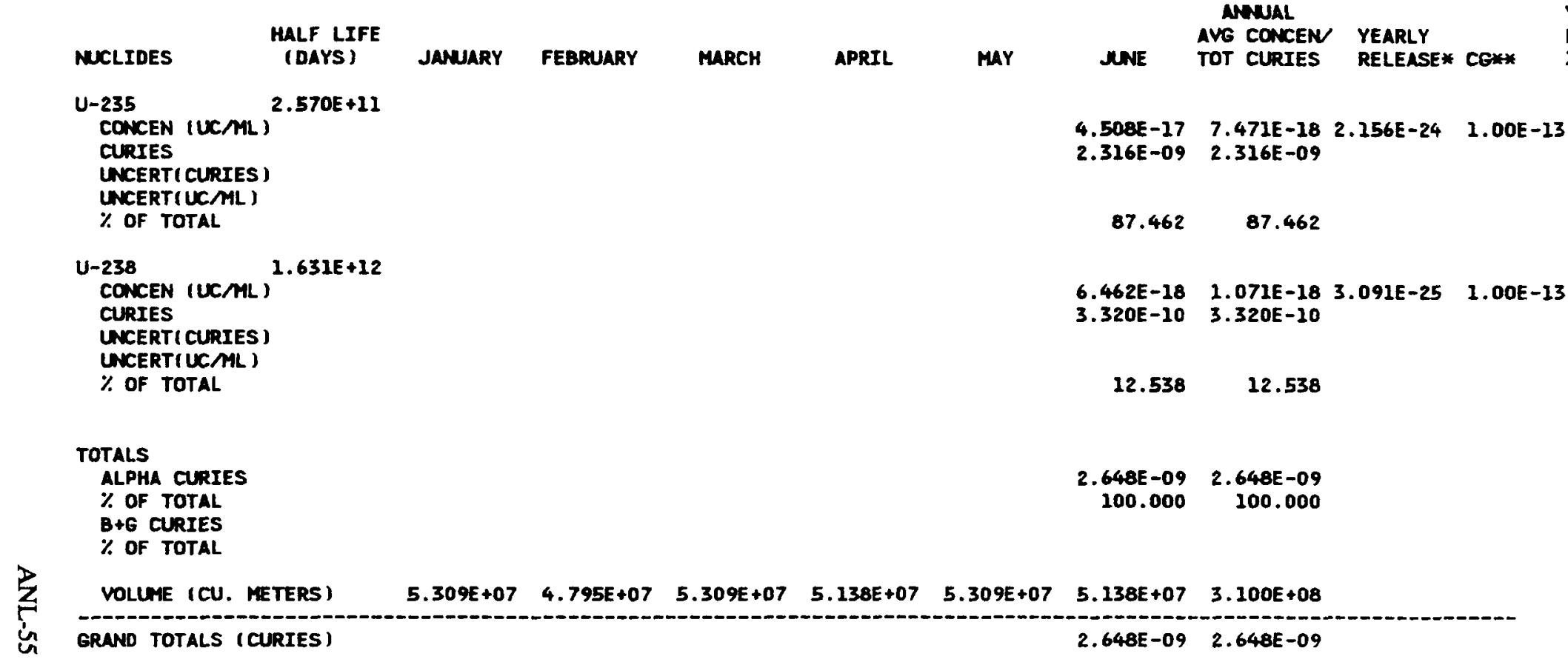

* This nuber is derived from a dispersion factor equation. The dispersion factor is darived from the straight line goussian model and is proveded by the National Oceanic Atwospheric Adninistration (NOAA).

** NOTE: DCG value obtainad from DOE ORDER 5400.5 dated Fabruary 8, 1990 , Figures III-1 and III-3 


\section{LEO MAIN STACK AIRBORNE WASTE SUMMARY} FOR JAN THROUGH JUN 1993

MCLIOES
EROS-BET-GNM
CONCEN (UCAL)
CURIES
UNCERTICURIES)
UNCERTIUCAL)
\% OF TOTAL

\section{HALF LIFE}

(OAYS)

JANUARY

FEBRUARY

MARCH

APRIL

MAY

JUNE

$4.860 E-14 \quad 9.900 E-15 \quad 3.930 E-14 \quad 3.440 E-14 \quad 2.590 E-15$

$1.287 E-06 \quad 2.904 E-07 \quad 1.115 E-06 \quad 1.009 E-06 \quad 7.350 E-08$

$98.809 \quad 100.000$

99.117

100.000

100.000

$3.500 E-16$

8.000E-16 5.860E-16

$9.933 E-09$

CONCEN (UCAL)

CURIES

UNERTI CURIES

UNCERTIUCAL

\% OF TOTAL

100.000

1.191

0.883
PAGE 1 RPT. 420 ANL-A

$\begin{array}{ccc}\text { ANUAL } & \text { ANL-A } & \text { YEARLY } \\ \text { AVG CONCEN } & \text { YEARLY } & \text { RELEASE }\end{array}$

TOT CURIES RELEASE* CG* \% OF CG

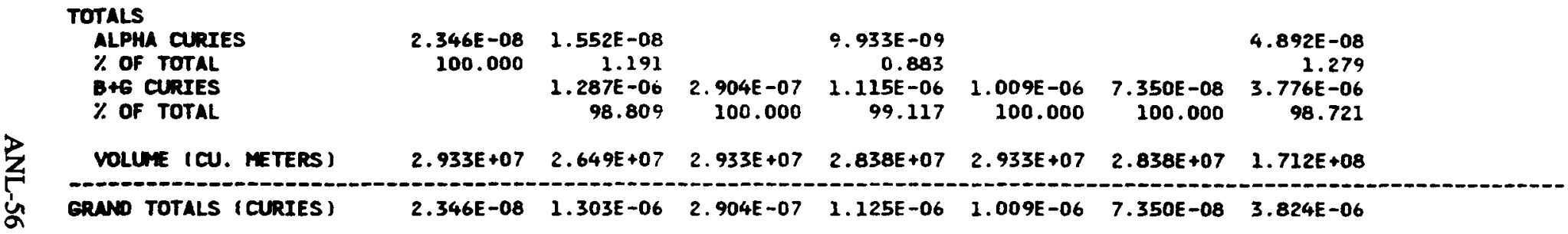

* This number is derived from a dispersion factor equation. The dispersion factor is derived from the straight line omuseian nodel and is proveded by the Nationel Dceanic Atmospheric Achinistration (NOAA).

** NOTE: DCG value obtained from DOE ORDER 5400.5 deted February 8, 1990, Figures III-1 and III-3 


\section{MUCLIDES \\ GROS-BET-GNM CONCEN (UCML)$$
\text { UNCERT (CURIES) }
$$$$
\text { UNCERT ( LCAL) }
$$ \\ \% OF total}

\section{HALF LIFE}

(DAYS)

\section{Gooss-ALPHA}

CONCEN (UCAL)

CURIES

UNCERT I CURIES I

UNCERTIUC/ML

$\%$ OF TOTAL

1. 500E -16

2.651E-09

100.000
NDA LABORATORY AIRBORNE WUSTE SLMMARY FOR JAN THROUBH JUN 1993

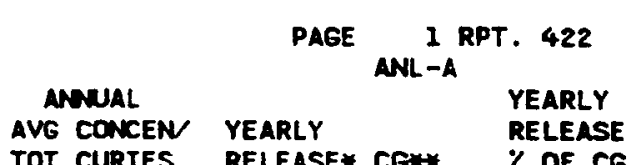

MARCH

APRIL

MaY

JUNE

TOT CURIES

$\begin{array}{llll}1.320 E-15 & 1.210 E-15 & 8.319 E-16 & 2.644 E-22\end{array}$

$2.620 E-15$

$4.182 E-08$

$2.332 E-08 \quad 2.069 E-08 \quad 8.583 E-08$

100.000

100.000

100.000

97.004

$2.569 E-178.166 E-24$

$2.651 E-09$

2.996

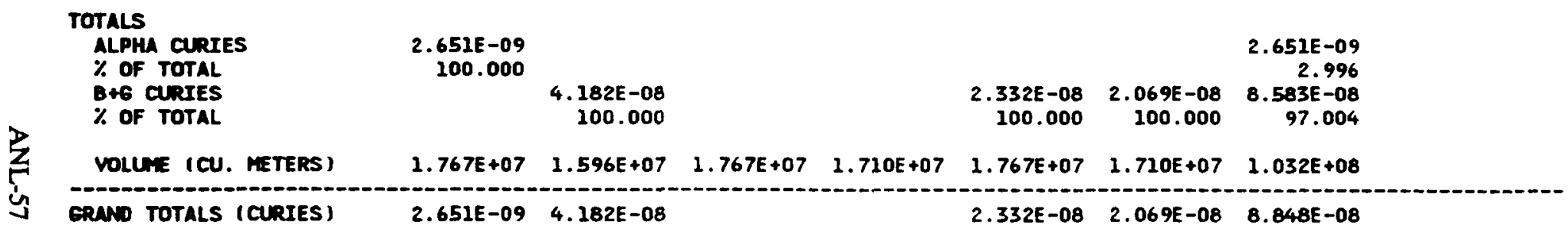

* This nuber is derived from a dispersion factor equation. The dispersion factor is derived from the straight line geuseien model and is proveded by the National Oceanic Atmospheric Administration (NOAA).

* NOTE: DCE value obtained from DOE ORDER 5400.5 dated Fobruary 8, 1990 , Figures III-I and III-3 
RADTOACTIVE WASTE MANAGEMENT INFORMATION SYSTEM

\section{RLMTF AIRBORNE WASTE SUMMARY} FOR JAN THROUGH JUN 1993

NUCLIDES
EROS-BET-GNM
CONCEN (UCML)
CURIES
UNCERT (CURIES)
UNCERT (UCML)
\% OF TOTAL

\section{GROSS-ALPHA}

CONCEN (UCML) CURIES

UNCERT (CURIES) UNCERT (UCML)

\% OF TOTAL

H-3
CONCEN (UCML)
CURIES
UNCERT(CURIES)
UNCERT(UCML)
\% OF TOTAL
TOTALS
ALPHA CURIES
\% OF TOTAL
B+G CURIES
$\%$ OF TOTAL
VOLUNE (CU. METERS )

\section{HALF LIFE}

DAYS ]

\section{FEBRUARY}

$$
\text { APRIL }
$$

MaY $\begin{array}{ll}9.330 E-15 & 1.240 E-14 \\ 3.362 E-08 & 4.323 E-08\end{array}$

99.187

0.001

$\begin{array}{llll}7.890 E-17 & 2.340 E-16 & 1.000 E-16 & 1.017 E-16\end{array}$

$2.843 E-10 \quad 7.614 E-10 \quad 3.603 E-10 \quad 3.545 E-10$

0.813
3.078E-10 3.038E-10 2.845E-09

$1.109 E-03 \quad 9.886 E-04 \quad 1.025 E-02$
100.000
100.000
100.000

$2.843 E-10 \quad 7.614 E-10 \quad 3.603 E-10 \quad 3.545 E-10$

$\begin{array}{rrrr}1.109 E-03 & 9.886 E-04 & 1.025 E-02 & 4.323 E-08\end{array}$ 100.000 100.000 $4.323 E-08$
99.187

JUNE

\begin{tabular}{|c|c|c|c|}
\hline & PAGE & $\operatorname{ANL}_{-1}^{1}$ & T. 490 \\
\hline $\begin{array}{l}\text { ANNUAL } \\
\text { VG CONCEN } \\
\text { OT CURIES }\end{array}$ & $\begin{array}{l}\text { YEARLY } \\
\text { RELEASE* }\end{array}$ & CG** & $\begin{array}{l}\text { YEARLY } \\
\text { RELEASE } \\
\% \text { OF CG }\end{array}$ \\
\hline
\end{tabular}

$\begin{array}{ll}9.900 E-16 & 4.460 E-15 \\ 3.567 E-09 & 1.555 E-08\end{array}$

$4.562 E-25 \quad 1.629 E-22$

0.003

0.001

$8.370 E-17$
$2.988 E-24$

1. 761E-09

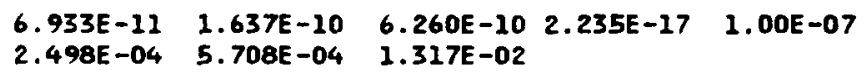

$99.999 \quad 99.997$

99.999

ERAN TOTALS (CuRTES)

$\begin{array}{lll}100.000 & 100.000 \quad 100.000\end{array}$

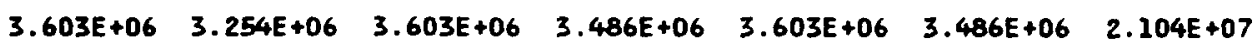

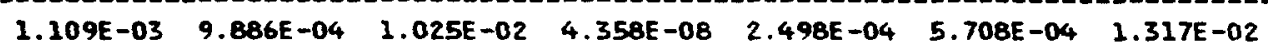

* This muber is derived from o dispersion factor equetion. The dispersion factor is derived from the straight line gevesian model and is proveded by the National Oceanic Atmospheric Adninistration (NOAA).

** NOTE: DCG value obtained from DOE ORDER 5400.5 dated February 8, 1990, Figures III-1 and III-3 


\section{SCMS AIRBORNE WASTE SUMYARY}

FOR JAN THROUGH JUN 1993

\section{MUCLIOES \\ EROS-BET-GNM \\ CONCEN (UCAL) \\ CURIES \\ UNCERTICURIES \\ UNCERTIUCALI) \\ $\%$ of TOTAL}

\begin{abstract}
HALF LIFE
\end{abstract}
(DAYS)

\section{CROSS-ALPHA}

CONCEN (UCML)

CURIES

UNCERTICURIES

UNCERTIUCAL ]

$\%$ OF TOTAL

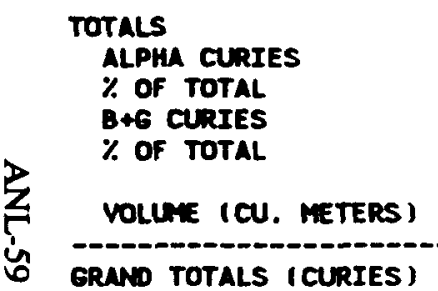

\section{$\begin{array}{llllllll}1.000 E-14 & 5.660 E-15 & 4.530 E-15 & 6.800 E-16 & 2.230 E-15 & 2.240 E-15 & 4.230 E-15 & 3.777 E-22\end{array}$ \\ $\begin{array}{lllllll}1.264 E-07 & 6.464 E-08 & 5.726 E-08 & 8.316 E-09 & 2.819 E-08 & 2.740 E-08 & 3.122 E-07\end{array}$}

100.000

\begin{abstract}
$92.665 \quad 100.000$
\end{abstract}
92.643

96.025

95.563

97.436 $\begin{array}{llllll}4.480 E-16 & 5.400 E-17 & 9.230 E-17 & 1.040 E-16 & 1.113 E-16 & 9.939 E-24 \\ 5.116 E-09 & 6.604 E-10 & 1.167 E-09 & 1.272 E-09 & 8.215 E-09\end{array}$

$\begin{array}{lllll}7.335 & 7.357 & 3.975 & 4.437 & 2.564\end{array}$
PAGE 1 RPT. 480 ANL $-\mathrm{A}$

* This muber is derived from a dispersion factor eqution. The dispersion factor is derivied from the straight line gaussian model and is proveded by the tional Oceanic Atmospheric Administration (NOAA).

* NOTE: DCG value obtainod from DOE ORDER 5400.5 dated February 8, 1990 , Figures III-1 and III-3 
IDAHO OPERATIONS OFFICE

U.S. DEPARTMENT OF ENERGY

RADIOACTIVE WASTE MUNAGEMENT INFORMATION SYSTEM

TREAT ATRBORNE WASTE SUMMARY FOR JAN THROUGH JUN 1993

HALF LIFE
MCLIDES

$A R-41$

CONCEN (UCAL)

CURIES

UNCERT ( CURIES

UNEERT(UCML)

$\%$ OF TOTAL

BA-140

CONCEN (UCAM)

CURIES

UNCERTI CURIES

UNERT (UCML)

$\%$ OF TOTAL

$\operatorname{cs}-140$

CONCEN IUCML)

CURIES

CLnIES

(ACERTIUCML)

\% OF TOTAL

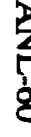

EROS-BET-GNM

CONCEN IUCML

CURIES

UNCERT (CURIES )

(NCERTIUCAL)

$\%$ OF TOTAL

\section{EROSS-ALPHA}

CONCEN (UCAL)

CURIES

LNCERTICURIES

UNCERTIUCAML

$\%$ OF TOTAL

KR-8EY $1.867 E-01$
CONCEN (UCML)
CURIES
UNCERT(CURIES)
UNCERT(UCML)
\% OF TOTAL
KR-87
CONCEN (UCML)
CURIES
UNCERTICURIES)
UNCERT(UCML)
\% OF TOTAL

$1.260 E+09$

$7.625 E-02$

4.374E-06 7.438E-06

2.862E+00 $1.056 E+00$

$\begin{array}{lllllll}13.247 & 13.246 & 13.247 & 13.247 & 13.246 & 13.246 & 13.246\end{array}$

$1.276 E+01$
0.001
0.001
0.002
0.001
0.001
0.001
0.002

$\begin{array}{llllllll}1.400 E-05 & 2.380 E-05 & 2.411 E-05 & 1.274 E-05 & 1.462 E-05 & 1.335 E-05 & 1.834 E-05 & 5.032 E-13\end{array}$

$\begin{array}{lllllll}2.368 E+00 & 9.158 E+00 & 3.379 E+00 & 2.163 E+00 & 2.928 E+00 & 1.286 E+00 & 2.128 E+01\end{array}$
42.389
42.387
42.387
42.385
42.386
42.375
42.386

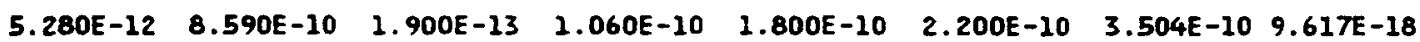

$\begin{array}{llllllll}8.934 E-07 & 3.305 E-04 & 2.663 E-08 & 1.799 E-05 & 3.606 E-05 & 2.119 E-05 & 4.067 E-04\end{array}$

0.002

0.001

0.001

0.001

$\begin{array}{lllll}5.800 E-14 & 4.600 E-14 & 1.300 E-13 & 2.721 E-14 & 7.468 E-22\end{array}$

$9.844 E-09 \quad 9.214 E-09 \quad 1.252 E-08 \quad 3.158 E-08$

$\begin{array}{lllllllll}1.749 E-08 & 2.976 E-08 & 3.014 E-08 & 1.593 E-08 & 1.827 E-08 & 1.669 E-08 & 2.293 E-08 & 6.291 E-16 & 1.00 E-07\end{array}$

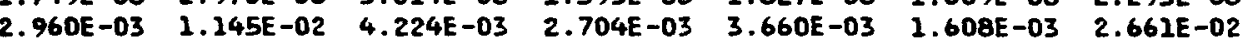
0.053
0.053
0.053
0.053
0.053
0.053
0.053

$\begin{array}{lllllllll}1.002 E-07 & 1.703 E-07 & 1.725 E-07 & 9.120 E-08 & 1.046 E-07 & 9.556 E-08 & 1.312 E-07 & 3.601 E-15 & 2.00 E-08\end{array}$

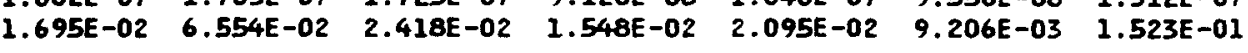
0.303
0.303
0.303
0.303
0.303
0.303
0.303

PAGE 1 RPT. 420 ANL-A

YEARLY RELEASE OF CG 
RADIOACTIVE WASTE MANAGEMENT INFORMATION SYSTEM

TREAT AIRBORNE WASTE SUMTARY FOR JAN THROUGH JUN 1993

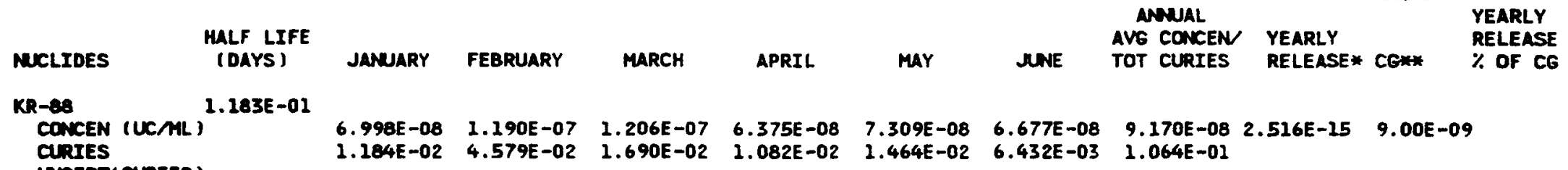

INEERTICURIES )

UNCERT(UCAL)

\% OF TOTAL
0.212
0.212
0.212
0.212
0.212
0.212
0.212

CONCEN (UCML) $1.678 E+00$

CURIES

$2.493 E-14 \quad 4.239 E-14 \quad 4.294 E-14 \quad 2.270 E-14$

$4.218 E-09 \quad 1.631 E-08$

$2.604 E-14 \quad 2.378 E-14 \quad 3.266 E-14 \quad 8.963 E-22 \quad 3.00 E-09$ UNCERTICURIES ) UNCERTIUCALI) \% OF TOTAL

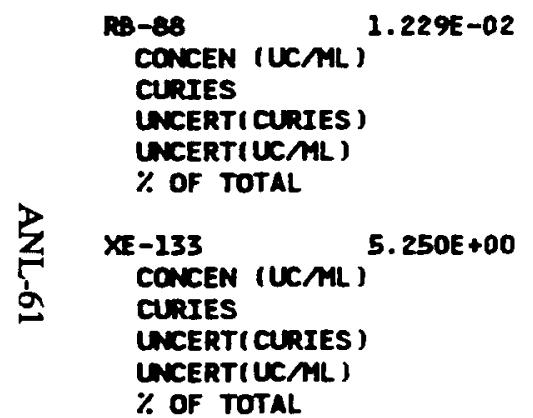

$\begin{array}{lllllllll}6.253 E-10 & 1.064 E-09 & 1.077 E-09 & 5.695 E-10 & 6.530 E-10 & 5.968 E-10 & 8.195 E-10 & 2.249 E-17 & 3.00 E-08\end{array}$ $\begin{array}{lllllll}1.058 E-04 & 4.093 E-04 & 1.510 E-04 & 9.667 E-05 & 1.308 E-04 & 5.749 E-05 & 9.511 E-04\end{array}$
0.002
0.002
0.002
0.002
0.002
0.002
0.002

$\begin{array}{lllllllll}8.747 E-09 & 1.488 E-08 & 1.505 E-08 & 7.965 E-09 & 9.136 E-09 & 8.346 E-09 & 1.146 E-08 & 3.145 E-16 & 5.00 E-07\end{array}$ $\begin{array}{lllllll}1.480 E-03 & 5.724 E-03 & 2.110 E-03 & 1.352 E-03 & 1.830 E-03 & 8.040 E-04 & 1.330 E-02\end{array}$
0.026
0.026
0.026
0.026
0.026
0.026
0.026

XE-135

CONCEN (UCML)

CURIES

UNCERTI CURIES

UNCERTIUCAML

\% OF TOTAL

3.792E-01

$x E-140$

CONCEN (UCAL) $1.570 E-04$

cunres

CURIES

CERTIUCAL $\begin{array}{lllllllll}1.749 E-08 & 2.976 E-08 & 3.025 E-08 & 1.593 E-08 & 1.827 E-08 & 1.744 E-08 & 2.300 E-08 & 6.312 E-16 & 8.00 E-08\end{array}$ $\begin{array}{lllllll}2.960 E-03 & 1.145 E-02 & 4.240 E-03 & 2.704 E-03 & 3.660 E-03 & 1.680 E-03 & 2.669 E-02\end{array}$
0.053
0.053
0.053
0.053
0.053
0.055
0.053

$\begin{array}{llllllll}1.443 E-05 & 2.455 E-05 & 2.486 E-05 & 1.314 E-05 & 1.508 E-05 & 1.377 E-05 & 1.891 E-05 & 5.190 E-13\end{array}$

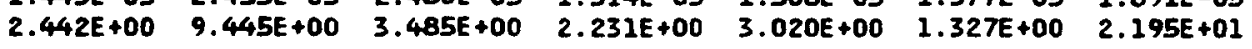
43.714
43.715
43.716
43.718
43.718
43.726
43.716 
RADIOACTIVE WASTE MANAGEMENT INFORMATION SYSTEM

TREAT AIREONONE MASTE SUMARY FOR JAN THROUEH JW 1993

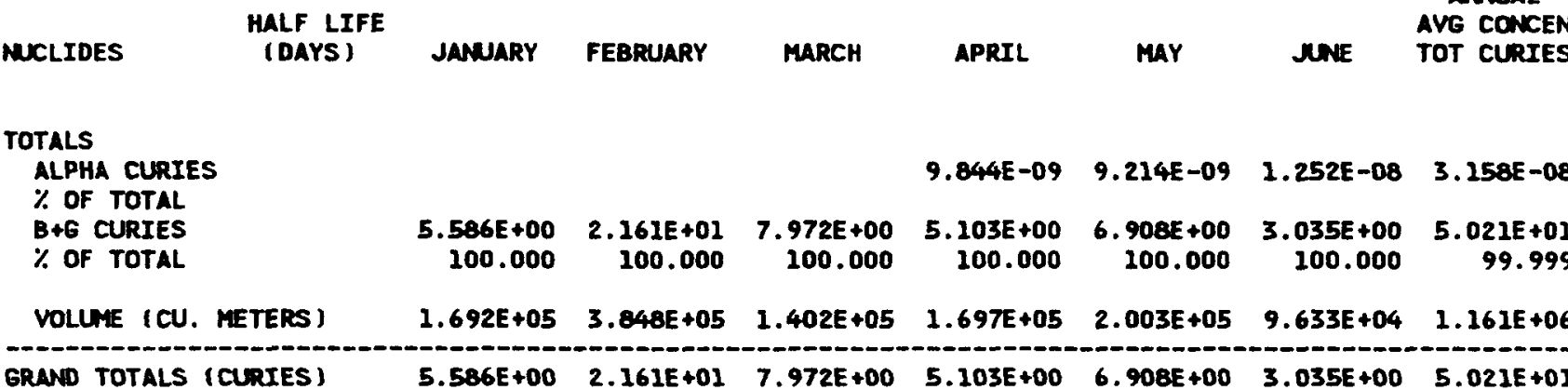

* This nuber is derived from a dispersion factor equetion. The dispersion factor is derived from the straight line goussian model and is provedad by the Notional Doeanic Atmospheric Administration (NOAA).

** NOTE: DCE value obtained from DOE ORDER 5400.5 dated Fabruary 8, 1990, Figures III-1 and III-3
PAGE 3 RPT. 410 ANL-A

YEARLY RELEASE 
ZPPR AIRBORNE MASTE SUMAARY

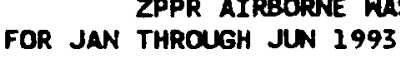

MCLIDES
CROS-BET-GNM
CONCEN (UCAL)
CURTES
UNCERTICURIES)
UNERT(UCML)
\% OF TOTAL

\section{MALF LIFE}

(DAYS)

JNWARY

\begin{abstract}
FEBRUARY
\end{abstract}
MARCH

APRIL

MAY

$\begin{array}{ll}2.890 E-16 & 5.030 E-15 \\ 1.584 E-09 & 3.052 E-08\end{array}$

100.000

100.000

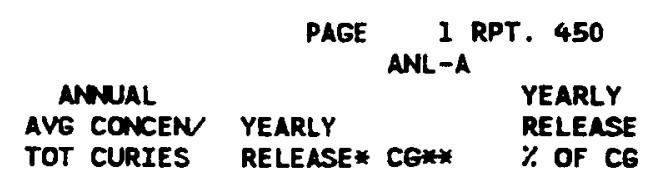

$6.360 E-16 \quad 1.012 E-15 \quad 3.490 E-23$

3.735E-09 3.584E-08

88.064

97.023

EROSS-ALPHA

CONCEN (UCML)

CURIES

UNCERT(CURIES)

UNCERT (UCAL)

$\%$ of Total

$9.780 E-17 \quad 8.620 E-17 \quad 3.104 E-17 \quad 1.071 E-24$

5.935E-10 5.062E-10 1.100E-09

100.000

11.936

2.977

\title{
TOTALS
}

ALPHA CURIES

\% OF TOTAL

B+G CURIES
$\%$ OF TOTAL

100.000

3. $052 E-08$

100.000

5. $935 E-10$

5.062E-10 1.100E-09

100.000

11.936

$3.735 E-09$

2.977

$\begin{array}{rr}88.064 & 3.584 E-08 \\ & 97.023\end{array}$

5. 935E-10 4.241E-09 3.694E-08

* This nubar is derived from a dispersion factor aquation. The dispersion factor is derived from the straight line onseien model and is proveded by the National Oceanic Atmospheric Administration (NOAA).

** NOTE: DCG velue obtainad from DOE ORDER 5400.5 dated february 8, 1990 , Figures III-1 and III-3 



\section{Central Facilities Area 1993 Detall Data}

Central Facilities Area (CFA) Bar Graphs of Annual Data by Month . . . . . . . . . CFA-3

CY1993 Bar Graphs ...................................

CFA Detail Data for Months July to December,

Annual Average Concentration and Total Curies for January to December ....... CFA-7

Report 160 -CFA Airborne Waste Summary ..................... CFA-9

Report 161-CFA Liquid Waste Summary ................... CFA-10

Report 162-CFA Disposed Solid Waste Summary .............. CFA-11

Report 166-CFA Solid Waste Summary, WERF Incinerator

Processed Waste ........................... CFA-13

Report 167-CFA Solid Waste Summary, Waste Sent to WERF

for Compaction ........................... CFA

CFA Detail Data for Months January to June,

Annual Average Concentration and Total Curies for January to June ......... CFA-15

Report 160 - CFA Airborne Waste Summary ................. CFA-17

Report 161 CFA Liquid Waste Summary ................... CFA-18

Report 162-CFA Disposed Solid Waste Summary ............... CFA-19

Report 166-CFA Solid Waste Summary, WERF Incinerator Processed Waste ........................ CFA-20

Report 167 - CFA Solid Waste Summary .................. CFA-21

CFA Facility Detail Data for Months July to December,

Annual Average Concentration and Total Curies for January to December ....... CFA-23

Report 185-CFA Laundry Airborne Waste Summary ............. CFA-25

Report 184 - CFA Respirator Airborne Waste Summary ............ CFA-26

Report 161 - CFA Liquid Waste Summary .................. CFA-27

CFA Facility Detail Data for Months January to June,

Annual Average Concentration and Total Curies for January to June . . . . . . . . CFA-29

Report 185-CFA Laundry Airborne Waste Summary .............. CFA-31

Report 184-CFA Respirator Airborne Waste Summary . . . . . . . . . . . CFA-32

Report 161 CFA Liquid Waste Summary ................... CFA-33 

Central Facilities Area (CFA) Bar Graphs of Annual Data by Month 

Central Facility Area (CFA) Monthly Details - CY 1993

㞼

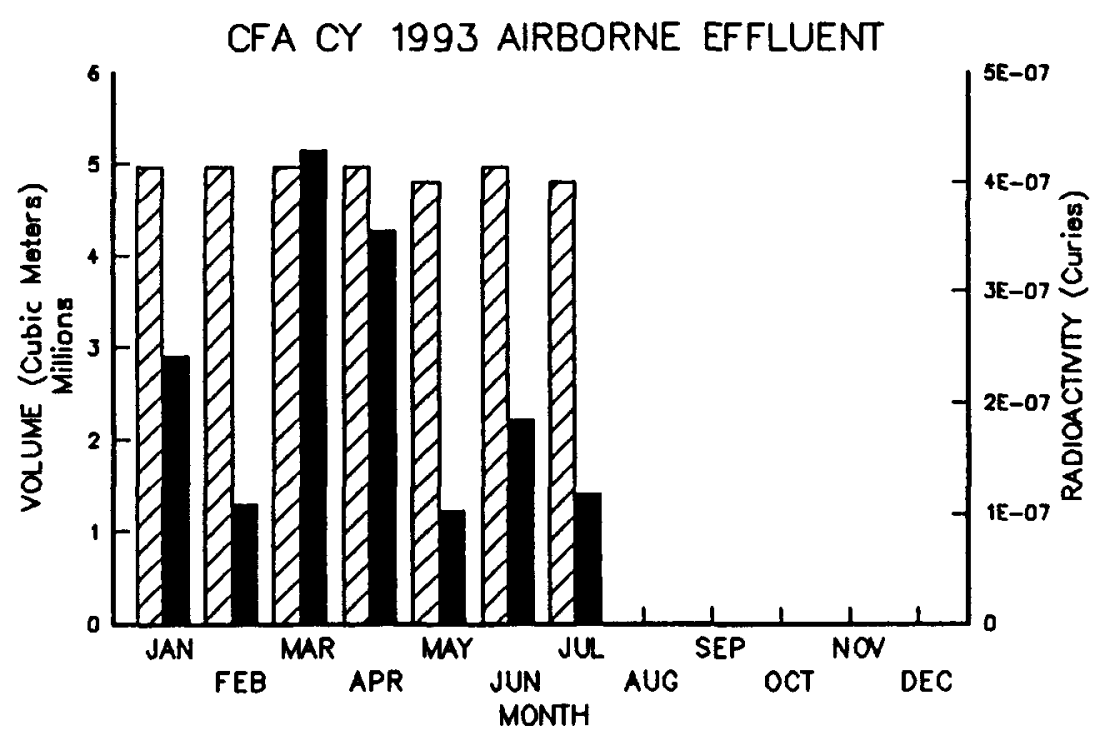

DVOLUME — RADIOACTMITY

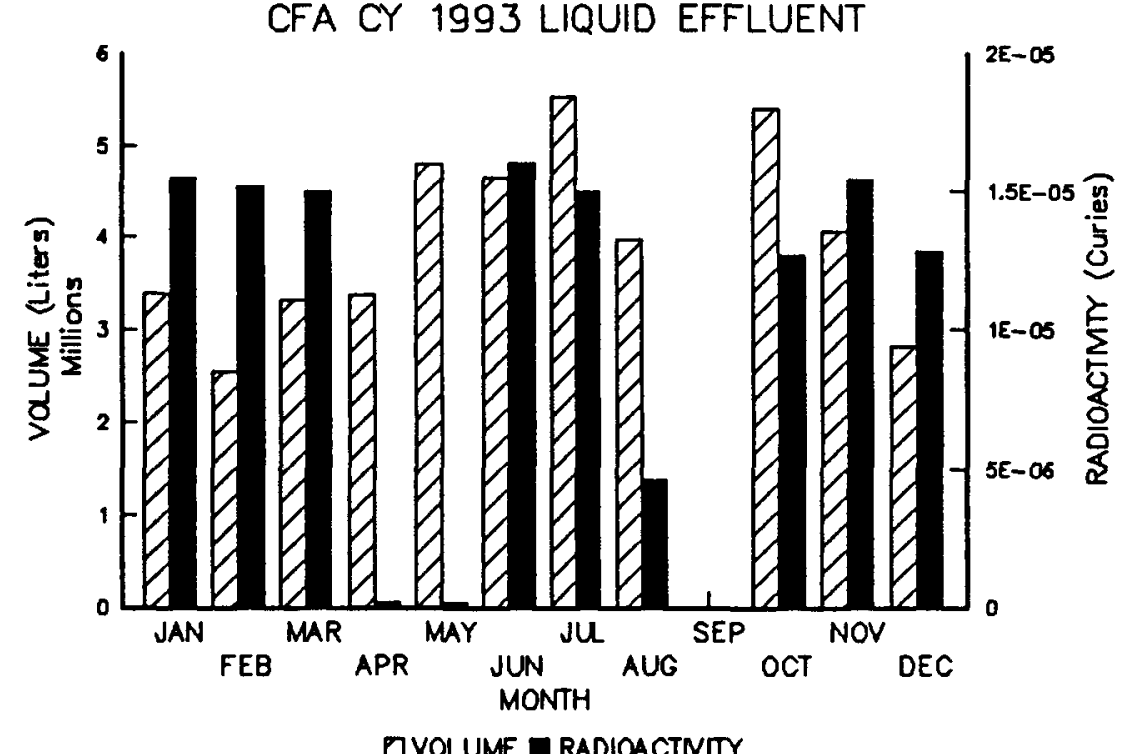

DVOLUME D RADIOACTNITY

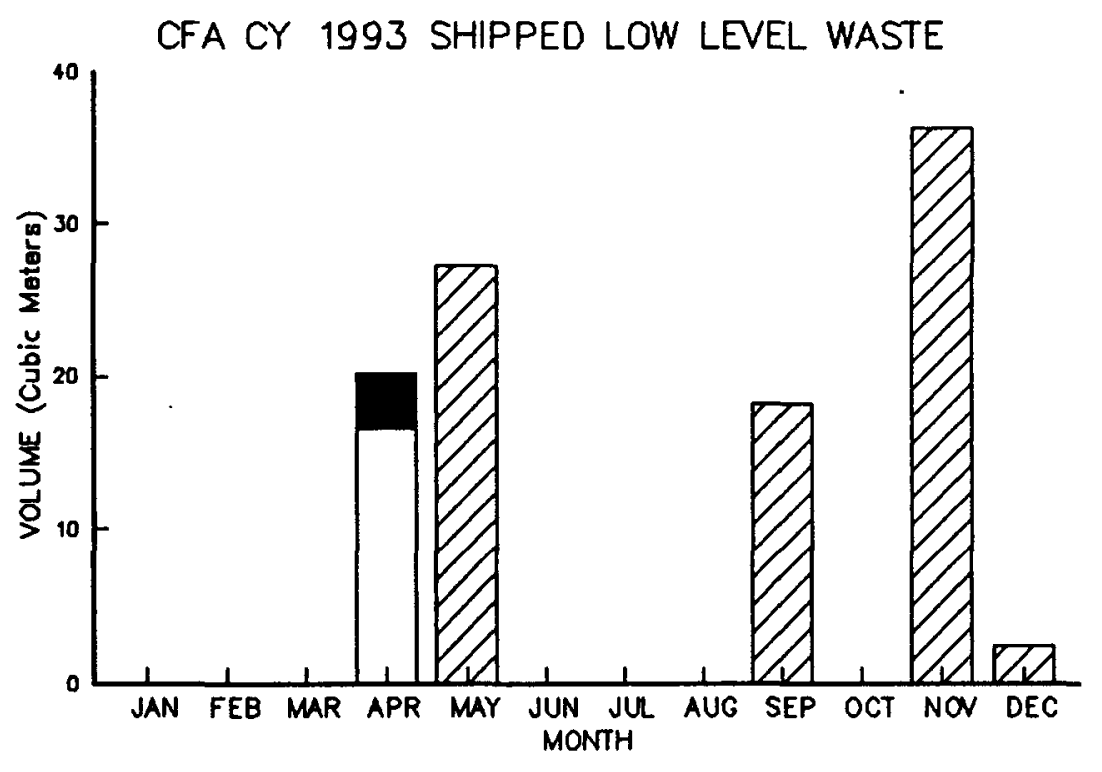

DINCINERATION D METAL SIZING I COMPACTION D DIRECT DISPOSAL 
CFA Detail Data for Months July to December Annual Average Concentration and Total Curies for January to December 
CFA AIRBOPNE WASTE SUMMARY

FOR JAN THROUGH DEC 1993

MALF LI
MUCLIDES

HALF LIFE
(DAYS )

JULY

AUEUST

SEPTEMBER

OCTOBER

NOVERBER

\section{$3.162 E-15$}

$1.517 E-08$

12.933

CONCEN

$\%$ OF TOTAL

2.111E-24

$1.013 E-07$

86.361

CONCEN (UCAL)

curres

SR-90 $1.059 E+04$

CONCEN (UCNL) $8.634 E-17$

CURIES $4.243 E-10$

$\begin{array}{lr}\% \text { OF TOTAL } & 0.353\end{array}$

$Y-90 \quad 2.667 E+00$

$8.634 E-17$
$4.143 E-10$

$\%$ OF TOTAL

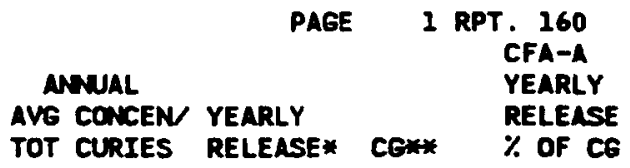

$5.291 E-15 \quad 1.311 E-21$

$1.819 E-07$

11.806

$3.741 E-14 \quad 9.275 E-21$

2.287E-06

83.488

1.055E-15 2.614E-22 $9.00 E-12$

3.627E-08

2.353

$\begin{array}{lll}1.055 E-15 & 2.614 E-22 \quad 1.00 E-09\end{array}$

$3.627 E-08$

$$
2.353
$$

$1.819 E-07$

11.806

$1.359 E-06$

88.194

$3.439 E+07$

$1.541 E-06$

* This muber is derived from a dispersion factor equation. The dispersion factor is derived from the straight line Goussian model and is provided by the National Oceanic Atmospheric Administration (NOMA).

* NOTE: DCE value obtained from DOE ORDER 5400.5 dated Fabruary 8, 1990, Figures III-1 and III-3 
RADIOACTIVE WASTE MANAGEMENT INFORMATION SYSTEM

CFA LIQUTD WUSTE SUMARY

FOR JAN THROUEH DEC 1993

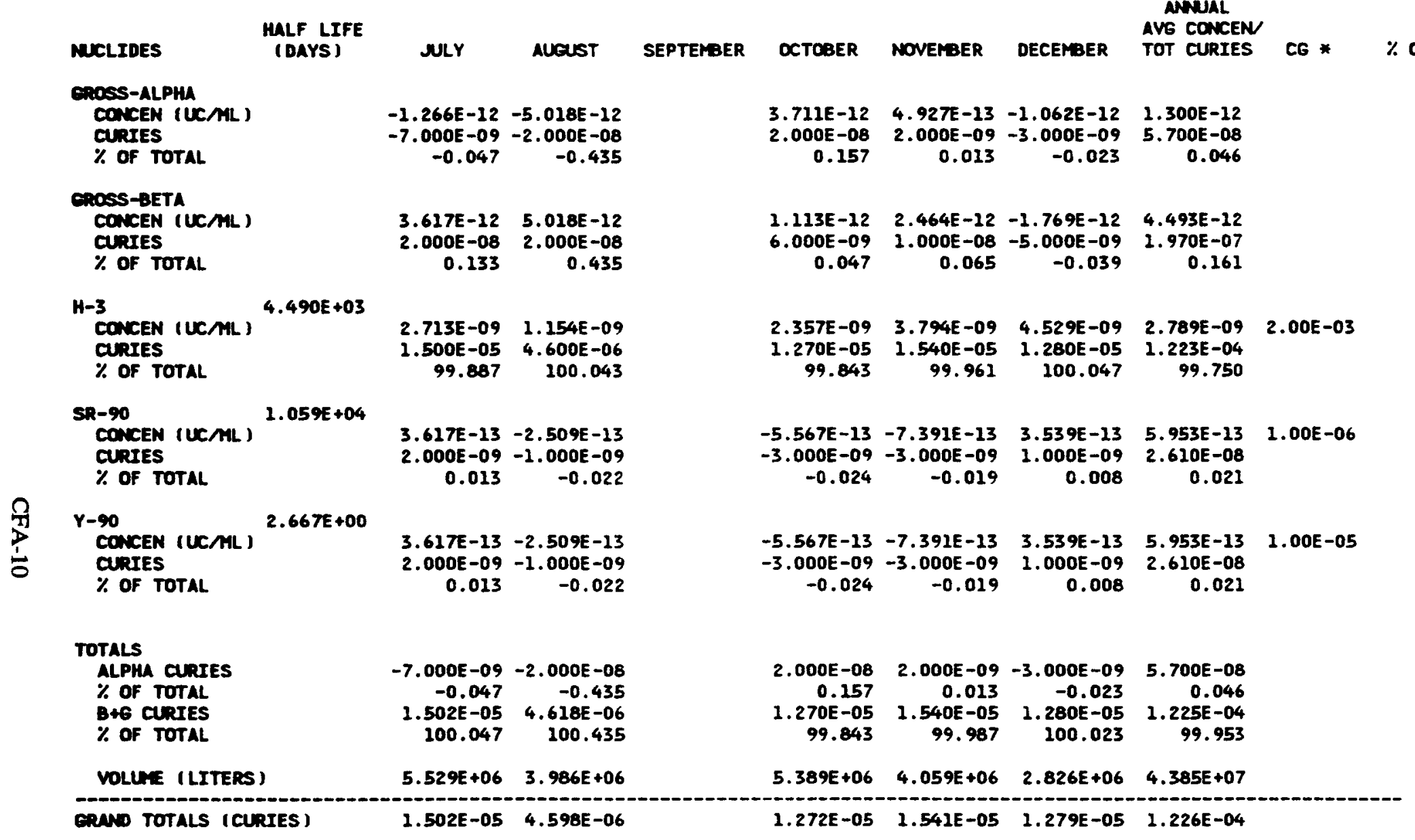

* NOTE: DCG value obtainad from DOE ORDER 5400.5 dated Fabruary8, 1990, Figuras III-1 and III-3 
CFA DISPOSED SOLID WASTE SUMMRY

FOR JAN THROUEH DEC 1993

MUCLIDES
AM-24I
CURIES
\% OF TOTAL
GRAMS

HALF LIFE

(DAYS)

JULY

Avast

SEPTERBER

OCTOBER

NOVEMER

MNUAL

TOTAL

1.577E+05

$2.844 E+02$

$$
\text { CURIES }
$$

$\%$ OF TOTAL

CO-60 $\quad 1.924 E+03$

CURIES

$\%$ OF TOTAL

CS-134

$$
\text { CURIES }
$$

$\%$ OF TOTAL

$7.537 E+02$

\section{CS-137}

$\%$ OF TOTAL

$1.101 E+04$

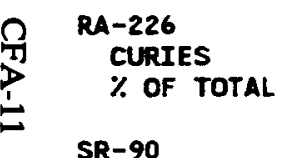

SR-90

$\%$ OF TOTAL

U-238

CURIES

$\%$ OF tOTAL

GRavs
$5.840 E+05$

$1.059 E+04$

$1.631 E+12$
4. 275E-05

$$
\text { 0.500 }
$$

2.821E-03

33.000

5.557E-03

65.000

8.550E-05

1.000

4. 275E-05

0.500
1. $272 E+02$

\section{4. $952 E-06$}

$$
0.031
$$

$1.440 E-06$

4.275E-05

$$
0.267
$$

$$
2.290 E-05 \quad 7.418 E-04 \quad 3.586 E-03
$$

$$
\begin{array}{rrr}
50.000 & 9.994 & 22.360
\end{array}
$$

$1.396 E-03 \quad 1.396 E-03$

$$
\begin{array}{rr}
18.807 & 8.704
\end{array}
$$

2.290E-05 1.801E-03 7.396E-03

$3.484 E-03 \quad 3.484 E-03$

$$
\begin{array}{rr}
46.937 & 21.723
\end{array}
$$

8.550E -05

0.533

4.275E-05

0.267

$1.272 E+02$ 
SCHED. NO. PGIPMOO9-G

IOAHO OPERATIONS OFFICE
U.S. DEPARTMENT OF ENERGY

RADIOACTIVE WUSTE MANAGEMENT INFORHATION SYSTEM

\section{CFA DISPOSED SOLID MASTE SUMYARY} FOR JUN THROUEH DEC 1993

NUCLIDES

\section{HALF LIFE}

(DAYS)

NULY

AUEUST

TOTALS

ALPHA CURIES

$\%$ OF TOTAL

B+G CURIES

$\%$ OF TOTAL

TOTAL GRAMS OF AM

TOTAL GRAYS OF AM

TOTAL GRAMS OF PU

TOTAL GRAMS OF PU-239+24I

TOTAL GRAMS OF $U$

TOTAL GRANS OF TH

TOTAL GRAMS OF NP

VOLUME (CU. METERS)

WEIGHT (KILOGRAMS)

GRAND TOTALS (CURIES)

OCTOBER

DECERER
RUN DATE : 05/12/94

PAGE 2 RPT 162

CFA-S 
SCHED. NO. P6IPMO09-N

IDAHO OPERATIONS OFFICE

U.S. DEPARTITS

RLN DATE: 05/11/94 MIOACTIVE WASTE MANAGEMENT IMFORPUTION SYSTEM

CFA SOLID MUSTE SUMURY

WERF INCINERATOR PROCESSED WASTE

FROM JAN THROUSH DEC 1993

MUCLIDES HALF LIFE

NLY

AUEUST

SEPTERBER

$1.924 E+03$

Co-60

CURIES

$\%$ OF TOTAL

CS-137

CURIES

$\%$ OF TOTAL

$1.102 E+04$

TOTALS

ALPHA CURIES

$\%$ OF TOTAL

$B+G$ CURIES

$\%$ of total

TOTAL GRAMS OF AM

TOTAL GRAHS OF PU

TOTAL GRNAS OF PU-239+241

TOTAL GRWIS OF $U$

TOTAL GRA'S OF TH

TOTAL GRANS OF NP

VOLUME ICU. METERS

WEIGHT (KILOGRAMS )

GRAND TOTALS ICURIES )
$1.657 E+01$

$8.260 E+02$

3.398E-06 
IDAHO OPERATIONS OFFICE

U.S. DEPARTMENT OF ENERGY

RADIOACTIVE MUSTE MANAGEMENT INFORMATION SYSTEM

\section{CFA SOLID WASTE SLMMARY}

WASTE SENT TO WERF FOR COMPACTION

$\begin{array}{lc}\text { MUCLIDES } & \text { HALF LIF } \\ \text { (DAYS) }\end{array}$

CURIES

\% OF TOTAL

CS- 137

CURIES

$\%$ of total

$1.101 E+09$

FROM JAN THROUEH DEC 1993

ULY

AUEUST

SEPTEYBER

OCTOBER

NOVEFBER
RUN DATE: 05/11/94

PAGE 1 RPT. 167

MUNAL

TOTAL

5. DOOE-04

$$
12.195
$$

$3.600 E-03$ 87.805

4. $100 E-03$ 100.000

AlPhM cuires

Bot curres

$\%$ of total

TOTAL CRAMS OF AM

TOTAL ERAS OF PU

TOTAL ERAYS OF PU-239+241

TOTAL CRNAS OF $U$

TOTAL erans of TH

TOTAL ERANS OF N

VOLUNE (CU. METERS)

VEIEHT (KILOSRNAS)

$3.625 E+00$

erano totals (CURIES)

$6.538 E+02$

4. $100 E-03$ 

RADIOACTIVE WASTE MANAGEMENT INFOPMUTION SYSTEM

CFA AIRBORNE MUSTE SUMUARY

FOR JAN THROUGH JUN 1993

\begin{tabular}{|c|c|c|c|c|c|c|c|c|c|c|}
\hline $\begin{array}{l}\text { HALF LIFE } \\
\text { (DAYS) }\end{array}$ & JANUARY & FEBRUARY & MARCH & APRIL & MAY & JNE & $\begin{array}{l}\text { MNUAL } \\
\text { AVG CONCEN } \\
\text { TOT CURIES }\end{array}$ & $\begin{array}{l}\text { YEARLY } \\
\text { RELEASE* }\end{array}$ & CGA* & $\begin{array}{l}\text { YEARLY } \\
\text { RELEASE } \\
\% \text { OF CE }\end{array}$ \\
\hline $\begin{array}{l}\text { GROSS-ALPHA } \\
\text { CONCEN (UCML) } \\
\text { CURIES } \\
\% \text { OF TOTAL }\end{array}$ & $\begin{array}{r}5.958 E-15 \\
2.954 E-08 \\
12.264\end{array}$ & $\begin{array}{r}1.273 E-14 \\
6.312 E-08 \\
58.671\end{array}$ & $\begin{array}{r}7.779 E-15 \\
3.857 E-08 \\
8.980\end{array}$ & $\begin{array}{r}3.972 E-15 \\
1.969 E-08 \\
5.527\end{array}$ & $\begin{array}{r}1.540 E-15 \\
7.388 E-09 \\
7.179\end{array}$ & $\begin{array}{r}1.702 E-15 \\
8.441 E-09 \\
4.575\end{array}$ & $\begin{array}{r}5.636 E-15 \\
1.668 E-07 \\
11.713\end{array}$ & 1.397E-21 & & \\
\hline $\begin{array}{l}\text { CROSS-BETA } \\
\text { CONCEN (UCML) } \\
\text { CORIES } \\
\% \text { OF TOTAL }\end{array}$ & $\begin{array}{r}4.135 E-14 \\
2.050 E-07 \\
84.413\end{array}$ & $\begin{array}{r}8.091 E-15 \\
4.012 E-08 \\
37.288\end{array}$ & $\begin{array}{r}7.961 E-14 \\
3.947 E-07 \\
91.898\end{array}$ & $\begin{array}{r}5.817 E-14 \\
2.884 E-07 \\
80.934\end{array}$ & $\begin{array}{r}1.814 E-14 \\
8.704 E-08 \\
84.574\end{array}$ & $\begin{array}{r}3.429 E-14 \\
1.700 E-07 \\
92.131\end{array}$ & $\begin{array}{r}4.006 E-14 \\
1.185 E-06 \\
83.251\end{array}$ & $9.930 E-21$ & & \\
\hline $\begin{array}{l}\text { SR-90 } \\
\text { CONCEN (UCAL) } \\
\text { CURIES } \\
\% \text { OF TOTAL }\end{array}$ & $\begin{array}{r}8.384 E-16 \\
4.157 E-09 \\
1.712\end{array}$ & $\begin{array}{r}4.385 E-16 \\
2.174 E-09 \\
2.021\end{array}$ & $\begin{array}{r}-3.806 E-16 \\
-1.887 E-09 \\
-0.439\end{array}$ & $\begin{array}{r}4.866 E-15 \\
2.412 E-08 \\
6.770\end{array}$ & $\begin{array}{r}8.845 E-16 \\
4.244 E-09 \\
4.124\end{array}$ & $\begin{array}{r}6.131 E-16 \\
3.040 E-09 \\
1.648\end{array}$ & $\begin{array}{r}1.212 E-15 \\
3.585 E-08 \\
2.518\end{array}$ & $3.004 E-22$ & $9.00 E-12$ & \\
\hline $\begin{array}{l}Y-90 \\
\text { COACEN (UCAL) } \\
\text { CURIES } \\
\% \text { OF TOTAL }\end{array}$ & $\begin{array}{r}8.384 E-16 \\
4.157 E-09 \\
1.712\end{array}$ & $\begin{array}{r}4.385 E-16 \\
2.174 E-09 \\
2.021\end{array}$ & $\begin{array}{r}-3.806 E-16 \\
-1.887 E-09 \\
-0.439\end{array}$ & $\begin{array}{r}4.866 E-15 \\
2.412 E-08 \\
6.770\end{array}$ & $\begin{array}{r}8.845 E-16 \\
4.244 E-09 \\
4.124\end{array}$ & $\begin{array}{r}6.131 E-16 \\
3.040 E-09 \\
1.648\end{array}$ & $\begin{array}{r}1.212 E-15 \\
3.585 E-08 \\
2.518\end{array}$ & $3.004 E-22$ & $1.00 E-09$ & \\
\hline $\begin{array}{l}\text { TOTALS } \\
\text { ALPHA CURIES } \\
\% \text { OF TOTAL } \\
\text { B+G CURIES } \\
\% \text { OF TOTAL } \\
\text { YOLLE (CU. METERS) }\end{array}$ & $\begin{array}{r}2.954 E-08 \\
12.164 \\
2.133 E-07 \\
87.836 \\
4.958 E+06\end{array}$ & $\begin{array}{r}6.312 E-08 \\
58.671 \\
4.446 E-08 \\
41.329 \\
4.958 E+06\end{array}$ & $\begin{array}{r}3.857 E-08 \\
8.980 \\
3.909 E-07 \\
91.020 \\
\\
4.958 E+06\end{array}$ & $\begin{array}{r}1.969 E-08 \\
5.527 \\
3.366 E-07 \\
94.473 \\
4.958 E+06\end{array}$ & $\begin{array}{r}7.388 E-09 \\
7.179 \\
9.553 E-08 \\
92.821 \\
4.798 E+06\end{array}$ & $\begin{array}{r}8.441 E-09 \\
4.575 \\
1.761 E-07 \\
95.425 \\
4.958 E+06\end{array}$ & $\begin{array}{r}1.668 E-07 \\
11.713 \\
1.257 E-06 \\
88.287 \\
2.959 E+07\end{array}$ & & & \\
\hline ERND TOTALS (CURIES) & $2.429 E-07$ & $1.076 E-07$ & $4.295 E-07$ & $3.563 E-07$ & $1.029 E-07$ & $1.845 E-07$ & $1.424 E-06$ & & & \\
\hline
\end{tabular}

- This nubar is derived from a dispersion factor equation. The disparsion factor is derived from the straight line Coussien model and is providad by the Nutional Dosanic Atwospharic Adainistration (IOMA).

* NOTE: DCE value obtained from DOE ORDER 5400.5 dated February 8, 1990 , figures III-1 and III-3 


\section{CFA LIqUID WASTE SUMUARY}

FOR JAN THROUGH JUN 1993

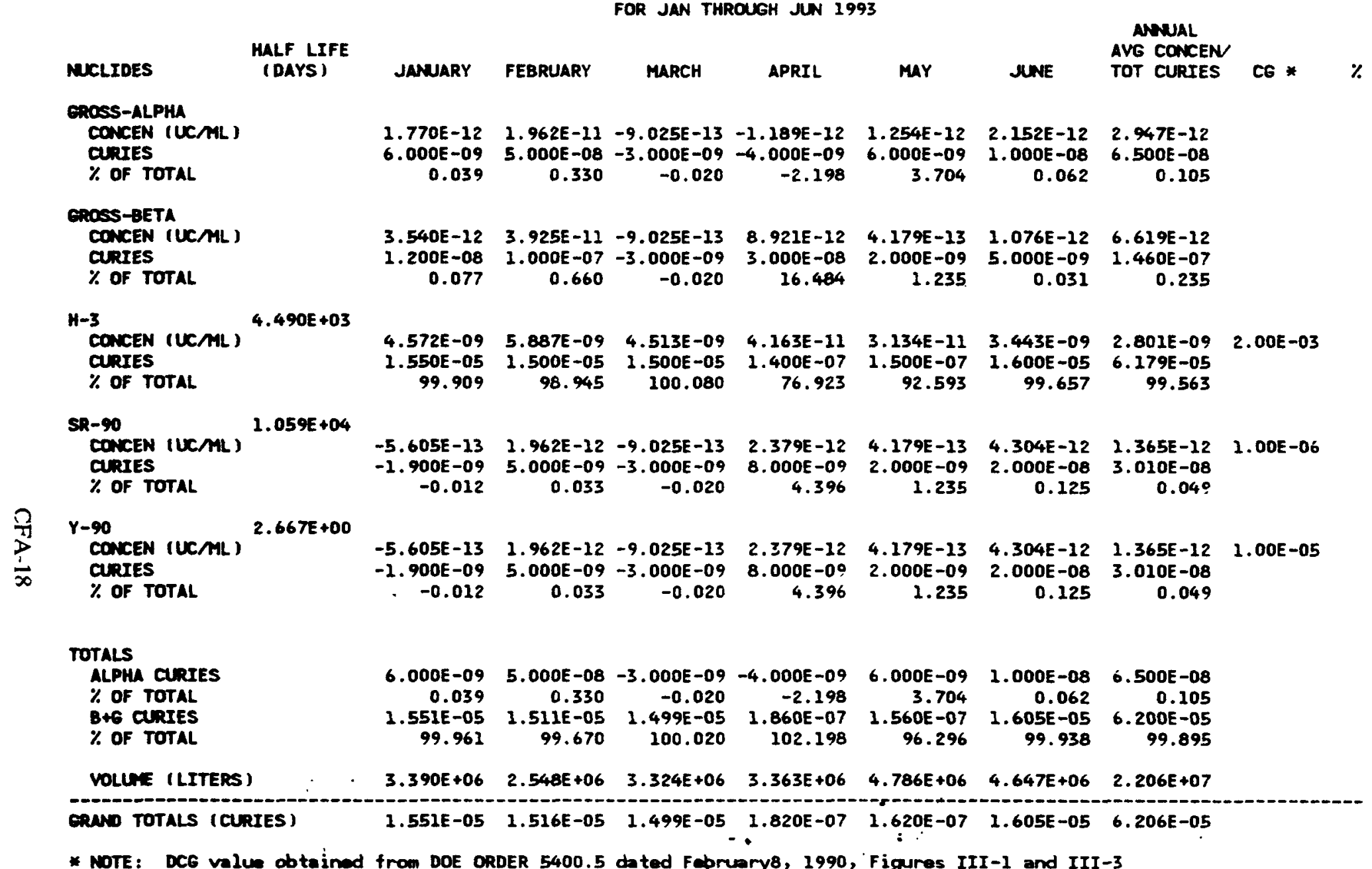

* NOTE: DCE value obtainad from DOE ORDER 5400.5 dated Fobriery8, 1990, Figures III-1 and III-3 
CFA DISPOSED SOLIO MUSTE SUMARY FOR JAN THROUGH JUN 1993

$\begin{array}{lc}\text { MUCLIDES } & \begin{array}{c}\text { MALF LIFE } \\ \text { (DAYS ) }\end{array} \\ \begin{array}{c}\text { NH-241 } \\ \text { CURIES } \\ \% \text { OF TOTAL } \\ \text { CQNAS }\end{array} & 2.577 E+05 \\ \begin{array}{c}\text { CS-137 } \\ \text { CURIES }\end{array} & 1.201 E+04 \\ \end{array}$

$\%$ of total

\section{TOTALS}

ALPHA CURIES

$\%$ OF TOTAL

B+6 cuares

\% OF TOTAL

TOTAL ERAMS OF AM

TOTAL ERANS OF AM

TOTAL ERNES OF PU

TOTAL ERAMS OF PU-239+241

TOTAL CRAMS OF $U$

TOTAL ERNIS Of TH

TOTAL GRANS OF NP

VOLCHE (CU. METERS)

VEIGIT (KILOERNAS)

JANUARY FEBRUARY

MARCH

APRIL

MAY

JUNE

PAGE I RPT 162

ANUAL

TOTAL

$\begin{array}{rr}4.952 E-06 & 4.952 E-06 \\ 25.027 & 25.027\end{array}$

25.027
$.40 E-06$

$1.483 E-05$
$1.483 E-05$

74.974
74.973

4. $952 E-06 \quad 4.952 E-06$

$25.027 \quad 25.027$

$1.483 E-05$

74.974

$1.483 E-05$

74.973

$1.440 E-06 \quad 1.440 E-06$

GRND TOTALS (CURIES)

8.873E+0z

$2.716 E+01$

$8.873 E+03$

1. $979 E-05$ 
SCHED. NO. P61PMOO9-N

IDAHO OPERATIONS OFFICE

U.S. DEPARTMENT OF ENERGY

RADIOACTIVE WASTE MANAGEMENT INFORMATION SYSTEM

\section{CFA SOLIO MASTE SUMMARY}

MERF INCINERATOR PROCESSED WASTE

$\begin{array}{lc}\text { NUCLIDES } & \begin{array}{c}\text { HALF LIFE } \\ \text { (DAYS ) }\end{array} \\ \begin{array}{c}\text { CO-60 } \\ \text { CURIES }\end{array} & 1.924 E+03 \\ \% \text { OF TOTAL } & \\ \begin{array}{c}\text { CS-137 } \\ \text { CURIES }\end{array} & 1.101 E+04\end{array}$

JANUARY

FEBRUARY

ROM JAN THROUGH UN 1993

$$
\text { APRIL MAY }
$$

3.252E-07

$$
9.570
$$

$3.073 E-06$

90.430

$\%$ OF TOTAL

\section{TOTALS}

ALPHA CURIES

$\%$ OF TOTAL

$B+G$ CURIES

$3.398 E-06$

100.000

$3.398 E-06$ 99.999

TOTAL GRAMS OF AM

TOTAL GRAMS OF PU

TOTAL GRAMS OF PU-239+241

TOTAL GRAMS OF $u$

TOTAL GRAIS OF TH

TOTAL GRAMA OF MP

TOTAL GRAMS OF

WEIGHT (KILOGRAHS)

GRAND TOTALS I CURIES

$1.657 E+01$

8. $260 E+02$

8. $260 E+02$

3.398E-06

$3.398 E-06$ 
CFA SOLID WASTE SUMYARY

WASTE SENT TO WERF FOR COMPACTION

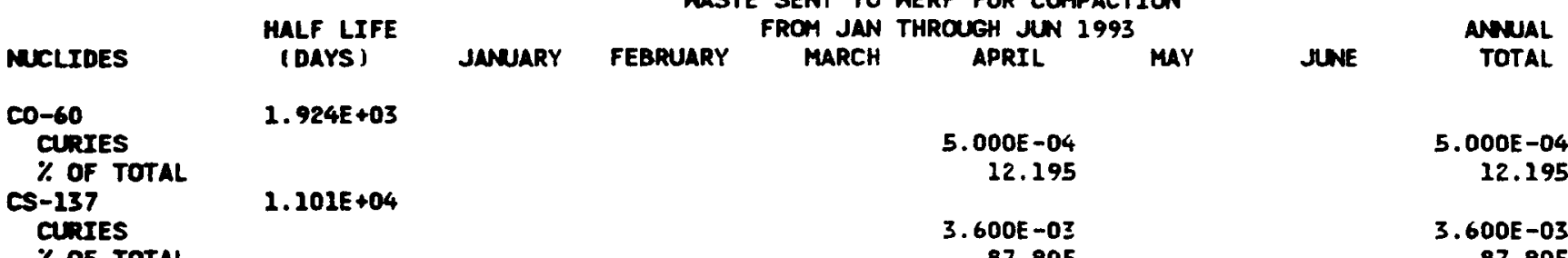

$\%$ OF TOTAL

87.805

87.805

TOTALS

ALPHA CLRIES

$\%$ OF TOTAL

B*6 curres

4. 100E-03

100.000

4. $100 E-03$

100.000

TOTAL CRAMS OF AM

TOTAL ERAMS OF PU

TOTAL ERUNS OF PU-239+241

TOTAL ERANS OF $U$

TOTAL Eevis of TH

TOTAL ERANS of NP

TOTAL ERANS OF NP
VOLUAE (CU. METERS

VOLUAE ICU. METERS
VEIEHT (KILOERNAS )

$3.625 E+00$

$6.538 E+02$

$3.625 E+00$

GRND TOTALS (CURIES)

4. $100 E-03$

$4.100 E-03$ 

CFA Facility Detail Data for Months July to December Annual Average Concentration and Total Curies for January to December 



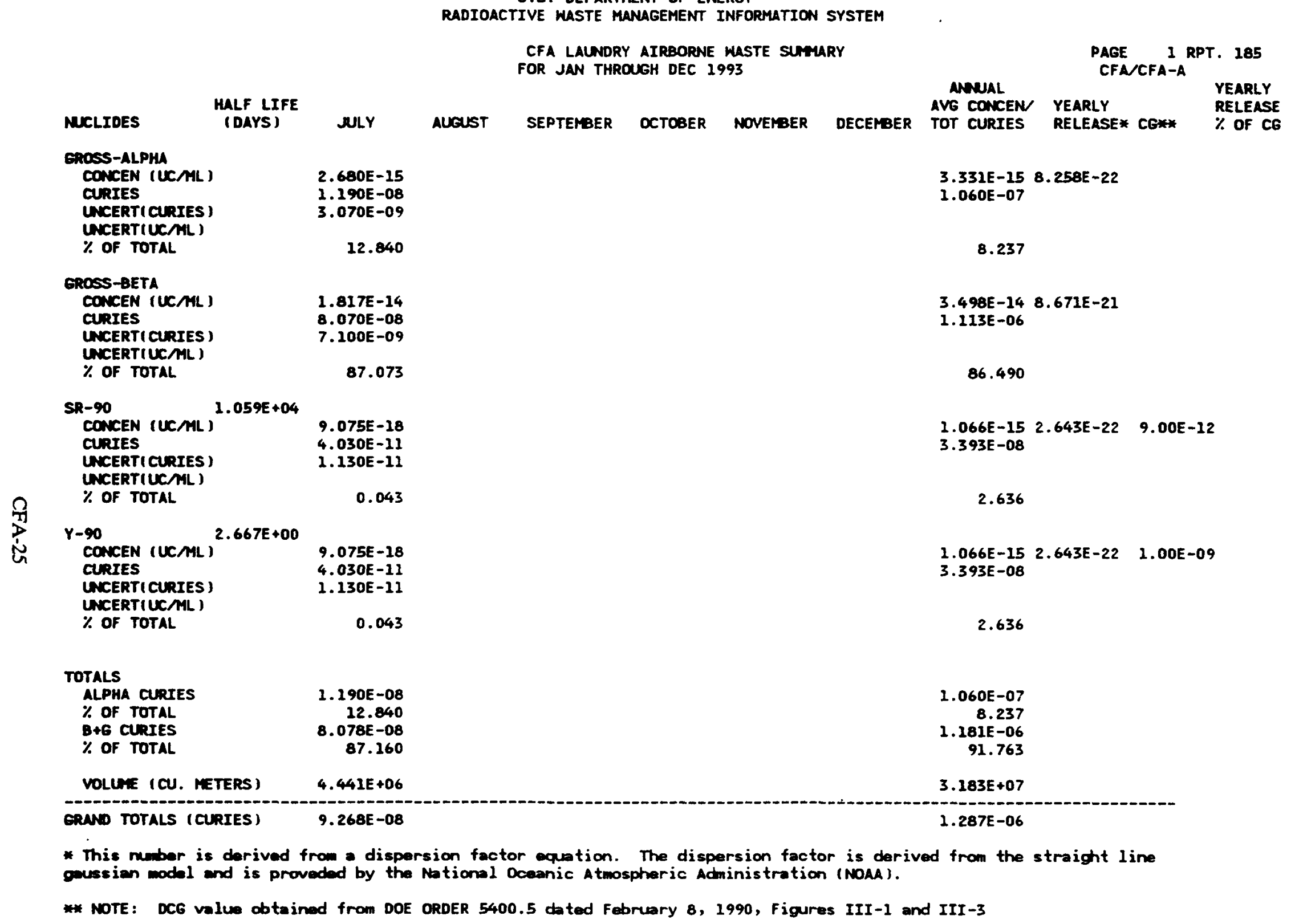


RADIOACTIVE WASTE MANAGEMENT INFORMATION SYSTEM

\begin{tabular}{|c|c|}
\hline $\begin{array}{l}\text { MALF LIFE } \\
\text { (OAYS) }\end{array}$ & suly \\
\hline $\begin{array}{l}\text { GROSS-ALPHA } \\
\text { CONCEN (UCML) } \\
\text { CURIES } \\
\text { UNCERT( CURIES) } \\
\text { UNCERT (UCAL) } \\
\text { \% OF TOTAL }\end{array}$ & $\begin{array}{r}9.155 E-15 \\
3.270 E-09 \\
5.140 E-10 \\
13.283\end{array}$ \\
\hline $\begin{array}{l}\text { GROSS-BETA } \\
\text { CONCEN (UCML) } \\
\text { CURIES } \\
\text { UNCERT(CURIES) } \\
\text { UNCERT (UCAL) } \\
\text { \% OF TOTAL }\end{array}$ & $\begin{array}{r}5.767 E-14 \\
2.060 E-08 \\
1.420 E-09 \\
83.679\end{array}$ \\
\hline $\begin{array}{l}\text { SR-90 } 1.059 E+04 \\
\text { CONCEN (UCML) } \\
\text { CURIES } \\
\text { UNCERT(CURIES) } \\
\text { (NCERT(UCAL) } \\
\% \text { OF TOTAL }\end{array}$ & $\begin{array}{r}1.047 E-15 \\
3.740 E-10 \\
1.170 E-10 \\
1.519\end{array}$ \\
\hline $\begin{array}{l}\text { Y-90 } 2.667 E+00 \\
\text { CONCEN (UCAL) } \\
\text { CURIES } \\
\text { UNCERTICURIES) } \\
\text { UNCERTIUCAML) } \\
\% \text { OF TOTAL }\end{array}$ & $\begin{array}{l}1.047 E-15 \\
3.740 E-10 \\
1.170 E-10\end{array}$ \\
\hline
\end{tabular}

CFA RESPIRATOR AIRBORNE WASTE SUMAARY FOR JAN THROUGH DEC 1993

PAGE I RPT 184 CFAVFA-A

MNUAL AVG CONCEN

AUGUST SEPTEMBER OCTOBER NOVEMBER DECEMBER

\section{YEARLY} YEARLY
RELEASE $\%$ OF CG

\section{TOTALS}

$\begin{array}{lr}\text { ALPHA CURIES } & 3.270 E-09 \\ \% \text { OF TOTAL } & 13.283\end{array}$

$\%$ of Tora

13.283

B+6 CURIES

$2.135 E-08$

86.717

$2.965 E-14 \quad 7.350 E-21$

$7.590 E-08$

29.893

$6.771 E-14 \quad 1.679 E-20$

$1.733 E-07$

68.267

9.125E-16 2.262E-22 $9.00 E-12$

$2.336 E-09$

0.920

$9.125 E-16 \quad 2.262 E-22 \quad 1.00 E-09$

2.336E-09

0.920

VOLUME (CU. METERS) 3.572E+05

GRAND TOTALS (CURIES) 2.462E-08

$7.590 E-08$

29.893

$1.780 E-07$

70.107

* This nuber is derived from a dispersion factor equation. The dispersion factor is derived from the straight line gaussian model and is provedod by the Notional Oceanic Atmospheric Administration (NOAA).

* NOTE: DCG value obtained from DOE ORDER 5400.5 dated February 8, 1990 , Figures III-1 and III-3 


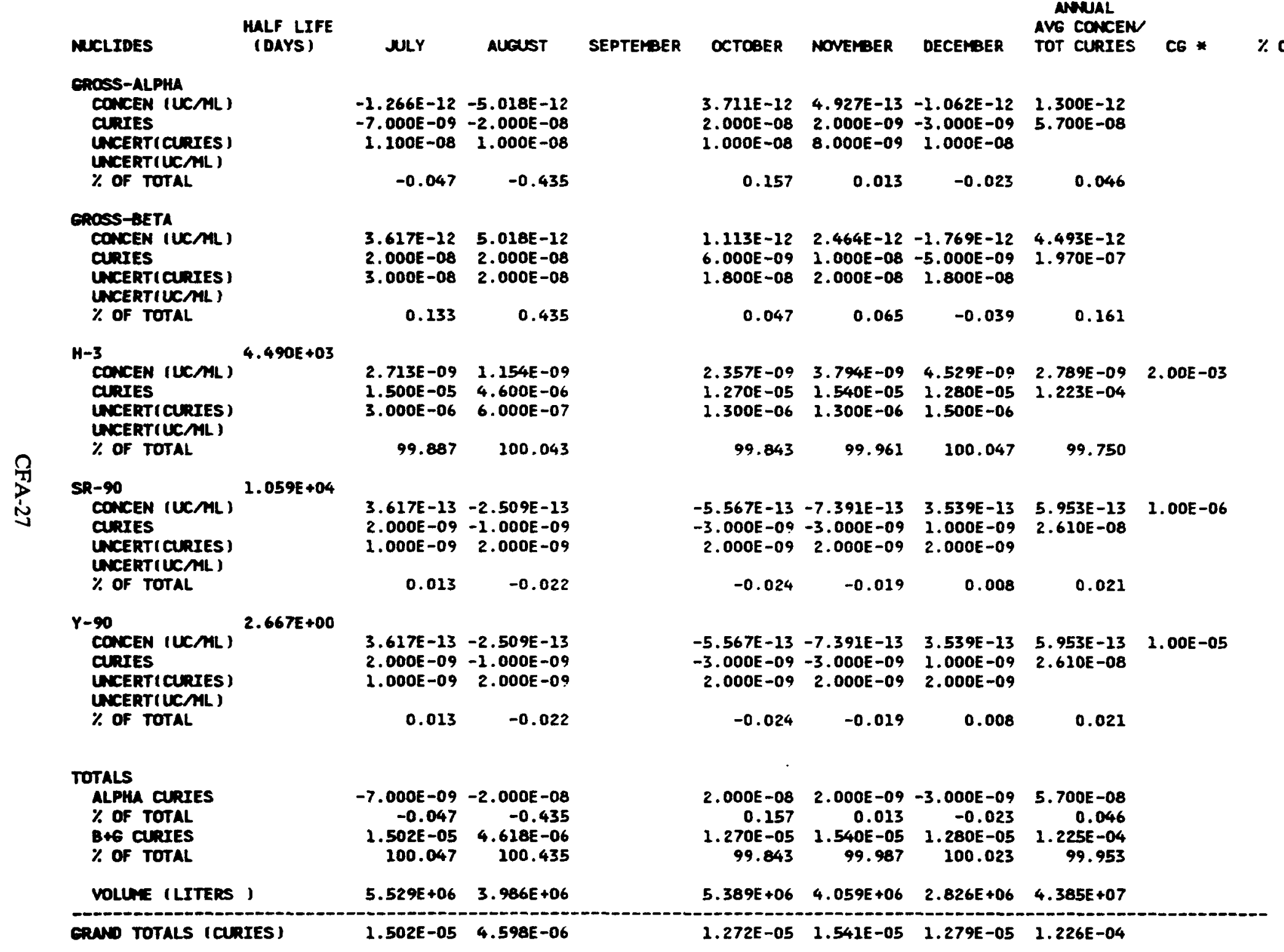

* NOTE: DCG value obtained from DOE ORDER 5400.5 hted Fabruary8, 1990 , Figures III-1 and III-3 

CFA Facility Detail Data for Months January to June Annual Average Concentration and Total Curies for January to June 

CFA LALUTRY ATPBOPNE WASTE SUMEARY

FOR JAN THROUGH JUN 1993

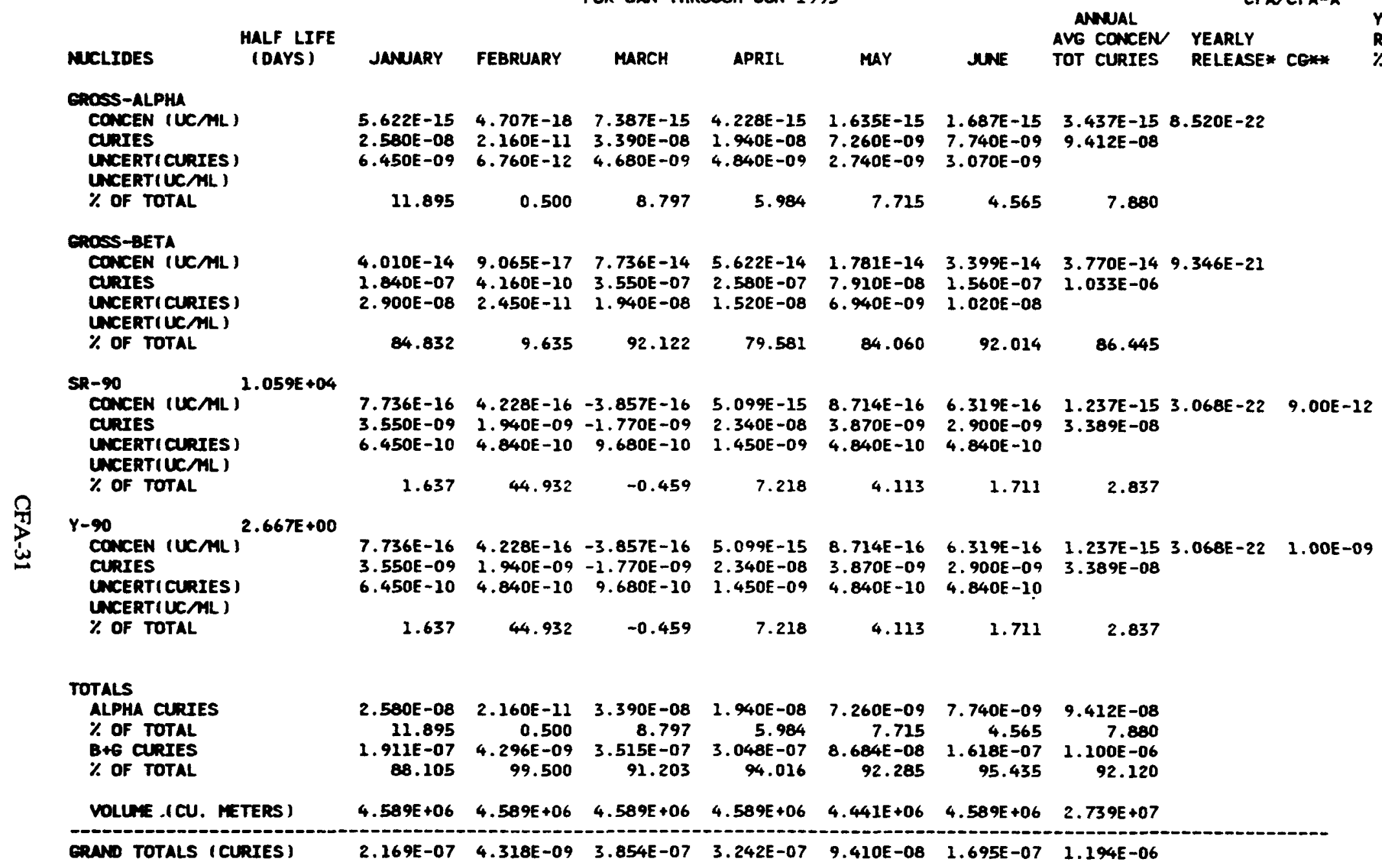

* This nuber is derived from a dispersion factor equation. The dispersion factor is derived from the straight line gemseien model and is proveded by the National Ocanic Atmospheric Administration (NOAA).

* NOTE: DCG value obtained from DOE ORDER 5400.5 dated February 8, 1990 , Figures III-I and III-3 
CFA RESPIRATOR AIPBORNE MASTE SUMMARY FOR JAN THROUGH JUN 1993

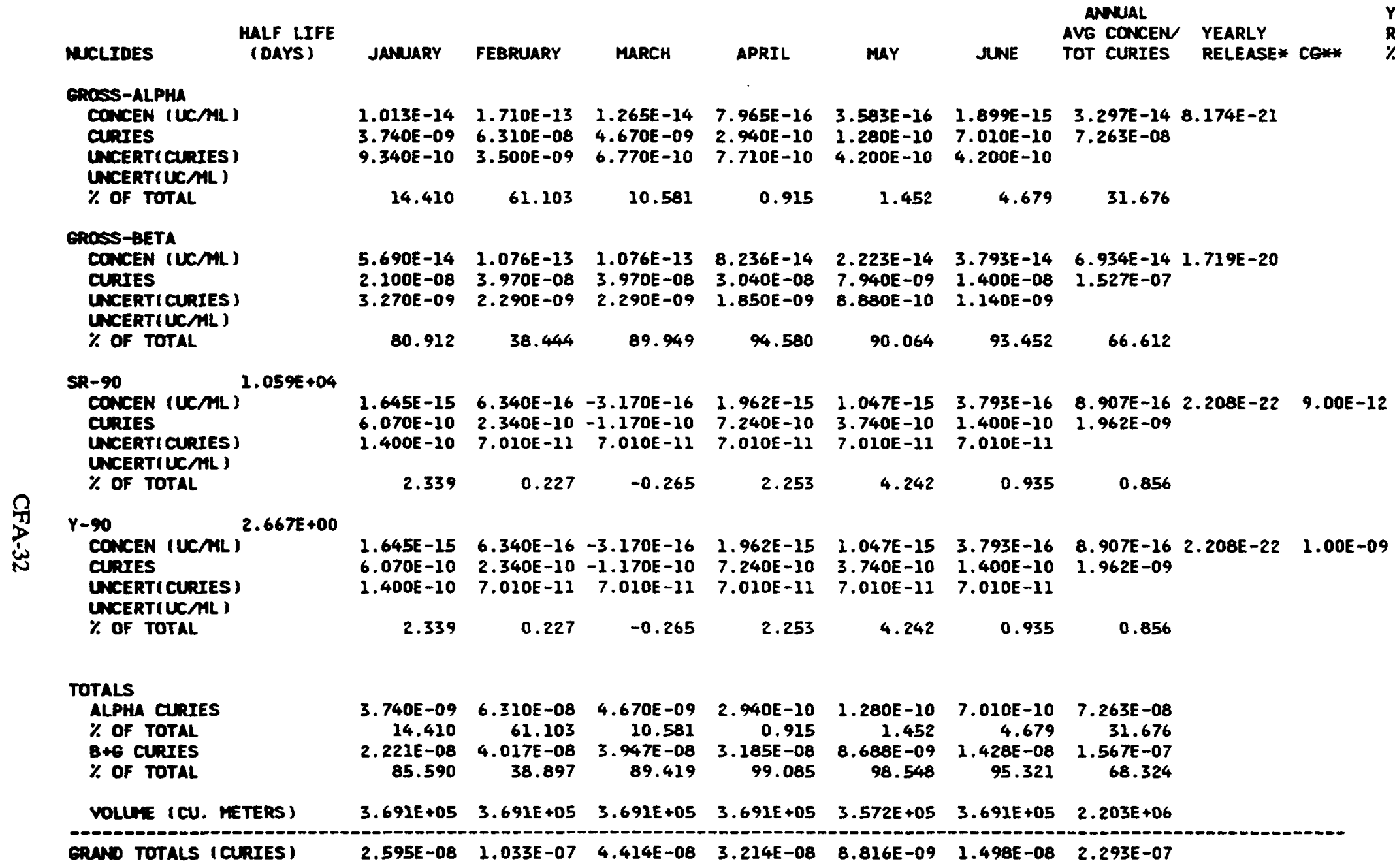

* This nuber is derived from a dispersion factor equation. The dispersion factor is derived from the straight line gevesian model and is proveded by the National Oceanic Atmospheric Administration (NOAA).

* NOTE: DCG value obtained from DOE ORDER 5400.5 deted February 8, 1990, Figures III-I and III-3 
CFA LIEUTO MASTE SUMARY

FOR JAN THROUGH JUN 1993

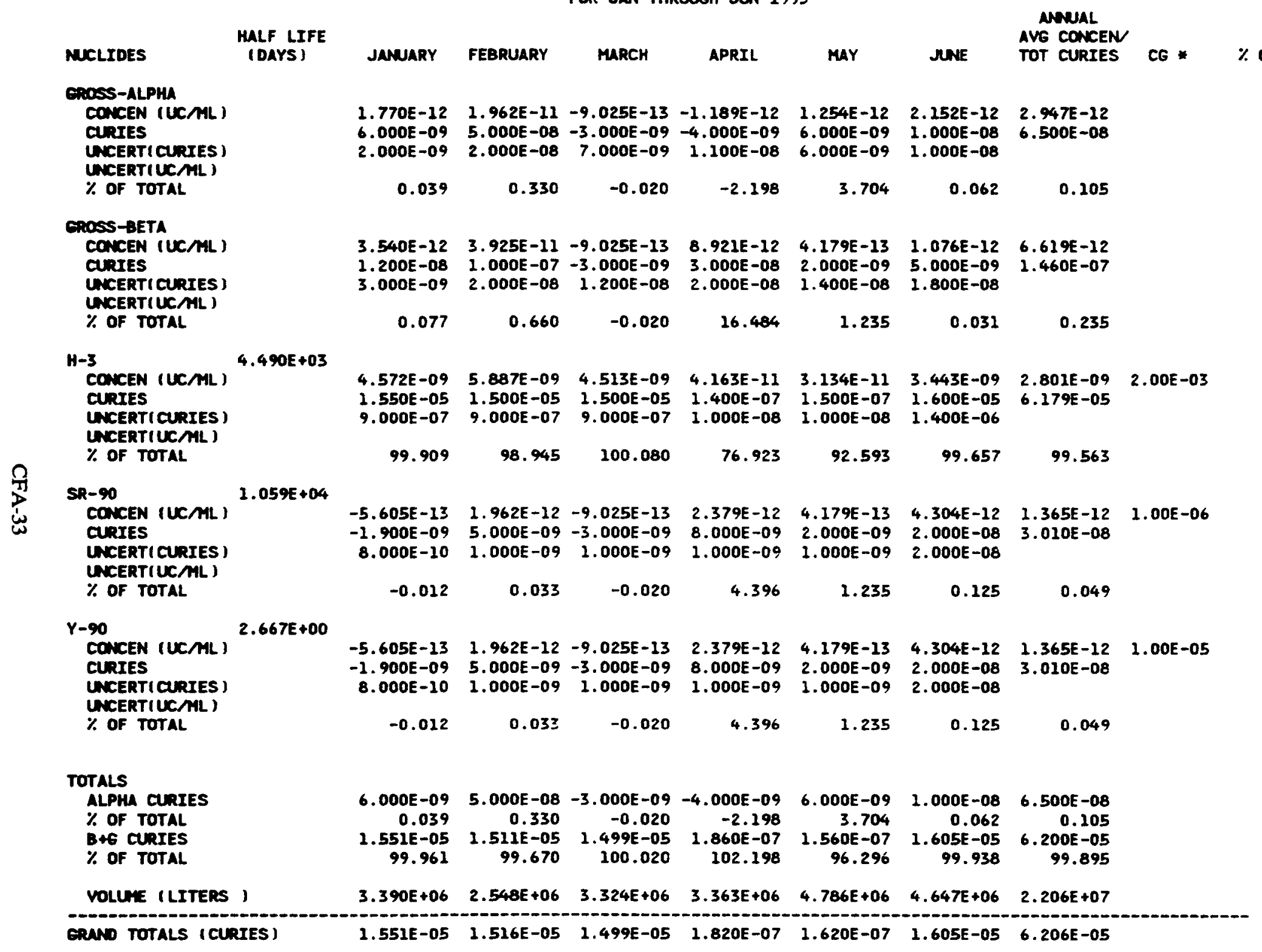

* NOTE: DCG value obtainad from DOE ORDER 5400.5 dated February8, 1990, Figures III-1 and III-3 


\section{Chemical Processing Plant 1993 Detail Data}

Chemical Processing Plant (CPP) Bar Graphs of Annual Data by Month ........ CPP-3

CY1993 Bar Graphs ........................... CPP-5

CPP Detail Data for Months July to December,

Annual Average Concentration and Total Curies for January to December ...... CPP-7

Report 210 - CPP Airborne Waste Summary ............... CPP-9

Report 211 - CPP Liquid Waste Summary ................... CPP-11

Report 212 - CPP Disposed Solid Waste Summary ............. CPP-12

Report 213-CPP Solid Waste Summary,

CPP Stored Wastes ..................... CPP-14

Report 223-CPP Solid Waste Summary,

WERF Incinerator Processed Waste $\ldots \ldots \ldots \ldots \ldots \ldots$ CPP-15

CPP Detail Data for Months January to June,

Annual Average Concentration and Total Curies for January to June $\ldots \ldots \ldots$ CPP-17

Report 210_CPP Airborne Waste Summary $\ldots \ldots \ldots \ldots \ldots \ldots \ldots$ CPP-19

Report 211 - CPP Liquid Waste Summary .................. CPP-21

Report 213 - CPP Solid Waste Summary, CPP Stored Wastes ........................ CPP-22

Report 223-CPP Solid Waste Summary, WERF Incinerator Processed Waste .............. CPP-23

CPP Facility Detail Data for Months July to December,

Annual Average Concentration and Total Curies for January to December ....... CPP-25

Report 221 -CPP FAST Stack Airborne Waste Summary . . . . . . . . . . . . CPP-27

Report 219 - CPP Main Stack Airborne Waste Summary .............. CPP-29

Report 220 - CPP NWCF Stack Airborne Waste Summary ............. CPP-32

Report 225 - CPP RAL Stack Airborne Waste Summary ............. CPP-33

Report 218 - CPP Liquid Waste to Infiltration Pond ............... CPP-34

CPP Facility Detail Data for Months January to June,

Annual Average Concentration and Total Curies for January to June ......... CPP-35

Report 221-CPP FAST Stack Airborne Waste Summary ............ CPP-37

Report 219 - CPP Main Stack Airborne Waste Summary ... . . . . . . . . . . CPP-38

Report 220 - CPP NWCF Stack Airborne Waste Summary . . . . . . . . . . . CPP-41

Report 225-CPP RAL Stack Airborne Waste Summary ............... CPP-42

Report 218 - CPP Liquid Waste to Infiltration Pond .............. CPP-43 
Chemical Processing Plant (CPP) Bar Graphs of Annual Data by Month 



\section{Chemical Processing Plant (CPP) Monthly Details - CY 1993}

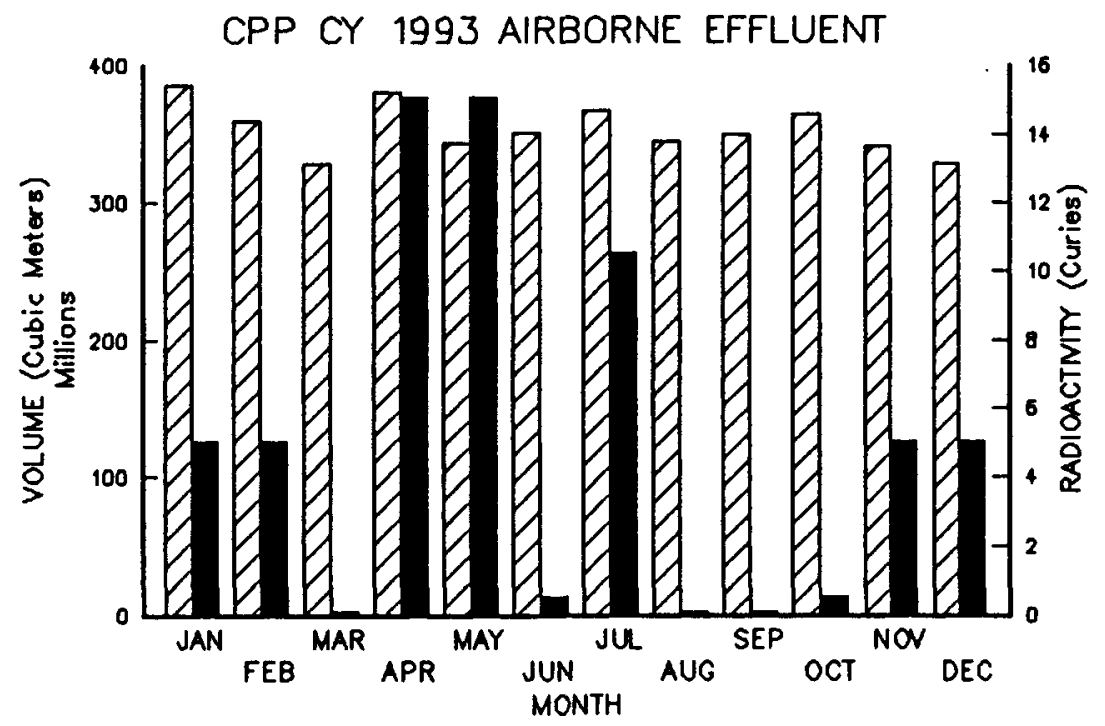

QVOLUME $\square$ RADIOACTIVTY

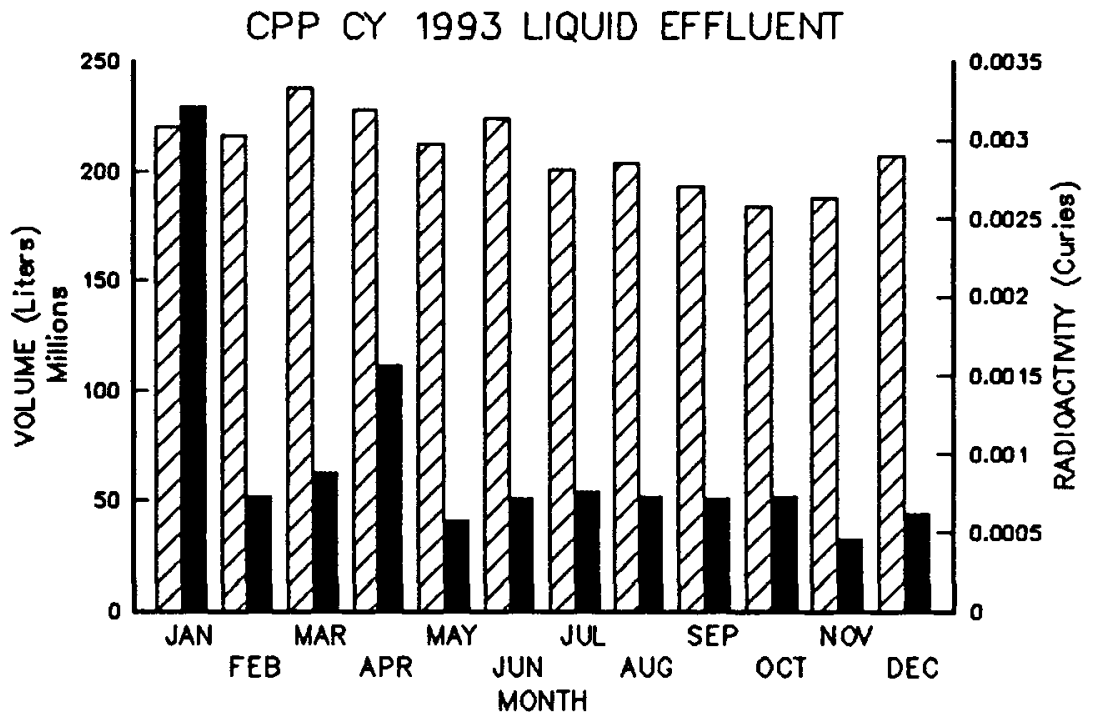

EVOLUME RADIOACTIVTY

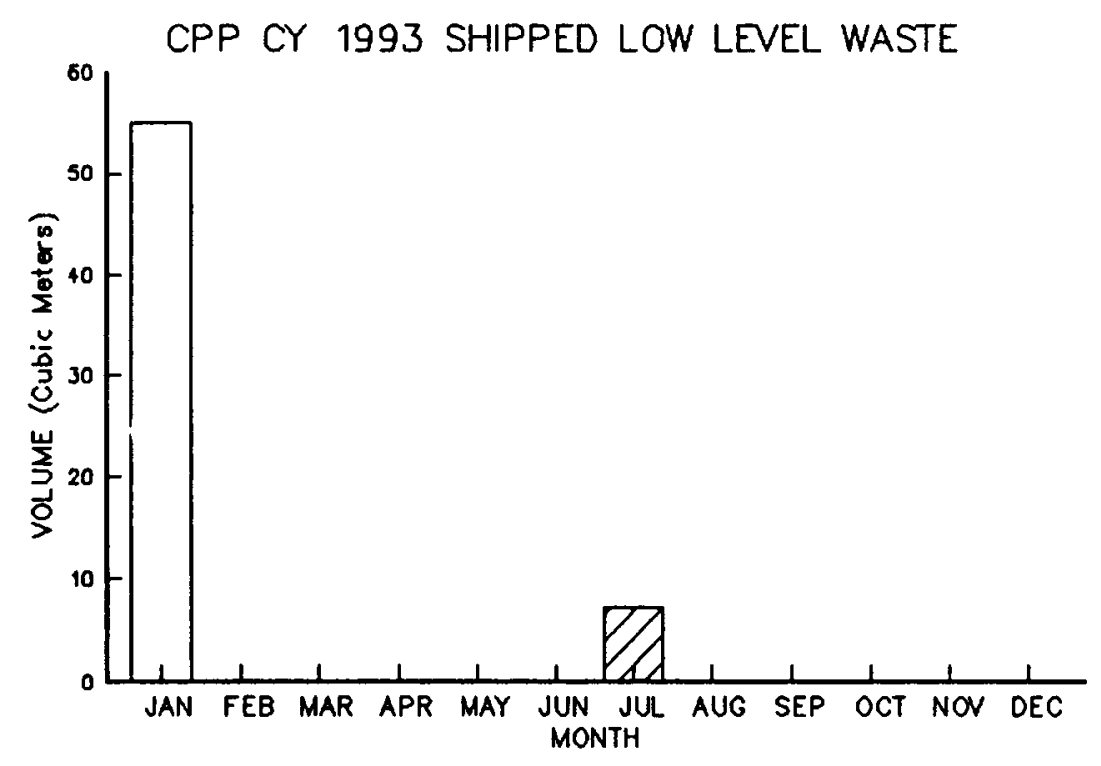

DINCINERATION EI METAL SIZING I COMPACTIONE DIRECT DISPOSAL 

CPP Detail Data for Months July to December Annual Average Concentration and Total Curies for January to December 

CPP AIPBORNE MUSTE SUMMARY

FOR JNN THROUEH DEC 1993

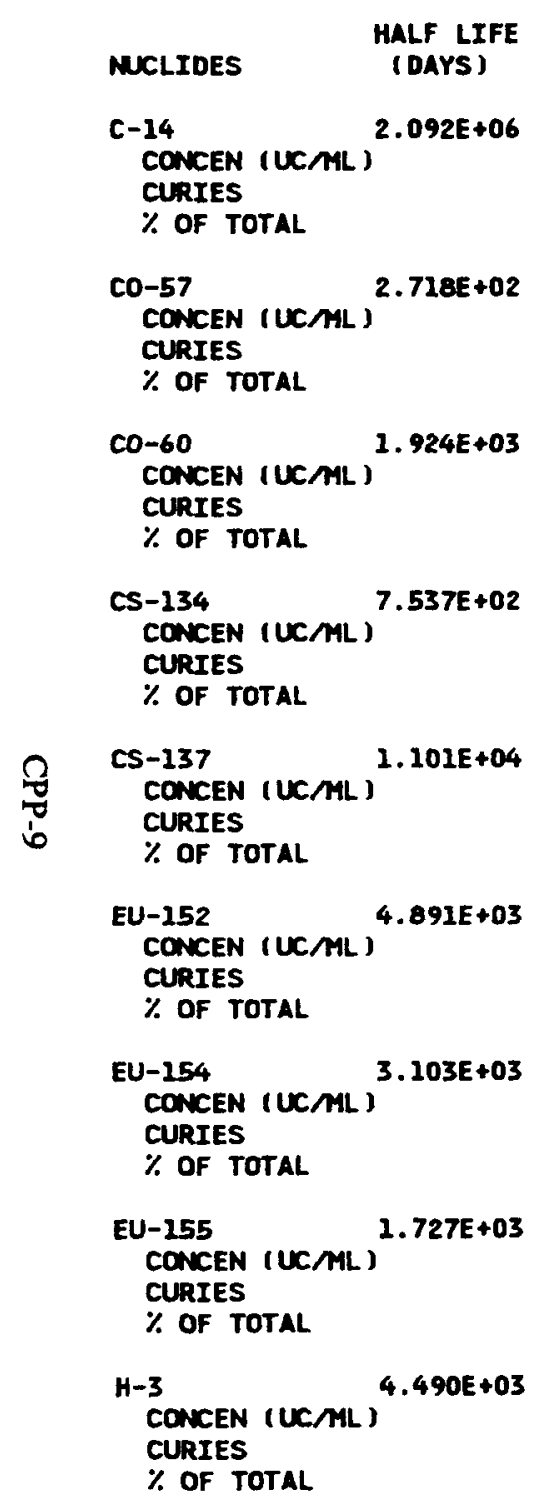

JULY

aucust

SEPTEMER

\section{$7.688 E-12$ \\ $2.800 E-03$}

0.525

$7.659 E-17 \quad 5.341 E-17$

$2.640 E-08 \quad 1.870 E-08$

\subsection{E-15 1.063E-16}

1.750E-06 3.720E-08

0.003

$\begin{array}{llll}2.250 E-16 & 7.978 E-14 & 4.170 E-15 & 5.848 E-16 \\ 8.240 E-08 & 2.750 E-05 & 1.460 E-06 & 2.130 E-07\end{array}$

0.040

0.002

$6.898 E-14 \quad 7.312 E-12$

$\begin{array}{lll}3.078 E-13 & 1.172 E-13\end{array}$

$\begin{array}{lllll}2.526 E-05 & 2.520 E-03 & 1.078 E-04 & 4.268 E-05\end{array}$ 3.643

0.163

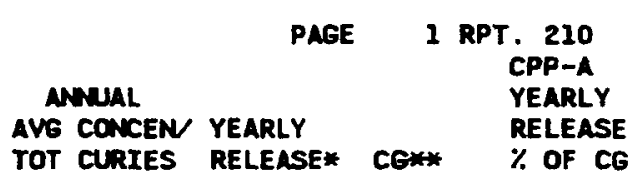

\section{$7.760 E-17$}

2.650E-08

$2.262 E-12 \quad 2.841 E-18 \quad 6.00 E-09$ $\begin{array}{lll}.000 E-03 & 1.000 E-03 & 9.600 E-03\end{array}$

$\begin{array}{lll}1.833 E-17 & 2.302 E-23 & 2.00 E-09 \\ 7.780 E-08 & & \end{array}$

$1.833 E-17$
$7.780 E-08$

$\begin{array}{lll}1.230 E-15 & 1.545 E-21 & 8.00 E-11\end{array}$

5. 220E-06

$\begin{array}{llll}1.072 E-16 & 2.060 E-14 & 2.587 E-20 & 2.00 E-10\end{array}$ 3.520E-08 8.741E-05

$7.664 E-14 \quad 2.432 E-14$ $617 E-05$ 0.001

$\begin{array}{lll}1.765 E-12 & 2.217 E-18 \quad 4.00 E-10\end{array}$ $.491 E-03$ 0.012

4.241E-16 5.327E-22 5.00E-11 1.800E-06

$1.546 \mathrm{E}-14$

$5.330 E-06$

0.008
$2.323 E-15 \quad 2.918 E-21 \quad 5.00 E-11$ $9.859 E-06$

$3.817 E-17 \quad 4.794 E-23 \quad 3.00 E-10$ $1.620 E-07$ $\begin{array}{lllllllll}2.873 E-08 & 1.832 E-10 & 1.803 E-10 & 1.441 E-09 & 1.480 E-08 & 1.539 E-08 & 1.461 E-08 & 1.835 E-14 & 1.00 E-07 \\ 1.052 E+01 & 6.314 E-02 & 6.314 E-02 & 5.247 E-01 & 5.055 E+00 & 5.055 E+00 & 6.202 E+01 & & \end{array}$ 
RADIOACTIVE WASTE MANAGEMENT INFORMATION SYSTEM

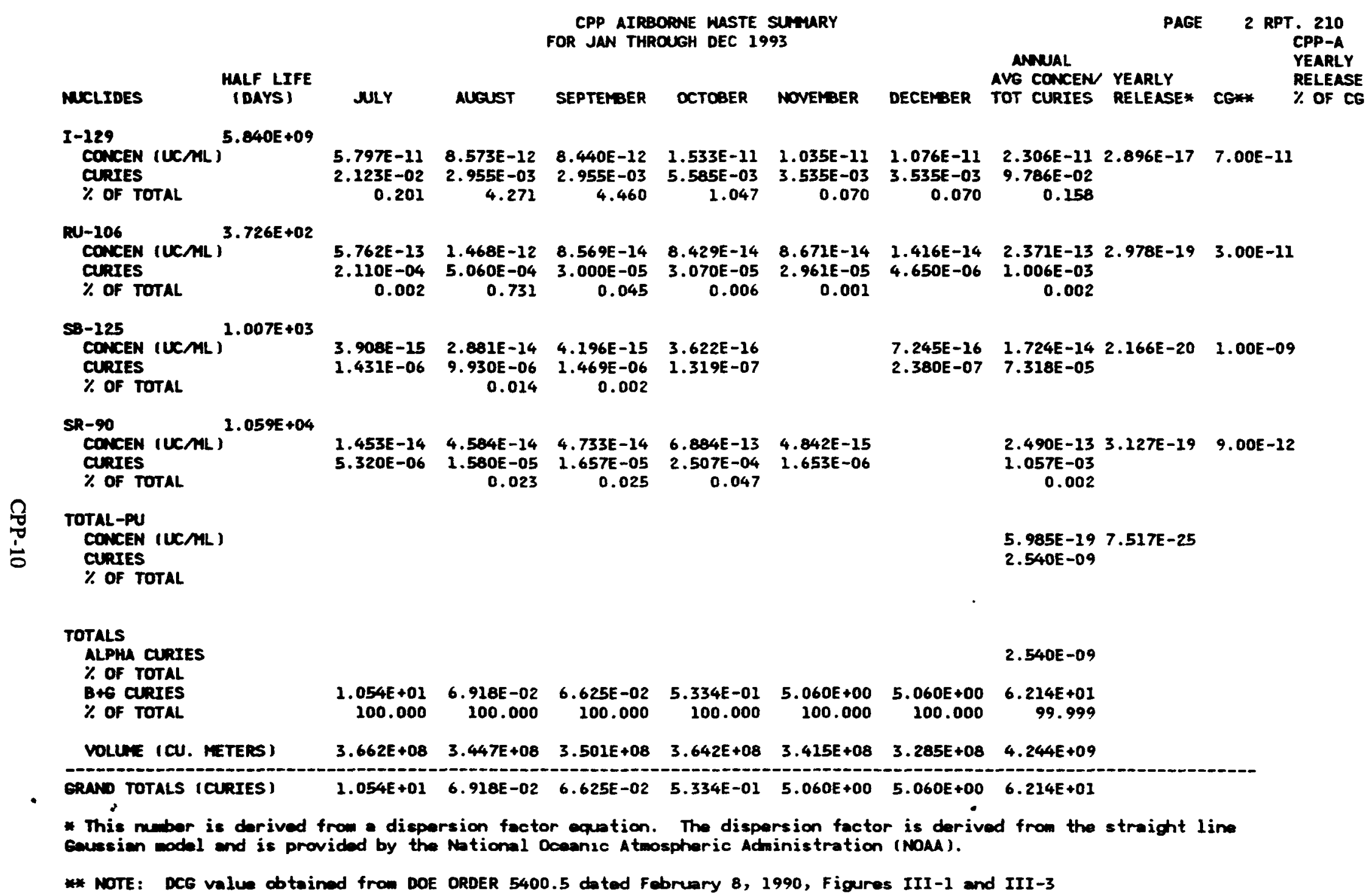


RADIOACTIVE WASTE MANAGEMENT INFORMATION SYSTEM

CPP LIOUTD WUSTE SUMMRY

FOR JAN THROUEH DEC 1993

$\begin{array}{lc}\text { NUCLIDES } & \begin{array}{c}\text { HLLF LIFE } \\ \text { (DAYS) }\end{array} \\ 00-60 & \begin{array}{c}1.924 E+03 \\ \text { CONCEN }(U C M L)\end{array}\end{array}$

CONCEN IUCML

CURIES

$\%$ OF TOTAL

cs-237

CONCEN IUC/ML $1.101 E+04$

CURIES

$\%$ OF TOTAL

SR-90

CONCEN IUCML

$\%$ OF TOTAL

$1.059 E+04$

TOTAL-PU

CONCEN (UCAL)

CURIES

$\%$ OF TOTAL

(1)

$3.101 E+04$

$1.065 E-10$
$\begin{array}{lllll}2.065 E-10 & 2.735 E-10 & 2.000 E-10 & 3.625 E-10 & 2.798 E-10 \\ 2.140 E-05 & 5.580 E-05 & 3.860 E-05 & 6.670 E-05 & 5.260 E-05\end{array}$
$\begin{array}{rrrrr}2.834 & 5.580 E-05 & 3.860 E-05 & 6.670 E-05 & 5.260 E-05 \\ & 7.707 & 5.391 & 9.191 & 11.481\end{array}$
$3.343 E-10$
$6.820 E-05$ 9.420

SEPTEMBER

OCTOBER

NOVERBER

DECEMER

ANG CONCEN

PAGE 1 RPT. 211

AVG CONCEN
TOT CURIES

CG * \% OF CG

CPP-L

$$
\begin{array}{rrrr}
2.207 E-10 & 2.489 E-10 & 3.383 E-10 & 1.918 E-10 \\
4.260 E-05 & 4.580 E-05 & 6.360 E-05 & 3.970 E-05 \\
5.950 & 6.311 & 13.882 & 6.406
\end{array}
$$

$5.00 E-06$

0.01

$002 E-03$

8.586

$\begin{array}{lllll}1.199 E-09 & 7.010 E-10 & 7.979 E-10 & 1.500 E-09\end{array}$

$\begin{array}{llll}1.199 E-09 & 7.010 E-10 & 7.979 E-10 & 1.500 E-09 \\ 2.410 E-04 & 1.430 E-04 & 1.540 E-04 & 2.760 E-04\end{array}$

$\begin{array}{rrrr}410 E-04 & 1.430 E-04 & 1.540 E-04 & 2.760 E-04 \\ 31.911 & 19.751 & 21.509 & 38.032\end{array}$

12.481

$8.657 E-10-3.00 E-06$

0.03

$2.177 E-03$

28.663

1. $900 E-11$

3.820E-06

$\begin{array}{rr}3.000 E-11 & 1.201 E-11 \\ 5.790 E-06 & 2.210 E-06 \\ 0.809 & 0.305\end{array}$

2. $101 E-11$

0.862
0
1.000E-09 $1.089 E-09 \quad 1.00 E-06$
$1.000 E-09$
$33.403 \quad 2.739 E-03$

$\begin{array}{llllllll}2.433 E-09 & 2.240 E-09 & 2.461 E-09 & 1.821 E-09 & 1.798 E-09 & 1.802 E-09 & 2.266 E-09\end{array}$

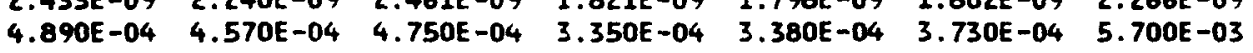

CONCEN (UCML)

CUNIES

64.749

63.122

66.342

46.162

73.775

60.190

48.860

\section{Totals}

ALPHA CURIES

$\%$ OF rotal

8+6 curres

$\%$ OF TOTAL

3.820E-06

0.506

$7.514 E-04$

$514 E-04$
99.494

5.790E-06 2.210E-06

$10 E-06$
0.305

$3.950 E-06$

102E-04 7.235E-04 4.542E-04

99.191

$\begin{array}{r}99.695 \\ \hline\end{array}$

$542 E-04$
99.138

6. 197E-04

4.815E-05

0.413

197E-04 1.162E-02

VOLURE (LITERS)

$\begin{array}{lllllll}2.010 E+08 & 2.040 E+08 & 1.930 E+08 & 1.840 E+08 & 1.880 E+08 & 2.070 E+08 & 2.515 E+09\end{array}$

eRANo Totals (CURIES )

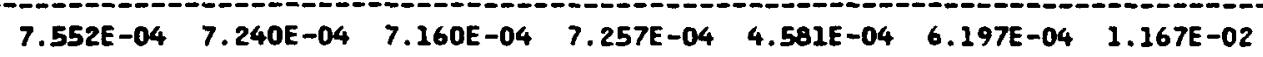

* NOTE: DCG velwe obtainad from DOE ORDER 5400.5 dated Fabruary8, 1990, Figures III-1 and III-3 
SCHED. NO. P6IPM009-G

IDAHO OPERATIONS OFFICE

U.S. DEPARTMENT OF ENERGY
RADIOACTIVE MUSTE MANAGEMENT INFORMATION SYSTEM

\section{CPP DISPOSED SOLID WASTE SUMARY}

FOR JAN THROUGH DEC 1993

\begin{tabular}{|c|c|c|}
\hline MCLIDES & $\begin{array}{l}\text { MALF LIFE } \\
\text { (DAYS) }\end{array}$ & JULY \\
\hline $\begin{array}{l}\text { CE - } 144 \\
\text { CURIES } \\
\% \text { OF TOTAL }\end{array}$ & $2.894 E+02$ & $\begin{array}{r}2.418 E-02 \\
19.700\end{array}$ \\
\hline $\begin{array}{l}\text { CS- } 237 \\
\text { CURIES } \\
\% \text { OF TOTAL }\end{array}$ & $1.101 E+04$ & $\begin{array}{r}7.200 E-03 \\
20.000\end{array}$ \\
\hline $\begin{array}{l}\text { NB-95 } \\
\text { CURIES } \\
\% \text { OF TOTAL }\end{array}$ & $3.498 E+01$ & $\begin{array}{r}2.232 E-03 \\
3.100\end{array}$ \\
\hline $\begin{array}{l}\text { PR-144 } \\
\text { CURIES } \\
\% \text { OF TOTAL }\end{array}$ & $1.200 E-02$ & $\begin{array}{r}1.418 E-02 \\
19.700\end{array}$ \\
\hline $\begin{array}{l}\text { RH-106 } \\
\text { CURIES } \\
\% \text { OF TOTAL }\end{array}$ & $3.441 E-04$ & $\begin{array}{r}7.200 E-03 \\
10.000\end{array}$ \\
\hline $\begin{array}{l}\text { RU-106 } \\
\text { CURIES } \\
\% \text { OF TOTAL }\end{array}$ & $3.726 E+02$ & $\begin{array}{r}7.200 E-03 \\
10.000\end{array}$ \\
\hline $\begin{array}{l}\text { S6-125 } \\
\text { CURIES } \\
\% \text { OF TOTAL }\end{array}$ & $1.007 E+03$ & $\begin{array}{r}3.168 E-03 \\
4.400\end{array}$ \\
\hline $\begin{array}{l}S R-90 \\
\text { CURIES } \\
\% \text { OF TOTAL }\end{array}$ & $1.059 E+04$ & $\begin{array}{r}7.200 E-03 \\
10.000\end{array}$ \\
\hline $\begin{array}{l}\text { Y-90 } \\
\text { CURIES } \\
\% \text { OF TOTAL }\end{array}$ & $2.667 E+00$ & $\begin{array}{r}7.200 E-03 \\
10.000\end{array}$ \\
\hline $\begin{array}{l}\text { ZR-95 } \\
\text { CURIES } \\
\% \text { OF TOTA }\end{array}$ & $6.403 E+01$ & $\begin{array}{r}2.232 E-03 \\
3.100\end{array}$ \\
\hline
\end{tabular}

RUN DATE: 05/11/94

PAGE 1 RPT 212

CPP-S

$1.418 E-02$

19.700

$7.200 E-03$

10.000

$2.232 E-03$

3.100

$1.418 E-02$ 19.700

7. $200 E-03$ 10.000

$7.200 E-03$ 10.000

$3.168 E-03$ 4.400

$7.200 E-03$ 10.000

$7.200 E-03$ 10.000

2. 232E-03

3.100 
CPP DISPOSED SOLID MUSTE SUVAARY FOR JAN THROUEH DEC 1993

NUCLIDES

\section{HALF LIFE}

(DAYS)

JuLY

AUEUST

DCtOBER
7.200E-02

100.000
$7.200 E-02$ 100.000

ALPHA CURIE

$B+G$ CURIES

TOTAL GRAMS OF AM

TOTAL GRMLS OF MM

TOTAL GRANS OF PU

TOTAL GRUYS OF PU-239+24I

TOTAL GRATS OF U

TOTAL GRANS OF TH

TOTAL GRAYS OF NP

WEIGHT (KILOGRAMS)

$7.250 E+00$

- MEIGHT IKILOGRMS)

$2.530 E+03$

$2.530 E+03$

GRAND TOTALS (CURIES)

7.200E-02

7.200E-02 
SCHED. NO. P6IPMOO9-K

IDAHO OPERATIONS OFFICE

RADIOACTIVE MASTE MAMAGEMENT INFORMATION SYSTEM

CPP SOLID WUSTE SUMMARY

FOR JAN THROUEH DEC 1993

CPP STORED MASTES

HALF LIF
MECLIOES (DAYS )

JULY

August

SEPTEMBER OCTOBER

MFP

CURIES

$\%$ OF TOTAL

$2.010 E+05$

100.000

$7.070 E+04$

(1)

$.070 E+05$

100.000

$3.440 E+03$

100.000

NOVERBER

DECEMBR

\section{ANANAL}

PAGE 1 RPT. 213

CPP-S

\section{TOTALS}

ALPHA CURIES

$\%$ OF TOTAL

B+G CURIES

$\%$ OF TOTAL

2.010E+05

$7.070 E+04 \quad 1.070 E+05 \quad 3.440 E+03$

$1.141 E+06$

100.000

TOTAL GRAMS OF AM

TOTAL GRANS OF PU

TOTAL ERAMS OF PU-239+241

TOTAL GRANS OF $U$

TOTAL GRANS OF TH

TOTAL GRAMS OF NP

VOLUME (CU. METERS)

$\begin{array}{llll}3.200 E+01 & 2.490 E+01 & 3.130 E+01 & 1.730 E+01\end{array}$

$1.965 E+02$ 
RADIOACTIVE WASTE MUUGEMENT INFORMATION SYSTEM

\section{CPP SOLID WUSTE SUMAARY}

WERF INCINERATOR PROCESSED WASTE

FROY JAN THROUGH DEC I993

$$
\begin{aligned}
& \text { HALF LIF } \\
& \text { (DAYS) }
\end{aligned}
$$

SULY

2. $844 E+02$

CE-144

CURIES

\% OF TOTAL

CS-137

CURIES

$\%$ OF TOTAL

$\mathrm{NB}-95$

CURIES

$\%$ Of TOTAL

PR-144

OF TOTAL

RH-106

CURIES

$\%$ OF TOTAL

RU-106

CURIES

$\%$ OF TOTAL

SB-125

CURIES

$1.101 E+04$

$3.498 E+01$

1. 200E-02

$3.441 E-04$

3. $726 E+02$

1. $007 E+03$

\% OF tOTAL

\& SR-90

$\%$ OF TOTAL

$Y-90$

CURIES

ZR-95

CURIES

\% OF TOTAL

$1.059 E+04$

$2.667 E+00$

6. $403 E+01$

TOTALS

ALPHA CURIES

$\%$ OF TOTAL.

$B+G$ CURIES

$\%$ OF TOTAL

TOTAL GRAMS OF AM

TOTAL GRAYS OF PU

TOTAL GRANS OF PU-239+241

TOTAL GRARS OF $U$

TOTAL GRANS OF TH

TOTAL GRAMS OF NP

VOLLME (CU. METERS )

NEIGHT (KILOGRNAS)
PAGE 2 RPT. 223

NWUAL

TOTAL

4. 137E-02

19.700

2. 100E-02 10.000

6.510E-03 3.100

4.137E-02

19.700

2. 100E-02 10.000

2.100E-02 10.000

9.240E-03 4.400

2. 100E-02 10.000

2. 100E-02 10.000

$6.510 E-03$

3.100

2.100E -01

100.000

GRAND TOTALS (CURIES)

$5.505 E+01$ 
CPP Detail Data for Months January to June Annual Average Concentration and Total Curies for January to June 

CPP AIRBOANE WUSTE SUMMARY FOR JAN THROUEH JUN 1993

MUCLIDES
(DALF LIFE
(DAYS

\section{JANEARY FEBRUARY}

MARCH

APRIL

MaY

JUNE

\begin{tabular}{|c|c|c|}
\hline & PAGE & 1 RPT $\begin{array}{r}210 \\
\text { CPP }-A\end{array}$ \\
\hline :N & $\begin{array}{l}\text { YEARLY } \\
\text { RELEASE* }\end{array}$ & $\begin{array}{l}\text { LY } \\
\text { ASE } \\
\text { CG }\end{array}$ \\
\hline
\end{tabular}

$\begin{array}{ll}2.592 E-12 & 2.782 E-12 \\ 1.000 E-03 & 1.000 E-03\end{array}$

$0.020 \quad 0.020$

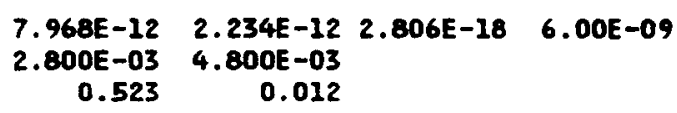

CO-57 CONCEN (UCAL)

CURIES

$\%$ of Total

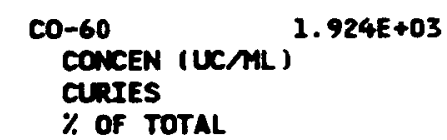

$\%$ of total

CS-134
CONCEN (UCML)
CURIES
$\%$ OF TOTAL

$7.537 E+02$

5- 137 1.101E+04

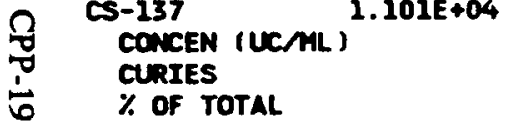

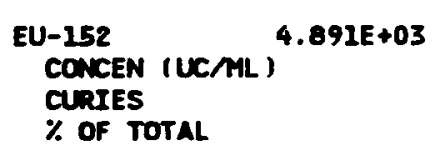

\section{EU-154 CONCEN IUCML \\ CURIES \\ $\%$ of total}

3. $103 E+03$
EU-155 CONCEN (UCA
CURIES
$\%$ OF TOTAL
$1.727 E+03$
H-3
CONCEN (UC/ML)
CurIES
\% OF total

$\begin{array}{lllll}1.905 E-15 & 1.953 E-14 & 1.509 E-14 & 3.607 E-14 & 3.027 E-14\end{array}$ $\begin{array}{lllll}7.350 E-07 & 7.020 E-06 & 4.960 E-06 & 1.370 E-05 & 1.040 E-05\end{array}$ 0.007
$9.306 E-17 \quad 1.522 E-17 \quad 1.911 E-23 \quad 2.00 E-09$ 3.270E-08 3.270E-08

$\begin{array}{lllllllll}9.098 E-17 & 5.415 E-16 & 3.697 E-15 & 1.723 E-15 & 3.415 E-15 & 1.585 E-15 & 1.991 E-21 & 8.00 E-11\end{array}$ 3.270E-08 $\quad 1.780 E-07 \quad 1.404 E-06 \quad 5.920 E-07 \quad 1.200 E-06 \quad 3.407 E-06$

$6.061 E-14 \quad 2.705 E-14 \quad 3.397 E-20 \quad 2.00 E-10$ $2.130 E-05 \quad 5.812 E-05$

0.004

$2.032 E-13 \quad 1.461 E-12 \quad 1.171 E-12 \quad 2.746 E-12 \quad 2.587 E-12$ $.838 E-05 \quad 5.252 E-04 \quad 3.848 E-04 \quad 1.043 E-03 \quad 8.890 E-04$ 0.002 0.010 0.007

$\begin{array}{llll}5.237 E-12 & 2.215 E-12 & 2.783 E-18 \quad 4.00 E-10\end{array}$ $840 E-03 \quad 4.760 E-03$

$$
0.344 \quad 0.012
$$

5.122E-15 8.377E-16 $1.052 E-21 \quad 5.00 E-11$ $1.800 E-06 \quad 1.800 E-06$

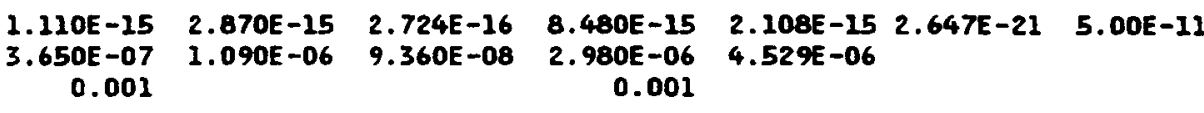

$\begin{array}{lllll}4.610 E-16 & 7.539 E-17 & 9.469 E-23 & 3.00 E-10\end{array}$ $1.620 E-07 \quad 1.620 E-07$

$\begin{array}{llllllllll}1.310 E-08 & 1.407 E-08 & 1.921 E-10 & 3.955 E-08 & 4.371 E-08 & 1.493 E-09 & 1.896 E-08 & 2.381 E-14 & 1.00 E-07 \\ 5.055 E+00 & 5.055 E+00 & 6.314 E-02 & 1.502 E+01 & 1.502 E+01 & 5.247 E-01 & 4.074 E+01 & & & \end{array}$ $\begin{array}{rrrrrrr}5.055 E+00 & 5.055 E+00 & 6.314 E-02 & 1.502 E+01 & 1.502 E+01 & 5.247 E-01 & 4.074 E+01 \\ 99.909 & 99.899 & 94.815 & 99.850 & 99.852 & 97.989 & 99.832\end{array}$ 
RADIOACTIVE WASTE MANAGEMENT INFORMATION SYSTEM

CPP AIRBORNE WASTE SUMUARY FOR JAN THROUGH JUN 1993

MCLIDES
$\begin{aligned} & \text { MLLF LIFE } \\ & \text { (DAYS) }\end{aligned}$
I-129
CONCEN (UCML)
CURIES
$\%$ OF TOTAL
RU-106
CONCEN (UCML)
CURIES
$\%$ OF TOTAL
SB-125
CONCEN (UCML)
CURIES
$\%$ OF TOTAL
SR-90
CONCEN (UCML)
CURIES
$\%$ OF TOTAL

JANUARY FEBRUARY MARCH APRIL MAY

\section{$3.535 E-03$}

\begin{abstract}
$35 E-03$
0.070
\end{abstract}
$2.240 E-14 \quad 4.090 E-14 \quad 3.925 E-14$

8.640E-06 1.470E-05 $1.290 E-05$

$$
0.019
$$

$1.198 E-14 \quad 2.822 E-14$

$\begin{array}{ll}4.620 E-06 & 6.550 E-06\end{array}$

$1.363 E-14$

$.480 E-06$
0.007

$3.373 E-14$

1.281E-05

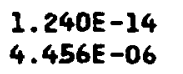

2. $750 \varepsilon-13$

$5.108 E-13$

$040 E-05$
0.136

$.108 E-13$
$.940 E-04$
MNE

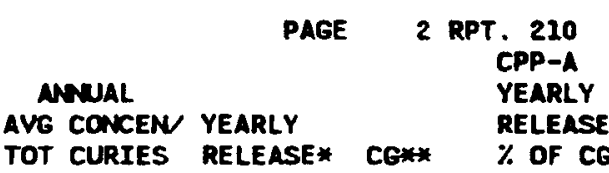

$3.984 E-24$
$1.369 E-05$

$1.783 E-05$

0.003

1.543E-13

$1.208 E-12$

5.302E-05

4. 246E-04

$3.567 E-13 \quad 4.481 E-19$ 0.001

0.079

$65 E-04$

0.002

\subsection{BE-18}

$2.540 E-09$

$1.182 E-18 \quad 1.485 E-24$
$2.540 E-09$
2.540E-09

$5.060 E+00$ 100.000

$5.060 E+00$ 100.000

$6.659 E-02$

$.659 E-02$
100.000

1. $504 E+01$

100.000

$.355 E-01$

2.540E-09

$3.058 E+08$

$3.594 E+$

. $287 E+08$

$3.798 E+08$

cRMn Totals Iaries

$$
\text { - }
$$

* This muber is dorived from a dispersion factor eqution. The dispersion factor is derived from the straight line Geussian modal and is provided by the Notional Oceanic Atmospheric Administration (NOAA).

* NOTE: DCE value obtained from DOE ORDER 5400.5 dated Fabruary 8, 1990, Figures III-I and III-3 


\section{RADIOACTIVE WASTE MPAMGEMENT LIOUTD MASTE SUMMARY}

FOR JAN THROUGH JN 1993

\begin{tabular}{|c|c|c|c|c|c|c|c|c|c|c|}
\hline MUCLIDES & $\begin{array}{l}\text { HALF LIFE } \\
\text { (DAYS) }\end{array}$ & JANUARY & FEBRUARY & MARCH & APRIL & MaY & JUNE & $\begin{array}{l}\text { ANUAL } \\
\text { AVG CONCEN } \\
\text { TOT CURIES }\end{array}$ & CG * & \% OF CG \\
\hline $\begin{array}{l}\text { CO-60 } \\
\text { CONCEN (UCML }) \\
\text { CURIES } \\
\% \text { OF TOTAL }\end{array}$ & $1.924 E+03$ & & & $\begin{array}{r}4.622 E-10 \\
1.100 E-04 \\
12.597\end{array}$ & $\begin{array}{r}2.355 E-09 \\
5.370 E-04 \\
34.297\end{array}$ & & $\begin{array}{r}4.228 E-10 \\
9.470 E-05 \\
13.164\end{array}$ & $\begin{array}{r}5.543 E-10 \\
7.417 E-04 \\
9.674\end{array}$ & $5.00 E-06$ & 0.01 \\
\hline $\begin{array}{l}\text { CS-137 } \\
\text { CONCEN (UCML ) } \\
\text { CURIES } \\
\% \text { OF TOTAL }\end{array}$ & $1.201 E+04$ & $\begin{array}{r}5.591 E-09 \\
1.230 E-03 \\
38.349\end{array}$ & $\begin{array}{r}9.861 E-11 \\
2.130 E-05 \\
2.945\end{array}$ & $\begin{array}{r}7.395 E-10 \\
1.760 E-04 \\
20.156\end{array}$ & $\begin{array}{r}1.798 E-09 \\
4.100 E-04 \\
26.186\end{array}$ & $\begin{array}{r}1.495 E-10 \\
3.270 E-05 \\
5.483\end{array}$ & $\begin{array}{r}3.263 E-10 \\
7.310 E-05 \\
10.162\end{array}$ & $\begin{array}{r}1.451 E-09 \\
1.942 E-03 \\
25.330\end{array}$ & $3.00 E-06$ & 0.05 \\
\hline $\begin{array}{l}\text { SR-90 } \\
\text { CONCEN (UCML) } \\
\text { CURIES } \\
\% \text { OF TOTAL }\end{array}$ & $1.059 E+04$ & $\begin{array}{r}6.500 E-09 \\
1.430 E-03 \\
44.585\end{array}$ & $\begin{array}{r}6.991 E-10 \\
1.510 E-04 \\
20.877\end{array}$ & & $\begin{array}{r}6.009 E-10 \\
1.370 E-04 \\
8.750\end{array}$ & & & $\begin{array}{r}1.284 E-09 \\
1.718 E-03 \\
22.407\end{array}$ & $2.00 E-06$ & 0.13 \\
\hline $\begin{array}{l}\text { TOTAL -PU } \\
\text { CONCEN (UCML) } \\
\text { CURIES } \\
\% \text { OF TOTAL }\end{array}$ & & $\begin{array}{r}2.900 E-11 \\
6.380 E-06 \\
0.199\end{array}$ & & $\begin{array}{r}2.601 E-11 \\
6.190 E-06 \\
0.709\end{array}$ & $\begin{array}{r}3.399 E-11 \\
7.750 E-06 \\
0.495\end{array}$ & $\begin{array}{r}4.000 E-11 \\
8.480 E-06 \\
1.467\end{array}$ & $\begin{array}{r}1.598 E-11 \\
3.580 E-06 \\
0.498\end{array}$ & $\begin{array}{r}2.420 E-11 \\
3.238 E-05 \\
0.422\end{array}$ & & \\
\hline $\begin{array}{l}\text { TOTAL-U } \\
\text { CONCEN (UCAL) } \\
\text { CURIES } \\
\% \text { OF TOTAL }\end{array}$ & & $\begin{array}{r}2.459 E-09 \\
5.410 E-04 \\
16.867\end{array}$ & $\begin{array}{r}2.551 E-09 \\
5.510 E-04 \\
70.179\end{array}$ & $\begin{array}{r}2.441 E-09 \\
5.810 E-04 \\
66.538\end{array}$ & $\begin{array}{r}2.079 E-09 \\
4.740 E-04 \\
30.273\end{array}$ & $\begin{array}{r}2.538 E-09 \\
5.380 E-04 \\
93.051\end{array}$ & $\begin{array}{r}2.446 E-09 \\
5.480 E-04 \\
76.177\end{array}$ & $\begin{array}{r}2.416 E-09 \\
3.233 E-03 \\
42.167\end{array}$ & & \\
\hline $\begin{array}{l}\text { TOTALS } \\
\text { ALPHA CURIES } \\
\% \text { OF TOTAL } \\
\text { B+6 CURIES } \\
\% \text { OF TOTAL }\end{array}$ & & $\begin{array}{r}6.380 E-06 \\
0.199 \\
3.201 E-03 \\
99.801\end{array}$ & $\begin{array}{r}7.233 E-04 \\
100.000\end{array}$ & $\begin{array}{r}6.190 E-06 \\
0.709 \\
8.670 E-04 \\
99.291\end{array}$ & $\begin{array}{r}7.750 E-06 \\
0.495 \\
1.558 E-03 \\
99.505\end{array}$ & $\begin{array}{r}8.480 E-06 \\
1.467 \\
5.697 E-04 \\
98.533\end{array}$ & $\begin{array}{r}3.580 E-06 \\
0.498 \\
7.158 E-04 \\
99.502\end{array}$ & $\begin{array}{r}3.238 E-05 \\
0.422 \\
7.635 E-03 \\
99.577\end{array}$ & & \\
\hline VOLUNE ILITERS & & $2.200 E+08$ & $2.160 E+08$ & $2.380 E+08$ & $2.280 E+08$ & $2.120 E+08$ & $2.240 E+08$ & $1.338 E+09$ & & \\
\hline ERAND TOTALS ICU: & RI & $3.207 E-03$ & $7.233 E-04$ & 8. $732 E-04$ & $1.566 E-03$ & $5.782 E-04$ & $7.194 E-04$ & $7.667 E-03$ & & \\
\hline
\end{tabular}

* NOTE: DCG value obtainad from DOE ORDER 5400.5 dated February8, 1990, figures IIII-1 and III-3 . 
SCHED. NO. P6IPNOO9-K

IDAHO OPERATIONS OFFICE
I.S. DEPARTMENT OF ENERGY

RADIOACTIVE WASTE MANAGEMENT INFORMATION SYSTEM

CPP SOLID WUSTE SUMYRY

FOR JAN THROUGH JUN 1993

HALF LIFE

NUCLIDES

(DAYS)

JANEARY

FEBRUARY

$$
\begin{aligned}
& \text { CPP ST } \\
& \text { MARCH }
\end{aligned}
$$

WASTES
APRIL

MaY

JUNE

ANWAL
$1.380 E+05$
2.790E +05
1. $940 E+05$
$1.480 E+05$
100.000
$7.590 E+05$ 100.000 100.000
100.000

RUN DATE: 05/12/94

PAGE I RPT. 213

CPP-S

\section{TOTALS}

ALPHA CURTES

$\%$ OF TOTAL

B+G CURIES

\% OF TOTAL

$1.380 E+05$

100.000

$2.790 E+05$

100.000

$1.940 E+05$

$1.480 E+05$

100.000

$.590 E+05$

TOTAL ERNS OF NH

TOTAL ERMIS OF PU

TOTAL ERAMS OF PU-239+24I

TOTAL GRAMS OF $U$

TOTAL GRAMS OF TH

TOTAL ERMIS DF NP

VOLUNE (CU. METERS )

$1.290 E+01 \quad 3.075 E+01 \quad 2.010 E+02 \quad 2.730 E+01 \quad 9.105 E+01$

WEIEHT (KILOERNA)

Gring Totals (curies)

$\begin{array}{lllll}1.380 E+05 & 2.790 E+05 & 1.940 E+05 & 1.480 E+05 & 7.590 E+05\end{array}$ 


$$
\text { CPP SOLID WASTE SUMARY }
$$

WERF INCINERATOR PROCESSED WUSTE

MCLIDES
CE-244
CURIES
$\%$ OF TOTAL
CS-237
CURIES
$\%$ OF TOTAL
N-95
CURIES
$\%$ OF TOTAL
PR-144
CURIES
$\%$ OF TOTAL
RH-106
CURIES
$\%$ OF TOTAL
PU-106
CURIES
$\%$ OF TOTAL
SB-125
CURIES
$\%$ OF TOTAL
SR-90
CURIES
$\%$ OF TOTAL
$Y-90$
CURIES
$\%$ OF TOTAL
$2 A-95$
CURIES
$\%$ OF TOTAL

HALF LIFE

(DAYS)

JANUARY

FEBRUARY

FROM JAN TI

APRIL

MaY

JUNE

4.137E-02

19.700

$1.101 E+04$

2. 100E-02

10.000

$3.498 E+01$

$6.510 E-03$

3.100

1. 200E-02

4. 137E-02

19.700

$3.441 E-04$

2.100E-02 10.000

3. $726 E+02$

2.100E-02 10.000

$1.007 E+03$

$9.240 E-03$

$$
4.400
$$

$1.059 E+04$

2. 100E-02 10.000

$2.667 E+00$

2. $100 E-02$

10.000

$6.403 E+01$

6.510E-03 3.100

$\%$ OF TOTAL

B+6 curIEs

100.000

$.100 E-01$

ANUAL

PAGE 1 RPT. 223

TOTAL

4. 137 E-02

19.700

2.100E-02

10.000

$6.520 E-03$

3.100

4.137E-02

19.700

2.100E-02 10.000

$2.100 E-02$
10.000

9. 240E-03

4.400

2.100E-02 10.000

$2.100 E-02$

$6.5105-03$ 3.100

TOTAL GRANS OF AM

TOTAL GRAMS Of PU

TOTAL ERANS OF PU

TOTAL ERAMS OF U

TOTAL ERANS OF NP

VOLURE (CU. METERS)

5. 505E +01

$5.505 E+01$

VEIGHT (KILOCRAMS)

$5.480 E+03$

$5.480 E+03$

ERAN TOTALS (CURIES)

2. 100E-01

2.100E-01 

.

(1)


CPP Facility Detail Data for Months July to December Annual Average Concentration and Total Curies for January to December 


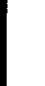




\section{IDAHO OPERATTONS OFFICE
IFPTE}

RUN DATE: 05/11/9

RADIOACTIVE WASTE MUNAGEMENT INFOPAATION SYSTEM

CPP FAST STACK AIRBORNE MASTE SUMMARY

FOR JAN THROUEH DEC 1993

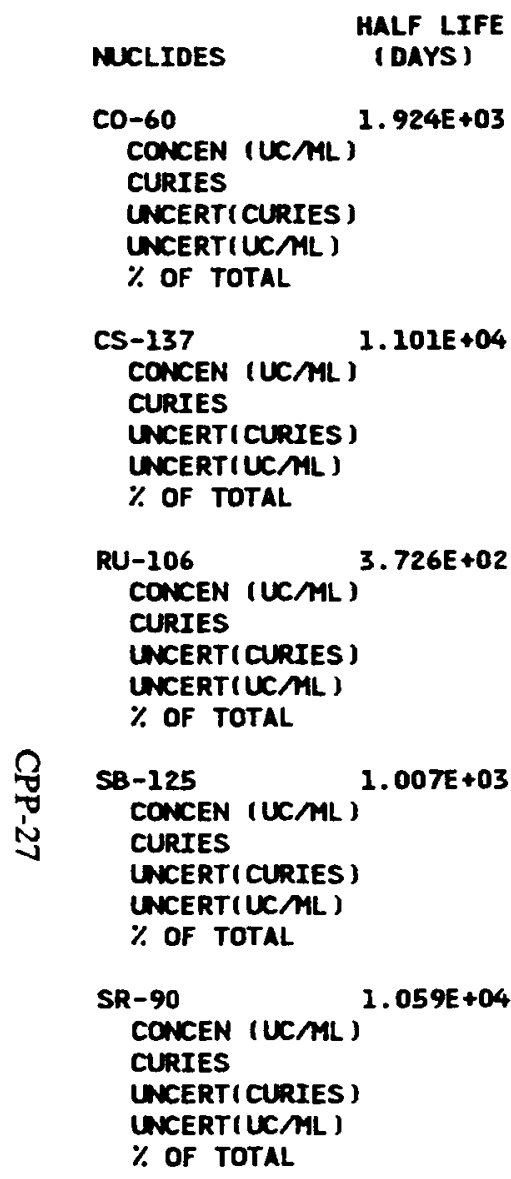

JuLY

Auaust

SEPTEMBER

OCTOBER

NOVERBER

$2.548 E-16$
$2.650 E-08$
$7.110 E-09$

PAGE $\underset{\text { CPP-A }}{1}$ RPT. 221

MNuAL

$\begin{array}{ll}\text { AVG CONCEN YEARLY } & \text { YEARLY } \\ \text { RELEASE }\end{array}$

TOT CURIES RELEASE* CG** \% OF CG

CONCEN (UCML)

$2.548 E-16$
$2.650 E-08$

6.578E-16 8.262E-22 8.00E-11

$8.315 E-07$

$\begin{array}{lllllllll}5.775 E-16 & 2.952 E-15 & 7.352 E-15 & 4.451 E-15 & 4.558 E-15 & 5.624 E-15 & 6.639 E-15 & 8.339 E-21 & 4.00 E-10 \\ 5.890 E-08 & 3.100 E-07 & 7.720 E-07 & 4.540 E-07 & 4.740 E-07 & 5.680 E-07 & 8.392 E-06 & & \end{array}$

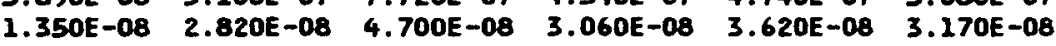
1.745
13.839
20.521
84.844
36.114
70.471
14.002

$7.808 E-15$

$8.120 E-07$

2. 280E-07

61.867

$6.424 E-16 \quad 8.068 E-22 \quad 3.00 E-11$

8.120E-07

1.355

4.382E-15 1.838E-14 9.905E-15 $7.951 E-16$

4.470E-07 1.930E-06 1.040E-06 8.110E-08

$\begin{array}{llll}6.510 E-08 & 1.650 E-07 & 1.040 E-07 & 2.890 E-08\end{array}$

$\begin{array}{llll}13.241 & 86.161 & 27.645 & 15.156\end{array}$

$2.356 E-15 \quad 1.664 E-14 \quad 2.089 E-20 \quad 1.00 E-09$

$2.380 E-07 \quad 2.103 E-05$

3.000E-08

29.529

35.085

$2.814 E-14$

$2.870 E-06$

8.140E-07

$1.857 E-14$
$1.950 E-06$

5.610E -07

85.014

51.834 $\begin{array}{lll}2.284 E-14 & 2.869 E-20 & 9.00 E-12 \\ 2.887 E-05 & & \end{array}$

48.171 
RADIOACTIVE WASTE MUNAGEMENT INFORHATION SYSTEM

CPP FAST STACK AIRBORNE WUSTE SUMMARY

FOR JAN THROUGH DEC 1993

NuCLIDES

HALF LIFE

(DAYS)
vLY

AUEUST

\section{SEPTEMrar}

OCTOBER NOVEYBER

\section{MNUAL} AVS CONCEN
TOT CURIES
PAGE 2 RPT. 221 CPP-A

YEARLY YEARLY RELEASE

\section{TOTALS}

ALPHA CURIES

$\%$ OF TOTAL

B $+G$ CURIES

$\%$ OF total

3.376E-06 2.240E-06

3.762E-06

DeCEMBER

\% OF CG

\begin{tabular}{llllllll} 
VOLUNE (CU. METERS) & $1.020 E+08$ & $1.050 E+08$ & $1.050 E+08$ & $1.020 E+08$ & $1.040 E+08$ & $1.010 E+08$ & $1.264 E+09$ \\
\hline GRAND TOTALS (CURIES) & $3.376 E-06$ & $2.240 E-06$ & $3.762 E-06$ & $5.351 E-07$ & $1.312 E-06$ & $8.060 E-07$ & $5.993 E-05$
\end{tabular}

* This number is derived from a disparsion fector equation. The dispersion factor is derived from the straight line gaussian model and is provedad by the Nutional Dosenic Atmospheric Administration (NOM).

* NOTE: DCG valwe obtainad from DOE ORDER 5400.5 dated February 8, 1990, figures III-1 and III-3 


\section{RADIOACTIVE WUSTE MUUGEMENT IMFOPRATION SYSTEM}

CPP MUIN STACK AIRBORNE MUSTE SUMHARY

FOR JAN THROUEH DEC 1993

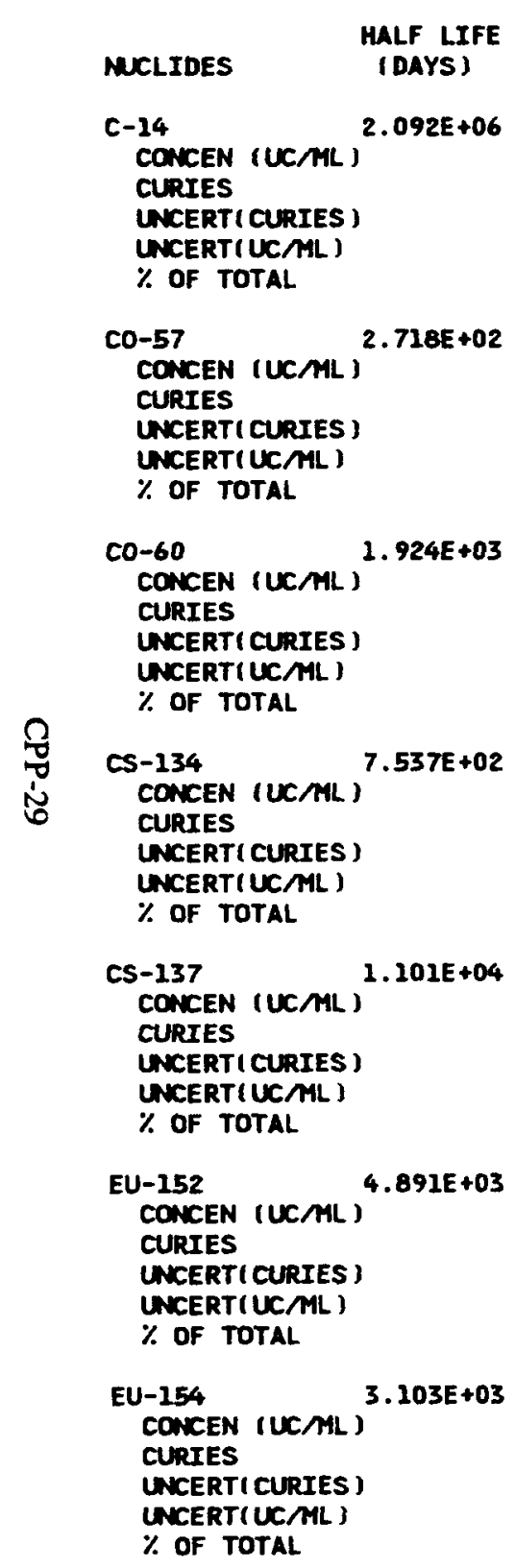

MLY

AUEUST

SEPTEMBER

$\begin{array}{ccc}\text { OCTOBER NOVEMBER } & \text { DECEMBER } \\ & & \\ 2.353 E-11 & 8.264 E-12 & 8.475 E-12 \\ 2.800 E-03 & 1.000 E-03 & 1.000 E-03\end{array}$

MNUAL

PAGE 1 RPT. 219

AVG CONCEN CPP-A

YEARLY

TOT CURIES RELEASE* CGN* \% OF CG

CONCEN IUCAL

CURIES

UNCERTICURIES I

$\%$ OF TOTAL
0.525
0.020
0.020
0.015

$2.146 E-16 \quad 1.496 E-16$

$2.640 E-08 \quad 1.870 E-08$

$7.280 E-09 \quad 4.790 E-09$

$\begin{array}{lll}5.190 E-17 & 4.365 E-23 \quad 2.00 E-09\end{array}$

$7.780 E-08$

$\begin{array}{ll}1.423 E-14 & 2.976 E-16 \\ 1.750 E-06 & 3.720 E-08\end{array}$

$\begin{array}{ll}1.750 E-06 & 3.720 E-08 \\ 1.340 E-07 & 6.430 E-09\end{array}$

$2.928 E-15 \quad 2.462 E-21 \quad 8.00 E-11$

0.003

$4.389 E-06$

0.003

$6.983 E-16 \quad 2.236 E-13 \quad 1.168 E-14 \quad 1.790 E-15$

$8.240 E-08 \quad 2.750 E-05 \quad 1.460 E-06 \quad 2.130 E-07$

8.320E-09 $8.960 E-07 \quad 3.460 E-08 \quad 2.060 E-08$

2. $983 E-16 \quad 5.831 E-14 \quad 4.904 E-20 \quad 2.00 E-10$ 3.520E-08 8.741E-05

1. $110 E-08$

$0.040 \quad 0.002$

$\begin{array}{lllll}2.136 E-13 & 2.049 E-11 & 8.560 E-13 & 3.546 E-13\end{array}$

$2.520 E-05 \quad 2.049 E-11$ 8.560E-13

2.780E-07

$2.520 E-03$

.070E-04 4.220E-05

4. 220E-05
$5.040 E-07$

$2.124 E-13$

$2.570 E-05 \quad 7.420 E-06$

3.643

0.162

0.008

0.001

$7.420 E-06$
$1.140 E-07$

$4.991 E-12 \quad 4.198 E-18 \quad 4.00 E-10$

0.012

$1.201 E-15$ 1.010E-21 5.00E-11

2.800E-06

$4.333 E-14$
$5.330 E-06$

5.330E-06

6.577E-15 5.531E-21 5.00E-11

$.060 E-07$

9.859E-06

0.008 
RADTOACTIVE WUSTE MUNAGEMENT INFORHATION SYSTEM

\section{CPP MAIN STACK AIRBORNE MUSTE SUMARY}

FOR JAN THROUGH DEC 1993

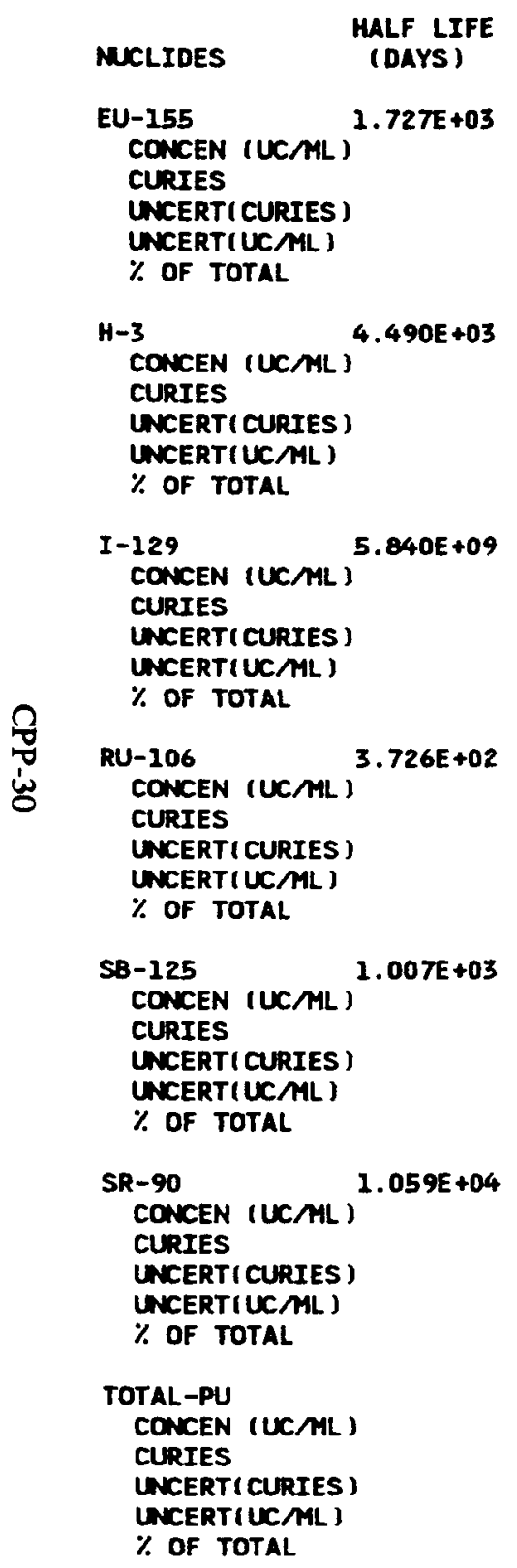$$
\% \text { OF TOTAL }
$$
$\begin{array}{lllllllll}8.915 E-08 & 5.133 E-10 & 5.051 E-10 & 4.409 E-09 & 4.178 E-08 & 4.284 E-08 & 4.137 E-08 & 3.480 E-14 & 1.00 E-07 \\ 1.052 E+01 & 6.314 E-02 & 6.314 E-02 & 5.247 E-01 & 5.055 E+00 & 5.055 E+00 & 6.202 E+01 & & \end{array}$

$$
\begin{array}{lllllll}
99.796 & 91.270 & 95.308 & 98.379 & 99.909 & 99.910 & 99.812
\end{array}
$$

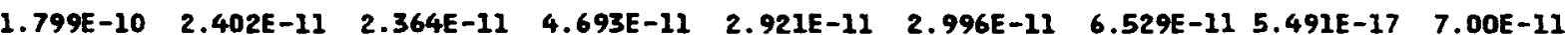
$\begin{array}{lllllll}2.123 E-02 & 2.955 E-03 & 2.955 E-03 & 5.585 E-03 & 3.535 E-03 & 3.535 E-03 & 9.786 E-02\end{array}$
0.201
4.272
4.460
1.047
0.070
0.070
0.158 $\begin{array}{lllllllll}1.788 E-12 & 4.114 E-12 & 2.400 E-13 & 2.580 E-13 & 2.380 E-13 & 3.941 E-14 & 6.708 E-13 & 5.641 E-19 & 3.00 E-11 \\ 2.110 E-04 & 5.060 E-04 & 3.000 E-05 & 3.070 E-05 & 2.880 E-05 & 4.650 E-06 & 1.005 E-03 & & \end{array}$ $\begin{array}{llllll}6.140 E-06 & 2.100 E-05 & 8.320 E-07 & 8.560 E-07 & 6.470 E-07 & 2.380 E-07\end{array}$
0.002
0.731
0.045
0.006
0.001
0.002

$\begin{array}{llll}8.339 E-15 & 6.504 E-14 & 3.432 E-15 & 4.269 E-16\end{array}$

$9.890 E-07 \quad 8.000 E-06 \quad 4.290 E-07 \quad 5.080 E-08$

$\begin{array}{llll}7.300 E-08 & 6.710 E-07 & 5.500 E-08 & 2.000 E-08\end{array}$

$$
0.012 \quad 0.001
$$

$\begin{array}{lllll}2.076 E-14 & 1.285 E-13 & 1.168 E-13 & 1.588 E-12 & 1.339 E-14\end{array}$ $\begin{array}{lllll}2.450 E-06 & 1.580 E-05 & 1.460 E-05 & 1.890 E-04 & 1.620 E-06\end{array}$ $\begin{array}{lllll}6.480 E-07 & 3.330 E-06 & 2.110 E-06 & 6.050 E-06 & 4.330 E-07\end{array}$

$$
\begin{array}{lll}
0.023 & 0.022 & 0.035
\end{array}
$$

$3.479 E-14 \quad 2.926 E-20 \quad 1.00 E-09$ 5.215E-05

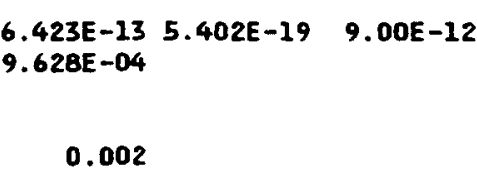

0.002

YEARLY RELEASE $\%$ OF CG 
RADIOACTIVE WUSTE MAKMGEMENT INFORMATION SYSTEM

CPP MUIN STACK AIRBOPNE MUSTE SUMARY FOR JAN THROUEH DEC 1993

MUCLIDES HALF LIFE (DAYS)
JULY

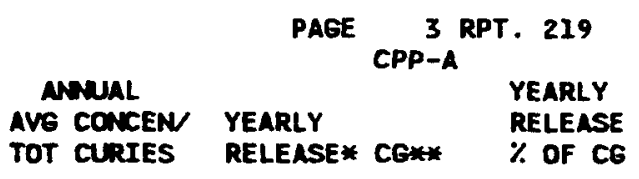

\section{TOTALS}

ALPHA CURIES

$\%$ OF TOTAL

$B+G$ CURIES

$\%$ OF TOTAL

VOLUME (CU. METERS)

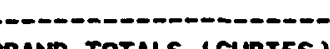

GRAND TOTALS (CURIES )

* This number is cherived

gaussian model and is proveded by the National Doeanic Atmospheric Aduinistration (NovA).

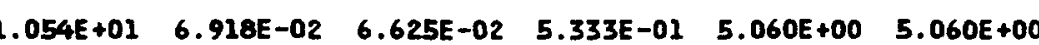

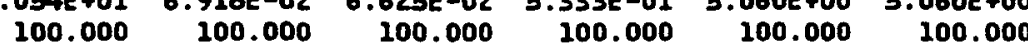

$2.540 E-09$

$6.214 E+01$

** NOTE: DCG value obtained from DOE ORDER 5400.5 datod Fabruery 8, 1990, Figures III-1 and III-3 
DAHO OPERATIONS OFFICE

U.S. DEPARTMENT OF ENERGY

RAOTOACTIVE WASTE MAMAGEMENT INFORMATION SYSTEM

\section{CPP NACF STACK AIRBOPNE WUSTE SUMMARY}

FOR JAN THROUEH DEC 1993

MUCLIDES
(DALF LIFE
SR-90
CONCEN (UCML)
CURIES
UNCERT(CURIES)
UNCERT (UCML)
\% OF TOTAL

\section{TOTALS}

ALPHA CURIES

$\%$ OF TOTAL

$B+G$ CURIES

$\%$ OF TOTAL

2.100E-08

100.000

$1.260 E+08$

$9.590 E+07 \quad 9.930 E+07 \quad 1.230 E+08$

$3.340 E-08$

100.000

1. $103 E-07$

100.000

VOLUNE ICU. METERS )

2. $100 E-08$

$9.570 E+07 \quad 8.930 E+07 \quad 1.236 E+09$

GRAND TOTALS (CURIES)

3.340E-08

$1.103 E-07$

* This nuber is derived from a dispersion factor eqpetion. The dispersion factor is derived from the straight line goussian model and is provedad by the National Doeanic Atmospheric Adainistration (NOAA). 
 \\ U.S. DEPARTMENT OF ENERGY}

RADIOACTIVE WASTE MANAGEMENT INFORMATION SYSTEM

CPP RAL STACK AIPBONAE WUSTE SUMARY

FOR JAN THROUEH DEC 1993

\begin{tabular}{|c|c|}
\hline CLIDES & $\begin{array}{l}\text { HALF LIFE } \\
\text { (DAYS) }\end{array}$ \\
\hline $\begin{array}{l}\text { CS-137 } \\
\text { CONCEN (UCML) } \\
\text { CURIES } \\
\text { UNCERT (CURIES) } \\
\text { UNCERT (UCML) } \\
\text { \% OF TOTAL }\end{array}$ & $1.101 E+04$ \\
\hline $\begin{array}{l}\text { SR-90 } \\
\text { CONCEN (UCML) } \\
\text { CURIES } \\
\text { UNCERT (CURIES) } \\
\text { UNCERT (UCML ) } \\
\% \text { OF TOTAL }\end{array}$ & $1.059 E+04$ \\
\hline
\end{tabular}

JULY

Aurust

\section{OCTOBER NOVEMER}

\section{$1.257 E-15$
$2.540 E-08$ \\ $2.540 E-08$
$1.310 E-08$ \\ 0.041
$6.170 E-05$
2.710E-06 \\ 3. $054 \mathrm{E}-12$ \\ 99.959}

\section{$\cos (\cos )$}

AVG CONCEN
TOT CURIES
PAGE 1 RPT. 225 CPP-A

YEARLY

RELEASE $\%$ OF CG

\section{TOTALS \\ ALPHA CURIES \\ $\%$ OF TOTAL \\ B+G CURIES \\ $\%$ OF TOTAL}

$6.173 E-05$
100.000

$6.570 E-05$

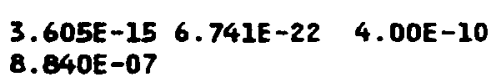

1.345

$2.644 E-13 \quad 4.943 E-20 \quad 9.00 E-12$

6. $482 E-05$

98.655

\section{VOLUME (CU. METERS)} $2.020 E+07 \quad 2.080 E+07 \quad 2.080 E+07$ GRAND TOTALS (CURIES)

* This numbar is darived from a dispersion factor equation. The dispersion factor is derived from the straight 1 ine goussian model and is proveded by the Nitional Dceanic Atmospheric Adrinistration (NOMA).

* NOTE: DCG value obtained from DOE ORDER 5400.5 dated Fabruary 8, 1990 , Figures III-1 and III-3 
RADIOACTIVE WASTE MANAGEMENT INFORMATION SYSTEM

CPP IIEUTD MASTE TO INFILTRATTON POND FOR JAN THROUGH DEC 1993

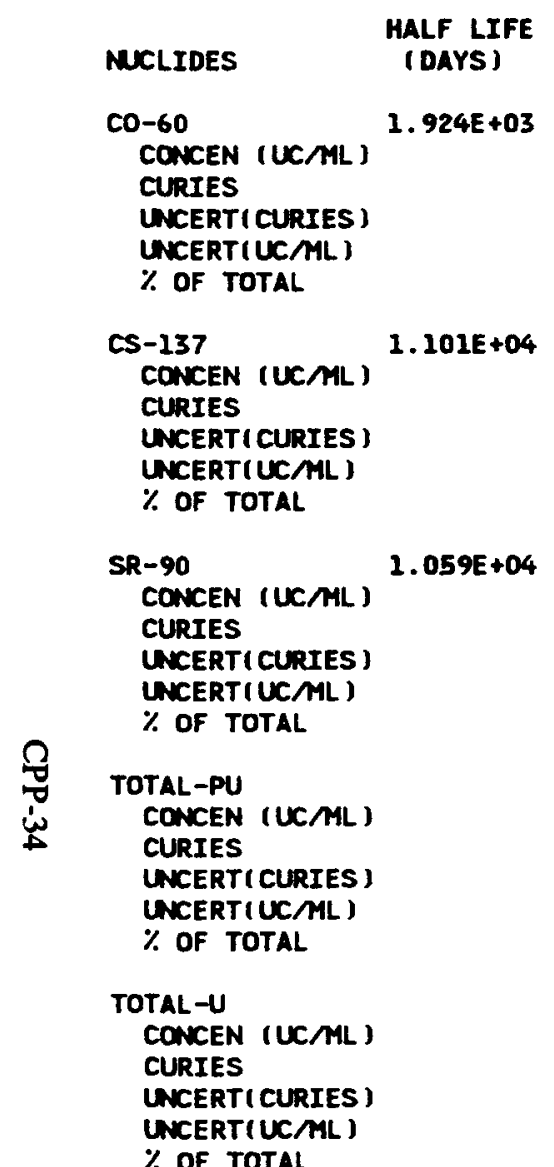

2.207E-10
2.834

7.707

5.391

9.191

11.481

1.199E-09 7.010E-10 7.979E-10 $1.500 E-09$

$2.410 E-04 \quad 1.430 E-04 \quad 1.540 E-04 \quad 2.760 E-04$

4.020E-05 4.080E-05 3.860E-05 5.520E-05

31.911

19.751

21.509

38.032

$1.900 E-11$

$3.820 E-06$

$1.010 E-06$

0.506

$2.433 E-09$
$4.890 E-04$

$4.890 E-04$
$4.500 E-05$

$4.570 E-04$

$4.560 E-05$

4.750E -0

$4.750 E-04$
$4.330 E-05$

$3.000 E-11$

$\begin{array}{lll}930 E-06 & 9.200 E-07 \quad 1.130 E-06\end{array}$

0.809

0.305

0.862
64.749

63.122

66.342

73.775

\section{SEPTEMBER \\ OCTOBER \\ NOVEMER \\ DECERER}

$3.343 \mathrm{E}-10$

$6.820 E-05$

$6.820 E-05$
$1.300 E-05$

9.420

$2.489 E-10 \quad 3.383 E-10$

$4.580 E-05 \quad 6.360 E-05$

$1.918 E-10$

$\begin{array}{ll}580 E-05 & 6.360 E-05 \\ 170 E-05 & 1.320 E-05\end{array}$

$3.970 E-05$

$.970 E-05$
$.130 E-06$

5.950

6.311

13.882

6.406
1.065E-10

$2.140 E-05$

2. $735 E-10$

$1.570 E-0.5$

8.586

$\begin{array}{lll}2.000 E-10 & 3.625 E-10 & 2.798 E-10 \\ 3 & 6.670 E-05 & 5.260 E-05\end{array}$

$3.860 E-05 \quad 6.670 E-05 \quad 5.260 E-05$

ANNUAL

AVG CONCEN

PAgE 1 RPT. 218

3.760E-05 6.210E-05

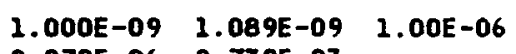

33.403

23.479

60.190

48.860

\begin{tabular}{|c|c|c|c|c|c|c|c|}
\hline $\begin{array}{l}\text { TOTALS } \\
\text { ALPHA CURIES } \\
\% \text { OF TOTAL } \\
\text { B+G CURIES } \\
\% \text { OF TOTAL }\end{array}$ & $\begin{array}{r}3.820 E-06 \\
0.506 \\
7.514 E-04 \\
99.494\end{array}$ & $\begin{array}{r}7.240 E-04 \\
100.000\end{array}$ & $\begin{array}{r}5.790 E-06 \\
0.809 \\
7.102 E-04 \\
99.191\end{array}$ & $\begin{array}{r}2.210 E-06 \\
0.305 \\
7.235 E-04 \\
99.695\end{array}$ & $\begin{array}{r}3.950 E-06 \\
0.862 \\
4.542 E-04 \\
99.138\end{array}$ & $\begin{array}{r}6.197 E-04 \\
100.000\end{array}$ & $\begin{array}{r}4.815 E-05 \\
0.413 \\
1.162 E-02 \\
99.587\end{array}$ \\
\hline VOLLME (LITERS ) & $2.010 E+08$ & $2.040 E+08$ & $1.930 E+08$ & $1.840 E+08$ & $1.880 E+08$ & $2.070 E+08$ & $2.515 E+09$ \\
\hline GRAND TOTALS (CURIES) & $7.552 E-04$ & $7.240 E-04$ & $7.160 E-04$ & $7.257 E-04$ & $4.581 E-04$ & $6.197 E-04$ & $1.167 E-02$ \\
\hline
\end{tabular}

TOTALS (CURIES)

7.552E-04 7.240E-04 $\quad 7.160 E-04 \quad 7.257 E-04 \quad 4.581 E-04 \quad 6.197 E-04 \quad 1.167 E-02$

* NOTE: DCG value obtained from DOE ORDER 5400.5 dated February8, 1990, Figures III-1 and III-3 

CPP FIST STACK ATPEODNE WASTE SUMARY FOR JAN THROUGH JUN 1993

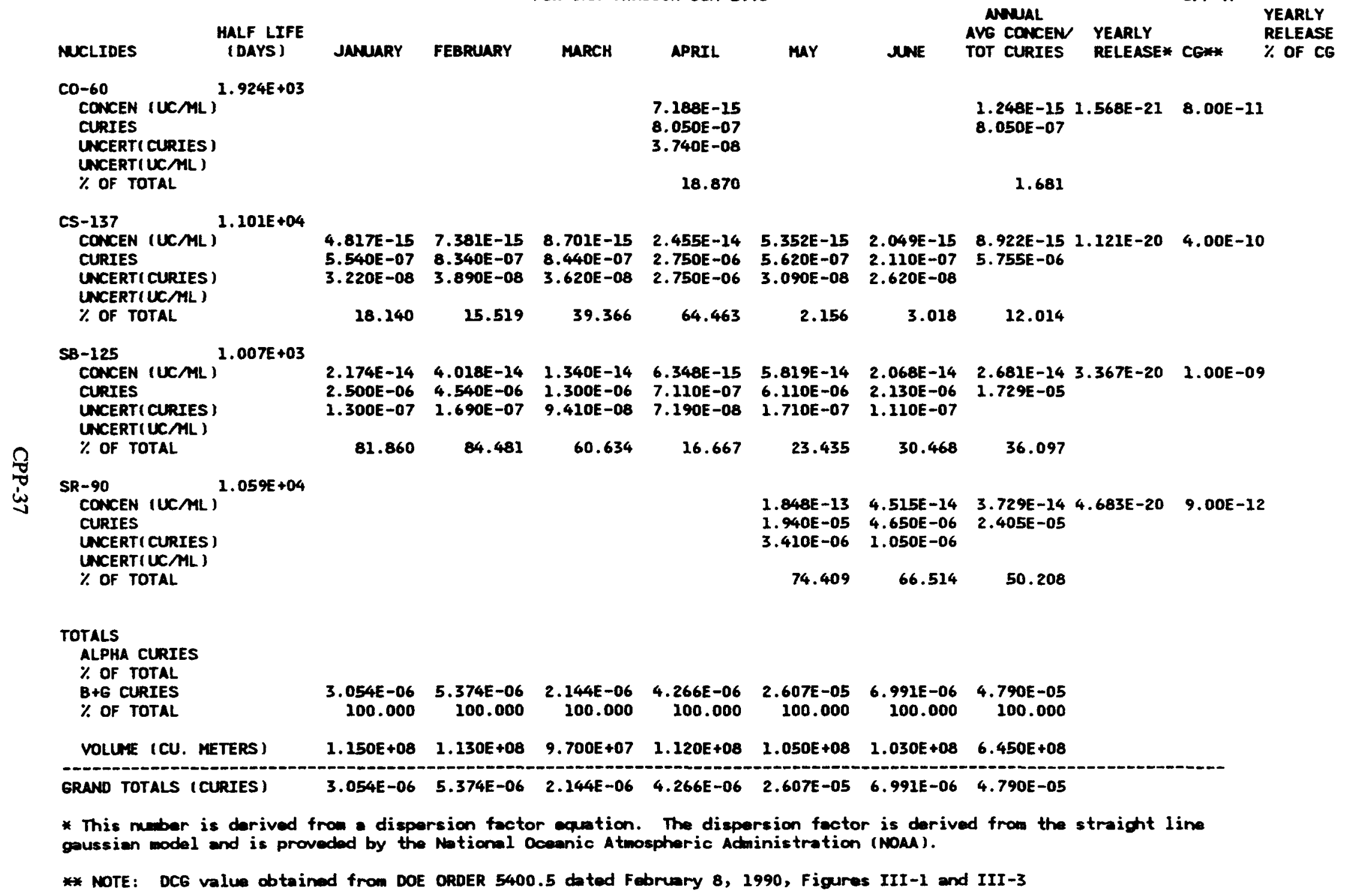


IDAHO OPERATIONS OFFICE

U.S. DEPARTMENT OF ENEREY

RADIOACTIVE WASTE MANAGEMENT INFORMATION SYSTEM

CPP MAIN STACK AIRBORNE WASTE SUMMARY

FOR JAN THROUGH JUN 1993

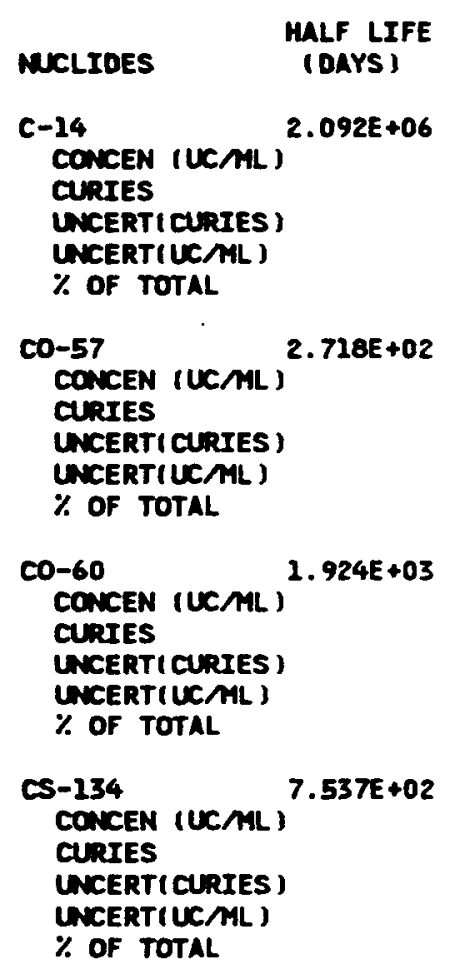

JANARY FEBRUARY

$1.000 E-03 \quad 2.000 E-03$

MARCH APRIL

maY

JUNE

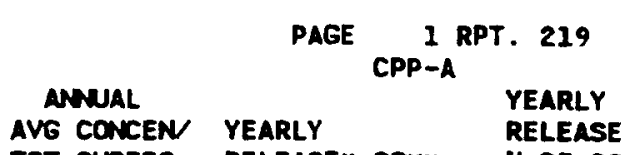

$\begin{array}{cll}\text { ANWAL } & \text { YEARLY } \\ \text { AVG CONCEN } & \text { YEARLY } & \text { RELEASE } \\ \text { TOT CURIES } & \text { RELEASE* CE** } & \% \text { OF CG }\end{array}$
$1.101 E+04$

CS-237
CONCEN (UCML)
CURIES
UNCERT(CURIES)
UNCERTIUCAML)
\% OF TOTAL
EU-152
CONCEN (UCML)
CURIES
UNCERT(CURIES)
UNCERT(UCML)
\% OF TOTAL

EU-154

3. 203E+03

CONCEN (UCAL)

CURIES

UNCERT ( CURIES)

UNCERT (UCAL)

\% OF TOTAL
$7.812 E-12 \quad 7.463 E-12$

$0.020 \quad 0.020$

$\begin{array}{lllllllll}5.742 E-15 & 5.239 E-14 & 4.099 E-14 & 1.022 E-13 & 8.125 E-14 & 1.638 E-13 & 7.499 E-14 & 6.307 E-20 & 2.00 E-10\end{array}$

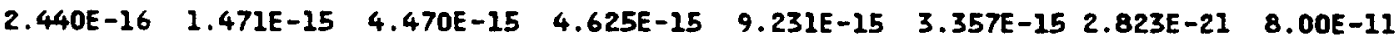

$\begin{array}{llllll}3.270 E-08 & 1.780 E-07 & 5.990 E-07 & 5.920 E-07 & 1.200 E-06 & 2.602 E-06\end{array}$

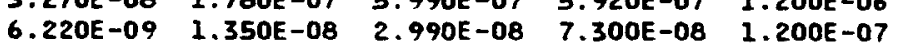

$2.154 E-11 \quad 6.194 E-12 \quad 5.209 E-18 \quad 6.00 E-09$ $\begin{array}{ll}2.154 E-11 & 6.194 E-12 \\ 2.800 E-03 & 4.800 E-03\end{array}$

$0.523 \quad 0.012$

$2.515 E-16 \quad 4.219 E-17 \quad 3.549 E-23 \quad 2.00 E-09$ $3.270 E-08 \quad 3.270 E-08$

8.320E-09

$2.640 E-010$

$\begin{array}{llll}1.280 E-07 & 2.240 E-07 & 3.560 E-07 & 3.530 E-07\end{array}$

0.007

0.004

$6.078 E-13 \quad 3.910 E-12 \quad 3.174 E-12 \quad 7.761 E-12 \quad 6.938 E-12 \quad 1.415 E-11 \quad 6.134 E-12 \quad 5.159 E-18 \quad 4.00 E-10$ $\begin{array}{llllllll}7.780 E-05 & 5.240 E-04 & 3.840 E-04 & 1.040 E-03 & 8.880 E-04 & 1.840 E-03 & 4.754 E-03\end{array}$

$\begin{array}{llllll}7.230 E-07 & 1.010 E-05 & 6.800 E-06 & 1.210 E-05 & 2.490 E-05 & 1.810 E-05\end{array}$

0.002

0.010

0.577

0.007

0.006

0.344

0.012

$1.385 E-14 \quad 2.323 E-15 \quad 1.953 E-21 \quad 5.00 E-11$ $1.800 E-06 \quad 1.800 E-06$

$7.030 E-08$ $\begin{array}{lllllll}3.017 E-15 & 8.134 E-15 & 7.313 E-16 & 2.292 E-14 & 5.843 E-15 & 4.915 E-21 & 5.00 E-11 \\ 3.650 E-07 & 1.090 E-06 & 9.360 E-08 & 2.980 E-06 & 4.529 E-06 & \end{array}$

$\begin{array}{llll}3.380 E-08 & 5.580 E-06 & 1.900 E-08 & 2.090 E-07\end{array}$

0.001

0.001 
 \\ U.S. DEPARTMENT OF ENERGY}

RADIOACTIVE WASTE MANAGEMENT INFORMATION SYSTEM

\section{CPP MAIN STACK AIRBORNE MASTE SUMTARY}

FOR JAN THROUGH JUN 1993

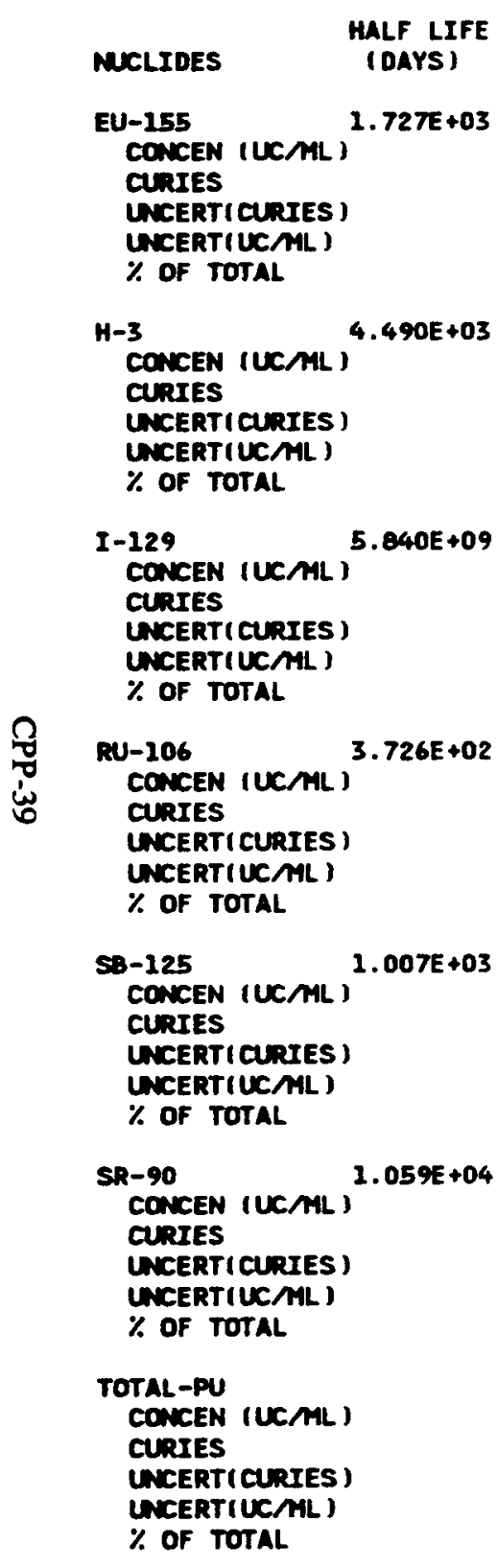

J

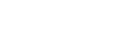


SCHED. NO. P6IPMO09-D

\section{RADIOACTIVE WASTE MANAGEMENT INFORMATION SYSTEM \\ CPP MUIN STACK AIRBORNE WASTE SUMUARY FOR JAN THROUEH JUN 1993}

.S. DEPARTMENT OF ENERGY

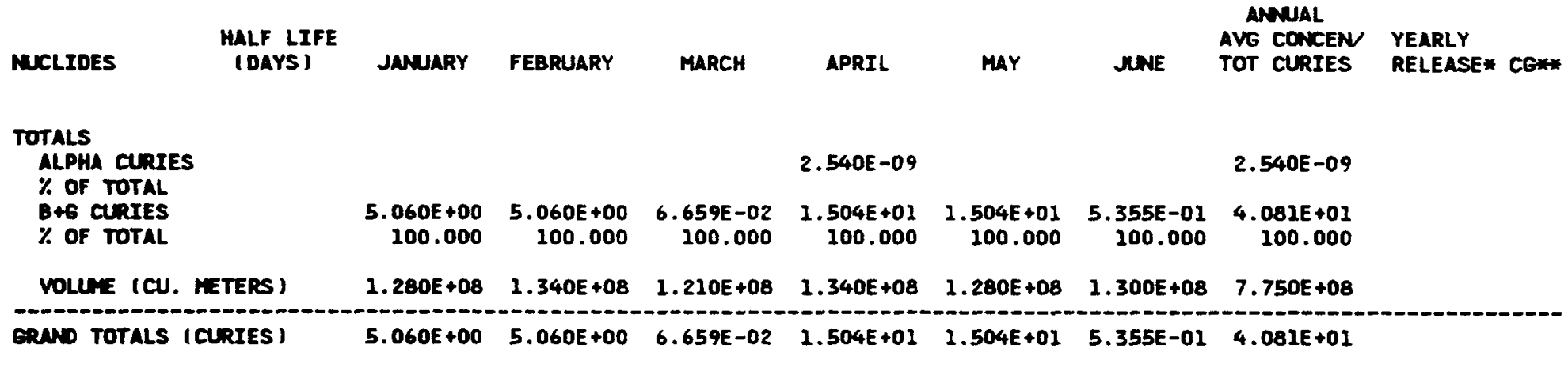

RUN DATE: 05/12/94

AAGE 3 RPT. 219 CPP-A

YEARLY

RELEASE

\% OF CG

* This muber is derived from a disparsion factor equation. The disparsion factor is derived from the straight line oussien model and is proveded by the National Oceanic Atmospheric Administration (NOAA).

* NOTE: DCG value abtained from DOE ORDER 5400.5 dated Fabruary 8, 1990, Figures III-1 and III-3 
CPP NUCF STACK AIRBORNE MUSTE SUMPARY FOR JAN THROUGH JUN 1993

MUCLIDES MALF LIFE
(DAYS)
SR-90
CONCEN (UCML)
CURIES
UNCERT(CURIES)
UNCERT(UCAL)
\% OF TOTAL

\section{Totals}
ALPHA CURIES
$\%$ Of total
B+6 CURIES
\% Of total

5. 590E-08

100.000

6. $103 E-16$

$1.650 E-08$

100.000

MARCH

APRIL

maY

JUNE

ANNALL
AVG CONCEN

PAgE 1 RPT. 220

AVG CONCEN

TOT CURIES

CPP-A

YEARLY

RELEASE

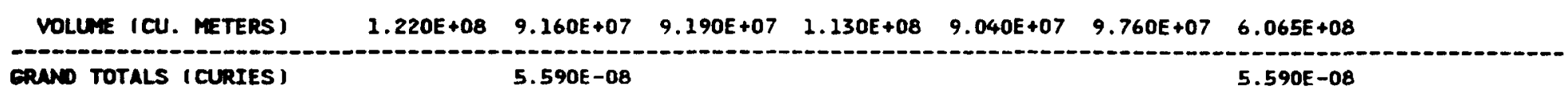

* This nubar is derived from a dispersion factor oquation. The dispersion factor is derived from the straight line goussien model and is proveded by the National Oceanic Atmospheric Administration (NOAA).

\# NOTE: DCG value obtained from DOE ORDER 5400.5 dated february 8, 1990 , figures III-I and III-3 
CPP RAL STACK ATPROPNE MASTE SUMMARY FOR JAN THROUGH JUN 1993

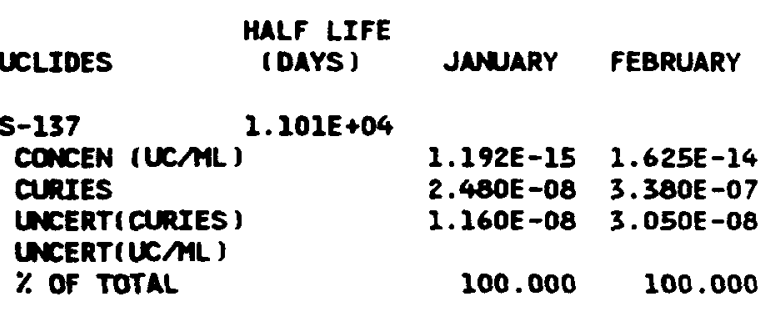
$1.059 E+04$

SR-90

\section{CURIES}

(

UNCERTIUCML)

$\%$ OF TOTAL

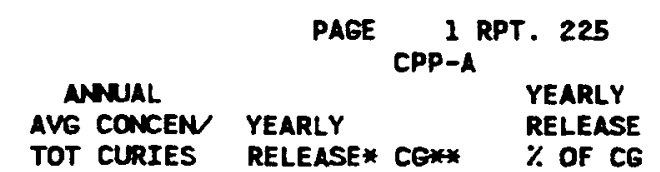

$\begin{array}{llllll}2.292 E-14 & 1.577 E-15 & 7.026 E-15 & 1.314 E-21 & 4.00 E-10 \\ 4.630 E-07 & 3.280 E-08 & 8.586 E-07 & & \end{array}$

$12.922 \quad 100.000 \quad 21.580$
$1.545 E-13$
$3.120 E-06$
1.150E-06

87.078

$2.553 E-14 \quad 4.774 E-21 \quad 9.00 E-12$

$3.120 E-06$

78.420

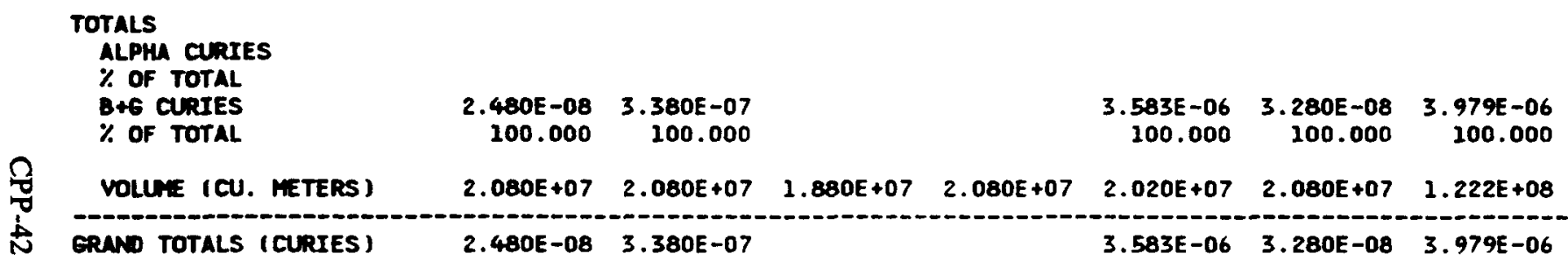

* This nuber is derived from a dispersion factor equation. The dispersion factor is darived from the straight line goussian model and is provedad by the National Doeanic Atmospheric Administration (NOAA).

* NOTE: DCE value obtained from DOE ORDER 5400.5 dated February 8, 1990, Figures III-I and III-3 


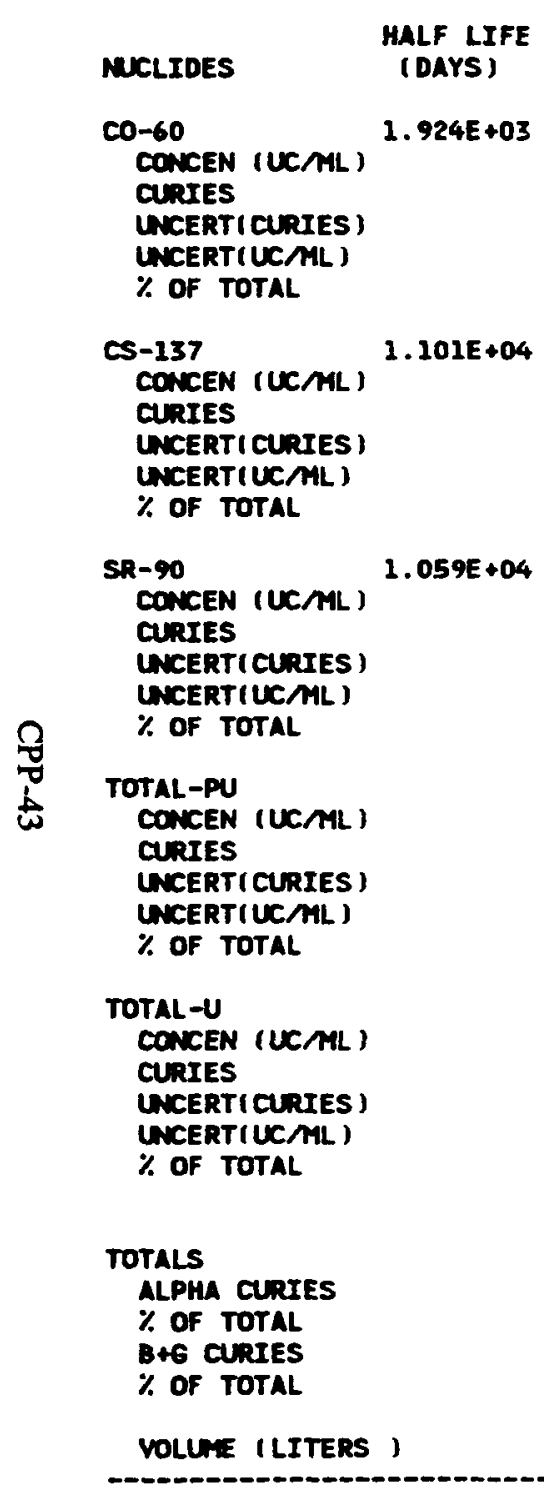

\section{CPP LIQUTD WASTE TO INFILTRATION POND}

FOR JAN THROUGH JUN 1993
JANUARY FEBRUARY

$\begin{array}{cc}\text { MARCH } & \text { APRIL } \\ & \cdot \\ 4.622 E-10 & 2.355 E-09 \\ 1.100 E-04 & 5.370 E-04 \\ 9.770 E-06 & 2.200 E-05\end{array}$

12.597

34.297

5.591E-09 $9.861 E-1 I$

$\begin{array}{ll}1.230 E-03 & 2.130 E-05 \\ 5.050 E-05 & 1.060 E-05\end{array}$

$\begin{array}{ll}1.760 E-04 & 4.100 E-04 \\ 2.540 E-05 & 2.110 E-05\end{array}$

38.349

2.945

20.156

26.186

$3.170 E-05$

$1.020 E-05$

MaY

JNE

PAGE 1 RPT. 218
CPP-L
ANUAL

ANNAL

AVG CONCEN
TOT CURIES

CG * $\%$ OF CG

$\begin{array}{ll}6.500 E-09 & 6.991 E-10 \\ 1.430 E-03 & 1.510 E-04\end{array}$

$\begin{array}{ll}1.430 E-03 & 1.510 E-04 \\ 1.980 E-04 & 4.320 E-05\end{array}$

44.585

20.877

$6.009 E-10$
$1.370 E-04$
$4.560 E-05$

5.483

4.228E-10 5.543E-10 5.00E-06

$9.470 E-0.5 \quad 7.417 E-04$

$1.620 E-05$

13.164

9.674

2. $900 E-11$

$6.380 \mathrm{E}-06$

$1.760 E-06$

0.199

2.601E-11 $3.399 E-11$

$6.190 E-06 \quad 7.750 E-06$

6.190E-06 $1.820 E-06$

0.709

0.495

2.467

$3.580 E-06$

$3.238 E-05$

$\begin{array}{ll}2.459 E-09 & 2.551 E-09 \\ 5.410 E-04 & 5.510 E-04\end{array}$

$\begin{array}{ll}5.410 E-04 & 5.510 E-04 \\ 4.930 E-05 & 6.130 E-05\end{array}$

2.441E-0～2.079E-09

5.810E-04 4.740E-04

$\begin{array}{lll}2.538 E-09 & 2.446 E-09 & 2.416 E-09 \\ 5.380 E-04 & 5.480 E-04 & 3.233 E-03\end{array}$

$16.867 \quad 76.179$

$66.538 \quad 30.273$

93.051

76.177

42.167

$\begin{array}{lllllll}6.380 E-06 & 6.190 E-06 & 7.750 E-06 & 8.480 E-06 & 3.580 E-06 & 3.238 E-05\end{array}$ $\begin{array}{lllllr}0.199 & 0.709 & 0.495 & 1.467 & 0.498 & 0.422\end{array}$ $\begin{array}{llllllll}3.201 E-03 & 7.233 E-04 & 8.670 E-04 & 1.558 E-03 & 5.697 E-04 & 7.158 E-04 & 7.635 E-03\end{array}$ $\begin{array}{lllllll}99.801 & 100.000 & 99.291 & 99.505 & 98.533 & 99.502 & 99.577\end{array}$ ERAND TOTALS (CURIES)

$\begin{array}{lllllll}2.200 E+08 & 2.160 E+08 & 2.380 E+08 & 2.280 E+08 & 2.120 E+08 & 2.240 E+08 & 1.338 E+09\end{array}$

$\begin{array}{lllllll}3.207 E-03 & 7.233 E-04 & 8.732 E-04 & 1.566 E-03 & 5.782 E-04 & 7.194 E-04 & 7.667 E-03\end{array}$

* NOTE: DCE valu obtainod from DOE ORDER 5400.5 doted Fabruary8, 1990, Figures III-I and III-3 


\section{Decontamination and Decommissioning 1993 Detail Data}

Decontamination and Decomissioning (D\&D) Bar Graph of Annual Data by Month

CY1993 Bar Graph D\&D-5

D\&D Detail Data for Months July to December,

Annual Average Concentration and Total Curies for January to December $D \& D-7$

Report 713-D\&D Disposed Solid Waste Summary D\&D-9 D\&D Detail Data for Months January to June, Annual Average Concentration and Total Curies for January to June D\&D-11

Report 713-D\&D Disposed Solid Waste Summary D\&D-13 

Decontamination and Decommissioning (D\&D) Monthly Details_CY 1993

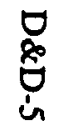

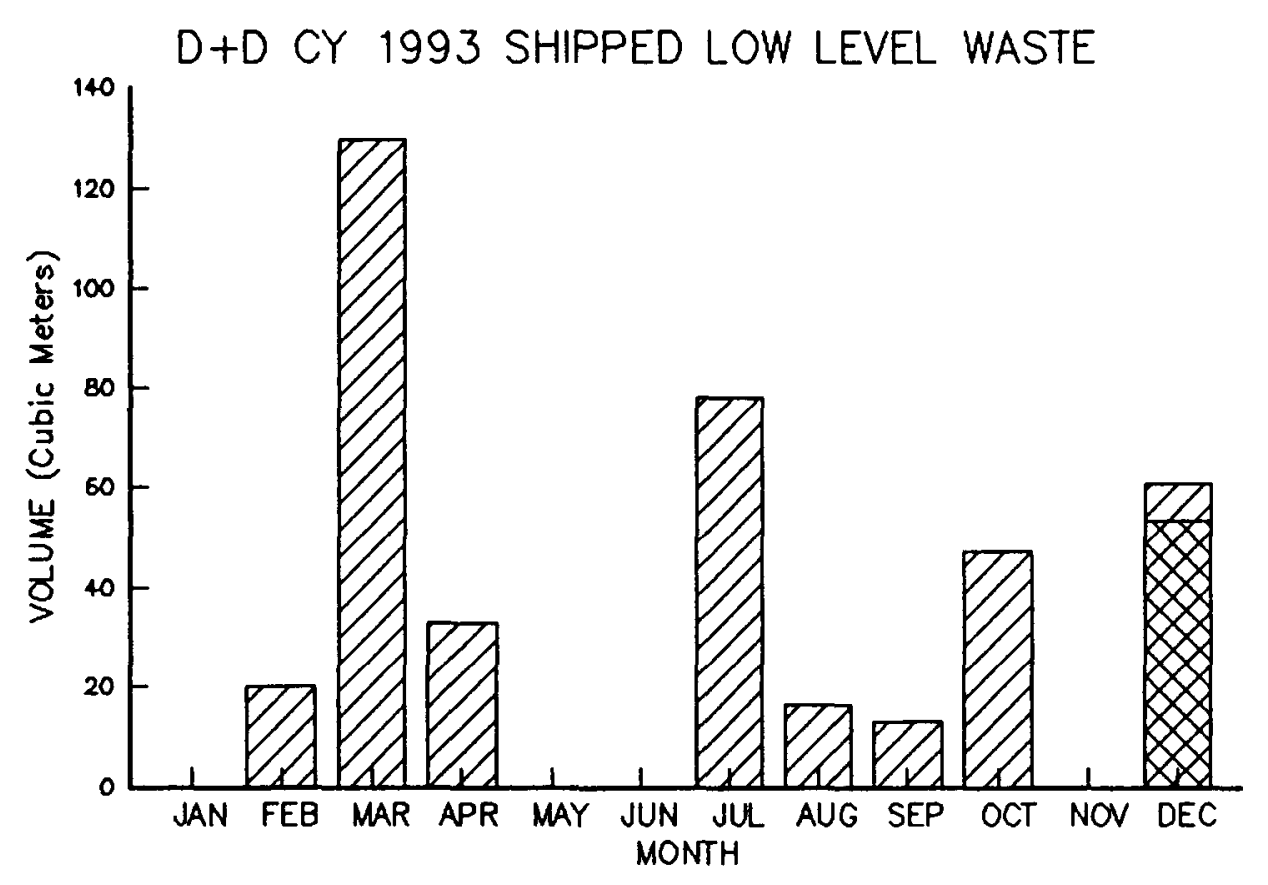

DINCINERATION $\triangle$ METAL SIZING COMPACTIONUDIRECT DISPOSAL 

D\&D Detail Data for Months July to December Annual Average Concentration and Total Curies for January to December 

D+D DISPOSED SOLIO MUSTE SUMMRY FOR JAN THROUEH DEC 1993

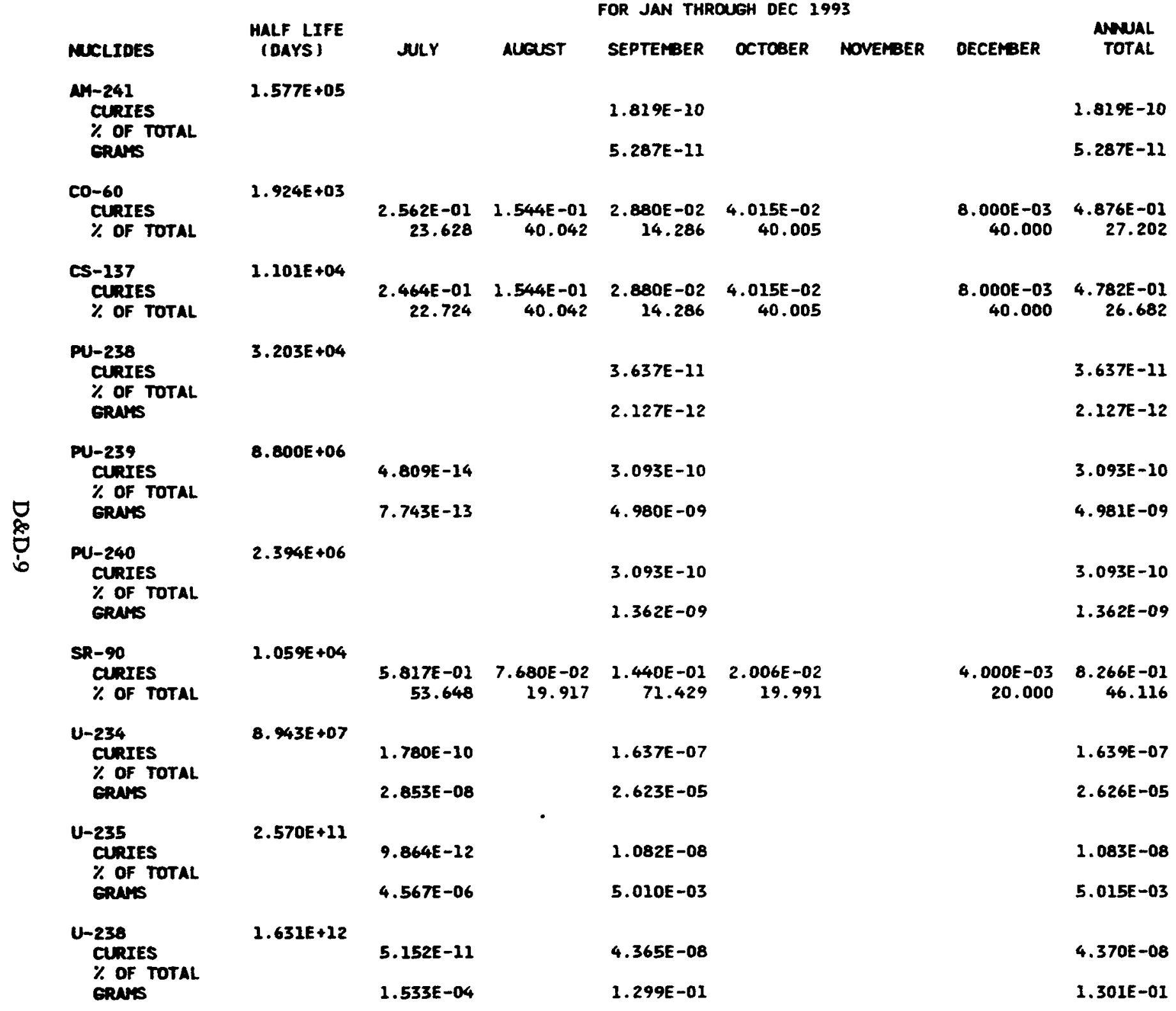

PAGE 1 RPT 713

$D+D-S$ 
SCHED. NO. P6IPW009-G

U.S. DEPARTMENT OF ENERGY

RADIOACTIVE WASTE MANAGEMENT INFORMATION SYSTEM

D+D DISPOSED SOLID MASTE SUMAARY FOR JAN THROUGH DEC 1993

\section{HALF LIFE}

(DAYS)

NLY

AUEUST

SEPTEMER

NOVEMBER

DECERBR

ANMAL

RUN DATE: $05 / 11 / 94$

PAge 2 RPT 713

$D+D-S$

TOTAL

\section{TOTALS}

ALPHA CURIES

$\%$ of total

o+g curies

$\%$ of total

TOTAL ERArS OF AM
2.395E-10

1. $084 E+00$
2.190E-07

$\begin{array}{rrr}3.856 E-01 & 2.016 E-01 & 1.004 E-01\end{array}$
2.192E-07

$2.000 E-02 \quad 1.792 E+00$

5.287E-11

$1.351 E-01$

$7.240 E+00 \quad 3.440 E+02$ $2.948 E+00 \quad 2.016 E+05$ $3.812 E+04 \quad 7.802 E+03 \quad 4.049 E+03 \quad 1.778 E+04$

$2.000 E-02$ 1.792E+00 
D\&D Detail Data for Months January to June Annual Average Concentration and Total Curies for January to June 
D+D DISPOSED SOLID MASTE SUMMARY

FOR JAN THROUGH JUN 1993

$\begin{array}{lc}\text { MUCLIDES } & \begin{array}{c}\text { MALF LIFE } \\ \text { (DAYS ) }\end{array} \\ \begin{array}{c}\text { CS-137 } \\ \text { CURIES }\end{array} & 1.201 E+04 \\ & \end{array}$

JANUARY

FEBRUARY

MARCH

APRIL

MAY

JNE

4.171E -05

$385 E-04$
100.000

$1.820 E-06$

4.171E-05 4.385E-04 $1.820 E-06$

$\begin{array}{rrr}171 E-05 & 4.385 E-04 & 1.820 E-06 \\ 100.000 & 100.000 & 100.000\end{array}$
PAGE 1 RPT 713

ASUAL

TOTAL

$4.820 E-04$

100.000

$4.820 E-04$

100.000

TOTAL ERAMS of AM

TOTAL ERNAS OF NM

TOTAL CRNAS Of PU

TOTAL ERANS Of PU-239+241

TOTAL ERAMS OF $U$

TOTAL ERANS OF TH

TOTAL ERNIS OF NP

VOLUAE (CU. METERS)

$1.988 E+01 \quad 1.296 E+02 \quad 3.285 E+01$

$1.065 E+04 \quad 8.144 E+04 \quad 4.176 E+04$

$1.823 E+02$

VEIEH (KILOERNIS)

-

$4.820 E-04$

ERAN TOTALS (CURIES)

4.171E-05 4.385E-04 1.820E-06 


\section{Naval Reactor Facility 1993 Detail Data}

Naval Reactor Facility (NRF) Bar Graphs of Annual Data by Month .......... NRF-3 CY1993 Bar Graphs '.................................... NRF-5

NRF Detail Data for Months July to December

Annual Average Concentration and Total Curies for January to December ....... NRF-7

Report 301-NRF Airborne Waste Summary $\ldots \ldots \ldots \ldots \ldots \ldots \ldots$ NRF-9

Report 305-NRF Disposed Solid Waste Summary ................ NRF-10

Report 308-NRF Solid Waste Summary,

Waste Sent To WERF For Metallic Processing .......... NRF-13

Report 309-NRF Solid Waste Summary,

WERF Incinerator Processed Waste $\ldots \ldots \ldots \ldots \ldots \ldots$ NRF-14

Report 313-NRF Solid Waste Summary,

Waste Sent To WERF for Compaction .............. NRF-16

NRF Detail Data for Months January to June

Annual Average Concentration and Total Curies for January to June ......... NRF-19

Report 301-NRF Airborne Waste Summary $\ldots \ldots \ldots \ldots \ldots \ldots \ldots$ NRF-21

Report 305-NRF Disposed Solid Waste Summary ............... NRF-22

Report 308-NRF Solid Waste Summary,

Waste Sent To WERF For Metallic Processing .......... NRF-25

Report 309-NRF Solid Waste Summary,

WERF Incinerator Processed Waste $\ldots \ldots \ldots \ldots \ldots \ldots$ NRF-26

Report 313-NRF Solid Waste Summary,

Waste Sent To WERF For Compaction .............. NRF-28

NRF Facility Detail Data for Months July to December

Annual Average Concentration and Total Curies for January to December ....... NRF-31

Report 320-A1W Airborne Waste Summary $\ldots \ldots \ldots \ldots \ldots \ldots \ldots$ NRF-33

Report 322-A1W Disposed Solid Waste Summary $\ldots \ldots \ldots \ldots \ldots \ldots$ NRF-34

Report 326-A1W-RWDS Airborne Waste Summary .............. NRF-36

Report 330_ECF Airborne Waste Summary .................. NRF-37

Report 332-ECF Disposed Solid Waste Summary ............... NRF-38

Report 343-Misc. Sources Airborne Waste Summary $\ldots \ldots \ldots \ldots \ldots . .$. NRF-41

Report 310-S1W Airborne Waste Summary $\ldots \ldots \ldots \ldots \ldots \ldots \ldots$ NRF-42

Report 312-S1W Disposed Solid Waste Summary $\ldots \ldots \ldots \ldots \ldots \ldots$ NRF-43

Report 340-S5G Airborne Waste Summary ................... NRF-44

Report 342-S5G Disposed Solid Waste Summary ............... NRF-45

NRF Facility Detail Data for Months January to June

Annual Average Concentration and Total Curies for January to June .......... NRF-47

Report 320_A1W Airborne Waste Summary .................. NRF-49

Report 322-A1W Disposed Solid Waste Summary ................ NRF-50

Report 326-A1W-RWDS Airborne Waste Summary ............... NRF-52

Report 330-ECF Airborne Waste Summary .................. NRF-53

Report 332-ECF Disposed Solid Waste Summary ............... NRF-54

Report 343-Misc. Sources Airborne Waste Summary $\ldots \ldots \ldots \ldots \ldots \ldots$ NRF-57

Report 310-S1W Airborne Waste Summary $\ldots \ldots \ldots \ldots \ldots \ldots \ldots$ NRF-58

Report 312-S1W Disposed Solid Waste Summary $\ldots \ldots \ldots \ldots \ldots \ldots$ NRF-59

Report 340-S5G Airborne Waste Summary .................... NRF-60

Report 342-S5G Disposed Solid Waste Summary ............... NRF-61 

Naval Reactor Facility (NRF) Bar Graphs of Annual Data by Month 
. 
Naval Recators Facility (NRF) Monthly Details - CY 1993

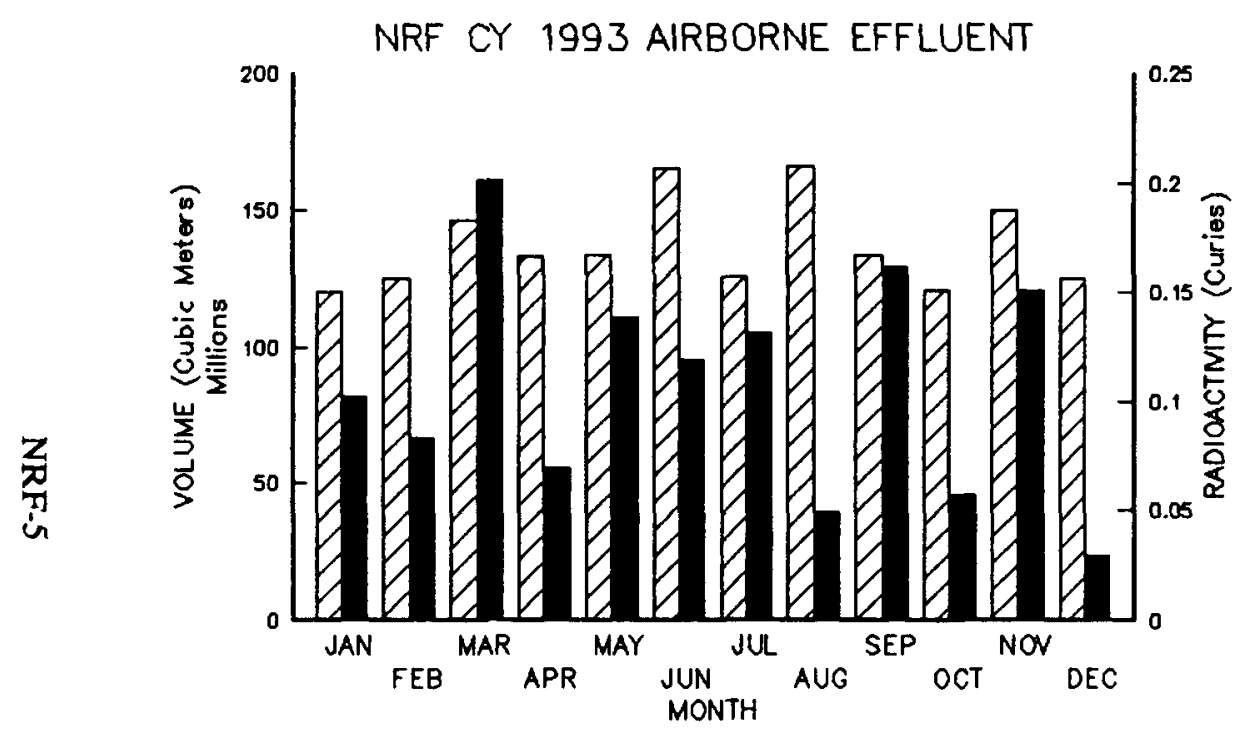

I VOLUME I RADIOACTIVTT

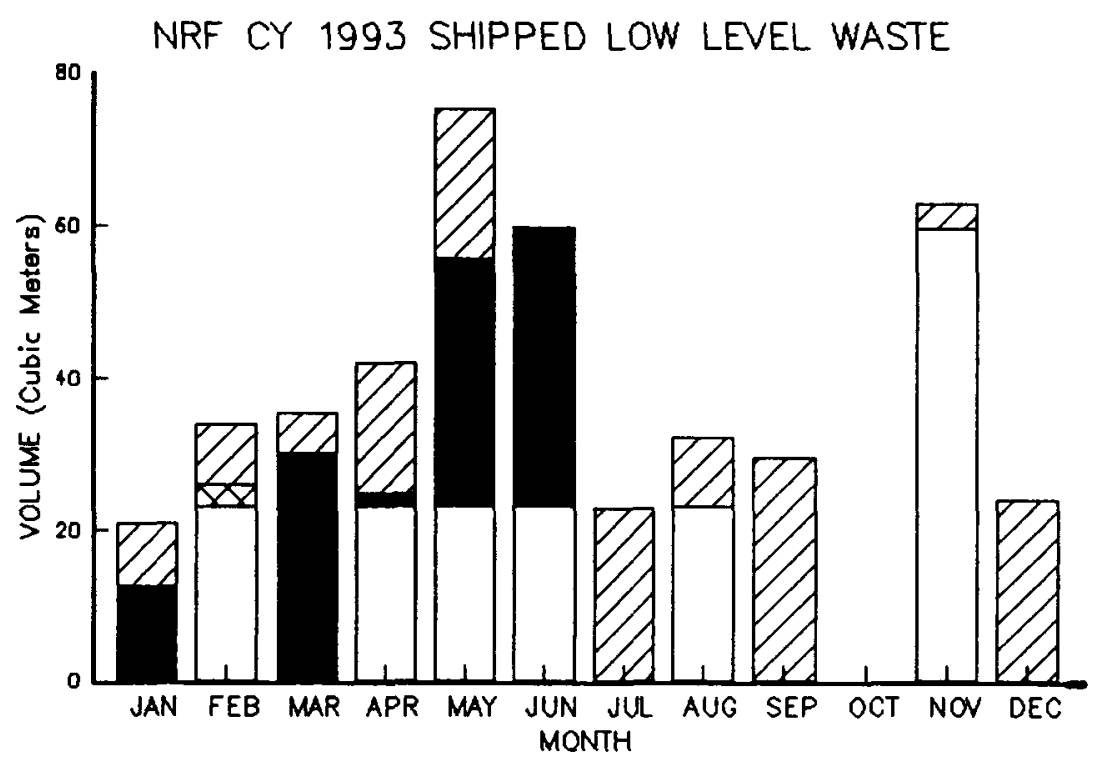

口INCINERATION $\square$ METAL SIZING I COMPACTION $\square$ DIRECT DISPOSAL 

NRF Detail Data for Months July to December Annual Average Concentration and Total Curies for January to December 

RADIOACTIVE WASTE MANAGEMENT INFORMATION SYSTEM

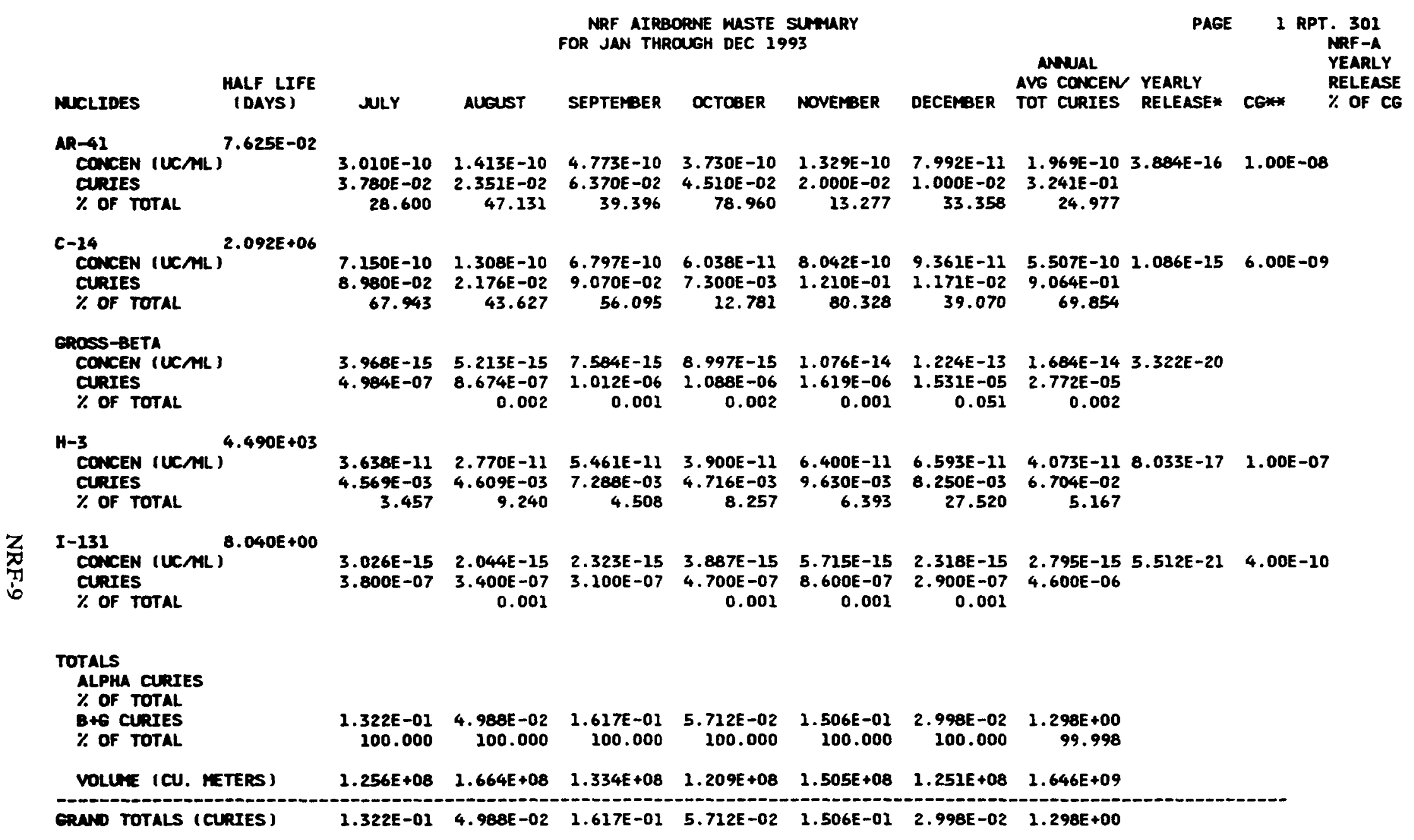

* This nuber is darived from a dispersion factor equation. The dispersion factor is derived from the straight line Gaussian wodel and is provided by the National Dceanic atmospheric Administretion (NOAA).

* NOTE: DCG velue obtained from DOE ORDER 5400.5 deted February 8, 1990, Figures III-I and III-3 
SCHEO. NO. P61PM009-6

IOAHO OPERATIONS OFFICE

U.S. DEPARTMENT OF ENERGY

RADIOACTIVE WASTE MANGGEMENT INFOPMATION SYSTEM

NRF DISPOSED SOLID WASTE SUMMARY

FOR JAN THROUGH DEC 1993

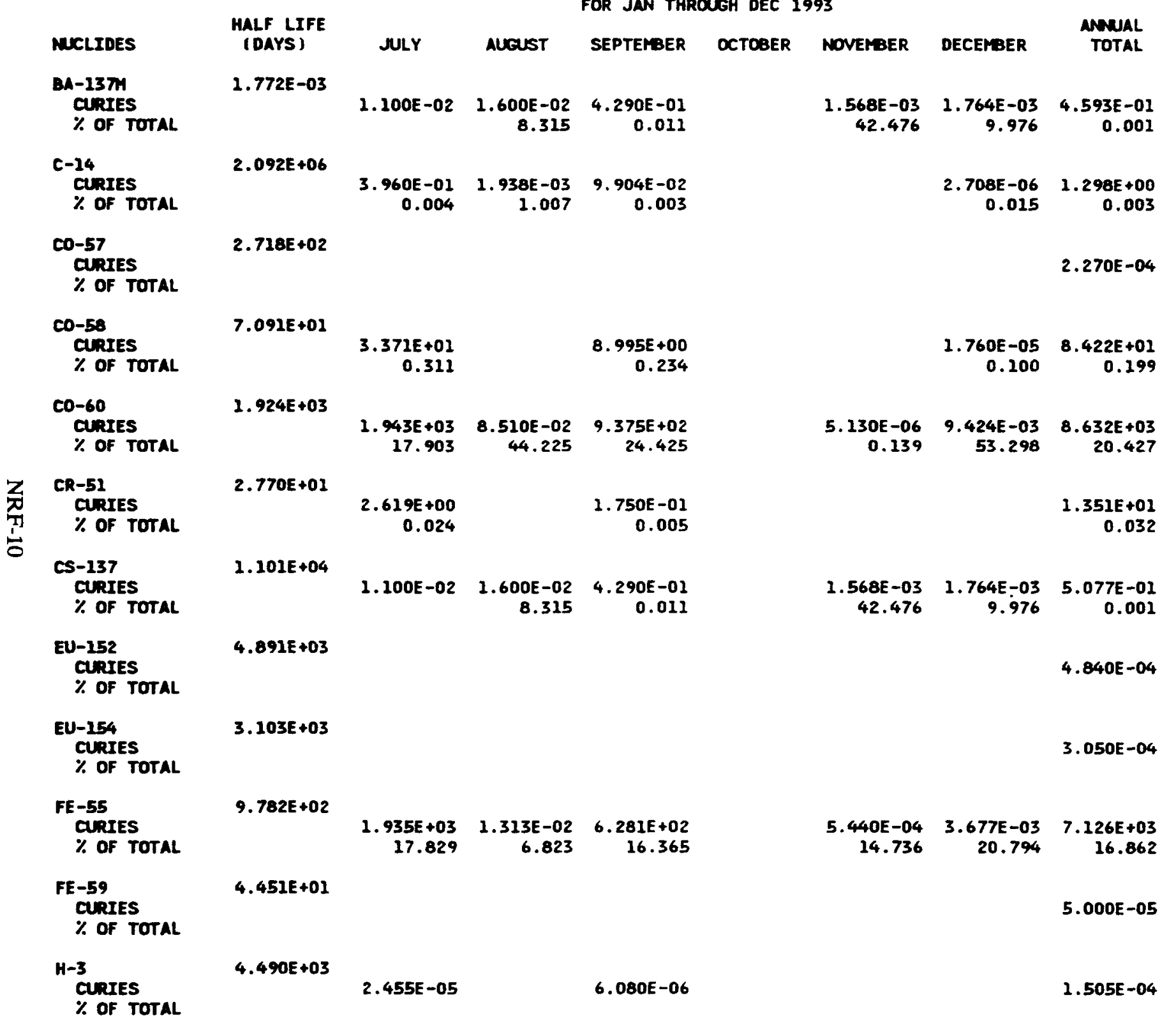

RUN DATE: 05/11/94

PAge 1 RPT 305 


\section{NRF DISPOSED SOLID WUSTE SUMYARY} FOR JAN THROUGH DEC 1993

\section{MUCLIDES}

HALF LIFE
(DAYS )

I-229

CURIES

$\%$ OF TOTAL

M-54

CURIES

\% OF TOTAL

Na-95

CURIES

$\%$ OF TOTAL

\section{NI -59}

Curres

$\%$ OF TOTAL

NI -63

CURIES

$\%$ Of TOTAL

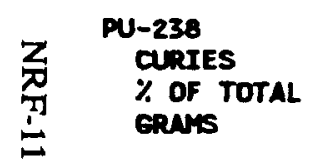

mU-106

CurIES
$\%$ OF TOTAL

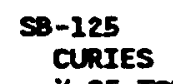

$\%$ of Total

\section{SN-1194}

CURIES

$\%$ OF TOTAL

SN-121M

CURIES

SR-90
CURIES
$\%$ OF TOTAL

TC-99

CURIES
$\%$ OF TOTAL
5. $840 E+09$

1.611E-11 7.780E-09 $\quad 1.460 E-10$

$3.122 E+02$

1.684E -05

$8.000 E-04$

$3.498 E+01$

2. $278 E+02$

1.178

$3.010 E+01$
0.784

$1.530 E+01$

0.399

$20 E+01$
0.564

$020 E+03$

$\begin{array}{rrr}6.070 E+03 & 5.260 E-02 & 2.020 E+03 \\ 55.928 & 27.335 & 52.628\end{array}$

$3.203 E+04$

$3.726 E+02$

$1.500 E-05$

5.847E-06

1.007E+03

$1.422 E+02$

1.310

$2.930 E+02$

$4.450 E+02$

4.100

$4.620 E+01$

1.204

$1.370 E+02$

3.569

$2.008 E+04$

$1.059 E+04$

$2.500 E-03 \quad 3.854 E-03 \quad 9.900 E-02$ $2.003 \quad 0.003$

$7.775 E+07$
PAgE 2 RPT 305

MNUAL

NRF-S 
SCHED. NO. P61PM009-G

NUCLIDES
Y-90
CURIES
$\%$ OF TOTAL

ZR-95

CuRIES
$\%$ of total

IDAHO OPERATIONS OFFICE

U.S. DEPARTMENT OF ENERGY

RADIOACTIVE WASTE MANAGEMENT INFORHATION SYSTEM

NRF DISPOSED SOLID WASTE SUMMARY FOR JAN THROUGH DEC 1993

$\begin{array}{ccrr}\begin{array}{c}\text { HALF LIFE } \\ \text { (DAYS ) }\end{array} & \text { NLY } & \text { AUGUST } & \text { SEPTEMBER } \\ 2.667 E+00 & & & \\ & 2.500 E-03 & \begin{array}{r}3.800 E-03 \\ 1.975\end{array} & \begin{array}{r}9.900 E-02 \\ 0.003\end{array} \\ 6.403 E+01 & & & \\ & 9.234 E+01 & & 1.370 E+01 \\ 0.851 & & 0.357 \\ & & & \\ & & & \\ & & & \\ & & & \\ & 1.085 E+04 & 1.924 E-01 & 3.838 E+03 \\ 100.000 & 100.000 & 100.000\end{array}$

TOTALS

ALPHA CURIES

$\%$ OF TOTAL

$\%$ OF TOTAL

100.000

100.000

100.000

OCTOBER

NOVEMBER DECEMBR

ANAUAL

TOTAL

TOTAL GRAMS OF NM

TOTAL ERNS OF AM

TOTAL GRNTS OF PU

TOTAL ERNAS OF PU

TOTAL ERUis OF $U$

TOTAL ERAMS OF TH

TOTAL ERUMS OF NP

VOLUME (CU. METERS )

MEIEAT (KILOGRATS )

$2.270 E+01 \quad 9.061 E+00 \quad 2.950 E+01$ $2.626 E+04 \quad 3.357 E+03 \quad 1.619 E+04$

3.200E-06 3.600E-06 $\quad 1.053 E-01$ 0.087 0.020

GRAN TOTALS (CURIES)

$1.085 E+04 \quad 1.924 E-01 \quad 3.838 E+03$
$2.271 E+02$

$7.890 E-08$

3.692E-03 $\quad 1.768 E-02 \quad 4.226 E+04$ $\begin{array}{rrr}100.000 & 100.000 & 99.999\end{array}$
$3.328 E+00 \quad 2.383 E+01 \quad 1.464 E+02$ $5.539 E+03 \quad 1.966 E+04 \quad 1.303 E+05$

$3.692 E-03 \quad 1.768 E-02 \quad 4.226 E+04$
RUN DATE: 05/11/94

PAge 3 RPT 305

NRF-S 
SCHED. NO. PGIPHOOO-H

IDAHO OPERATIONS OFFICE

U.S. DEPARTMENT OF ENERGY

RUN DATE: 05/12/94

NRF SOLID WASTE SUMUARY

RADIOACTIVE WASTE MANAGEMENT INFORMATION SYSTEM

WUSTE SENT TO WERF FOR METALLIC PROCESSING

MALF LIFE
(OAYS)

suLY

Aueust

FROM JAN THROUGH DEC 1993

MUCLIDES

$1.924 E+03$

CURrEs

$\%$ Of TOTAL

FE-55

CURIES

$\%$ OF TOTAL

NI-63

CURIEs

$9.782 E+02$

TOTALS

ALPHA CURIES

$\%$ OF TOTAL

$\%$ OF TOTAL

$7.165 E-05$

100.000

TOTAL CRMYS OF AM

TOTAL ERNS OF PU

TOTAL ERAMS OF PU-239+241

TOTAL GRAYS OF $U$

Total canis of

TOTAL ERNAS OF NP

VOLUNE (CU. METERS)

VEIEHT (KILOGRAYS)

ERND TOTALS (CURIES)
PAGE 1 RPT. 308

ANNUAL

TOTAL

4. $900 E-05$

68.383

2. $915 E-06$

4.068

1. $974 E-05$

27.549
2. $268 E+02$

$7.265 E-05$ 
SCHED. NO. P61PHOO9-N

DOAO OPERATIONS OFFICE

RUN DATE: 05/11/94

RADIOACTIVE WASTE MANAGEMENT INFORMATION SYSTEM

NRF SOLID WASTE SUMARY

INCINERATOR PROCESSED WASTE

MALF LIF
(DAYS)

JULY

AUGUST

SEPTEMBER

OCTOBER NOVEMBER DECEMBER

ANMAL

PAGE 1 RPT. 309

$\%$ of rotaL

$\%$ of
co-57

CURIES

$\%$ OF TOTAL

CO-58

curres

$\%$ OF TOTAL

CO-60

CuRIES

$\%$ OF TOTAL

CS-137

curres

$\%$ OF TOTAL

EU-152

CURIES

$\%$ of total

EU-154

CURIES

Z \% OF TOTAL

FE- 55

CURIES

$\%$ OF TOTAL

I-129

$2.092 E+06$

OF TOTAL

MN-54

curies

$\%$ OF TOTAL

3-95

CURIES

\% OF total

NI-63

curres

$\%$ OF TOTAL

RU-106

CURIES

$\%$ of total.

TC-99

CURIES

ZR-95

CURIES

$\%$ OF tOTAL
$7.908 E-06$

0.086

$2.718 E+02$

$7.091 E+01$

$1.924 E+03$

$1.101 E+04$

$4.891 E+03$

3. $103 E+03$

$9.782 E+02$

5. $840 E+09$

3.031E-05

0.074

2.030E-06

0.005

0.030

5. 701E-05
0.617

2.011E-04

0.489

$2.622 E-02$ 63.716

$738 E-03$

62.099

5.130E-06

0.056

$3.780 E-06$

0.009

4.340E-06

0.064

$3.705 E-06$

0.040

2.635E-03

28.517

2. $900 E-11$

$3.122 E+02$

3.411E-05

0.369

5.130E-06

0.056

6. $448 \mathrm{E}-04$

6.978

$3.726 E+02$

9.793E-05

1.060

7. $908 E-09$

2. 755E-06 0.030
.730E-06 0.007

$1.127 \mathrm{E}-02$ 27.394

1.111E-10

1.190E-04

0.289

3.780E-06

0.009

$2.838 E-03$

6.896

4.514E-04

1.097

$3.031 E-08$

$2.030 E-06$

0.005
2. 145E-04

0.138

4.059E-05 0.026

1.478E-03

8. 814E-02 56.496

$7.571 E-05$ 0.049

8.763E-05

0.056

5.424E-05 0.035

$5.162 \mathrm{E}-02$ 33.085

$7.890 E-10$

8.792E-04 0.564

7.571E-05 0.049

1.195E-02 7.663

$1.355 E-03$ 0.868

$2.145 E-07$

4.059E-05 0.026 
RADIOACTIVE WASTE MANAGEMENT INFORMATION SYSTEM

MRF SOLID WASTE SUMYARY

WERF INCINERATOR PROCESSED MASTE FROM JAN THROUGH DEC 1993

HALF LIFE
(DAYS)

NLY

AveUst

993
$9.240 E-03$

300.000

\section{4. $115 E-02$}

100.000

PAGE

2 RPT. 309

MNUAL
TOTAL

\section{TOTALS}

$$
\text { \% OF TOTAL }
$$

TOTAL ERAYS OF AM

TOTAL ERAHS Of PU

TOTAL Eevis of PU-239+241

TOTAL ERNA OF $U$

TOTAL GRNS OF TH

TOTAL ENAS OF

VOLUNE (CU. METERS

NEIEHT (KILOERNIS)

$2.294 E+01 \quad 5.950 E+01 \quad 1.742 E+02$

$3.653 E+03$

ERMD TOTALS ICURIES 
SCHED. NO. P6IPHOO9-0 IDAHO OPERATIONS OFFICE
U.S. DEPARTMENT OF ENERGY

RADIOACTIVE WASTE MANAGEMENT INFORMATION SYSTEM

NRF SOLID WASTE SUMMARY

WASTE SENT TO WERF FOR COMPACTION

FROM JAN THROUEH DEC 1993

MWNAL

MALF LIFE
MCLIDES (DAYS)

NUY

AUGUST

SEPTEMBER

OCTOBER NOVEMBER DECEMBER

$1.772 E-03$

CURIES

$\%$ Of Total

C-14

curres

$\%$ OF TOTAL

CO-57

CURIES

$\%$ OF TOTAL

co-58

CURIES

\% OF TOTAL

c0-60

CurIEs

$\%$ of total

CR-51

CuRres

$\%$ OF TOTAL

$\operatorname{cs}-134$

CURIES

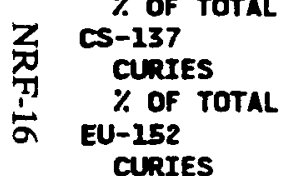

$\%$ OF TOTAL

EU-154

CURIES

$\%$ of total

FE -55

CURIES

$\%$ OF TOTAL

FE-59

curres

$\%$ of total

I-129

CURIES

$\%$ of total.

$K-40$

curres

$\%$ OF TOTAL

MN-54

curres

$\%$ of total

N-95

CURIES

$\%$ of total
$2.092 E+06$

$2.718 E+02$

$7.091 E+01$

$1.924 E+03$

$2.770 E+01$

$7.537 E+02$

1.101E +04

$4.891 E+03$

3. 103E+03

$9.782 E+02$

$4.451 E+01$

5.840E+09

$4.709 E+11$

$3.122 E+02$

$3.498 E+01$
RUN DATE: 05/11/94

PAGE 1 RPT. 313

ANUAL
TOTAL

2.067E-03

$$
0.150
$$

3.627E-03

$$
0.263
$$

$1.369 E-03$ 0.099

3.940E-02 2.861

5. $489 E-01$ 39.860

4. $040 E-03$ 0.293

4. OOOE-07

$4.616 E-03$ 0.335

2. $925 E-03$ 0.212

$1.840 E-03$ 0.134

6.113E-01 44.386

6.600E-04 0.048

$1.330 E-08$

6.000E-05

0.004

1.804E-02 1.310

$6.858 E-03$ 0.498 
MRF SOLID MASTE SUMYRY

WUSTE SENT TO WERF FOR COMPACTION

FROM JAN THROUGH DEC 1993

JULY

AUEUST

SEPTEMBER

\section{$3.650 E+04$}

NI -63

CURIES

$\%$ OF TOTAL

Pu-238

CURIES

$\%$ of total

\% Of Total

$3.203 E+04$

PU-239

CURIES

$\%$ OF tOTAL

enums

$8.800 E+06$

RU-106

CURIES

$\%$ Of total

SR-90

CuRIES

$\%$ OF TOTAL

TC-99

Z⿱乛龰 $\%$ OFIES TOTAL

$\gamma-90$

CURIES

$\%$ OF tOTAL

2R-95

CURIES

$\%$ of total

$3.726 E+02$

$1.059 E+04$

7.775E+07

$2.667 E+00$

$6.403 E+01$

\section{TOTALS}

ALPHA CURIES

$\%$ OF TOTAL

B+G curIEs

$\%$ of total

TOTAL ERMS OF AM

TOTAL GRANS OF PU

TOTAL ERANS OF PU-239+241

TOTAL ERNIS OF $u$

TOTAL ERNAS OF $T H$

TOTAL ERNAS OF TH

TOTAL GRAMS OF NP

VOLUNE (CU. METERS )
WEIEHT (KILOERNAS)

GRANO TOTALS (CURIES)

$1.141 E+02$

$1.557 E+04$

PAGE 2 RPT. 313

ANEUAL

TOTAL

$1.226 E-01$

2. 140E-06

6.667E-08

5.400E-07

$8.696 E-06$

4.144E-03

$6.880 E-04$

0.050

3.627E-06

$6.880 E-04$

0.050

$3.309 E-03$
0.240

$1.680 E-06$

$1.377 E+00$

99.999

o

$1.377 E+00$ 

NRF Detail Data for Months January to June

Annual Average Concentration and Total Curies for January to June 


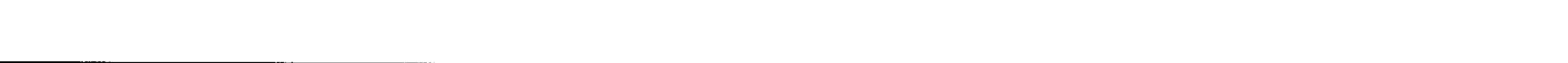




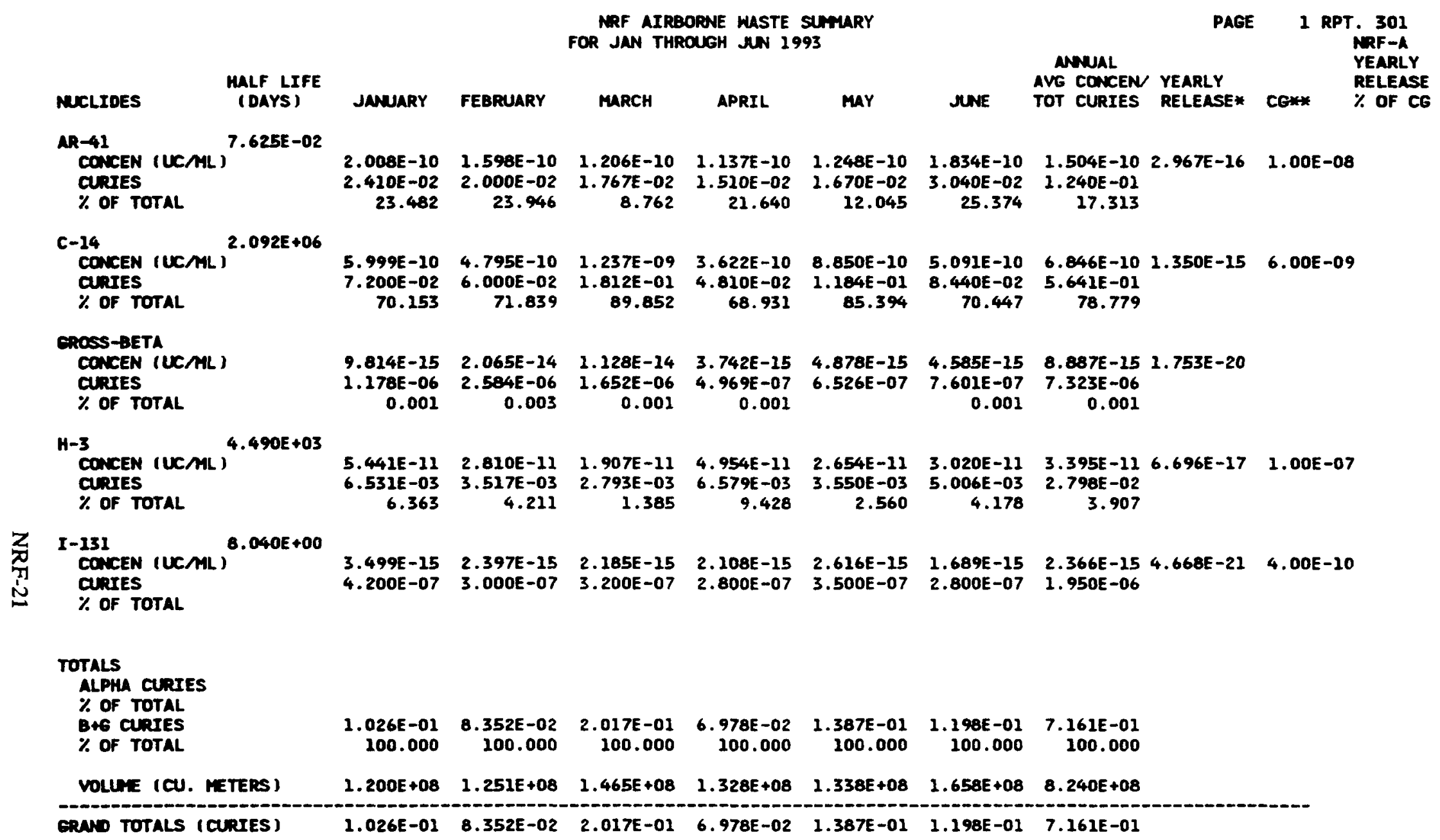

* This nuber is dorivad from a dispersion factor equation. The dispersion factor is derived from the straight line Cacusien wodel and is provided by the Mtional Oceanic Atmospheric Adninistration (NOAA).

** NOTE: DCE value abtained from DOE ORDER 5400.5 dated February 8, 1990, Figures III-1 and III-3 
IDAHO OPERATIONS OFFICE

U.S. DEPARTMENT OF ENERGY

RADIOACTIVE WASTE MANAGEMENT INFORMATION SYSTEM

NRF DISPOSED SOLIO MASTE SUMAARY FOR JAN THROUGH JUN 1993

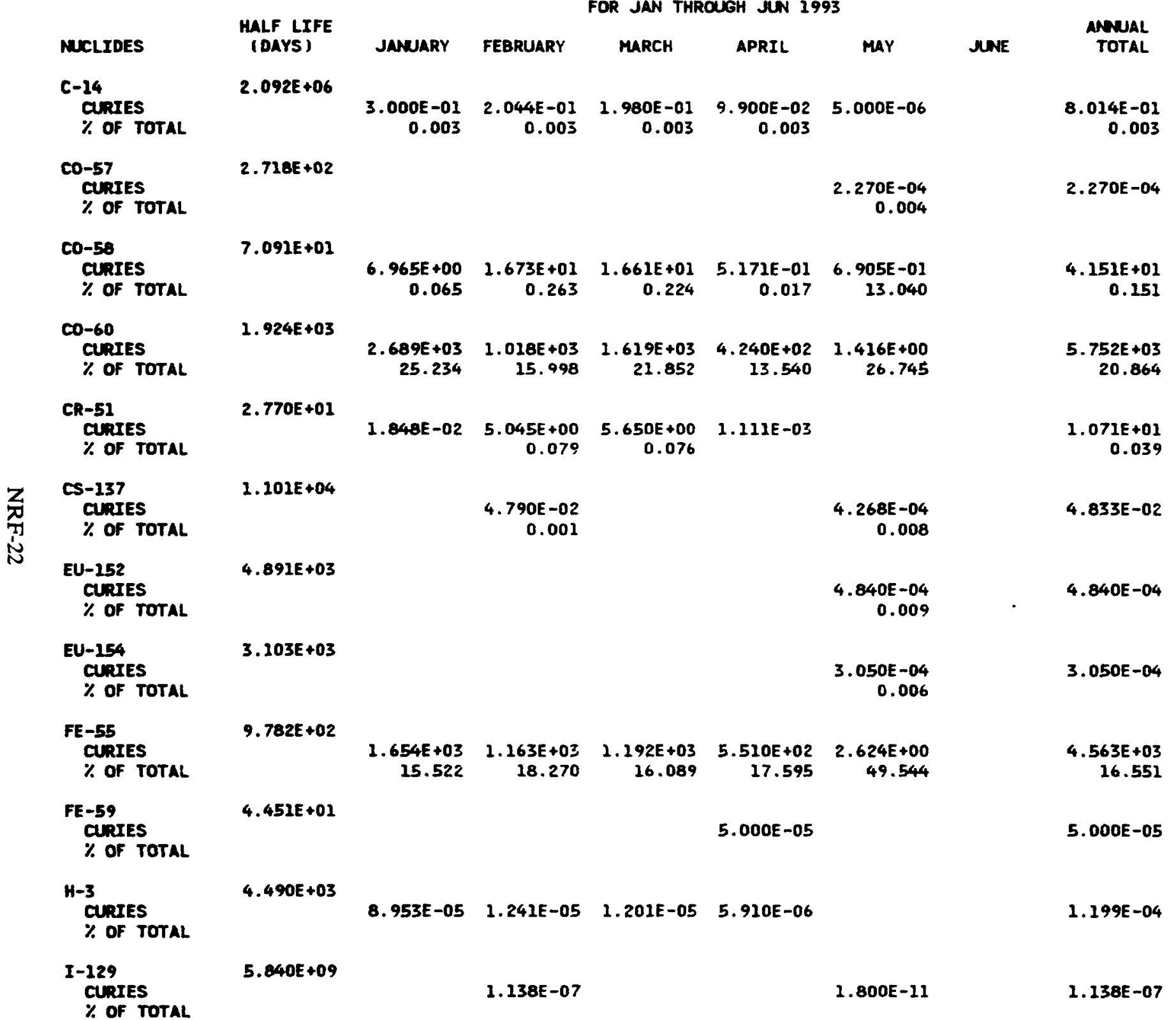

RUN DATE : 05/12/94

PAGE 1 RPT 305

NRF-S 

RADIOACTIVE WASTE MANAGEMENT INFORMATION SYSTEM

FOR JAN THROUGH JNN 1993

MUCLIOES
M-54
CURIES
$\%$ OF TOTAL

\section{ANUAL}

$\%$ of total

$$
\text { CURIEs }
$$

$\%$ OF TOTAL

\section{NI -59}

cunies

NI-63

CURIES

\% OF TOTAL

Pu-238

CURIES

$\%$ OF TOTAL

envis

\begin{tabular}{|c|}
\hline $\begin{array}{l}\text { RU-106 } \\
\text { CURIES } \\
\% \text { OF TOTAL }\end{array}$ \\
\hline
\end{tabular}

SB-125
CURIES
$\%$ OF TOTAL

SN-119M

CURIES

\% of TOTAL

\section{SN-121M}

$\%$ OF TOTAL

SR-90
CURIES
$\%$ OF TOTAL

TC-99

CURIES

$\%$ Of total

\section{CURIES}

$\%$ OF TOTAL

MALF LIF
(DAYS)
$3.122 E+0$

$3.498 E+01$

JANUARY FEBRUARY

MARCH

APRIL

MaY

JUNE

TOTAL

$9.800 E-02$
0.002

$2.000 E-04 \quad 9.833 E-02$
1.857

1.965E-01

0.001

$2.666 E+02$

0.967

$$
\begin{array}{r}
66+01 \\
0.184
\end{array}
$$

$$
\begin{array}{r}
97 E+02 \\
1.880
\end{array}
$$

$245 E+02$
1.681

$691 E+00$
0.086

.220E-04

$\begin{array}{llll}4.420 E+01 & 2.720 E+01 & 3.060 E+01 & 1.530 E+01\end{array}$

0.415

0.427

0.413

0.489

$1.173 E+02$

0.425

$\begin{array}{lllll}5.820 E+03 & 3.601 E+03 & 4.030 E+03 & 2.010 E+03 & 4.644 E-01\end{array}$

$\begin{array}{rrrrr}54.617 & 56.565 & 54.394 & 64.184 & 8.769\end{array}$

$2.546 E+04$

56.085

$3.203 E+04$$$
54.394
$$

$7.890 E-08$

4.614E-09

$7.890 E-08$

4.614E-09

$2.660 E-04$

0.005

$2.660 E-04$

2.007E+03

$\begin{array}{rrrr}1.244 E+02 & 8.520 E+01 & 8.780 E+01 & 4.060 E+01 \\ 1.167 & 1.338 & 1.185 & 1.296\end{array}$

$3.380 E+02$

1.226

2. $930 E+02$

$2.886 E+02$

$2.750 E+02 \quad 2.460 E+02 \quad 8.620 E+01$

$\begin{array}{rrrr}2.708 & 4.320 & 3.320 & 2.753\end{array}$

8. $958 E+02$

3.249

1. 200E-02

1. 200E-02

$1.059 E+04$

2.440E-06 4.574E-03

4.576E-03

$7.775 E+07$

\begin{tabular}{l}
$9.620 E-05 \quad 8.780 E-05 \quad 0.660 E-05 \quad 3.330 E-05 \quad 5.100 E-09$ \\
\hline
\end{tabular}

$2.839 E-04$

$6.403 E+01$

$\begin{array}{rrrrr}8.945 E+00 & 5.431 E+01 & 5.659 E+01 & 1.220 E+00 & 2.270 E-04 \\ 0.084 & 0.853 & 0.764 & 0.039 & 0.004\end{array}$
0.084
0.853
0.764
0.039

1.211E+02 
SCHED. NO. P61PMO09-G

IDAHO OPERATIONS OFFICE

U.S DEPARTMENT OF ENERGY

RADIOACTIVE WASTE MANAGEMENT INFORMATION SYSTEM

NRF DISPOSED SOLID WASTE SUMARY

FOR JAN THROUGH JUN 1993

MUCLIDES

HALF LIFE

(DAYS)

JANUARY FEBRUARY

MARCK

APRIL

MAY

JUNE

AMNUAL

TOTAL

TOTALs

ALPHA CURIES

\% OF TOTAL

B+6 CURIES

$\%$ OF TOTAL
$7.890 E-08$
5. $295 E+00$
$1.066 E+04 \quad 6.366 E+03$
100.000
100.000
$7.409 E+03$
100.000
$3.132 E+03$
100.000

100.000

$7.890 E-08$

2. $757 E+04$ 99.999

TOTAL ERMS OF M

TOTAL ERNS OF AM

TOTAL GRAMS OF PU

TOTAL GRAYS OF PU-239+241

TOTAL ERANS of $U$

TOTAL ERAMS OF TH

TOTAL GRAMS OF NP

VOLUAE (CU. METERS)

VEIGHT (KILOGRANS)

$\begin{array}{lllll}7.980 E+00 & 7.982 E+00 & 5.320 E+00 & 1.716 E+01 & 1.958 E+01\end{array}$

eromo rotals $1.116 E+04 \quad 1.912 E+04 \quad 7.926 E+03 \quad 2.098 E+04 \quad 1.010 E+04$

$1.066 E+04 \quad 6.366 E+03 \quad 7.409 E+03 \quad 3.132 E+03 \quad 5.295 E+00$

$5.802 E+01$ $5.928 E+04$

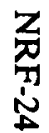




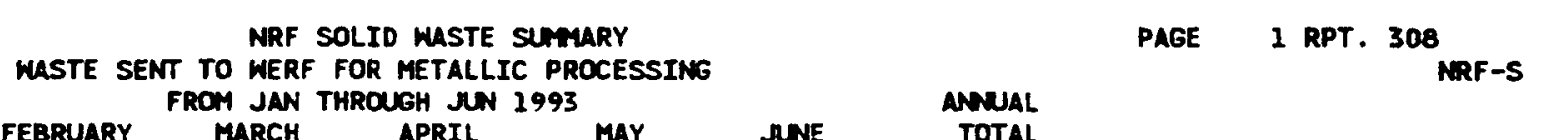

$\begin{array}{cc}\text { MUCLIDES } & \begin{array}{c}\text { HALF LIFE } \\ \text { (DAYS) }\end{array} \\ \text { CO-60 } & \\ \text { CURIES } & 1.924 E+03 \\ \% \text { OF TOTAL } & \\ \text { FE -55 } & \\ \text { CURIES } & 9.782 E+02 \\ \text { \% OF TOTAL } & \\ \text { NI -63 } \\ \text { CURIES }\end{array}$

JNWARY FEBRUARY

MARCH THROUGH JUN 2993

TOTAL

4. $900 E-05$

68.383

2. 915E-06

4.068

$1.974 E-05$

27.549

$7.165 E-05$

100.000
4. $900 E-05$

68.383

2. 915E-06

4.068

$1.974 E-05$

27.549

\section{265E-05}

100.000

TOTAL ERMY OF AM

TOTAL eruHs of PU

TOTAL ERANS OF PU-239+241

TOTAL GRNAS OF $U$

TOTAL ERNAS OF TH

TOTAL ERNAS OF NP

VOLUNE (CU. METERS)

2. $922 E+00$

$2.268 E+02$

2. $922 E+00$

VEIET (KILOEDNS )

$7.165 E-05$

$2.268 E+02$

ERUN TOTALS (CURIES)

$7.165 E-05$ 
IDAHO OPERATIONS OFFICE

U.S. DEPARTMENT OF ENERGY

RADIOACTIVE MASTE MUNAGEMENT INFORMATION SYSTEM

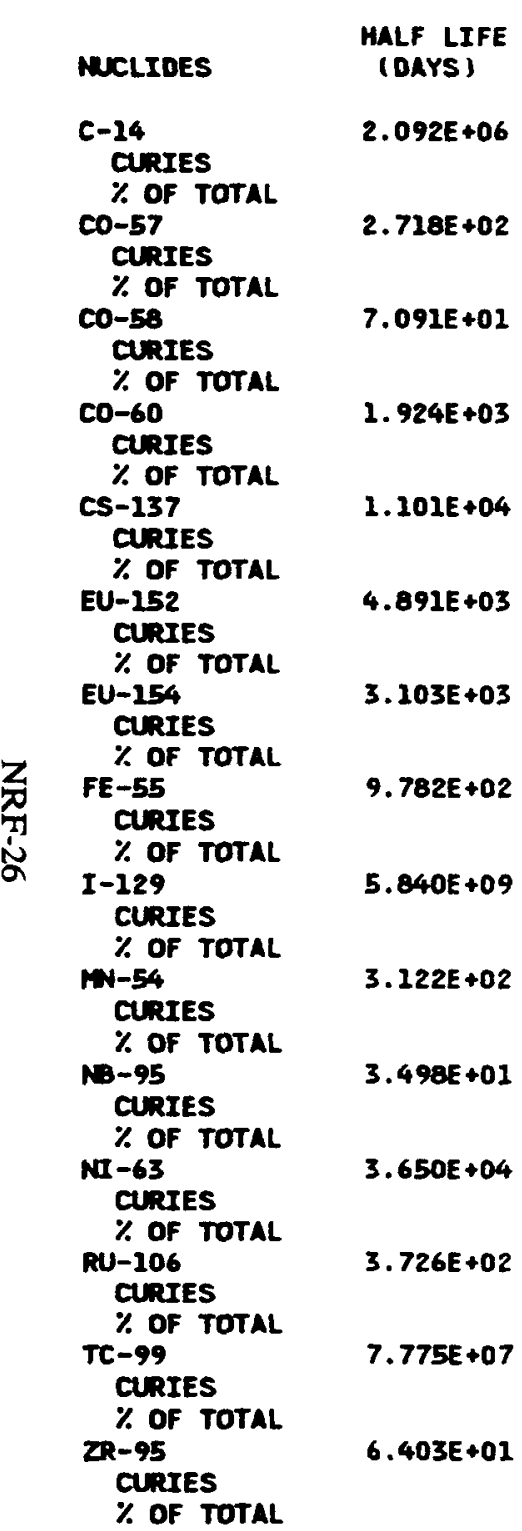

NRF SOLIO WASTE SUMUARY

WERF INCINERATOR PROCESSED WASTE

FROM JAN THROUGH JUN 1993

JANUARY FEBRUARY MARCK APRIL MAY JWE TWE TOTAL 0.162

$1.200 E-05$ 0.072

$1.990 E-04$

$$
1.189
$$

6. $938 E-03$ 53.412

$2.300 E-05$
0.137

$600 E-05$

$.600 E-05$
0.155

1. $600 \mathrm{E}-05$ 0.096

5.897E-03 35.240

$9.800 E-11$

$1.200 E-04$ 0.717

2.300E-05

$$
0.137
$$

$1.308 E-03$ 7.816

$1.330 E-04$ 0.795

2. 700E-08

1. 200E-05 0.072
9.200E-05 3.

$$
0.194
$$

$3.800 E-05$

0.170

1. $932 E-05$

0.102

1.763E-04

1. 600 E-05

0.034

$.000 E-06$
0.009

$5.800 E-06$
0.031

$0.031 \quad 0.034$

6.320E-04 2.510E-04 $1.374 E-04 \quad 1.219 E-03$

$$
1.331
$$$$
1.121
$$$$
0.723
$$

1.154

2.385E-02 1.193E-02 1.147E-02 5.619E-02 $\begin{array}{rrrr}50.229 & 53.246 & 60.345 & 53.194\end{array}$

$\begin{array}{rrrr}2.900 E-05 & 4.000 E-06 & 1.080 E-05 & 6.680 E-05 \\ 0.061 & 0.018 & 0.057 & 0.063\end{array}$

$3.400 E-05 \quad 5.000 E-06 \quad 1.240 E-05 \quad 7.740 E-05$ $\begin{array}{rrrr}0.072 & 0.022 & 0.065 & 0.073\end{array}$

$2.200 E-05 \quad 3.000 E-06 \quad 7.800 E-06 \quad 4.780 E-05$ $\begin{array}{rrrr}0.044 & 0.013 & 0.041 & 0.045\end{array}$

$1.809 E-02 \quad 8.028 E-03 \quad 5.690 E-03 \quad 3.771 E-02$ $\begin{array}{llll}38.107 & 35.839 & 29.936 & 35.701\end{array}$

$3.400 E-10 \quad 1.400 E-10 \quad 7.084 E-11 \quad 6.488 E-10$

3.760E-04 1.480E-04 8.206E-05 7.261E-04 $\begin{array}{rrrr}0.792 & 0.661 & 0.432 & 0.687\end{array}$

$2.900 E-05 \quad 4.000 E-06 \quad 2.080 E-05 \quad 6.680 E-05$ $\begin{array}{llll}0.061 & 0.018 & 0.057 & 0.063\end{array}$

3.978E-03 1.821E-03 1.365E-03 8.472E-03 $\begin{array}{rrrr}6.378 & 8.129 & 7.181 & 8.021\end{array}$

3.150E-04 1.670E-04 1.903E-04 8.053E-04 $\begin{array}{llll}0.663 & 0.746 & 1.002 & 0.762\end{array}$

$9.200 E-08 \quad 3.800 E-08 \quad 1.932 E-08 \quad 3.763 E-07$

$\begin{array}{llll}1.600 E-05 & 2.000 E-06 & 5.800 E-06 & 3.580 E-05\end{array}$ $\begin{array}{rrrr}0.034 & 0.009 & 0.031 & 0.034\end{array}$
PAGE 1 RPT. 309

NRF-S 
SCHED. M. PG1P4009-N

IDAHO OPERATIONS OFFICE

IDAHO DPERATIONS OFFICE

RUN DATE: 05/12/94

NRF SOLXD WUSTE SUMUARY FROY JAN THROUGH JUN 1993

MCLIDES

MALF LIFE

JANEARY

$$
\text { APRIL }
$$

JNE

NWUAL

TOTAL
PAge

2 RPT. 309

TOTALS

ALPHA CURIES

$\%$ OF total

B+G CURIES

$\%$ OF TOTAL

1.673E-02

100.000

TOTAL GRAMS OF AM

TOTAL ERAVS Of PU

TOTAL GRAHS Of PU-239+241

TOTAL GRAMS of $u$

TOTAL GRANS OF TH

TOTAL GRAS OF NP

VOLUNE (CU. METERS)

MEIEHT (KILOGRNAS)

GRAD TOTALS (CURIES)
$2.251 E+03$

1.673E-02

$$
\begin{array}{rrrr}
4.748 E-02 & 2.240 E-02 & 1.901 E-02 & 1.056 E-01 \\
100.000 & 100.000 & 100.000 & 99.999
\end{array}
$$




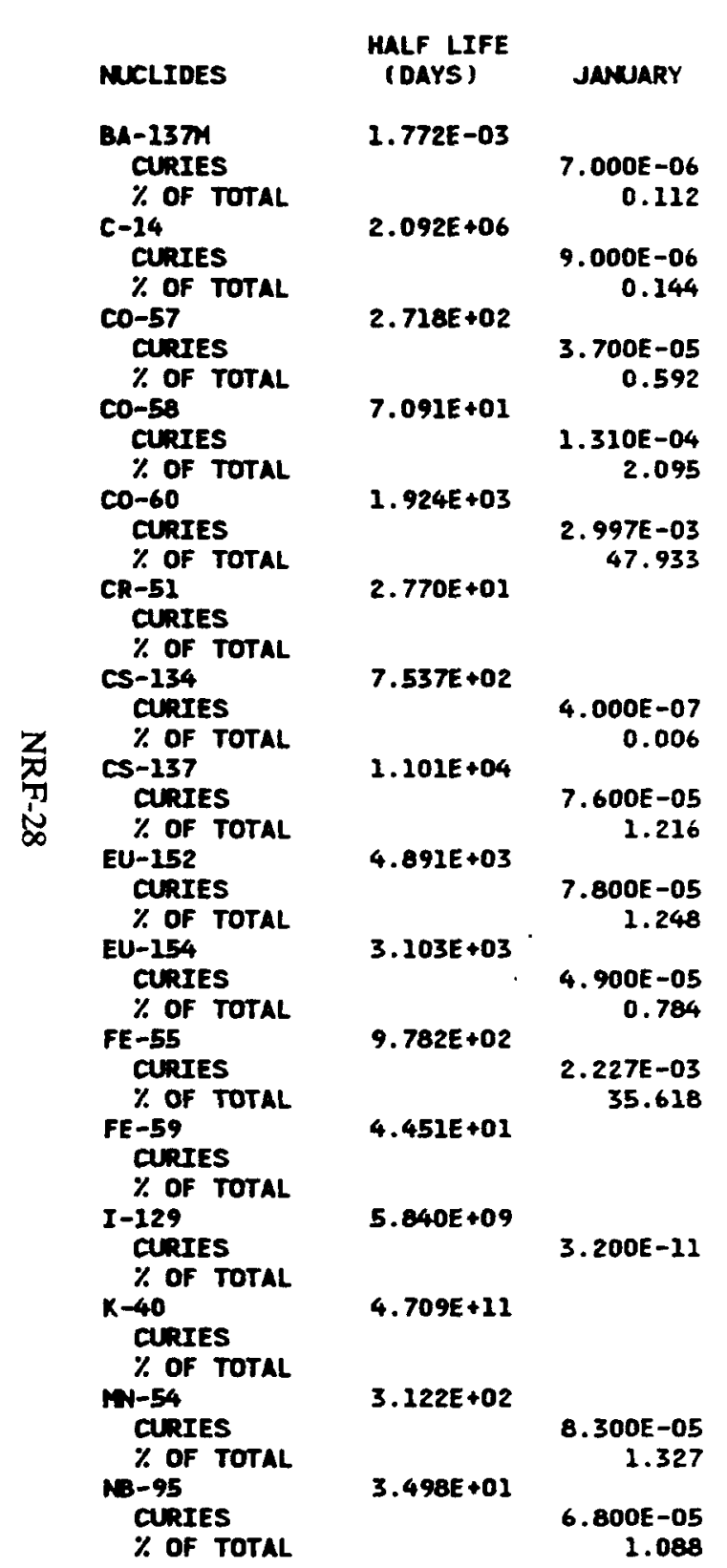

NRF SOLID WASTE SUMUARY
WASTE SENT TO WERF FOR COMPACTION

FROM JAN THROUGH JUN 1993

FEBRUARY MARCH APRIL 1993 MAY

$$
\begin{array}{r}
2.060 E-03 \\
1.595
\end{array}
$$

ANNAL

PAgE 1 RPT. 313

NRF-S

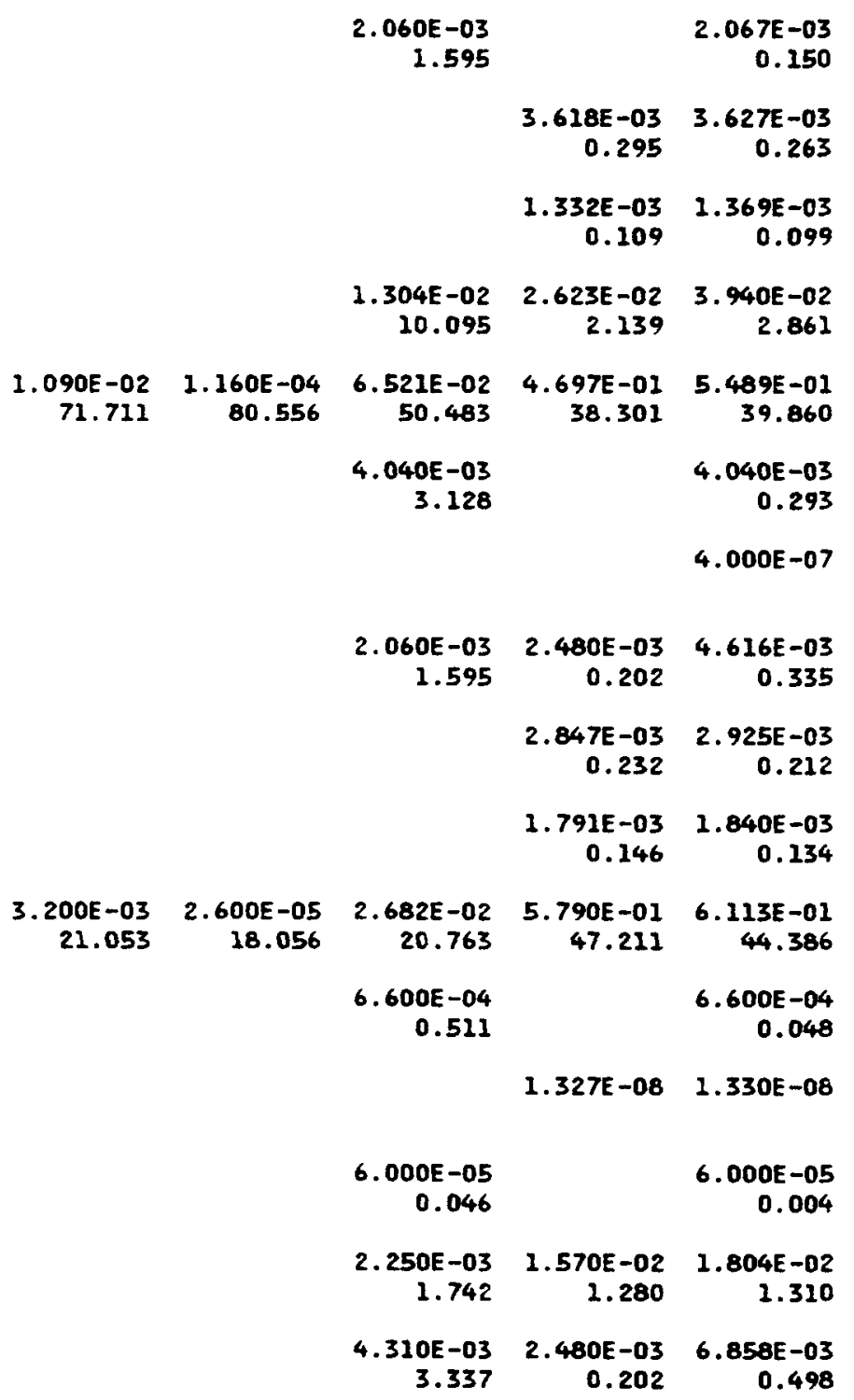




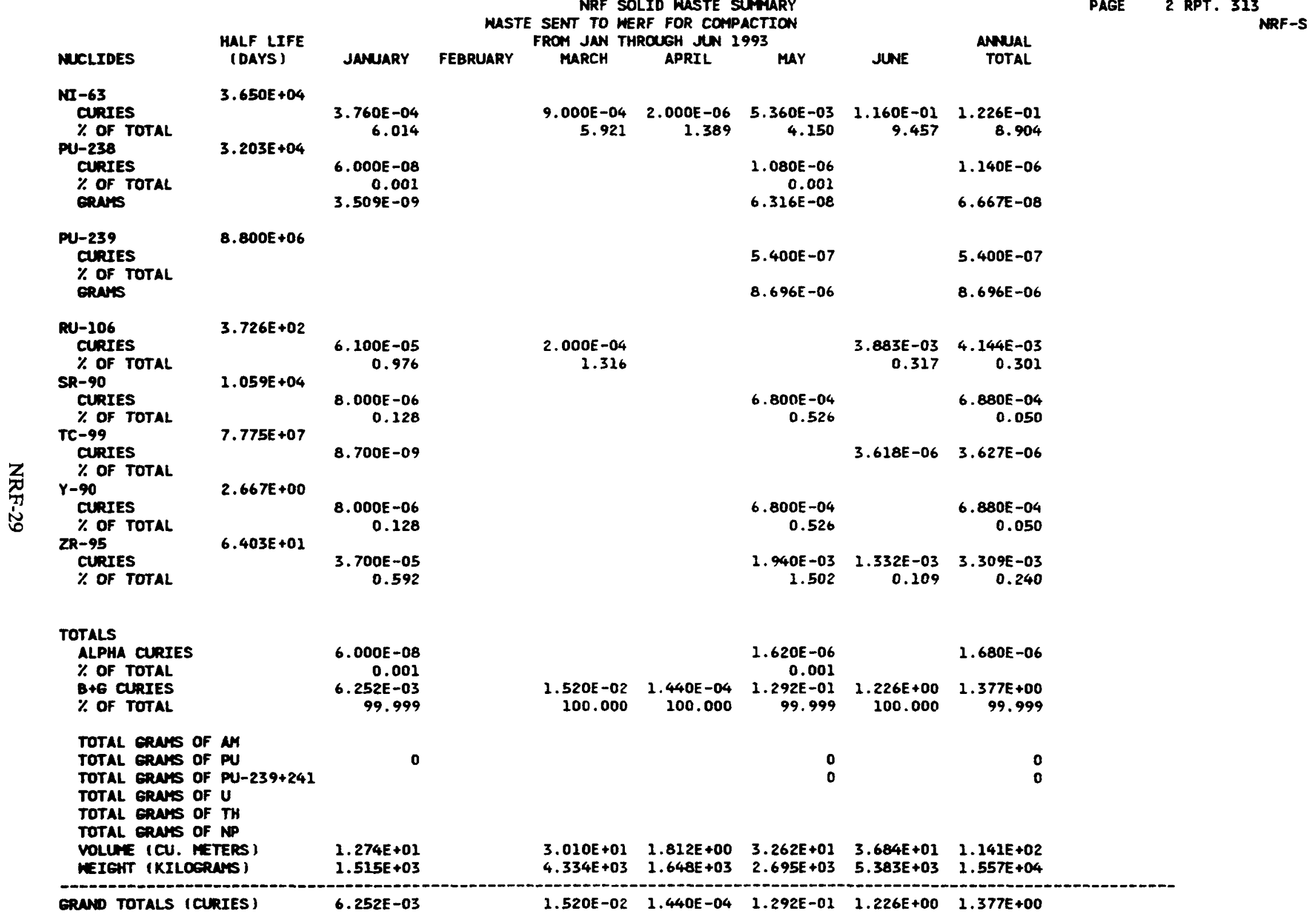


NRF Facility Detail Data for Months July to December Annual Average Concentration and Total Curies for January to December 



\section{AIN AIRBORNE WASTE SUTURY} FOR JAN THROUGH DEC 2993

\begin{tabular}{|c|c|}
\hline MCLIDES & $\begin{array}{l}\text { HALF LI } \\
\text { (DAYS) }\end{array}$ \\
\hline $\begin{array}{l}\text { AR-4I } \\
\text { CONCEN (UCAL) } \\
\text { CURIES } \\
\text { UNCERT(CURIES) } \\
\text { UNCERT(UCAML) } \\
\% \text { OF TOTAL }\end{array}$ & $7.625 \mathrm{E}$ \\
\hline
\end{tabular}

\section{EROSS-BETA}

\section{CONCEN (UCAL) CURIES}

UNCERTICURIES

UNCERTIUCML

$\%$ OF TOTAL

$\mathrm{H}-3$

CONCEN (UCML)

\section{CURrES}

CURIES

UNCERT (UCARL)

UNCERTIUCATI
$\%$ OF TOTAL

$4.490 E+03$

$$
\text { \% of rotal }
$$

TOTALS

ALPHA CURIES

$\%$ OF TOTAL

\% OF TOTAL

$\%$ OF TOTAL

VOLUME (CU. METERS)

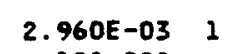

5. 926E-12 1.244E-11

\begin{tabular}{ccc} 
& \multicolumn{2}{c}{ PAGE I RPT. 320} \\
NRF/NRF-A & \\
ANNAL & \multicolumn{1}{c}{ YEARLY } \\
AVG CONCEN YEARLY & RELEASE
\end{tabular}

$\begin{array}{ccccccc}2.700 E+07 & 4.500 E+07 & 3.600 E+07 & 2.300 E+07 & 2.900 E+07 & 2.400 E+07 & 3.760 E+08\end{array}$

ERAND TOTALS (CURIES)

$\begin{array}{lllllll}2.960 E-03 & 1.070 E-03 & 3.010 E-03 & 4.820 E-03 & 4.005 E-04 & 2.044 E-03 & 2.551 E-02\end{array}$

* This nuber is derived from a dispersion factor equation. The dispersion factor is derived from the straight line gouseian modal and is proveded by the National Oceanic Atmospheric Administration (NOAA).

* NOTE: DCG value obtainad from DOE ORDER 5400.5 dated February 8, 1990 , Figures III-1 and III-3 
SCHED. NO. P61PMOO9-H

IDAHO OPERATIONS OFFICE
U.S. DEPARTMENT OF ENERGY

RADIOACTIVE WASTE MANAGEMENT INFORMATION SYSTEM

AIW DISPOSED SOLID WASTE SUMARY

FOR JAN THROUGH DEC 1993

MUCLIDES
BA-13M
CURIES
$\%$ OF TOTAL
CO-58
CURIES
$\%$ OF TOTAL

co-60

CURIES

CR-5I

CURIES

$\%$ OF total

HALF LIFE

(DAYS)

ULY

AUEUST

SEPTEMBER

octoser noverber

7. 091E+01

1. $924 E+03$

$2.770 E+01$
RUN DATE: 05/11/94

PAGE 1 RPT. 322 NRF/AIH-S

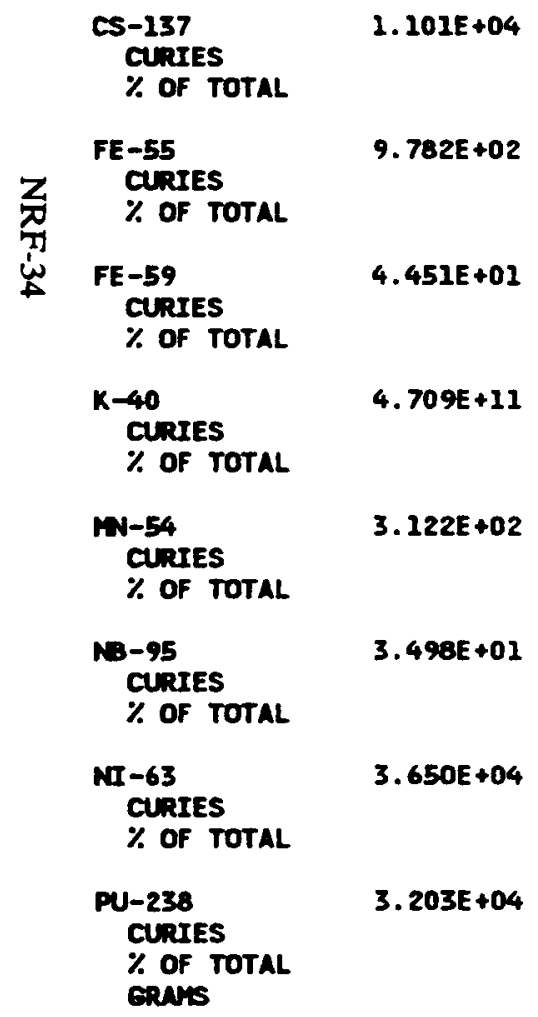

DECEMER

ANNUAL

TOTAL

$2.060 E-03$

1.537

1.336E-02

9.966

6. 741E-02

50.283

4.160E-03

3.103

2.060E-03

1.537

2.863E-02

21.356

$7.100 E-04$

0.530

6.000E-05 0.045

2. $450 E-03$

1.828

4. 360E-03 3.252

5. 480 E- -03 4.088

1.080E-06 0.001

$6.316 E-08$ 
RADIOACTIVE WASTE MANAGEMENT INFORMATION SYSTEM

AIW DISPOSED SOLID MASTE SUMMARY

PAGE 2 RPT. 322 FOR JAN THROUGH DEC 1993

NRF/AIW-S

\begin{tabular}{|c|c|}
\hline MCLIDES & $\begin{array}{l}\text { HALF LIFE } \\
\text { (DAYS) }\end{array}$ \\
\hline $\begin{array}{l}\text { PU-239 } \\
\text { cuRIES } \\
\text { \% OF TOTAL } \\
\text { CRMIS }\end{array}$ & $8.800 E+06$ \\
\hline $\begin{array}{l}\text { SR-90 } \\
\text { CURIES } \\
\text { \% OF TOTAL }\end{array}$ & $1.059 E+04$ \\
\hline $\begin{array}{l}Y-90 \\
\text { CURIES } \\
\% \text { OF TOTAL }\end{array}$ & $2.667 E+00$ \\
\hline $\begin{array}{l}\text { ZR-95 } \\
\text { CURIES }\end{array}$ & $6.403 E+01$ \\
\hline
\end{tabular}

MLL

SEPTEMBER OCTOBER NOVEMBER DECEMBER

ANEAL

TOTAL

$5.400 E-07$

$8.696 E-06$

$6.800 E-04$ 0.507

$6.800 E-04$ 0.507

$1.960 E-03$ 1.462

\section{Totals}

ALPHA CURIES

$\%$ OF TOTAL

B+G CURIES

贫 \% OF TOTAL

$1.620 E-06$ 0.001

$1.341 E-01$

99.998

TOTAL ERAS OF AM

TOTAL ERAYS OF PU

TOTAL ERNSS OF PU-239+241

$759 E-06$

TOTAL ERATS OF $U$

TOTAL ERAMS OF TH

TOTAL ERAMS OF NP

VOLUAE (CU. METERS)

8.759E-06
$8.696 E-06$

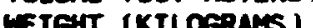

$3.987 E+01$

$6.906 E+03$

crmo Totals (cuRTES)

$1.341 E-01$ 
RADIOACTIVE WASTE MANAGEMENT INFORMATION SYSTEM

AIW-PWDS AIRBOBNE WASTE SUMMRY

FOR JAN THROUGH DEC 1993

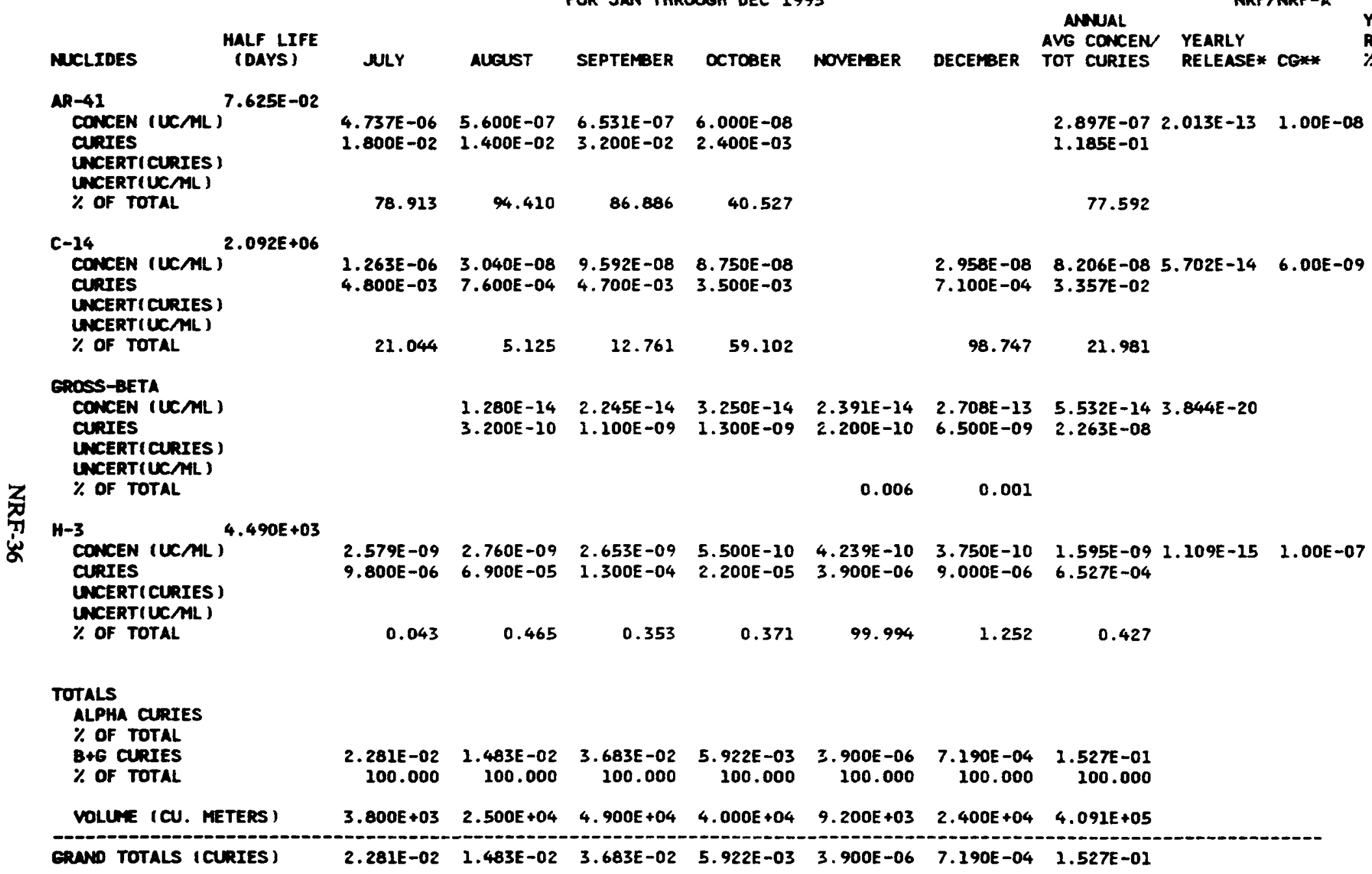

* This nubar is darived from a dispersion factor equation. The dispersion factor is derived from the straight line ouseian modal and is proveded by the National Doeanic Atmospheric Administration (NOAA).

* NOTE: DCG value obtained from DOE ORDER 5400.5 dated February 8, 1990, Figures III-1 and III-3 
IVE WASTE MANAGEMENT INFORMATION SYSTEM

FOR JAN THROUGH DEC 1993

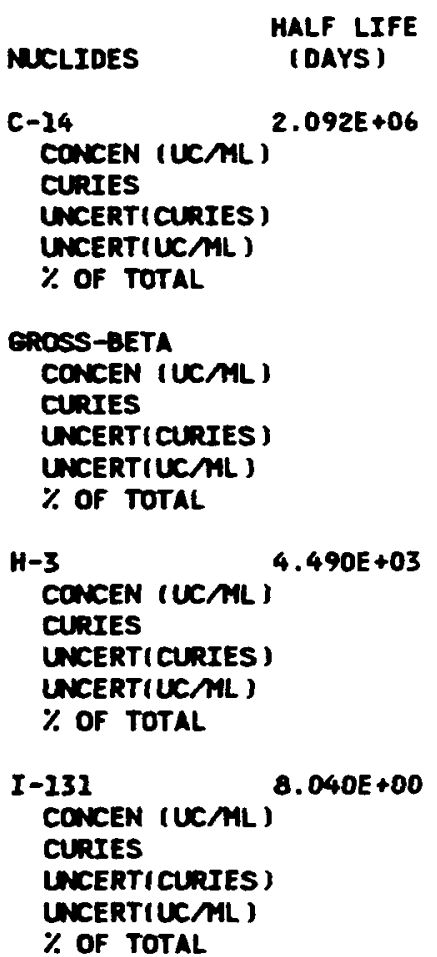

$\boldsymbol{u}$

$\begin{array}{lllllllll}1.042 E-09 & 2.442 E-14 & 8.857 E-10 & 1.343 E-15 & 1.264 E-09 & 3.611 E-14 & 8.207 E-10 & 1.619 E-15 & 6.00 E-09 \\ 7.400 E-02 & 2.100 E-06 & 6.200 E-02 & 9.400 E-08 & 1.100 E-01 & 2.600 E-06 & 7.460 E-01 & & \end{array}$
95.237
0.06290 .510
0.002
92.669
0.035
92.726

$\begin{array}{lllllllll}5.634 E-15 & 5.581 E-15 & 7.286 E-15 & 1.014 E-14 & 1.126 E-14 & 1.389 E-13 & 1.950 E-14 & 3.847 E-20\end{array}$ $\begin{array}{llllllll}4.000 E-07 & 4.800 E-07 & 5.100 E-07 & 7.100 E-07 & 9.800 E-07 & 1.000 E-05 & 1.773 E-05\end{array}$
0.001
0.014
0.001
0.017
0.001
0.135
0.002

$\begin{array}{lllllllll}5.211 E-11 & 3.953 E-11 & 9.286 E-11 & 5.857 E-11 & 1.000 E-10 & 1.028 E-10 & 6.436 E-11 & 1.269 E-16 & 1.00 E-07\end{array}$ $\begin{array}{lllllll}3.700 E-03 & 3.400 E-03 & 6.500 E-03 & 4.100 E-03 & 8.700 E-03 & 7.400 E-03 & 5.850 E-02\end{array}$
4.762
99.914
9.489
99.969
7.329
99.826
7.271

5.352E-15 $3.953 E-15 \quad 4.429 E-15 \quad 6.714 E-15 \quad 9.885 E-15 \quad 4.028 E-15 \quad 5.061 E-15 \quad 9.981 E-21 \quad 4.00 E-10$

$\begin{array}{lllllll}3.800 E-07 & 3.400 E-07 & 3.100 E-07 & 4.700 E-07 & 8.600 E-07 & 2.900 E-07 & 4.600 E-06\end{array}$
0.010
0.011
0.001
0.004
0.001

\section{TOTALS}

ALPHA CURIES

$\%$ OF TOTAL

B+G CURIES

$\%$ of total

7.770E-02 3.403E-03

$403 E-03$
100.000

$.850 E-02$

4. $101 E-03$

$1.187 E-01$

8. 045E-01

$\begin{array}{llllllll}\text { VOLUNE (CU. METERS) } \quad 7.200 E+07 & 8.600 E+07 & 7.000 E+07 & 7.000 E+07 & 8.700 E+07 & 7.200 E+07 & 9.090 E+08\end{array}$

GRND TOTALS (CURIES ]

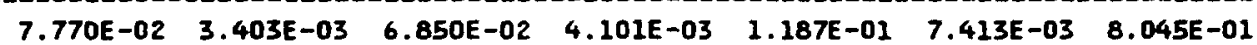

* This muber is darived from a dispersion factor equation. The dispersion factor is derived from the straight line oussian modal and is provedad by the National Dceanze Atwospheric Administration (NOAA).

* NOTE: DCG value obtained from DOE ORDER 5400.5 dated February 8, 1990, figures III-I and III-3 
ECF DISPOSED SOLID WASTE SUMMARY

FOR JAN THROUGH DEC 1993

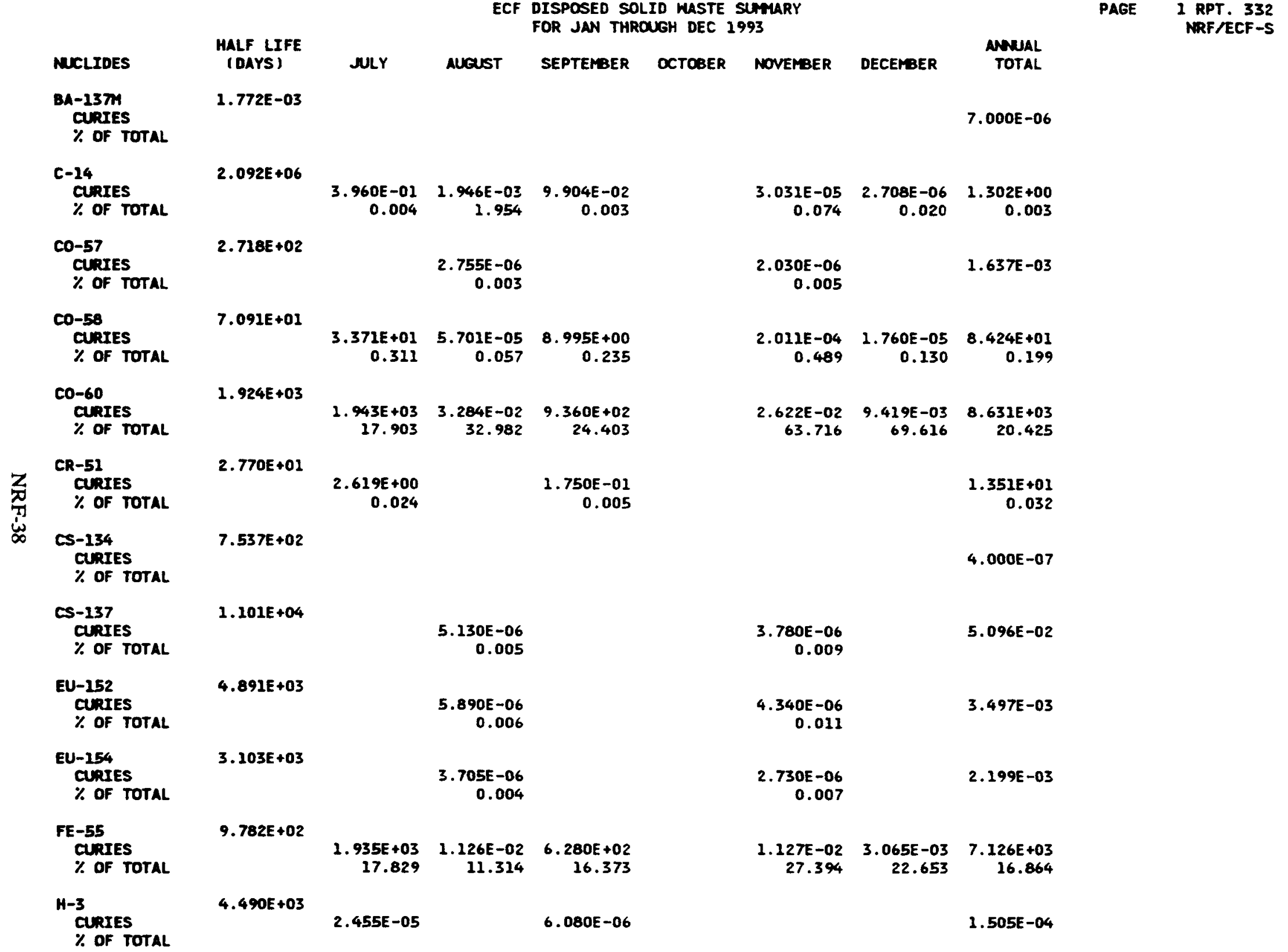




\section{ECF DISPOSED SOLID MASTE SUMARY}

FOR JAN THROUEH DEC 1993

\section{NUCLIDES}

I-129

CURIES

$\%$ OF total

\section{MN-54
CURIES}

$\%$ Of TOTAL

\section{M-95
CURres}

CurIES
$\%$ OF TOTAL

\section{NI-59}

Curres
\% OF TOTAL

NI -63

CURIES

$\%$ Of TOTAL

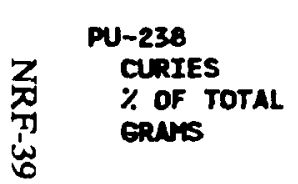

RU-106

CURIES

$\%$ Of TOTAL

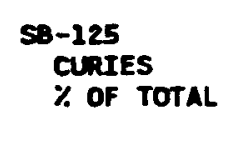

SN-119M

CURIES
$\%$ OF TOTAL

SN-121M

CURIES

$\%$ OF TOTAL
SR-90
curres
$\%$ OF total

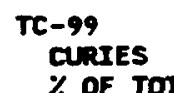

CURIES

\section{HALF LIFE (DAYS) \\ $5.840 E+09$}

$1.611 E-11 \quad 7.809 E-09 \quad 1.460 E-10$

3.122E+02

1.684E-05 3.411E-05 8.000E-04 0.034

$3.498 E+01$

$1.278 E+02 \quad 5.130 E-06 \quad 3.010 E+01$

$\begin{array}{rrr}1.178 & 0.005 & 0.785\end{array}$

$2.774 E+07$

$6.120 E+01$

$1.530 E+01$

0.564

0.399

$3.650 E+04$

6. $070 E+03$

55.928

$5.324 E-02 \quad 2.020 E+03$

$53.478 \quad 52.665$

$3.203 E+04$

$3.726 E+02$

$\begin{array}{rr}2.500 E-05 \quad 9.793 E-05 & 5.847 E-06 \\ 0.098 & \end{array}$

$1.007 E+03$

$1.422 E+02$

$4.620 E+01$

1.205

$2.930 E+02$

4. $450 E+02$

4.200

$1.370 E+02$

3.572

$2.008 E+04$

$1.0596+04$

5. 440E-05

0.055

$7.775 E+07$
$1.111 E-10 \quad 4.400 E-12 \quad 1.359 E-07$

$1.190 E-04 \quad 1.038 E-05 \quad 2.138 E-01$

$$
\begin{array}{rrr}
90 E-04 & 1.038 E-05 & 2.138 E-01 \\
0.289 & 0.077 & 0.001
\end{array}
$$

$\begin{array}{rr}3.780 E-06 & 4.245 E+02 \\ 0.009 & 1.004\end{array}$

1. $938 E+02$

0.459

$\begin{array}{ccc}2.838 E-03 & 8.407 E-04 & 2.355 E+04\end{array}$

$$
\begin{array}{rrr}
6.896 & 6.214 & 55.732
\end{array}
$$

$1.389 E-07$

8. 123E-09

4.514E-04 $1.746 E-04 \quad 5.960 E-03$ $\begin{array}{rr}1.097 & 1.291\end{array}$

$5.264 E+02$

1.246

$1.478 E+03$

3.497

1. 200E-02

4. $639 E-03$

3.031E-08 2.708E-09 4.562E-04
PAgE 2 RPT. 332

NRF/ECF-S 
SCHED. NO. P6IPMOO9-H

IDAHO OPERATIONS OFFICE

U.S. DEPARTMENT OF ENERGY

RADIOACTIVE WASTE MANAGEMENT INFORMATION SYSTEM

ECF DISPOSED SOLID WASTE SUMARY

FOR JAN THROUGH DEC 1993

\section{MUCLIDES}

HALF LIFE

(oars)

JULY

Aucust

SEPTEMBER

OCTOBER

NOVERER

DECEMBR

aNNUAL

TOTAL

8. 000E-06

CLRIES
$\%$ OF TOTAL

$2 R-95$

CUREs

$\%$ OF TOTAL

$6.403 E+01$

$9.234 E+01$

0.851

$2.755 E-06$
0.003

$1.370 E+01$

0.357

Torals

ALPHA CURIES

$\%$ OF TOTAL

B+G CURIES

$\%$ OF TOTAL

TOTAL GRAMS OF AM

TOTAL ERAMS OF PU

TOTAL ERAMS OF PU-239+241

TOTAL ERNAS OF $U$

TOTAL ERNis OF TH

TOTAL eRAMS of MP

VOLUNE (CU. METERS)

MEIGHT (KILOERANS)

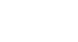
$1.085 E+04 \quad 9.956 E-02 \quad 3.836 E+03$ $100.000 \quad 100.000 \quad 100.000$

$1.704 E+01 \quad 2.634 E+01 \quad 1.818 E+01$

5.950E+01 $1.988 E+01 \quad 3.588 E+02$

$7.374 E+03,355 E+04 \quad 1.252 E+05$
RUN DATE: 05/11/94

PAGE 3 RPT. 332 NRF/ECF $-S$

$1.085 E+04 \quad 9.956 E-02 \quad 3.836 E+03$

4.115E-02 1.353E-02 4.226E+04 
MISC. SOURCES AIRBORNE WASTE SUMMARY

FOR JAN THROUGH DEC 1993

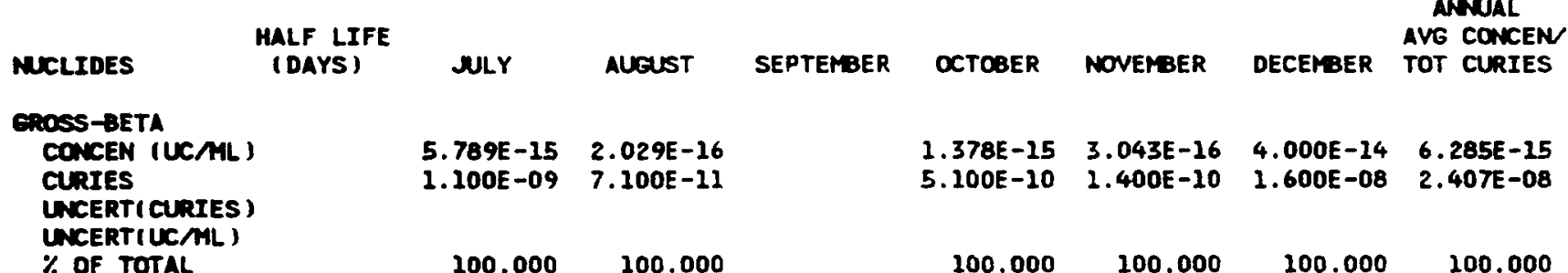

\section{TOTALS}

ALPHA CURIES
$\%$ OF TOTAL

B+G CURIES

$100.000 \quad 100.000$

100.000

100.000

100.000

100.000

Volune ICU. METERS ]

1.100E-09 7.200E-11

100.000

5.100E-10 $\quad 1.400 E-10 \quad 1.600 E-08 \quad 2.407 E-08$

$\begin{array}{llll}100.000 & 100.000 & 100.000 & 100.000\end{array}$

Volure rCU. Reters

$1.900 E+05 \quad 3.500 E+05$

$3.700 E+05 \quad 4.600 E+05 \quad 4.000 E+05 \quad 3.830 E+06$

CRAND TOTALS ICURIES )

1. $100 E-09 \quad 7.100 E-11$

5. $100 \mathrm{E}-10 \quad 1.400 \mathrm{E}-10 \quad 1.600 \mathrm{E}-08 \quad 2.407 E-08$

* This nuber is derived from a disparsion factor equation. The disparsion factor is derived from the straight line omussian wodal and is proveded by the National Oceanic Atmospheric Administration (NOAA).

* MTE: DCG value obtained from DOE ORDER 5400.5 dated Fobruary 8, 1990, Figures III-1 and III-3 
RADIOACTIVE WASTE MANAGEMENT INFORMATION SYSTEM

SIW AIRBORNE WASTE SUMMARY FOR JAN THROUGH DEC 1993

MUCLIDES
EROSS-BETA
CONCEN (UCML)
CURIES
UNCERT(CURIES)
UNCERT(UCML)
\% OF TOTAL

\title{
HALF LIFE
} (DAYS)

JULY

AUEUST

SEPTERBER

OCTOBER

$6.900 E-08$

8.182E-15

$1.000 E-14$

$1.071 E-14$

0.448

\begin{abstract}
0.362
\end{abstract}
0.448

1.014

0.646

CONCEN (UCML)

\section{CURIES}

CURIES

UNCERTICURIES
UNCERTIUCAML

\% OF TOTAL

$2.262 E-12 \quad 1.818 E-12 \quad 9.762 E-13$

$1.900 E-05$

$1.818 E-12$

$9.762 E-13$

$1.647 E-12$

$1.600 E-12$

$1.264 E-12$

$2 \quad 1.395 E-12 \quad 2.134 E-19 \quad 1.00 E-07$

$99.638 \quad 99.552$

98.986

99.354

99.256

90.164

TOTALS

ALPHA CURIES

$\%$ of total

B+G curIES

$\%$ OF TOTAL

$Z$ VOLUME (CU. METERS)

$1.907 E-05,2.009 E-05 \quad 8.2$

$2.009 E-05$

8. $284 E-06$

$1.409 E-05$

$1.612 E-05$

$\begin{array}{rr}1.220 E-05 & 1.524 E-04 \\ 100.000 & 100.000\end{array}$

ERND TOTALS (CuRIES)

$\begin{array}{lllllll}8.400 E+06 & 1.100 E+07 & 8.400 E+06 & 8.500 E+06 & 1.000 E+07 & 8.700 E+06 & 1.077 E+08\end{array}$

$1.907 E-05 \quad 2.009 E-05 \quad 8.284 E-06 \quad 1.409 E-05 \quad 2.612 E-05 \quad 1.220 E-05 \quad 1.524 E-04$

* This nuber is derived from a dispersion factor equation. The dispersion factor is derived from the straight line goussian nodel and is proveded by the Notional Doeanic Atmospheric Administration (NOAA).

** NOTE: DCE value abtained from DOE ORDER 5400.5 dated February 8, 1990 , Figures III-I and III-3 
RADIOACTIVE WASTE MANAGEMENT INFORMATION SYSTEM

SIW DISPOSED SOLID WASTE SUMMARY

\begin{tabular}{|c|c|}
\hline MCLIDES & $\begin{array}{l}\text { HALF LIFE } \\
\text { (DAYS) }\end{array}$ \\
\hline $\begin{array}{l}\text { BA-137M } \\
\text { CURIES } \\
\% \text { OF TOTAL }\end{array}$ & $1.772 E-03$ \\
\hline $\begin{array}{l}\text { CO-60 } \\
\text { CURIES } \\
\% \text { Of TOTAL }\end{array}$ & $1.924 E+03$ \\
\hline $\begin{array}{l}\text { CS-137 } \\
\text { cURIES } \\
\% \text { OF TOTAL }\end{array}$ & $1.101 E+04$ \\
\hline $\begin{array}{l}\text { FE-55 } \\
\text { cURIES } \\
\% \text { OF TOTAL }\end{array}$ & $9.782 E+02$ \\
\hline $\begin{array}{l}\text { NT-63 } \\
\text { CURIES } \\
\% \text { OF TOTAL }\end{array}$ & $3.650 E+04$ \\
\hline $\begin{array}{l}\text { SR-90 } \\
\text { CURIES } \\
\% \text { OF TOTAL }\end{array}$ & $1.059 E+04$ \\
\hline $\begin{array}{l}Y-90 \\
\text { CURIES } \\
\% \text { OF TOTAL }\end{array}$ & $2.667 E+00$ \\
\hline
\end{tabular}

\section{TOTALS}

ALPHA CURIES

$\%$ OF TOTAL

B+G CURIES

$\%$ OF TOTAL

TOTAL GRANS OF AM

TOTAL ERANS OF PU

TOTAL GRANS of PU-239+241

TOTAL ERAYS OF $U$

TOTAL ERANS OF TH

TOTAL ERMNS OF NP

VOLUAE (CU. METERS )

VEIGHT (KILOERANS)

eRNo TOTALS (CURIES) FOR JAN THROUGH DEC 1993

JULY

Aucust

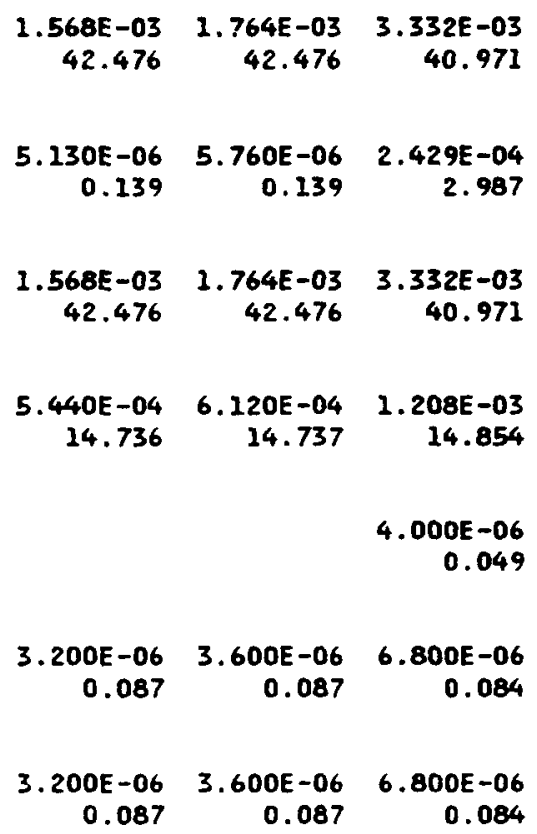

$3.328 E+00 \quad 3.947 E+00 \quad 1.271 E+01$

$5.539 E+03 \quad 6.110 E+03 \quad 1.500 E+04$

3.692E-03 4.153E-03 8.132E-03 
$\begin{array}{ccccccccc}8.947 E-10 & 3.750 E-10 & 1.526 E-09 & 2.000 E-09 & 6.333 E-10 & 4.200 E-10 & 7.353 E-10 & 1.483 E-16 & 1.00 E-08\end{array}$ 1.700E-02 9.000E-03 2.900E-02 3.800E-02 2.000E-02 B.400E-03 1.831E-01
59.275
29.450
54.368
89.919
63.472
42.445
58.199

$\begin{array}{ccccccccc}5.789 E-10 & 8.750 E-10 & 1.263 E-09 & 2.000 E-10 & 4.583 E-10 & 5.500 E-10 & 5.092 E-10 & 1.027 E-16 & 6.00 E-09\end{array}$

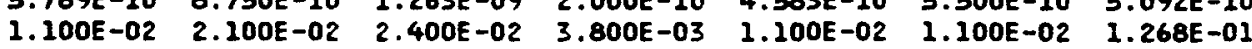
38.354
68.717
44.994
8.992
34.910
55.583
40.304
$\begin{array}{llllllll}1.421 E-15 & 7.083 E-16 & 8.947 E-16 & 1.842 E-15 & 7.917 E-16 & 1.450 E-14 & 2.948 E-15 & 5.943 E-22\end{array}$ $\begin{array}{lllllll}2.700 E-08 & 1.700 E-08 & 1.700 E-08 & 3.500 E-08 & 1.900 E-08 & 2.900 E-07 & 7.340 E-07\end{array}$

\subsection{1}

$\begin{array}{lllllllll}3.579 E-11 & 2.333 E-11 & 1.789 E-11 & 2.421 E-11 & 2.125 E-11 & 1.950 E-11 & 1.892 E-11 & 3.814 E-18 & 1.00 E-07\end{array}$ $\begin{array}{lllllll}6.800 E-04 & 5.600 E-04 & 3.400 E-04 & 4.600 E-04 & 5.100 E-04 & 3.900 E-04 & 4.711 E-03\end{array}$
2.37
1.832
0.637
1.086
1.619
1.971
1.497

TOTALS

ALPHA CURIES
$\%$ OF TOTAL

$\%$ OF TOTAL
B+G CURIES

$\%$ OF TOTAL

2.868E-02 3.0

3.056E-02

$100.000 \quad 100.000$

.334E-02

$226 E-02$
00.000

$3.151 E-02$
100.000

3. $979 E-02$

$3.146 E-01$

VOLUAE (CU. METERS )

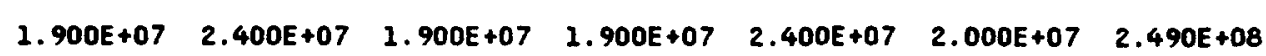

CRND TOTALS (CURTES)

$2.868 E-02 \quad 3.056 E-02 \quad 5.334 E-02 \quad 4.226 E-02 \quad 3.151 E-02 \quad 1.979 E-02 \quad 3.146 E-01$

* This nubar is derived from a dispersion factor eqution. The dispersion factor is derived from the straight line coussian wodel and is proveded by the Mtional Oceanic Atmospheric Administration (NOAl).

* NOTE: DCG value obtained from DOE ORDER 5400.5 dated February 8, 1990, Figures III-1 and III-3 
RADIOACTIVE WASTE MANAGEMENT INFORMATION SYSTEM

S5G DISPOSED SOLID MASTE SUMMARY

FOR JAN THROUGH DEC 1993

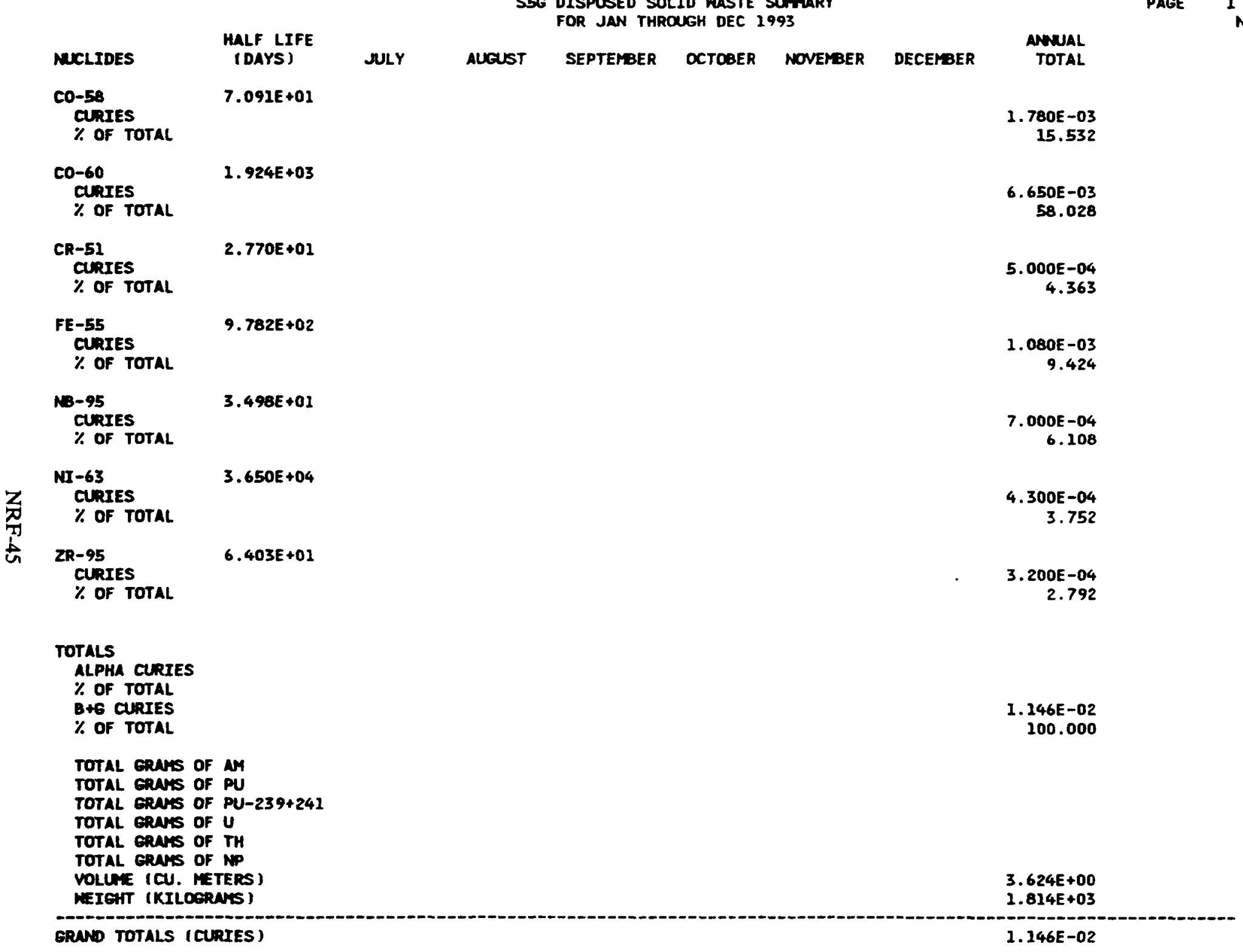



NRF Facility Detail Data for Months January to June Annual Average Concentration and Total Curies for January to June 
. 


\section{RADIOACTIVE MASTE MANAGEMENT INFORMATION SYSTEM}

AIW AIRBORNE WASTE SUMARY
FOR JAN THROUGH JUN 1993

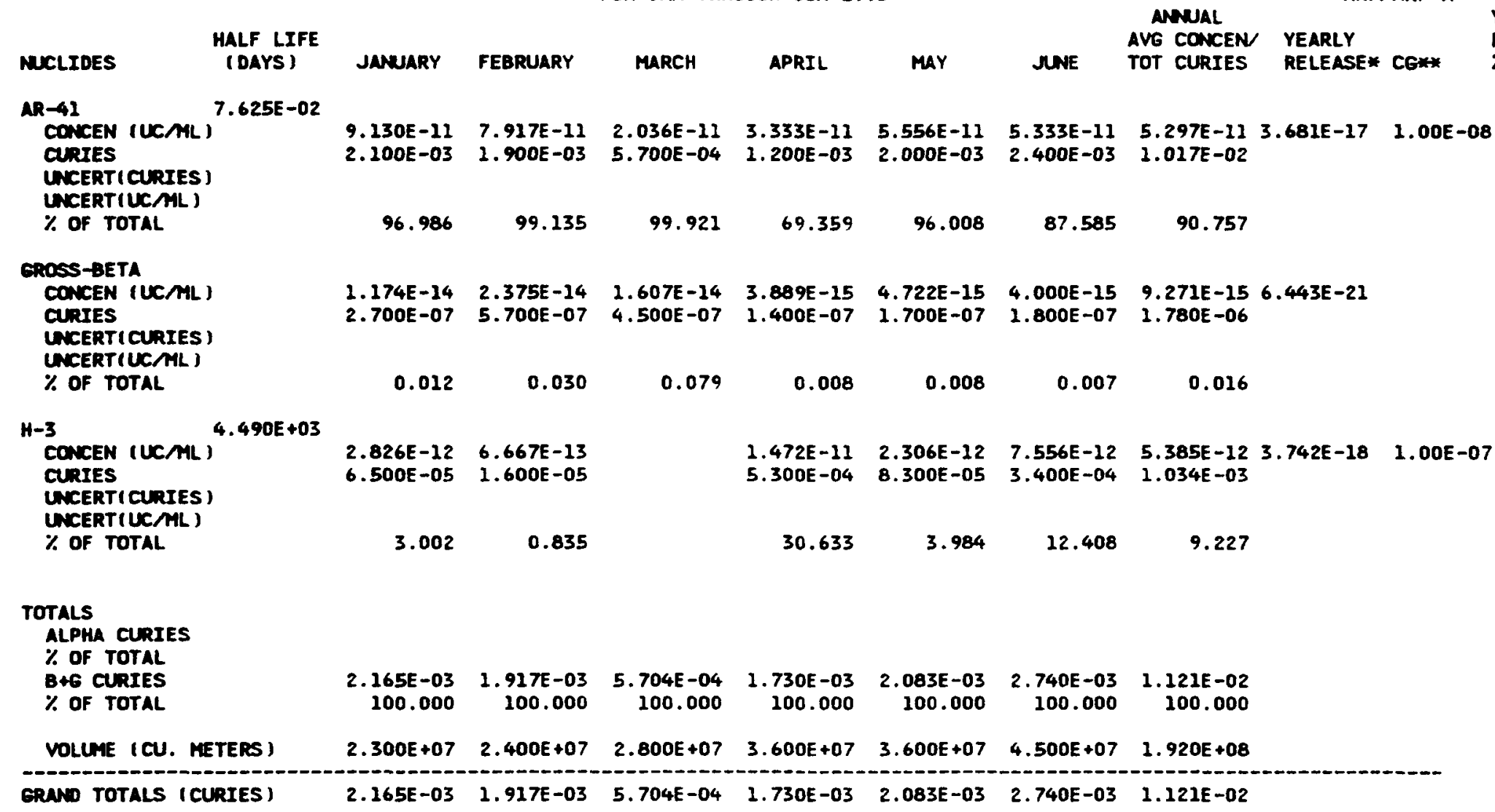

* This muber is derived from a dispersion factor equation. The dispersion factor is derived from the straight line onuseien model and is proveded by the National Dceanic Atmospheric Adninistration (NOAa).

* NOTE: DCG value obtained from DOE ORDER 5400.5 dated Fabruary 8,1990 , Figures III-I and III-3 
AIW DISPOSED SOLID WASTE SUMMARY

$$
\text { FOR JAN THROUGH NUN } 1993
$$

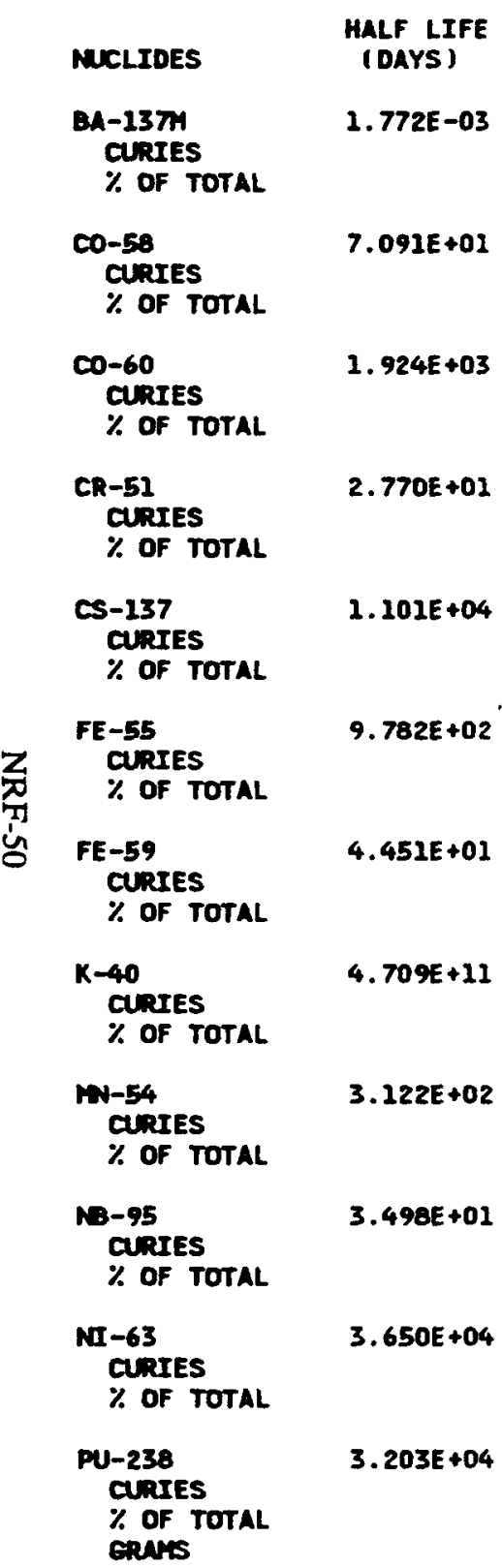

JANUARY FEBRUARY

MARCH

APRIL

MaY

JUNE

ANUAL

TOTAL

2.060E-03

$2.060 E-03$ 2.537

$\begin{array}{rrr}3.200 E-04 & 1.304 E-02 & 1.336 E-02 \\ 6.544 & 10.095 & 9.966 \\ & & \\ 2.200 E-03 & 6.521 E-02 & 6.741 E-02 \\ 44.990 & 50.483 & 50.283\end{array}$

$\begin{array}{lll}1.200 E-04 & 4.040 E-03 & 4.160 E-03\end{array}$

3.103

2.060E-03

1.537

37.014

$2.682 E-02$

20.763

$2.863 E-02$ 21.356

5.000E-05 6.600E-04 1.022 0.511

$7.100 E-04$ 0.530

$6.000 E-05$ 0.046

6.000E-05 0.045

$2.000 E-04 \quad 2.250 E-03$ $4.090 \quad 1.742$

$2.450 E-03$ 1.828

$\begin{array}{rr}5.000 E-05 & 4.310 E-03 \\ 1.022 & 3.337\end{array}$

4. $360 E-03$ 3.252

1.200E-04 5.360E-03 $2.454 \quad 4.150$

5. $480 E-03$ 4.088

$\begin{array}{rr}1.080 E-06 & 1.080 E-06 \\ 0.001 & 0.001 \\ 6.316 E-08 & 6.316 E-08\end{array}$

PAge 1 RPT. 322 NRF/AIH-S 

RADIOACTIVE WASTE MANAGEMENT INFORMATION SYSTEM

AIN DISPOSED SOLID WASTE SUMMARY

FOR JAN THROUGH JUN 1993

\begin{tabular}{|c|c|}
\hline MULIDES & $\begin{array}{l}\text { HALF LIFE } \\
\text { (DAYS) }\end{array}$ \\
\hline $\begin{array}{l}\text { PU-239 } \\
\text { CURIES } \\
\% \text { OF TOTAL } \\
\text { GRUYS }\end{array}$ & 8. $800 E+06$ \\
\hline $\begin{array}{l}\text { SR-90 } \\
\text { CURIES } \\
\% \text { OF TOTAL }\end{array}$ & $1.059 E+04$ \\
\hline $\begin{array}{l}Y-90 \\
\text { CURIES } \\
\% \text { OF TOTAL }\end{array}$ & $2.667 E+00$ \\
\hline $\begin{array}{l}\text { ZR-95 } \\
\text { CURIES }\end{array}$ & $6.403 E+01$ \\
\hline
\end{tabular}

$\%$ OF TOTAL

\section{TOTALS}

ALPHA CURIES

$\%$ OF TOTAL

B+6 CLRIES

$\%$ OF TOTAL

TOTAL GRNS OF AM

TOTAL ERAMS OF PU

TOTAL ERANS of PU-239+241

TOTAL GRANS OF $U$

TOTAL ERAMS OF TH

TOTAL ERATS OF NP

VOLLAE (CU. METERS)

MEIEHT (KILOGRASS

JANUARY FEBRUARY

MARCH

APRIL

MaY

JNE

5. 400E-07

$5.400 E-07$

8.696E-06

$8.696 E-06$

6. $800 E-04$ 0.526

$6.800 E-04$ 0.507

$6.800 E-04$
0.526

2.000E-0E

1. $940 E-03$ 0.409 2.502 1.462

$\begin{array}{rrr}1.620 E-06 & 1.620 E-06 \\ 4.890 E-03 & 1.292 E-01 & 0.001 \\ 100.000 & 99.999 & 1.341 E-01 \\ & & 99.998 \\ & 8.759 E-06 & \\ 8.696 E-06 & 8.759 E-06 \\ & & 8.696 E-06\end{array}$

$7.248 E+00 \quad 3.262 E+01$ 4.211E+03 2.695E+03

$.987 E+01$ 6. $906 E+03$ 
RADIOACTIVE WASTE MUNAGEMENT INFORMATION SYSTEM

AIW-RWDS AIRBORNE WLSTE SUMMARY FOR JAN THROUGH JUN 1993

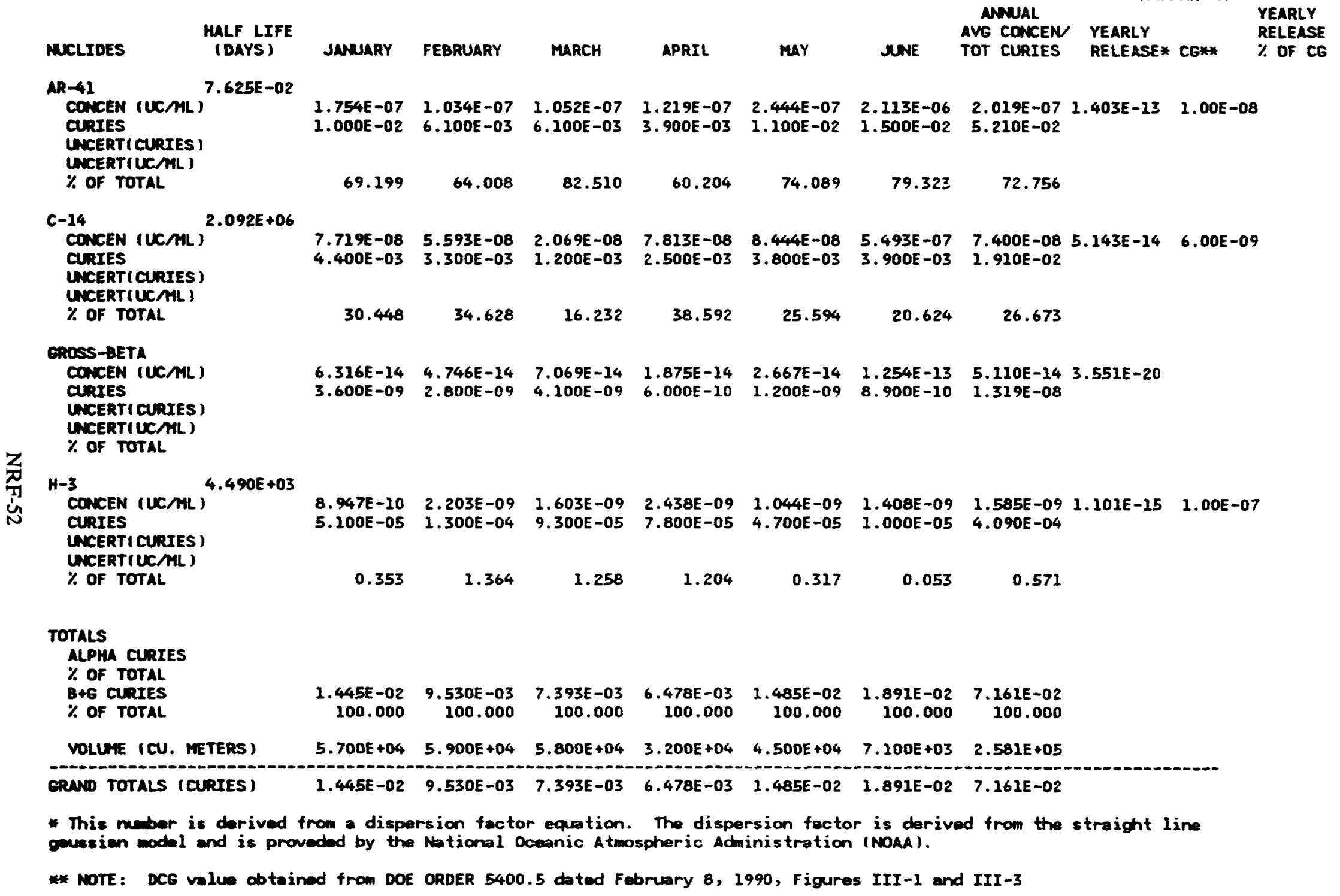


RADIOACTIVE WASTE MANAGEMENT INFORMATION SYSTEM

FOR JAN THROUGH JUN 1993

MULIDES MLLF LIFE
(DAYS)
C-14
CONCEN (UCML)
CURIES
UNCERTICURIES)
UNCERTIUCAML)
\% OF TOTAL
EROSS-BETA
CONCEN (UCML)
CURIES
UNCERTICURIES)
UNCERTIUCAML)
\% OF TOTAL

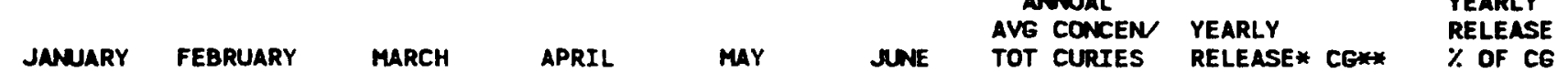

H-3
CONCEN (UCML)
CURIES
UNCERT(CURIES)
UNCERT(UCML)
\% OF TOTAL
I-131
CONCEN (UCML)
CURIES
UNCERT(CURIES)
UNCERT(UCML)
\% OF TOTAL
8.857E-10
$6.200 E-02$
6. $944 E-10 \quad 2.000 E-09$
$5.217 E-10 \quad 1.571 E-09$
8. $276 E-10$
$1.104 E-09 \quad 2.177 E-15 \quad 6.00 E-09$

$$
91.175
$$

93.805

98.436

86.955

97.258

$1.157 E-14$
$8.100 E-07$

$2.361 E-14 \quad 1.176 E-14$

4. $203 E-15$

0.001

0.003

0.001

0.001

$5.571 E-15 \quad 5.287 E-15$

95.291

$\begin{array}{llllllllll}8.571 E-11 & 4.583 E-11 & 3.176 E-11 & 7.826 E-11 & 4.429 E-11 & 4.828 E-11 & 5.453 E-11 & 1.075 E-16 & 1.00 E-07\end{array}$ $\begin{array}{lllllll}6.000 E-03 & 3.300 E-03 & 2.700 E-03 & 5.400 E-03 & 3.100 E-03 & 4.200 E-03 & 2.470 E-02\end{array}$
8.823
6.191
1.563
13.043
2.741
$\mathbf{5 . 5 1 2}$
4.707

$6.000 E-15 \quad 4.167 E-15 \quad 3.765 E-15 \quad 4.058 E-15 \quad 5.000 E-15 \quad 3.218 E-15 \quad 4.305 E-15 \quad 8.490 E-21 \quad 4.00 E-10$ $\begin{array}{lllllll}4.200 E-07 & 3.000 E-07 & 3.200 E-07 & 2.800 E-07 & 3.500 E-07 & 2.800 E-07 & 1.950 E-06\end{array}$
0.00
0.001
0.001

\section{TOTALS}

\begin{tabular}{|c|c|c|c|c|c|c|c|}
\hline $\begin{array}{l}\text { B+G curies } \\
\% \text { of total }\end{array}$ & $\begin{array}{r}6.800 E-02 \\
100.000\end{array}$ & $\begin{array}{r}5.330 E-02 \\
100.000\end{array}$ & $\begin{array}{r}1.727 E-01 \\
100.000\end{array}$ & $\begin{array}{r}4.140 E-02 \\
100.000\end{array}$ & $\begin{array}{r}1.131 E-01 \\
100.000\end{array}$ & $\begin{array}{r}7.620 E-02 \\
100.000\end{array}$ & $\begin{array}{r}5.247 E-01 \\
100.000\end{array}$ \\
\hline VOLUME (CU. METERS) & $7.000 E+07$ & $7.200 E+07$ & $8.500 E+07$ & $6.900 E+07$ & $7.000 E+07$ & $8.700 E+07$ & $4.530 E+08$ \\
\hline & $6.800 E-02$ & $330 E-02$ & $2.727 E-01$ & $4.140 E-02$ & $1.232 E-01$ & $7.620 E-02$ & $5.247 E-01$ \\
\hline
\end{tabular}

* This nubar is derived from a dispersion factor equetion. The dispersion factor is derived from the straight line goussian model and is proveded by the National Doeanic Atmospheric Administration (NOMA).

* NOTE: DCG value obtained from DOE ORDER 5400.5 dated February 8, 1990 , Figures III-1 and III-3 
IDAHO OPERATIONS OFFICE

U.S. DEPARTMENT OF ENERGY

RADIOACTIVE MUSTE MANAGEMENT INFORMATION SYSTEM

ECF DISPOSED SOLID WUSTE SUMAARY FOR JAN THROUEH JUN 2993

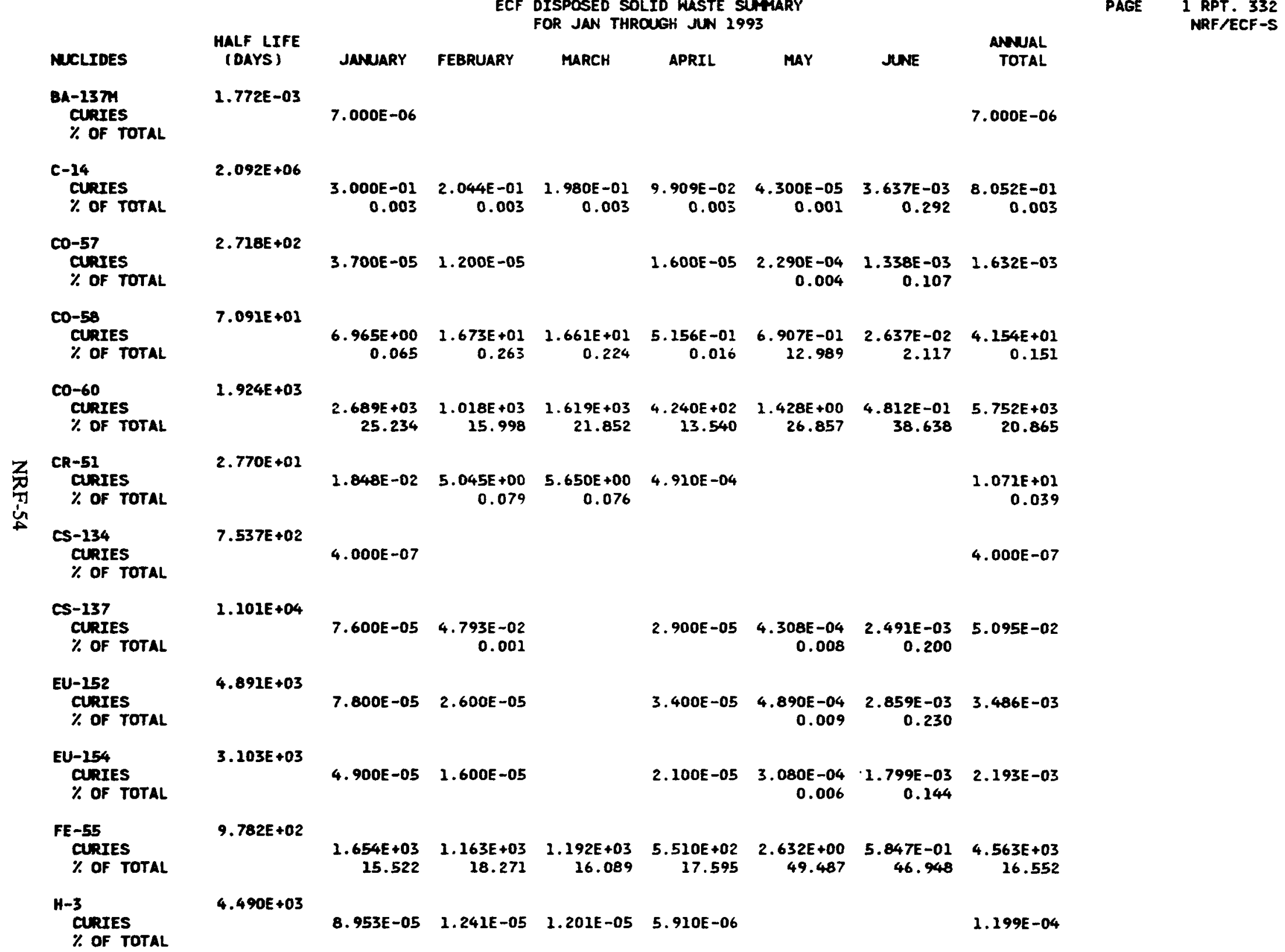


ECF DISPOSED SOLIO WASTE SUMMARY

FOR JAN THROUGH JUN 1993

\begin{tabular}{|c|c|c|c|c|c|c|c|c|}
\hline MUCLIDES & $\begin{array}{l}\text { HALF LIFE } \\
\text { (DAYS) }\end{array}$ & JANUARY & FEBRUARY & MARCH & APRIL & MAY & JUNE & $\begin{array}{l}\text { ANNUAL } \\
\text { TOTAL }\end{array}$ \\
\hline $\begin{array}{l}\text { I- } 129 \\
\text { CURIES } \\
\% \text { OF TOTAL }\end{array}$ & $5.840 E+09$ & $3.200 E-11$ & $1.139 E-07$ & & $3.400 E-10$ & $1.580 E-10$ & $1.334 E-08$ & $1.278 E-07$ \\
\hline $\begin{array}{l}\text { MN-54 } \\
\text { CURIES } \\
\% \text { OF TOTAL }\end{array}$ & $3.122 E+02$ & $8.300 E-05$ & $\begin{array}{r}9.812 E-02 \\
0.002\end{array}$ & & $3.760 E-04$ & $\begin{array}{r}9.848 E-02 \\
1.852\end{array}$ & $\begin{array}{r}1.579 E-02 \\
1.268\end{array}$ & $\begin{array}{r}2.128 E-01 \\
0.001\end{array}$ \\
\hline $\begin{array}{l}\text { M-95 } \\
\text { CURIES } \\
\% \text { OF TOTAL }\end{array}$ & $3.498 E+01$ & $\begin{array}{r}1.966 E+01 \\
0.184\end{array}$ & $\begin{array}{r}1.197 E+02 \\
1.880\end{array}$ & $\begin{array}{r}1.245 E+02 \\
1.681\end{array}$ & $\begin{array}{r}2.690 E+00 \\
0.086\end{array}$ & $\begin{array}{r}4.260 E-04 \\
0.008\end{array}$ & $\begin{array}{r}2.491 E-03 \\
0.200\end{array}$ & $\begin{array}{r}2.666 E+02 \\
0.967\end{array}$ \\
\hline $\begin{array}{l}\text { NI-59 } \\
\text { CURIES } \\
\% \text { OF TOTAL }\end{array}$ & $2.774 E+07$ & $\begin{array}{r}4.420 E+01 \\
0.425\end{array}$ & $\begin{array}{r}2.720 E+01 \\
0.427\end{array}$ & $\begin{array}{r}3.060 E+01 \\
0.413\end{array}$ & $\begin{array}{r}1.530 E+01 \\
0.489\end{array}$ & & & $\begin{array}{r}1.173 E+02 \\
0.425\end{array}$ \\
\hline $\begin{array}{l}\text { NI- } 63 \\
\text { CURIES } \\
\% \text { OF TOTAL }\end{array}$ & $3.650 E+04$ & $\begin{array}{r}5.820 E+03 \\
54.617\end{array}$ & $\begin{array}{r}3.601 E+03 \\
56.565\end{array}$ & $\begin{array}{r}4.030 E+03 \\
54.394\end{array}$ & $\begin{array}{r}2.010 E+03 \\
64.183\end{array}$ & $\begin{array}{r}4.662 E-01 \\
8.766\end{array}$ & $\begin{array}{r}1.173 E-01 \\
9.422\end{array}$ & $\begin{array}{r}1.546 E+04 \\
56.082\end{array}$ \\
\hline
\end{tabular}

$\begin{array}{ll} & \text { PU-238 } \\ \text { Z } & \text { CURIES } \\ \text { Tu } & \% \text { OF TOTAL } \\ \text { in } & \text { ERATS } \\ \text { in } & \end{array}$

$3.203 E+04$

$6.000 E-08$

3.509E-09

$7.890 E-08$

$1.389 E-07$

RU-106

CURIES
$\%$ OF TOTAL

3. $726 E+02$

6. $100 E-$

SB-125

$\%$ OF TOTAL

SN-IIMH

CURIES
$\%$ OF TOTAL

SN-121M

$\%$ OF TOTAL

$5 R-90$

CURIES

$\%$ OF TOTAL

1.007E +03

$1.244 E+02$

$$
\begin{array}{rrrr}
244 E+02 & 8.520 E+01 & 8.780 E+01 & 4.060 E+01 \\
1.167 & 1.338 & 1.185 & 1.296
\end{array}
$$

4.614E-09

8. $123 E-09$

$2.930 E+02$

\section{$2.886 E+02$}

$$
2.708
$$

$2.750 E+02$

4.320

$\begin{array}{rr}2.460 E+02 & 8.620 E+01 \\ 3.320 & 2.753\end{array}$

2.753

$\begin{array}{rrr}4.330 E-04 & 4.073 E-03 & 5.215 E-03 \\ 0.008 & 0.327 & \end{array}$

$2.008 E+04$

1.200E-02

$3.380 E+02$

1.226

$8.958 E+02$

3.249

1.200E-02

$1.059 E+04$

$2.044 E-05 \quad 4.574 E-03$

4. $584 E-03$

CURIES

$\%$ OF TOTAL

$7.775 E+07$

$\begin{array}{lllllll}9.621 E-05 & 8.783 E-05 & 6.660 E-05 & 3.339 E-05 & 4.310 E-08 & 3.637 E-06 & 2.877 E-04\end{array}$

PAGE 2 RPT. 332

NRF/ECF-S 
SCHED. NO. P61PMOO9-H

U.S. DEPARTMENT OF ENERGY

RADIOACTIVE WASTE MANAGEMENT INFORMATION SYSTEM

ECF OISPOSED SOLID NASTE SUMAARY FOR JAN THROUGH JUN 1993

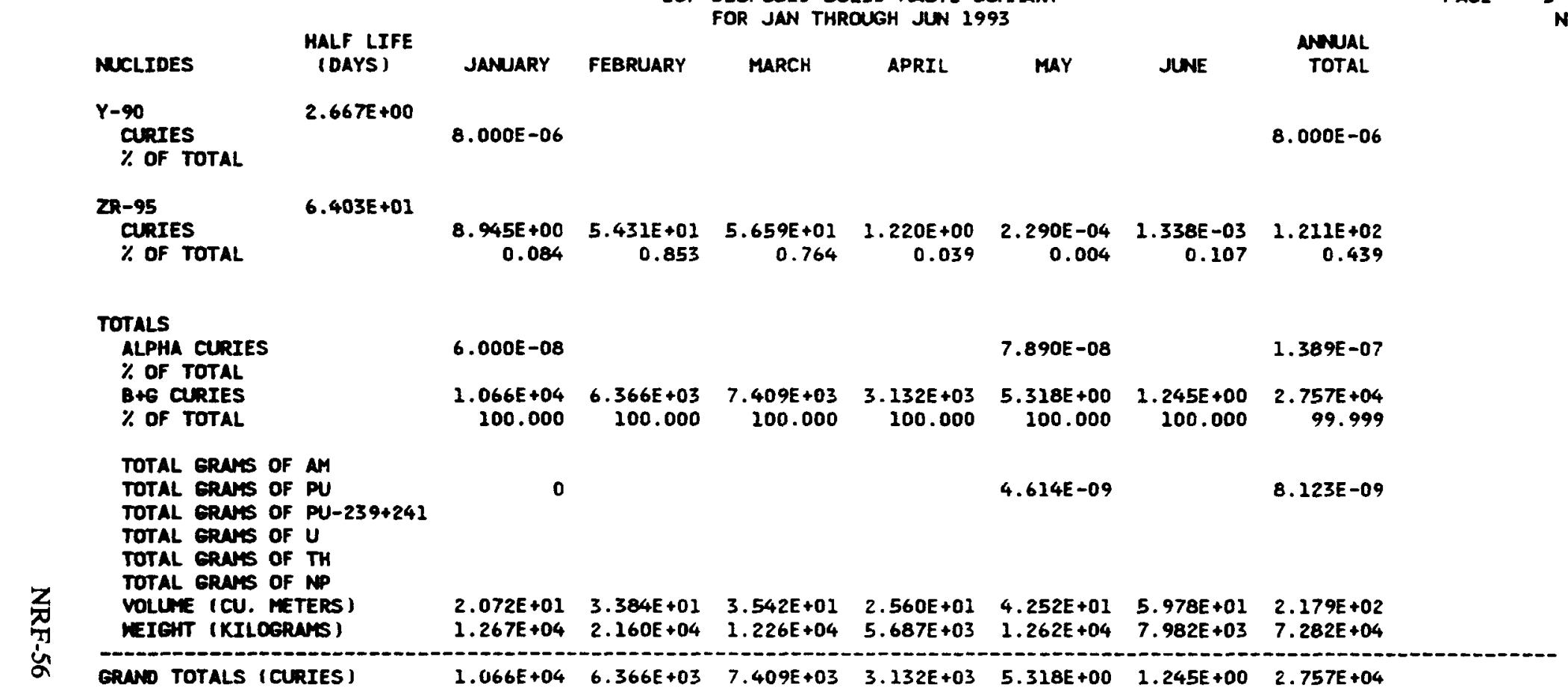

RUN DATE: 05/12/94

3 RPT. 332
NRF/ECF-S 
RADIOACTIVE MUSTE MANAGEMENT INFORMATION SYSTEM

MISC. SOURCES AIRBORNE WASTE SUMHARY FOR JAN THROUGH JUN 1993

NUCLIDES
ERCSS-BETA
CONCEN (UCAL)
CURIES
UNCERT (CURIES)
UNCERT (UCAL)
\% OF TOTAL

\section{HALF LIFE}

(DAYS)
MARCH

APRIL

MAY

$6.486 E-15 \quad 3.421 E-15$

$\begin{array}{ll}6.406 E-15 & 3.421 E-15 \\ 2.400 E-09 & 1.300 E-09\end{array}$

$3.810 E-15$
$1.600 E-09$

$\begin{array}{ll}8.919 E-16 & 1.542 E-15 \\ 3.300 E-10 & 3.700 E-10\end{array}$

100.000

100.000

100.000

100.000
ANNUAL

AVG CONCEN TOT CURIES

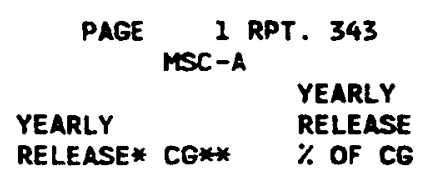

\section{TOTALS}

$$
\begin{aligned}
& \text { ALPHA CURIES } \\
& \% \text { OF TOTAL } \\
& \text { B+G CURIES } \\
& \% \text { OF TOTAL }
\end{aligned}
$$
$2.400 E-09 \quad 1.3$

VOLUHE (CU. METERS)

100.000

$\begin{array}{lllllll}3.700 E+05 & 3.800 E+05 & 4.200 E+05 & 3.700 E+05 & 2.400 E+05 & 2.800 E+05 & 2.060 E+06\end{array}$

$1.600 E-09$ $\begin{array}{rr}600 E-09 & 3.300 E-10 \\ 100.000 & 100.000\end{array}$

. $700 E-10$

$2.500 E-10$

6. $250 E-09$

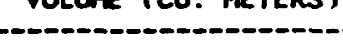

GRAND TOTALS (CURIES) $2.400 E-09$ 1.300E-09 $1.600 E-09 \quad 3.300 E-10 \quad 3.700 E-10 \quad 2.500 E-10 \quad 6.250 E-09$

* This muber is derived from a dispersion factor equation. The dispersion factor is derived from the straight line coussian modal and is proveded by the National Oceanic Atmospheric Administration (NOAa).

* NOTE: DCG value obtainod from DOE ORDER 5400.5 dated february 8, 1990, Figures III-1 and III-3 
RADTOACTIVE WASTE MANAGEMENT INFORMATION SYSTEM

SIW AIRBORNE MASTE SUMMARY FOR JAN THROUEH JUN 1993

UCLIDES
OAOSS-BETA
CONCEN (UCML)
CURIES
UNCERTICURIES)
UNCERTIUCML)
\% OF TOTAL

$H-3$

COACEN (UCML) 4.490E+03

CONCEN (UCML)

CURIES

UNCERT (UCML)

$\%$ of total

\section{HALF LIFE}

(DAYS)

JANUARY FEBRUARY

MARCH

APRIL

MaY

JNE

$\begin{array}{llllll}9.605 E-15 & 1.724 E-14 & 1.400 E-14 & 5.595 E-15 & 6.471 E-15\end{array}$

$\begin{array}{lllll}7.300 E-08 & 1.500 E-07 & 1.400 E-07 & 4.700 E-08 & 5.500 E-08\end{array}$

$8.421 E-15 \quad 1.034 E-14 \quad 1.583 E-21$

$0.484 \quad 100.000 \quad 100.000$

0.223

100.000

0.307

0.871

$1.974 E-12$

2.500E-12

$2.100 E-05$

$\begin{array}{llll}2.737 E-12 & 1.176 E-12 & 1.801 E-19 & 1.00 E-07 \\ 2.600 E-05 & 6.200 E-05 & \end{array}$

99.516

99.777

99.693

99.129

\section{TOTALS}

ALPHA CURIES

$\%$ OF TOTAL

B+e curres

$\%$ OF TOTAL

507E-05 $\quad 1.500 E-07 \quad 1.400 E-07$

$\begin{array}{lrr}100.000 & 100.000 \quad 100.000\end{array}$

2. $105 E-05$ 100.000

5.500E-08 100.000

$2.608 E-05$ 100.000

$6.254 E-05$ 100.000

Z VOLUNE (CU. METERS)

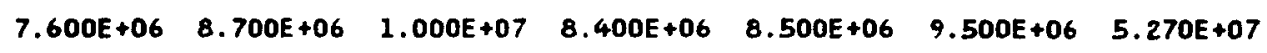
GRAN TOTALS (CURIES)

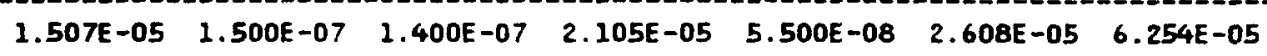

* This nuber is derived from a dispersion factor eqation. The dispersion factor is derived from the straight line oussian model and is proveded by the National Oceanic Atmospheric Adninistration (NOAA).

* NOTE: DCG value obtainod from DOE OROER 5400.5 dated february 8, 1990 , Figures III-1 and III-3 PAGE I RPT. 310
NRF/SIW-A

YEARLY RELEASE

$\%$ OF CG 
SCHEO. NO. P61PMOO9-H

IDAHO OPERATIONS OFFICE

U.S. DEPARTMENT OF ENERGY

RUN DATE: 05/12/94

RADIOACTIVE WASTE MANAGEMENT INFORMATION SYSTEM

SIK DISPOSED SOLID WASTE SUMTARY

FOR JAN THROUGH JUN 2993

MALF LIFE
(OAYS)

$1.924 E+03$

co-60

CURIES

$\%$ OF TOTAL

\section{FE-55 \\ $9.782 E+02$}

CURIES

$\%$ OF TOTAL

MI-63

\section{CURIES}

$\%$ OF TOTAL

$3.650 E+04$

TOTALS

ALPHA CURIES

$\%$ OF TOTAL

B+G CURIES

$\%$ OF TOTAL

TOTAL ERMS OF AM

TOTAL ERANS Of PU

TOTAL ERAYS OF PU-239+241

TOTAL ERATS OF $U$

TOTAL ERAHS OF TH

TOTAL ERAYS OF NP

VOLUAE (CU. METERS )

MEIGHT (KILOERANS)

$2.880 E-04$

100.000

2. 880E-04

100.000

$5.436 E+00$

$5.436 E+00$

$3.355 E+03$

$3.355 E+03$

ERND TOTALS (CURIES )

$2.880 E-04$

2.880E-04 
RADIOACTIVE WASTE MANAGEMENT INFORMATION SYSTEM

FOR JAN THROUGH JUN 1993

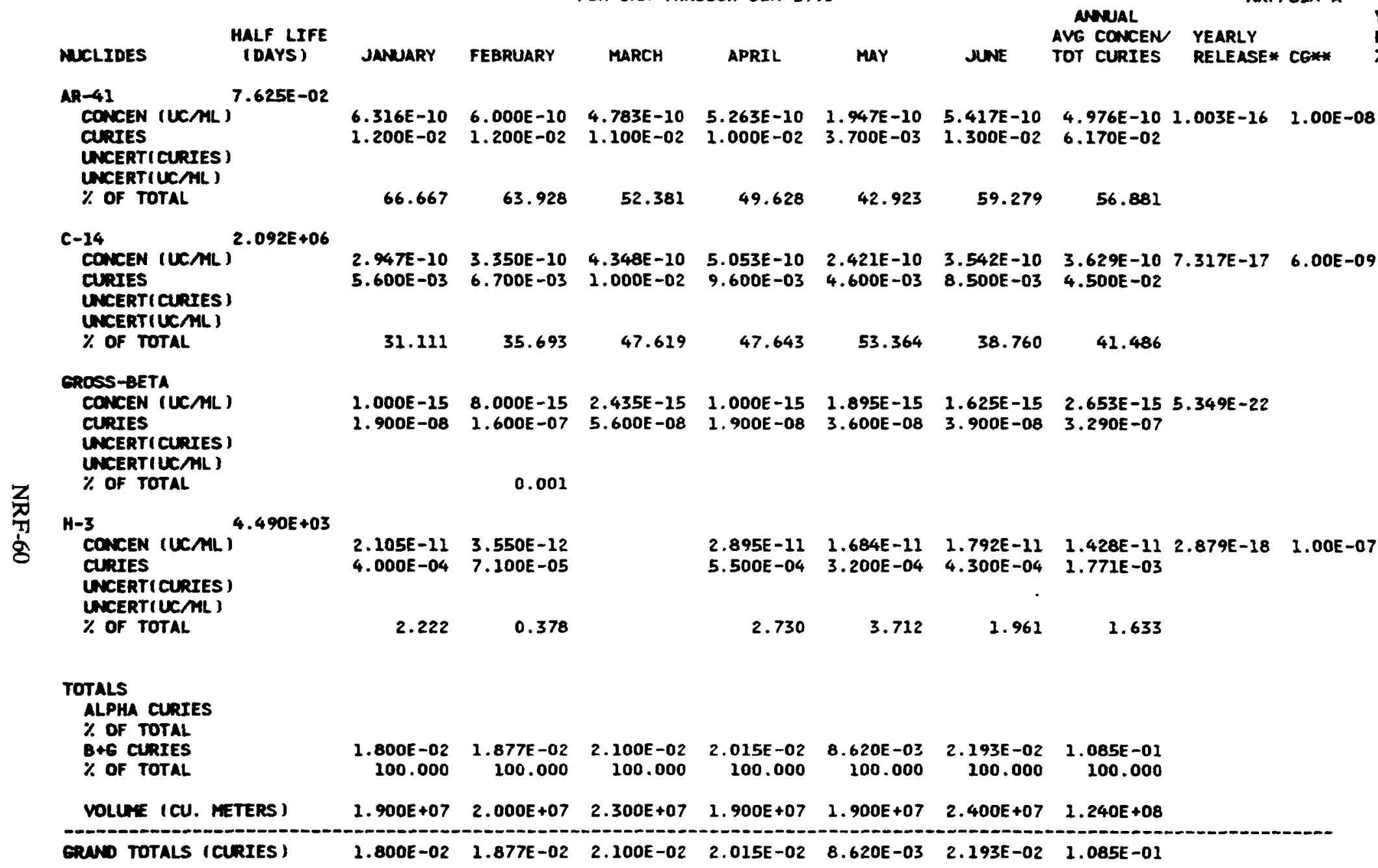

* This nuber is darived from a dispersion factor equation. The dispersion factor is derived from the straight line goussien model and is proveded by the National Oceanic Atmospheric Administration (NOAA).

* NOTE: DCE value obtained from DOE ORDER 5400.5 doted Februery 8, 1990, Figures III-I and III-3 


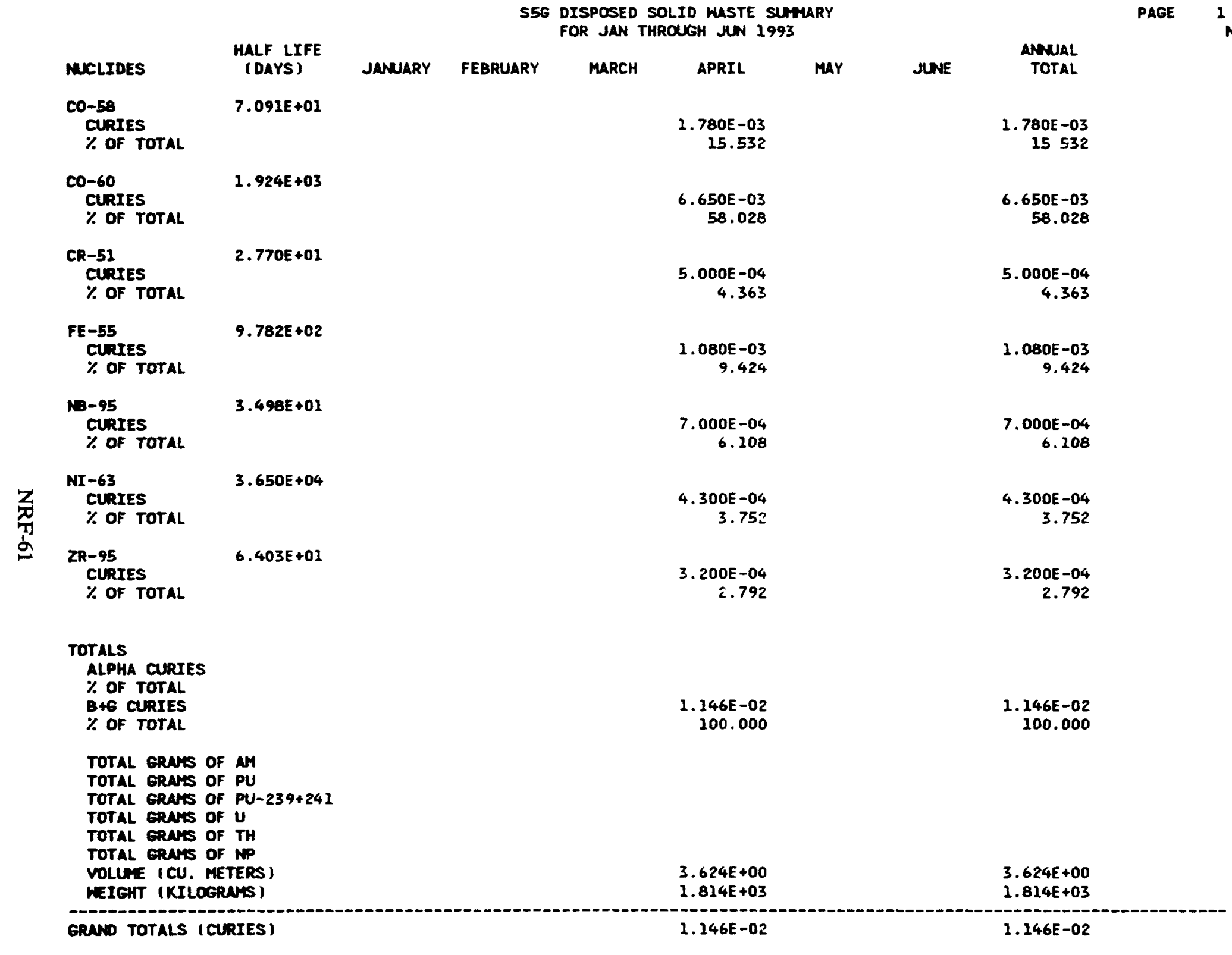




\section{Power Burst Facility 1993 Detail Data}

Power Burst Facility (PBF) Bar Graph of Annual Data by Month . . . . . . . . . . PBF-3

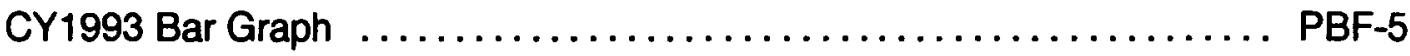

PBF Detail Data for Months July to December

Annual Average Concentration and Total Curies for January to December ....... PBF-7

Report 130_PBF Airborne Waste Summary ................ PBF-9

PBF Detail Data for Months January to June

Annual Average Concentration and Total Curies for January to June ........ PBF-11

Report 130-PBF Airborne Waste Summary ................ PBF-13 

Power Burst Facility (PBF) Bar Graph of Annual Data by Month 

Power Burst Facility (PBF) Monthly Details - CY 1993

苗

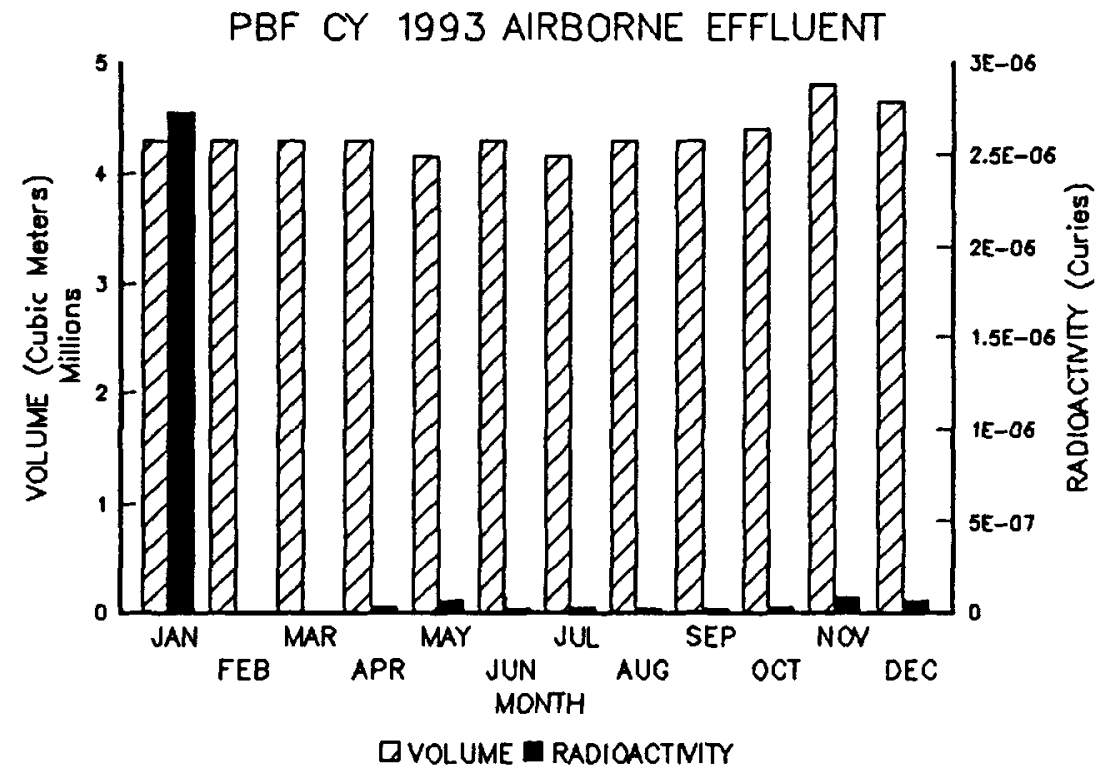



PBF Detail Data for Months July to December Annual Average Concentration and Total Curies for January to December 

RADIOACTIVE WASTE MANAGEMENT INFORMATION SYSTEM

MUCLIDES
CROSS-ALPHA
CONCEN (UCML)
CURIES
UCERTICURIES)
UXERT (UCMM)
\% OF TOTAL

\section{EROSS-BETA}

CONCEN (UCML)

UNCERT I CURIES )

UNEERT (UCAML )

$\%$ OF TOTAL

SR-90
CONCEN (UCML)

CURIES

UNERT C CRIES I

UNCERTIUCAM

\% OF TOTAL

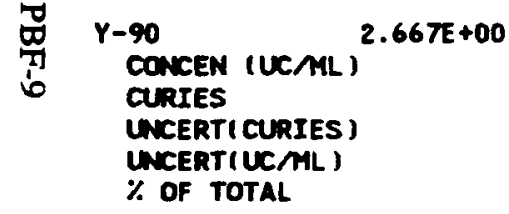

JLY

AUEUST
$1.315 E-15$
$5.470 E-09$
$2.330 E-09$

$1.291 E-15 \quad 5.305 E-16$

$\begin{array}{ll}5.550 E-09 & 2.280 E-09 \\ 2.340 E-09 & 1.930 E-09\end{array}$

$1.038 \mathrm{E}-15$

$4.570 E-09$

$140 E-0$
FOR JAN THROUGH DEC 1993

PBF AIRBORNE MASTE SUMmLRY

MLLF LIFE (DAYS)

\begin{abstract}
SEPTEMER
\end{abstract}
23.769

11.585

15.097

$2.200 E-08$
$3.900 E-09$

\begin{abstract}
26.961
\end{abstract}
$3.549 E-15$

$1.650 E-08$
$4.070 E-09$

\begin{abstract}
18.011
\end{abstract}

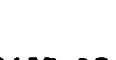

5. $987 E-15$

$2.490 E-08$
$3.630 E-09$

$4.141 E-15$
$1.780 E-08$
$3.620 E-09$

$4.048 E-15$
$1.740 E-08$
$3.180 E-09$

$5.836 E-15$
$2.570 E-08$
$3.520 E-09$

$1.241 E-14$
$5.960 E-08$

5. $960 E-08$
$5.290 E-09$

$4.0630 E-08$
$5.260 E-09$

81.989

76.231

88.415

84.903

73.039

74.962

95.202

\begin{tabular}{|c|c|c|c|}
\hline & \multicolumn{3}{|c|}{ PAGE $\underset{\text { PBF-A }}{1}$ RPT. 130} \\
\hline $\begin{array}{l}\text { EN } \\
\text { ES }\end{array}$ & $\begin{array}{l}\text { YEARLY } \\
\text { RELEASE* }\end{array}$ & CG** & $\begin{array}{l}\text { YEARLY } \\
\text { RELEASE } \\
\% \text { OF CE }\end{array}$ \\
\hline
\end{tabular}

\section{TOTALS}

ALPHA CURIES
$\%$ OF TOTAL
B+6 CURIES
$\%$ OF TOTAL

5.470E-09 18.011
$490 E-08$

5.550E-09 23.769
$780 E-08$

2. 280E-09 11.585
$740 E-08$
4.570E-09 15.097 $.570 E-08$
84.903 $.490 E-08$
81.989 $.780 E-08$
76.231

88.415

2. 200E-0B 26.961
$960 E-08$ $730 E-08$
73.039

1.650E-08 25.038 $940 E-08$

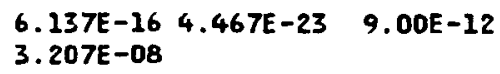
3. $207 E-08$

1.035

$6.137 E-16 \quad 4.467 E-23 \quad 1.00 E-09$ 3. 207E-08

1.035 $4.159 E+06 \quad 4.298 E+06 \quad 4.298 E+06 \quad 4.404 E+06$ $4.803 E+06 \quad 4.649 E+06 \quad 5.225 E+07$ equid TOTALS (CURIES) $\begin{array}{lllllll}3.037 E-08 & 2.335 E-08 & 1.968 E-08 & 3.027 E-08 & 8.160 E-08 & 6.590 E-08 & 3.099 E-06\end{array}$

* This nuber is darived from a dispersion factor equetion. The dispersion factor is derived from the straight line geussien wodel and is proveded by the Notional Doeanic Atmospheric Administration (NDMA).

* NOTE: DCG value obtained from DOE ORDER 5400.5 doted February 8, 1990 , Figures III-1 and III-3 

PBF Detail Data for Months January to June Annual Average Concentration and Total Curies for January to June 

RADIOACTIVE WASTE MANAGEMENT INFORMATION SYSTEM

PBF AIRBORNE MASTE SUMMARY

FOR JAN THROUGH JUN 1993

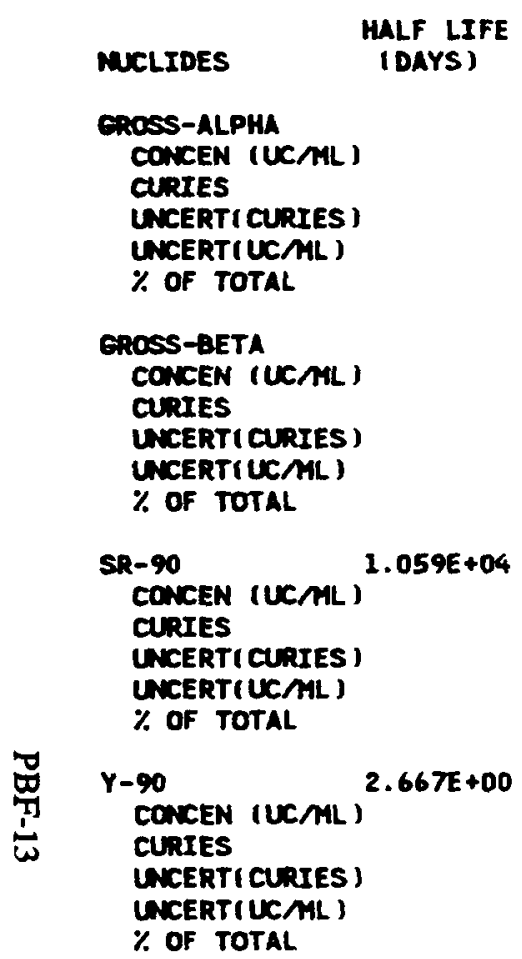

JANUARY FEBRUARY

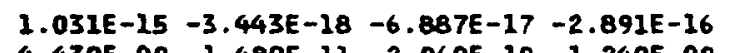

$4.430 E-09-2.480 E-11-2.960 E-10-1.240 E-09$

$2.960 E-09 \quad 1.030 E-10$

0.162

$-97.36 \%$

$-12.521$

$-3.967$

$1.760 E-08$

maY

JNE

$.430 E-07 \quad 7.390 E-10$

$4.210 E-09$

$97.478 \quad 1947.381$

112.521

103.967

71.975

66.464

$7.492 E-15-3.094 E-17$

$3.220 E-08-1.330 E-10$

2.070E-09 2.960E-11

$1.180-875.006$

7.492E-15 - $3.094 E-17$

$3.220 E-08-1.330 E-10$

$\begin{array}{ll}2.070 E-09 & 2.960 E-11\end{array}$

$1.180 \quad-875.006$
1.460E-09 $3.250 E-08$
PAGE

1 RPT. 130 PBF-A

MNUAL AVG CONCEN YEARLY

YEARLY TOT CURIES RELEASE* CG** \% \% DF CG

\begin{tabular}{|c|c|c|c|c|c|c|c|}
\hline $\begin{array}{l}\text { OTALS } \\
\text { ALPHA CURIES } \\
\% \text { OF TOTAL } \\
\text { B+G CURIES } \\
\% \text { OF TOTAL }\end{array}$ & $\begin{array}{r}4.430 E-09 \\
0.162 \\
2.724 E-06 \\
99.838\end{array}$ & $\begin{array}{r}-1.480 E-11 \\
-97.369 \\
3.000 E-11 \\
197.369\end{array}$ & $\begin{array}{r}-2.960 E-10 \\
-12.521 \\
2.660 E-09 \\
112.521\end{array}$ & $\begin{array}{r}-1.240 E-09 \\
-3.967 \\
3.250 E-08 \\
103.967\end{array}$ & $\begin{array}{r}1.760 E-08 \\
28.025 \\
4.520 E-08 \\
71.975\end{array}$ & $\begin{array}{r}7.720 E-09 \\
33.536 \\
1.530 E-08 \\
66.464\end{array}$ & $\begin{array}{r}2.820 E-08 \\
0.990 \\
2.820 E-06 \\
99.010\end{array}$ \\
\hline VOLUNE (CU. METERS) & $4.298 E+06$ & $4.298 E+06$ & $4.298 E+06$ & $4.289 E+06$ & $4.259 E+06$ & $4.298 E+06$ & $2.564 E+07$ \\
\hline TALS 10 & $2.729 E-06$ & $1.520 E-11$ & $2.364 E-09$ & $3.126 E-08$ & $6.280 E-08$ & $2.302 E-08$ & $2.848 E-06$ \\
\hline
\end{tabular}

TOTALS ICURYES

$\begin{array}{lllllll}2.729 E-06 & 1.520 E-11 & 2.364 E-09 & 3.126 E-08 & 6.280 E-08 & 2.302 E-08 & 2.848 E-06\end{array}$

$1.251 E-15 \quad 9.102 E-23 \quad 9.00 E-12$ 3.207E-08

1.126

$1.251 E-15 \quad 9.102 E-23 \quad 1.00 E-09$ 3.207E-08

1.126

- This muber is derived from a dispersion factor aquetion. The dispersion factor is derived from the straight line geuseian modal and is provedad by the National Doeanic Atmospheric Administration (NOAA).

* NOTE: DCG value obtained from DOE ORDER 5400.5 dated February B, 1990 , Figures III-1 and III-3 


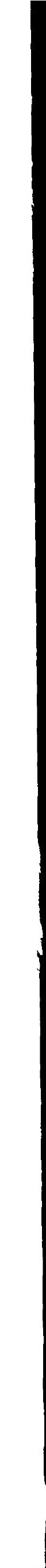




\section{Radioactive Waste Management Complex 1993 Detail Data}

Radioactive Waste Management Complex (WMC) Bar Graph of Annual

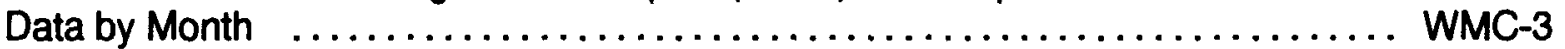

CY1993 Bar Graph WMC-5

WMC Detail Data for Months July to December

Annual Average Concentration and Total Curies for January to December WMC-7

Report 708-DrumVent Stack Airborne Waste Summary WMC-9

WMC Detail Data for Months January to June

Annual Average Concentration and Total Curies for January to June WMC-11

Report 708-DrumVent Stack Airborne Waste Summary WMC-13 


Waste Management Complex (WMC) Monthly Details - CY 1993

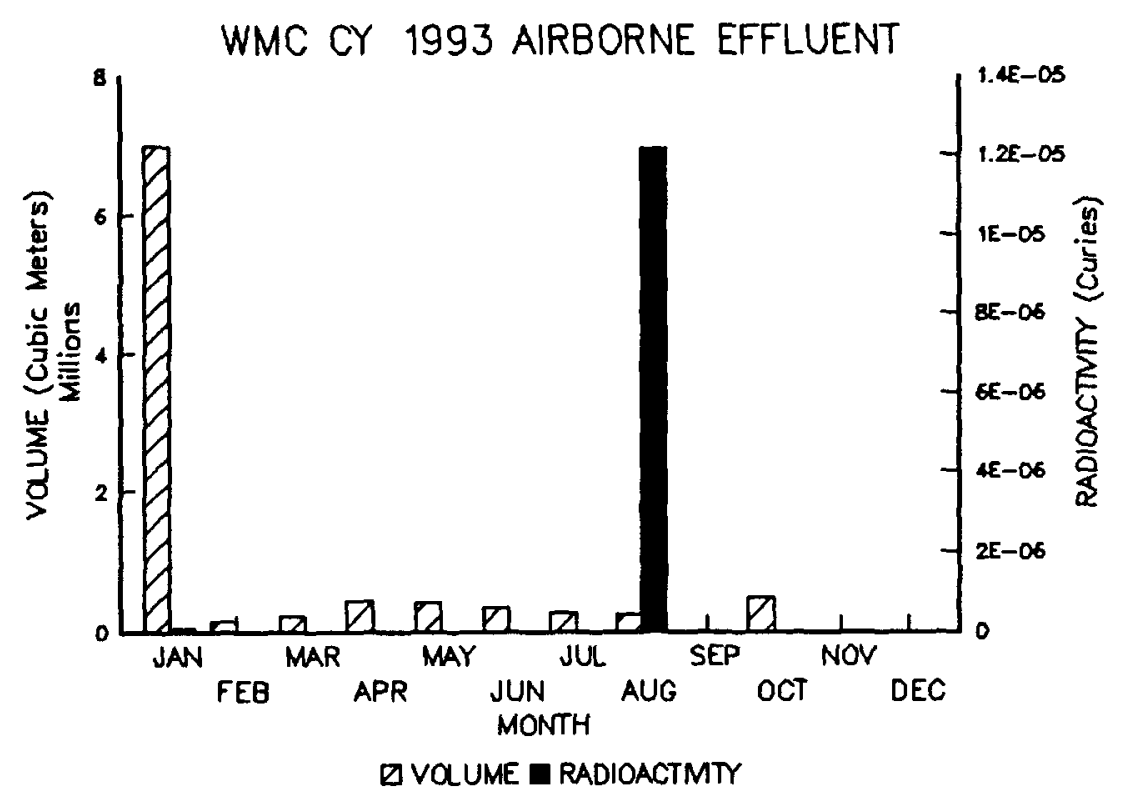




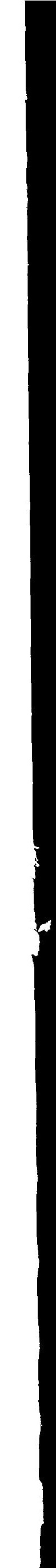


WMC Detail Data for Months July to December Annual Average Concentration and Total Curies for January to December 

RADIOACTIVE WUSTE MANAGEMENT INFORMATION SYSTEM

DRLMVENT STACK AIRBORNE WASTE SUMTIARY

FOR JAN THROUGH DEC 1993

MUCLIDES
GROSS-ALPHA
CONCEN (UCML)
CURIES
UNCERTICURIES)
UNCERTIUCML)
\% OF TOTAL

HALF LIFE
(DAYS )

JLY AUGUST

SEPTEMBER

DCtober

NOVEMBER

\subsection{E-16 \\ $3.390 E-10$}

$4.860 E-10 \quad 1.926 E-12$

$2.120 E-10 \quad 6.600 E-07$

11.183

9.836

$\begin{array}{ll}1.394 E-14 & 4.516 E-11 \\ 3.860 E-09 & 1.100 E-05\end{array}$

3.800E-20 1.400E-06

88.817

90.164

$\begin{array}{rr}4.860 E-10 & 1.200 E-06 \\ 11.183 & 9.836\end{array}$

11.183

$3.860 E-09$
88.817

9.836
$100 E-05$

$100 E-05$
90.164

$2.770 E+05 \quad 2.436 E+05$

$4.346 E-09 \quad 1.220 E-05$

10.183

6. $048 E-15$

2. $990 E-09$

89.817
$4.346 E-09 \quad 1.220 E-05$

$3.3296-09$

$3.390 E-10$

10.183

2. $990 E-09$

89.817
PAGE I RPT. 708

MKC MMC-A

ANMAL

AVG CONCEN

DECEMBER

TOT CURIES

YEARLY

RELEASE* CG*

YEARLY

RELEASE

$\%$ OF total

$\%$ Of TOTAL

$1.254 E-13 \quad 1.279 E-20$

$1.199 E-06$

9.743

$1.162 E-12 \quad 1.185 E-19$

$2.211 E-05$

90.257

GRMD TOTALS (CURIES )

* This muber is derived from a dispersion factor equation. The dispersion factor is derived from the straight line

$1.199 E-06$

$111 E-05$
90.257 coussian model and is proveded by the National Oceanic Atmospheric Administration (NOAA).

* NOTE: DCG value obtained from DOE ORDER 5400.5 dated February 8, 1990 , figures III-1 and III-3 
WMC Detail Data for Months January to June Annual Average Concentration and Total Curies for January to June 



\section{RADIOACTIVE WASTE MANAGEMENT INFORMATION SYSTEM}

DRUINENT STACK AIRBORNE WASTE SUMMARY FOR JAN THROUGH JUN 1993

MUCLIDES
ERCSS-ALPHA
CONCEN (UCML)
CURIES
UNCERTICURIES)
UNCERT(UCAL)
\% OF TOTAL

\section{HALF LIFE (DAYS)}

JANUARY FEBRUARY

MARCH

APRIL

MaY

$\begin{array}{lll}-4.285 E-10 & 1.466 E-15 & 2 \\ -2.990 E-09 & 2.280 E-10 & 4\end{array}$

$2.990 E-09$
$3.950 E-09$

1. $220 E-10$

$2.153 E-15$

$-3.505$

4.752

$4.710 E-10$
$1.800 E-10$

JUNE

\begin{tabular}{lll} 
& \multicolumn{2}{c}{ PAGE I RPT. 708} \\
MNUAL & \multicolumn{1}{c}{ INCNMC-A } & YEARLY \\
AVG CONCEN & YEARLY & RELEASE \\
TOT CURIES & RELEASE* CG** & $\%$ OF CG
\end{tabular}

GROSS-BETA

CONCEN (UCAL)

CURIES

UNCERT (CURIES)

UNCERT ( UCML )

$\%$ OF TOTAL
$\begin{array}{llllllll}1.265 E-14 & 2.939 E-14 & 2.669 E-14 & 4.876 E-15 & 6.426 E-15 & 2.681 E-15 & 1.222 E-14 & 1.246 E-21\end{array}$
$\begin{array}{llllllll}8.830 E-08 & 4.570 E-09 & 5.840 E-D 9 & 2.170 E-09 & 2.700 E-09 & 8.910 E-10 & 1.045 E-07\end{array}$
$\begin{array}{llllll}7.800 E-09 & 2.870 E-10 & 3.770 E-10 & 5.330 E-10 & 4.500 E-10 & 1.930 E-10\end{array}$
103.505
95.248
92.537
80.880
94.870
82.196
101.397

\section{TOTALS}

\begin{tabular}{|c|c|c|c|c|c|c|c|}
\hline $\begin{array}{l}\text { ALPHA CURIES } \\
\% \text { OF TOTAL } \\
\text { B+E CURIES } \\
\% \text { OF TOTAL }\end{array}$ & $\begin{array}{r}-2.990 E-09 \\
-3.505 \\
8.830 E-08 \\
103.505\end{array}$ & $\begin{array}{r}2.280 E-10 \\
4.752 \\
4.570 E-09 \\
95.248\end{array}$ & $\begin{array}{r}4.710 E-10 \\
7.463 \\
5.840 E-09 \\
92.537\end{array}$ & $\begin{array}{r}5.130 E-10 \\
19.120 \\
2.170 E-09 \\
80.880\end{array}$ & $\begin{array}{r}1.460 E-10 \\
5.130 \\
2.700 E-09 \\
94.870\end{array}$ & $\begin{array}{r}1.930 E-10 \\
17.804 \\
8.910 E-10 \\
82.196\end{array}$ & $\begin{array}{r}-1.439 E-09 \\
-1.397 \\
1.045 E-07 \\
101.397\end{array}$ \\
\hline VOLUME (CU. METERS) & $6.978 E+06$ & $1.555 E+05$ & $2.188 E+05$ & $4.450 E+05$ & $4.202 E+0.5$ & $3.323 E+0.5$ & $8.550 E+06$ \\
\hline$S 10$ & $8.531 E-08$ & $4.798 E-00$ & $6.311 E-09$ & $2.683 E-09$ & $2.846 E-09$ & $1.084 E-09$ & $1.030 E-07$ \\
\hline
\end{tabular}

* This nuber is derived from a dispersion factor equation. The dispersion factor is derived from the straight line omussian model and is proveded by the National Dceanic Atmospheric Administration (NOAA).

* NOTE: OCG value obtained from DOE ORDER 5400.5 dated february 8, 1990 , Figures III-1 and III-3 



\section{Special Manufacturing Capability 1993 Detail Data}

Special Manufacturing Capability (SMC) Bar Graph of Annual

Data by Month $\ldots \ldots \ldots \ldots \ldots \ldots \ldots \ldots \ldots \ldots \ldots \ldots \ldots \ldots \ldots \ldots \ldots \ldots \ldots$, SMC-3

CY1993 Bar Graphs . ................................ SMC-5

SMC Detail Data for Months July to December

Annual Average Concentration and Total Curies for January to December ....... SMC-7

Report 731-SMC Airborne Waste Summary $\ldots \ldots \ldots \ldots \ldots \ldots \ldots$ SMC-9

SMC Detail Data for Months January to June

Annual Average Concentration and Total Curies for January to June ......... SMC-11

Report 731-SMC Airborne Waste Summary $\ldots \ldots \ldots \ldots \ldots \ldots \ldots$ SMC-13

SMC Facility Detail Data for Months July to December

Annual Average Concentration and Total Curies for January to December ...... SMC-15

Report 741-Exhaust Fan P1 Airborne Waste Summary ............. SMC-17

Report 742-Laser Punch P1 Airborne Waste Summary .............. SMC-18

Report 754-Liquid Reclaim Airborne Waste Summary ............... SMC-19

Report 738-MDF Prod Floor Airborne Waste Summary ............. SMC-20

Report 739-MDF QC Lab Airborne Waste Summary ............... SMC-21

Report 748-N. Process S9 Airborne Waste Summary . ............. SMC-22

Report 749-N. Process S10 Airborne Waste Summary .............. SMC-23

Report 750-N. Process S11 Airborne Waste Summary $\ldots \ldots \ldots \ldots \ldots .$. SMC-24

Report 753-PRF Stack S14 Airborne Waste Summary ............. SMC-25

Report 751-Scrap Handling Airborne Waste Summary $\ldots \ldots \ldots \ldots \ldots$. SMC-26

Report 745-S. Process S6 Airborne Waste Summary $\ldots \ldots \ldots \ldots \ldots \ldots$. SMC-27

Report 746-S. Process S7 Airborne Waste Summary .............. SMC-28

Report 747-S. Process S8 Airborne Waste Summary ............. SMC-29

Report 744-2B Area Phase 1 Airbome Waste Summary ............ SMC-30

SMC Facility Detail Data for Months January to June

Annual Average Concentration and Total Curies January to June ........... SMC-31

Report 741-Exhaust Fan P1 Airborne Waste Summary ............. SMC-33

Report 742-Laser Punch P1 Airborne Waste Summary .............. SMC-34

Report 754-Liquid Reclaim Airborne Waste Summary ................ SMC-35

Report 738-MDF Prod Floor Airborne Waste Summary ............. SMC-36

Report 739-MDF QC Lab Airborne Waste Summary .............. SMC-37

Report 748-N. Process S9 Airborne Waste Summary .............. SMC-38

Report $749-N$. Process S10 Airborne Waste Summary .............. SMC-39

Report 750-N. Process S11 Airborne Waste Summany ............. SMC-40

Report 753-PRF Stack S14 Airborne Waste Summary ............. SMC-41

Report 751 -Scrap Handling Airborne Waste Summary ............ SMC-42

Report 745-S. Process S6 Airborne Waste Summary .............. SMC-43

Report 746-S. Process S7 Airborne Waste Summary .............. SMC-44

Report 747-S. Process S8 Airborne Waste Summary .............. SMC-45

Report 744-2B Area Phase 1 Airbome Waste Summary ............ SMC-46 


Special Manufacturing Capability (SMC) Monthly Details - CY 1993

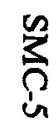

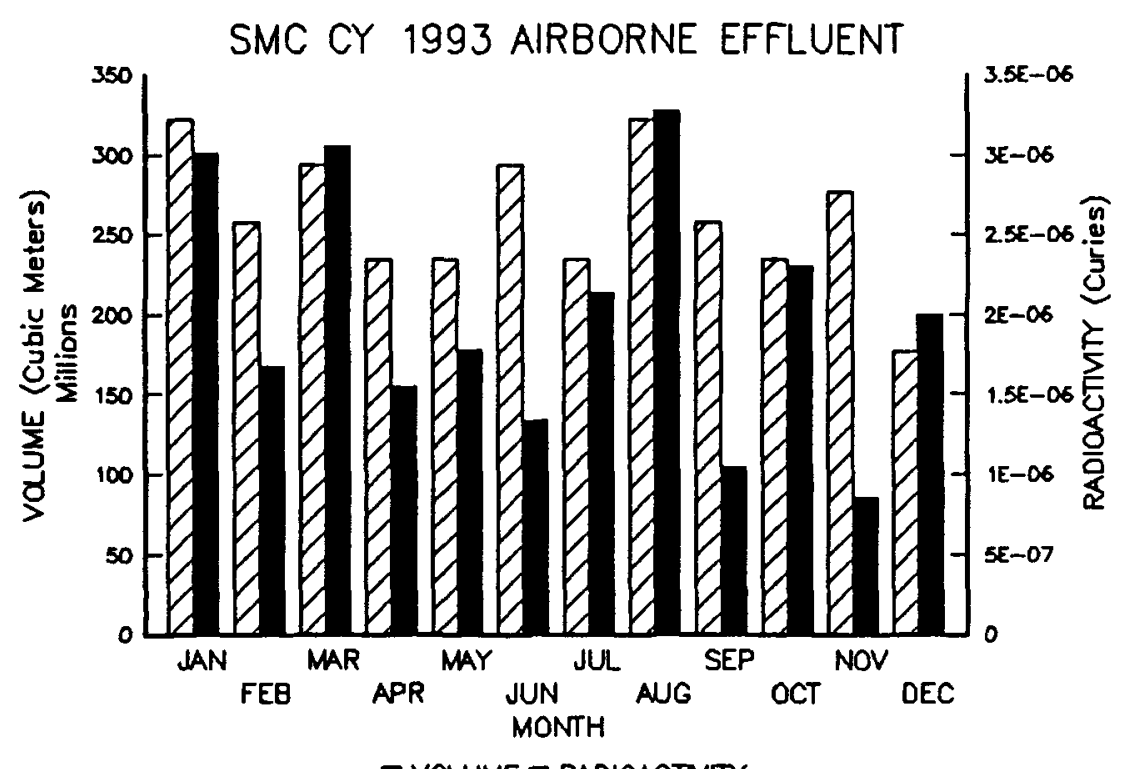

I VQLUME RADIOACTMITY 


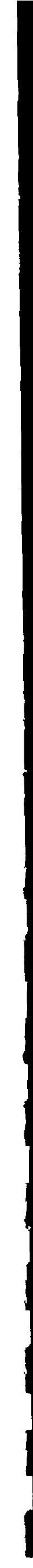


SMC Detail Data for Months July to December Annual Average Concentration and Total Curies for January to December 

RADIOACTIVE WASTE MAMAGEMENT INFORMATION SYSTEM

SUC ATREOPHE MUSTE SUMURY FOR JAN THROUGH DEC 1993

\begin{tabular}{|c|c|c|c|c|c|c|c|c|c|}
\hline $\begin{array}{l}\text { HALF LIFE } \\
\text { (DAYS) }\end{array}$ & JULY & AUEUST & SEPTEMBER & OCTOBER & NOVEMBER & DECEMBER & $\begin{array}{l}\text { ANUAL } \\
\text { AVG CONCEN } \\
\text { TOT CURIES }\end{array}$ & $\begin{array}{l}\text { YEARLY } \\
\text { RELEASE* }\end{array}$ & $\cos x \cdot$ \\
\hline $\begin{array}{l}\text { PA-234 } \\
\text { CONCEN (UCML) } \\
\text { CORIES } \\
\% \text { OF TOTAL }\end{array}$ & $\begin{array}{r}2.908 E-15 \\
6.830 E-07 \\
31.916\end{array}$ & $\begin{array}{r}3.245 E-15 \\
1.045 E-06 \\
31.938\end{array}$ & $\begin{array}{r}1.292 E-15 \\
3.330 E-07 \\
31.989\end{array}$ & $\begin{array}{r}3.127 E-15 \\
7.350 E-07 \\
31.943\end{array}$ & $\begin{array}{r}9.815 E-16 \\
2.720 E-07 \\
31.925\end{array}$ & $\begin{array}{r}3.620 E-15 \\
6.390 E-07 \\
31.966\end{array}$ & $\begin{array}{r}2.441 E-15 \\
7.667 E-06 \\
31.943\end{array}$ & $1.746 E-21$ & $2.00 E-08$ \\
\hline $\begin{array}{l}\text { TH-234 } \\
\text { CONCEN }(U \mathrm{CML})^{2.410 E+01} \\
\text { CURIES } \\
\% \text { OF TOTAL. }\end{array}$ & $\begin{array}{r}2.908 E-15 \\
6.830 E-07 \\
31.916\end{array}$ & $\begin{array}{r}3.245 E-15 \\
1.045 E-06 \\
31.938\end{array}$ & $\begin{array}{r}1.292 E-15 \\
3.330 E-07 \\
31.989\end{array}$ & $\begin{array}{r}3.127 E-15 \\
7.350 E-07 \\
31.943\end{array}$ & $\begin{array}{r}9.815 E-16 \\
2.720 E-07 \\
31.925\end{array}$ & $\begin{array}{r}3.620 E-15 \\
6.390 E-07 \\
31.966\end{array}$ & $\begin{array}{r}2.441 E-15 \\
7.667 E-06 \\
31.943\end{array}$ & $1.746 \mathrm{E}-21$ & $4.00 E-10$ \\
\hline $\begin{array}{l}\text { U-234 } \\
\text { CONCEN (UCML) } \\
\text { CURIES } \\
\% \text { OF TOTAL }\end{array}$ & $\begin{array}{r}3.874 E-16 \\
9.100 E-08 \\
4.252\end{array}$ & $\begin{array}{r}4.254 E-16 \\
1.370 E-07 \\
4.287\end{array}$ & $\begin{array}{r}1.630 E-16 \\
4.200 E-08 \\
4.035\end{array}$ & $\begin{array}{r}4.085 E-16 \\
9.600 E-08 \\
4.172\end{array}$ & $\begin{array}{r}1.299 E-16 \\
3.600 E-08 \\
4.225\end{array}$ & $\begin{array}{r}4.645 E-16 \\
8.200 E-08 \\
4.102\end{array}$ & $\begin{array}{r}3.187 E-16 \\
1.001 E-06 \\
4.170\end{array}$ & $2.279 E-22$ & $9.00 E-14$ \\
\hline $\begin{array}{l}U-238 \\
\text { CONCEN (UCML) } \\
\text { CURIES } \\
\% \text { OF TOTAL }\end{array}$ & $\begin{array}{r}2.908 E-15 \\
6.830 E-07 \\
31.916\end{array}$ & $\begin{array}{r}3.245 E-15 \\
1.045 E-06 \\
31.938\end{array}$ & $\begin{array}{r}1.292 E-15 \\
3.330 E-07 \\
31.989\end{array}$ & $\begin{array}{r}3.127 E-15 \\
7.350 E-07 \\
31.943\end{array}$ & $\begin{array}{r}9.815 E-16 \\
2.720 E-07 \\
31.925\end{array}$ & $\begin{array}{r}3.620 E-15 \\
6.390 E-07 \\
31.966\end{array}$ & $\begin{array}{r}2.441 E-15 \\
7.667 E-06 \\
31.943\end{array}$ & $1.746 E-21$ & $1.00 E-1$ \\
\hline $\begin{array}{l}\text { TOTALS } \\
\text { ALPHA CURIES } \\
\% \text { OF TOTAL } \\
\text { B+G CURIES } \\
\% \text { OF TOTAL }\end{array}$ & $\begin{array}{r}7.740 E-07 \\
36.169 \\
1.366 E-06 \\
63.832\end{array}$ & $\begin{array}{r}1.182 E-06 \\
36.125 \\
2.090 E-06 \\
63.875\end{array}$ & $\begin{array}{r}3.750 E-07 \\
36.023 \\
6.660 E-07 \\
63.977\end{array}$ & $\begin{array}{r}8.310 E-07 \\
36.115 \\
1.470 E-06 \\
63.885\end{array}$ & $\begin{array}{r}3.080 E-07 \\
36.250 \\
5.440 E-07 \\
63.850\end{array}$ & $\begin{array}{r}7.210 E-07 \\
36.068 \\
1.278 E-06 \\
63.932\end{array}$ & $\begin{array}{r}8.668 E-06 \\
36.113 \\
1.533 E-05 \\
63.886\end{array}$ & & \\
\hline IRMO TOTALS ICU & $. .240 E-06$ & $.272 E-06$ & $1.041 E-06$ & $2.301 E-06$ & B.520E-07 & $1.999 E-06$ & $.400 E-05$ & & \\
\hline
\end{tabular}

This maber is darived from dispersion factor aquetion. The dispersion factor is derivod from the straight line Gaussien model and is providad by the thional Oceanic Atmospheric Administration (NOAA).

* NOTE: DCE value obtainod from DOE ORDER 5400.5 dated February 8, 1990, Figures III-1 and III-3 

SMC Detail Data for Months January to June Annual Average Concentration and Total Curies for January to June 



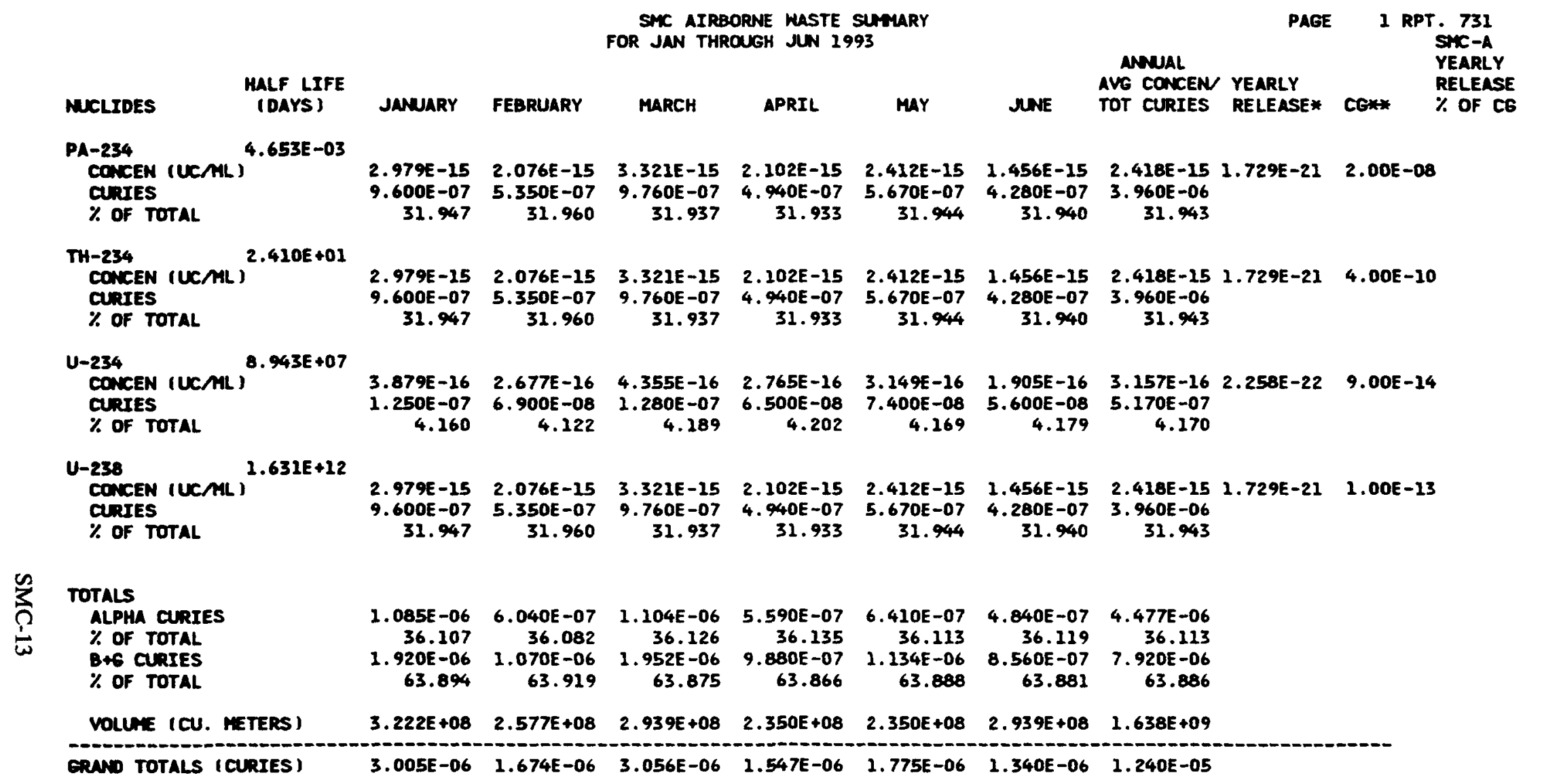

* This nuber is derived fron a dispersion factor equation. The dispersion factor is derived from the straight line Geussian model and is provided by the Mtional Oceanic Atmospheric Achinistration (NOMA).

** NOTE: DCE value obtained from DOE ORDER 5400.5 dated Fabruary 8, 1990 , Figures III-1 and III-3 

SMC Facility Detail Data for Months July to December Annual Average Concentration and Total Curies for January to December 


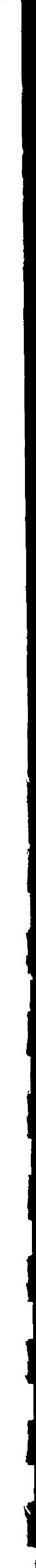


EXHAUST FAN PI AIRBORNE MLSTE SUMMARY FOR JAN THROUGH OEC 1993

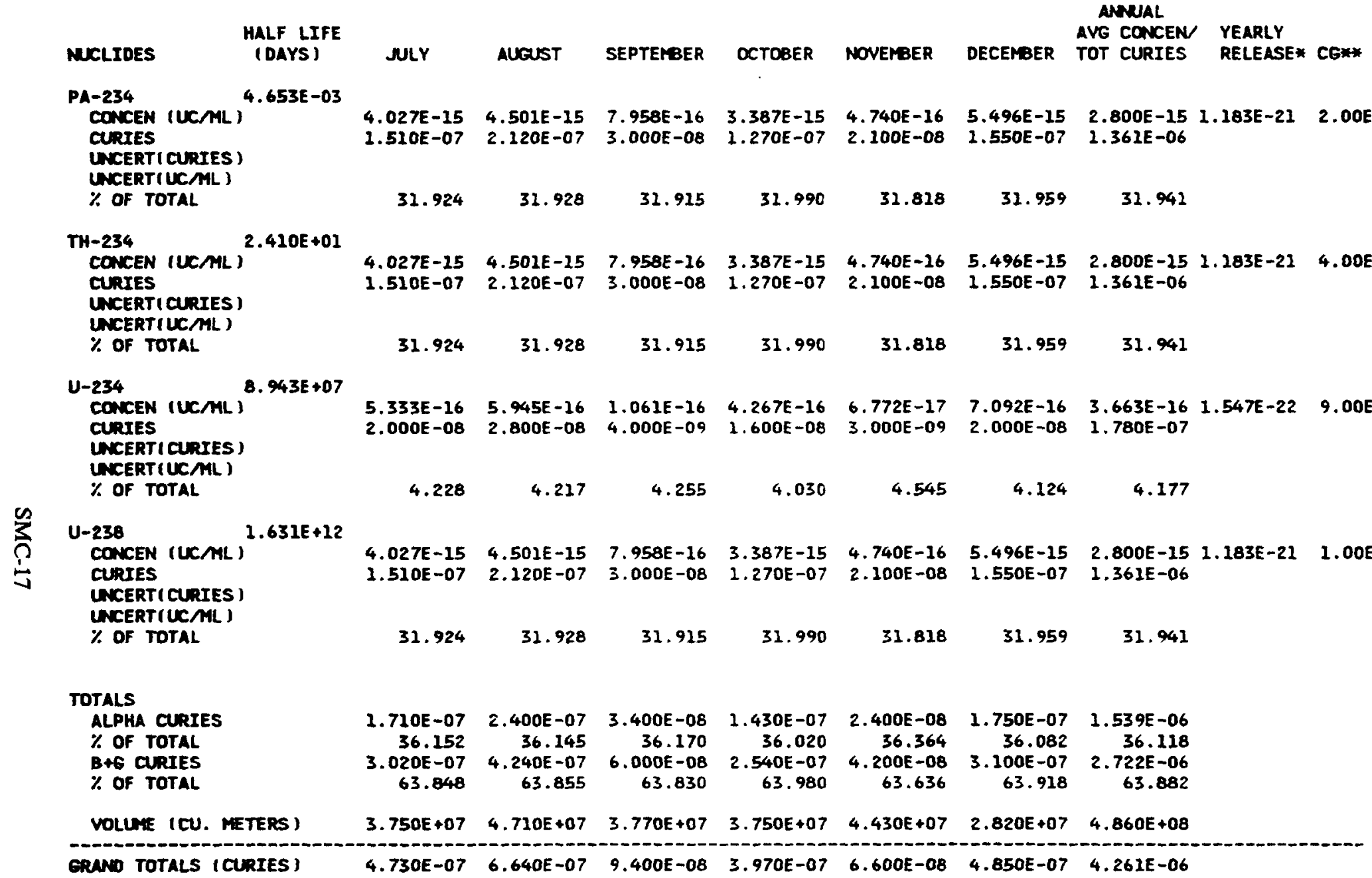

* This nuber is darived from a dispersion factor quation. The dispersion factor is derived from the straight line gaussian model and is proveded by the National Oceanic Atmospheric Adninistration INOAA.

* NOTE: DCG valu abtained from DOE ORDER 5400.5 dated Fabruary 8, 1990 , Figures III-1 and III-3 
LASER PUNCH PI AIRBORNE MASTE SLMMARY FOR JAN THROUGH DEC 1993

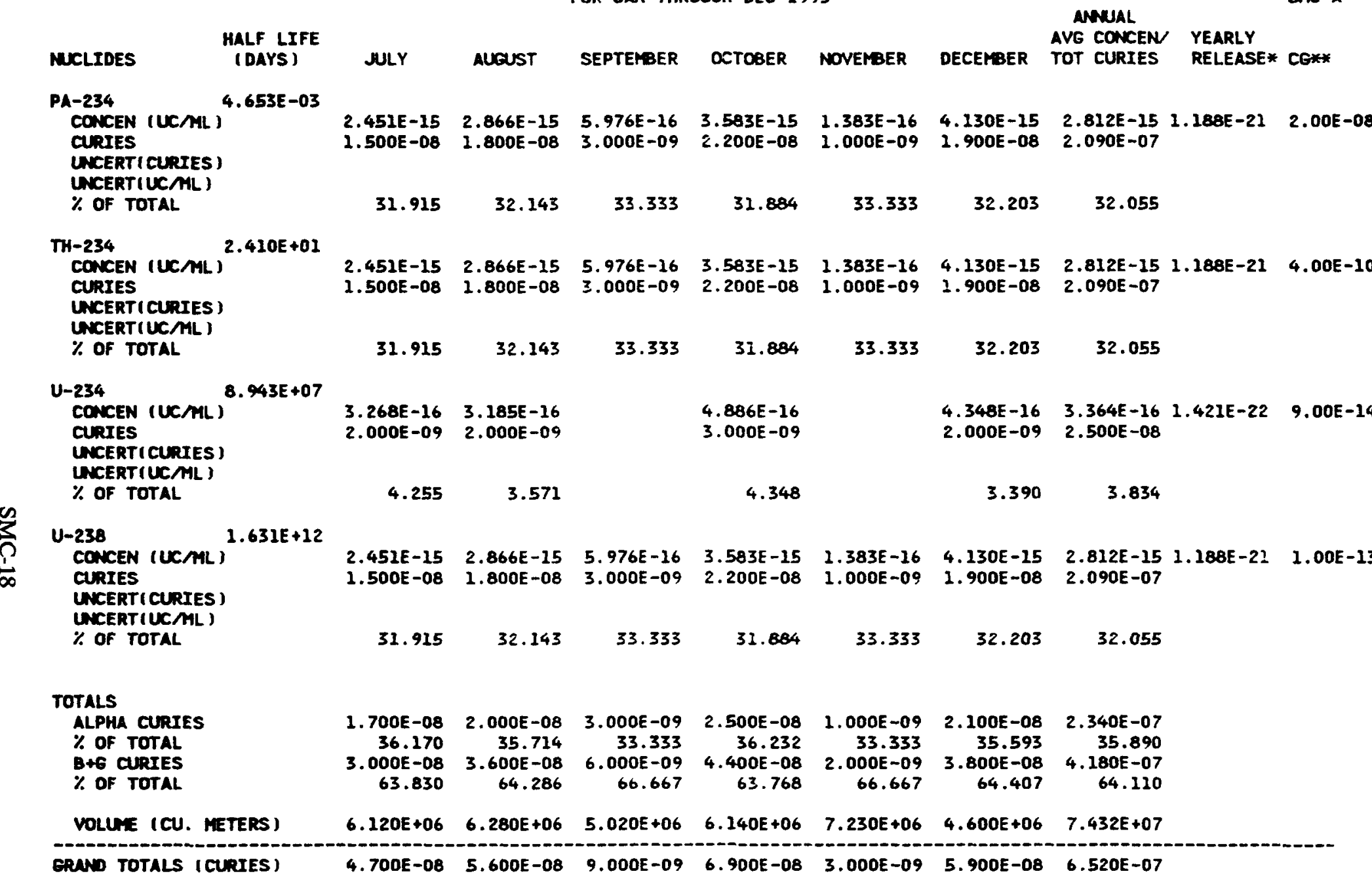

* This nuber is derived from a dispersion factor equation. The dispersion factor is derived from the straight line oausian model and is proveded by the National Oceanic Atmospharic Administration (MOAA).

* NOTE: DCG value obtainad from DOE OROER 5400.5 dated february 8, 1990, Figures III-1 and III-3 
RADIOACTIVE WASTE MUNAGEMENT INFORMATION SYSTEM

LIQUID RECLAIM AIRBORNE WASTE SUMMARY FOR JAN THROUGH DEC 1993

MUCLIDES HALF LIFE
(DAYS)

JULY

Augrst

SEPTEYBER

$2.000 E-15$
$3.700 E-08$

$2.597 E-15$

ANNAL
AVG CONCEN YEARLY
YET CURIES RELY

PAGE 2 RPT. 754
31.897
31.915
32.000
31.958

$\begin{array}{lllllllll}2.00 D E-15 & 2.597 E-15 & 1.730 E-15 & 2.108 E-15 & 3.670 E-16 & 4.029 E-15 & 1.910 E-15 & 5.134 E-22 & 4.00 E-10\end{array}$ $\begin{array}{lllllll}3.700 E-08 & 6.000 E-08 & 3.200 E-08 & 3.900 E-08 & 8.000 E-09 & 5.600 E-08 & 4.570 E-07\end{array}$
31.897
31.915
32.000
31.967
32.000
32.000
31.958

u-234

8. $943 E+07$

CONCEN (UCML)

$\begin{array}{lllllll}2.703 E-16 & 3.463 E-16 & 2.162 E-16 & 2.703 E-16 & 4.587 E-17 & 5.036 E-16\end{array}$

5.000E-09 8.000E-09 $4.000 E-09$ 5.000E-09 $1.000 E-09 \quad 7.000 E-09$

$2.466 E-16 \quad 6.628 E-23 \quad 9.00 E-14$ CURIES UNCERT CURIES ) UNCERT I UCML $\%$ OF TOTAL

$\mathrm{U}-238$ $1.631 E+12$

CONCEN (UCML)

CURIES

4.310

4.255

4.000

4.098

4.000

4.000

4.126

LNCERTI CURIES )

UNCERTIUCAL I

$\%$ OF TOTAL

$\begin{array}{llll}2.000 E-15 & 2.597 E-15 & 1.730 E-15 & 2.108 E-15\end{array}$

$3.700 E-08 \quad 6.000 E-08 \quad 3.200 E-08$

$3.900 E-08$

$3.670 E-16 \quad 4.029 E-15$

1.910E-15 5.234E-22 1.00E-13
31.897
31.915
32.000
31.967
32.000
32.000
31.958

\section{TOTALS}

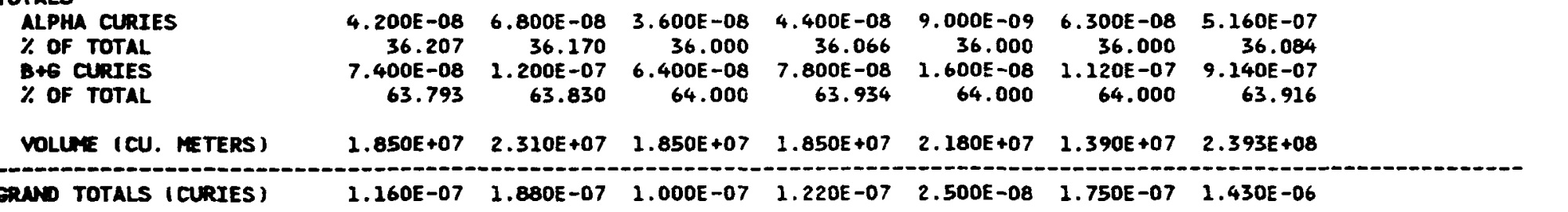

* This nuber is derived from a dispersion factor equation. The dispersion factor is derived from the straight line coussian model and is provedad by the National Dceanic Atmospheric Administration (NOAA).

* NOTE: DCG value obtained from DOE ORDER 5400.5 dated February 8, 1990, Figures III-I and III-3 
RADIOACTIVE WASTE MANAGEMENT INFORMATION SYSTEM

MDF PROD FLOOR AIRBOPNE MASTE SUMMARY FOR JAN THROUGH DEC 1993

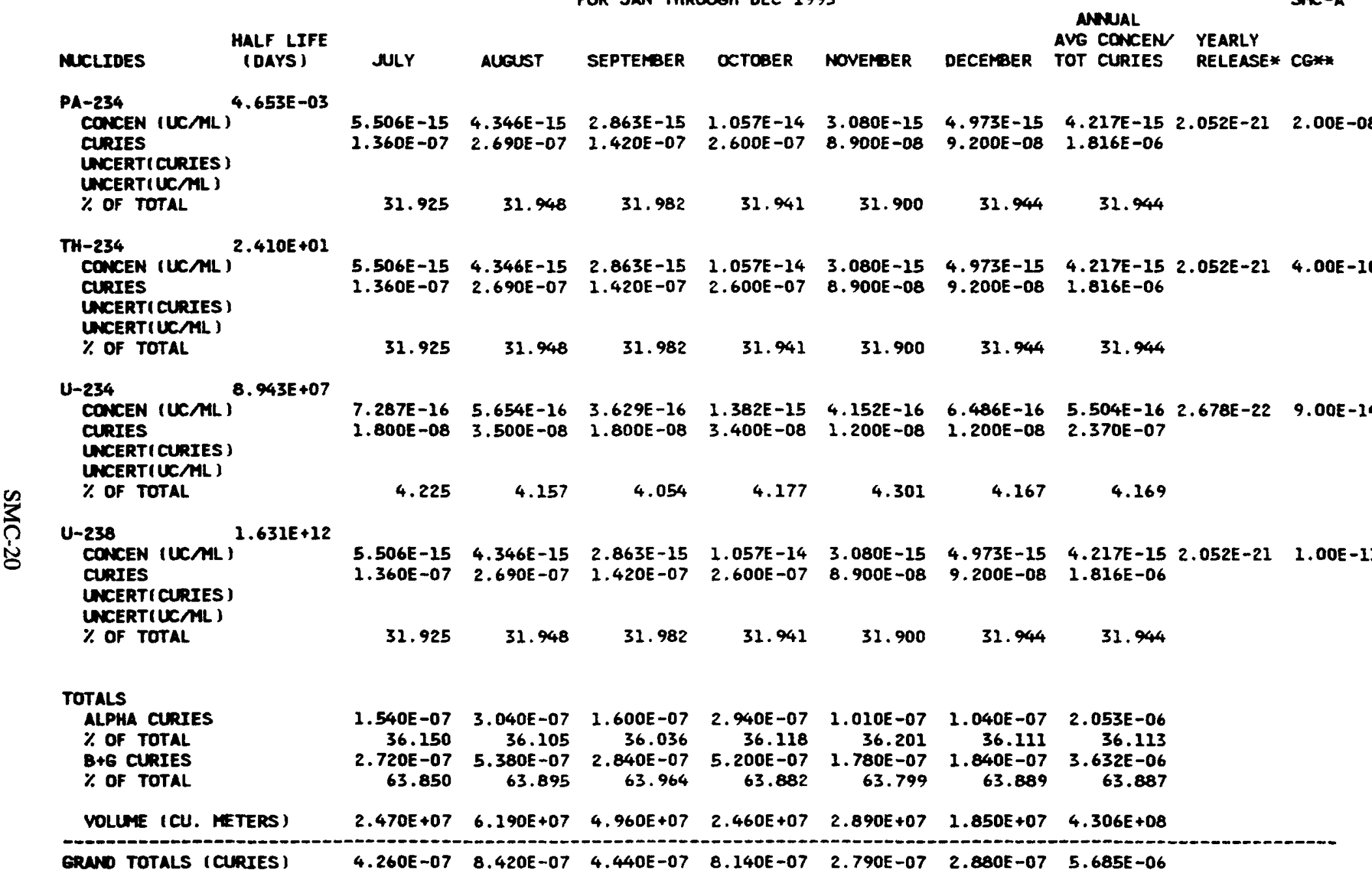

* This number is derived from a dispersion factor equation. The dispersion factor is derived from the straight line omsesian model and is provedad by the Notional Dceanic Atmospheric Administration (NOAL).

* NOTE: DCG value obtained from DOE ORDER 5400.5 dated February 8, 1990, Figures III-I and III-3 
MDF OC LAB AIRBORNE WASTE SLYMARY FOR JAN THROUGH DEC 1993

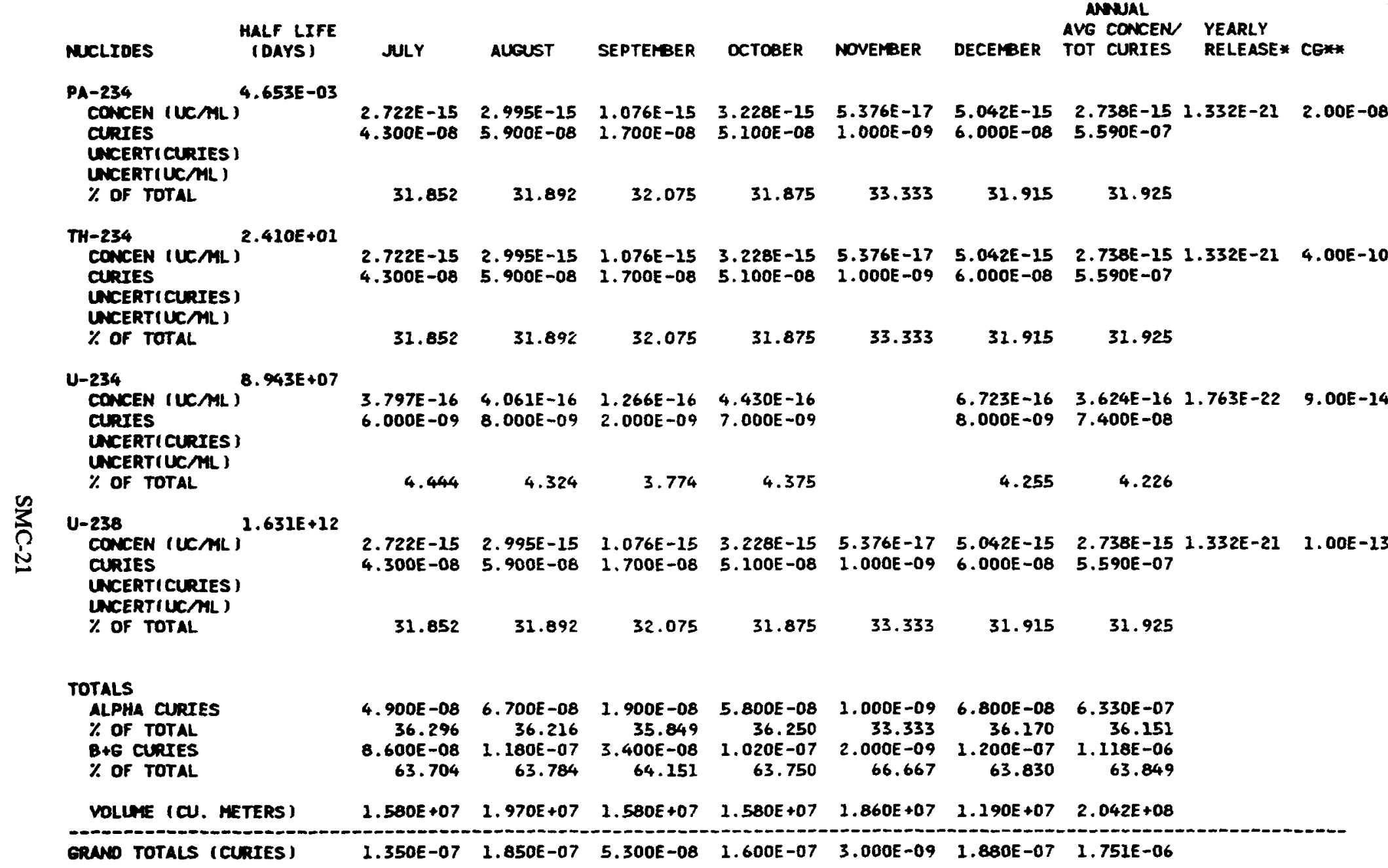

* This muber is derived from a dispersion factor eqution. The dispersion factor is derivod from the straight line gusesian modsl and is proveded by the National Oceanic Atmospheric Administration (MOAA).

* NOTE: DCG volus obtained from DOE ORDER 5400.5 doted february 8, 1990, figures III-I and III-3 
N. PROCESS S9 AIPBORNE WASTE SUMYARY FOR JAN THROUGH DEC 1993

\begin{tabular}{|c|c|c|c|c|c|c|c|c|c|}
\hline $\begin{array}{l}\text { MALF LIFE } \\
\text { (DAYS) }\end{array}$ & JULY & AUEUST & SEPTEMBER & OCTOBER & NOVERBER & DECEMBER & $\begin{array}{l}\text { MNUAL } \\
\text { AVE CONCEN } \\
\text { TOT CURIES }\end{array}$ & $\begin{array}{l}\text { YEARLY } \\
\text { RELEASE* }\end{array}$ & $\sin x-A$ \\
\hline $\begin{array}{l}\text { PA-234 } \\
\text { CONCEN (UCAL) } \\
\text { CORIES } \\
\text { UNCERT(CURIES) } \\
\text { UNCERT(UCAML) } \\
\% \text { OF TOTAL }\end{array}$ & $\begin{array}{l}1.931 E-15 \\
2.800 E-08\end{array}$ & $\begin{array}{l}1.492 E-15 \\
2.700 E-08\end{array}$ & $\begin{array}{l}1.103 E-15 \\
1.600 E-08\end{array}$ & $\begin{array}{l}1.034 E-15 \\
1.500 E-08\end{array}$ & $\begin{array}{l}6.433 E-16 \\
1.100 E-08\end{array}$ & $\begin{array}{l}3.486 E-15 \\
3.800 E-08\end{array}$ & $\begin{array}{l}1.505 E-15 \\
2.820 E-07\end{array}$ & $1.076 E-21$ & $2.00 E-08$ \\
\hline $\begin{array}{l}\text { TH-234 } \\
\text { CONCEN (UCAL) } \\
\text { CURIES } \\
\text { UNCERT(CURIES) } \\
\text { UNCERT(UCML) } \\
\% \text { OF TOTAL }\end{array}$ & $\begin{array}{l}1.931 E-15 \\
2.800 E-08\end{array}$ & $\begin{array}{l}1.492 E-15 \\
2.700 E-08\end{array}$ & $\begin{array}{l}1.103 E-15 \\
1.600 E-08\end{array}$ & $\begin{array}{r}1.034 E-15 \\
1.500 E-08 \\
31.915\end{array}$ & $\begin{array}{r}6.433 E-16 \\
1.100 E-08 \\
32.353\end{array}$ & $\begin{array}{r}3.486 E-15 \\
3.800 E-08 \\
31.933\end{array}$ & $\begin{array}{r}1.505 E-15 \\
2.820 E-07 \\
31.937\end{array}$ & $1.076 E-21$ & $4.00 E-10$ \\
\hline $\begin{array}{l}\text { U-234 } \\
\text { CONCEN IUCML) } \\
\text { CURIES } \\
\text { UNCERTICURIES ) } \\
\text { UNCERTIUCMLIL) } \\
\text { \% OF TOTAL }\end{array}$ & $\begin{array}{r}2.759 E-16 \\
4.000 E-09 \\
4.545\end{array}$ & $\begin{array}{l}2.210 E-16 \\
4.000 E-09\end{array}$ & $\begin{array}{l}1.379 E-16 \\
2.000 E-09\end{array}$ & $\begin{array}{r}1.379 E-16 \\
2.000 E-09 \\
4.255\end{array}$ & $\begin{array}{r}5.848 E-17 \\
1.000 E-09 \\
2.941\end{array}$ & $\begin{array}{r}4.587 E-16 \\
5.000 E-09 \\
4.202\end{array}$ & $\begin{array}{r}1.974 E-16 \\
3.700 E-08 \\
4.190\end{array}$ & $1.412 E-22$ & $9.00 E-14$ \\
\hline $\begin{array}{l}\text { CONCEN (UCAL) } \\
\text { CURIES } \\
\text { UNCERTI CURIES) } \\
\text { UNCERT(UCAML) } \\
\% \text { OF TOTAL }\end{array}$ & $\begin{array}{l}1.931 E-15 \\
2.800 E-08\end{array}$ & $\begin{array}{l}1.492 E-15 \\
2.700 E-08\end{array}$ & $\begin{array}{l}1.103 E-15 \\
1.600 E-08\end{array}$ & $\begin{array}{l}1.034 E-15 \\
1.500 E-08\end{array}$ & $\begin{array}{r}6.433 E-16 \\
1.100 E-08 \\
32.353\end{array}$ & $\begin{array}{r}3.486 E-15 \\
3.800 E-08 \\
31.933\end{array}$ & $\begin{array}{r}1.505 E-15 \\
2.820 E-07 \\
31.937\end{array}$ & $1.076 E-21$ & $1.00 E-13$ \\
\hline $\begin{array}{l}\text { TOTALS } \\
\text { ALPHA CURIES } \\
\% \text { OF TOTAL } \\
\text { B+G CURIES } \\
\% \text { OF TOTAL } \\
\text { VOLUNE (CU. METERS ) }\end{array}$ & $\begin{array}{r}3.200 E-08 \\
36.364 \\
5.600 E-08 \\
63.636 \\
1.450 E+07\end{array}$ & $\begin{array}{r}3.100 E-08 \\
36.471 \\
5.400 E-08 \\
63.529 \\
1.810 E+07\end{array}$ & $\begin{array}{r}1.800 E-08 \\
36.000 \\
3.200 E-08 \\
64.000 \\
1.450 E+07\end{array}$ & $\begin{array}{r}1.700 E-08 \\
36.170 \\
3.000 E-08 \\
63.830 \\
\\
1.450 E+07\end{array}$ & $\begin{array}{r}1.200 E-08 \\
35.294 \\
2.200 E-08 \\
64.706 \\
1.710 E+07\end{array}$ & $\begin{array}{r}4.300 E-08 \\
36.134 \\
7.600 E-08 \\
63.866 \\
\\
1.090 E+07\end{array}$ & $\begin{array}{r}3.190 E-07 \\
36.127 \\
5.640 E-07 \\
63.873 \\
\\
1.874 E+08\end{array}$ & & \\
\hline ERND TOTALS (CURIES) & $8.800 E-08$ & $8.500 E-08$ & $5.000 E-08$ & $4.700 E-08$ & $3.400 E-08$ & $1.190 E-07$ & $8.830 E-07$ & & \\
\hline
\end{tabular}

* This nuber is derived from a dispersion factor equation. The dispersion factor is derived from the straight line goussien modal and is proveded by the National Dceanic Atmospheric Administration (NOAA).

* NOTE: DCG value obtained from DOE ORDER 5400.5 dated February 8, 1990, Figures III-1 and III-3

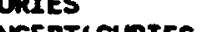

UNCERT(UCML

$2.420 E+01$

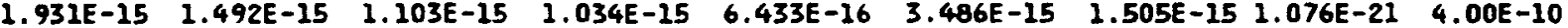

UNCERTICURIES

UNCERT(LCML)

8. $943 E+07$

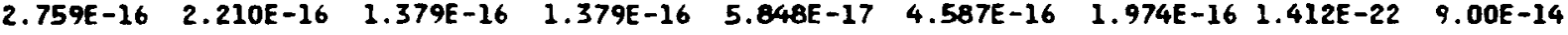

4.545

\section{YEARLY}

RELEASE 
N. PROCESS S2O AIRBORNE WASTE SUMMARY FOR JAN THROUGH DEC 1993
PAGE 2 RPT. 749 SME-h

\section{ANWUAL} YEARLY AVG CONCEN YEARLY RELEASE

\begin{tabular}{|c|c|c|c|c|c|c|c|c|c|}
\hline & $\begin{array}{l}\text { HALF LIFE } \\
\text { (DAYS) }\end{array}$ & & & & & & & $\begin{array}{l}\text { AVE CONCEN } \\
\text { TOT CURIES }\end{array}$ & $\begin{array}{l}\text { YEARLY } \\
\text { RELEASE* CG** }\end{array}$ \\
\hline vel & & NLY & ALEUST & SEPTEMBER & OCTOBER & NOVEMBER & DECEMBER & & \\
\hline
\end{tabular}

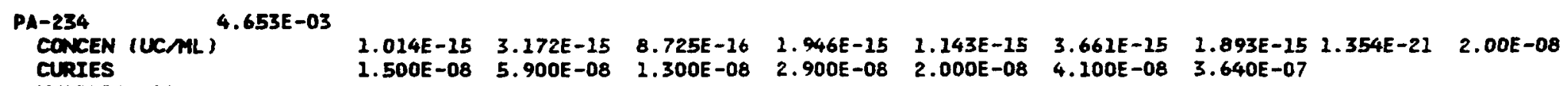

UNCERTI CURIES

UNCERT (UCAL)

$\%$ OF TOTAL
31.925
31.892
31.707
31.868
31.746
32.031
31.930

\section{$\mathrm{TH}-234$}

$2.410 E+01$

CONCEN (UCML)

Curies

$1.014 E-15 \quad 3.172 E-15$

8. $725 E-16,2.946 E-15$

.500E -08 5.900E -08

$1.946 E-15 \quad 1.143 E-15 \quad 3.661 E-15$

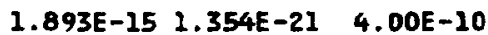

UAERT C CuRIES

UNCERTIUCML

$\%$ of total
31.892
31.707

31.868

31.746

32.031

31.930

$U-234$ 8. $943 E+07$

CONCEN (UCML)

CURIES

$\begin{array}{lllllllll}1.351 E-16 & 4.301 E-26 & 1.342 E-16 & 2.685 E-16 & 1.714 E-16 & 4.464 E-16 & 2.496 E-16 & 1.785 E-22 & 9.00 E-14 \\ 2.000 E-09 & 8.000 E-09 & 2.000 E-09 & 4.000 E-09 & 3.000 E-09 & 5.000 E-09 & 4.800 E-08 & & \end{array}$

UNCERTI CURTES

(NCERT (UCML)

$2 \%$ OF TOTAL

$1.631 E+12$

CONCEN (UCML)

CORTES

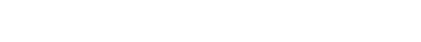

$$
\text { 4. } 255
$$

4.324

4.878

4.396

4.762

3.906

4.211

$\begin{array}{lllllllll}2.024 E-15 & 3.172 E-15 & 8.725 E-16 & 1.946 E-15 & 1.143 E-15 & 3.661 E-15 & 1.893 E-15 & 1.354 E-21 & 1.00 E-13\end{array}$ $\begin{array}{lllllll}1.500 E-08 & 5.900 E-08 & 1.300 E-08 & 2.900 E-08 & 2.000 E-08 & 4.100 E-08 & 3.640 E-07\end{array}$

UNCERT ( CURIES )

(NCERT (UCML)

$\%$ OF TOTAL
31.915
31.892

31.707

31.868

31.746

32.031

31.930

\section{TotALS}

$\begin{array}{lrrrrrrr}\text { ALPHA CURIES } & 1.700 E-08 & 6.700 E-08 & 1.500 E-08 & 3.300 E-08 & 2.300 E-08 & 4.600 E-08 & 4.120 E-07 \\ \% \text { OF TOTAL } & 36.170 & 36.216 & 36.585 & 36.264 & 36.508 & 35.938 & 36.140 \\ \text { B+G CURIES } & 3.000 E-08 & 1.280 E-07 & 2.600 E-08 & 5.800 E-08 & 4.000 E-08 & 8.200 E-08 & 7.280 E-07 \\ \% \text { OF TOTAL } & 63.830 & 63.784 & 63.415 & 63.736 & 63.492 & 64.063 & 63.860\end{array}$

VOLUNE (CU. METERS) $1.480 E+07 \quad 1.860 E+07 \quad 1.490 E+07 \quad 1.490 E+07 \quad 1.750 E+07 \quad 1.120 E+07 \quad 1.923 E+08$

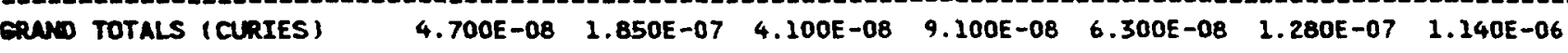

* This nuber is derived from a dispersion factor equetion. The dispersion factor is derived from the straight line ouserien model and is provedad by the National Dceanic Atmospheric Administration (NOAA).

** NOTE: DCG velue obtained from DOE ORDER 5400.5 doted February 8, 1990, Figures III-I and III-3 
IDAHO OPERATIONS OFFICE

U.S. DEPARTMENT OF ENERGY

RADIOACTIVE WUSTE MANAGEMENT INFORMATION SYSTEM

N. PROCESS SII AIRBORNE WASTE SUMMARY FOR JAN THROUGH DEC 1993

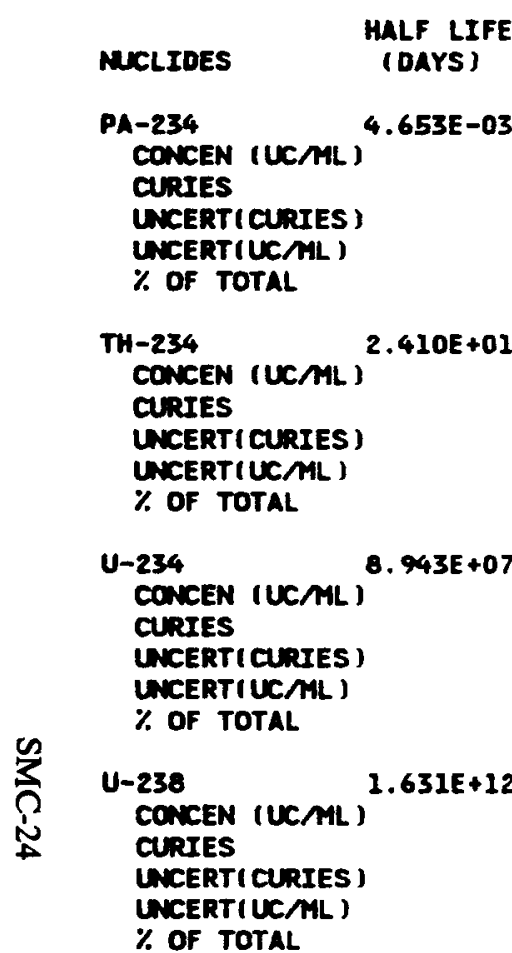
AVG CONCEN

PAGE 1 RPT. 750 $\sin -A$

\section{YEARLY} RELEASE $\%$ OF CG $\begin{array}{lllllllll}1.216 E-15 & 2.228 E-15 & 7.143 E-16 & 2.365 E-25 & 1.714 E-16 & 2.973 E-15 & 1.385 E-15 & 9.908 E-22 & 2.00 E-08\end{array}$ $\begin{array}{lllllll}1.800 E-08 & 4.300 E-08 & 1.100 E-08 & 3.500 E-08 & 3.000 E-09 & 3.300 E-08 & 2.690 E-07\end{array}$
32.143
31.852
32.353
31.818
33.333
32.039
31.948

$\begin{array}{llll}1.216 E-15 & 2.228 E-15 & 7.143 E-16 & 2.365 E-15\end{array}$ $\begin{array}{lllllll}1.800 E-08 & 4.300 E-08 & 1.100 E-08 & 3.500 E-08 & 3.000 E-09 & 3.300 E-08 & 2.690 E-07\end{array}$
32.243
32.852
32.353
31.818
33.333
32.039
31.948

$\begin{array}{llll}1.351 E-16 & 3.109 E-16 & 6.494 E-17 & 3.378 E-16\end{array}$ 2.000E-09 6.000E-09 1.000E-09 5.000E-09

$3.604 E-16 \quad 1.802 E-16 \quad 1.289 E-22 \quad 9.00 E-14$ 4. DOOE -09 3.500E-08
3.571
4.444
2.941
4.545
3.883
4.157

$\begin{array}{lllllllll}1.216 E-15 & 2.228 E-15 & 7.143 E-16 & 2.365 E-15 & 1.714 E-16 & 2.973 E-15 & 1.385 E-15 & 9.908 E-22 & 1.00 E-13\end{array}$ $\begin{array}{lllllll}1.800 E-08 & 4.300 E-08 & 1.100 E-08 & 3.500 E-08 & 3.000 E-09 & 3.300 E-08 & 2.690 E-07\end{array}$

$$
\begin{array}{lllllll}
32.143 & 31.852 & 32.353 & 31.818 & 33.333 & 32.039 & 31.948
\end{array}
$$

\section{TOTALS}

$\begin{array}{lrrrrrrr}\text { ALPHA CURIES } & 2.000 E-08 & 4.900 E-08 & 1.200 E-08 & 4.000 E-08 & 3.000 E-09 & 3.700 E-08 & 3.040 E-07 \\ \text { \% OF TOTAL } & 35.714 & 36.296 & 35.294 & 36.364 & 33.333 & 35.922 & 36.104 \\ \text { B+G CURIES } & 3.600 E-08 & 8.600 E-08 & 2.200 E-08 & 7.000 E-08 & 6.000 E-09 & 6.600 E-08 & 5.380 E-07 \\ \% \text { OF TOTAL } & 64.286 & 63.704 & 64.706 & 63.636 & 66.667 & 64.078 & 63.895\end{array}$

$\begin{array}{lllllllll}\text { VOLAAE (CU. METERS) } & 1.480 E+07 & 1.930 E+07 & 1.540 E+07 & 1.480 E+07 & 1.750 E+07 & 1.120 E+07 & 1.942 E+08\end{array}$

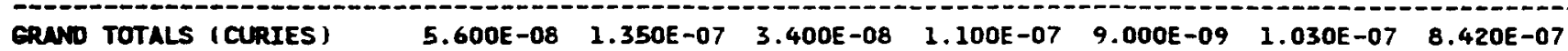

* This muber is derived from - dispersion factor equation. The dispersion factor is derived from the straight line geussien model and is provedod by the National Dceanic Atmospheric Administration (MOAA).

* NOTE: DCG value obtained from DOE ORDER 5400.5 dated Fabruary 8, 1990, Figures III-1 and III-3 
PRF STACK S14 AIPROPNE WASTE SUMMARY

FOR JAN THROUGH DEC 1993

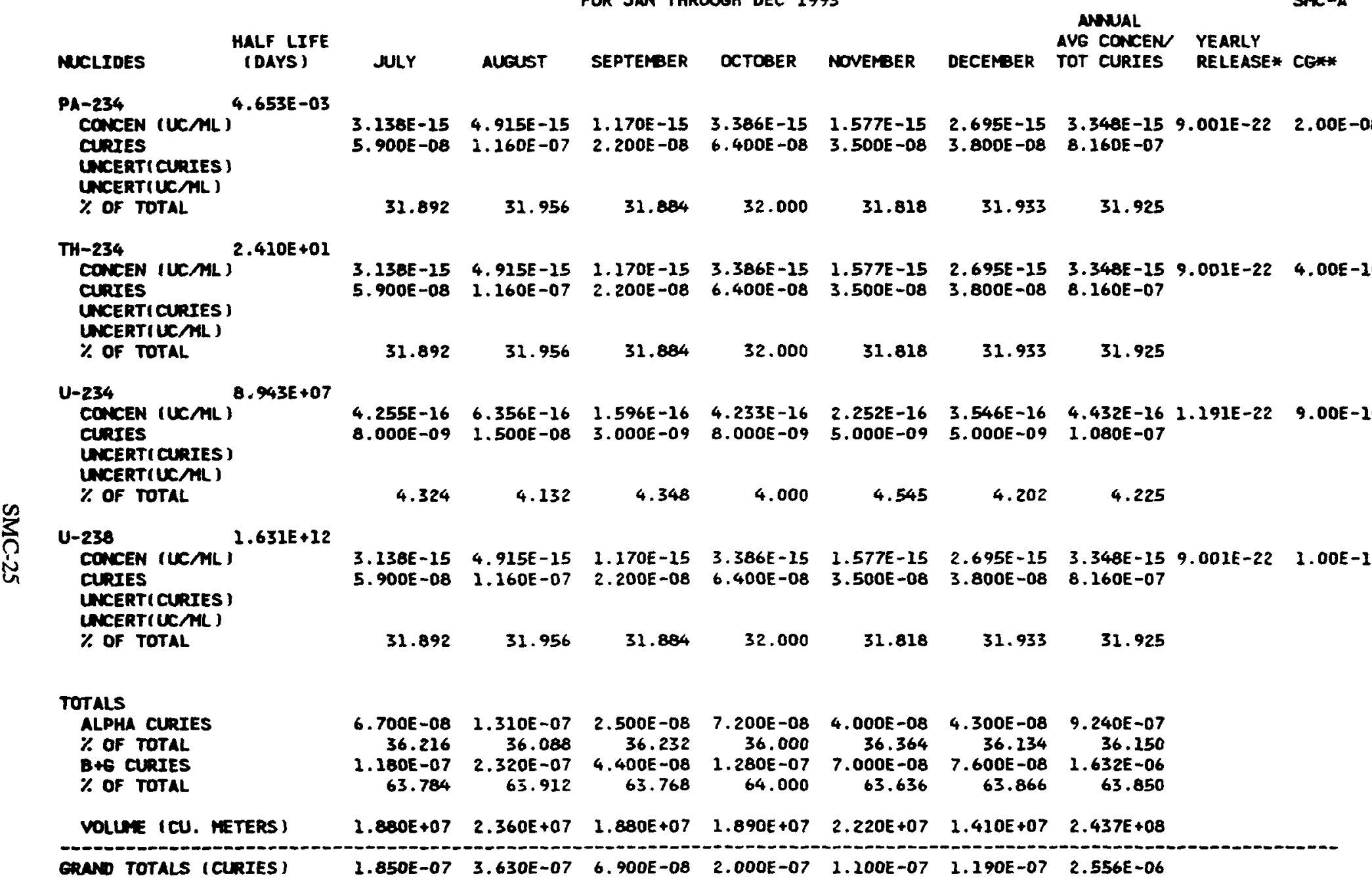

* This nuber is darived from a dispersion factor equation. The dispersion factor is derived from the straight line anusian modal and is proveded by the Netional Dceanic Atmospheric Administration iNOMA).

** NOTE: DCG value obtained from DOE ORDER 5400.5 dated February 8, 1990 , Figures III-1 and III-3 
SCRAP HANOL ING ATPBOPNE MASTE SLMMARY FOR JAN THROUGH DEC 1993

\begin{tabular}{|c|c|c|c|c|c|c|c|c|c|}
\hline $\begin{array}{l}\text { HALF LIFE } \\
\text { ( DAYS ) }\end{array}$ & WLY & AUGUST & SEPTEMBER & OCTOBER & NOVEMBER & DECEMBER & $\begin{array}{l}\text { ANUAL } \\
\text { AVG CONCEN } \\
\text { TOT CURIES }\end{array}$ & $\begin{array}{l}\text { YEARLY } \\
\text { RELEASE* }\end{array}$ & CGX* \\
\hline $\begin{array}{l}\text { PA-234 } \\
\text { CONCEN (UCML) } \\
\text { CURIES } \\
\text { UNCERTICURIES) } \\
\text { UNCERT(UCML) } \\
\text { \% OF TOTAL }\end{array}$ & $\begin{array}{l}2.240 E-15 \\
4.100 E-08\end{array}$ & $\begin{array}{l}1.410 E-15 \\
3.200 E-08\end{array}$ & $\begin{array}{l}6.044 E-16 \\
1.100 E-08\end{array}$ & $\begin{array}{l}5.464 E-16 \\
1.000 E-08\end{array}$ & $\begin{array}{l}3.241 E-16 \\
7.000 E-09\end{array}$ & $\begin{array}{l}2.246 E-15 \\
3.100 E-08\end{array}$ & $\begin{array}{l}1.091 E-25 \\
2.580 E-07\end{array}$ & $2.933 E-22$ & $2.00 E-08$ \\
\hline $\begin{array}{l}\text { TH-234 } \\
\text { CONCEN (UCAM) } \\
\text { CURIES } \\
\text { UNCERT(CURIES) } \\
\text { UNCERT(UCALI) } \\
\% \text { OF TOTAL }\end{array}$ & $\begin{array}{l}2.240 E-15 \\
4.100 E-08\end{array}$ & $\begin{array}{l}1.410 E-15 \\
3.200 E-08\end{array}$ & $\begin{array}{l}6.044 E-16 \\
1.100 E-08\end{array}$ & $\begin{array}{l}5.464 E-16 \\
1.000 E-08\end{array}$ & $\begin{array}{l}3.241 E-16 \\
7.000 E-09\end{array}$ & $\begin{array}{l}2.246 E-15 \\
3.100 E-08\end{array}$ & $\begin{array}{r}1.091 E-15 \\
2.580 E-07 \\
32.010\end{array}$ & $2.933 E-22$ & $4.00 E-10$ \\
\hline $\begin{array}{l}\text { U-234 } \\
\text { CONCEN (UCML) } \\
\text { CURIES } \\
\text { UNCERT(CURIES) } \\
\text { UNCERT(UCML) } \\
\% \text { OF TOTAL }\end{array}$ & $\begin{array}{l}2.732 E-16 \\
5.000 E-09\end{array}$ & $\begin{array}{l}1.762 E-16 \\
4.000 E-09\end{array}$ & $\begin{array}{l}5.495 E-17 \\
1.000 E-0^{\circ}\end{array}$ & $\begin{array}{l}5.464 E-17 \\
1.000 E-09\end{array}$ & $\begin{array}{l}4.630 E-17 \\
1.000 E-09\end{array}$ & $\begin{array}{l}2.899 E-16 \\
4.000 E-09\end{array}$ & $\begin{array}{r}1.353 E-16 \\
3.200 E-08 \\
3.970\end{array}$ & $3.637 E-23$ & $.00 E$ \\
\hline $\begin{array}{l}\text { U-238 } \\
\text { CONCEN (UCML) } \\
\text { CURIES } \\
\text { UNCERT(CURIES) } \\
\text { UNCERT(UCML) } \\
\% \text { OF TOTAL }\end{array}$ & $\begin{array}{l}2.240 E-15 \\
4.100 E-08\end{array}$ & $\begin{array}{l}1.410 E-15 \\
3.200 E-08\end{array}$ & $\begin{array}{l}6.044 E-16 \\
1.100 E-08\end{array}$ & $\begin{array}{l}5.464 E-16 \\
1.000 E-08\end{array}$ & $\begin{array}{l}3.241 E-16 \\
7.000 E-09\end{array}$ & $\begin{array}{l}2.246 E-15 \\
3.100 E-08\end{array}$ & $\begin{array}{l}1.091 E-15 \\
2.580 E-07\end{array}$ & $2.933 E-22$ & $.00 E$ \\
\hline $\begin{array}{l}\text { TOTALS } \\
\text { ALPHA CURIES } \\
\% \text { OF TOTAL } \\
\text { B+6 CURIES } \\
\% \text { OF TOTAL } \\
\text { VOLUME (CU. METERS) }\end{array}$ & $\begin{array}{r}4.600 E-08 \\
35.938 \\
8.200 E-08 \\
64.063 \\
1.830 E+07\end{array}$ & $\begin{array}{r}3.600 E-08 \\
36.000 \\
6.400 E-08 \\
64.000 \\
2.270 E+07\end{array}$ & $\begin{array}{r}1.200 E-08 \\
35.294 \\
2.200 E-08 \\
64.706 \\
1.820 E+07\end{array}$ & $\begin{array}{r}1.100 E-08 \\
35.484 \\
2.000 E-08 \\
64.516 \\
1.830 E+07\end{array}$ & $\begin{array}{r}8.000 E-09 \\
36.364 \\
1.400 E-08 \\
63.636 \\
2.160 E+07\end{array}$ & $\begin{array}{r}3.500 E-08 \\
36.083 \\
6.200 E-08 \\
63.918 \\
1.380 E+07\end{array}$ & $\begin{array}{r}2.900 E-07 \\
35.980 \\
5.160 E-07 \\
64.020 \\
2.365 E+08\end{array}$ & & \\
\hline RWD TOTALS ICURIES) & $1.280 E-07$ & $1.000 E-07$ & $3.400 E-08$ & $3.100 E-08$ & $2.200 E-08$ & $9.700 E-08$ & $8.060 E-07$ & & \\
\hline
\end{tabular}

* This muber is darived from a dispersion factor equation. The dispersion factor is derived from the straight line geussian model and is proveded by the National Dceanic Atmospharic Administration (NOAA).

* NOTE: DCG value obtained from DOE ORDER 5400.5 dated February 8, 1990, Figures III-1 and III-3 
RADIOACTIVE WASTE MANAGEMENT INFORALTION SYSTEM

S. PROCESS S6 AIRBORNE MASTE SUMTARY FOR JAN THROUGH DEC 1993

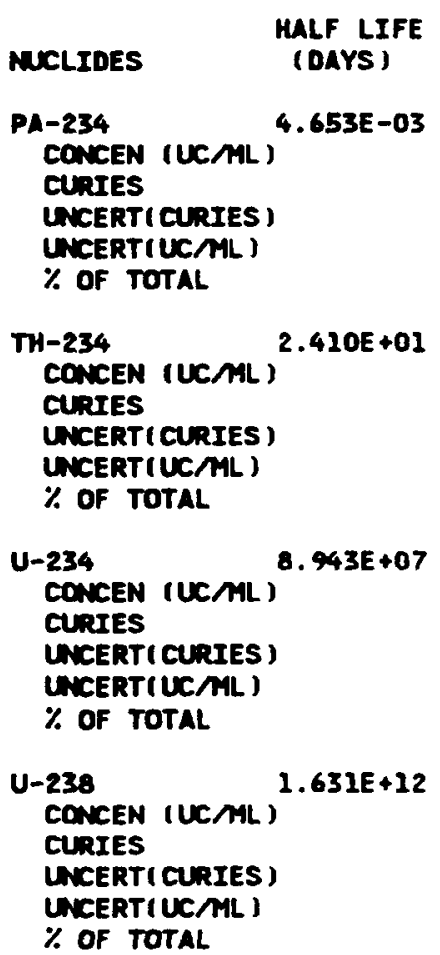

JULY

AUEUST

SEPTEMBER

$1.824 E-15 \quad 1.946 E-15$

$2.700 E-08 \quad 3.600 E-08$

$1.082 E-15$
$1.600 E-08$

$1.400 E-08$

31.765

31.858

32.000

31.818

31.959

32.353 $\begin{array}{lllllllll}1.824 E-15 & 1.946 E-15 & 1.081 E-25 & 9.459 E-16 & 1.771 E-15 & 9.910 E-16 & 1.797 E-15 & 1.286 E-21 & 4.00 E-10 \\ 2.700 E-08 & 3.600 E-08 & 1.600 E-08 & 1.400 E-08 & 3.100 E-08 & 1.100 E-08 & 3.440 E-07 & & \end{array}$
31.765
31.858
32.000
31.818
31.959
32.353
31.911

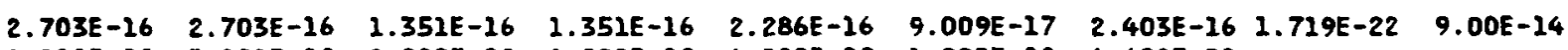
4.000E-09 5.000E-D9 2.000E-09 2.000E-09 4.000 -09 $1.000 E-09 \quad 4.600 E-08$
4.706
4.425
4.000
4.545
4.124
2.941
4.267

$\begin{array}{lllllllll}1.824 E-15 & 1.946 E-15 & 1.081 E-15 & 9.459 E-16 & 1.771 E-15 & 9.910 E-16 & 1.797 E-15 & 1.286 E-21 & 1.00 E-13\end{array}$ $2.700 E-08 \quad 3.600 E-08 \quad 1.600 E-08 \quad 1.400 E-08 \quad 3.100 E-08 \quad 1.100 E-08 \quad 3.440 E-07$
31.765
31.858
32.000
31.818
31.959
32.353
31.911

\section{Totals}

\begin{tabular}{|c|c|c|c|c|c|c|c|}
\hline $\begin{array}{l}\text { ALPHA CURIES } \\
\% \text { OF TOTAL } \\
\text { B.G CURTES } \\
\% \text { OF TOTAL }\end{array}$ & $\begin{array}{r}3.100 E-08 \\
36.471 \\
5.400 E-08 \\
63.529\end{array}$ & $\begin{array}{r}4.100 E-08 \\
36.283 \\
7.200 E-08 \\
63.717\end{array}$ & $\begin{array}{r}1.800 E-08 \\
36.000 \\
3.200 E-08 \\
64.000\end{array}$ & $\begin{array}{r}1.600 E-08 \\
36.364 \\
2.800 E-08 \\
63.636\end{array}$ & $\begin{array}{r}3.500 E-08 \\
36.083 \\
6.200 E-08 \\
63.918\end{array}$ & $\begin{array}{r}1.200 E-08 \\
35.294 \\
2.200 E-08 \\
64.706\end{array}$ & $\begin{array}{r}3.900 E-07 \\
36.178 \\
6.880 E-07 \\
63.822\end{array}$ \\
\hline VOLUME ICU. ME & $1.480 E+07$ & $1.850 E+07$ & $1.480 E+07$ & $1.480 E+07$ & $1.750 E+07$ & $1.110 E+07$ & $1.914 E+08$ \\
\hline
\end{tabular}

* This nuber is derived from a dispersion factor eqution. The dispersion factor is derived from the straight line coussian model and is proveded by the National Oceanic Atmospheric Administration (NOAA).

* NOTE: DCE value obtained from DOE ORDER 5400.5 dated February 8, 1990 , Figures III-1 and III-3 
S. PROCESS S7 AIRBORNE MASTE SUMMARY

FOR JAN THROUGH DEC 1993

GRAND TOTALS (CURIES) $\quad 1.570 E-07 \quad 1.720 E-07 \quad 1.600 E-08 \quad 6.300 E-08 \quad 5.000 E-08 \quad 7.200 E-08 \quad 1.090 E-06$

* This muber is derived from a dispersion factor equation. The dispersion factor is derived from the straight line goussian model and is provedad by the National Oceanic Atmospheric Administration (NOAA).

* NOTE: DCG velue obtained from DOE ORDER 5400.5 dated Fabruary 8, 1990, Figures III-1 and III-3 
RADIOACTIVE WASTE MUNAGEMENT INFORHATION SYSTEM

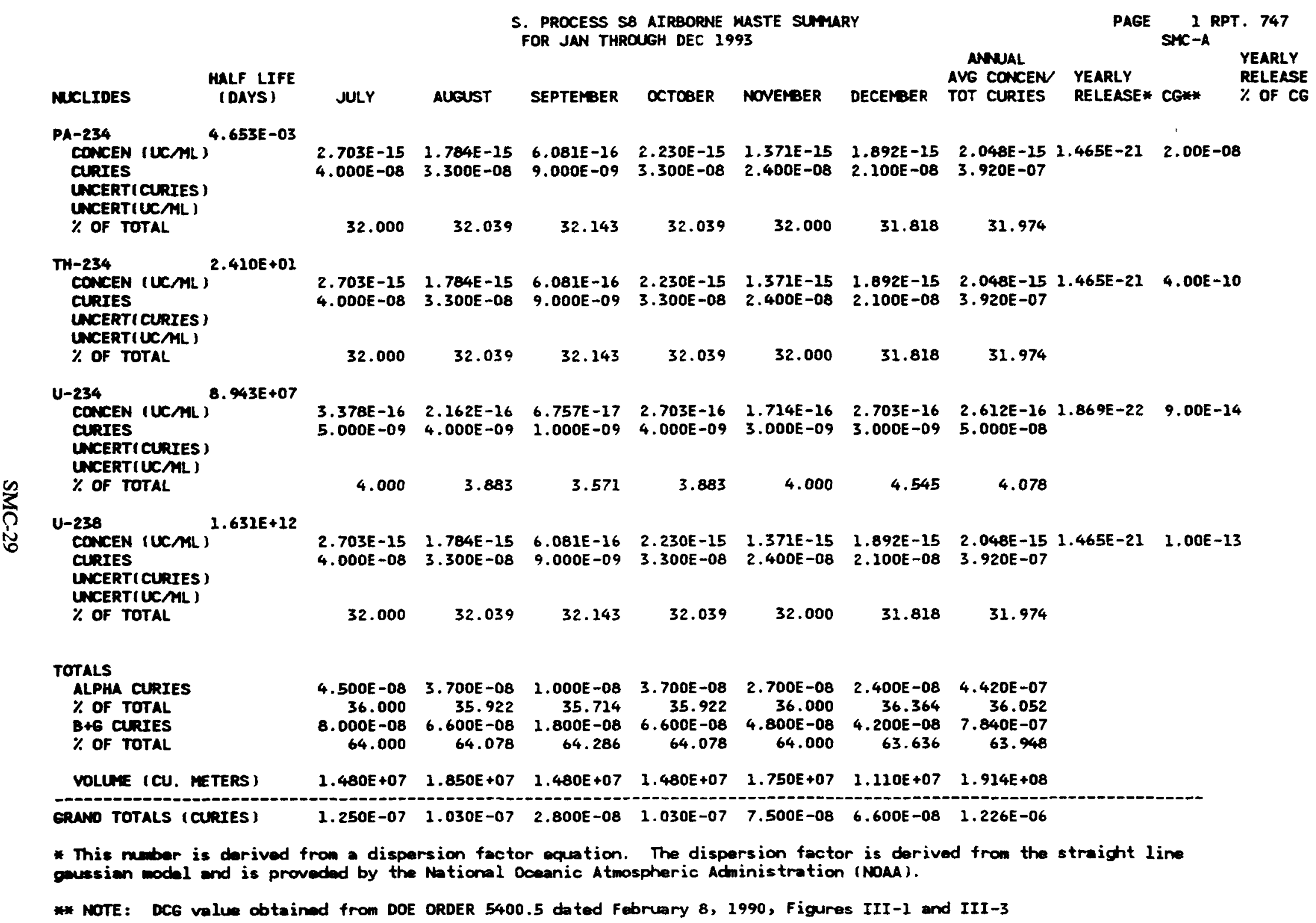


RADIOACTIVE WASTE MANAGEMENT INFORMATION SYSTEM

2B AREA PHASEI AIRBORNE MASTE SUMMARY FOR JAN THROUEH DEC 1993

\begin{tabular}{|c|c|c|c|c|c|c|c|c|c|}
\hline $\begin{array}{l}\text { HALF LIFE } \\
\text { (DAYS) }\end{array}$ & NLY & AUGUST & SEPTEMBER & OCTOBER & NOVERBER & DECERBER & $\begin{array}{l}\text { ANNUAL } \\
\text { AVG CONCEN } \\
\text { TOT CURIES }\end{array}$ & $\begin{array}{l}\text { YEARLY } \\
\text { RELEASE* }\end{array}$ & CEX: \\
\hline $\begin{array}{l}\text { PA-234 } \\
\text { CONCEN (UCAL) } \\
\text { CURIES } \\
\text { UNCERTICURIES) } \\
\text { UNCERT(UCAL) } \\
\text { \% OF TOTAL }\end{array}$ & $\begin{array}{l}3.443 E-15 \\
2.300 E-08\end{array}$ & $\begin{array}{l}4.235 E-15 \\
2.600 E-08\end{array}$ & $\begin{array}{l}1.222 E-15 \\
6.000 E-09\end{array}$ & $\begin{array}{l}2.392 E-15 \\
1.600 E-08\end{array}$ & $\begin{array}{l}6.337 E-16 \\
5.000 E-09\end{array}$ & $\begin{array}{l}4.183 E-15 \\
2.100 E-08\end{array}$ & $\begin{array}{l}2.446 E-15 \\
1.920 E-07\end{array}$ & $1.033 E-21$ & $2.00 E-08$ \\
\hline $\begin{array}{l}\text { TH-234 } \\
\text { CONCEN (UCML) } \\
\text { CURIES } \\
\text { UNCERT(CURIES) } \\
\text { UNCERTIUCAL) } \\
\text { \% OF TOTAL }\end{array}$ & $\begin{array}{l}3.443 E-15 \\
2.300 E-08\end{array}$ & $\begin{array}{l}4.235 E-15 \\
2.600 E-08\end{array}$ & $\begin{array}{l}1.222 E-15 \\
6.000 E-09\end{array}$ & $\begin{array}{l}2.392 E-15 \\
1.600 E-08\end{array}$ & $\begin{array}{l}6.337 E-16 \\
5.000 E-09\end{array}$ & $\begin{array}{l}4.183 E-15 \\
2.100 E-08\end{array}$ & $\begin{array}{l}2.446 E-15 \\
1.920 E-07\end{array}$ & $1.033 E-21$ & $4.00 E-10$ \\
\hline $\begin{array}{l}\text { U-234 } \\
\text { CONCEN (UCML) } \\
\text { CURIES } \\
\text { UNCERT(CURIES) } \\
\text { UNCERT(UCML) } \\
\text { \% OF TOTAL }\end{array}$ & $\begin{array}{l}4.491 E-16 \\
3.000 E-09\end{array}$ & $\begin{array}{l}4.886 E-16 \\
3.000 E-09\end{array}$ & $\begin{array}{l}2.037 E-16 \\
1.000 E-09\end{array}$ & $\begin{array}{l}2.990 E-16 \\
2.000 E-09\end{array}$ & $\begin{array}{l}1.267 E-16 \\
1.000 E-09\end{array}$ & $\begin{array}{l}5.976 E-16 \\
3.000 E-09\end{array}$ & $\begin{array}{l}3.313 E-16 \\
2.600 E-08\end{array}$ & $1.399 E-22$ & $9.00 E-14$ \\
\hline $\begin{array}{l}\text { U-238 } \\
\text { CONCEN (UCML) } \\
\text { CURIES } \\
\text { UNCERT(CURIES) } \\
\text { UNCERT(UCAML) } \\
\text { \% OF TOTAL }\end{array}$ & $\begin{array}{l}3.443 E-15 \\
2.300 E-08\end{array}$ & $\begin{array}{l}4.235 E-15 \\
2.600 E-08\end{array}$ & $\begin{array}{l}1.222 E-15 \\
6.000 E-09\end{array}$ & $\begin{array}{l}2.392 E-15 \\
1.600 E-08\end{array}$ & $\begin{array}{l}6.337 E-16 \\
5.000 E-09\end{array}$ & $\begin{array}{l}4.183 E-15 \\
2.100 E-08\end{array}$ & $\begin{array}{r}2.446 E-15 \\
1.920 E-07 \\
31.894\end{array}$ & $1.033 E-21$ & $1.00 E-13$ \\
\hline $\begin{array}{l}\text { TOTALS } \\
\text { ALPHA CURIES } \\
\% \text { OF TOTAL } \\
\text { B+G CURIES } \\
\% \text { OF TOTAL } \\
\text { VOLLAE (CU. METERS) }\end{array}$ & $\begin{array}{r}2.600 E-08 \\
36.111 \\
4.600 E-08 \\
63.889 \\
\\
6.680 E+06\end{array}$ & $\begin{array}{r}2.900 E-08 \\
35.802 \\
5.200 E-08 \\
64.198 \\
6.140 E+06\end{array}$ & $\begin{array}{r}7.000 E-09 \\
36.842 \\
1.200 E-08 \\
63.158 \\
4.910 E+06\end{array}$ & $\begin{array}{r}1.800 E-08 \\
36.000 \\
3.200 E-08 \\
64.000 \\
6.690 E+06\end{array}$ & $\begin{array}{r}6.000 E-09 \\
37.500 \\
1.000 E-08 \\
62.500 \\
7.890 E+06\end{array}$ & $\begin{array}{r}2.400 E-08 \\
36.364 \\
4.200 E-08 \\
63.636 \\
5.020 E+06\end{array}$ & $\begin{array}{r}2.180 E-07 \\
36.213 \\
3.840 E-07 \\
63.787 \\
\\
7.849 E+07\end{array}$ & & \\
\hline CRUND TOTALS (CURIES) & $7.200 E-08$ & $8.100 E-08$ & $1.900 E-08$ & $5.000 E-08$ & $1.600 E-08$ & $6.600 E-08$ & $6.020 E-07$ & & \\
\hline
\end{tabular}

* This nuber is darived from a dispersion fector equation. The dispersion factor is derived from the straight line omesian model and is proveded by the Nationel Dceanic Atmospheric Administration (NOAA).

* NOTE: DCG value obtained from DOE ORDER 5400.5 dated February 8, 1990, Figures III-I and III-3 
SMC Facility Detail Data for Months January to June Annual Average Concentration and Total Curies for January to June 

EXHAUST FAN PI ATRBORNE WASTE SUMMARY FOR JAN THROUGH JUN 1993

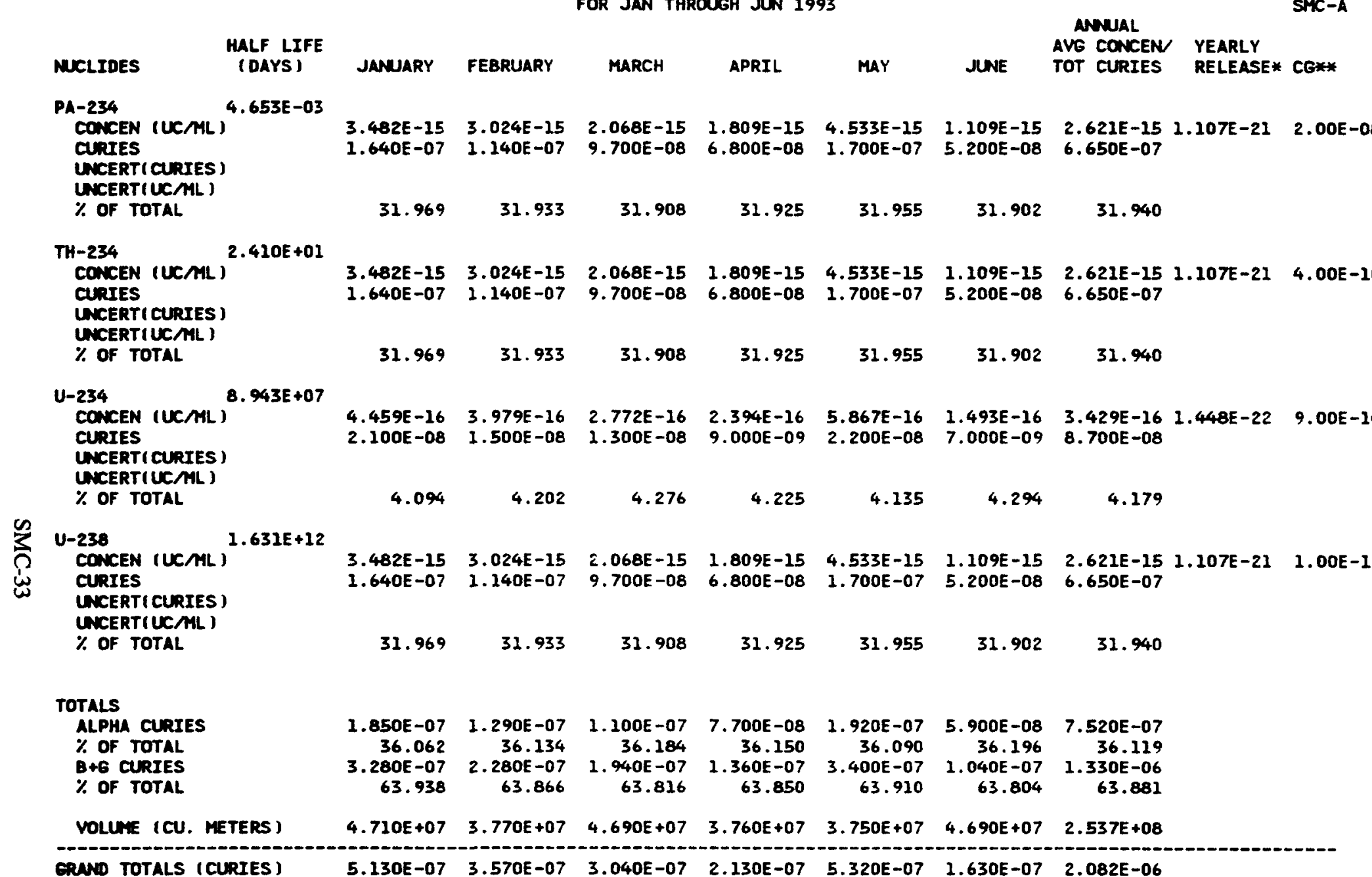

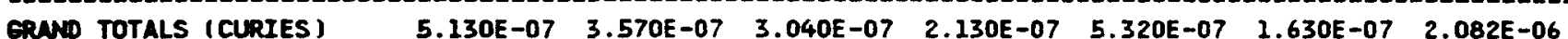

* This nubar is darived from a dispersion factor equation. The dispersion factor is derived from the straight line omsesian model and is proveded by the National Oceanic atmospheric Adninistration (NOAA).

** NOTE: DCG velue obtainad from DOE ORDER 5400.5 dated February 8, 1990, Figures III-1 and III-3 
RADIOACTIVE WASTE MANAGEMENT INFORMATION SYSTEM

LASER PUNCH P1 ATRBORNE MASTE SUMARY FOR JAN THROUGH JUN 1993

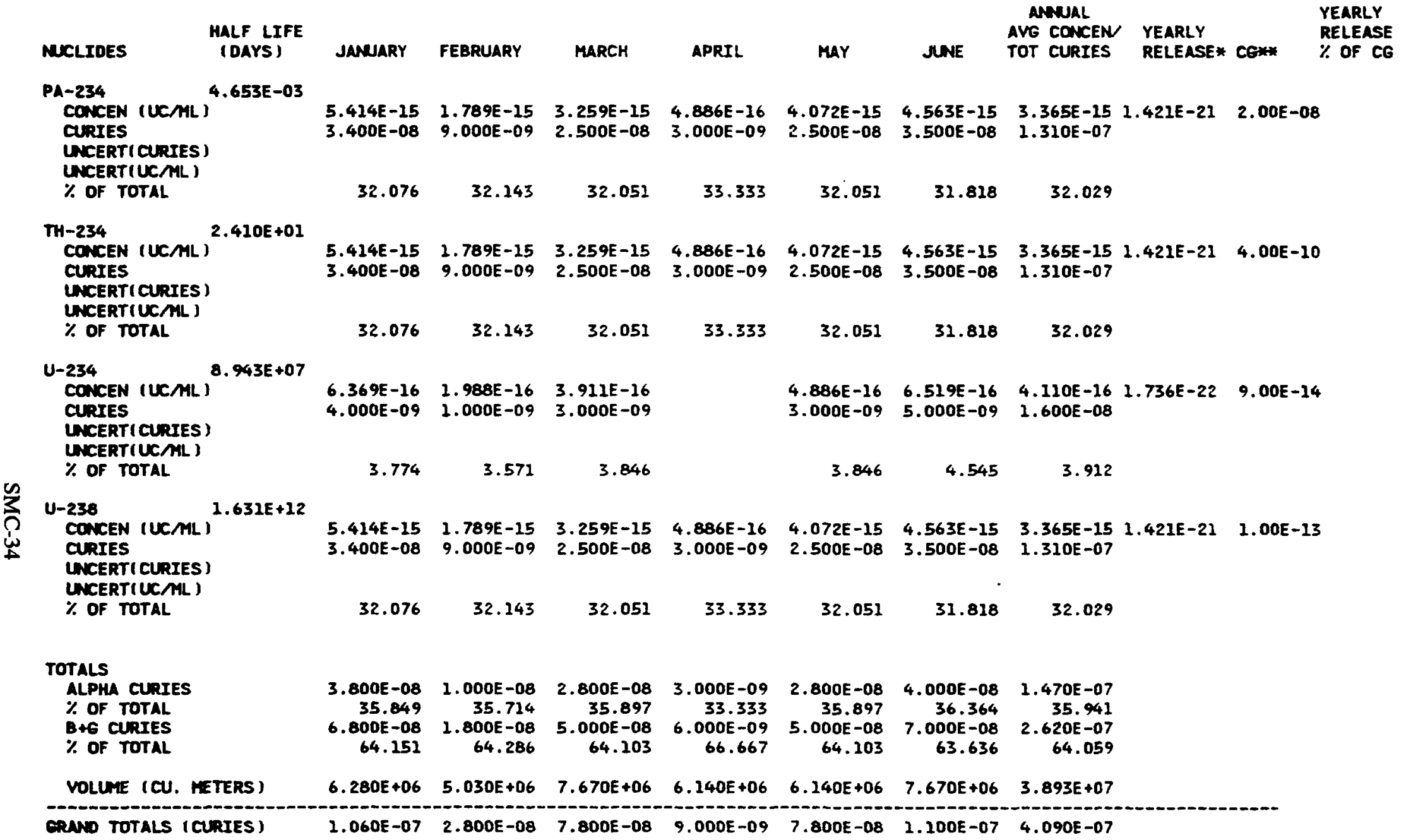

* This nubar is derived from a dispersion factor eqution. The dispersion factor is derived from the straight line onssian model and is proveded by the Netional Oceanic Atmospheric Adwinistration (NOAA).

** NOTE: DCE value obtained from DOE ORDER 5400.5 dated February 8, 1990, Figures III-1 and III-3 
LIQUID RECLAIM AIRBORNE WASTE SUMYARY FOR JAN THROUGH JUN 1993

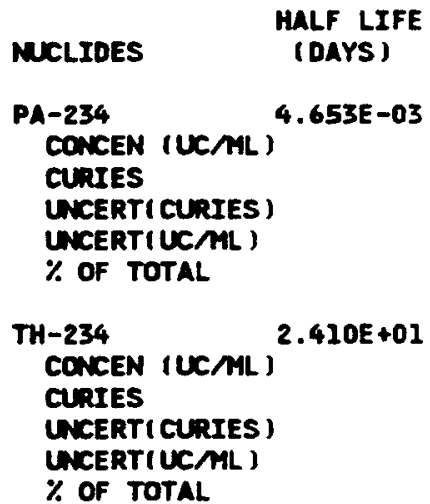

$U-234$

8. $943 E+07$

CONCEN (UCML) CURIES UNCERTI CURIES UNCERTIUCAL $\%$ OF TOTAL

$1.631 E+12$

CONCEN (UCAML)

CURIES

1.631E+12

$$
\mathbf{3 . 4 6 3}
$$$$
\begin{array}{lllll}
3.463 E-16 & 2.162 E-16 & 3.879 E-16 & 2.162 E-16 & 2.162 E-16 \\
8.000 E-09 & 4.000 E-09 & 9.000 E-09 & 4.000 E-09 & 4.000 E-09
\end{array}
$$
UNCERTI CURIES UNCERT ( UCALI ) $\%$ OF TOTAL

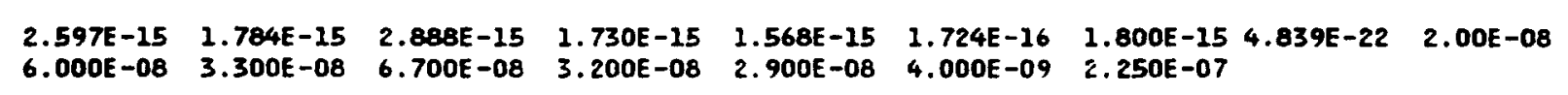

$$
\begin{array}{lllllll}
31.915 & 32.039 & 31.905 & 32.000 & 31.868 & 33.333 & 31.960
\end{array}
$$$$
\begin{array}{lllllllll}
2.597 E-15 & 1.784 E-15 & 2.888 E-15 & 1.730 E-15 & 1.568 E-15 & 1.724 E-16 & 1.800 E-15 & 4.839 E-22 & 4.00 E-10
\end{array}
$$

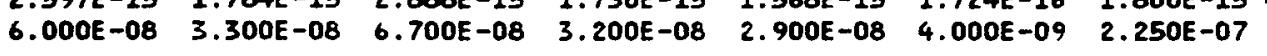
32.039
31.905
32.000
31.868
33.333
31.960

$2.320 E-16 \quad 6.237 E-23 \quad 9.00 E-14$ 2. $900 E-08$
4.255
3.883
4.286
4.000
4.396
4.119 $\begin{array}{lllllllll}2.597 E-15 & 1.784 E-15 & 2.888 E-15 & 1.730 E-15 & 1.568 E-15 & 1.724 E-16 & 1.800 E-15 & 4.839 E-22 & 1.00 E-13 \\ 6.000 E-08 & 3.300 E-08 & 6.700 E-08 & 3.200 E-08 & 2.900 E-08 & 4.000 E-09 & 2.250 E-07 & & \end{array}$
$31.915 \quad 32.039$
31.905
32.000
31.868
33.333

\begin{tabular}{|c|c|c|c|c|c|c|c|}
\hline $\begin{array}{l}\text { OTALS } \\
\text { ALPHA CURIES } \\
\% \text { OF TOTAL } \\
\text { B+G CURIES } \\
\% \text { OF TOTAL }\end{array}$ & $\begin{array}{r}6.800 E-08 \\
36.170 \\
1.200 E-07 \\
63.830\end{array}$ & $\begin{array}{r}3.700 E-08 \\
35.922 \\
6.600 E-08 \\
64.078\end{array}$ & $\begin{array}{r}7.600 E-08 \\
36.190 \\
1.340 E-07 \\
63.810\end{array}$ & $\begin{array}{r}3.600 E-08 \\
36.000 \\
6.400 E-08 \\
64.000\end{array}$ & $\begin{array}{r}3.300 E-08 \\
36.264 \\
5.800 E-08 \\
63.736\end{array}$ & $\begin{array}{r}4.000 E-09 \\
33.333 \\
8.000 E-09 \\
66.667\end{array}$ & $\begin{array}{r}2.540 E-07 \\
36.080 \\
4.500 E-07 \\
63.920\end{array}$ \\
\hline VOLUNE (CU. METERS) & $2.310 E+07$ & $1.850 E+07$ & $2.320 E+07$ & $1.850 E+07$ & $1.850 E+07$ & $2.320 E+07$ & $1.250 E+08$ \\
\hline and & $.880 E-07$ & $30 E-07$ & $100 E-07$ & $.000 E-07$ & $9.100 E-08$ & $1.200 E-08$ & $.040 E-07$ \\
\hline
\end{tabular}
31.960

* This muber is derived from a dispersion factor equetion. The dispersion factor is derived from the straight line goussien model and is proveded by the National Oceanic Atmospheric Adhinistration (MOAA).

* NOTE: DCG value obtainod from DOE ORDER 5400.5 doted fobruary 8, 1990, figures III-1 and III-3 
MOF PROD FLOOR AIPBORNE WASTE SUMMARY

FOR JAN THROUGH JUN 1993

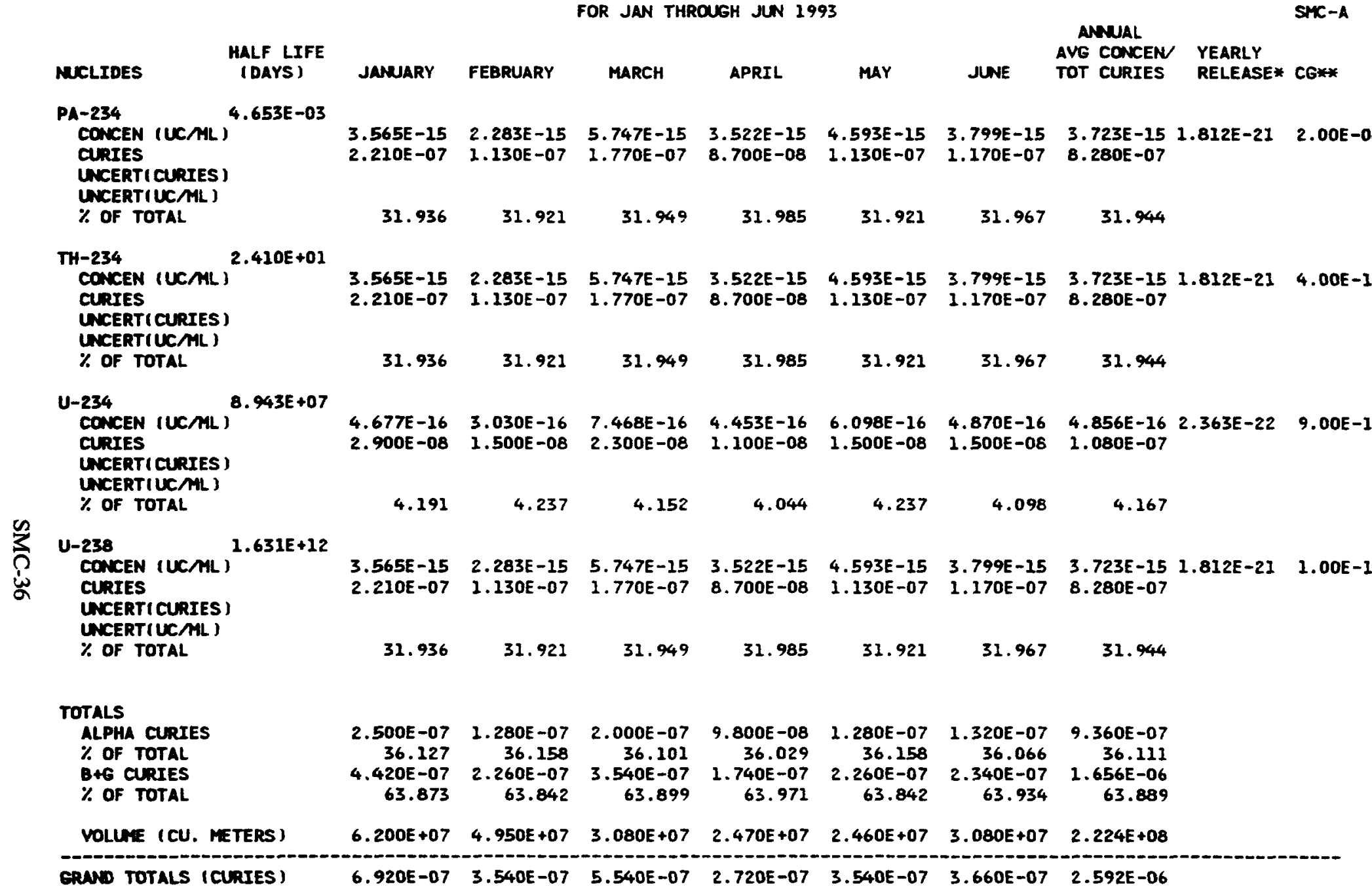

* This nuber is derived from a dispersion factor eqution. The dispersion factor is derived from the straight line gaussian model and is proveded by the Nbtional Oceanic Atmospheric Adninistration (NOAA).

** NOTE: DCG velue abtained from DOE ORDER 5400.5 dated February 8, 1990, Figures III-I and III-3 
MDF EC LAB AIRBOPNE MASTE SUMMARY

FOR JAN THROUGH JUN 1993

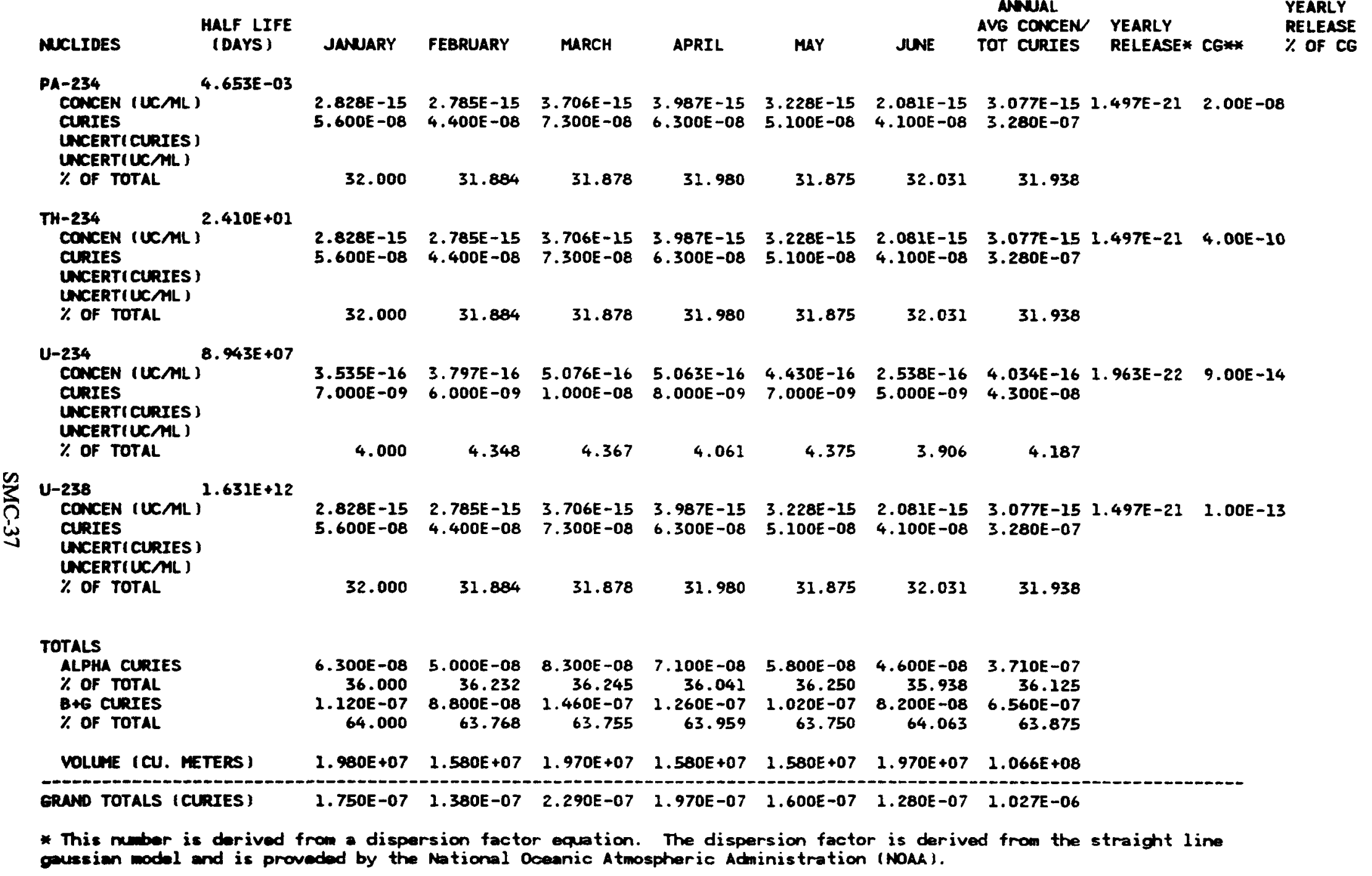

* NOTE: DCG value obtainad from DOE ORDER 5400.5 dated Fabmary 8, 1990 , Figures III-1 and III-3 
RADIOACTIVE WASTE MANAGEMENT INFORMATION SYSTEM

N. PROCESS S9 AIRBORNE MASTE SUMARY FOR JAN THROUGH JUN 1993

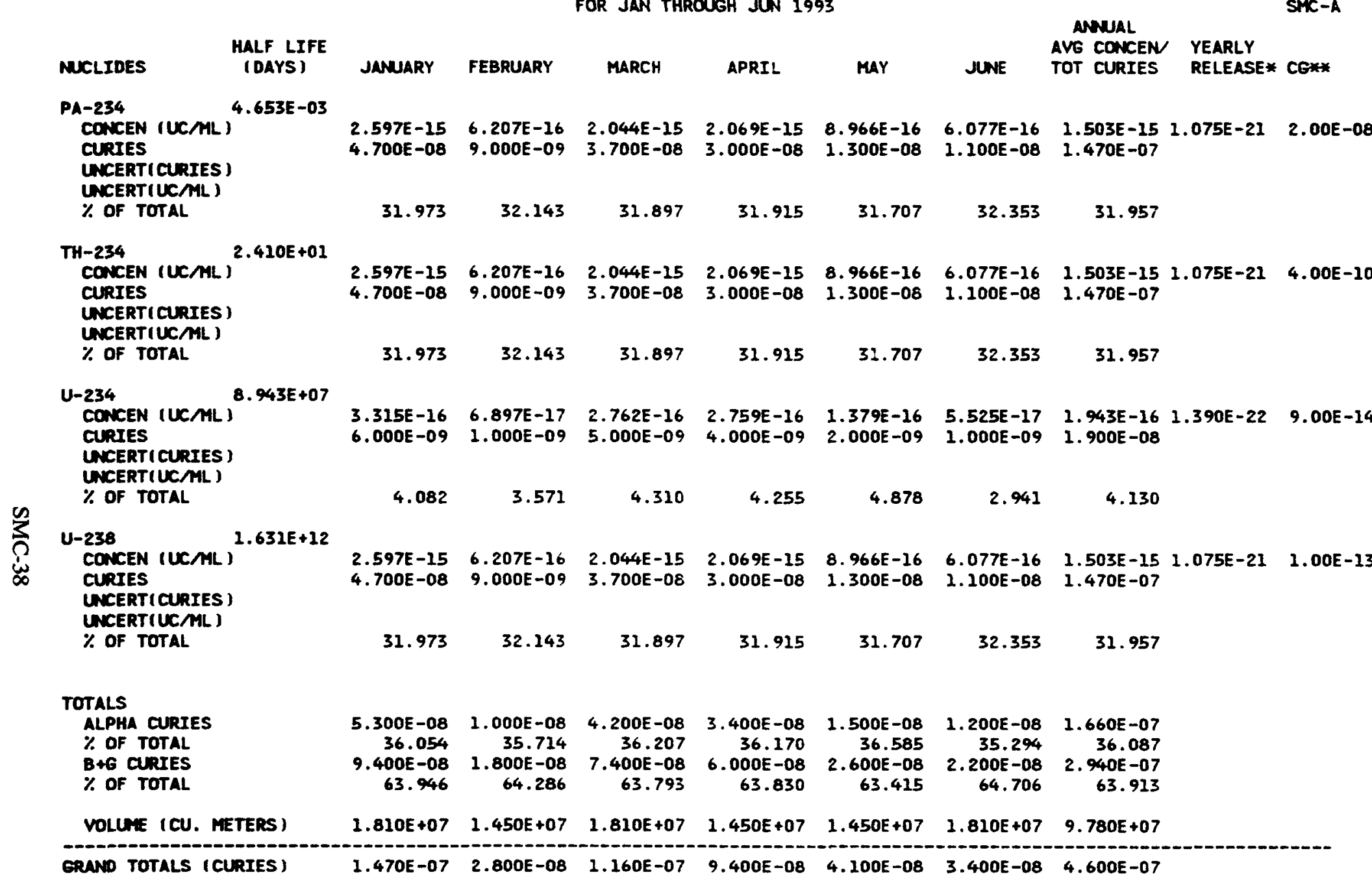

* This nuber is derived from a dispersion factor equetion. The dispersion factor is derived from the straight line omssian model and is proveded by the National Dceanic Atmospheric Adninistration (NOAA).

* NOTE: DCG value obtained from DOE ORDER 5400.5 dated February 8, 1990, Figures III-I and III-3 
N. PROCESS S10 AIRBORNE MASTE SUMMARY FOR JAN THROUGH JUN 1993

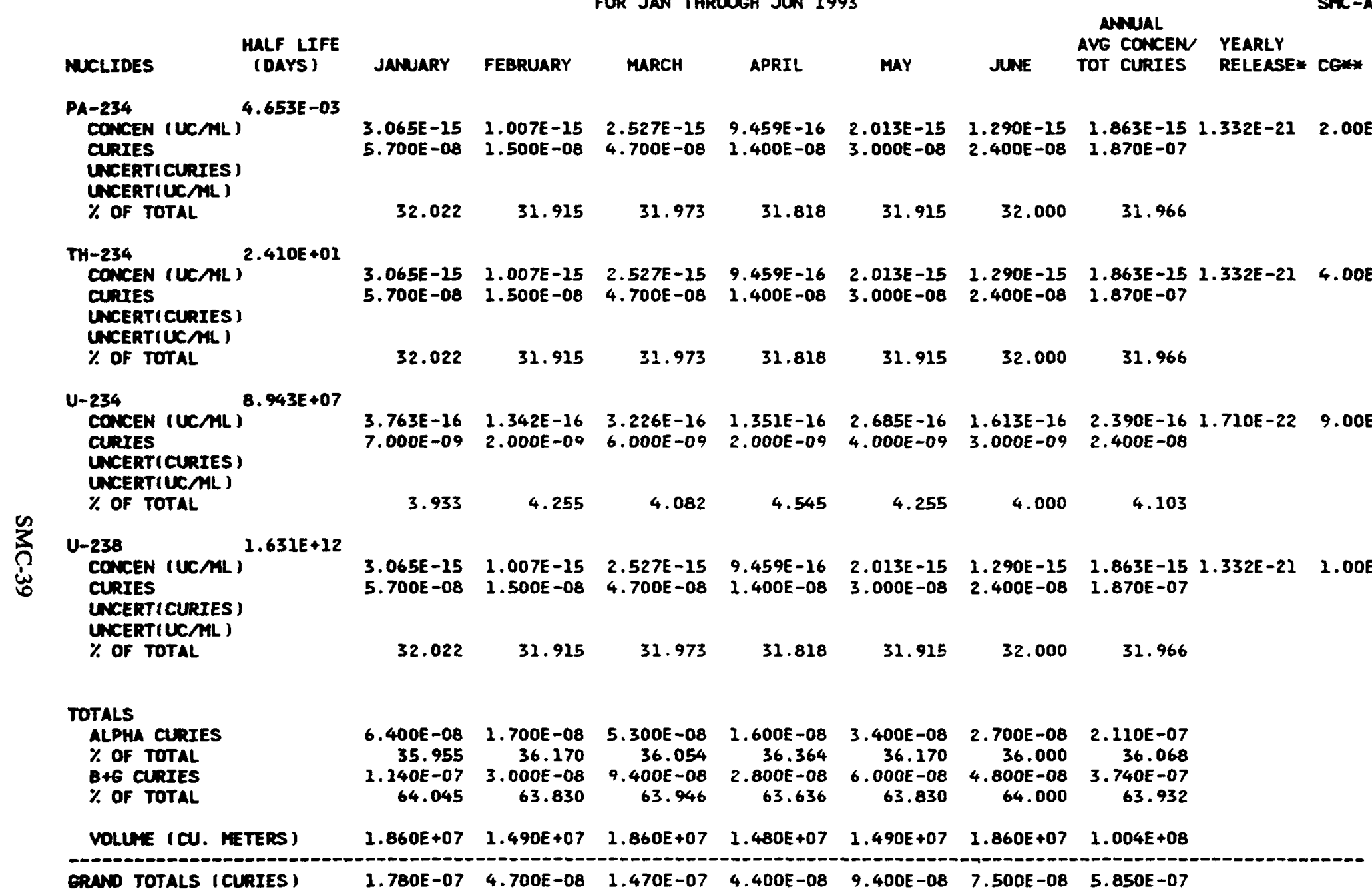

* This mubor is derived from a dispersion factor equation. The dispersion factor is derived from the straight line goussian wodel and is proveded by the National Oceanic Atmospharic Adninistration (NOAA).

* NOTE: DCG value obtained from DOE ORDER 5400.5 dated february 8, 1990, Figures III-1 and III-3 
RADIOACTIVE WASTE MANAGEMENT INFORMATION SYSTEM

N. PROCESS SII AIRBORNE WASTE SUMYARY FOR JAN THROUGH JUN 1993

MUCLIDES HALF LIFE
(DAYS)
PA-234
CONCEN (UCML)
CURIES
UNCERT(CURIES)
UNCERTIUCML)
\% OF TOTAL
TH-234
CONCEN (UCML)
CURIES
UNCERT(CURIES)
UNCERT(UCAML)
\% OF TOTAL

$\mathrm{u}-234$

8. $943 E+07$

CONCEN (LCML) CURIES

UNCERT (CURIES)

UNCERT (UCAML

$\%$ OF TOTAL

U-238

CURIES

$1.631 E+12$

UNERT ( CURIES )

UNCERTIUCML)

$\%$ OF TOTAL

JANARY FEBRUARY

MARCH

MAY

JUNE

$\begin{array}{lllll}1.399 E-15 & 2.143 E-15 & 1.784 E-15 & 2.703 E-16 & 1.149 E-15\end{array}$ 2.700E-08 $3.300 E-08 \quad 3.300 E-08 \quad 4.000 E-09 \quad 1.700 E-08 \quad 1.200 E-08$

\begin{tabular}{|c|c|c|c|}
\hline & & $\sin ^{1}-1$ & 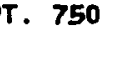 \\
\hline EEN & $\begin{array}{l}\text { YEARLY } \\
\text { RELEASE }\end{array}$ & & $\begin{array}{l}\text { YEARLY } \\
\text { RELEASE } \\
\% \text { OF CG }\end{array}$ \\
\hline
\end{tabular}

$\begin{array}{lllllllll}1.399 E-15 & 2.143 E-15 & 1.784 E-15 & 2.703 E-16 & 1.149 E-15 & 6.486 E-16 & 1.244 E-15 & 8.897 E-22 & 4.00 E-10\end{array}$ $\begin{array}{lllllll}2.700 E-08 & 3.300 E-08 & 3.300 E-08 & 4.000 E-09 & 1.700 E-08 & 1.200 E-08 & 1.260 E-07\end{array}$

$\begin{array}{lllllll}31.765 & 32.039 & 32.039 & 30.769 & 32.075 & 31.579 & 31.899\end{array}$

$\begin{array}{lllllllll}2.073 E-16 & 2.597 E-16 & 2.162 E-16 & 6.757 E-17 & 1.351 E-16 & 1.081 E-16 & 1.678 E-16 & 1.200 E-22 & 9.00 E-14\end{array}$ $4.000 E-09 \quad 4.000 E-09 \quad 4.000 E-09 \quad 1.000 E-09 \quad 2.000 E-09 \quad 2.000 E-09 \quad 1.700 E-08$

$\begin{array}{lllllll}4.706 & 3.883 & 3.883 & 7.692 & 3.774 & 5.263 & 4.304\end{array}$

$\begin{array}{lllllllll}1.399 E-15 & 2.143 E-15 & 1.784 E-15 & 2.703 E-16 & 1.149 E-15 & 6.486 E-16 & 1.244 E-15 & 8.897 E-22 & 1.00 E-13\end{array}$ $\begin{array}{llllllll}2.700 E-08 & 3.300 E-08 & 3.300 E-08 & 4.000 E-09 & 1.700 E-08 & 1.200 E-08 & 1.260 E-07\end{array}$
31.765
32.039
32.039
$30.769 \quad 32.075$
31.579
31.899

\section{TOTALS}

\begin{tabular}{|c|c|c|c|c|c|c|c|}
\hline $\begin{array}{l}\text { ALPHA CURIES } \\
\% \text { OF TOTAL } \\
\text { B+6 CURIES } \\
\% \text { OF TOTAL }\end{array}$ & $\begin{array}{r}3.100 E-08 \\
36.471 \\
5.400 E-08 \\
63.529\end{array}$ & $\begin{array}{r}3.700 E-08 \\
35.922 \\
6.600 E-08 \\
64.078\end{array}$ & $\begin{array}{r}3.700 E-08 \\
35.922 \\
6.600 E-08 \\
64.078\end{array}$ & $\begin{array}{r}5.000 E-09 \\
38.462 \\
8.000 E-09 \\
61.538\end{array}$ & $\begin{array}{r}1.900 E-08 \\
35.849 \\
3.400 E-08 \\
64.251\end{array}$ & $\begin{array}{r}1.400 E-08 \\
36.842 \\
2.400 E-08 \\
63.158\end{array}$ & $\begin{array}{r}1.430 E-07 \\
36.202 \\
2.520 E-07 \\
63.797\end{array}$ \\
\hline VOLLAE (CU. METERS) & $1.930 E+07$ & $1.540 E+07$ & $1.850 E+07$ & $1.480 E+07$ & $1.480 E+07$ & $1.850 E+07$ & $1.013 E+08$ \\
\hline RAND TOTA & $8.500 E-08$ & $1.030 E-07$ & $1.030 E-07$ & $1.300 E-08$ & $5.300 E-08$ & $3.800 E-08$ & $3.950 E-07$ \\
\hline
\end{tabular}

* This nuber is derived from a dispersion factor equation. The dispersion factor is derived from the straight lime ocussian model and is proveded by the National Oceanic Atmospheric Administration (NOAA).

* NOTE: DCG value obtained from DOE ORDER 5400.5 dated Februery 8, 1990 , Figures III-1 and III-3 
PRF STACK S14 AIRBORNE MASTE SUMMARY FOR JAN THROUGH JUN 1993

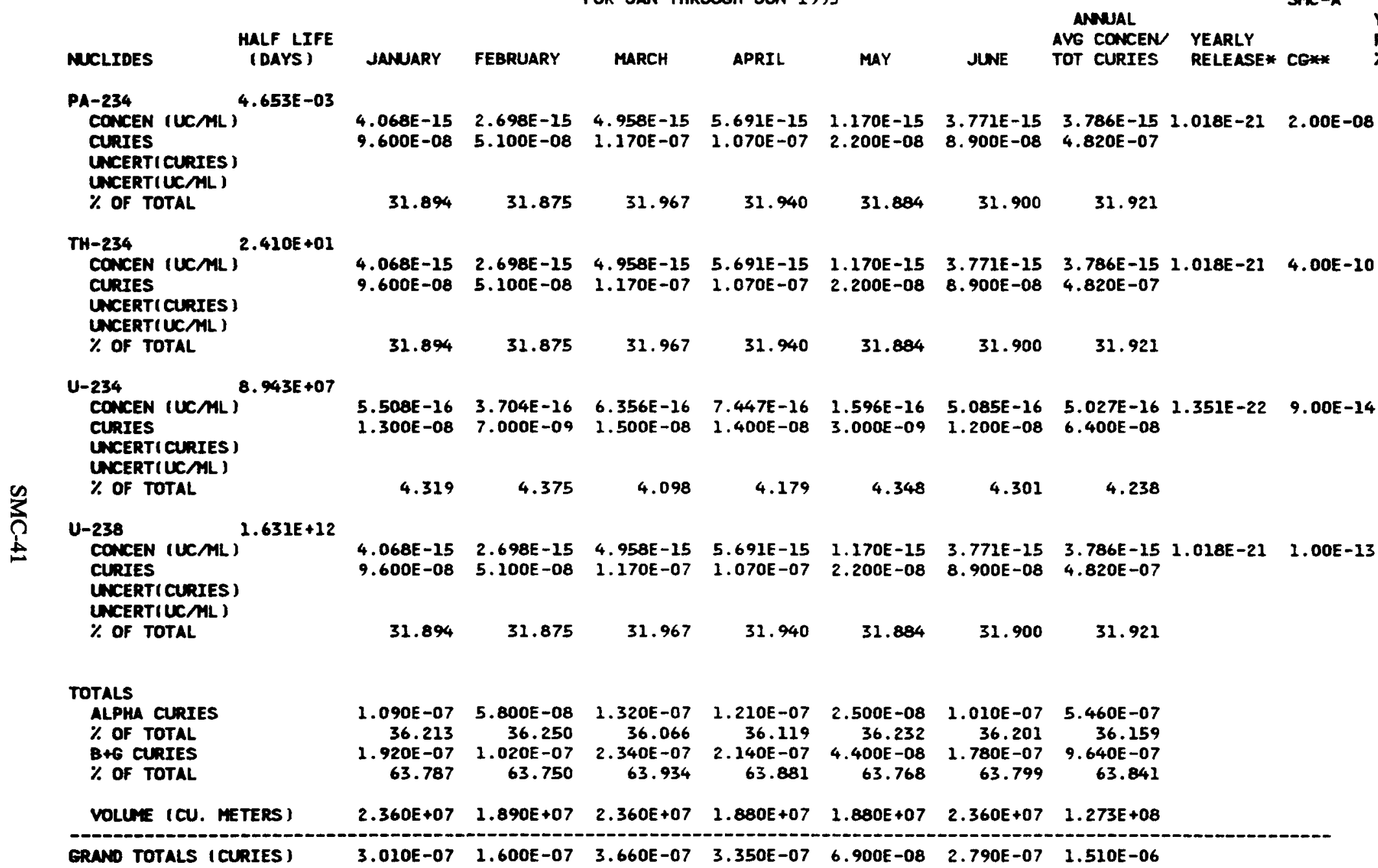

* This number is derived from a dispersion factor equation. The dispersion factor is derived from the straight line omssian model and is provedod by the National Oceanic Atmospheric Administration (NOAA).

** NOTE: DCG value obtained from DOE ORDER 5400.5 doted February 8, 1990, Figures III-1 and III-3 
RADIOACTIVE WASTE MANAGEMENT INFORMATION SYSTEM

SCRAP HANDLING AIRBORNE WASTE SUMTARY FOR JAN THROUGH JUN 1993

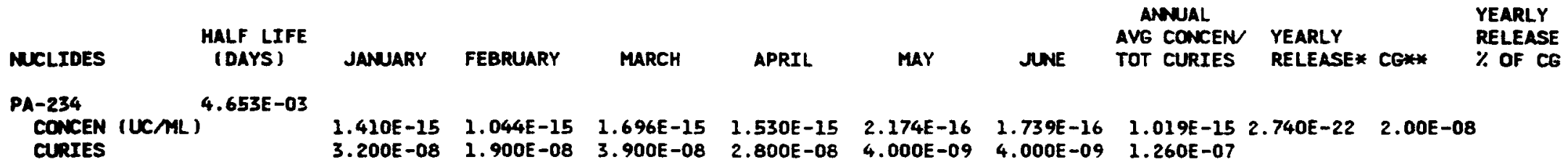

$3.200 E-08 \quad 1.900 E-08 \quad 3.900 E-08 \quad 2.800 E-08$

$32.000 \quad 32.203$

31.967

31.818

33.333

30.769

31.980

TH-234

$2.410 E+01$

CONCEN (UCAL)

CURIES

$\begin{array}{lllllllll}1.410 E-15 & 1.044 E-15 & 1.696 E-15 & 1.530 E-15 & 2.174 E-16 & 1.739 E-16 & 1.019 E-15 & 2.740 E-22 & 4.00 E-10 \\ 3.200 E-08 & 1.900 E-08 & 3.900 E-08 & 2.800 E-08 & 4.000 E-09 & 4.000 E-09 & 1.260 E-07 & & \end{array}$

UNCERT (CURIES

UNCERT (UCML)

$\%$ OF TOTAL

$32.000 \quad 32.203$

31.967

31.818

33.333

30.769

31.980

U-234 $8.943 E+07$

CONCEN (UCAL)

CURIES

$1.762 E-16 \quad 1.099 E-16 \quad 2.174 E-16 \quad 2.186 E-16$

4.000E-09 2.000E-09 5.000E-09 4.000E-09

$4.348 E-17 \quad 1.294 E-16 \quad 3.480 E-23 \quad 9.00 E-14$ UNCERT ( CURIES ) UNCERT (UCAL) $\%$ OF TOTAL

$1.631 E+12$

$U-238$

CONCEN (UCML)

\section{CURIES}

UNCERTICURIES

UNCERT (UCML)

$\%$ Of TOTAL

.000

3.390

4.098

4.545

$1.000 E-09 \quad 1.600 E-08$

$\begin{array}{lllllllll}1.410 E-15 & 1.044 E-15 & 1.696 E-15 & 1.530 E-15 & 2.174 E-16 & 1.739 E-16 & 1.019 E-15 & 2.740 E-22 & 1.00 E-13\end{array}$ $\begin{array}{lllllll}3.200 E-08 & 1.900 E-08 & 3.900 E-08 & 2.800 E-08 & 4.000 E-09 & 4.000 E-09 & 1.260 E-07\end{array}$
32.000
32.203
31.967
31.818
33.333
30.769
31.980

\section{TOTALS}

$\begin{array}{lrrrrrrr}\text { ALPHA CURIES } & 3.600 E-08 & 2.100 E-08 & 4.400 E-08 & 3.200 E-08 & 4.000 E-09 & 5.000 E-09 & 1.420 E-07 \\ \text { \% OF TOTAL } & 36.000 & 35.593 & 36.066 & 36.364 & 33.333 & 38.462 & 36.041 \\ \text { B+6 CURTES } & 6.400 E-08 & 3.800 E-08 & 7.800 E-08 & 5.600 E-08 & 8.000 E-09 & 8.000 E-09 & 2.520 E-07\end{array}$

B+6 CURIES

$6.400 E-08$

$800 E-08$

7.800E-08

$.000 E-09$

64.000

63.934

63.636

66.667

61.538

63.959

VOLLNE (CU. METERS)

GRAN TOTALS (CURIES)

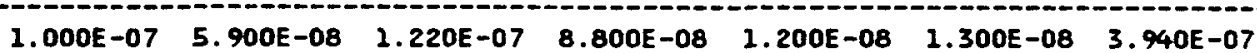

* This number is derived from a dispersion factor equation. The dispersion factor is derived from the straight line seussian model and is proveded by the tional Oceanic Atmospheric Administration (NOAA).

* NOTE: DCG value obtained from DOE ORDER 5400.5 dated Febreary 8, 1990, Figures III-1 and III-3 
RADIOACTIVE WASTE MANAGEMENT INFORMATION SYSTEM

S. PROCESS S6 AIRBORNE WASTE SUMMARY FOR JAN THROUGH JUN 1993

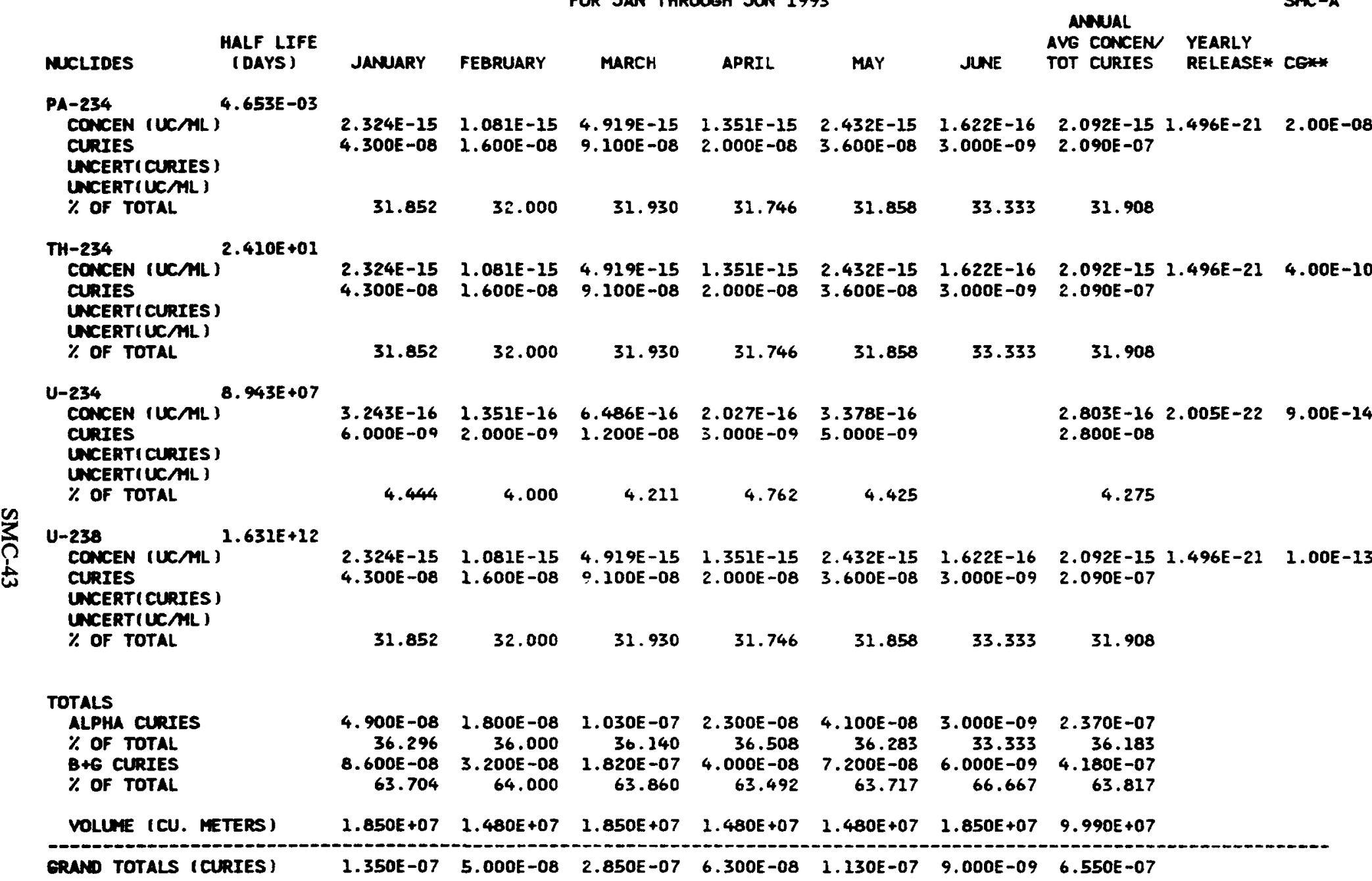

* This nuber is derived from a dispersion factor equation. The dispersion factor is derived from the straight line oussian model and is provedad by the National Oceanic Atmospheric Administration (NOMA).

* NOTE: DCG value obtainad from DOE ORDER 5400.5 dated Fabruary 8, 1990 , Figures III-1 and III-3 
S. PROCESS S7 AIRBORNE WASTE SUMMARY FOR JAN THROUGH JUN 1993

\begin{tabular}{|c|c|c|c|c|c|c|c|c|c|}
\hline $\begin{array}{l}\text { HALF LIFE } \\
\text { (DAYS) }\end{array}$ & JANUARY & FEBRUARY & MARCH & APRIL & MAY & JUNE & $\begin{array}{l}\text { ANNUAL } \\
\text { AVG CONCEN } \\
\text { TOT CURIES }\end{array}$ & $\begin{array}{l}\text { YEARLY } \\
\text { RELEASE* }\end{array}$ & $\cos *$ \\
\hline $\begin{array}{l}\text { PA-234 }{ }^{4.653 E-03} \\
\text { CONCEN (UCML) } \\
\text { CURIES } \\
\text { UNCERTICURIES) } \\
\text { UNCERT(UCAML) } \\
\% \text { OF TOTAL }\end{array}$ & $\begin{array}{l}3.405 E-15 \\
6.300 E-08\end{array}$ & $\begin{array}{l}1.081 E-15 \\
1.600 E-08\end{array}$ & $\begin{array}{l}2.865 E-15 \\
5.300 E-08\end{array}$ & $\begin{array}{l}1.014 E-15 \\
1.500 E-08\end{array}$ & $\begin{array}{l}1.824 E-15 \\
2.700 E-08\end{array}$ & $\begin{array}{l}2.703 E-16 \\
5.000 E-09\end{array}$ & $\begin{array}{l}1.792 E-15 \\
1.790 E-07\end{array}$ & $1.282 E-21$ & $2.00 E-08$ \\
\hline $\begin{array}{l}\text { TH-234 } \\
\text { CONCEN (UC/ML) } \\
\text { CURIES } \\
\text { UNCERT(CURIES) } \\
\text { UNCERT(UCAML) } \\
\text { \% OF TOTAL }\end{array}$ & $\begin{array}{l}3.405 E-15 \\
6.300 E-08\end{array}$ & $\begin{array}{l}1.081 E-15 \\
1.600 E-08\end{array}$ & $\begin{array}{l}2.865 E-15 \\
5.300 E-08\end{array}$ & $\begin{array}{l}1.014 E-15 \\
1.500 E-08\end{array}$ & $\begin{array}{l}2.824 E-15 \\
2.700 E-08\end{array}$ & $\begin{array}{r}2.703 E-16 \\
5.000 E-09 \\
31.250\end{array}$ & $\begin{array}{l}1.792 E-15 \\
1.790 E-07\end{array}$ & $1.282 E-21$ & $4.00 E-10$ \\
\hline $\begin{array}{l}\text { U-234 } \\
\text { CONCEN (UCML) } \\
\text { CURIES } \\
\text { UNCERT(CURIES) } \\
\text { UNCERT(UCML) } \\
\% \text { OF TOTAL }\end{array}$ & $\begin{array}{l}4.324 E-16 \\
\text { B. } 000 E-09\end{array}$ & $\begin{array}{l}1.351 E-16 \\
2.000 E-09\end{array}$ & $\begin{array}{l}3.784 E-16 \\
7.000 E-09\end{array}$ & $\begin{array}{l}1.351 E-16 \\
2.000 E-09\end{array}$ & $\begin{array}{l}2.027 E-16 \\
3.000 E-09\end{array}$ & $\begin{array}{r}5.405 E-17 \\
1.000 E-09 \\
6.250\end{array}$ & $\begin{array}{r}2.302 E-16 \\
2.300 E-08 \\
4.107\end{array}$ & $1.647 E-22$ & $9.00 E-14$ \\
\hline $\begin{array}{l}\text { U-238 } \\
\text { CONCEN (UCML) } \\
\text { CURIES } \\
\text { UNCERTICURIES) } \\
\text { UNCERTIUCML) } \\
\text { \% OF TOTAL }\end{array}$ & $\begin{array}{l}3.405 E-15 \\
6.300 E-08\end{array}$ & $\begin{array}{l}1.081 E-15 \\
1.600 E-08\end{array}$ & $\begin{array}{l}2.865 E-15 \\
5.300 E-08\end{array}$ & $\begin{array}{l}1.014 E-15 \\
1.500 E-08\end{array}$ & $\begin{array}{l}1.824 E-15 \\
2.700 E-08\end{array}$ & $\begin{array}{l}2.703 E-16 \\
5.000 E-09\end{array}$ & $\begin{array}{l}1.792 E-15 \\
1.790 E-07\end{array}$ & $1.282 E-21$ & $1.00 E-13$ \\
\hline $\begin{array}{l}\text { TOTALS } \\
\text { ALPHA CURIES } \\
\% \text { OF TOTAL } \\
\text { B+G CURIES } \\
\% \text { OF TOTAL }\end{array}$ & $\begin{array}{r}7.100 E-08 \\
36.041 \\
1.260 E-07 \\
63.959\end{array}$ & $\begin{array}{r}1.800 E-08 \\
36.000 \\
3.200 E-08 \\
64.000\end{array}$ & $\begin{array}{r}6.000 E-08 \\
36.145 \\
1.060 E-07 \\
63.855\end{array}$ & $\begin{array}{r}1.700 E-08 \\
36.170 \\
3.000 E-08 \\
63.830\end{array}$ & $\begin{array}{r}3.000 E-08 \\
35.714 \\
5.400 E-08 \\
64.286\end{array}$ & $\begin{array}{r}6.000 E-09 \\
37.500 \\
1.000 E-08 \\
62.500\end{array}$ & $\begin{array}{r}2.020 E-07 \\
36.071 \\
3.580 E-07 \\
63.929\end{array}$ & & \\
\hline VOLUNE (CU. METERS) & $1.850 E+07$ & $1.480 E+07$ & $2.850 E+07$ & $1.480 E+07$ & $1.480 E+07$ & $1.850 E+07$ & $9.990 E+07$ & & \\
\hline
\end{tabular}

* This nuber is derived from a dispersion factor equation. The dispersion factor is derived from the straight line omsesian model and is proveded by the National Dosanic Atmospheric Administration (NOAA).

** NOTE: DCG value obtained from DOE ORDER 5400.5 dated February 8, 1990, Figures III-1 and III-3 
S. PROCESS SG AIRBORNE WASTE SUMARY FOR JAN THROUGH JUN 1993

NUCLIDES MALF LIFE
(DAYS)

\section{Jar} 2.48

$\begin{array}{ll}2.486 E-15 & 3.716 E-15 \\ 4.600 E-08 & 5.500 E-D 8\end{array}$

MARCH APRIL MAY JUNE

\begin{tabular}{|c|c|c|c|}
\hline & PAGE & $\sin ^{2}-1$ & T. 747 \\
\hline $\begin{array}{l}\text { ANNAL } \\
\text { AVG CONCEN }\end{array}$ & $\begin{array}{l}\text { YEARLY } \\
\text { RELEASE* }\end{array}$ & 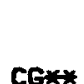 & $\begin{array}{l}\text { YEARLY } \\
\text { RELEASE }\end{array}$ \\
\hline
\end{tabular}

$$
\begin{array}{lllllll}
31.944 & 31.977 & 31.915 & 32.353 & 31.707 & 32.075 & 31.956
\end{array}
$$

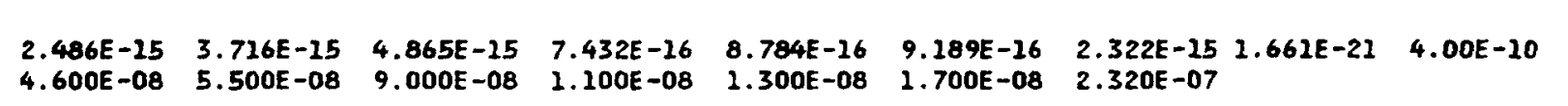
31.944
31.977
$31.915 \quad 32.353$
31.707
32.075
31.956

U-234 8. $943 E+07$

CONCEN (UCML) CURIES

$\begin{array}{lllllllll}3.243 E-16 & 4.730 E-16 & 6.486 E-16 & 6.757 E-17 & 1.351 E-16 & 1.081 E-16 & 3.003 E-16 & 2.248 E-22 & 9.00 E-14\end{array}$ UNCERTICURIES)

UNCERT(UCAL)

$\%$ OF TOTAL

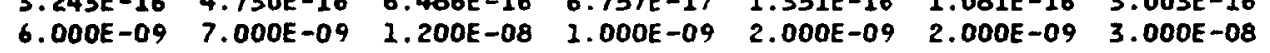
4.167
4.070
4.255
2.941
4.878
3.774
4.132
$\overbrace{2}^{u-238}$ CONEEN (UCML)
CURIES
$1.631 E+12$
(NCERT (CURIES)
UNCERT (UCANI)
$\%$ Of TOTAL

$\begin{array}{llllllllll}2.486 E-15 & 3.716 E-15 & 4.865 E-15 & 7.432 E-16 & 8.784 E-16 & 9.189 E-16 & 2.322 E-15 & 1.661 E-21 & 1.00 E-13\end{array}$ $\begin{array}{lllllll}4.600 E-08 & 5.500 E-08 & 9.000 E-08 & 1.100 E-08 & 1.300 E-08 & 1.700 E-08 & 2.320 E-07\end{array}$

$$
\begin{array}{lllllll}
31.944 & 31.977 & 31.915 & 32.353 & 31.707 & 32.075 & 31.956
\end{array}
$$

\begin{tabular}{|c|c|c|c|c|c|c|c|}
\hline $\begin{array}{l}\text { ALPHA CURIES } \\
\% \text { OF TOTAL } \\
\text { B+G CURIES } \\
\% \text { OF TOTAL }\end{array}$ & $\begin{array}{r}5.200 E-08 \\
36.121 \\
9.200 E-08 \\
63.889\end{array}$ & $\begin{array}{r}6.200 E-08 \\
36.047 \\
1.100 E-07 \\
63.953\end{array}$ & $\begin{array}{r}1.020 E-07 \\
36.170 \\
1.800 E-07 \\
63.830\end{array}$ & $\begin{array}{r}1.200 E-08 \\
35.294 \\
2.200 E-08 \\
64.706\end{array}$ & $\begin{array}{r}1.500 E-08 \\
36.585 \\
2.600 E-08 \\
63.415\end{array}$ & $\begin{array}{r}1.900 E-08 \\
35.849 \\
3.400 E-08 \\
64.151\end{array}$ & $\begin{array}{r}2.620 E-07 \\
36.088 \\
4.640 E-07 \\
63.912\end{array}$ \\
\hline & $n E$ \& & 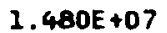 & $50 \mathrm{~F}+\mathrm{n}$ & anf tr & $E+$ & DEt & 9905 \\
\hline
\end{tabular}

\section{TOTALS}

$\begin{array}{llllllll}\text { VOLUE (CU. METERS) } & 1.850 E+07 & 1.480 E+07 & 1.850 E+07 & 1.480 E+07 & 1.480 E+07 & 1.850 E+07 & 9.990 E+07\end{array}$

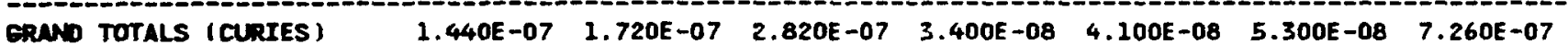

* This nuber is darived from a dispersion factor equation. The dispersion factor is derived from the straight line gausian model and is proveded by the National Oceanic Atmospheric Adninistration (NOMA).

* NOTE: DCG value obtained from DOE ORDER 5400.5 dated Fabruary 8, 1990 , Figures III-I and III-3 
RADIOACTIVE WASTE MANAGEMENT INFORMATION SYSTEM

2B AREA PHASEI AIRBORNE WLSTE SUMARA FOR JAN THROUGH JUN 1993

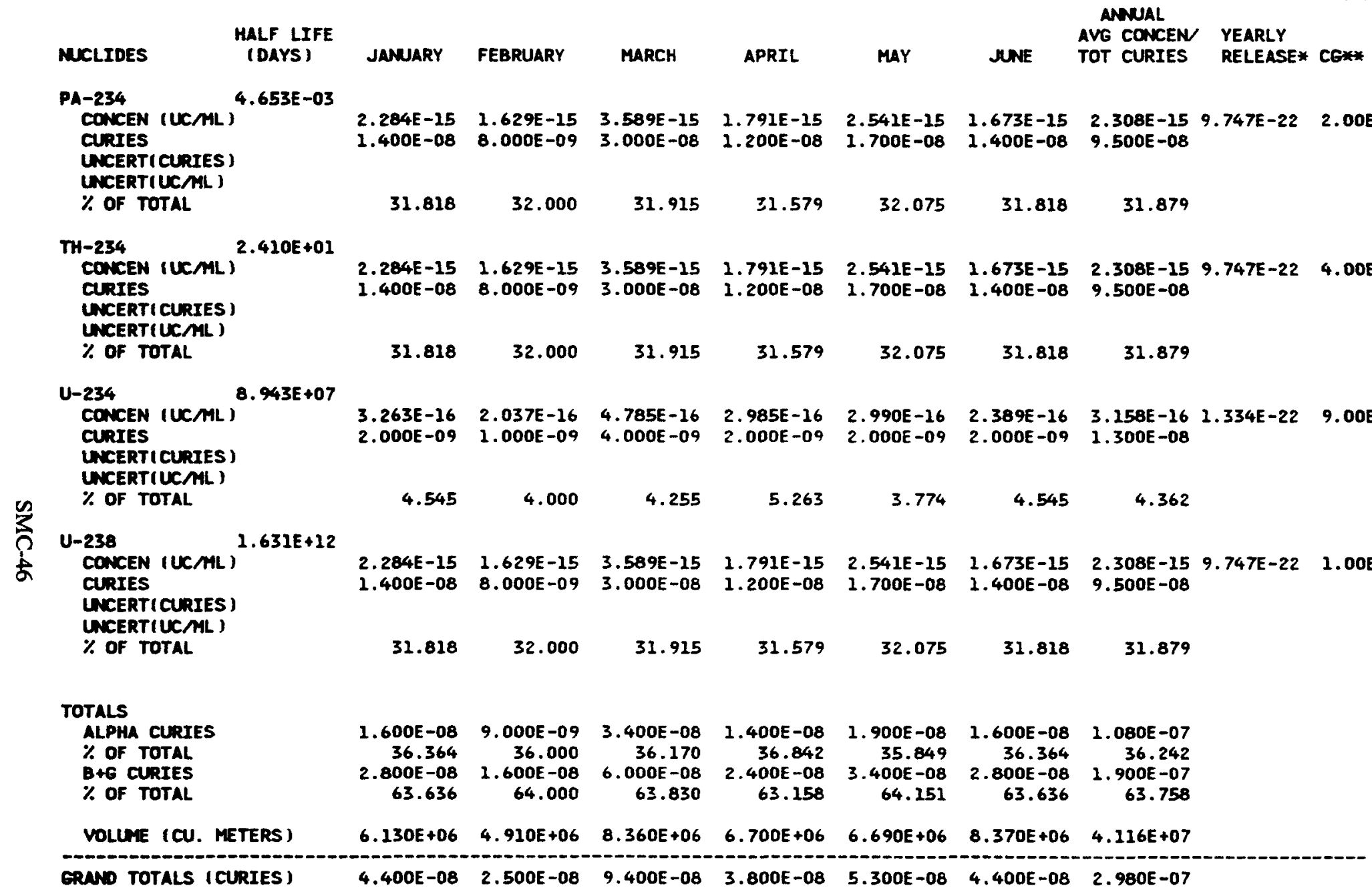

* This number is chrived from a dispersion factor equation. The dispersion factor is derived from the straight line goussien model and is proveded by the National Doennic Atmospheric Administration (NOAA).

* NOTE: DCG value obtained from DOE ORDER 5400.5 dated Fabruary 8, 1990, Figures III-1 and III-3 
Special Power Excursion Reactor 1993 Detail Data

Special Power Excursion Reactor Test (PER) Bar Graph of Annual Data by Month PER-3

CY1993 Bar Graph PER-5

PER Detail Data for Months July to December

Annual Average Concentration and Total Curies for January to December PER-7

Report 170-PER Solid Waste Summary, WERF Incinerator Processed Waste ............... PER-9 Report 168-PER Solid Waste Summary, Waste Sent to WERF for Compaction .............. PER-10 Report 169-PER Solid Waste Summary, Waste Sent to WERF for Metallic Processing ........... PER-11

PER Detail Data for Months January to June

Annual Average Concentration and Total Curies for January to June

PER-13

Report 170-PER Solid Waste Summary,

WERF Incinerator Processed Waste

PER-15

Report 168-PER Solid Waste Summary,

Waste Sent to WERF for Compaction

PER-16

Report 169_PER Solid Waste Summary,

Waste Sent to WERF for Metallic Processing

PER-17 




\section{Special Power Excursion Reactor Test (PER) Monthly Details - CY 1993}

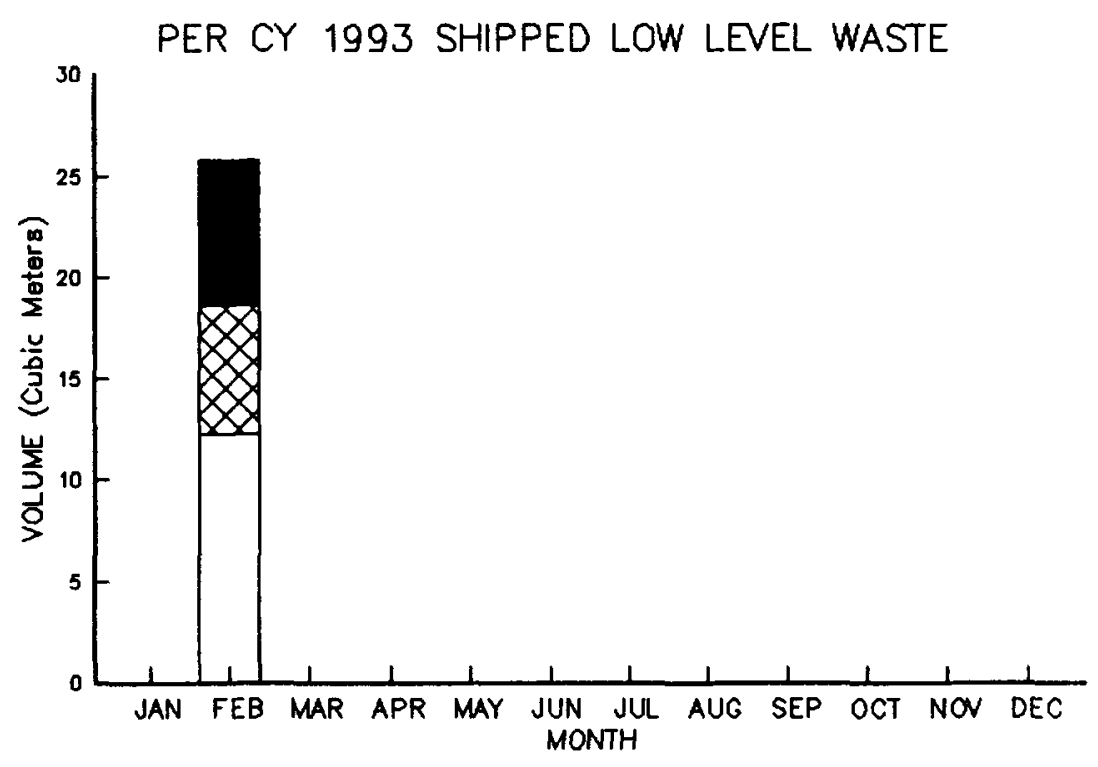

口INCINERATION $\square$ METAL SIZING $\square$ COMPACTION $\square$ DIRECT DISPOSAL 

PER Detail Data for Months July to December Annual Average Concentration and Total Curies for January to December 

SCHED. ND. P6IPMOOO-N

IDAHO OPERATIONS OFFICE

US DEPARTMENT OF

ADIOACTIVE WASTE MWNAGEMENT INFORMATION SYSTEM

PER SOLID NASTE SUMARY

WERF INCINERATOR PROCESSED WASTE FROM JAN THROUGH DEC 1993

MClides

HALF LIFE

JULY

1. $924 E+03$

CO-60

$\%$ OF TOTAL

CS-137

CURIES

$\%$ OF TOTAL

\section{TOTALS}

ALPHA CURIES

\% OF TOTAL

$B+G$ CURIES

$\%$ OF TOTAL

TOTAL ERNAS OF AM

TOTAL ERAMS OF PU

TOTAL ERAMS OF PU-239+241

TOTAL GRMYS OF $U$

TOTAL GRMS OF $U$

TOTAL ERMAS OF TH

TOTAL ERANS OF NP

VOLUME (CU. METERS
VEIGHT (KILOGRAMS)

SEPTEMBER OCTOBER NOVEMBER DECEMBER

ANNAL

TOTAL

$5.520 E-07$

30.296

$1.270 E-06$

69.704

$1.822 E-06$

100.000

RUN DATE: 05/11/94

PAge

1 RPT. 170

PER-S

$\pi$ (1)

$3.678 E+02$

1. 822E-06 
SCHED. NO. P6IPMO09-0

IDAHO OPERATIONS OFFICE

U.S. DEPARTMENT OF ENERGY

RADIOACTIVE MASTE MANAGEMENT INFORMATION SYSTEM

PER SOLID MASTE SUMYARY

WASTE SENT TO WERF FOR COMPACTION

HALF LIFE FROM JAN THROUGH DEC 1993

MUCLIDES (DAYS )

$1.924 E+03$

CO-60

CFr TOTAL

$\operatorname{cs}-134$

CURIES

$\%$ OF TOTAL

CS-137

CURIES

$\%$ OF TOTAL

EU-154

CURIES

$\%$ OF TOTAL

s8-125

CURIES

NLY

AUEUST

SEPTEMBER

OCTOBER

NOVEMER

DECEMBER

ANMAL

TOTAL

$2.140 E-04$

56.704

$7.537 E+02$

$1.101 E+04$

$3.103 E+03$

1.007E+03

TOTALS

ALPHA CURIES

$\%$ OF tOTAL

B+G CuRIES

T) OF TOTAL

D TOTAL GRAMS OF AM

TOTAL GRNAS OF PU

TOTAL ERAMS OF PU-239+241

TOTAL GRANS OF $U$

TOTAL ERNAS OF TH

TOTAL ERAMS OF NP

VOLUME (CU. METERS)

WEIGHT (KILOERANS)

$7.250 E+00$

.810E +02

3.774E-04

ERAND TOTALS (CURIES)

3.774E-04

$1.498 E-04$

$3.180 E-06$ 0.843

. $920 E-06$

2.364
RUN DATE: 05/11/94

PAGE 1 RPT. 168

PER-S 
SCHED. NO. P61PMO09-M

IDAHO OPERATIONS OFFICE

U.S. DEPARTIENT OF ENEREY

RUN DATE: $05 / 1 \overline{1 / 94}$

PER SOLID MUSTE SUMTARY

WASTE SENT TO WERF FOR METALLIC PROCESSING

\begin{tabular}{|c|c|}
\hline MCLIDES & $\begin{array}{l}\text { HALF LIFE } \\
\text { (DAYS) }\end{array}$ \\
\hline $\begin{array}{l}\text { CO-60 } \\
\text { CURIES } \\
\% \text { OF TOTAL }\end{array}$ & $1.924 E+03$ \\
\hline $\begin{array}{l}\text { CS-134 } \\
\text { CURIES } \\
\% \text { OF TOTAL }\end{array}$ & $7.537 E+02$ \\
\hline $\begin{array}{l}\text { CS }-137 \\
\text { CURIES } \\
\% \text { OF TOTAL }\end{array}$ & $1.101 E+04$ \\
\hline $\begin{array}{l}\text { EU-154 } \\
\text { CURIES } \\
\% \text { OF TOTAL }\end{array}$ & $3.103 E+03$ \\
\hline $\begin{array}{l}\text { S8-125 } \\
\text { CURIES } \\
\% \text { OF TOTAL }\end{array}$ & $1.007 E+03$ \\
\hline
\end{tabular}

FROM JAN THROUEH DEC 1993

TOTAL

TOTAL

$4.451 E-05$

52.832

$3.200 E-07$

0.368

3.693E-05

43.834

6.590E-07

0.782

$1.840 E-06$

2.184

8.425E-0.5 100.000

\section{TOTALS}

ALPHA CURIES

$\%$ OF TOTAL

in $\%$ OF TOTAL

D TOTAL GRAMS OF AM

TOTAL GRAMS OF PU

TOTAL GRAMS OF PU-239+241

TOTAL GRAMS OF U

TOTAL GRAMS OF TH

TOTAL GRAMS OF NP

VOLUME (CU. METERS )

WEIGHT (KILOGRANS)

$6.343 E+00$

GRAND TOTALS (CURIES) 


SCHED. NO. P61PMO09-N

IOAHO OPERATIONS OFFICE

U.S. DEPARTMENT OF ENERGY

RUN DATE : 05/12/94

RADIOACTIVE MASTE MANAGEMENT INFORMATION SYSTEM

PER SOLID WASTE SUMARY

WERF INCINERATOR PROCESSED MUSTE

$\begin{array}{lc}\text { MUCLIDES } & \begin{array}{c}\text { HALF LIFE } \\ \text { (DAYS ) }\end{array} \\ \begin{array}{c}\text { C0-60 } \\ \text { CURIES }\end{array} & 1.924 E+03 \\ \% \text { OF TOTAL } & \\ \text { CS- } 137 \\ \text { CURIES }\end{array}$

JANUARY FEBRUARY

FROM JAN THROUGH JUN 1993

MARCH APRIL

5.520E -07

30.296

$1.270 E-06$

69.704

JUNE

ANUAL

TOTAL

PAGE

1 RPT. 170

PER-S

\section{TOTALS}

ALPHA CURIES

$\%$ OF TOTAL

B+G CURIES

1. 822E-06

100.000

$1.822 E-06$

100.000

TOTAL GRAMS OF AM

TOTAL GRUYS OF PU

TOTAL ERANS OF PU-239+241

TOTAL ERNAS OF $U$

TOTAL ERNAS OF $U$

TOTAL ERUAS OF TH

TOTAL ERUNS OF NP

VOLUNE ICU. METERS
VEIGHT (KILOERNIS )

1. $223 E+01$

3.680E+02

$1.223 E+01$

and TOTALS (CURTES )

1. 822E-06

1.822E-06 
RADIOACTIVE WASTE MANAGEMENT INFORMATION SYSTEM

PER SOLID WASTE SUMMARY

WUSTE SENT TO WERF FOR COMPACTION

$\begin{array}{ll}\text { MUCLIDES } & \begin{array}{c}\text { HALF LIFE } \\ \text { (DAYS ) }\end{array} \\ \begin{array}{c}\text { CO-60 } \\ \text { CURIES }\end{array} & 1.924 E+03 \\ \% \text { OF TOTAL } \\ \begin{array}{c}\text { CS-134 } \\ \text { CURIES }\end{array} & 7.537 E+02 \\ \% \text { OF TOTAL } & \\ \text { CS-137 } \\ \text { CURIES } \\ \% \text { OF TOTAL } & 1.102 E+04 \\ \text { EU-154 } \\ \text { CURIES } \\ \% \text { OF TOTAL } & 3.103 E+03 \\ \text { SB-125 } \\ \text { CURIES }\end{array}$
FROM JAN THROUGH JUN 1993
FA

MARCH APRIL 1993 MAY

MAY

HNE

PAGE 1 RPT. 168

JANUARY FEBRUARY

MARCK

2.140E-04

56.704

$1.498 E-06$

0.397

$1.498 E-04$

39.693

ANUEAL

TOTAL

3. 180E-06

0.843

2. 140E-04

56.704

$1.498 E-06$ 0.397

$1.498 E-04$ 39.693

3. 180E-06

0.843

8. $920 E-06$ 2.364

8. $920 E-06$ 2.364

\section{TOTALS}

ALPHA CURIES

$\%$ OF total

B+6 curIEs

营 $\%$ OF TOTAL

TOTAL ERAMS of AM

TOTAL ERNIS OF PU

TOTAL ERANS of PU-239+24I

TOTAL ERNAS OF $u$

TOTAL ERAN OF TH

VOLUE (CU. METERS)

VEIEHT (KILOERUIS I

100.000

$3.774 E-04$

100.000

$7.250 E+00$ $6.810 E+02$

7. $250 E+00$

GRND TOTALS (CURIES)

3. 774E-04

3.774E-04 
RADIOACTIVE WASTE MUNAGEMENT INFORMATION SYSTEM

PAGE 1 RPT. 169

WASTE SENT TO WERF FOR METALLIC PROCESSING FROM JAN THROUGH JUN 1993

HALF LIFE

JANUARY

MARCH

APRIL

MAY

JUNE

ANNAL

c0-60

cunres

$\%$ OF TOTAL

cs-134

$1.924 E+03$

$7.537 E+02$

$\%$ OF TOTAL

CS-137

CURIES

$\%$ OF TOTAL

EU-154

cuntes

$3.100 E-07$

52.832

$1.201 E+04$

$\%$ of total

SB-125

$\%$ OF TOTAL

$1.007 E+03$

\section{TOTALS}

ALPHA CURIES

$\%$ of total

$B+6$ CURIES

$\%$ OF TOTAL

TOTAL ERAMS OF AM

TOTAL ERAYS OF PU

TOTAL ERANS OF PU-239+241

TOTAL ERAMS OF $U$

TOTAL GRAMS OF TH

TOTAL ERNAS OF NP

VOLUNE (CU. METERS)

VEIEYT (KILOERNAS)

8. $425 E-05$

8. 425E-05

100.000

$5.424 \mathrm{E}+02$

$6.343 E+00$
$4.451 E-05$

52.832

$3.100 E-07$
0.368

$3.693 E-05$

6.590E-07

0.782

$.840 E-06$
2.184

ERAND TOTALS (CURIES )

8. $425 E-05$

$8.425 E-05$

8.425E-05 



\section{Test Area North 1993 Detail Data}

Test Area North (TAN) Bar Graphs of Annual Data by Month $\ldots \ldots \ldots \ldots \ldots$ TAN-3

CY1993 Bar Graphs . .................................. TAN-5

TAN Detail Data for Months July to December

Annual Average Concentration and Total Curies for January to December ...... TAN-7

Report 150-TAN Airborne Waste Summary .................. TAN-9

Report 151_TAN Liquid Waste Summary $\ldots \ldots \ldots \ldots \ldots \ldots \ldots \ldots$ TAN-10

Report 152-TAN Disposed Solid Waste Summary ............... TAN-11

TAN Detail Data for Months January to June

Annual Average Concentration and Total Curies for January to June ........ TAN-13

Report 150-TAN Airborne Waste Summary $\ldots \ldots \ldots \ldots \ldots \ldots \ldots \ldots$ TAN-15

Report 151-TAN Liquid Waste Summary $\ldots \ldots \ldots \ldots \ldots \ldots \ldots \ldots \ldots$ TAN-16

Report 152-TAN Disposed Solid Waste Summary .............. TAN-17

TAN Facility Detail Data for Months July to December

Annual Average Concentration and Total Curies for January to December ...... TAN-19

Report 155-TSF Exhaust Airborne Waste Summary ............. TAN-21

Report 151-TAN Liquid Waste Summary $\ldots \ldots \ldots \ldots \ldots \ldots \ldots \ldots \ldots$ TAN-22

Report 192-CTF(LOF) Disposed Solid Waste Summary ............ TAN-24

TAN Facility Detail Data for Months January to June

Annual Average Concentration and Total Curies for January to June $\ldots \ldots \ldots$ TAN-25

Report 155-TSF Exhaust Airborne Waste Summary $\ldots \ldots \ldots \ldots \ldots \ldots$ TAN-27

Report 151 -TAN Liquid Waste Summary $\ldots \ldots \ldots \ldots \ldots \ldots \ldots \ldots$ TAN-29 

Test Area North (TAN) Bar Graphs of Annual Data by Month 
Test Area North (TAN) Monthly Details - CY 1993

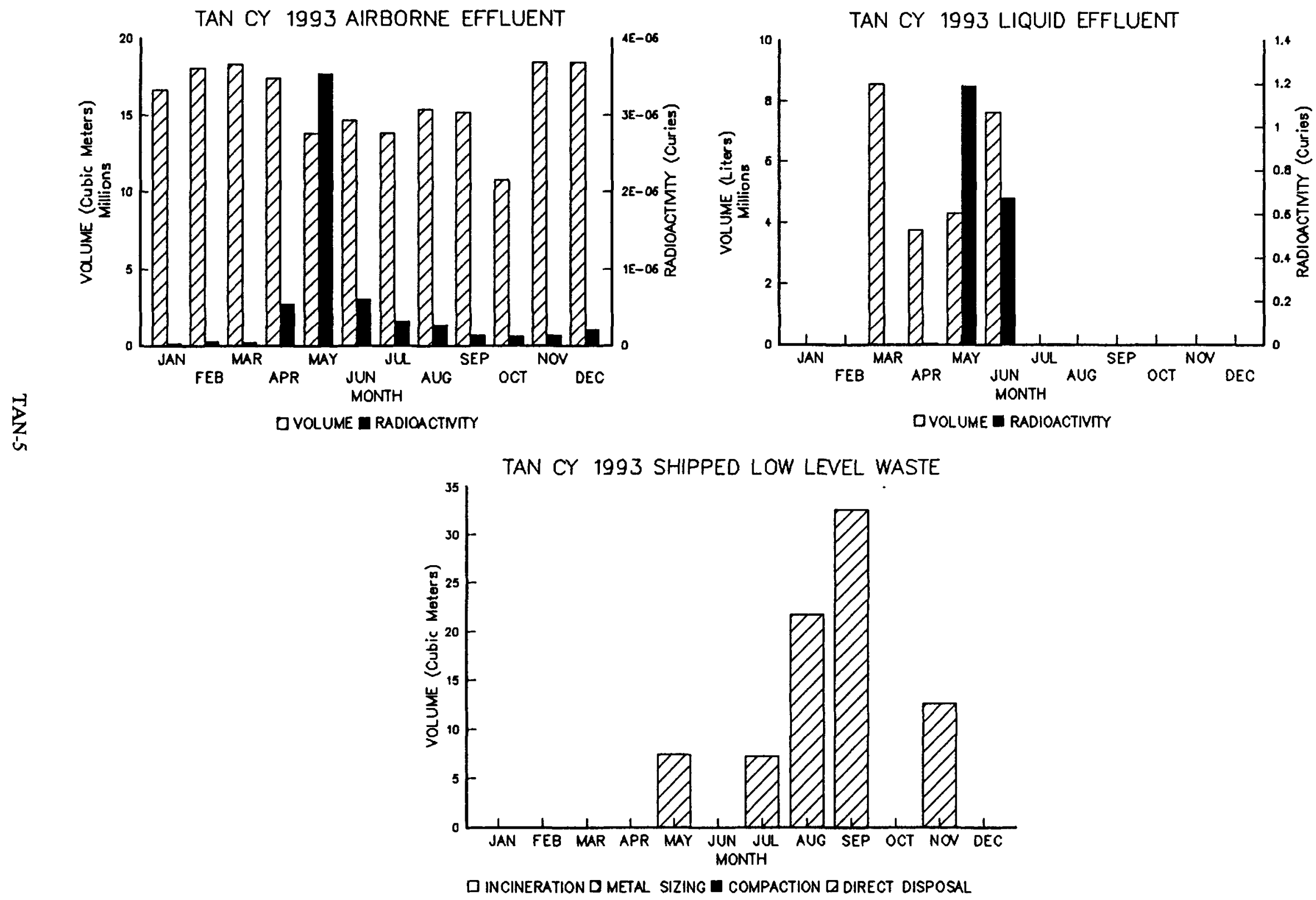




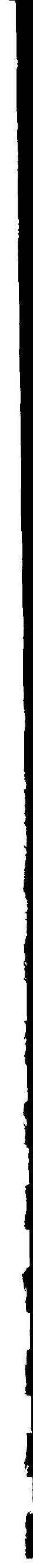


TAN Detail Data for Months July to December

Annual Average Concentration and Total Curies for January to December 

TAN AIRBORNE WASTE SUMHARY

FOR JAN THROUGH DEC 1993

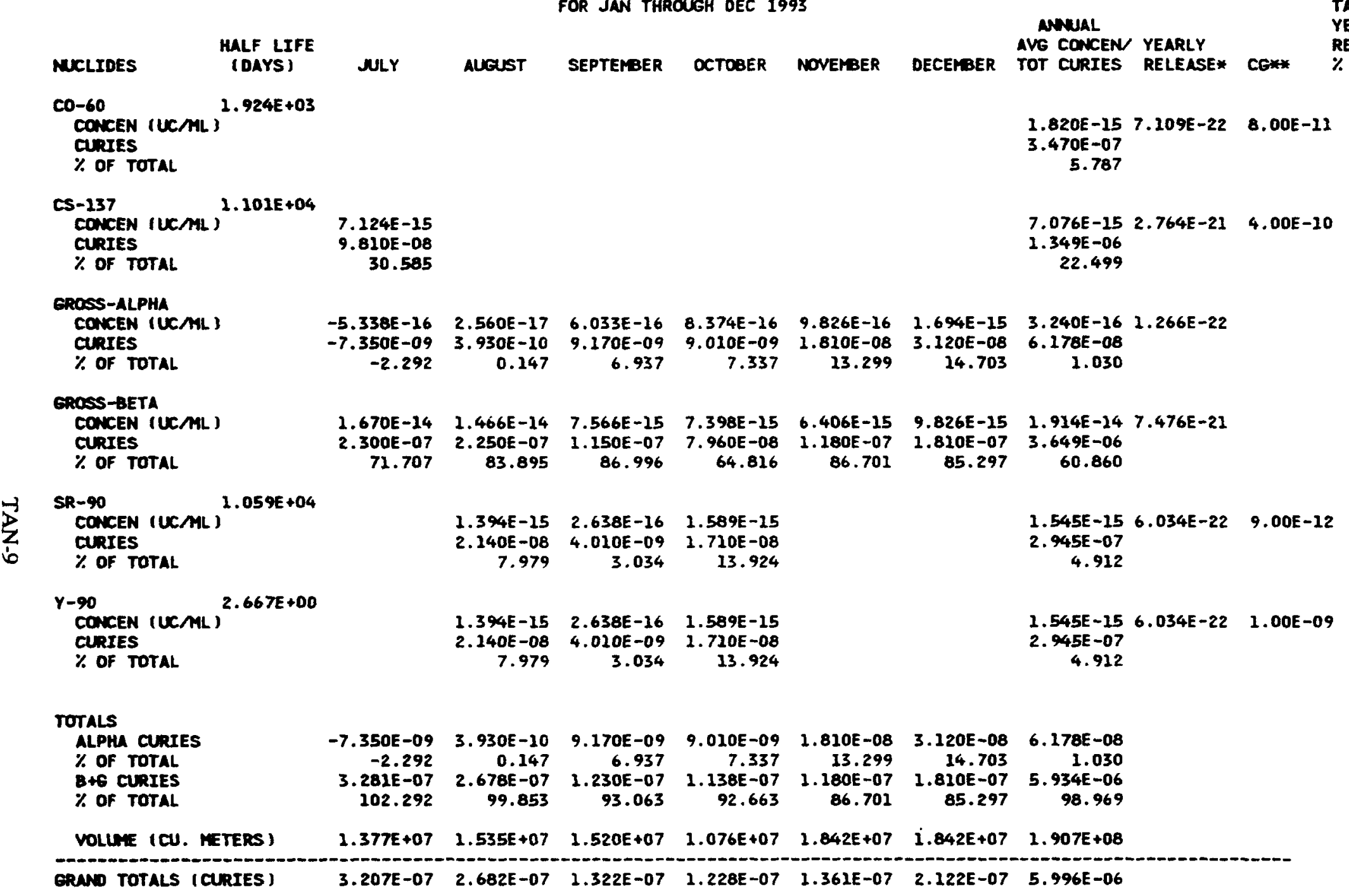

* This muber is derived from a dispersion factor eqution. The dispersion factor is derived from the straight line Gaussian model and is providad by the Netional Ocaanic Atmospheric Adninistration (NOAA).

** NOTE: DCG value obtainad from DOE ORDER 5400.5 dated February 8, 1990, Figures III-1 and III-3 
SCHED. NO. P6IPMOO9-E

IDAHO OPERATIONS OFFICE

U.S. DEPARTMENT OF ENERGY

RADIOACTIVE WASTE MUNAGEMENT INFORMATION SYSTEM

TAN LIQUID WASTE SUMYARY

FOR JAN THROUGH DEC 1993

MCLIDES
CROSS-ALPHA
CONCEN (UCML)
CURIES

HALF LIFE
(DAYS)

JULY

ALeUsT

SEPTEMBER

OCTOBER

\section{3}

NOVEMBER
CURIES

(DAYS) JULY AUEUST SEPTEFBR DCTOER NOVEMBER DECEMER

\% OF TOTAL
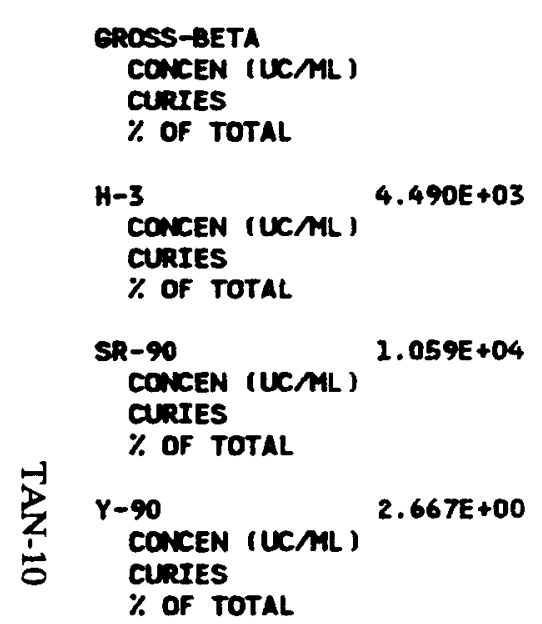

\section{TOTALS \\ ALPHA CURIES \\ \% OF TOTAL \\ B+6 CURIES \\ \% OF TOTAL}

\section{(2)}

DECEMER
ANMAL

AVG CONCEN

TOT CURIES

$-1.508 E-11$

$-3.660 E-07$

\subsection{E-09}

$7.310 E-05$

0.004

7.671E-05

$2.862 E+00$

99.986

$2.00 E-03$

3.84

4.004E-09 $1.00 E-06$

$9.720 E-05$

0.005

4.004E-09 1.00E-05

$9.720 E-05$

0.005

$-3.660 E-07$

$1.863 E+00$

100.000

$2.428 E+07$

\section{VOUUE (UTERS)}

$1.863 E+00$

ERW TOTALS (CURIES)

* NOTE: DCG velue abtainad from DOE ORDER 5400.5 dated February8, 1990, Figures III-I and III-3 
RADIOACTIVE WASTE MAMAGEMENT INFORMATION SYSTEM

TAN DISPOSEO SOLID WASTE SUMMARY FOR JAN THROUGH DEC 1993

HALF LIFE
(DAYS)

NLY

ALEUST

SEPTEMER

OCTOBER

NOVERBER

DECERBER

3.572E -05

81.880

24.760

0.884

\% OF TOTAL

cs-137

Cunies

$\%$ OF TOTAL

$1.101 E+04$

FE -55

ClRIES

H-3

CURIES

$\%$ Of TOTAL

NI -63

CURIES

$\%$ OF TOTAL

PU-238
CURIES
\% OF TOTAL
ERANS

PU-239

CURIES

$\%$ OF TOTAL

GRNA

s8-125

CURIES

$\%$ OF TOTAL

SR-90

CURIES

$\%$ OF total

$U-234$

CuRres

$\%$ OF TOTAL

Geurs

U-238

CURIES

$\%$ of total

GRAMS
$1.007 E+03$

$\begin{array}{rrr}75.066 & 99.116 & 100.000\end{array}$

$9.782 E+02$

$4.490 E+03$

$3.650 E+04$

$3.203 E+04$

8. 800E+06

$6.108 E-03$

0.174

$1.059 E+04$

8. $943 E+07$

$1.631 E+12$

\section{$2.632 E+00 \quad 1.046 E-03 \quad 1.112 E-06$}

PAGE 1 RPT 152

TAN-S
ANUAL

TOTAL

$1.116 E+00$

0.518

$2.633 E+00$

1.222

$4.860 E+01$

22.549

$1.428 E+00$ 0.662

$1.617 E+02$ 75.020

$2.797 E-06$

1.635E-07

$1.654 E-06$

2.664E-05

$6.108 E-03$ 0.003

5. 409E-02 0.025

1.300E-06

2.083E-04

2.332E-03

$$
0.001
$$

$6.939 E+03$ 
RADIOACTIVE WASTE MANAGEMENT INFORMATION SYSTEM

TAN DISPOSED SOLID WASTE SUMMARY FOR JAN THROUGH OEC 1993

MALF LIFE
(DAYS)

JULY

AUSUST

SEPTERBER

OCTOBER

NOVEMBER

DECEMER

\section{MNUAL}

PAGE 2 RPT 252

TOTAL

\section{TOTALS}

ALPHA CURIES

$\%$ OF TOTAL

B+G curies

$\%$ OF TOTAL
$3.507 E+00 \quad 1.055 E-03 \quad 1.112 E-06$ 100.000
4. $363 E-05$

100.000
2.337E-03

$$
0.001
$$

2.155E+02

99.998

TOTAL GRAM OF AM

TOTAL GRNAS OF AM

TOTAL GRAMS OF PU

TOTAL ERANS OF PU-239+241

TOTAL GRAMS OF $U$

TOTAL GRAMS OF U

TOTAL ERAMS OF NP

VOLURE (CU. METERS

VEICHT (KILOERNAS)

$7.248 E+00 \quad 2.175 E+01 \quad 3.262 E+01$

$3.629 E+03 \quad 5.897 E+03 \quad 2.329 E+04$

0
0
$6.939 E+03$

GND TOTALS (CURTES

3.507E +00 1.055E-03 1.112E-06

$1.263 E+01 \quad 8.164 E+01$

$5.669 E+03 \quad 4.436 E+04$


TAN Detail Data for Months January to June 

TAN AIRBORNE WASTE SUMMARY

FOR JAN THROUGH JUN 1993

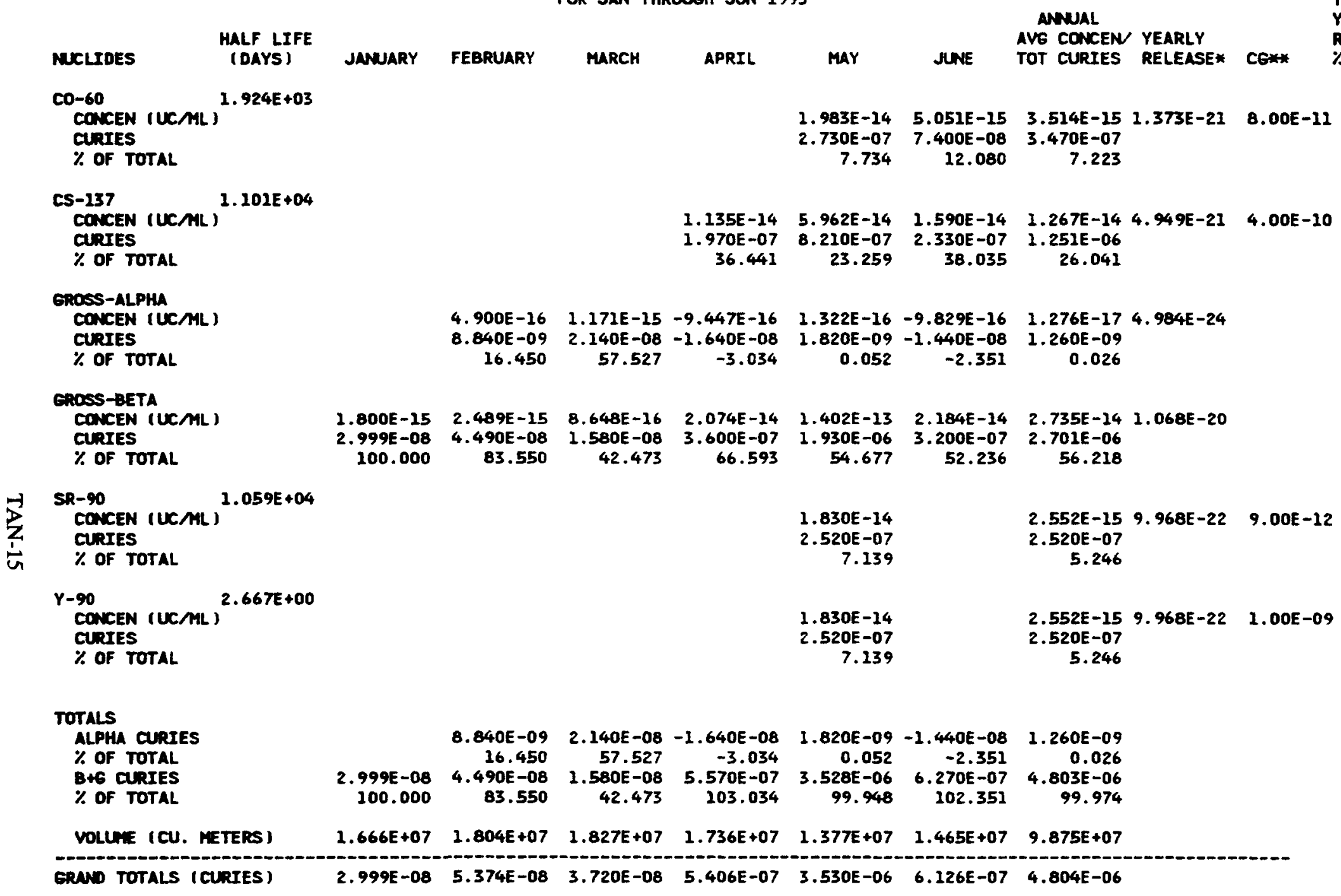

* This mubar is darived from a disparsion factor equetion. The dispersion factor is derived from the straight line Eewsian model and is provided by the Nional ocoanic Atmospheric Adwinistration (NOAA).

* NOTE: DCG value abtained from DOE ORDER 5400.5 dated February 8, 1990, Figures III-I and III-3 
RADIOACTIVE WASTE MANAGEMENT INFORMATION SYSTEM

TAN LIEUID MASTE SUMMARY

FOR JAN THROEGH JU 1993

MUCLIOES
EROSS-ALPHA
CONCEN (UCML)
CURIES
$\%$ OF TOTAL

HALF LIFE
(DAYS ]

\section{JANUARY FEBRUARY}

MARCH

APRIL

MaY

JUNE

$\begin{array}{lll} & \text { PAGE } & 1 \text { RPT. } 151 \text { TAN-L } \\ \text { AMNUAL } \\ \text { AVG CONCEN } \\ \text { TOT CURIES CG } * & \% \text { OF CG }\end{array}$

$-1.508 E-11$

$-9.685 E-11$

$\%$ OF TOTAL

EAOSS-BETA

CONCEN (UCAL)

CURIES

$\%$ of total

CONCEN (UCML)

CONCEN

$\%$ OF TOTAL

$4.490 E+03$

$\%$ or

$1.059 E+04$

CONCEN (UCAL)

CURIES

$\%$ of total

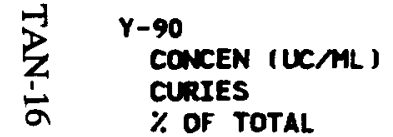

$2.667 E+00$

$$
\text { CURIES }
$$

$\%$ of total

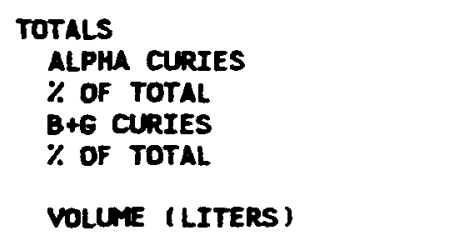

TOTALS
ALPHA CLRIES
$\%$ OF TOTAL
B+G CURIES
$\%$ OF TOTAL
VOLUNE (LITERS)

\begin{tabular}{|c|c|c|c|c|c|c|}
\hline & $\begin{array}{r}1.934 E-08 \\
7.310 E-05 \\
14.489\end{array}$ & & & $\begin{array}{r}3.011 E-09 \\
7.310 E-05 \\
0.004\end{array}$ & & \\
\hline & $\begin{array}{r}9.685 E-08 \\
3.660 E-04 \\
72.542\end{array}$ & $\begin{array}{r}2.765 E-04 \\
1.190 E+00 \\
99.998\end{array}$ & $\begin{array}{r}8.803 E-05 \\
6.720 E-01 \\
99.99 Z\end{array}$ & $\begin{array}{r}7.671 E-05 \\
1.862 E+00 \\
99.986\end{array}$ & $2.00 E-03$ & 3.84 \\
\hline $\begin{array}{r}3.329 E-09 \\
2.850 E-05 \\
50.000\end{array}$ & $\begin{array}{r}8.706 E-09 \\
3.290 E-05 \\
6.521\end{array}$ & $\begin{array}{r}2.997 E-09 \\
1.290 E-05 \\
0.001\end{array}$ & $\begin{array}{r}3.000 E-09 \\
2.290 E-05 \\
0.003\end{array}$ & $\begin{array}{r}4.004 E-09 \\
9.720 E-05 \\
0.005\end{array}$ & $1.00 E-06$ & 0.40 \\
\hline $\begin{array}{r}3.329 E-09 \\
2.850 E-05 \\
50.000\end{array}$ & $\begin{array}{r}8.706 E-09 \\
3.290 E-05 \\
6.521\end{array}$ & $\begin{array}{r}2.997 E-09 \\
1.290 E-05 \\
0.001\end{array}$ & $\begin{array}{r}3.000 E-0 \% \\
2.290 E-0.5 \\
0.003\end{array}$ & $\begin{array}{r}4.004 E-09 \\
9.720 E-05 \\
0.005\end{array}$ & $1.00 E-05$ & 0.04 \\
\hline $\begin{array}{r}5.700 E-05 \\
100.000\end{array}$ & $\begin{array}{r}-3.660 E-07 \\
-0.073 \\
5.049 E-04 \\
100.073\end{array}$ & $\begin{array}{r}1.190 E+00 \\
100.000\end{array}$ & $\begin{array}{r}6.720 E-01 \\
100.000\end{array}$ & $\begin{array}{r}-3.660 E-07 \\
1.863 E+00 \\
100.000\end{array}$ & & \\
\hline $.561 E+06$ & $3.779 E+06$ & $4.304 E+06$ & $7.634 E+06$ & $2.428 E+07$ & & \\
\hline $5.700 E-05$ & $5.045 E-04$ & $1.190 E+00$ & $6.720 E-01$ & $1.863 E+00$ & & \\
\hline
\end{tabular}

ERAND TOTALS (CURIES)

$\begin{array}{lllll}5.700 E-05 & 5.045 E-04 & 1.190 E+00 & 6.720 E-01 & 1.863 E+00\end{array}$

* NOTE: DCG value abtained from DOE ORDER 5400.5 dated February8, 1990, Figures III-1 and III-3 
TAN DISPOSED SOLID WASTE SUMMARY FOR JAN THROUEH JW 1993

\begin{tabular}{|c|c|}
\hline MUCLIDES & $\begin{array}{l}\text { HALF LIFE } \\
\text { (DAYS) }\end{array}$ \\
\hline $\begin{array}{l}\text { CO-60 } \\
\text { CURIES } \\
\% \text { OF TOTAL }\end{array}$ & $1.924 E+03$ \\
\hline $\begin{array}{l}\text { FE-55 } \\
\text { CURIES } \\
\% \text { OF TOTAL }\end{array}$ & $9.782 E+02$ \\
\hline $\begin{array}{l}\text { H-3 } \\
\text { CURIES } \\
\% \text { OF TOTAL }\end{array}$ & $4.490 E+03$ \\
\hline $\begin{array}{l}\text { NI - } 63 \\
\text { CURIES } \\
\% \text { OF TOTAL }\end{array}$ & $3.650 E+04$ \\
\hline $\begin{array}{l}\text { PU-238 } \\
\text { CURIES } \\
\text { \% OF TOTAL } \\
\text { GRAMS }\end{array}$ & $3.203 E+04$ \\
\hline $\begin{array}{l}\text { PU-239 } \\
\text { CURIES } \\
\text { \% OF TOTAL } \\
\text { GRAMS }\end{array}$ & $8.800 E+06$ \\
\hline $\begin{array}{l}\text { SR-90 } \\
\text { CURIES } \\
\% \text { OF TOTAL }\end{array}$ & $1.059 E+04$ \\
\hline $\begin{array}{l}\text { U-234 } \\
\text { CURIES } \\
\text { \% OF TOTAL } \\
\text { GRAMS }\end{array}$ & $8.943 E+07$ \\
\hline $\begin{array}{l}\text { U-238 } \\
\text { CURIES } \\
\% \text { OF TOTAL } \\
\text { ERAYS }\end{array}$ & $1.631 E+12$ \\
\hline
\end{tabular}

JANUARY FEBRUARY MARCH APRIL MAY

\begin{tabular}{rr}
$2.473 E-01$ & $2.473 E-01$ \\
0.117 & 0.117 \\
$4.860 E+01$ & \\
\hline & $4.860 E+01$
\end{tabular}

$22.922 \quad 22.922$

$\begin{array}{rr}1.428 E+00 & 1.428 E+00 \\ 0.673 & 0.673\end{array}$

$\begin{array}{rr}1.617 E+02 & 1.617 E+02 \\ 76.262 & 76.262\end{array}$

$2.797 E-06 \quad 2.797 E-06$

$\begin{array}{ll}1.635 E-07 & 1.635 E-07\end{array}$

$1.654 E-06 \quad 1.654 E-06$

$2.664 E-05 \quad 2.664 E-05$

5.409E-02 $\quad 5.409 E-02$

0.026

$1.300 E-06 \quad 1.300 E-06$

$2.083 E-04 \quad 2.083 E-04$

$\begin{array}{rr}2.332 E-03 & 2.332 E-03 \\ 0.001 & 0.001 \\ 6.939 E+03 & 6.939 E+03\end{array}$

PAGE 1 RPT 152 TAN-S 
SCHED. NO. P6IPNO09-G

$$
\text { IDAHO OPERATIONS OFFICE }
$$

U.S. DEPARTMENT OF ENERGY

RADIOACTIVE WASTE MANAGEMENT INFORMATION SYSTEM

TAN DISPOSED SOLID MASTE SUMTARY FOR JAN THROUGH JN 1993

\section{HALF LIFE}

(DAYS)

JANARY

FEBRUARY

MARCH

APRIL

MaY

JNE
$2.337 \mathrm{E}-03$
0.001

$2.120 E+02$
RIN DATE: 05/12/94

PAGE

2 RPT 152

ANUAL

$2.337 E-03$$$
0.001
$$

$2.120 E+02$

$\%$ OF TOTAL

TOTAL GRAMS OF AM

TOTAL ERAMS OF AM

TOTAL ERAMS OF PU

TOTAL ERAMS OF PU-239+241

TOTAL GRANS OF $U$

TOTAL GRNAS OF TH

TOTAL ERAYS OF NP

VOLLAE (CU. METERS)

99.999

99.998

$\begin{array}{rr}0 & 0 \\ 0 & 0 \\ 6,939 & 6,939\end{array}$

$7.399 E+00$ $5.871 \mathrm{E}+03$

$7.399 E+00$ $5.871 E+03$ 
TAN Facility Detail Data for Months July to December Annual Average Concentration and Total Curies for January to December 

IDAHO OPERATIONS OFFICE

RUN DATE: 05/11/94 RADIOACTIVE WASTE MANAGEMENT INFORMATION SYSTEM

MALLIDES
(DAYS )
CO-60
CONCEN (UCML)
CURIES
UNCERT(CURIES)
UNCERT(UCML)
\% OF TOTAL

CS-137 $\quad 1.101 E+04$ CONCEN (UCAL)

CIRIES

UNCERTI CURIES )

UNCERT (UCML)

$\%$ OF TOTAL

CONCEN (UCAL)

CURIES

UNCERT (CURIES )

UNCERT (UCML)

$\rightarrow \quad \%$ OF TOTAL

\section{GROSS-BETA}

CONCEN (UCML)

CURIES

UNCERTICURIES )

UNCERT ( UCML I

$\%$ OF TOTAL

$1.059 E+04$

SR-90

CONCEN (UCML)

CURIES

UNCERTI CURIES

UNCERT ( UCAML

\% OF TOTAL

$Y-90 \quad 2.667 E+00$

CONCEN (UCAL)

CURIES

UNCERTI CURIES

UNCERT(UC/ML

$\%$ OF TOTAL
TSF EXHAUST AIRBORNE WASTE SUMARY FOR JAN THROUEH DEC 1993

7. 124E-15

$9.810 E-08$

$2.130 E-08$

30.585
JULY

Avars

\section{OCTOBER}

\section{NONERBER}

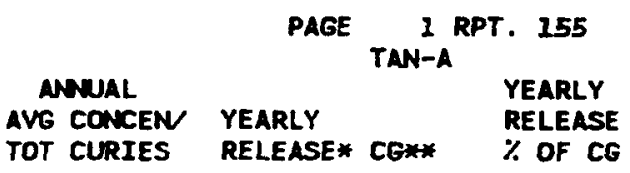

1.820E-15 7.209E-22 8.00E-11

$3.470 E-07$

5.787

$\begin{array}{lll}7.076 E-15 & 2.764 E-2 I \quad 4.00 E-10\end{array}$

$1.349 E-06$

22.499

$\begin{array}{lllllll}-7.330 E-16 & 2.560 E-17 & 6.033 E-16 & 8.374 E-16 & 9.826 E-16 & 1.694 E-15 \\ -7.350 E-09 & 3.930 E-10 & 9.170 E-09 & 9.010 E-09 & 1.810 E-08 & 3.120 E-08\end{array}$

6. $100 E-09 \quad 6.850 E-09 \quad 6.300 E-09 \quad 4.980 E-09 \quad 1.250 E-08 \quad 1.320 E-08$

$-2.292$

0.147

6.937

7.337

13.299

14.703

$3.240 E-16$
$6.178 E-08$

$\begin{array}{lllll}1.670 E-14 & 1.466 E-14 & 7.566 E-15 & 7.398 E-15\end{array}$

$\begin{array}{llll}2.300 E-07 & 2.250 E-07 & 1.150 E-07 & 7.960 E-08 \\ 1.610 E-08 & 1.610 E-08 & 1.430 E-08 & 1.030 E-08\end{array}$

$6.406 E-15$

$1.870 E-08 \quad 2.010 E-08$

71.707

83.895

86.996

64.816

86.701

85.297

60.860
OF CG
$1.610 E-08 \quad 1.610 E-08$
$1.545 E-15 \quad 6.034 E-22 \quad 9.00 E-12$ 2. 945E-07

4.912 2. $945 E-07$

4.912 $\begin{array}{lll}1.545 E-15 & 6.034 E-22 & 1.00 E-09\end{array}$

$\begin{array}{lll}1.394 E-15 & 2.638 E-16 & 1.589 E-15\end{array}$

$\begin{array}{lll}2.140 E-08 & 4.010 E-09 & 1.710 E-08 \\ 2.890 E-09 & 3.440 E-09 & 1.710 E-09\end{array}$

$\begin{array}{lll}7.979 & 3.034 & 13.924\end{array}$ 
RADIOACTIVE WASTE MANAGEMENT INFORMATION SYSTEM

TSF EXHAUST ATRBORNE WASTE SUMMARY

FOR JAN THROUEH DEC 1993

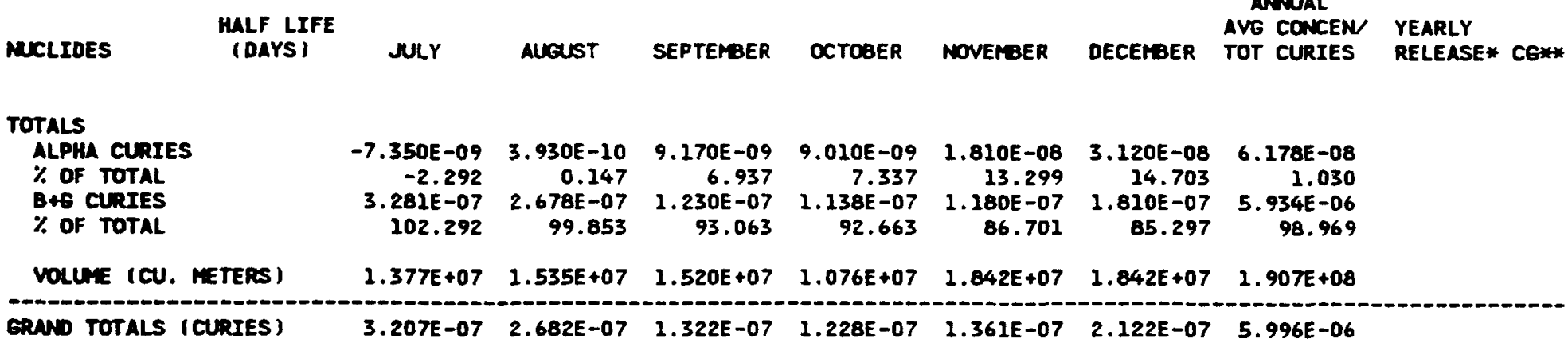

* This number is derived from a dispersion factor equation. The dispersion factor is derived from the straight line ousesien modal and is provedad by the National Dceanic Atmospharic Administrotion (NOAA).

* NOTE: DCG value obtainod from DOE ORDER 5400.5 dated February 8, 1990 , Figures III-I and III-3 


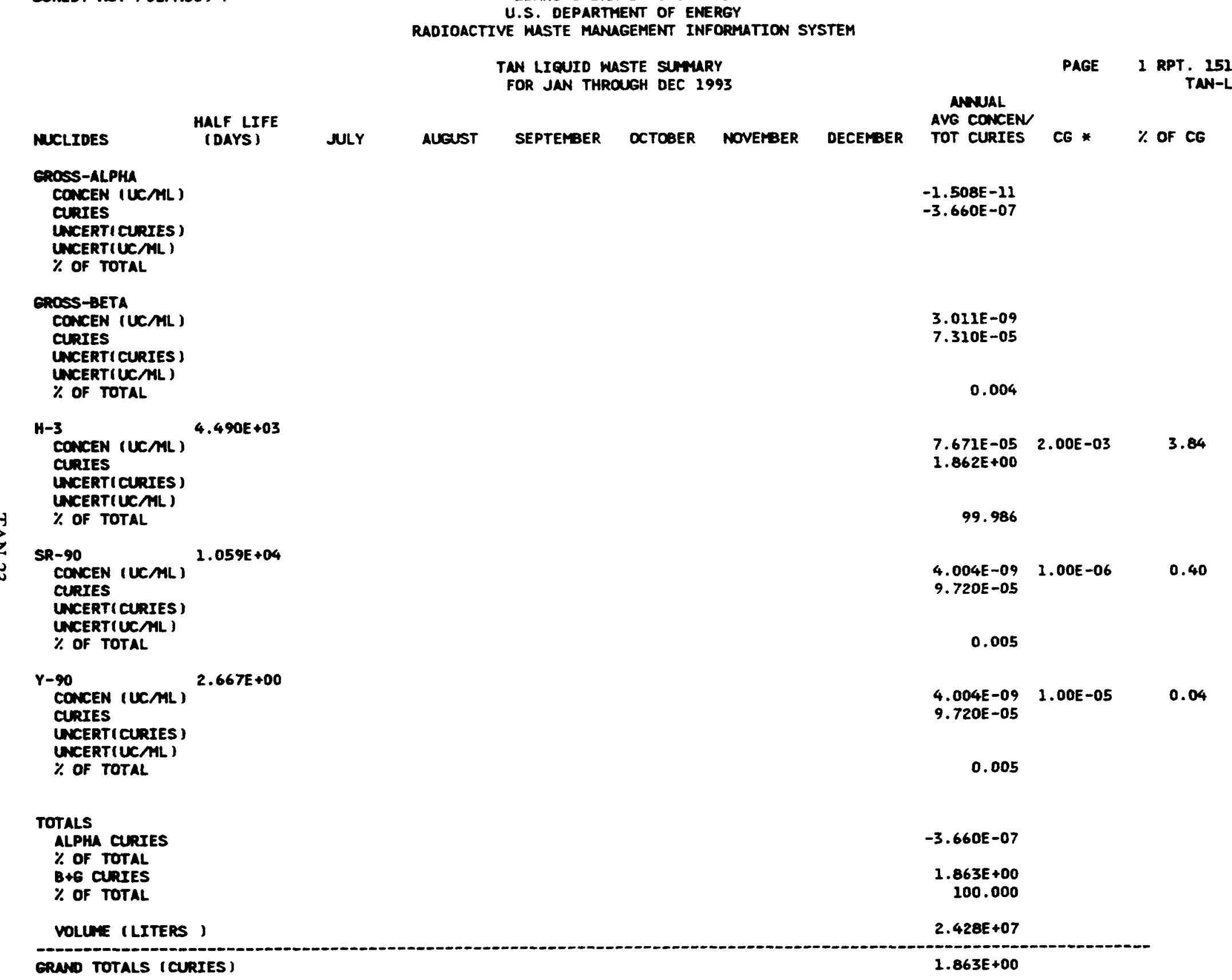

* NOTE: DCG value obtainad from DOE ORDER 5400.5 dated February8, 1990, Figures III-1 and III-3 
SCHED. NO. P61PMOO9-G

IDAHO OPERATIONS OFFICE

U.S. DEPARTMENT OF ENEREY

RADIOACTIVE WUSTE MANAGEMENT INFORMATION SYSTEM

CTFILOF) DISPOSED SOLID WASTE SUMUARY FOR JAN THROUEH DEC 1993

\begin{tabular}{|c|c|}
\hline MCLIOES & $\begin{array}{l}\text { HALF LIFE } \\
\text { (OAYS) }\end{array}$ \\
\hline $\begin{array}{l}\text { CE-144 } \\
\text { CURIES } \\
\% \text { OF TOTAL }\end{array}$ & $2.894 E+02$ \\
\hline $\begin{array}{l}\text { CO-60 } \\
\text { CURIES } \\
\% \text { OF TOTAL }\end{array}$ & $1.924 E+03$ \\
\hline $\begin{array}{l}\text { CS-137 } \\
\text { CURIES } \\
\% \text { OF TOTAL }\end{array}$ & $1.101 E+04$ \\
\hline $\begin{array}{l}\text { EU-155 } \\
\text { CURIES } \\
\% \text { OF TOTAL }\end{array}$ & $1.72 \pi+03$ \\
\hline $\begin{array}{l}\text { MU-206 } \\
\text { CURIES } \\
\% \text { OF TOTAL }\end{array}$ & $3.726 E+02$ \\
\hline $58-125$ & $1.007 E+03$ \\
\hline
\end{tabular}

CURIES

OF TOTAL

$\stackrel{n}{A}$

Totals

ALPHA CURIES

$\%$ Of TOTAL

B*G CURIES

$\%$ of total

TOTAL ERANS OF MM

TOTAL ERArS OF AM

TOTAL CRNYS OF PU

TOTAL ERAMS OF PU-239+241

TOTAL ERNAS OF $u$

TOTAL ERNES OF $U$

TOTAL ERAS OF TH
TOTAL ERATS OF NP

TOTAL ERAS OF NP
VOLUAY (CU. METERS )

VOLUME (CU. METERS)
MEIEHT (KILOGRAMS)

NULY

ALEUST

SEPTEMBER

OCTCBER

NOVERBER

DECEMER

2.600E-02

10.942

2.600E-02

10.942

$1.717 E-01$

72.262

1. $950 E-03$

0.821

$5.980 E-03$

2.517

$5.980 E-03$

2.517

2.376E-01

100.000

2.376E-01 99.999

$5.436 E+01$

$3.304 E+04$

$5.436 E+01$
RUN DATE: 05/11/94

PAGE

1 RPT 192

CTF( LOF )-S

CRND TOTALS (CURIES)

2.376E-01

$2.376 E-01$ 
TAN Facility Detail Data for Months January to June Annual Average Concentration and Total Curies for January to June 

RADIOACTIVE WASTE MANAGEMENT INFORMATION SYSTEM

MUCLIDES
MALF LIFE
(DAYS)
CO-60
CONCEN (UCML)
CURTES
UNERT(CURIES)
UNCERT(UCAML)
$\%$ OF TOTAL

\section{CS- 137}

CONCEN IUCAL

CURIES

UNCERTICURIES

UNCERT ( UCAML)

\% OF TOTAL

$1.101 E+04$

\section{EROSS-ALPHA}

CONCEN (UCAL)

CURIES

CNCERT ( CURIES )

UNERT (UCML

\section{enoss-beta}

CONCEN (UCML)

CURIES

36.441

23.259

4.900E-16 $1.171 E-15-9.447 E-16 \quad 1.322 E-16-9.829 E-16$ $8.840 E-09$ 2.140E-08 $-1.640 E-08$ 1.820E-09 $-1.440 E-08$ $\begin{array}{rrrrr}8.840 E-09 & 2.140 E-08 & -1.640 E-08 & 1.820 E-09 & -1.440 E-08 \\ 5.650 E-09 & 1.100 E-08 & 9.160 E-09 & 6.440 E-09 & 6.630 E-09\end{array}$

57.527

$-3.034$

0.052

$1.800 E-15$

$\begin{array}{llllll}2.489 E-15 & 8.648 E-16 & 2.074 E-14 & 1.402 E-13 & 2.184 E-14\end{array}$ $\begin{array}{lllll}4.490 E-08 & 1.580 E-08 & 3.600 E-07 & 1.930 E-06 & 3.200 E-07\end{array}$ $2.999 E-08$

UNCERT (CURIES )

UNCERT(UCML)

$\%$ OF TOTAL

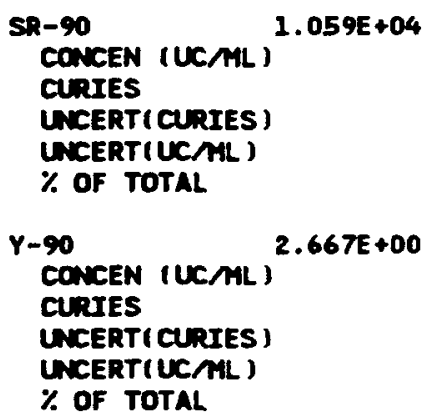

TSF EXHALST AIRBORNE MASTE SUMMARY FOR JAN THROUGH JUN 1993

JANGARY FEBRUARY MARCH APRIL MAY JWE

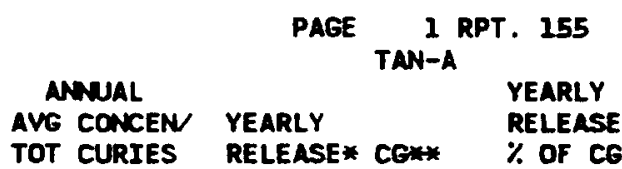

TOT CURTES

$\begin{array}{lllll}1.983 E-14 & 5.051 E-15 & 3.514 E-15 & 1.373 E-21 & 8.00 E-11\end{array}$

$2.730 E-07 \quad 7.400 E-08 \quad 3.470 E-07$

$3.110 E-08$ 1.920E-08

$\begin{array}{lll}7.734 & 12.080 & 7.223\end{array}$

$1.135 E-14 \quad 5.962 E-14 \quad 1.590 E-14$

$\begin{array}{lll}1.970 E-07 & 8.210 E-07 & 2.330 E-07\end{array}$

$\begin{array}{lll}4.230 E-08 & 6.770 E-08 & 3.190 E-08\end{array}$

38.035

$1.267 E-14 \quad 4.949 E-21 \quad 4.00 E-10$ $1.251 E-06$

$1.276 E-174.984 E-24$

$-2.351$

0.026

$2.735 E-14 \quad 1.068 E-20$

$1.800 E-15$
100.000

83.550

42.473

66.593

54.677

52.236

56.218

$1.830 E-14$

$2.520 E-07$

$1.610 E-08$

7.139

$2.552 E-25 \quad 9.968 E-22 \quad 9.00 E-12$

$2.520 E-07$

5.246

$1.830 E-14$

$2.520 E-07$

$1.610 E-08$

7.139

5.246 
RADIOACTIVE WASTE MANAGEMENT INFORMATION SYSTEM

\section{TSF EXHAUST AIRBORNE WASTE SUMHARY}

FOR JAN THROUGH JUN 1993

MUCLIDES
TOTALS
ALPHA CURIES
$\%$ OF TOTAL
B+G CURIES
$\%$ OF TOTAL

\section{HALF LIFE}

(DAYS)

\section{JANARY FEBRUARY}

\begin{abstract}
MARCH
\end{abstract}
APRIL
maY

JUNE

\begin{tabular}{|c|c|c|c|}
\hline & \multicolumn{3}{|c|}{ PAGE $\underset{\text { TAN-A }}{2}$ RPT. 155} \\
\hline $\begin{array}{l}\text { NWUAL } \\
\text { AVG CONKEN } \\
\text { TOT CURIES }\end{array}$ & $\begin{array}{l}\text { YEARLY } \\
\text { RELEASE* }\end{array}$ & CQ** & $\begin{array}{l}\text { YEARLY } \\
\text { RELEASE } \\
\text { \% OF CG }\end{array}$ \\
\hline
\end{tabular}

\begin{tabular}{llllllll} 
VOLUME (CU. METERS) & $2.666 E+07$ & $1.804 E+07$ & $1.827 E+07$ & $1.736 E+07$ & $1.377 E+07$ & $1.465 E+07$ & $9.875 E+07$ \\
\hline ERAND TOTALS (CURIES) & $2.999 E-08$ & $5.374 E-08$ & $3.720 E-08$ & $5.406 E-07$ & $3.530 E-06$ & $6.126 E-07$ & $4.804 E-06$
\end{tabular}

* This nuber is derived from a dispersion factor equetion. The dispersion factor is derived from the straight 1 ine onussian model and is proveded by the National Oceanic Atmospheric Administration (NOAA).

** NOTE: DCG value obtained from DOE ORDER 5400.5 dated February 8,1990 , Figures III-1 and III-3 
TAN LIQUTD WUSTE SUMURY
FOR JAN THROUGH JN 1993

\begin{tabular}{|c|c|}
\hline $\begin{array}{l}\text { MCLIOES } \\
\text { EAOSS-ALPHA } \\
\text { CONCEN (UCML) } \\
\text { CURIES } \\
\text { UNCERT(CURIES) } \\
\text { UCERT(UCML) } \\
\text { \% OF TOTAL }\end{array}$ & $\begin{array}{l}\text { HALF LIFE } \\
\text { (DAYS ) }\end{array}$ \\
\hline $\begin{array}{l}\text { EROSS-BETA } \\
\text { CONCEN (UCML) } \\
\text { CURIES } \\
\text { UNCERTICURIES) } \\
\text { UNCERT (UCMM ) } \\
\% \text { OF TOTAL }\end{array}$ & \\
\hline $\begin{array}{l}\text { H-3 } \\
\text { CONCEN (UCML) } \\
\text { CURIES } \\
\text { UNCERT(CURIES) } \\
\text { UNCERT (UCML) } \\
\text { \% OF TOTAL }\end{array}$ & $4.490 E+03$ \\
\hline $\begin{array}{l}\text { SR-90 } \\
\text { CONCEN (UCAL) } \\
\text { CURIES } \\
\text { UNCERT(CURIES) } \\
\text { UNCERT (UCML) } \\
\% \text { OF TOTAL }\end{array}$ & $1.059 E+04$ \\
\hline $\begin{array}{l}Y-90 \\
\text { CONCEN (UCML) } \\
\text { CURIES } \\
\text { UNCERTICURIES) } \\
\text { UCERTIUCML) } \\
\% \text { OF TOTAL }\end{array}$ & $2.667 E+00$ \\
\hline
\end{tabular}

\section{JANUARY FEBRUARY \\ MARCH \\ MAY}

$$
\begin{array}{r}
-9.685 E-11 \\
-3.660 E-07 \\
2.560 E-06
\end{array}
$$$$
-0.073
$$

$$
\begin{aligned}
& 1.934 E-08 \\
& 7.310 E-05
\end{aligned}
$$$$
\text { 3.660E-0.5 }
$$

$$
14.489
$$

\subsection{E-08 \\ $3.660 E-04$}

$3.660 E-04$

$$
72.542
$$$$
1.28
$$

$3.329 E-09$

2. $850 E-05$

$7.130 E-06$

50.000

$3.290 E-05$

(1)

$3.329 E-09$
$2.850 E-05$

$2.850 E-05$
$7.130 E-06$

6.521

\begin{abstract}
50.000
\end{abstract}
$2.765 E-04$
$1.190 E+00$

. $9405-02$

02

8.803E-05

.720E-01

99.993

$\begin{array}{ll}2.997 E-09 & 3.000 E-09 \\ 1.290 E-05 & 2.290 E-05 \\ 4.300 E-06 & 7.630 E-06\end{array}$

0.001

0.003

$9.720 E-05$

$3.011 E-09$

$7.310 E-05$

0.004

$7.671 E-05$

$2.00 E-03$

PAGE 1 RPT. 251

CONCEN

$-1.508 E-11$

$-3.660 E-07$

$\begin{array}{lll}\text { 8.706E }-09 & 2.997 E-09 & 3.000 E-09\end{array}$

$3.290 E-05$ 1.290E-05 2.290E-05

$\begin{array}{ll}1.290 E-05 & 2.290 E-05 \\ 4.300 E-06 & 7.630 E-06\end{array}$

\section{(2.7.}

0.005

TAN-L

CG * \% OF CG

\section{TOTALS}

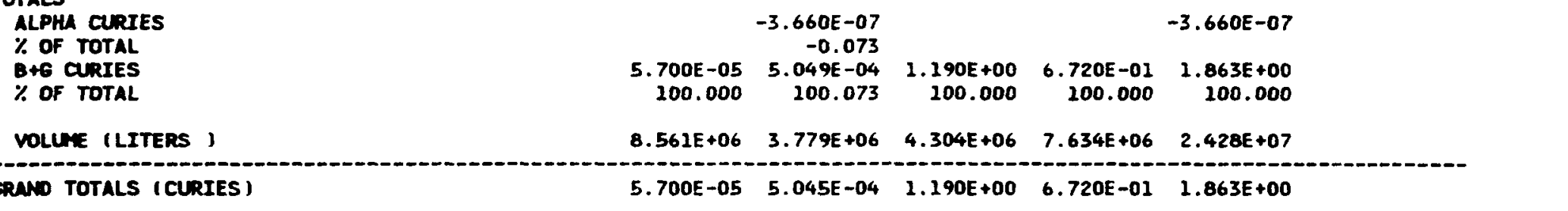

* NOTE: DCG value obtained from DOE ORDER 5400.5 dated Fabruary8, 1990, Figures III-1 and III-3 



\section{Test Reactoringa 1993 Detail Data}

Test Reactor $=($ TRA) Bar Graphs of Annual Data by Month $\ldots \ldots \ldots \ldots . .$. TRA-3

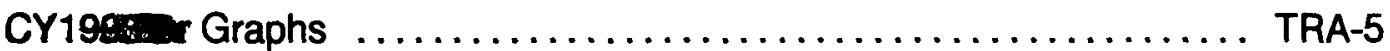

TRA Detail Danthe Monty to December

Annual Averasomentration and Total Curies for January to December ....... TRA-7

Reporans -TRA Airborne Waste Summary $\ldots \ldots \ldots \ldots \ldots \ldots \ldots$ TRA-9

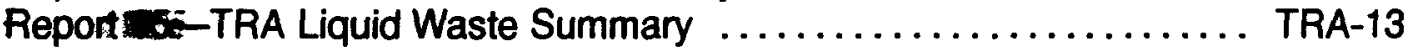

Reportis-TRA Disposed Solid Waste Summary ............... TRA-16

TRA Detail DufserMonths January to June

Anmual Averanectacentration and Total Curies for January to June ......... TRA-21

Reporte 4 TRA Airborne Waste Summary $\ldots \ldots \ldots \ldots \ldots \ldots \ldots \ldots$ TRA-23

Report $\ldots$-TRA Liquid Waste Summary $\ldots \ldots \ldots \ldots \ldots \ldots \ldots \ldots$ TRA-27

Report s-TRA Disposed Solid Waste Summary $\ldots \ldots \ldots \ldots \ldots \ldots$ TRA-30

TRA Facility Butata for Months July to December

Annual Avera

Report wes Alpha Lab Air Airborne Waste Summary .............. TRA-35

Reporten -ATR Airborne Waste Summary $\ldots \ldots \ldots \ldots \ldots \ldots \ldots \ldots$ TRA-36

Report ATR Disposed Solid Waste Summary $\ldots \ldots \ldots \ldots \ldots \ldots$ TRA-41

Repor nos-Chem Lab Add. Airborne Waste Summary .............. TRA-45

Report ETR Airborne Waste Summary $\ldots \ldots \ldots \ldots \ldots \ldots \ldots \ldots$ TRA-46

Report th ETR Disposed Solid Waste Summary $\ldots \ldots \ldots \ldots \ldots \ldots$ TRA-47

Report tes MTR Airborne Waste Summary $\ldots \ldots \ldots \ldots \ldots \ldots \ldots \ldots$ TRA-48

Report

Report 4RA Hot Cell Solid Waste Summary ................. TRA-51

Reportas -TRA Liquid Waste Summary $\ldots \ldots \ldots \ldots \ldots \ldots \ldots \ldots$ TRA-53

TRA Facility D.trata for Months January to June

Annual Avera Concentration and Total Curies for January to June ......... TRA-57

Reportes-Alpha Lab Air Airborne Waste Summary ............. TRA-59

Report ed-ATR Airborne Waste Summary $\ldots \ldots \ldots \ldots \ldots \ldots \ldots \ldots$ TRA-60

Report -ATR Disposed Solid Waste Summary $\ldots \ldots \ldots \ldots \ldots \ldots$ TRA-65

Report ins-Chem Lab Add. Airborne Waste Summary .............. TRA-68

Report -ETR Airborne Waste Summary $\ldots \ldots \ldots \ldots \ldots \ldots \ldots \ldots$ TRA-69

Report ETR Disposed Solid Waste Summary $\ldots \ldots \ldots \ldots \ldots \ldots \ldots$ TRA-70

Report 2 MTR Airborne Waste Summany $\ldots \ldots \ldots \ldots \ldots \ldots \ldots \ldots$ TRA-71

Repont us-TRA Hot Cell Airborne Waste Summary $\ldots \ldots \ldots \ldots \ldots \ldots$ TRA-73

Report $=$-TRA Liquid Waste Summary $\ldots \ldots \ldots \ldots \ldots \ldots \ldots \ldots$ TRA-74 

, 
Text Reactor Area (TRA) Monthly Details - CY 1993

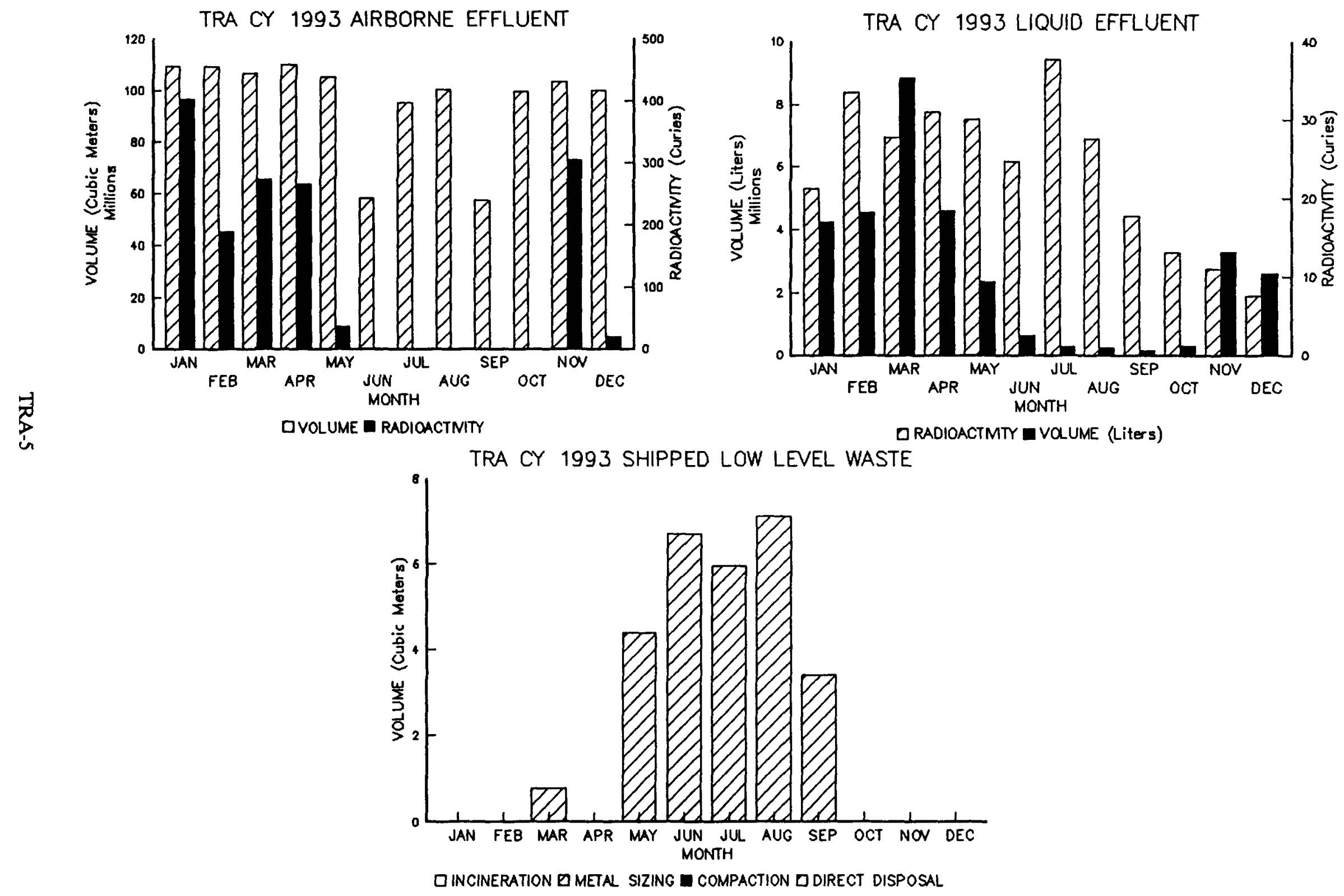




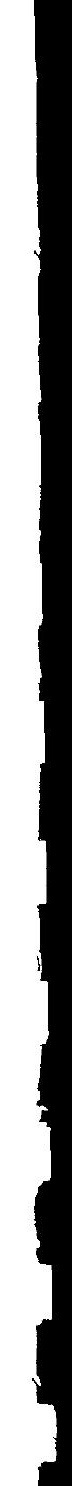


TRA Detail Data for Months July to December Annual Average Concentration and Total Curies for January to December 
RADIOACTIVE WASTE MANAGEMENT INFORMATION SYSTEM

TRA AIRBORNE MUSTE SUMYARY

FOR JAN THROUGH DEC 1993

MUCLIDES
AR-4L
CONCEN (DAYS)
CURIES
$\%$ OF TOTAL

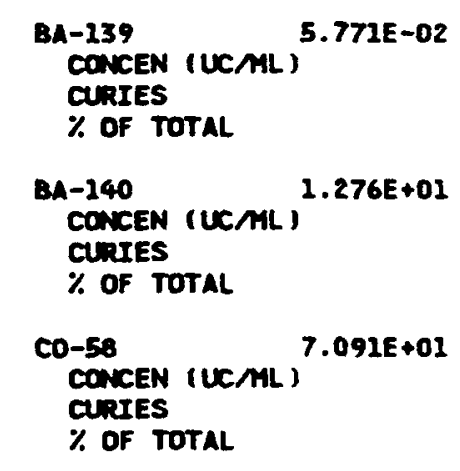

wLY

AUGUST

OCTOBER

4. $250 \mathrm{E}-04$

\section{$1.924 E+03$ \\ CONCEN (UCMIL) \\ CURIES}

$\%$ of rotal

CR-51

$\left.{ }_{\text {CONCEN }(U C / M L}\right)^{2.770 E+01}$

CURIES

\% of total

$1.101 E+04$

CS-137

\% OF total

SEPTEMBER

NOVERBR

\subsection{E-06 \\ $2.762 E+02$ \\ 90.740}

$83 E-03$
0.002

2. $906 \mathrm{E}-11$

$.906 E-03$
0.014

(1)

ANeuaL

PAGE 1 RPT. 104

NWUAL
AVG CONCEN

DECEMER TOT CORIEN YEARLY

TOT CURIES RELEASE* CEK*

TRA-A

YEARLY

RELEASE

1.823E-07 $\quad 1.142 E-06 \quad 3.147 E-13 \quad 1.00 E-08$ 23E+01 1.319E+03

88.203
5. $110 E-11$

Cs-138

CONCEN (UCML)

CURIES

\% OF TOTAL

EROSS-ALPHA

CONCEN (UCAL)

CURIES

$\%$ of total

\subsection{E-13} $3.539 E-05$ 71.913

$3.448 E-13$

$3.462 E-05$ 49.472

$2.635 E-13$

$1.518 E-05$

4.184E-13 $170 \mathrm{E}-0 \mathrm{C}$

$7.100 E-14 \quad 1.258 E-13$ 85.614 0.369

\section{5. $732 E-24$}

$5.468 E-06$

11.111

2. 762E-14

$2.635 E-06$

5.354
$9.009 E-15$

8. $980 E-07$

0.008

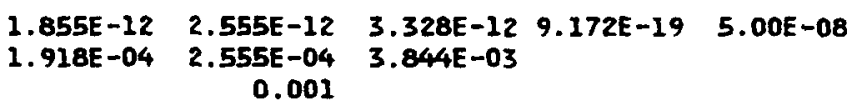

$6.909 E-16 \quad 1.904 E-22 \quad 2.00 E-09$ $7.981 E-07$

5.405E-15 1.490E-21 3.00E-09 $6.243 E-06$

$5.290 E-15 \quad 1.458 E-21 \quad 4.00 E-10$ $6.110 E-06$

$\begin{array}{lllll}7.725 E-10 & 4.034 E-10 & 5.985 E-10 & 1.650 E-16 & 8.00 E-09\end{array}$ 7.987E-02 4.034E-02 6.913E-01

$$
\begin{array}{rrr}
0.026 & 0.034 E-02 & 6.913 E-01 \\
& 0.198 & 0.046
\end{array}
$$

$\begin{array}{llllllll}-1.202 E-16 & -4.413 E-17 & 3.400 E-16 & 1.353 E-16 & -6.431 E-17 & 2.907 E-15 & 7.035 E-16 & 1.939 E-22\end{array}$

$\begin{array}{llllllll}-1.051 E-08 & -4.430 E-09 & 1.959 E-08 & 1.349 E-08 & -6.649 E-09 & 2.907 E-07 & 8.126 E-07\end{array}$ 


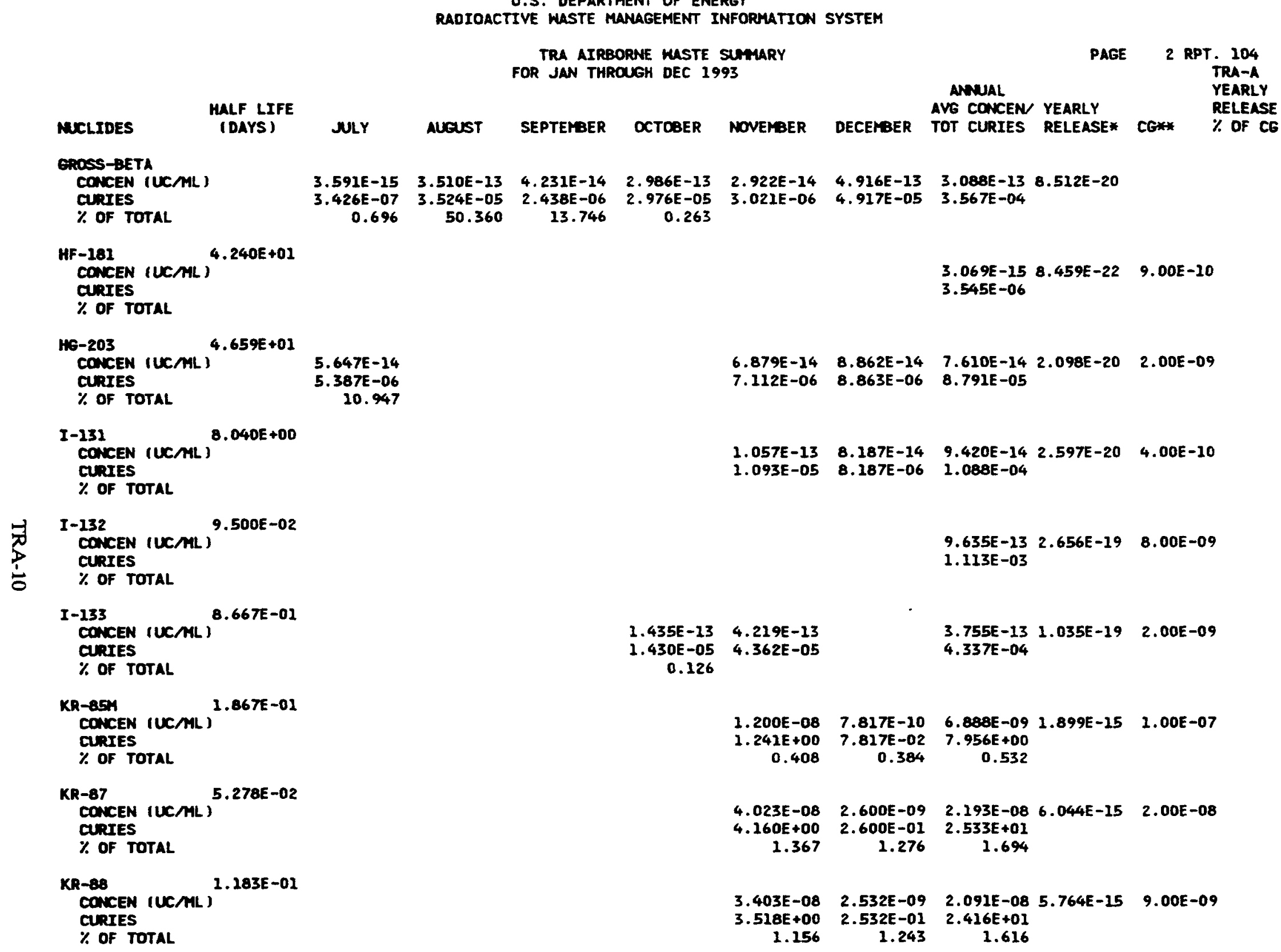

TRA ATRBORNE MASTE SLMMAY FOR JAN THROUGH DEC 1993

MUCLIDES
(DAYS)
GAOSS-BETA
CONCEN (UCML)
CURIES
$\%$ OF TOTAL
HF-181
CONCEN (UCML)
CURIES
$\%$ OF TOTAL
HG-203
CONCEN (UCML)
CURIES
$\%$ OF TOTAL
I-132
CONCEN (UCML)
CURIES
$\%$ OF TOTAL

I-133 8.667E-0I CONCEN (UCNL)

CURIES

$\%$ of total

NLY

Aumest

SEPTEIBER

3.591E-15 3.510E-13

$3.426 E-07 \quad 3.524 E-05$ 0.696

4. $231 E-14 \quad 2.986 E-13$

$438 E-06$
13.746 0.263

$2.922 E-14 \quad 4.916 E-13$ 50.360

5.647E-14 $5.387 E-06$ 10.947 
RADIOACTIVE WASTE MANAGEMENT INFORMATION SYSTEM

TRA AIRBORNE WASTE SUMMARY

FOR JAN THROUGH DEC 1993

\begin{tabular}{|c|c|}
\hline MUCLIDES & $\begin{array}{l}\text { HALF LIFE } \\
\text { (DAYS) }\end{array}$ \\
\hline $\begin{array}{l}\text { LA-140 } \\
\text { CONCEN (UCML) } \\
\text { CURIES } \\
\% \text { OF TOTAL }\end{array}$ & $1.678 E+00$ \\
\hline $\begin{array}{l}\text { Ma-24 } \\
\text { CONCEN }(U \mathrm{C} M L) \\
\text { CURIES } \\
\% \text { OF TOTAL }\end{array}$ & $6.237 E-01$ \\
\hline $\begin{array}{l}\text { R日-88 } \\
\text { CONCEN (UCML) } \\
\text { CURIES } \\
\% \text { OF TOTAL }\end{array}$ & $1.229 E-02$ \\
\hline $\begin{array}{l}\text { RB-89 } \\
\text { CONCEN (UCML) } \\
\text { CURIES } \\
\% \text { OF TOTAL }\end{array}$ & $1.056 E-02$ \\
\hline $\begin{array}{l}\text { RE-168 } \\
\text { CONCEN (UCAL) } \\
\text { CURIES } \\
\% \text { OF TOTAL }\end{array}$ & $7.075 E-01$ \\
\hline $\begin{array}{l}\text { SR-90 } \\
\text { CONCEN (UC/ML) } \\
\text { CURIES } \\
\% \text { OF TOTAL. }\end{array}$ & $1.059 E+04$ \\
\hline $\begin{array}{l}\text { TC-99M } \\
\text { COMCEN (UCML) } \\
\text { CURIES } \\
\text { \% OF TOTAL }\end{array}$ & $2.504 E-01$ \\
\hline $\begin{array}{l}\text { XE-133 } \\
\text { CONCEN (UCML) } \\
\text { CURIES } \\
\% \text { OF TOTAL }\end{array}$ & $5.250 E+00$ \\
\hline $\begin{array}{l}\text { XE-135 } \\
\text { CONCEN (UCML) } \\
\text { CURIES } \\
\% \text { OF TOTAL }\end{array}$ & $3.792 E-01$ \\
\hline
\end{tabular}

JLY

AUEUST

SEPTEMBER

OCTOBER
$6.076 E-16 \quad 8.157 E-16$

$6.100 E-08 \quad 4.700 E-08$

$0.087 \quad 0.265$
NOVEMER

DECEMER

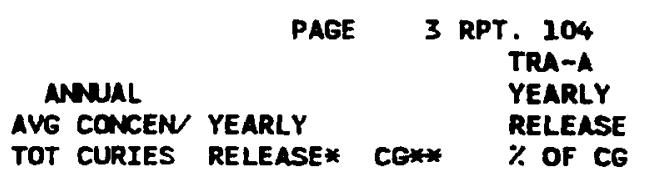

$\begin{array}{lll}7.385 E-15 & 2.036 E-21 \quad 3.00 E-09\end{array}$

8.531E-06
$3.761 E-12 \quad 2.467 E-12$
$3.889 E-04 \quad 2.467 E-04$
0.001
$6.431 E-10 \quad 3.563 E-10 \quad 4.462 E-10 \quad 1.230 E-16 \quad 3.00 E-08$ $6.649 E-02$ 3.563E-10
3.563E-02 5.154E-01

$\begin{array}{rrr}0.022 & 0.175 & 0.034\end{array}$
$\begin{array}{llllll}1.084 E-10 & 8.812 E-10 & 4.473 E-10 & 6.300 E-10 & 1.737 E-16 & 9.00 E-09\end{array}$ $1.080 E-02 \quad 9.111 E-02 \quad 4.473 E-02 \quad 7.278 E-01$

$$
\begin{array}{rrrr}
95.477 & 9.111 E-02 & 4.473 E-02 & 7.278 E-01 \\
& 0.030 & 0.220 & 0.049
\end{array}
$$

$2.899 E-14 \quad 7.991 E-21 \quad 6.00 E-09$ $3.349 E-05$
$9.894 E-16$

$1.023 E-07$
$2.280 E-12 \quad 2.035 E-12 \quad 1.866 E-12 \quad 5.144 E-19 \quad 1.00 E-07$ $2.357 E-04 \quad 2.035 E-04 \quad 2.156 E-03$$$
0.001
$$

5.790E-09 1.120E-09 3.603E-09 9.932E-16 5.00E-07 $5.986 E-01 \quad 1.120 E-01 \quad 4.162 E+00$
4.428E-08 2.934E-09 2.422E-08 $\quad 6.675 E-15 \quad 8.00 E-08$ $4.568 E+00 \quad 2.934 E-01 \quad 2.797 E+01$

$$
\begin{array}{rrr}
1.501 & 1.440 & 1.871
\end{array}
$$


RADIOACTIVE WASTE MANAGEMENT INFORMATION SYSTEM

\begin{tabular}{|c|c|}
\hline MUCLIOES & $\begin{array}{l}\text { MALF LIFE } \\
\text { (DAYS ) }\end{array}$ \\
\hline $\begin{array}{l}\text { XE-135M } \\
\text { CONCEN (UCML) } \\
\text { CURIES } \\
\% \text { OF TOTAL }\end{array}$ & $1.062 E-02$ \\
\hline $\begin{array}{l}\text { XE-238 } \\
\text { CONCEN (UCML) } \\
\text { CURIES } \\
\% \text { OF TOTAL }\end{array}$ & $9.792 E-03$ \\
\hline $\begin{array}{l}Y-90 \\
\text { CONCEN (UCAL) } \\
\text { CURIES } \\
\% \text { OF TOTAL }\end{array}$ & $2.667 E+00$ \\
\hline $\begin{array}{l}Y-91 M \\
\text { CONCEN }(U C M L)^{3} \\
\text { CURIES } \\
\% \text { OF TOTAL }\end{array}$ & $3.451 E-02$ \\
\hline
\end{tabular}

$\begin{array}{ll}6.076 E-16 & 8.157 E-16 \\ 6.100 E-08 & 4.700 E-08\end{array}$

$\begin{array}{rr}100 E-08 & 4.700 E-08 \\ 0.087 & 0.265\end{array}$
TRA ATPBOPNE WASTE SUMMARY

FOR JAN THROUGH DEC 1993

wLY

AUEUST SEPTEMBER OCTOBER NOVEMER

PAGE 4 RPT. 104
TRA-A

AMNAL

AVG CONCEN YEARLY YEARLY DECEMBER TOT CURIES RELEASE* CG** \% OF CG

\section{Totals}

ALPHA CuRIES

$\%$ OF TOTAL

B+G curies

$-1.051 E-08-4.430 E-09$

$-0.021$

\% OF TOTAL

4. 922E-05

$-0.006$
2.715E-08 $\quad 1.500 E-09 \quad 1.190 E-08 \quad 3.279 E-15 \quad 5.00 E-08$
$2.807 E+00 \quad 1.500 E-01 \quad 1.374 E+01$

$\begin{array}{rrr}0.922 & 0.736 & 0.919\end{array}$

VOLUME (CU. METERS)

$100.021 \quad 6.900 .006$

$1.959 E-08$
0.110
$1.771 E-05$

1.131E-02

$\begin{array}{lllll}1.069 E-07 & 8.717 E-09 & 6.152 E-08 & 1.696 E-14 & 2.00 E-08\end{array}$

$1.105 E+01 \quad 8.718 E-01 \quad 7.106 E+01$

3.630

4.279

4.753

$9.894 E-16$

$1.023 E-07$

$9.810 E-16 \quad 2.704 E-22 \quad 2.00 E-09$ $1.133 E-06$

$\begin{array}{lllll}1.728 E-12 & 2.348 E-12 & 1.361 E-12 & 3.750 E-19 & 4.00 E-08\end{array}$

$1.787 E-04 \quad 2.348 E-04 \quad 1.572 E-03$

0.001

ERMD TOTALS (CURIES)

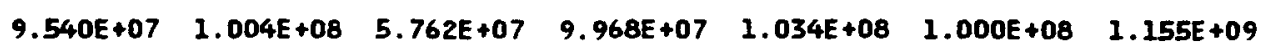

* This muber is derived from a dispersion factor equation. The dispersion factor is derived from the straight line Gaussian modal and is provided by the Notional Oceanic Atmospheric Administration (NOAA).

* NOTE: DCG value obtained from DOE ORDER 5400.5 dated Fobruary 8, 1990 , Figures III-1 and III-3 
TRA LIQUID MUSTE SUMMARY

FOR JAN THROUGH DEC 1993

\begin{tabular}{|c|c|}
\hline MUCLIDES & $\begin{array}{l}\text { HALF LIFE } \\
\text { (DAYS) }\end{array}$ \\
\hline $\begin{array}{l}\text { CE-142 } \\
\text { CONCEN (UCML) } \\
\text { CURIES } \\
\% \text { OF TOTAL }\end{array}$ & $3.250 E+01$ \\
\hline $\begin{array}{l}\text { CE-144 } \\
\text { CONCEN (UCML) } \\
\text { CURIES } \\
\% \text { OF TOTAL }\end{array}$ & $2.844 E+02$ \\
\hline $\begin{array}{l}\text { CO-58 } \\
\text { CONCEN (UCML) } \\
\text { CURIES } \\
\% \text { OF TOTAL }\end{array}$ & $7.091 E+01$ \\
\hline $\begin{array}{l}\text { CO-60 } \\
\text { CONCEN (UCAL) } \\
\text { CURIES } \\
\% \text { OF TOTAL }\end{array}$ & $1.924 E+03$ \\
\hline
\end{tabular}

$\underset{\frac{1}{2}}{\frac{1}{\omega}}$

\section{JULY}

August

SEPTE

$$
\begin{array}{r}
3.863 E-07 \\
2.663 E-03 \\
0.268
\end{array}
$$

$3.080 E-07 \quad 1.449 E-07$

.900E-03 $9.985 E-04$

$0.270 \quad 0.101$

$2.530 E-05 \quad 9.165 E-05 \quad 1.840 E-05 \quad 1.800 E-05$

$2.383 E-01$

$6.318 E-01$

63.690

$148 E-02$
13.228

$5.870 E-02$
5.020

2.310E-OE

$6.304 E-02$
0.481

6. $190 E-05$

1.

$\begin{array}{lllllll}7.550 E-06 & 3.191 E-06 & 6.850 E-07 & 5.660 E-07 & 1.640 E-05 & 1.920 E-04 \\ 7.110 E-02 & 2.200 E-02 & 3.033 E-03 & 1.846 E-03 & 4.476 E-02 & 3.660 E-01\end{array}$

0.492

0.158

$\%$ OF TOTAL 6.627

2.217

$$
0.492
$$

\section{ANNUAL \\ AVG CONCEN}

TOT CURIES
PAGE I RPT. 105

TRA-L

$90 E-05$
$80 E-01$
1.141

$7.172 E-08$

$5.068 E-03$

$$
0.004
$$

1.228E-07 7.00E-06

e.680E-03

0.007

0.341

3.539

4.075E-07 4.00E-05

$2.880 E-02$

$$
0.022
$$

.901E-05 5.00E-06

$051 E+00$

1.600

CG * $\%$ OF CG

$7.537 E+02$

CS-134

1.673E-08 2.00E-06

$1.182 E-03$

0.001

\% OF TOTAL

\section{CS-137 \\ CONCEN ILCML \\ CORIES \\ $\%$ OF TOTAL}

$1.201 E+04$

$\begin{array}{lll}2.230 E-06 & 1.798 E-06 & 2.520 E-06 \\ 2.100 E-02 & 1.240 E-02 & 1.116 E-02\end{array}$

$\begin{array}{rrr}.100 E-02 & 1.240 E-02 & 1.116 E-02 \\ 1.957 & 1.250 & 1.812\end{array}$

\section{EROSS-ALPHA \\ CONCEN (UCAL) \\ CURIES}

$\%$ OF TOTAL

\section{aROSS-BETA}

CONCEN (UCML)

CURIES

$\%$ Of TOTAL

$\begin{array}{rrrrrrr} & & & & & & \begin{array}{r}7.074 E-08 \\ 5.000 E-03 \\ 0.004\end{array} \\ & & & & & & \\ 2.100 E-05 & 1.602 E-05 & 2.300 E-05 & 1.400 E-05 & 1.300 E-05 & 5.400 E-05 & 2.311 E-05 \\ 1.978 E-01 & 1.104 E-01 & 1.018 E-01 & 4.565 E-02 & 3.548 E-02 & 1.029 E-01 & 1.634 E+00 \\ 18.433 & 11.134 & 16.535 & 3.905 & 0.271 & 0.995 & 1.275\end{array}$

0.24

1.75

1.02

580.25 
RADIOACTIVE WASTE MANAGEMENT INFORMATION SYSTEM

FOR JAN THROUGH DEC 1993

$\begin{array}{ll}\text { MUCLIDES } & \begin{array}{c}\text { HALF LIFE } \\ \text { (DAYS) }\end{array} \\ \text { H-3 } & \begin{array}{c}4.490 E+03 \\ \text { CONCEN (UCML) }\end{array} \\ \text { CURIES } & \\ \% \text { OF TOTAL } \\ \text { M-54 } \\ \text { CONCEN (UCML) } \\ \text { CURIES } \\ \% \text { OF TOTAL }\end{array}$

JULY

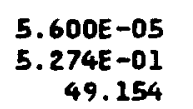

PAGE 2 RPT. 105 TRA-L

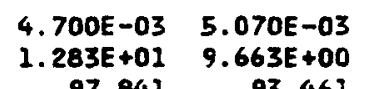

86.32 95.204

$M-24$ 6.237E-01 CONCEN (UCML)

CURIES

$\%$ Of TOTAL

\begin{tabular}{|c|c|}
\hline $\begin{array}{l}\text { MB-95 } \\
\text { CONCEN (UCML) } \\
\text { CURIES } \\
\% \text { OF TOTAL }\end{array}$ & $3.498 E+01$ \\
\hline $\begin{array}{l}\text { SB-124 } \\
\text { CONCEN (UCML) } \\
\text { CURIES } \\
\% \text { OF TOTAL }\end{array}$ & $6.020 E+01$ \\
\hline $\begin{array}{l}\text { SR-89 } \\
\text { CONCEN (UCML) } \\
\text { CURIES } \\
\% \text { OF TOTAL }\end{array}$ & $5.052 E+01$ \\
\hline $\begin{array}{l}\text { SA-90 } \\
\text { CONCEN (UCML) } \\
\text { CURIES } \\
\% \text { OF TOTAL }\end{array}$ & $1.059 E+04$ \\
\hline $\begin{array}{l}\text { TA-182 } \\
\text { CONCEN (UCML) } \\
\text { CURIES } \\
\% \text { OF TOTAL }\end{array}$ & $1.145 E+02$ \\
\hline $\begin{array}{l}Y-90 \\
\text { CONCEN (UCML) } \\
\text { CURIES } \\
\% \text { OF TOTAL }\end{array}$ & $2.667 E+00$ \\
\hline
\end{tabular}

2.117E-07

$1.459 E-03$

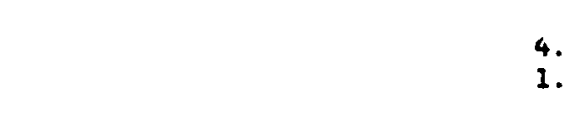

0.006

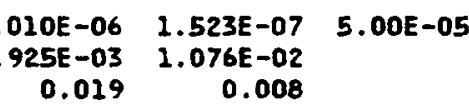

0.006

$\%$ of Torat 
SCHED. NO. P61PMOOS-E

IDAHO OPERATIONS OFFICE

U.S. DEPARTMENT OF ENERGY

RUN DATE: 05/11/94

RADIOACTIVE WASTE MANAGEMENT INFORMATION SYSTEM

TRA LIQUTO WASTE SUTHRY

FOR JAN THROUEH DEC 1993

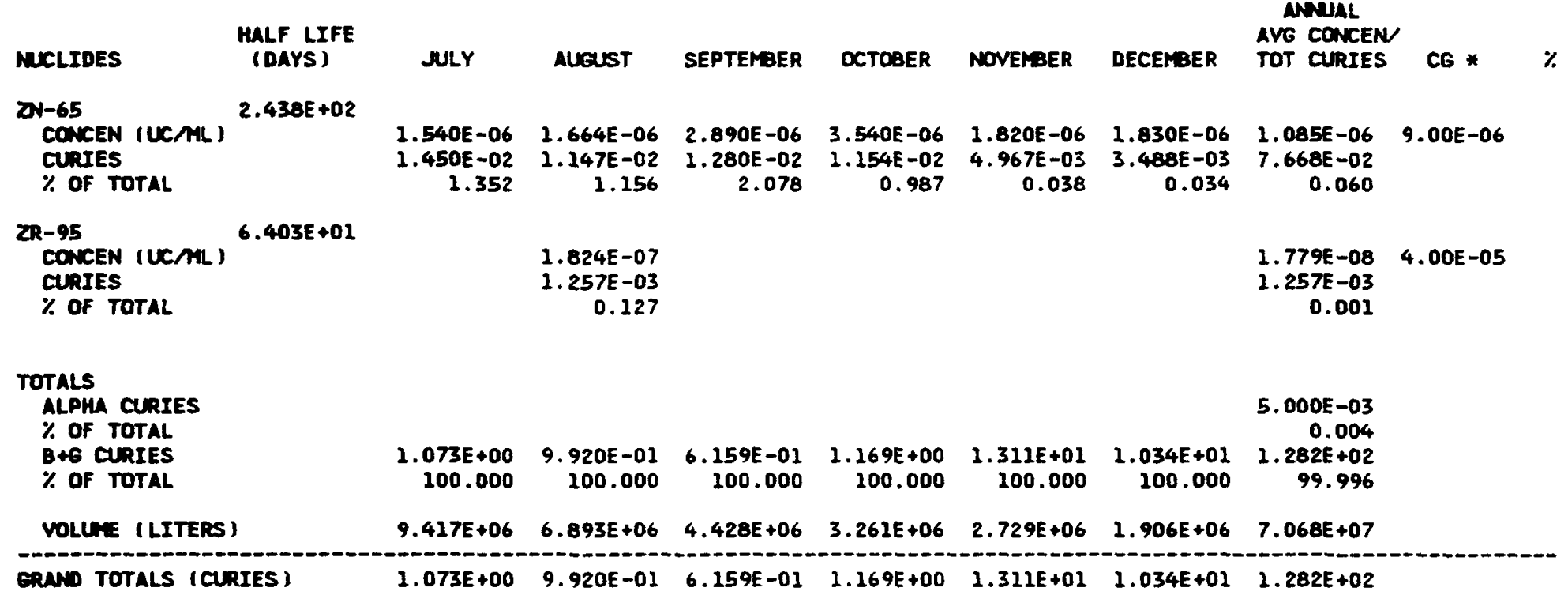

* NOTE: DCG value obteined from DOE ORDER 5400.5 deted February8, 1990, Figures III-1 and III-3

*** The CG linit is being approached or has been exceeded during the current month. please verity the acouracy of the reported data. If the data is in error, please send correct data to Waste Information. 
SCHED. NO. P61PM009-G

IDAHO OPERATIONS OFFICE

U.S. DEPARTMENT OF ENERGY

RADIOACTIVE WASTE MANAGEMENT INFORMATION SYSTEM

TRA DISPOSED SOLID WASTE SUMARY FOR JAN THROUGH DEC 1993

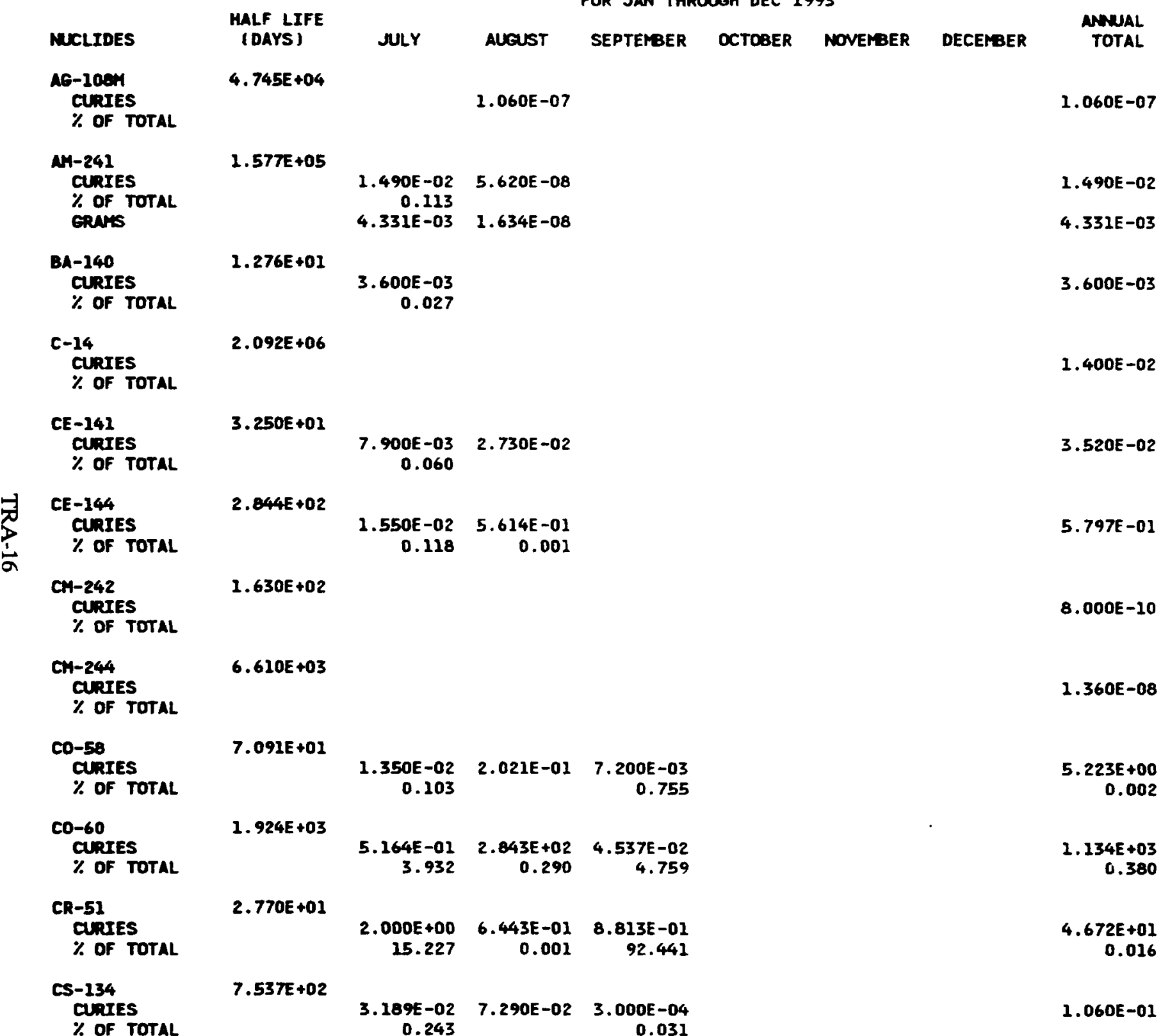

RUN DATE : 05/11/94

PAgE 1 RPT 106 TRA-S 
SCHED. NO. P61PMOO9-G

IDAHO OPERATIONS OFFICE U.S. DEPARTMENT OF ENERGY

RADIOACTIVE WASTE MANAGEMENT INFORMUTION SYSTEM

TRA DISPOSED SOLID MASTE SUMMARY

FOR JAN THROUGH DEC 1993

MCLIDES
CS- 237
CURIES
$\%$ OF TOTAL

HALF LIFE

(DAYS)

JUY

Aueust

SEPTERBER

\section{oCtose}

NOVEMBER

$1.261 E+00 \quad 2.087 E-01$

EU-152

CURTES
$\%$ OF TOTAL

EU-254

CURIES

U-155

CURIES

\% of total

FE-55

CURIES

$\%$ of total

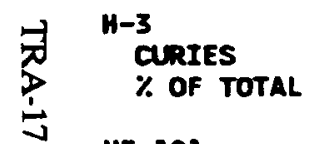

HF-181
CURIES

\% OF TOTAL

I-232

CURTES

IR-192

curres

$\%$ of total

LA-140

$\%$ of total

M-54

CURTES

$\%$ of total

no-99

CURIES

\% OF TOTAL
9.601

$1.101 E+04$

$4.891 E+03$

$1.090 E-12 \quad 6.820 E-06$

3.203E+03

3.760E-02 2.690E-07

0.134

$1.727 E+03$

8. $700 E-13$

$8.700 E-13$

$6.900 E+02$

0.231

$4.490 E+03$

6.200E-03 9.682E+04 $0.047 \quad 98.668$

$4.240 E+01$

$1.500 E-03 \quad 2.740 E-02$

$$
0.011
$$

\section{$2.000 E-04$}

0.021

1. $900 \mathrm{E}-03$

0.014

$1.678 E+00$

\section{$3.600 E-03$}

$$
0.027
$$

$3.122 E+02$

2.110E-02 1.445E-01

$$
0.161
$$

1. $900 E-02$
$2.930 E+05$

98.273

$3.480 E-02$

$6.600 E-03$

$1.900 E-03$

$3.600 E-03$

$17 E+01$
RUN DATE: 05/11/94

PAGE 2 RPT 206

$$
\text { TRA-S }
$$


SCHED. NO. P61PMO09-G

MUCLIDES

$3-94$

$\%$ OF TOTAL

N-95

CURIES

$\%$ OF TOTAL

NI-59

CURIES

$\%$ OF TOTAL

NI -63

CURIES

$\%$ OF TOTAL

PR-144

CURIES

$\%$ Of TOTAL

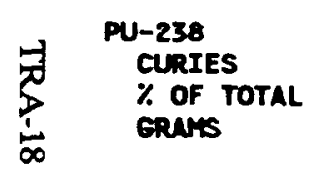

Pu-239

CURIES

$\%$ OF TOTAL

gRents

RU-103
CURIES
$\%$ OF TOTAL

3. 924E+01

S8-124

CURIES

$\%$ OF TOTAL

sB-125

$\%$ OF TOTAL

$s c-46$

CURIES

$\%$ OF TOTAL

SE-75

CURIES

$\%$ OF TOTAL

(DAYS)

$3.498 E+01$

$2.774 E+07$

$3.650 E+04$

1.200E-02

3. 203E+04

8.800E+06

$6.020 E+01$

$1.007 E+03$

$8.382 E+01$

$1.298 E+02$
IDAHO OPERATIONS OFFICE

U.S DEPARTMENT OF ENERGY

RADIOACTIVE WASTE MANAGEMENT INFORMATION SYSTEM

TRA DISPOSED SOLID MASTE SUMARY

FOR JAN THROUGH DEC 1993

MNNAL
TOTAL

JULY

AUEUST

SEPTEMBER

OCTOBER

NOVEMBER

DECEMBER

9.0DOE-07

$1.330 E-02 \quad 9.520 E-02$

0.101

$1.020 E+03$

1.039

$2.537 E-01$

$3.075 E+03$
1.031

2.537E-01

4. 280E-06

$2.503 E-07$

3.800E-07

$6.119 E-06$

5.600E-03

$900 E-03$
0.030

$2.180 E-08$

0.002

$4.600 E-03$

5.600E-03

$9.000 E-03$

5.000E-04
RUN DATE: 05/11/94

PAge

3 RPT 106

TRA-S 

RADIOACTIVE WASTE MAMAGEMENT INFORMATION SYSTEM

TRA DISPOSED SOLID WASTE SUMAARY

FOR JAN THROUGH DEC 1993

\section{MUCLIDES}

HALF LIFE

(DAYS)

NULY

AUGUST

SEPTEMBER

OCTOBER

NOVEMBER DECEMBER

SN-119M

CURIES

$2.930 E+02$

$\%$ OF TOTAL

$$
\begin{aligned}
& \text { SR-89 } \\
& \text { CURIES } \\
& \% \text { OF TOTAL }
\end{aligned}
$$

sR-90

CURIES

$\%$ Of total.

TC-99

$\%$ OF TOTAL

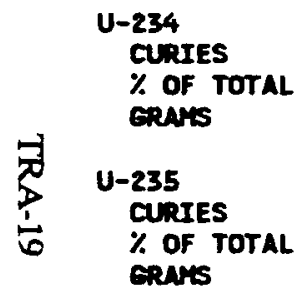

ZN-65

CURIES

2R-95

CURIES

$\%$ Of Total
$5.052 E+01$

\subsection{E-01}

6.102

$1.059 E+04$

8.241E+00 2.816E-0I

62.742

$7.775 E+07$

8. $943 E+07$

$4.340 E-07$

6. $955 E-05$

2. $270 E-08$

1.051E-02

$2.438 E+02$

1.494E-01 3.223E-01

1.137

$6.403 E+01$

$9.000 E-03 \quad 7.080 E-02$ 0.069

\section{ANUAL}

PAGE 4 RPT 106

TRA-S
$1.500 E+02$

0.005

8.015E-01

$8.526 E+00$ 0.003

$2.500 E-05$

4.340E-07

$6.955 E-05$

2. $270 E-08$

1. 051E-02

8. 247E+01

0.028

8. 730 -02 
SCHED. NO. P6LPMOOS-G

IDAHO OPERATIONS OFFICE

U.S. DEPARTMENT OF ENERGY

RUN DATE: 05/11/94

RADTOACTIVE WASTE MANAGEMENT INFORMATION SYSTEM

TRA DISPOSED SOLID WASTE SUMYARY

FOR JAN THROUGH DEC 2993

$\begin{array}{lrrrr}\text { MUCLIDES } & \begin{array}{c}\text { MALF LIFE } \\ \text { (DAYS) }\end{array} & \text { JULY } & \text { AUGUST } & \text { SEPTEMBER } \\ \text { TOTALS } & & & & \\ \text { ALPHA CURIES } & & 1.490 E-02 & 5.129 E-07 & \\ \% \text { OF TOTAL } & & 0.113 & & \\ \text { B+G CURIES } & & 1.312 E+01 & 9.813 E+04 & 9.534 E-01 \\ \% \text { OF TOTAL } & & 99.887 & 100.000 & 100.000\end{array}$

OCTOBER

NOVERBER

DECEMBER

ANNAL

TOTAL

PAGE 5 RPT 106 TRA-S

$1.491 E-02$

$\begin{array}{rrr}912 E+01 & 9.813 E+04 & 9.534 E-01 \\ 99.887 & 100.000 & 100.000\end{array}$

2. $982 E+05$

100.000

TOTAL ERANS OF M

TOTAL ERAMS OF MM

TOTAL ERAMs of PU

TOTAL ERANS Of PU-239+241

TOTAL ERNAS OF $U$

TOTAL. ERANS OF TH

TOTAL GRNAS OF NP

VOLLME ICU. METERS

NEIEHT (KILOERAMS)

$5.964 E+00 \quad 7.125 E+00 \quad 3.398 E+00$

$6.391 E+03 \quad 6.509 E+03 \quad 7.710 E+03$

CRAm Totals (CLnTEs)

$1.313 E+01 \quad 9.813 E+04 \quad 9.534 E-01$

$4.331 E-03$

$6.369 E-06$

6.119E-06

$1.058 E-02$

$2.982 E+05$ 

Annual Average Concentration and Total Curies for January to June 
TRA AIRBORNE WASTE SUMARY

FOR JAN THROUGH JUN 1993

MALF LIFE
(DAYS)
$\begin{aligned} & \text { AR-4IDES } \\ & \text { CONCEN (UCML) } \\ & \text { CORIES }\end{aligned}$

CURIES

\% Of TOTAL

5.771E-02

84-139 CONCEN IUCML)

CURIES

$\%$ of rotal

JAMUARY

FEBRUARY

APRIL

maY

JNE

$\begin{array}{lllll}3.162 E-06 & 1.532 E-06 & 2.271 E-06 & 2.158 E-06 & 3.026 E-07 \\ 3.45 B E+02 & 1.672 E+02 & 2.421 E+02 & 2.373 E+02 & 3.188 E+01\end{array}$

85.755

88.119

88.408

88.979

86.646

$9.509 E-11 \quad 6.354 E-11$

1.040E-02

$6.935 E-03$

0.004

$5.795 E-11$
$6.179 E-03$

$6.565 E-11$
$7.218 E-03$

$1.352 E-10$

0.002

0.003

$424 E-02$
0.039

$5.708 E-14$

6. $243 E-06$

CONIEN (LCML)

curres
\% OF total

$1.276 E+03$

co-58

$7.091 E+01$

CONCEN (UCML)

CURIES

$\%$ OF TOTAL

CO-60

$1.924 E+03$

CONCEN (UCML)

CURIES

\% of total

$2.697 E-14$

$2.014 E-14$

$7.575 E-15$

7. $981 E-07$

$4.548 E-06$

$2.770 E+01$

CR-51 CONCEN (UCAL)

CURIES

$\%$ OF TOTAL

5.535E-12

$6.053 E-04$

$7.061 E-12 \quad 1.034 E-11$

6.553E-12

$21.486 E-12$

$1.566 E-04$

CS-137

$1.101 E+04$

CONCEN (UCAM)

CURIES

\% OF TOTAL
CS-138
CONCEN (UCML)
CURIES
$\%$ OF TOTAL

ERoss-AlPHA

CONEEN (UCML)

CURIES

$\%$ OF TOTAL
$1.644 E-14$
$1.808 E-06$

$\begin{array}{llllll}1.139 E-09 & 7.847 E-10 & 8.331 E-10 & 8.643 E-10 & 1.680 E-09\end{array}$

$1.246 E-01 \quad 8.564 E-02 \quad 8.883 E-02 \quad 9.503 E-02 \quad 1.770 E-01$ $\begin{array}{rrrrr}0.031 & 0.045 & 0.032 & 0.036 & 0.481\end{array}$
$4.167 E-14$
$4.548 E-06$

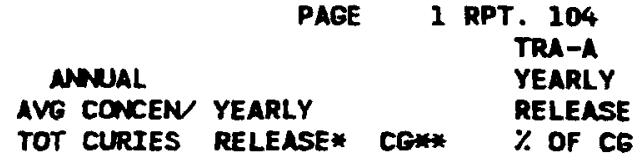

$1.711 E-06 \quad 4.716 E-13 \quad 1.00 E-08$

$1.024 E+03$

87.522

$7.513 E-11 \quad 2.071 E-17 \quad 7.00 E-08$ 4.497E-02 0.004

$1.043 E-14 \quad 2.875 E-21 \quad 3.00 E-09$ $6.243 E-06$

$\begin{array}{lll}1.333 E-15 & 3.675 E-22 & 2.00 E-09\end{array}$ $7.981 E-07$

$.625 E-13 \quad 4.480 E-20 \quad 8.00 E-11$ $754 E-05 \quad 9.731 E-05$ 63.905

$\begin{array}{llllllll}-5.723 E-16 & 2.194 E-15 & 1.929 E-15 & 2.814 E-16 & 8.631 E-16 & 1.023 E-16 & 8.526 E-16 & 2.350 E-22\end{array}$

$\begin{array}{lllllll}-6.259 E-08 & 2.395 E-07 & 2.057 E-07 & 3.094 E-08 & 9.093 E-08 & 5.950 E-09 & 5.104 E-07\end{array}$
$9.540 E-10 \quad 2.630 E-16 \quad 8.00 E-09$ $5.711 E-01$ 0.049 
TRA AIRBORNE MUSTE SUMARY FOR JAN THROUEH JUN 1993

MUCLIDES MALF LIFE
(DAYS)
EROSS-BETA
CONCEN (UCML)
CURIES
$\%$ OF TOTAL
HF-181
CONEEN (UCML)
CURIES
$\%$ OF TOTAL
WO-203
COMCEN (UCML)
CURIES
$\%$ OF TOTAL

JANUARY FEBRUARY MARCH APRIL MAY

$6.788 E-14 \quad 7.130 E-13 \quad 1.373 E-14 \quad 1.108 E-12 \quad 2.362 E-13$ $\begin{array}{lllll}7.424 E-06 & 7.781 E-05 & 1.464 E-06 & 1.218 E-04 & 2.488 E-05\end{array}$

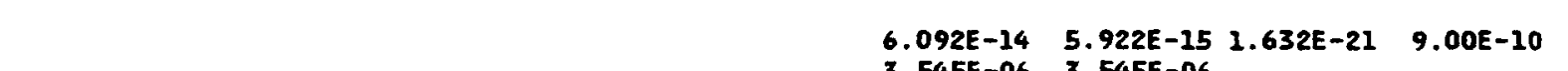

$\begin{array}{llllll}9.774 E-13 & 8.807 E-13 & 3.721 E-13 & 9.540 E-13 & 2.679 E-13\end{array}$ $\begin{array}{lllll}1.069 E-04 & 9.612 E-05 & 3.968 E-05 & 1.049 E-04 & 2.823 E-05\end{array}$

1.642

$\begin{array}{lllll}7.728 E-08 & 2.774 E-08 & 3.722 E-08 & 3.914 E-08 & 6.008 E-09\end{array}$ $8.452 E+00 \quad 3.027 E+00 \quad 3.969 E+00 \quad 4.304 E+00 \quad 6.330 E-01$

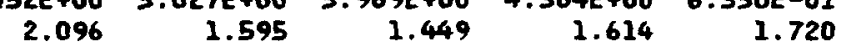

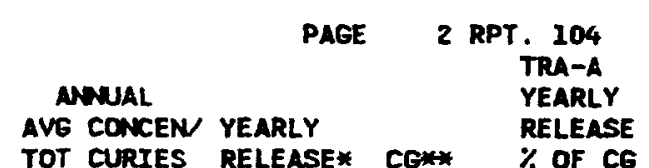

$5.783 E-14 \quad 3.955 E-13 \quad 1.090 E-19$ 2.457

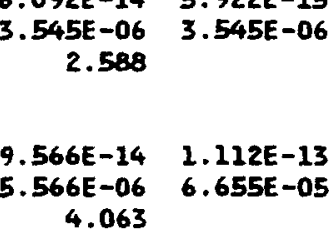

$1.109 E-08 \quad 3.056 E-15 \quad 1.00 E-07$ $6.637 E+00$ 0.567

3.492E-08 9.627E-15 2.00E-08 $2.091 \mathrm{E}+01$

1.786

3.405E-08 9.386E-15 9.00E-09 $2.038 E+01$ 
TRA AIRBORNE WUSTE SUMMARY

FOR JAN THROUEH JUN 1993

\begin{tabular}{|c|c|}
\hline NUCLIDES & $\begin{array}{l}\text { MALF LIFE } \\
\text { (DAYS) }\end{array}$ \\
\hline $\begin{array}{l}\text { LA-140 } \\
\text { CONCEN }(U \mathrm{C} / \mathrm{ML})^{1} \\
\text { CURIES } \\
\% \text { OF TOTAL }\end{array}$ & $1.678 E+00$ \\
\hline $\begin{array}{l}\text { M-24 } \\
\text { CONCEN (UCML) } \\
\text { CURIES } \\
\% \text { OF TOTAL }\end{array}$ & $6.237 E-01$ \\
\hline $\begin{array}{l}\text { PB-88 } \\
\text { CONCEN }(U \mathrm{CML})^{1} \\
\text { CURIES } \\
\% \text { OF TOTAL }\end{array}$ & $1.229 E-02$ \\
\hline $\begin{array}{l}\text { PB-89 } \\
\text { CONCEN (UCML) } \\
\text { CURIES } \\
\text { \% OF TOTAL }\end{array}$ & $1.056 E-02$ \\
\hline $\begin{array}{l}\text { RE-188 } \\
\text { CONCEN (UCML) } \\
\text { CURIES } \\
\% \text { OF TOTAL }\end{array}$ & $7.075 E-01$ \\
\hline $\begin{array}{l}\text { SR-90 } \\
\text { CONCEN (UCAL) } \\
\text { CURIES } \\
\% \text { OF TOTAL }\end{array}$ & $1.059 E+04$ \\
\hline $\begin{array}{l}\text { TC-99N } \\
\text { CONCEN }(U C / M L)^{2} \\
\text { CURIES } \\
\% \text { OF TOTAL }\end{array}$ & $2.504 E-01$ \\
\hline $\begin{array}{l}\text { XE-133 } \\
\text { CONCEN (UCML) } \\
\text { CURIES } \\
\% \text { OF TOTAL }\end{array}$ & $5.250 E+00$ \\
\hline $\begin{array}{l}\text { XE-135 } \\
\text { CONCEN (UCML) } \\
\text { CURIES } \\
\% \text { OF TOTAL }\end{array}$ & $3.792 E-01$ \\
\hline
\end{tabular}

$7.800 E-14$

8.531E-06

$5.882 E-12$ $6.432 E-04$

$4.876 E-12 \quad 4.356 E-12$ 0.001 $5.199 \mathrm{E}-04$ $4.790 E-04$ $.678 E-04$ 0.001
0.04

8.996E-10 4.885E-10 $\quad 6.828 E-10 \quad 6.216 E-10 \quad 1.143 E-09$ $9.839 E-02 \quad 5.331 E-02 \quad 7.281 E-02 \quad 6.835 E-02 \quad 1.204 E-01$ $\begin{array}{rrrrr}0.024 & 0.028 & 0.027 & 0.026 & 0.327\end{array}$

$\begin{array}{lllll}1.307 E-09 & 6.629 E-10 & 9.269 E-10 & 8.397 E-10 & 1.658 E-09\end{array}$ $\begin{array}{lllll}1.429 E-01 & 7.235 E-02 & 9.883 E-02 & 9.233 E-02 & 1.747 E-01\end{array}$ $\begin{array}{rrrrr}0.035 & 0.038 & 0.036 & 0.035 & 0.475\end{array}$

$3.141 E-13$

$3.349 E-05$

$4.574 E-16 \quad 4.319 E-15 \quad 2.468 E-15$

4. $992 E-08 \quad 4.605 E-07 \quad 2.724 E-07$

$\begin{array}{llllll}2.599 E-12 & 4.831 E-12 & 4.140 E-12 & 3.467 E-12 & 7.838 E-13\end{array}$ $\begin{array}{llllll}842 E-04 & 5.272 E-04 & 4.414 E-04 & 3.812 E-04 & 8.258 E-05\end{array}$

1.325E-08 5.151E-09 8.630E-09 4.731E-09 $1.449 E+00 \quad 5.622 E-01 \quad 9.202 E-01 \quad 5.202 E-01$

$\begin{array}{rrrr}0.359 & 0.296 & 0.336 & 0.195\end{array}$

8.129E-08 3.164E-08 5.042E-08 $\quad 4.300 E-08 \quad 6.320 E-09$ $\begin{array}{lllll}8.129 E-08 & 3.164 E-08 & 5.042 E-08 & 4.300 E-08 & 6.320 E-09 \\ 8.890 E+00 & 3.453 E+00 & 5.376 E+00 & 4.728 E+00 & 6.658 E-01\end{array}$ $\begin{array}{rrrrr}2.205 & 3.453 E+00 & 5.376 E+00 & 4.728 E+00 & 6.658 E-01 \\ & 1.820 & 1.963 & 1.773 & 1.810\end{array}$
$2.475 E-11$
PAGE 3 RPT. 104 TRA-A YEARLY $\begin{array}{ll}\text { ANUAL } & \text { YEARLY } \\ \text { AVG CONCEN YEARLY } & \text { RELEASE }\end{array}$ AVG CONCEN YEARLY

MNE

$1.425 E-14 \quad 3.928 E-21 \quad 3.00 E-09$ 8.531E-06

$7.702 E-12 \quad 2.123 E-18 \quad 4.00 E-09$ 4.611E-03

6. $903 E-10 \quad 1.903 E-16 \quad 3.00 E-08$ 4.133E-01

$$
0.035
$$

$9.707 E-10 \quad 2.676 E-16 \quad 9.00 E-09$ $5.811 E-01$

$$
0.050
$$

$5.594 E-14 \quad 1.542 E-20 \quad 6.00 E-09$ $3.349 E-05$

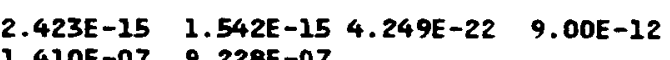

0.103

$2.868 E-12 \quad 7.904 E-29 \quad 1.00 E-07$ $1.717 E-03$

5.766E-09 1.589E-15 5.00E-07 $3.452 E+00$

$$
0.295
$$

3.861E-08 1.064E-14 8.00E-08 $2.311 E+01$ 
TRA AIRBOPNE WASTE SUMARY FOR JAN THROUGH JUN 1993

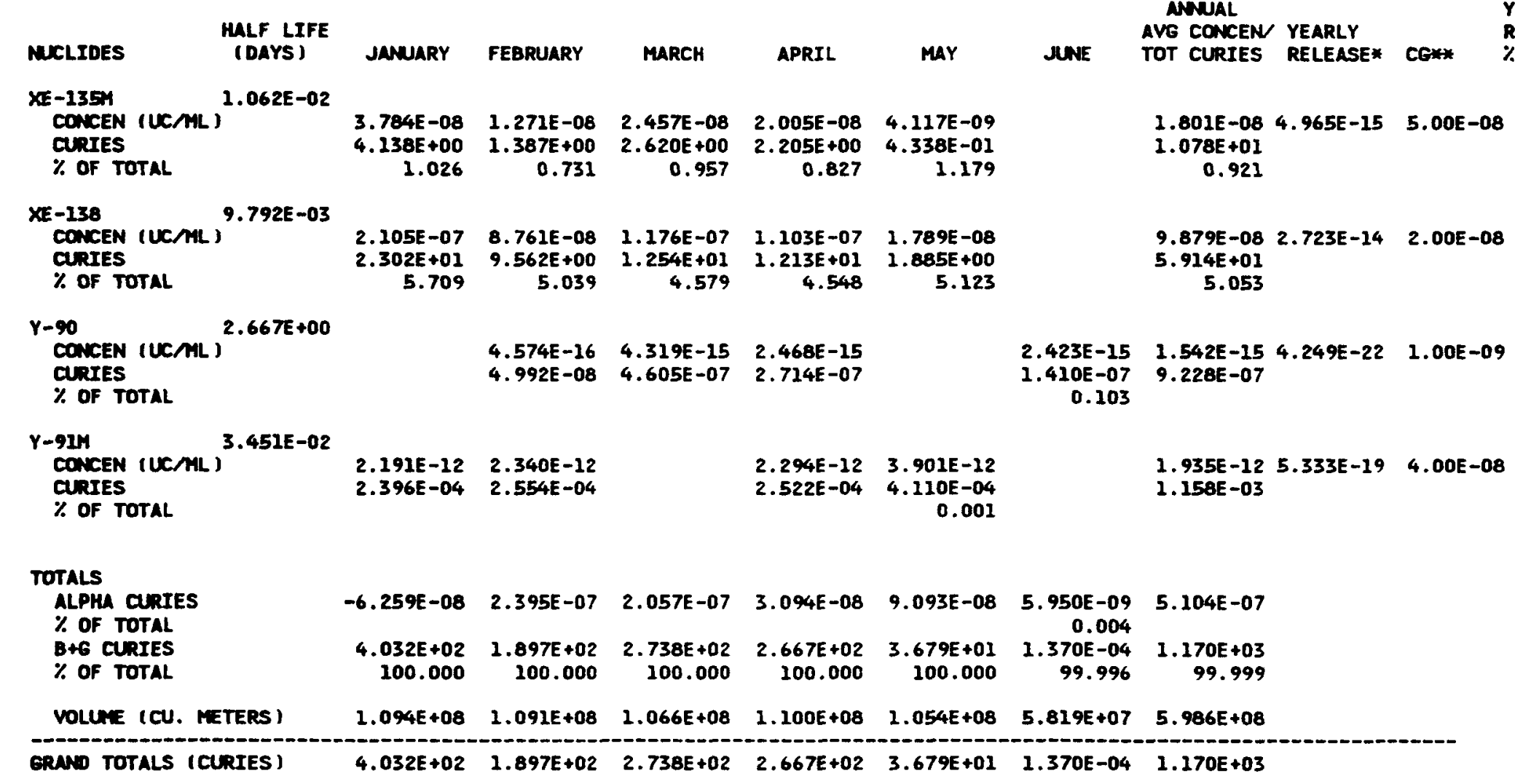

* This nubar is derived from a dispersion factor equation. The dispersion factor is derived from the straight line Goussian model and is provided by the Mtional Dosonic Atmospheric Administration (NOAA).

** NOTE: DCG value obtained from DOE ORDER 5400.5 dated February 8, 1990, Figures III-1 and III-3 


\section{TRA LIOUID WASTE SUMARY}

FOR JAN THROUEH JUN 1993

\begin{tabular}{|c|c|}
\hline MUCLIDES & $\begin{array}{l}\text { HALF LIFE } \\
\text { (DAYS ) }\end{array}$ \\
\hline $\begin{array}{l}\text { CE-141 } \\
\text { CONCEN (UCML) } \\
\text { CURIES } \\
\% \text { OF TOTAL }\end{array}$ & $3.250 E+01$ \\
\hline $\begin{array}{l}\text { CE-144 } \\
\text { CONCEN (UCML) } \\
\text { CURIES } \\
\% \text { OF TOTAL }\end{array}$ & $2.844 E+02$ \\
\hline $\begin{array}{l}\text { CO-58 } \\
\text { CONCEN (UCML) } \\
\text { CURIES } \\
\% \text { OF TOTAL }\end{array}$ & $7.091 E+01$ \\
\hline $\begin{array}{l}\text { CO-60 } \\
\text { CONCEN IUCML) } \\
\text { CURIES } \\
\% \text { OF TOTAL }\end{array}$ & $1.924 E+03$ \\
\hline $\begin{array}{l}\text { CR-51 } \\
\text { CONCEN (UCML) } \\
\text { CURIES } \\
\% \text { OF TOTAL }\end{array}$ & $2.770 E+01$ \\
\hline
\end{tabular}

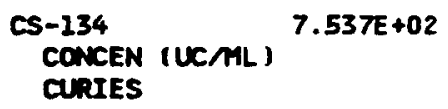

CURIES

$\%$ OF TOTAL

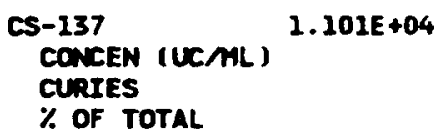

\section{EnOSS-ALPHA \\ CONCEN (UCML) \\ CURIES \\ $\%$ OF TOTAL}

\section{EROSS-BETA \\ CONCEN (LCML) \\ CURIES}

$\%$ OF TOTAL

$\begin{array}{rrr}\text { JANUARY } & \text { FEBRUARY } & \text { MARCH } \\ & & \\ 5.270 E-07 & 2.710 E-07 & \\ 2.799 E-03 & 2.269 E-03 & \\ 0.016 & 0.012 & \\ & & \\ & & \\ 2.850 E-07 & 3.310 E-07 & 2.490 E-07 \\ 1.514 E-03 & 2.772 E-03 & 1.732 E-03 \\ 0.009 & 0.015 & 0.005\end{array}$

$$
\text { APRIL }
$$$$
\text { MaY }
$$$$
\text { JUNE }
$$$$
\begin{array}{r}
32 E-03 \\
0.005
\end{array}
$$

PAGE I RPT. 105 TRA-L
ANRAL
AVG CONCEN

TOT CURIES
$2.205 E-07 \quad 5.00 E-05$
$5.068 E-03$
0.005

$1.431 E-07$ 7.00E-06
$6.017 E-03$

$$
0.006
$$

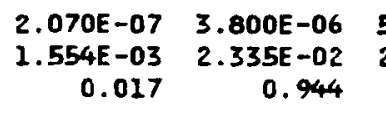

$\begin{array}{lllllll}3.200 E-06 & 1.600 E-05 & 1.700 E-05 & 5.500 E-06 & 2.000 E-05 & 9.400 E-05 & 2.472 E-05\end{array}$ $\begin{array}{lllllll}1.700 E-02 & 1.340 E-01 & 1.182 E-01 & 4.266 E-02 & 1.501 E-01 & 5.775 E-01 & 1.039 E+00\end{array}$
23.360
1.031 
RADIOACTIVE WASTE MANAGEMENT INFORMATION SYSTEM

TRA ITOUTD WASTE SIMMARY FOR JAN THROUGH JUN 1993

\begin{tabular}{|c|c|c|c|c|c|c|c|c|c|c|}
\hline MUCLIOES & $\begin{array}{l}\text { MALF LIFE } \\
\text { (DAYS ) }\end{array}$ & JANUARY & FEBRUARY & MARCH & APRIL & MAY & MUNE & $\begin{array}{l}\text { ANWUAL } \\
\text { AVG CONCEN } \\
\text { TOT CURIES }\end{array}$ & CG * & $\%$ OF CG \\
\hline $\begin{array}{l}\text { H-3 } \\
\text { CONCEN }(U C / M L) \\
\text { CURIES } \\
\% \text { OF TOTAL }\end{array}$ & $4.490 E+03$ & $\begin{array}{r}3.180 E-03 \\
1.689 E+01 \\
99.063\end{array}$ & $\begin{array}{r}2.110 E-03 \\
1.767 E+01 \\
97.011\end{array}$ & $\begin{array}{r}5.000 E-03 \\
3.477 E+01 \\
98.397\end{array}$ & $\begin{array}{r}2.350 E-03 \\
1.823 E+01 \\
99.080\end{array}$ & $\begin{array}{r}1.210 E-03 \\
9.081 E+00 \\
96.585\end{array}$ & $\begin{array}{r}1.280 E-04 \\
7.864 E-01 \\
31.810\end{array}$ & $\begin{array}{r}2.317 E-03 \\
9.742 E+01 \\
96.583\end{array}$ & 2.00E-03 & $\begin{array}{r}115.86 \\
* \quad * *\end{array}$ \\
\hline $\begin{array}{l}M A-24 \\
\text { CONCEN (UCML) } \\
\text { CURIES } \\
\% \text { OF TOTAL }\end{array}$ & $6.237 E-01$ & $\begin{array}{r}1.060 E-06 \\
5.630 E-03 \\
0.033\end{array}$ & $\begin{array}{r}2.040 E-06 \\
2.708 E-02 \\
0.094\end{array}$ & & & & & $\begin{array}{r}5.402 E-07 \\
2.271 E-02 \\
0.023\end{array}$ & $1.00 E-04$ & 0.54 \\
\hline $\begin{array}{l}\text { S8-124 } \\
\text { CONCEN (UCML) } \\
\text { CURIES } \\
\% \text { OF TOTAL }\end{array}$ & $6.020 E+01$ & & & & & & $\begin{array}{r}2.510 E-07 \\
1.542 E-03 \\
0.062\end{array}$ & $\begin{array}{r}3.668 E-08 \\
1.542 E-03 \\
0.002\end{array}$ & 1.00E-05 & 0.37 \\
\hline $\begin{array}{l}\text { SR-90 } \\
\text { CONCEN (UCML) } \\
\text { CURIES } \\
\% \text { OF TOTAL }\end{array}$ & $1.059 E+04$ & & & $\begin{array}{r}7.900 E-07 \\
5.494 E-03 \\
0.016\end{array}$ & $\begin{array}{r}2.890 E-06 \\
2.242 E-02 \\
0.122\end{array}$ & $\begin{array}{r}6.800 E-06 \\
5.103 E-02 \\
0.543\end{array}$ & & $\begin{array}{r}1.878 E-06 \\
7.895 E-02 \\
0.078\end{array}$ & $1.00 E-06$ & $\begin{array}{l}187.76 \\
* * * * * *\end{array}$ \\
\hline $\begin{array}{l}\text { TA-182 } \\
\text { CONCEN (UCML) } \\
\text { CURIES } \\
\% \text { OF TOTAL }\end{array}$ & $1.145 E+02$ & & & $\begin{array}{r}1.480 E-07 \\
1.029 E-03 \\
0.003\end{array}$ & & & & $\begin{array}{r}2.448 E-08 \\
1.029 E-03 \\
0.001\end{array}$ & $2.00 E-05$ & 0.12 \\
\hline $\begin{array}{l}Y-90 \\
\text { CONCEN (UCML) } \\
\text { CURIES } \\
\% \text { OF TOTAL }\end{array}$ & $2.667 E+00$ & & & $\begin{array}{r}7.900 E-07 \\
5.494 E-03 \\
0.016\end{array}$ & $\begin{array}{r}2.890 E-06 \\
2.242 E-02 \\
0.122\end{array}$ & $\begin{array}{r}6.800 E-06 \\
5.103 E-02 \\
0.543\end{array}$ & & $\begin{array}{r}1.878 E-06 \\
7.895 E-02 \\
0.078\end{array}$ & $1.00 E-05$ & 18.78 \\
\hline $\begin{array}{l}2 N-65 \\
\text { CONCEN (UCML) } \\
\text { CURIES } \\
\% \text { OF TOTAL }\end{array}$ & $2.438 E+02$ & $\begin{array}{r}2.060 E-07 \\
1.094 E-03 \\
0.006\end{array}$ & $\begin{array}{r}5.390 E-07 \\
4.514 E-03 \\
0.025\end{array}$ & $\begin{array}{r}6.110 E-07 \\
4.249 E-03 \\
0.012\end{array}$ & & $\begin{array}{r}1.400 E-07 \\
1.051 E-03 \\
0.011\end{array}$ & $\begin{array}{r}1.140 E-06 \\
7.004 E-03 \\
0.283\end{array}$ & $\begin{array}{r}4.260 E-07 \\
1.791 E-02 \\
0.018\end{array}$ & $9.00 E-06$ & 4.73 \\
\hline $\begin{array}{l}\text { TOTALS } \\
\text { ALPHA CURIES } \\
\% \text { OF TOTAL } \\
\text { B+G CURIES } \\
\% \text { OF TOTAL }\end{array}$ & & $\begin{array}{r}1.705 E+01 \\
100.000\end{array}$ & $\begin{array}{r}1.821 E+01 \\
100.000\end{array}$ & $\begin{array}{r}3.534 E+01 \\
100.000\end{array}$ & $\begin{array}{r}1.629 E-03 \\
0.009 \\
1.840 E+01 \\
99.991\end{array}$ & $\begin{array}{r}2.327 E-03 \\
0.025 \\
9.400 E+00 \\
99.975\end{array}$ & $\begin{array}{r}1.044 E-03 \\
0.042 \\
2.471 E+00 \\
99.958\end{array}$ & $\begin{array}{r}5.000 E-03 \\
0.005 \\
1.009 E+02 \\
99.995\end{array}$ & & \\
\hline VOLUAE ILITERS & & $5.311 E+06$ & $8.374 E+06$ & $6.954 E+06$ & $7.757 E+06$ & $7.505 E+06$ & $6.144 E+06$ & $4.205 E+07$ & & \\
\hline GRANO TOTALS ICU & RIES ] & $1.705 E+01$ & $1.821 E+01$ & $3.534 E+01$ & $1.840 E+01$ & $9.402 E+00$ & $2.472 E+00$ & $1.009 E+02$ & & \\
\hline
\end{tabular}

TRA-L

* NOTE: DCG value obtained from DOE ORDER 5400.5 deted February8, 1990, Figures III-I and III-3 
RADIOACTIVE WASTE MANAGEMENT INFORMATION SYSTEM

TRE LIOUID WESTE SUMARY

FOR JAN THROUGH JUN 1993

PAGE 3 RPT. 105

MCLIDES

HALF LIFE

JANUARY FEBRUARY

MARCH

APRIL

MaY

JUNE

AVG CONCEN

TOT CURIES CG * \% OF CG

* The CG limit is baing approached or has bean exceedbd during the current month. Please verify the accuracy of the reported date. If the data is in arror, please send correct data to laste Information. 
SCHED. NO. P62PM009-G

IDAHO OPERATIONS OFFICE

U.S. DEPARTMENT OF ENERGY

RADIOACTIVE WASTE MANAGEMENT INFORMATION SYSTEM

TRA DISPOSEO SOLID WASTE SUMARY

FOR JAN THROUGH JUN 1993

MUCLIDES
M-241
CURIES
$\%$ OF TOTAL
ERMS

C-14

CURIES

$\%$ OF TOTAL

CE-144

CURIES

$\%$ Of TOTAL

CH-242

CURIES

$\%$ OF TOTAL

CM-244

CURIES

$\%$ OF TOTAL

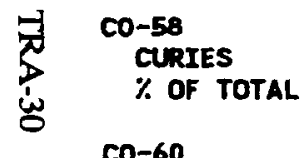

c0-60

$\%$ OF TOTAl

CR-51

CURIES

$\%$ OF TOTAL

CS-134
CURIES

$\%$ OF TOTAL

CS-137

CURIES

$\%$ of total

$\begin{array}{ll}\text { FE-55 } & 9.782 E+02 \\ \text { CURIES } & \\ \% \text { OF TOTAL } & \\ \begin{array}{l}\text { H-3 } \\ \text { CURIES }\end{array} & 4.490 E+03\end{array}$

\section{HALF LIFE}

(DAYS )

JANUARY FEBRUARY

MARCH

APRIL

MAY

$7.540 E-08$

JUNE

\section{ANNUAL}

TOTAL

$2.192 E-08$

$7.540 E-08$

$2.192 E-08$

$1.400 \mathrm{E}-02$

0.001

$2.844 E+02$

$1.630 E+02$

$6.610 E+03$

$7.091 E+01$

$1.924 E+03$

$2.770 E+01$

$7.537 E+02$

$1.101 E+04$

$5.000 E+00$

0.424

$2.850 E+02$

24.250

$6.900 E+02$

$100 E-04$
RUN DATE: 05/12/94

PAGE 1 RPT 106

TRA-S 
RADIOACTIVE WASTE MANAGEMENT INFORMATION SYSTEM

TRA DISPOSED SOLID MASTE SUMMRY

FOR JAN THROUGH JUN 1993

MCLIDES
HF-181
CURIES
$\%$ OF TOTAL
I-13I
CURIES
$\%$ OF TOTAL

\section{WN-54}

$\%$ OF TOTAL

HALF LIFE
(DAYS )

JANAARY FEBRUARY

MARCH

APRIL

MaY

JUNE

ANMAL

4. $240 E+01$

$8.040 E+00$

$3.122 E+02$

N3-94

CURIES

$\%$ OF TOTAL

$7.300 E+06$

$\mathrm{NB}-95$

CURIES

$\%$ OF TOTAL

$3.498 E+01$

$2.774 E+07$

II -59

\% OF TOTAL

心 NI-63

CURIES

$\%$ of total

PU-238

CURIES

$\%$ of total

ERAMS

CURIES

$\%$ OF TOTAL

earus

RU-103

CURIES

$\%$ OF TOTAL

SB-124

$\%$ OF TOTAL

s8-125

CURIES

$\%$ of total
8. $800 E+06$

3. $924 E+01$

$3.650 E+04$

$3.203 E+04$

$6.020 E+01$

$1.007 E+03$
$5.900 E-03 \quad 5.900 E-03$

$6.400 E-03 \quad 6.400 E-03$

$8.300 E+01$

4. 000E-04 8.300E+01

0.041

$9.000 E-07$

$4.360 E-02 \quad 4.360 E-02$

1. 120E-D1

$1.120 E-01$

0.009

$1.500 E+01$

$1.020 E+03$

$1.020 E+03 \quad 2.055 E+03$

1.025

$1: 027$

1.027

4. 280E-06

4.280E-06

2.503E-07

2.503E-07

3.800E-07

$3.800 E-07$

$6.119 E-06$

6.119E-06

$1.700 E-03 \quad 1.700 E-03$

5. 000E-04 5.000E-04

5. $000 E+00$

0.424
$9.000 E-07$
PAGE 2 RPT 106

TRA-S 

RADIOACTIVE WASTE MANAGEMENT INFORMATION SYSTEM

TRA DISPOSEC SOLID KASTE SUMMARY FOR JAN THROUGH JUN 1993

\begin{tabular}{|c|c|}
\hline MUCLIDES & $\begin{array}{l}\text { HALF LIFE } \\
\text { (DAYS) }\end{array}$ \\
\hline $\begin{array}{l}\text { SC- } 96 \\
\text { CURIES } \\
\% \text { OF TOTAL }\end{array}$ & $8.382 E+01$ \\
\hline $\begin{array}{l}\text { SE }-75 \\
\text { CURIES } \\
\% \text { OF TOTAL }\end{array}$ & $1.198 E+02$ \\
\hline $\begin{array}{l}\text { SN-IIIM } \\
\text { CURIES } \\
\% \text { OF TOTAL }\end{array}$ & $2.930 E+02$ \\
\hline $\begin{array}{l}\text { SR-90 } \\
\text { CURIES } \\
\% \text { OF TOTAL }\end{array}$ & $1.059 E+04$ \\
\hline $\begin{array}{l}\text { TC- } 99 \\
\text { CURIES } \\
\% \text { OF TOTAL }\end{array}$ & $7.775 E+07$ \\
\hline $\begin{array}{l}2 \mathrm{~N}-65 \\
\text { CURIES } \\
\% \text { OF TOTAL }\end{array}$ & $2.438 E+02$ \\
\hline $\begin{array}{l}\text { ZR-95 } \\
\text { CURIES } \\
\% \text { OF TOTAL }\end{array}$ & $6.403 E+01$ \\
\hline
\end{tabular}

\section{JANUARY FEBRUARY}

\begin{abstract}
MARCH
\end{abstract}
APRIL

MaY

JUNE

ANNAL

PAGE 3 RPT 106

TRA-S

APRIL

TOTAL

1.000E-03 2.000E-03

$9.000 E-03 \quad 9.000 E-03$

$1.500 E+01$

1.271

$1.500 E+01$

0.007

$2.500 E-09$

$3.400 E-03 \quad 3.400 E-03$

$2.500 E-0.5$

2.500E-05

8. $200 E+01$

$.600 E-03 \quad 8.200 E+01$

6.948

0.041

7.500 E-03 7.500E-03

\section{TOTALS}

ALPHA CURIES

$\%$ OF TOTAL

B+G CURIES

$\%$ of total

TOTAL ERANS OF MM

TOTAL ERANS OF M

TOTAL ERNAS Of PU

TOTAL ERANS OF PU-239+241

TOTAL ERANS OF PU

TOTAL ERAYS OF TH

TOTAL ERANS OF NP

VOLUME (CU. METERS )

WEIGI (KILOERUNS)

$7.640 E-01$

$\begin{array}{lll}4.384 E+00 & 6.709 E+00 & 1.186 E+01 \\ 5.965 E+02 & 8.459 E+03 & 9.464 E+03\end{array}$

GRUNO TOTALS (CURIES)

$4.080 E+02$

4.750E-06 4.750E-06

$1.180 E+03$

100.000

$9.950 \mathrm{E}+04$
100.000

$.933 E+04$

100.000

$.000 E+05$

100.000

1.180E+03

$9.950 E+04 \quad 9.933 E+04 \quad 2.000 E+05$ 
TRA Facility Detail Data for Months July to December Annual Average Concentration and Total Curies for January to December 
. 
RADIOACTIVE WASTE MANAGEMENT INFORMATION SYSTEM

\section{ALPHA LAB AIR AIRBORNE WUSTE SUMAARY} FOR JAN THROUEH DEC 1993

MUCLIDES

MALF LIF

EROSS-ALPHA

CONCEN (UCAL)

CORIES

UACERTICURIES

(NCERTIUCMU

$\%$ OF TOTAL

CONCEN (UCML)

UACERT ( CURIES

UNCERTILCAL

$\%$ OF TOTAL

(DAYS)

JULY

Aumst

SEPTEMBER

OCTOaER
enoss-8ETA

$\begin{array}{lllllll}3.564 E-16 & -6.651 E-17 & 1.187 E-16 & 3.599 E-17 & -1.708 E-16 & -1.913 E-16 & -5.212 E-17-1.109 E-23 \\ 7.210 E-09 & -1.390 E-09 & 2.480 E-09 & 7.280 E-10 & -3.570 E-09 & -3.870 E-09 & -1.293 E-08\end{array}$

4.250E-09 4.490E-09 4.DOOE-09 4.930E-09 6.310E-09 5.8BOE-09
10.154
$-1.871$
1.930
1.467
$-8.787$
$-15.777$
$-1.467$

$\begin{array}{lllllllll}3.154 E-15 & 3.622 E-15 & 6.029 E-15 & 2.417 E-15 & 2.215 E-15 & 1.404 E-15 & 3.606 E-15 & 7.675 E-22 \\ 6.380 E-08 & 7.570 E-08 & 1.260 E-07 & 4.890 E-08 & 4.420 E-08 & 2.840 E-08 & 8.947 E-07 & \end{array}$

$\begin{array}{llllll}7.610 E-09 & 8.730 E-09 & 9.690 E-09 & 7.640 E-09 & 9.320 E-09 & 8.130 E-09\end{array}$

$\begin{array}{lllllll}89.847 & 101.871 & 98.070 & 98.533 & 108.787 & 115.777 & 101.467\end{array}$

\section{TOTALS}

ALPHA CURIES

$\%$ OF TOTAL

B to curies

$\%$ OF TOTAL$$
10.154
$$

10.154
$380 E-08$

$6.380 E-08$
89.847

$390 E-09$
-1.871

570E-08
PAGE 1 RPT. 115 TRATRA-A

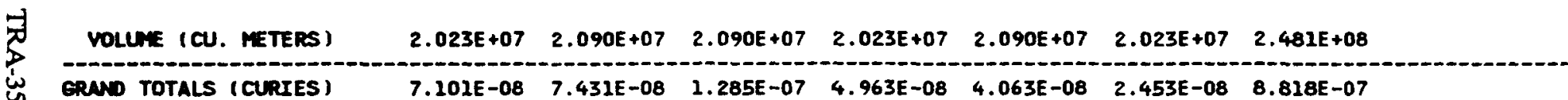

* This nuber is darived from a dispersion factor aqution. The dispersion factor is derived from the straight line gavesien model and is proveded by the National Oceanic Atmospheric Adninistration (NOAA).

* NOTE: DCG value obtainad from DOE ORDER 5400.5 dated Fabruary 8, 1990, Figures III-1 and III-3 
RADIOACTIVE WASTE MUNAGEMENT INFORMATION SYSTEM

\section{ATR AIRBORNE WASTE SUMTARY} FOR JAN THROUGH DEC 1993

$\begin{array}{ll}\text { MUCLIDES } & \begin{array}{c}\text { MALF LIFE } \\ \text { (DAYS) }\end{array} \\ \text { AR-41 } & \\ \text { CONCEN (UCAML) } & 7.625 E-02 \\ \text { CURIES } & \text { UNCERT(CURIES) } \\ \text { UNCERT (UCAML) } \\ \text { \% OF TOTAL }\end{array}$

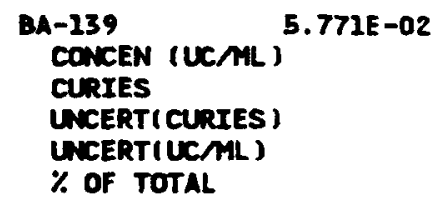

BA-140
CONCEN (UCML)
CURIES
UNCERT(CURIES)
UNCERT(UCML)
\% OF TOTAL
CO-58
CONCEN (UCML)
CURIES
UNCERT(CURIES)
UNCERT(UCML)
\% OF TOTAL
CO-60
CONCEN (UCML)
CLRIES
UNCERT(CURIES)
UNCERT(UCAL)
\% OF TOTAL

JULY

AUEAST

SEPTEMBER

BER OCTOBER

S. DEPARTMENT OF ENERGY

PAGE 1 RPT. 120 TRA ATR-A

ANNUAL

$\begin{array}{lll}\text { AVG CONCEN } & \text { YEARLY } & \text { RELEASE } \\ \text { TOT CURIES } & \text { RELEASE* CG* } & \% \text { OF CG }\end{array}$

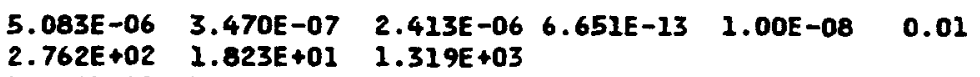

$\begin{array}{llllll}5.083 E-06 & 3.470 E-07 & 2.413 E-06 & 6.651 E-13 & 1.00 E-08 & 0.01\end{array}$
90.740
89.481
88.203

8.142E-12 9.722E-11 5.532E-11

$9.806 E-11 \quad 2.703 E-17 \quad 7.00 E-08$ $4.250 E-04 \quad 5.283 E-03 \quad 2.906 E-03 \quad 5.359 E-02$

$8.660 E-05 \quad 1.460 E-04 \quad 1.720 E-04$
3.758
0.002
0.014
0.004

$1.142 E-14 \quad 3.149 E-21 \quad 3.00 E-09$ 6. $243 E-06$

$1.460 E-15 \quad 4.025 E-22 \quad 2.00 E-09$ $7.981 E-07$

$\begin{array}{lllllllll}7.385 E-13 & 6.743 E-13 & 1.696 E-12 & 7.989 E-13 & 1.325 E-13 & 2.395 E-13 & 4.413 E-13 & 1.216 E-19 & 8.00 E-11\end{array}$ $\begin{array}{llllllll}3.539 E-05 & 3.462 E-05 & 1.452 E-05 & 4.170 E-05 & 7.146 E-06 & 1.258 E-05 & 2.411 E-04\end{array}$

$1.390 E-06 \quad 8.200 E-07 \quad 1.310 E-06 \quad 1.710 E-06 \quad 7.100 E-07 \quad 9.500 E-07$
72.402
49.786
98.905
0.369

FARLY

OF CG

CR-51 $2.770 E+01$ CONCEN (UCML) CunIEs

1. 141E-13 $5.468 E-06$ 2.050E-06 UNCERTCURIES \% OF TOTAL

11.187 $\begin{array}{lllll}3.530 E-12 & 4.822 E-12 & 7.029 E-12 & 1.938 E-18 & 5.00 E-08 \\ 1.918 E-04 & 2.533 E-04 & 3.841 E-03 & & \end{array}$

5.500E-06 6.700E-06

0.001

CS-137

$1.101 E+04$

CONCEN (UC/ML)

CURIES

$5.499 E-14$

$2.635 E-06$

$7.890 E-07$

5.391
$1.720 E-14$
$8.980 E-07$
4.210E-07

0,008

$\%$ OF total

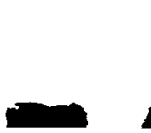


RADIOACTIVE WASTE MANAGEMENT INFORMATION SYST
ATR AIRBORNE WASTE SUMMARY FOR JAN THROUGH DEC 1993

MUCLIDES
$\begin{aligned} & \text { MLLF LIFE } \\ & \text { (DAYS) }\end{aligned}$
CS-138
CONCEN (UCML)
CURIES
UNCERT(CURIES)
UNCERT(UCAL)
\% OF TOTAL

\section{GROSS-ALPHA}

CONCEN (UCML)

CURIES

UNCERTICURIES

UNCERT (UCAIL

$\%$ OF TOTAL

\section{GROSS-BETA}

CONCEN (UCML)

CURIES

UNERT CURIES

UNCERT UCAM

\section{HF-181} $4.240 E+01$

CONCEN (UCAL)

CURIES

UNCERTICURIES )

UNCERT (UCML)

$\%$ OF TOTAL

H6-203
CONCEN (UCML)
CURIES
UNCERT(CURIES)
UNERT(UCAL)
\% OF TOTAL
I-131
CONCEN (UCML)
CURIES
UNERTICURIES)
UNCERT(UCAL)
\% OF TOTAL
I-132
CONCEN (UCML)
CURIES
UNCERTICURTES)
UNCERT(UCAL)
\% OF TOTAL

AUMUST SEPTEMBER OCTOBER MOVEMER DECEMER

NLY

1. $124 E-13$

5.387E-06

6.470E-07

11.021
$4.871 E-16$
$2.501 E-08$
$3.530 E-08$

0.036

\section{$\begin{array}{llllllll}6.772 E-13 & 7.796 E-15 & 5.421 E-13 & 4.367 E-14 & 9.252 E-13 & 6.259 E-13 & 1.725 E-19\end{array}$ \\ $\begin{array}{lllllll}3.477 E-05 & 6.676 E-08 & 2.830 E-05 & 2.373 E-06 & 4.860 E-05 & 3.421 E-04\end{array}$

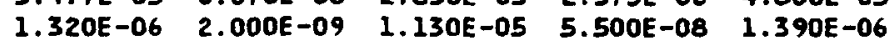

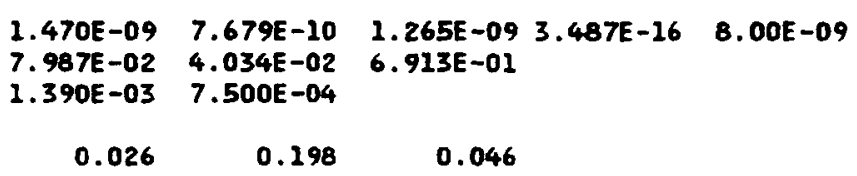

0.026

0.198

0.046

$\begin{array}{llll}3.627 E-17 & 4.093 E-15 & 1.225 E-15 & 3.377 E-22\end{array}$

$2.971 E-09 \quad 2.250 E-07 \quad 6.696 E-07$

$2.460 E-09 \quad 6.890 E-08$
PAGE 2 RPT. 120 TRNATR-A

YEARLY RELEASE \% OF CG
$6.487 E-15 \quad 1.788 E-21 \quad 9.00 E-10$

$3.545 E-06$

$1.309 E-13 \quad 1.687 E-13 \quad 1.609 E-23 \quad 4.434 E-20 \quad 2.00 E-09$ 7.112E-06 8.863E-06 8.791E-05

1.560E-06 9.540E-D7

2.011E-13 $\quad 1.559 E-13 \quad 1.991 E-13 \quad 5.488 E-20 \quad 4.00 E-10$ $3.093 E-05 \quad 8.187 E-06 \quad 2.088 E-04$

$2.020 E-06 \quad 1.720 E-06$
$2.036 E-12$
$1.113 E-03$ 
RADIOACTIVE WASTE MANAGEMENT INFORMATION SYSTEM

ATR ATRBORNE WASTE SUMYARY FOR JAN THROUGH DEC 1993

\begin{tabular}{|c|c|}
\hline MCLIDES & $\begin{array}{l}\text { HALF LIFE } \\
\text { (DAYS) }\end{array}$ \\
\hline $\begin{array}{l}\text { I-133 } \\
\text { CONCEN (UCML) } \\
\text { CURIES } \\
\text { UNCERT (CURIES) } \\
\text { UNCERT (UCAML) } \\
\text { \% OF TOTAL }\end{array}$ & $8.667 E-01$ \\
\hline $\begin{array}{l}\text { KR-85M } \\
\text { CONCEN (UCML) } \\
\text { CURIES } \\
\text { UNCERT(CURIES) } \\
\text { UNCERT(UCML) } \\
\% \text { OF TOTAL }\end{array}$ & $1.867 E-01$ \\
\hline $\begin{array}{l}\text { KR-87 } \\
\text { CONCEN (UCML) } \\
\text { CURIES } \\
\text { UNCERT(CURIES) } \\
\text { UNCERT(UCML) } \\
\text { \% OF TOTAL }\end{array}$ & 5.278E-02 \\
\hline $\begin{array}{l}\text { KR-8B } \\
\text { CONCEN (UCML) } \\
\text { CURIES } \\
\text { UNCERT(CURIES) } \\
\text { UNCERT(UC/ML) } \\
\text { \% OF TOTAL }\end{array}$ & $1.183 E-01$ \\
\hline $\begin{array}{l}\text { LA-240 } \\
\text { CONCEN (UCML) } \\
\text { CURIES } \\
\text { UNCERT (CURIES) } \\
\text { UNCERT (UCML) } \\
\% \text { OF TOTAL }\end{array}$ & $1.678 E+00$ \\
\hline $\begin{array}{l}\text { MA-24 } \\
\text { CONCEN (UCML) } \\
\text { CURIES } \\
\text { UNCERT(CURIES) } \\
\text { UNCERT (UCML) } \\
\text { \% OF TOTAL }\end{array}$ & $6.237 E-01$ \\
\hline $\begin{array}{l}\text { PB-8B } \\
\text { CONCEN (UCML) } \\
\text { CURIES } \\
\text { UNCERT (CURIES) } \\
\text { UNCERT(UCML) } \\
\text { \% OF TOTAL }\end{array}$ & $1.229 E-02$ \\
\hline
\end{tabular}

JULY AUGUST SEPTEMBER OCTOBER NOVEMBER DECEMBR
PAGE 3 RPT. 120 TRNATR-A

ANUAL AVG CONCE YEARLY YEARLY RELEASE \% OF CG

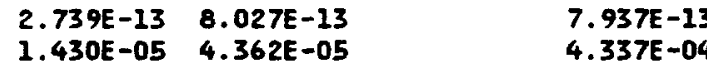

0.126

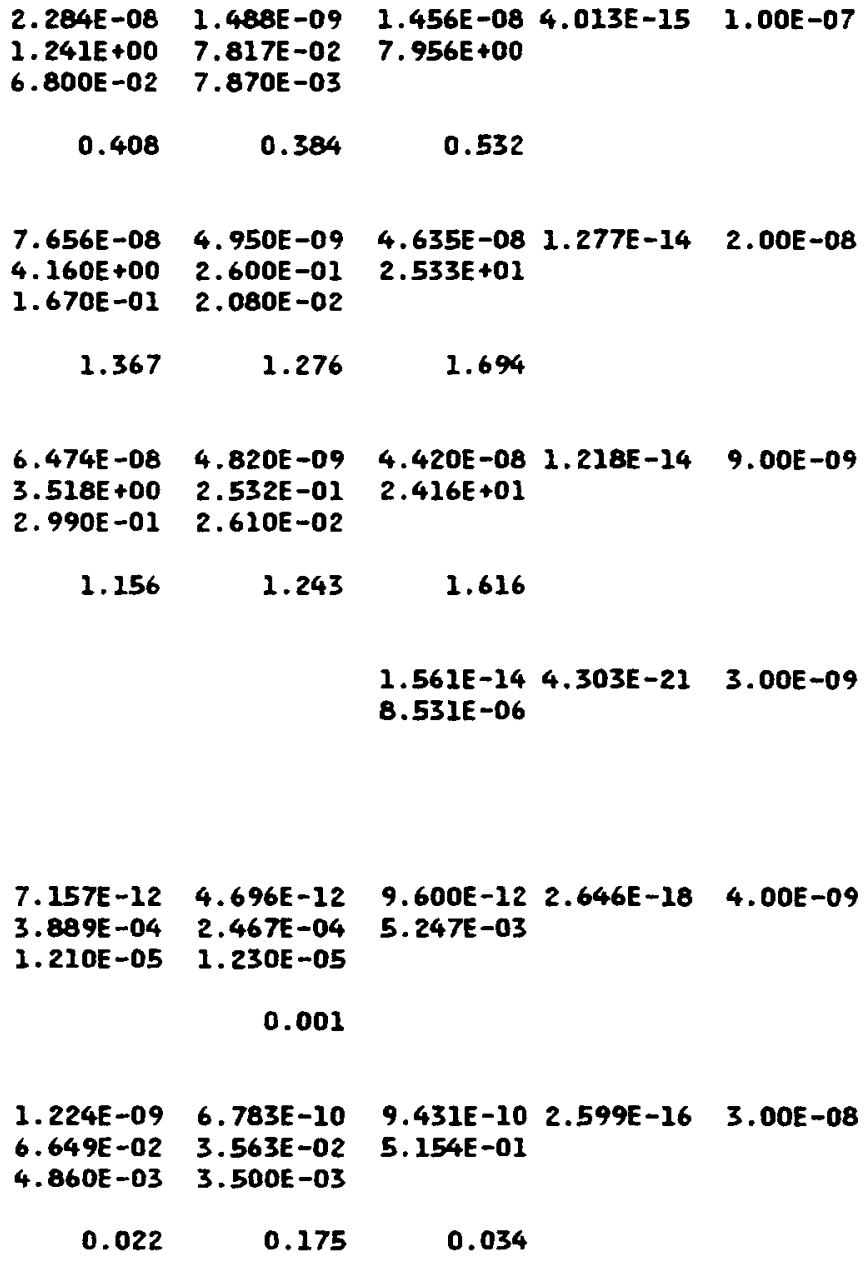
0.022
0.175
0.034 

FOR JAN THROUGH DEC 1993

ATR AIRBORNE WASTE SUMARY

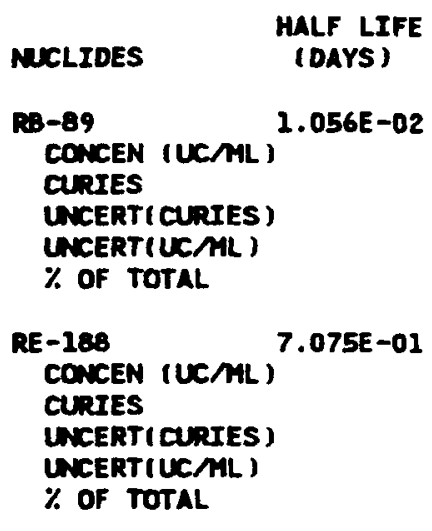

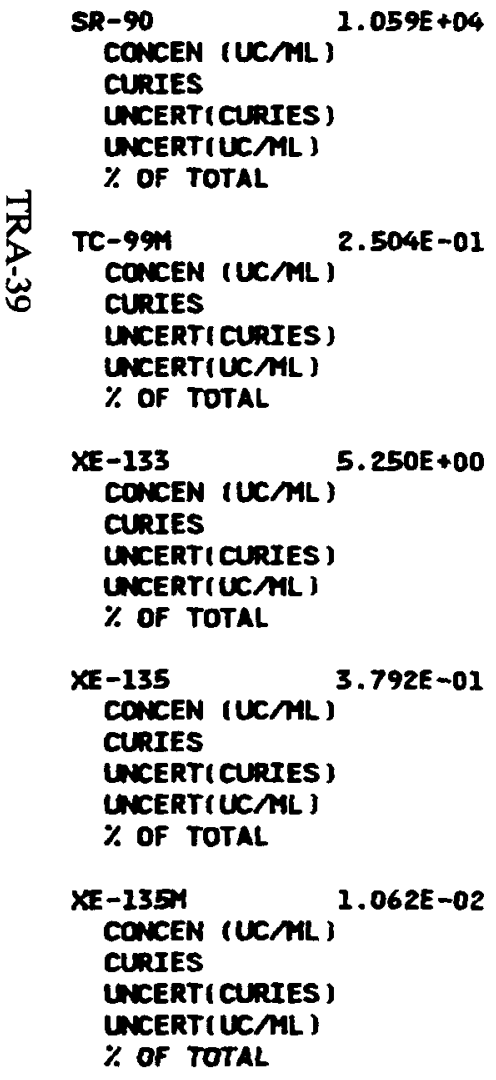

$1.188 E-15 \quad 5.489 E-15$

$6.100 E-08 \quad 4.700 E-08$

$1.220 E-08 \quad 2.400 E-08$
0.088
0.320

PAGE 4 RPT. 120 TRAATR-A

ANUAL AVE CONCEN YEARLY YEARLY TOT CURIES RELEASE* CG** $\%$ OF CG $\begin{array}{llllll}2.069 E-10 & 1.677 E-09 & 8.515 E-10 & 1.332 E-09 & 3.671 E-16 & 9.00 E-09\end{array}$

$1.080 E-02 \quad 9.121 E-02 \quad 4.473 E-02 \quad 7.278 E-01$

\begin{tabular}{l}
$1.530 E-03 \quad 3.160 E-03 \quad 2.060 E-03$ \\
\hline
\end{tabular}
95.489
0.030
0.220
0.049

$6.128 E-14 \quad 1.689 E-20 \quad 6.00 E-09$ $3.349 E-05$

$1.815 E-15 \quad 5.004 E-22 \quad 9.00 E-12$

$1.883 E-15$

$1.023 E-07$

$9.921 E-07$

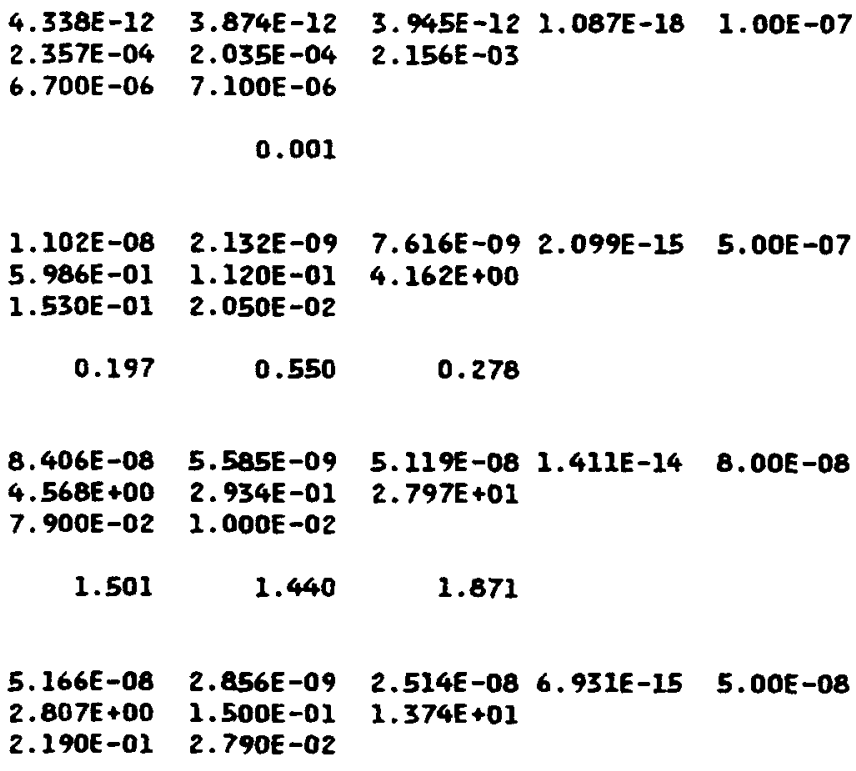

0.001

$\begin{array}{lllll}1.102 E-0 B & 2.132 E-09 & 7.616 E-09 & 2.099 E-15 & 5.00 E-07\end{array}$ 5.986E-01 1.120E-01 4.162E+00

$1.530 E-01 \quad 2.050 E-02$

$\begin{array}{lll}0.197 & 0.550 & 0.278\end{array}$

8.406E-08 5.585E-09 5.119E-08 $1.411 E-14 \quad 8.00 E-08$ $4.56 B E+00 \quad 2.934 E-01 \quad 2.797 E+01$

7.900E-02 1.000E-02

$\begin{array}{lll}1.501 & 1.440 & 1.871\end{array}$

5.166E-08 2.856E-09 2.514E-08 6.931E-15 5.00E-08 $2.807 E+00 \quad 1.500 E-01 \quad 1.374 E+01$

$2.190 E-012.790 E-02$
0.922
0.736
0.919 
RADIOACTIVE WUSTE MANAGEMENT INFORMATION SYSTEM

FOR JN THROUGH DEC 1993

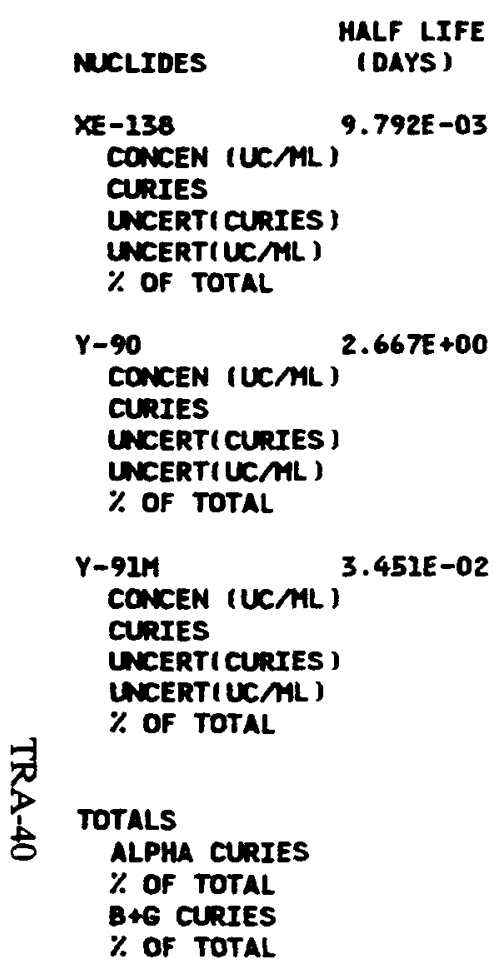

AUEUST

SEPTEMBER

OCTOBER

$1.188 E-15 \quad 5.489 E-15$

6.100E $-08 \quad 4.700 E-08$

1.220E-OB $2.400 E-0 B$

0.088

0.320

NOVEFBER

DECEMER

5 RPT. 120

ANNAL TRNATR-A

AVG CONCEN YEARLY

VATR-A YEARLY

TOT CURIES RELEASE* CG** RELEASE

$2.033 E-07 \quad 1.660 E-08 \quad 1.300 E-07 \quad 3.584 E-14 \quad 2.00 E-08$

$1.105 E+01 \quad 8.718 E-01 \quad 7.106 E+01$

4.400E-01 5.620E-02

3.630

4.279

4.753

1. $883 E-15$

$1.023 E-07$

$1.815 E-15$
$5.004 E-22 \quad 1.00 E-09$

$\begin{array}{lllll}3.289 E-12 & 4.470 E-12 & 2.876 E-12 & 7.927 E-19 & 4.00 E-08\end{array}$ $1.787 E-04 \quad 2.34 B E-04$ 1.572E -03

$7.630 E-05 \quad 7.590 E-05$

0.001

$\begin{array}{rrrrrrr} & 2.501 E-08 & & & 1.971 E-09 & 2.150 E-07 & 6.696 E-07 \\ 4.888 E-05 & 6.951 E-05 & 1.468 E-05 & 1.131 E-02 & 3.044 E+02 & 2.037 E+01 & 1.495 E+03 \\ 100.000 & 99.964 & 100.000 & 100.000 & 100.000 & 100.000 & 99.999\end{array}$

VOLUME (CU. METERS)

$\begin{array}{llll}5.220 E+07 & 5.434 E+07 & 5.253 E+07 & 5.465 E+08\end{array}$

ERND TOTALS (CURIES)

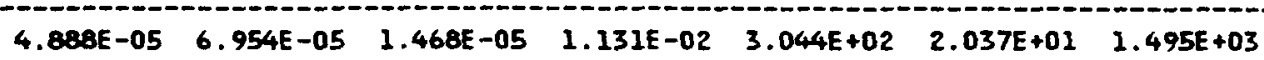

* This number is derived from a dispersion factor equetion. The dispersion factor is derived from the straight line ourseien model and is proveded by the National Oceanic Atmospheric Administration (NOAA).

** NOTE: DCG value obtainad fron DOE ORDER 5400.5 dated February 8, 1990 , Figures III-1 and III-3 


HALF LIFE
(DAYS)

N4-241

CURIES

\% OF TOTAL

epars

BA-140

CURIES

$\%$ OF TOTAL

C- 14

CURIES

$\%$ OF TOTAL

\section{CE-141}

CURIES

$\%$ Of TOTAL

CE-144

CURIES

$\%$ Of TOTAL

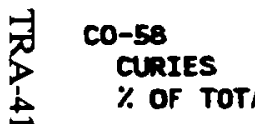

$\%$ OF TOTAL

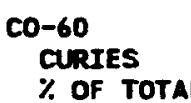

CR-51

CURIES
$\%$ OF TOTAL

CS-134

CURIES

$\%$ of total

CS-137

$\%$ OF TOTAL

EU-154

CURIES

$\%$ of total

FE-55

CURIES

\% OF TOTAL

$1.924 E+03$

$\begin{array}{rrr}5.164 E-01 & 2.843 E+02 & 4.537 E-02 \\ 3.932 & 0.290 & 4.759\end{array}$

$2.770 E+01$

2.000E+00 6.443E-01 8.813E-01

$$
\begin{array}{rrr}
15.227 & 0.001 & 92.441
\end{array}
$$

$7.537 E+02$

3.189E-02 7.290E-02 3.000E-04

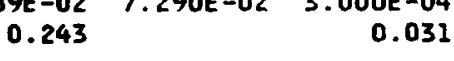

$1.261 E+00 \quad 2.086 E-01$

9.601

1. 760E-02

$$
0.134
$$

$9.782 E+02$
ATR DISPOSED SOLID WASTE SUMARY

FOR JAN THROUGH DEC 1993

$2.577 E+0.5$

JUL

1.490E-02

0.113

4.331E-03

$1.276 E+01$

3.600E -03

0.027

$2.092 E+06$

$3.250 E+01$

7. 900E-03 2.730E-02 0.060

$2.844 E+02$

$$
\begin{array}{rr}
1.550 E-02 & 5.614 E-01 \\
0.218 & 0.001
\end{array}
$$

$7.091 E+01$

$$
\begin{array}{r}
350 E-02 \\
0.103
\end{array}
$$

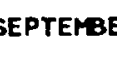

OCTOBER NOVEMBER DECEMBER

MANAL

TOTAL

PAgE I RPT. 122

TRUATR-S

$1.490 E-02$

4.331E-03

$3.600 E-03$

$1.400 E-02$

$3.520 E-02$

5.797E-01

$5.223 E+00$

0.002

$1.134 E+03$

0.380

4.672E+01

0.016

$1.060 E-01$

$1.475 E+00$

1.760E-02 
SCHED. NO. P61PMOO9-H

IDAHO OPERATIONS OFFICE

MCLIDES

$\%$ OF TOTAL

HF-181

CURIES

$\%$ OF total

I-131

CURIES

$\%$ OF TOTAL

IR-192

CURIES

\% of total

LA-140

CURIES

$\%$ of total

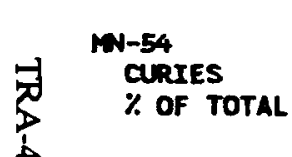

no-99

CURIES

$\%$ OF total

N3-94

Curres

\% OF TOTAL

M-95
CURIES

$\%$ OF TOTAL

NI -59

CURIES

\%. OF TOTAL

NI-63

CURIES

$\%$ OF TOTAL

PR-194

CURIES

$\%$ of Total

1. 900 E-02

$$
\begin{array}{r}
30 E-02 \\
0.201
\end{array}
$$

$9.520 E-02$

$2.774 E+07$

$3.650 E+04$

$1.020 E+03$

1.039

$2.537 E-01$
U.S. DEPARTMENT OF ENERGY

RADIOACTIVE WASTE MANAGEMENT INFORMATION SYSTEM

ATR DISPOSED SOLID MASTE SUMMARY

FOR JAN THROUGH DEC 1993

HALF LIFE

(DAYS)

JULY

AUEUST

SEPTEMBER

OCTOBER

NOVEMER

$6.200 E-03 \quad 9.682 E+04$

$0.047 \quad 98.668$

$4.240 E+01$

1.500E-03 2.740E-02 0.011

$8.040 E+00$

$7.383 E+01$

$1.900 E-03$ 0.014

$1.678 E+00$

$3.600 E-03$

0.027

$3.122 E+02$

$2.110 E-02 \quad 1.445 E-01$ 0.161

$2.000 E-04$

0.021

RUN DATE: 05/11/94

PAGE

2 RPT. 122

TRAATR-S

MNNUAL

TOTAL

$2.930 E+0.5$

98.273

$3.480 E-02$

$6.600 E-03$

$1.900 E-03$

$3.600 E-03$

$8.317 E+01$

0.028

1. 900E-02

$9.000 E-07$

1.521E-01

1.120E-01

$3.075 E+03$

1.031 
RADIOACTIVE WASTE MANAGEMENT INFORMATION SYSTEM

ATR DISPOSED SOLID MASTE SUMMARY FOR JAN THROUGH DEC 1993

MUCLIOES
RU-103

CURIES

$\%$ of total

SB-124

CURIES

$\%$ OF TOTAL

58-125

CURIES

$\%$ OF TOTAL

SC-46

$\%$ OF TOTAL

sE -75

CURIES

$\%$ OF TOTAL.

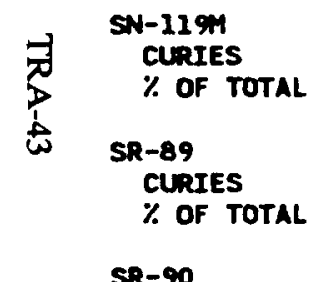

SR-90

$\%$ OF TOTAL

TC-99

CURIES

$\%$ OF TOTAL

QN-65

CURIES

$\%$ OF TOTAL

ZR-95
CURIES

$\%$ OF TOTAL 0.069
HALF LIFE
(DAYS)

sulr

ALEUST

SEPTEMBER

OCTOBER

$3.900 E-03$

0.030

$6.020 E+01$

2.007E+03

$8.382 E+01$

$1.198 E+02$

2. $930 E+02$

$5.052 E+01$

8.015E-01

6.102

$1.059 E+04$

$8.241 E+00$

$2.816 E-01$

62.742

4.600E-03

NOVEMBR

ANUAL

5.600E-03

5. 000E-04

$5.000 E+00$

0.002

5. 6OOE-03

$9.000 E-03$

$1.500 E+01$

0.005

$8.015 E-01$

$8.526 E+00$

0.003

$7.775 E+07$

$2.438 E+02$

$\begin{array}{r}1.494 E-01 \\ 1.237\end{array} \quad 3.223 E-01$

$2.500 E-05$

8.247E+01

0.028

8. $730 E-02$

$6.403 E+01$

9.000E-03 7.080E-02
PAGE 3 RPT. 122

TRA ATR-S 
SCHED. NO. P61PMDO9-H

OPERATIONS OFFICE

U.S. DEPARTMENT OF ENERGY

RADIOACTIVE WASTE MANAGEMENT INFORMATION SYSTEM
ATR DISPOSED SOLID WASTE SUMMARY

FOR JAN THROUGH DEC 2993

\begin{tabular}{|c|c|c|c|c|}
\hline MUCLIDES & $\begin{array}{l}\text { HALF LIFE } \\
\text { (DAYS) }\end{array}$ & NLY & AUEUST & \\
\hline $\begin{array}{l}\text { TOTALS } \\
\text { ALPHA CURIES } \\
\% \text { OF TOTAL } \\
\text { B+6 CURIES } \\
\% \text { OF TOTAL }\end{array}$ & & $\begin{array}{r}1.490 E-02 \\
0.113 \\
1.312 E+01 \\
99.887\end{array}$ & $\begin{array}{r}9.813 E+04 \\
100.000\end{array}$ & $\begin{array}{r}9.534 E-01 \\
100.000\end{array}$ \\
\hline
\end{tabular}

TOTAL ERMY OF AM

TOTAL GRNAS OF PU-239+24I

TOTAL GRNAS OF $U$

TOTAL GRAMS OF TH

TOTAL ERANS OF TH

VOUTE (CU. METERS )

VOLUHE (CU. METERS

5. $945 E+00 \quad 6.709 E+00 \quad 3.398 E+00$

$6.350 E+03 \quad 8.459 E+03 \quad 7.710 E+03$

OCTOBER

NOVERBER
TOTAL ERNAS OF PU
RUN DATE: $05 / 11 / 94$

PAGE

4 RPT. 122

TRA ATR-S
gRNo TOTALS (CURIES)

$1.313 E+019.813 E+04 \quad 9.534 E-03$

1.490E-02

2. $982 E+05$

100.000

$2.429 E+01$ $3.160 E+04$

$2.982 E+05$ 
RADIOACTIVE MASTE MANAGEMENT INFORMATION SYSTEM

CHEM LAB ADD ATPBOPNE WASTE SIMMARY

FOR JAN THROUGH DEC 1993

MULLIDES
EROSS-ALPHA
CONCEN (UCML)
CURIES
UNCERT(CURIES)
UNCERT (UCML)
\% OF TOTAL

\section{eross-BETA}

CONCEN (UCML)

CURIES

UNCERT (CURIES)

UNCERT (UCAL)

$\%$ OF TOTAL.

\section{JuLY}

$1.690 E-09 \quad 1.970 E-09 \quad 1.570 E-09 \quad 2.040 E-09 \quad 2.330 E-09 \quad 2.960 E-09$

2.800E-09 3.309E-09 2.890E-09 3.030E-09 3.750E-09 4.100E-09

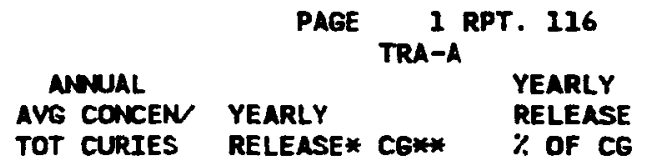

\section{TOTALS \\ ALPHA curres \\ $\%$ of total \\ B+G CURIES}

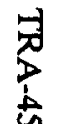

\% OF TOTAL

$\begin{array}{lllllllll}5.839 E-16 & -4.777 E-16 & -9.656 E-17 & -1.958 E-17 & -2.485 E-16 & 6.296 E-16 & 9.756 E-17 & 2.077 E-23\end{array}$

4.980E-09 $-4.210 E-09-8.510 E-10-1.670 E-10-2.190 E-09 \quad 5.370 E-09$ 1.021E-08

$\begin{array}{lllllll}32.592 & -194.907 & -10.072 & -1.527 & -18.084 & 15.996 & 7.409\end{array}$

$\begin{array}{llllllll}1.208 E-15 & 7.22 B E-16 & 1.055 E-15 & 1.301 E-15 & 1.623 E-15 & 3.306 E-15 & 1.219 E-15 & 2.595 E-22\end{array}$

$\begin{array}{lllllll}1.030 E-08 & 6.370 E-09 & 9.300 E-09 & 1.110 E-08 & 1.430 E-08 & 2.820 E-08 & 1.276 E-07\end{array}$

$\begin{array}{lllllll}67.408 & 294.907 & 110.072 & 101.528 & 118.084 & 84.004 & 92.591\end{array}$

\begin{tabular}{llllllll} 
VOLUE (CU. METERS) & $8.529 E+06$ & $8.813 E+06$ & $8.813 E+06$ & $8.529 E+06$ & $8.813 E+06$ & $8.529 E+06$ & $1.046 E+08$ \\
\hline COND TOTALS (CURIES) & $1.528 E-08$ & $2.160 E-09$ & $8.449 E-09$ & $1.093 E-08$ & $1.211 E-08$ & $3.357 E-08$ & $1.378 E-07$
\end{tabular}

* This number is derived from a dispersion factor equetion. The dispersion factor is derived from the straight line gouseian model and is proveded by the Netional Dosanic Atmospheric Administration (NOAA).

* NOTE: DCG value obtainad from DOE ORDER 5400.5 dated Fabrury 8, 1990 , Figures III-I and III-3 
RADIOACTIVE WASTE MANAGEMENT INFORMATION SYSTEM

FOR JAN THROUGH DEC 1993

MCLIDES
GROSS-ALPHA
CONCEN (UCML)
CURIES
UCERTICURIES)
UNCERTIUCML)
\% OF TOTAL

\section{HALF LIFE}

(DAYS)

JLY

Aveust

SEPTEMBE

OCTOBER

NOVEMBER

Decermer

\begin{tabular}{lll} 
& \multicolumn{2}{c}{ PAGE 1 RPT. 110} \\
TRNETR-A & \\
ANUAL & \multicolumn{2}{c}{ YEARLY } \\
AVG CONCEN & YEARLY & RELEASE \\
TOT CURIES & RELEASE* CE* & $\%$ OF CG
\end{tabular}

\section{$3.056 E-16 \quad 4.310 E-23$}

$7.650 E-09$

0.919

enoss-Beta

CONCEN (UCML)

CURIES

UNCERTI CURIES

UNCERT ( UCAML)

$\%$ OF TOTAL

\section{$3.296 E-14 \quad 4.648 E-21$}

8.250E-07

99.081

\section{TOTALS}

ALPHA CuRIES

$\%$ of total

B+G curres

$\frac{1}{8}$

$\%$ of total

7.650E-09

0.919

8.250E-07

99.081

VOLUNE (CU. METERS)

2.503E+07

8.326E-07

ERND TOTALS (CURIES)

* This muber is derived from a dispersion factor equation. The dispersion factor is derived from the straight line geussian model and is proveded by the National Dceanic Atmospheric Administration (NOAA).

** NOTE: DCG value obtained from DOE ORDER 5400.5 deted February 8, 1990 , Figures III-1 and III-3 
RADIOACTIVE MASTE MWUAEMENT INFORMATION SYSTEM

ETR DISPOSED SOLID WLSTE SUMMARY

FOR JAN THROUGH DEC 1993

MALF LIFE
(DAYS)

M-241

1.577E+05

CURIES

$\%$ OF TOTAL

ENArs

\section{CH-242 \\ CURIES}

$\%$ OF TOTAL

Cr-244

CURIES

$\%$ Of TOTAL

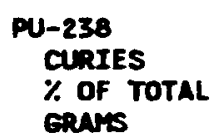

$\%$ Of TOTAL

epars

$1.630 E+02$

$6.610 E+03$

$3.203 E+04$

PU-239

curIEs

$\%$ OF TOTAL

붕 GRans

8. $800 E+06$

ULY

Auast

TOTALS

ALPHA CUNIES
$\%$ OF TOTAL

$\%$ OF TOTAL
B+G CuRIES

$\%$ Of TOTAL

TOTAL ERAMS OF AM

TOTAL GRANS OF PU

TOTAL ERANS Of PU-239+241

TOTAL ERNAS OF $U$

TOTAL ERASS OF TH

TOTAL ERANS OF NP

VOLUHE (CU. METERS)

VEIEHT (KILOGRNAS)

GRNO TOTALS (CURIES)
PAGE 1 RPT. 112

TRAETR-S
ANUAL

TOTAL

$7.540 E-08$
1.587

$2.292 E-08$

8.000E-10 0.017

$1.360 E-08$ 0.286

4. 280E-06

$$
90.109
$$

2.503E-07

3. 800E -07

$6.119 E-06$

4. 750E-06

200.000

2. $192 E-08$

$6.369 E-06$

6. $129 E-06$

$3.620 E+00$

$3.856 E+02$

$4.750 E-06$ 
RADIOACTIVE WASTE MANAGEMENT INFORMATION SYSTEM

MTR AIRBORNE WASTE SUMMARY

FOR JAN THROUGH DEC 1993

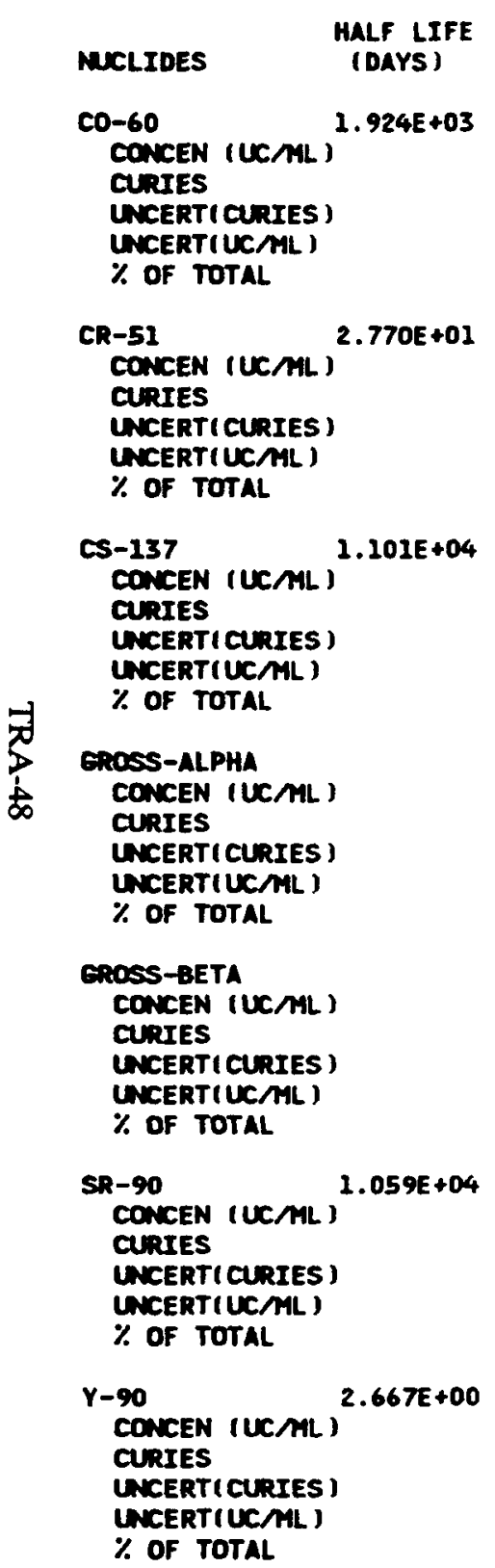

\section{WLY AUEUST SEPTEMBER OCTOBER NOVEMBER DECEMBER}

$\begin{array}{ll}4.370 E-14 & 1.285 E-14 \\ 6.630 E-07 & 1.950 E-07 \\ 6.500 E-08 & 2.870 E-08\end{array}$

$23.299 \quad 26.186$

\begin{tabular}{lll} 
& \multicolumn{2}{c}{ PAGE 1 RPT .123} \\
ANMALL & TRATRA-A & YEARLY \\
AVG CONCEN & YEARLY & RELEASE \\
TOT CURIES & RELEASE* CG** & $\%$ OF CG
\end{tabular}

$1.641 E-14 \quad 1.477 E-21 \quad 8.00 E-11$
$2.976 E-06$

16.703

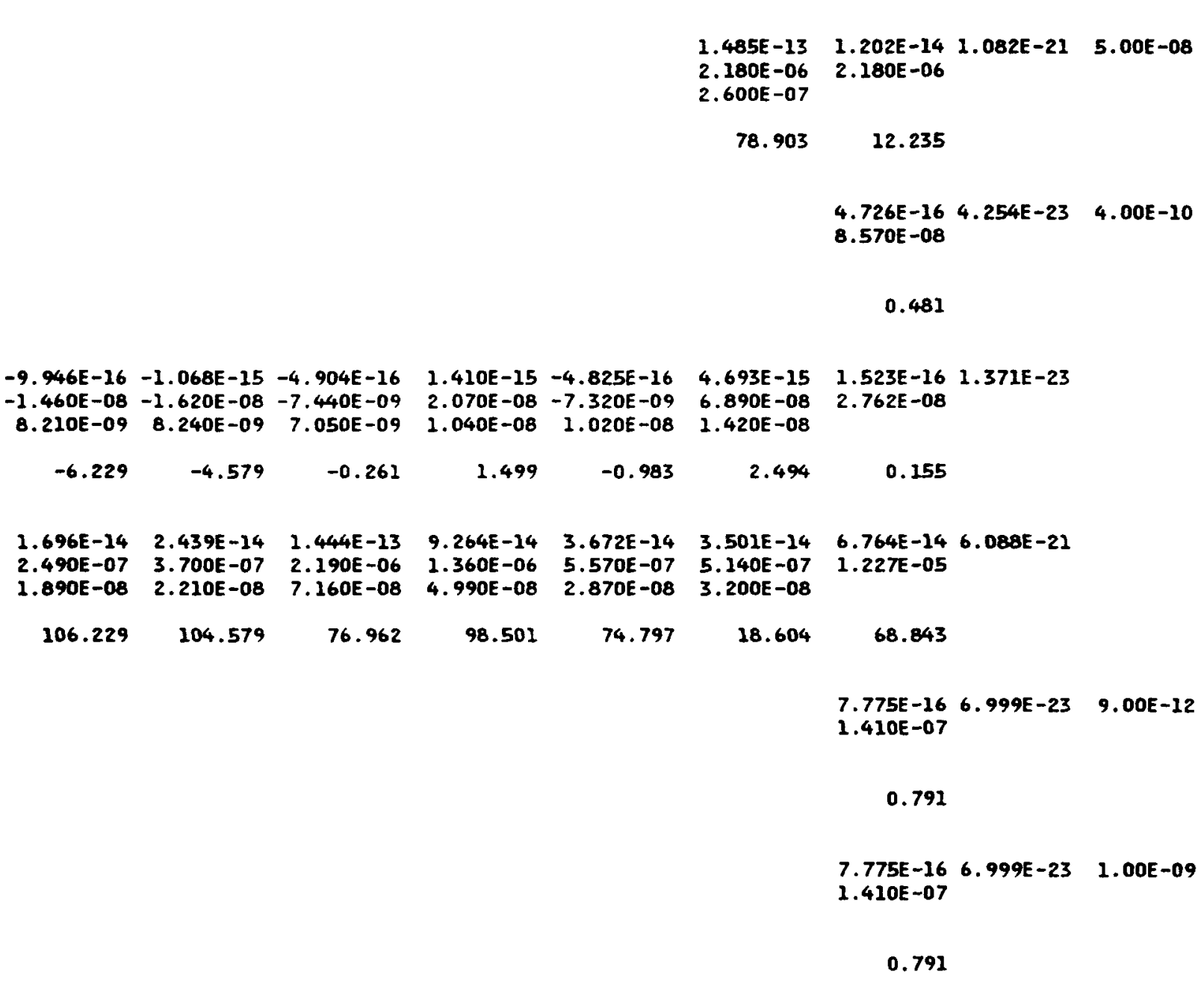

0.791

$7.775 E-16 \quad 6.999 E-23 \quad 1.00 E-09$ 1. $410 E-07$

0.791 
MTR AIRBORNE MUSTE SUMMARY

FOR JAN THROUGH DEC 2993

MALF LIFE
MUCLIDES (DAYS)

JulY AUEST

SEPTEMBER
OCTOBER

MONeriser

$-1.460 E-08-1.620 E-08-7.440 E-09$

$$
\begin{array}{rrr}
-6.229 & -4.579 & -0.261 \\
2.490 E-07 & 3.700 E-07 & 2.853 E-06 \\
106.229 & 104.579 & 100.261
\end{array}
$$

\% Of TOTAL

106.229

104.579
$\begin{array}{rr}2.070 E-08 & -7.320 E-09 \\ 1.499 & -0.983\end{array}$
$360 \mathrm{E}-06$

98.501

\section{ANUAL}

AVG CONCEN
PAGE 2 RPT. 123

TRATRA-A

\section{YEARLY}

RELEASE* CG**
YEARLY RELEASE

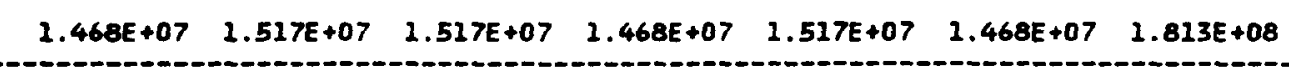

creno Torats

$2.344 E-07 \quad 3.538 E-07 \quad 2.846 E-06 \quad 1.381 E-06 \quad 7.447 E-07 \quad 2.763 E-06 \quad 1.782 E-05$

* This nuber is derived from a dispersion factor eqution. The dispersion factor is darived from the straight line goussian model and is proveded by the National Oceanic Atmospharic Administration (MOAA).

** NOTE: DCE value obtained from DOE ORDER 5400.5 dated February 8, 1990, Figures III-1 and III-3 


\section{RADIOACTIYE WASTE MANAGEMENT INFORMATION SYSTEM}

TRA HOT CELL AIRBORNE WASTE SUMMARY

FOR JAN THROUGH DEC 1993

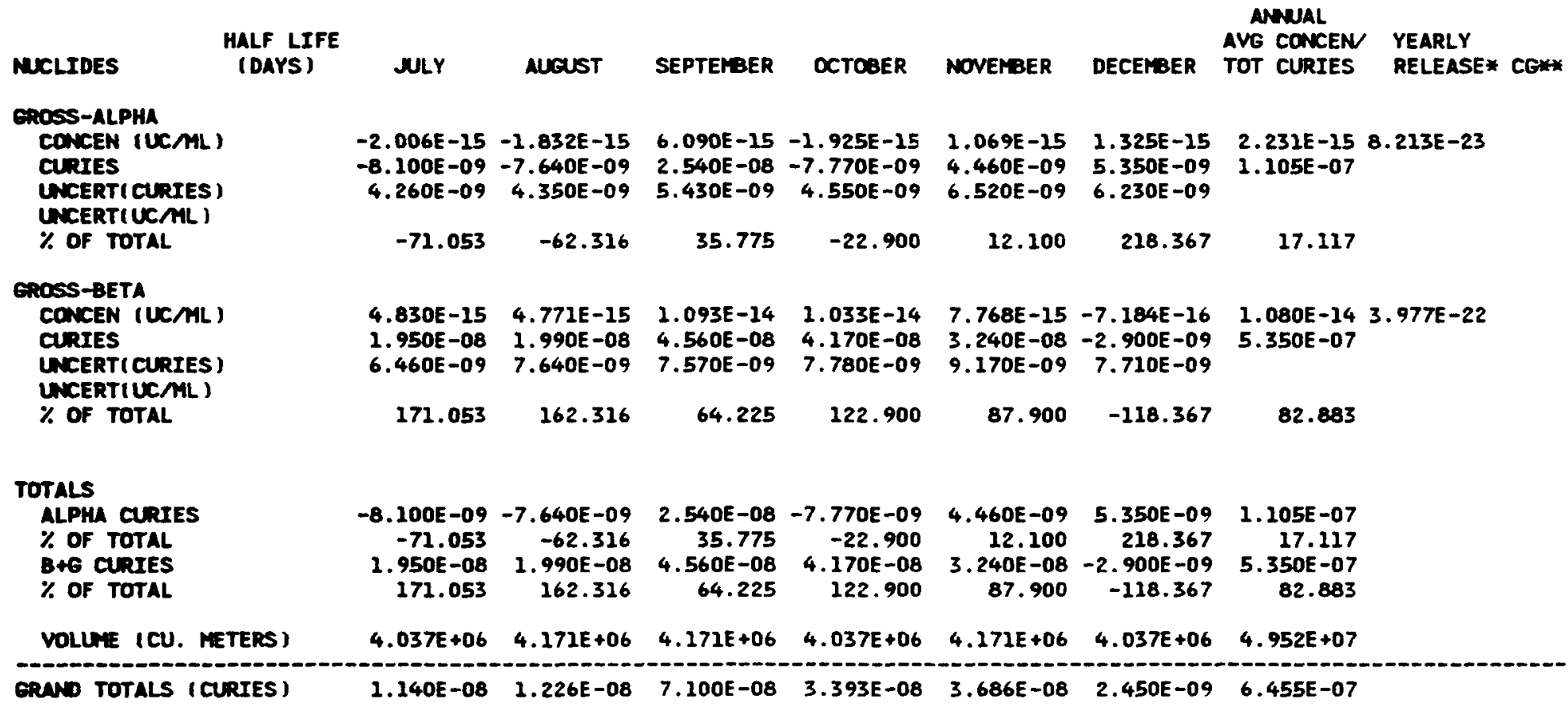

* This nuber is derived from a disparsion factor equation. The dispersion factor is derivad from the straight line oussian wodel and is proveded by the Notional Dceanic Atmospheric Administration (NOMA).

** NOTE: DCG velue obtained from DOE ORDER 5400.5 dated fabruary 8, 1990 , Figures III-1 and III-3 
TRA HOT CELL SOLTO WASTE SIMMARY

FOR JAN THROUGH DEC 1993

\begin{tabular}{|c|c|c|c|}
\hline MUCLIDES & $\begin{array}{l}\text { HALF LIFE } \\
\text { (DAYS) }\end{array}$ & MLY & AUGUST \\
\hline $\begin{array}{l}\text { AG-10EM } \\
\text { CURIES } \\
\% \text { OF TOTAL }\end{array}$ & $4.745 E+04$ & & $\begin{array}{r}1.060 E-07 \\
0.124\end{array}$ \\
\hline $\begin{array}{l}\text { AM-241 } \\
\text { CURIES } \\
\% \text { OF TOTAL } \\
\text { GRMIS }\end{array}$ & $1.577 E+05$ & & $\begin{array}{r}5.620 E-08 \\
0.066 \\
1.634 E-08\end{array}$ \\
\hline $\begin{array}{l}\text { CO-60 } \\
\text { CURIES } \\
\% \text { OF TOTAL }\end{array}$ & $1.924 E+03$ & $\begin{array}{r}2.910 E-12 \\
5.916\end{array}$ & $\begin{array}{r}2.090 E-05 \\
24.531\end{array}$ \\
\hline $\begin{array}{l}\text { CS- } 234 \\
\text { CURIES } \\
\% \text { OF TOTAL }\end{array}$ & $7.537 E+02$ & & $\begin{array}{r}4.210 E-0 \\
0.49\end{array}$ \\
\hline $\begin{array}{l}\text { CS-137 } \\
\text { CURIES } \\
\% \text { OF TOTAL }\end{array}$ & $1.101 E+04$ & $\begin{array}{r}4.120 E-11 \\
83.757\end{array}$ & $\begin{array}{r}6.300 \mathrm{E}-0 . \\
73.94\end{array}$ \\
\hline $\begin{array}{l}\text { EU-152 } \\
\text { CURIES } \\
\% \text { OF TOTAL }\end{array}$ & $4.891 E+03$ & $\begin{array}{r}1.090 E-12 \\
2.216\end{array}$ & $\begin{array}{r}6.820 E-08 \\
0.080\end{array}$ \\
\hline $\begin{array}{l}\text { EU-154 } \\
\text { CURIES } \\
\% \text { OF TOTAL. }\end{array}$ & $3.103 E+03$ & $\begin{array}{r}3.120 E-12 \\
6.343\end{array}$ & $\begin{array}{r}1.690 E-0 \\
0.19\end{array}$ \\
\hline $\begin{array}{l}\text { EU- } 155 \\
\text { CURIES } \\
\% \text { OF TOTAL }\end{array}$ & $1.727 E+03$ & $\begin{array}{r}8.700 E-13 \\
1.769\end{array}$ & \\
\hline $\begin{array}{l}58-125 \\
\text { CURIES } \\
\% \text { OF TOTAL }\end{array}$ & $1.007 E+03$ & & $\begin{array}{r}2.180 E-0 \\
0.02\end{array}$ \\
\hline $\begin{array}{l}\text { U-234 } \\
\text { CURIES } \\
\% \text { OF TOTAL } \\
\text { GRAMS }\end{array}$ & 8. $943 E+07$ & & $\begin{array}{r}4.340 E-07 \\
0.509 \\
6.955 E-05\end{array}$ \\
\hline $\begin{array}{l}\text { U-235 } \\
\text { CURIES } \\
\% \text { OF TOTAL }\end{array}$ & $2.570 E+11$ & & $\begin{array}{r}2.270 E-08 \\
0.027 \\
1.051 E-02\end{array}$ \\
\hline
\end{tabular}

PAGE 1 RPT. 124

ANWAL

TRA-S
TOTAL

$1.060 E-07$

$$
0.124
$$

$5.620 E-08$

$$
0.066
$$

$1.634 E-08$

$2.090 E-05$ 24.531

4.210E-07 0.494

$6.300 E-05$ 73.945

$6.820 E-08$ 0.080

$1.690 E-07$ 0.198

8. $700 E-13$

$2.180 E-08$ 0.026

4.340E-07$$
0.509
$$$$
\text { 6. } 955 \mathrm{E}-05
$$

2.270E -08 0.027

$1.051 E-02$ 
SCHED. NO. P6IPMOO9-H

IDAHO OPERATIONS OFFICE

U.S. DEPARTMENT OF ENERGY

RADIOACTIVE WASTE MANAGEMENT INFORMATION SYSTEM

TRA HOT CELL SOLID WASTE SUMMARY

FOR JAN THROUEH DEC 1993

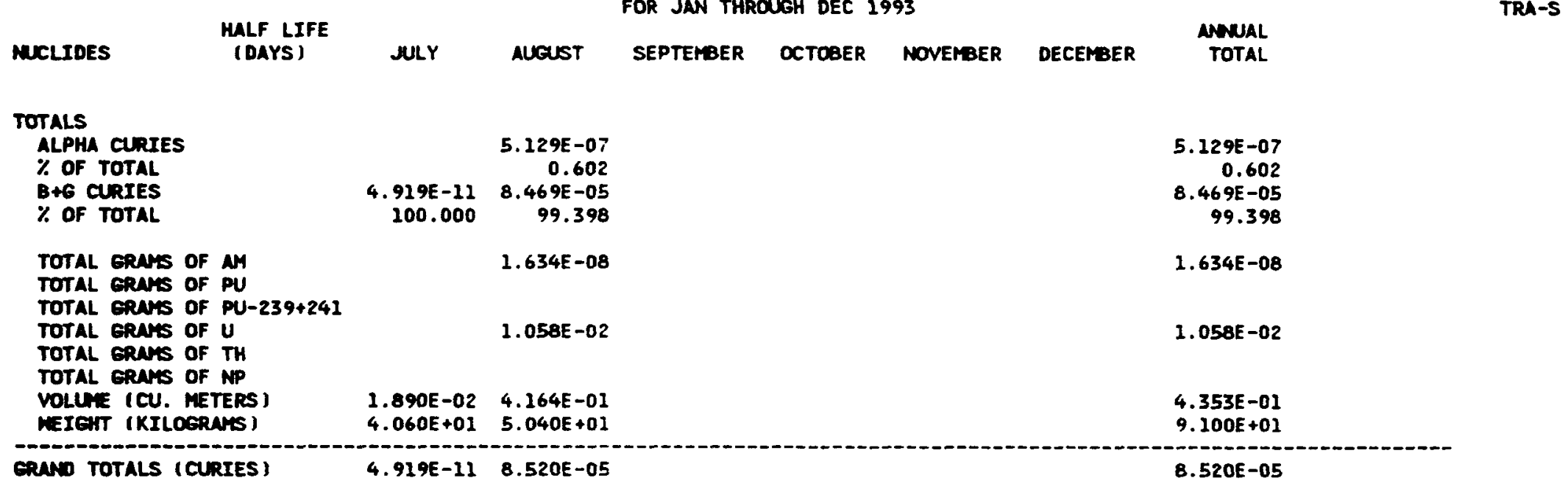

RUN DATE: 05/21/94

PAGE 2 RPT. 124 TRA-S 
RADIOACTIVE WASTE MANAGEMENT INFORMATION SYSTEM

TRA LIEUID WASTE SUMTARY

FOR JAN THROUGH DEC 1993

$\begin{array}{ll}\text { NUCLIDES } & \begin{array}{c}\text { MALF LIFE } \\ \text { (DAYS) }\end{array} \\ \text { CE-I4I } & \begin{array}{l}3.250 E+01 \\ \text { CONCEN (UCML) } \\ \text { CURIES }\end{array} \\ \text { UNCERTICURIES) } \\ \text { UNCERT(UCML) } \\ \text { \% OF TOTAL }\end{array}$

JULY AUEUST SEPTEMER OCTOBER NOVERBER DECEMBER

PAGE 1 RPT. 105
TRA-L
ANNAL
AVG CONCEN
TOT CURIES CG *

$2.844 E+02$

CE-144

CURIES

UNCERTI CURIES )

UACERT(UCML)

\% Of TOTAL

(2)

CURIES

$7.171 E-08 \quad 5.00 E-05$

0.14

$5.068 E-03$

0.004

$3.863 E-07$
$2.663 E-03$

$1.228 E-07 \quad 7.00 E-06$

1.75

1. 100E -07

$$
0.268
$$

0.007

CO-58
CONCEN (UCML)

CURIES

$7.091 E+01$

LNCERTI CURIES

UNCERT ( UCANL)

耑

$\%$ OF TOTAL

3.080E-07 $1.449 E-07$

$2.900 E-03 \quad 9.985 E-04$

2.500E-08 4.200E -08

$\begin{array}{rr}0.270 & 0.101\end{array}$

1. $924 E+03$

CONCEN (UCML)

CURIES

UNCERT I CURIES )

UNCERTIUCAL.

$\%$ OF TOTAL

$2.530 E-05 \quad 9.165 E-05 \quad 1.840 E-05 \quad 1.800 E-05$

$2.383 E-01 \quad 6.318 E-01$

$1.840 E-05$
$8.148 E-02$

5.870E-02

6.304E-02

1

$.000 E-07$

$200 E-06$
63.690

$.000 E-07$
13.228

O0OE -07
5.020

$1.000 E-07$
0.481

2.000E -07

1.141
1.600

CR-51

2.770E+01

CONCEN (UCML)

CURIES

$\begin{array}{llllllll}7.550 E-06 & 3.191 E-06 & 6.850 E-07 & 5.660 E-07 & 1.640 E-05 & 1.920 E-04 & 2.518 E-05 \\ 7.110 E-02 & 2.200 E-02 & 3.033 E-03 & 1.846 E-03 & 4.476 E-02 & 3.660 E-01 & 1.780 E+00 \\ & & & & & & & \\ 1.500 E-07 & 8.700 E-07 & 9.600 E-08 & 1.600 E-07 & 4.000 E-07 & 1.000 E-06 & \end{array}$

UNCERT (UCAL)

$\%$ of rotal

$\begin{array}{rrrrrr}1.500 E-07 & 8.700 E-07 & 9.600 E-08 & 1.600 E-07 & 4.000 E-07 & 1.000 E-06 \\ 6.627 & 2.217 & 0.492 & 0.158 & 0.341 & 3.539\end{array}$

1.389

$7.537 E+02$

CS- 134
CONCEN (UCML)

CURIES

UNCERT (CURIES )

UNERT (UCML)

$\%$ OF TOTAL

1.673E-08

$1.182 E-03$

$2.00 E-06$

0.84

0.001

CS-137 $1.101 E+04$

CONCEN (UCML)

CURIES

2.230E-06 $\quad 1.798 E-06 \quad 2.520 E-06$

2.100E-02 1.240E-02 $1.116 E-02$

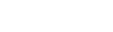

5.000E-08 2.300E-07 5.000E-08

$\begin{aligned} & 1.112 E-06 \\ & 7.856 E-02\end{aligned} \quad 3.00 E-06$

37.05

0.061

1.250 


\section{MULLIDES \\ EROSS-ALPHA \\ CONCEN (UCAL) \\ CURIES \\ CNCERTICURIES \\ NNERT ( UCML \\ \% OF TOTAL}

HALF LIFE

\section{GoSS-BETA}

CONCEN (UCML)

CURIES

UNCERT (CURIES )

UNCERT (UCML)

\% OF TOTAL

H-3

CONCEN IUCML)

CURIES

UNCERTICURIES )

UNCERTIUCAL)

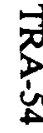

$4.490 E+03$ m-54

3.222E+02

CURIES

CNCERTI CURIES

UNCERTI UCAML

\% Of TOTAL

$\mathrm{M}-24$

CONCEN IUCML

CURIES

UNCERTI CURIES

UNCERTIUCAL)

\% OF TOTAL

13-95 3.498E+02

CONCEN (UCAML)

INCERTICURIES

UNCERTIUCAL I

$\%$ OF TOTAL

\section{8-124}

CONCEN (UCAM)

CURIES

UNCERT I CURIES

UNCERTIUCAML

\% OF TOTAL
TRA LIQUID WASTE SUMYARY
FOR JAN THROUEH DEC 1993
JULY ALGUST SEPTEMBER OCTOBER NOVEMBER DECEMBER
PAGE

2 RPT. 105

TRA-L 
RADIOACTIVE WASTE MANAGEMENT INFORMATION SYSTEM

TRA LIEUID WASTE SUMARY

FOR JAN THROUGH DEC 1993

PAGE 3 RPT. 105

$\begin{array}{ll}\text { MUCLIDES } & \begin{array}{c}\text { HALF LIFE } \\ \text { (DAYS) }\end{array} \\ \text { SR-89 } & \begin{array}{c}\text { 5.052E+0) } \\ \text { CONCEN (UCML) } \\ \text { CURIES }\end{array} \\ \text { UNCERTICURIES) } \\ \text { UNCERTIUCAML) } \\ \% \text { OF TOTAL }\end{array}$

ULY

August

\section{SEPTEMBER OCTOBER NOVEMBER}

NNUAL

AVG CONCEN

TOT CURIES

CG * $\%$ OF CG

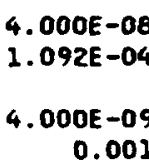

$\begin{array}{llll}2.278 E-06 & 2.970 E-06 & 8.700 E-07 & 2.780 E-07\end{array}$

$\begin{array}{llll}1.570 E-02 & 1.315 E-02 & 2.837 E-03 & 7.587 E-04\end{array}$

$\begin{array}{rrrr}1.500 E-07 & 1.800 E-07 & 5.000 E-08 & 1.600 E-08 \\ 1.583 & 2.135 & 0.243 & 0.006\end{array}$

$1.544 E-09 \quad 2.00 E-05$

0.01
$5 R-90$
CURIES
UNCERTI (CURIES)
UNCERT (UCAL)
$\%$ OF TOTAL

$1.059 E+04$

TA-182

CONCEN (UCML)

$1.245 E+02$

CURIES

UNCERTICURIES )

UNCERTIUCAL

$\%$ OF total

$2.667 E+00$

CONCEN (UCML)

CURIES
UNEERTICURIES )

UXEERTIUCALI)

$\%$ OF TOTAL

$2.438 E+02$

$\mathrm{ZN}-65$

CONCEN (UCAL)

CURIES

UNCERTICURIES )

UNCERTIUCALI

$\%$ Of TOTAL

$6.403 E+01$

ZR-95

CONCEN (UCML)

CURIES

0.006

1.576E-06 $\quad 1.00 E-06$

$1.114 E-01$

157.60

0.087

1.456E-08 2.00E-05

0.07

$1.029 E-03$

0.001

$\begin{array}{llll}2.278 E-06 & 2.970 E-06 & 8.700 E-07 & 2.780 E-07\end{array}$

$1.570 E-02 \quad 1.315 E-02 \quad 2.837 E-03 \quad 7.587 E-04$

1.576E-06 1.00E-05

15.76

$\begin{array}{llll}1.500 E-07 & 1.800 E-07 & 5.000 E-08 & 1.600 E-08\end{array}$

1.583

2.135

0.243

0.006

$1.114 E-01$

0.087

$\begin{array}{llllll}1.540 E-06 & 1.664 E-06 & 2.890 E-06 & 3.540 E-06 & 1.820 E-06 & 1.830 E-06\end{array}$

$1.450 E-02$

$1.147 E-02 \quad 1.280 E-02 \quad 2.154 E-02 \quad 4.967 E-03 \quad 3.488 E-03$

$1.085 E-06 \quad 9.00 E-06$

12.05

8.000E-08

5.000E -0

$1.000 E-07$

$1.700 E-07$

$600 E-07$
0.038

2.300E -07

0.034

0.060

$3.824 E-07$

1. 257E-03

1.779E-08 4.00E-05

$1.257 E-03$

0.04

$\%$ OF TOTAL
0.001

\section{DOE-07
0.127}


SCHED. NO. P61PM009-F

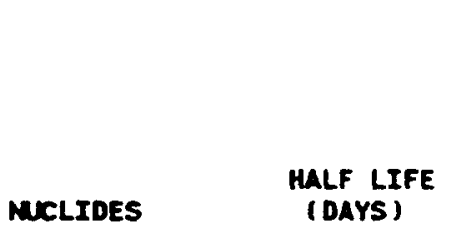

MCLIDES

(DAYS)
IOAHO OPERATIONS OFFICE

U.S. DEPARTMENT OF ENERGY

RAOIOACTIVE WISTE MANAGEMENT INFORMATION SYSTEM

TRA LIQUID WASTE SUMARY
FOR JAN THROUGH DEC 1993

NLY

Aveust
SEPTEMBER OCTOBER

93

\section{TOTALs}

ALPHA CURIES
$\%$ OF TOTAL

B+6 curIEs

$\%$ Of TOTAL

VOLUNE (LITERS)

Volure (LIters)

ERND TOTALS (CURIES)

* Note: DCG value obta

** The ce limit is being approached or has boan exceeded during the current month. Please verify the accuracy of the reported data. If the data is in error, please sand correct dato to vaste Information.

$1.073 E+00 \quad 9.920 E-01 \quad 6.159 E-01 \quad 1.169 E+00 \quad 1.311 E+01 \quad 1.034 E+01 \quad 1.282 E+02$

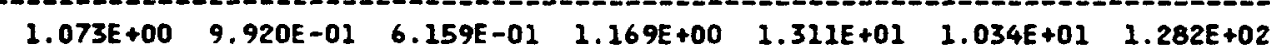

RUN DATE: 05/11/94

PAGE 4 RPT. 105
TRA-L
AVG CONCEN
TOT CURIES CG

$5.000 E-03$ 
TRA Facility Detail Data for Months January to June Annual Average Concentration and Total Curies for January to June 

ALPHA LAB ATR ATRBOPAE WASTE SUMMARY

FOR JAN THROUGH IN 1993

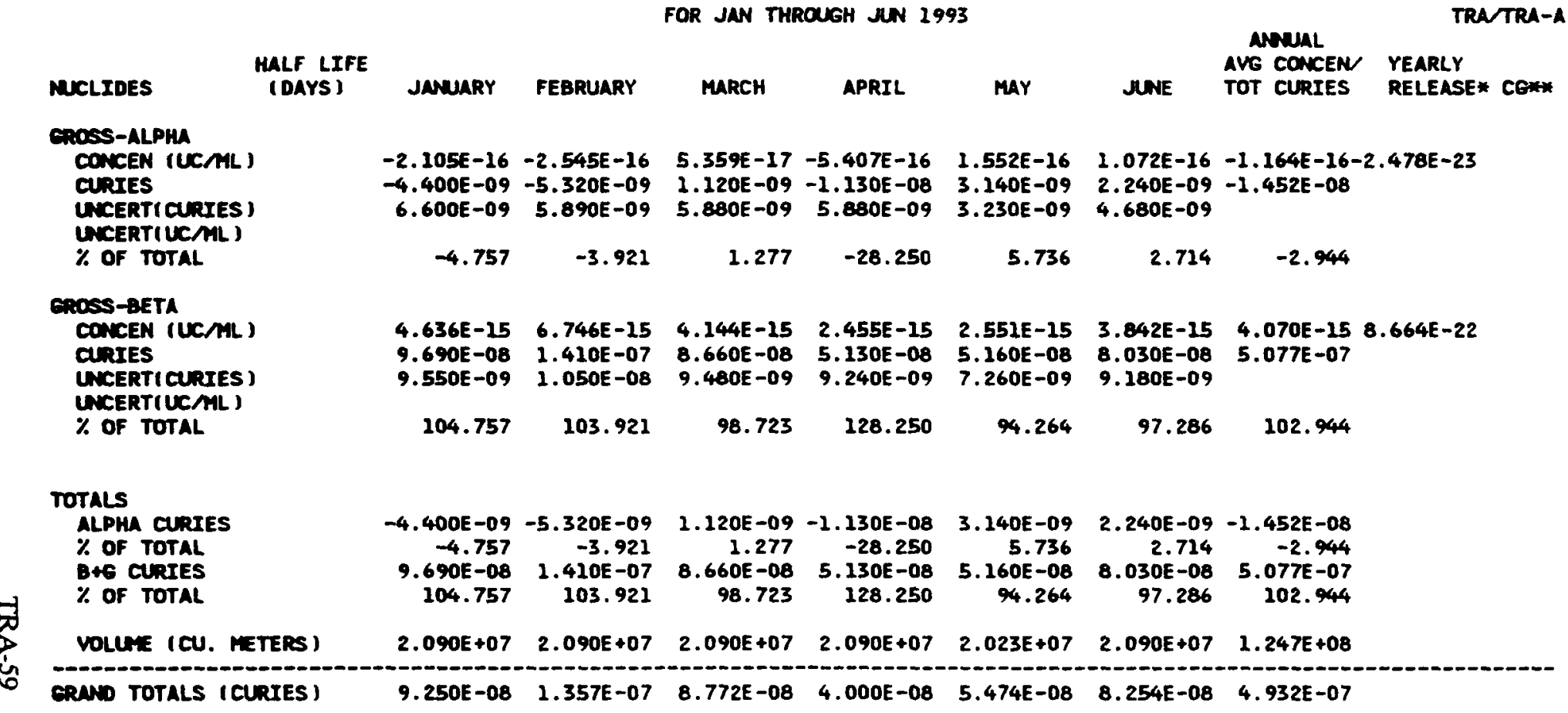

* This maber is derived from o dispersion factor eqution. The dispersion factor is derived from the straight line gevesien modbl and is proveded by the Notional Oceanic Atmospheric Administration (NOAA).

** NOTE: DCE velue obtainad from DOE ORDER 5400.5 dated Februery 8, 1990, Figures III-1 and III-3 
IDAHO OPERATIONS OFFICE

U.S. DEPARTMENT OF ENERGY

RADIOACTIVE WUSTE MANAGEMENT INFOPMATION SYSTEM

ATR AIRBORNE MASTE SUMMARY FOR JAN THROUGH JUN 1993

MCLIDES
AR-4I
CONCEN (UCML)
CURIES
UNCERT(CURIES)
UNCERT(UCAL)
\% OF TOTAL

MALF LIFE
(DAYS)

JANUARY FEBRUARY

MARCH

APRIL

MaY

$\begin{array}{llllll}6.654 E-06 & 3.232 E-06 & 4.918 E-06 & 3.979 E-06 & 5.508 E-07\end{array}$

$\begin{array}{lllll}3.458 E+02 & 1.672 E+02 & 2.421 E+02 & 2.373 E+02 & 3.188 E+01\end{array}$

$\begin{array}{lllll}9.000 E-01 & 5.000 E-01 & 7.000 E-01 & 7.000 E-01 & 2.400 E-01\end{array}$

85.755

88.119

88.408

88.979

86.646

5. 771E-02

BA-139
CONCEN (UCML)

$$
\text { CURIES }
$$

$2.001 E-10 \quad 1.340 E-10 \quad 1.255 E-10 \quad 1.210 E-10 \quad 2.460 E-10$ $1.040 E-02 \quad 6.935 E-03 \quad 6.179 E-03 \quad 7.218 E-03 \quad 1.424 E-02$

CURERT (CURIES)

UNCERTICURIES )
UNCERTIUCAL)

\% OF TOTAL

2.000E-04 $1.810 E-04 \quad 1.870 E-04$ 1.980E-04 $3.800 E-04$
0.003
0.004
0.002
0.003
0.039

1.201E-13

$6.243 E-06$

$1.690 E-06$

COnires

$1.276 E+01$

CURIES

UNCERTIUCALI

$\%$ OF TOTAL

年

c0-58

CONCEN IUCAL

$7.091 E+01$

CURIES

UNCERT (CURIES )

UNCERT (UCAL )

$\%$ OF TOTAL

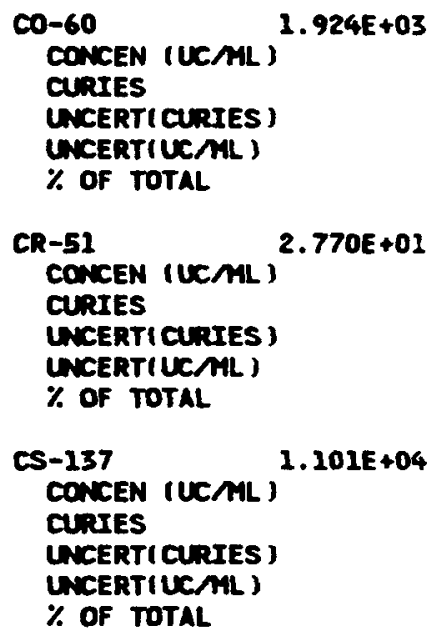

$1.379 E-14$
$7.981 E-07$

$1.620 E-07$

8.790E-14 $5.842 E-14 \quad 3.712 E-14$

$\begin{array}{lll}4.548 E-06 & 2.876 E-06 & 2.214 E-06\end{array}$

$\begin{array}{lll}6.380 E-07 & 6.370 E-07 & 5.630 E-07\end{array}$

$2.887 E-14$ $1.722 E-06$
$5.860 E-07$
RUN DATE: 05/12/94

PAGE 1 RPT. 120 TREATR-A

$\begin{array}{llll}\begin{array}{c}\text { MNUAL } \\ \text { AVG CONCEN }\end{array} & \begin{array}{l}\text { YRARLY } \\ \text { YEAR-A }\end{array} & \begin{array}{l}\text { YEARLY } \\ \text { RELERSE }\end{array} \\ \text { TOT CURIES } & \text { RELERSE* CEK* } & \% \text { OF CG } \\ 3.663 E-06 & 1.010 E-12 & 1.00 E-08 & 0.01 \\ 1.024 E+03 & & & \\ 87.522 & & & \end{array}$

$$
\begin{aligned}
& \begin{array}{l}
1.608 E-10 \\
4.497 E-02
\end{array} \\
& 0.434 E-17 \quad 7.00 E-08 \\
& 0.004
\end{aligned}
$$

$2.233 E-14 \quad 6.155 E-21 \quad 3.00 E-09$ 6.243E-06

$2.854 E-15 \quad 7.868 E-22 \quad 2.00 E-09$
$7.981 E-07$ $\begin{array}{llll}9.366 E-12 & 3.405 E-13 & 9.384 E-20 & 8.00 E-11 \\ 8.555 E-05 & 9.519 E-05 & & \end{array}$

2.260E-06

65.136

$\begin{array}{lllllllll}1.165 E-11 & 1.489 E-11 & 2.238 E-11 & 1.208 E-11 & 2.706 E-12 & 3.931 E-12 & 1.213 E-11 & 3.343 E-18 & 5.00 E-08\end{array}$ $\begin{array}{llllllll}6.053 E-04 & 7.706 E-04 & 1.202 E-03 & 7.205 E-04 & 1.566 E-04 & 3.591 E-05 & 3.391 E-03\end{array}$

$\begin{array}{llllll}8.900 E-06 & 1.020 E-05 & 1.500 E-05 & 1.090 E-05 & 8.200 E-06 & 2.660 E-06\end{array}$

27.341 $\begin{array}{lll}8.423 E-14 & 8.911 E-15 & 2.456 E-21 \quad 4.00 E-10\end{array}$ $7.694 E-07 \quad 2.491 E-06$

$2.100 E-07$

0.586 
ATR ATRBORNE WUSTE SUMHARY FOR JAN THROUGH JUN 1993

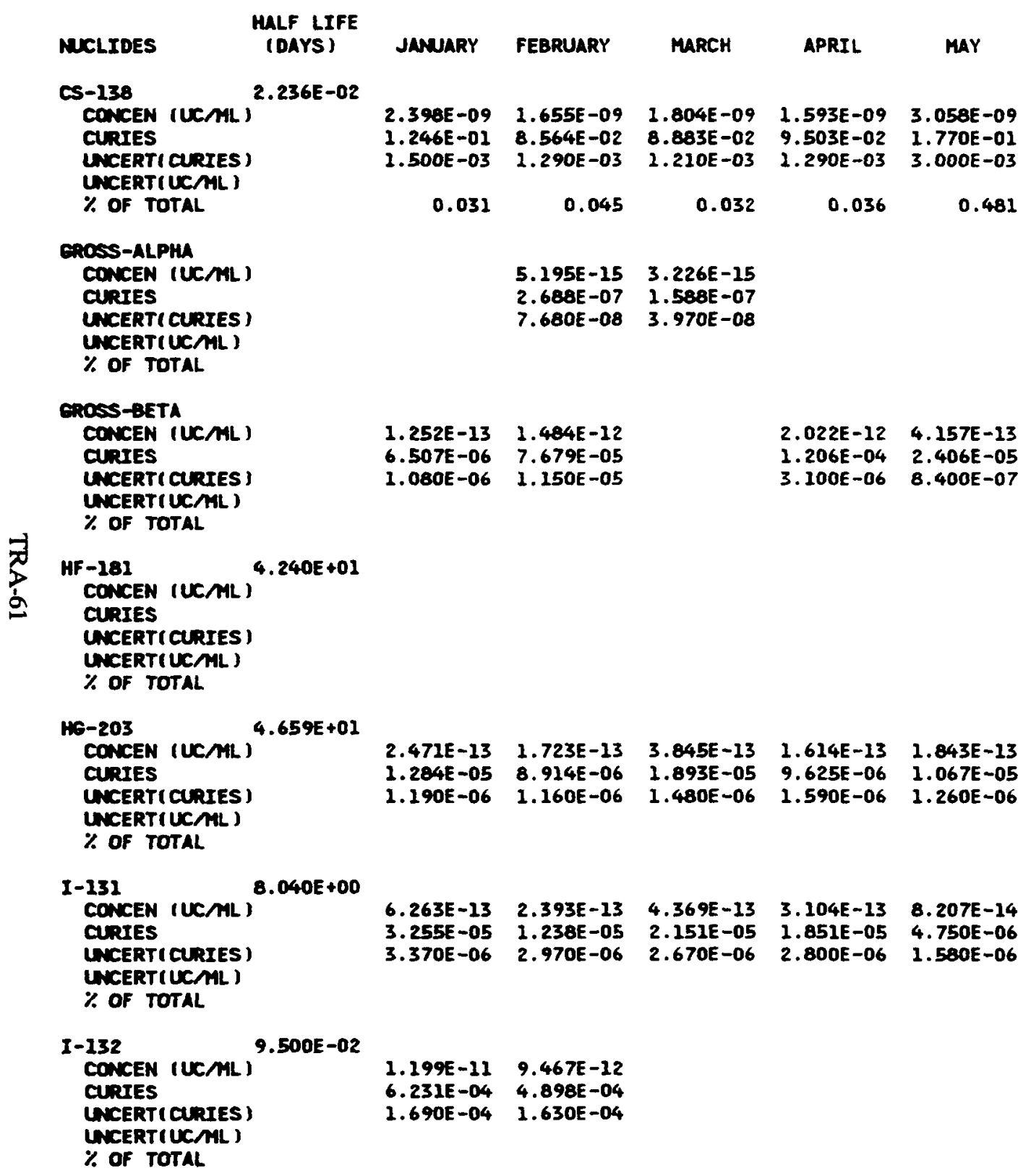

\section{JNE}

\begin{tabular}{lll} 
& \multicolumn{2}{c}{ PAGE 2 RPT. 120} \\
TRANATR-A & YNUAL \\
AVG CONCEN & YEARLY & $\begin{array}{l}\text { YEARLY } \\
\text { RELEASE }\end{array}$ \\
TOT CURIES & RELEASE* CG** & $\%$ OF CG
\end{tabular}

$3.881 E-13 \quad 1.268 E-14 \quad 3.495 E-21 \quad 9.00 E-10$ $3.545 E-06 \quad 3.545 E-06$

2.320E-07

2.043E-09 5.630E-16 8.00E-09

5.711E-01

0.049

$1.529 E-15 \quad 4.215 E-22$

$4.276 E-07$

$8.153 E-13 \quad 2.247 E-19$

2.280E-04

2.699

6.094E-13 2.380E-13 $\quad 6.560 E-20 \quad 2.00 E-09$

$5.566 E-06 \quad 6.655 E-05$

$6.680 E-07$

4.238

\author{
$3.208 E-13 \quad 8.843 E-20 \quad 4.00 E-10$ \\ 8.970E-05 \\ $3.980 E-12 \quad 1.097 E-18 \quad 8.00 E-09$ \\ $1.113 E-03$
}


RADIOACTIVE WASTE MUMAGEMENT INFORMATION SYSTEM

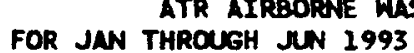

ATR AIRBORAE WLSTE SUMMARY

\begin{tabular}{|c|c|}
\hline MCLIDES & $\begin{array}{l}\text { HALF LIFE } \\
\text { (DAYS) }\end{array}$ \\
\hline $\begin{array}{l}\text { I-133 } \\
\text { CONCEN (UCAL) } \\
\text { CURIES } \\
\text { UNCERT(CURIES ) } \\
\text { UNCERTIUCML) } \\
\text { \% OF TOTAL }\end{array}$ & $8.66 \pi E-01$ \\
\hline $\begin{array}{l}\text { KR-85M } \\
\text { CONCEN (UCML) } \\
\text { CURIES } \\
\text { UNCERT(CURIES) } \\
\text { UNCERT(UCML) } \\
\text { \% OF TOTAL }\end{array}$ & $1.867 E-01$ \\
\hline $\begin{array}{l}\text { KR-B7 } \\
\text { CONCEN (LCML) } \\
\text { CURIES } \\
\text { UNCERT (CURIES) } \\
\text { UNCERT (UCML) } \\
\text { \% OF TOTAL }\end{array}$ & 5. 278E-02 \\
\hline $\begin{array}{l}\text { KR-B8 } \\
\text { CONCEN (UCML) } \\
\text { CURIES } \\
\text { UNCERT(CURIES) } \\
\text { UNCERTIUCALI) }\end{array}$ & $1.183 E-01$ \\
\hline
\end{tabular}

$\begin{array}{lllll}5.014 E-08 & 1.920 E-08 & 3.061 E-08 & 2.223 E-08 & 3.535 E-09\end{array}$ $\begin{array}{lllll}2.606 E+00 & 9.934 E-01 & 1.507 E+00 & 1.326 E+00 & 2.046 E-01\end{array}$ 6.500E-02 3.790E-02 4.400E-02 4.300E-02 $1.850 E-02$
0.646
0.524
0.550
0.497
0.556

$\begin{array}{llllll}1.637 E-07 & 6.448 E-08 & 9.230 E-08 & 6.563 E-08 & 1.044 E-08\end{array}$ $8.509 E+00 \quad 3.336 E+00 \quad 4.544 E+00 \quad 3.914 E+00 \quad 6.041 E-01$ $\begin{array}{lllll}8.509 E+00 & 3.336 E+00 & 4.544 E+00 & 3.914 E+00 & 6.041 E-01 \\ 1.700 E-01 & 9.400 E-02 & 1.220 E-01 & 1.030 E-01 & 4.590 E-02\end{array}$
2.110
1.758
1.659
1.468
1.642

1.626E-07 5.850E-08 8.062E-08 7.217E-08 $1.094 E-08$ $\begin{array}{lllll}8.452 E+00 & 3.027 E+00 & 3.969 E+00 & 4.304 E+00 & 6.330 E-01\end{array}$ $1.600 E-01 \quad 1.520 E-01 \quad 1.910 E-01$ 1.100E-01 $7.810 E-02$

$$
2.096
$$

1.595

1.449

1.614

1.720
$1.642 E-13$

8.531E-06

1.910E-06

$\begin{array}{ll}\text { LA-140 } & \\ \text { CONCEN (UCML) } & 1.678 E+00 \\ \text { CURIES } & 1.642 E-13 \\ \text { UNCERT (CURIES) } & 8.531 E-06 \\ \text { LNCERT (UCAL) } & 1.910 E-06\end{array}$

LACERT(UC)

$\%$ OF TOTAL

MA-24 $(U \mathrm{CML}) 6.237 E-01$

CURIES UNCERT (UCAL)

$\%$ OF TOTAL

$1.238 E-11 \quad 5.220 E-11 \quad 1.056 E-11 \quad 8.032 E-12 \quad 4.627 E-12$ 6.432E-04 $2.701 E-03 \quad 5.199 E-04 \quad 4.790 E-04 \quad 2.678 E-04$ $\begin{array}{lllll}1.390 E-05 & 3.200 E-05 & 1.660 E-05 & 1.760 E-05 & 5.620 E-05\end{array}$

0.001

0.001
PB-88 $1.229 E-02$

CONCEN (UCML)

CURIES

INCERTIUCA

$\%$ OF TOTAL $\begin{array}{lllll}1.093 E-09 & 1.030 E-09 & 1.479 E-09 & 1.246 E-09 & 2.080 E-09\end{array}$ $\begin{array}{llllll}9.839 E-02 & 5.331 E-02 & 7.281 E-02 & 6.835 E-02 & 1.204 E-01\end{array}$ $\begin{array}{lllll}5.460 E-03 & 4.520 E-03 & 1.220 E-02 & 1.200 E-02 & 8.100 E-03\end{array}$
0.024
0.028
0.027
0.026
0.327

PAgE 3 RPT. 120

TRA ATR-A

ANNUAL

AVG COUCEN YEARLY TOT CURIES RELEASE* CE* \% OF CG

$1.344 E-12 \quad 3.705 E-19 \quad 2.00 E-09$ $3.758 E-04$

$2.374 E-08 \quad 6.543 E-15 \quad 1.00 E-07$ $6.637 E+00$

0.567

$\begin{array}{lll}7.478 E-08 & 2.061 E-14 & 2.00 E-08\end{array}$ $2.091 E+01$

1.786

7.291E-08 2.010E-14 9.00E-09 $2.038 E+01$

1.742

$3.051 E-14 \quad 8.410 E-21 \quad 3.00 E-09$ 8.531E-06

1.649E-11 4.546E-18 4.00E-09 4.611E-03

$1.478 E-09 \quad 4.074 E-16 \quad 3.00 E-08$ 4.133E-01

0.035 
FOR JAN THROUGH SUN 1993

MCLIDES $\begin{gathered}\text { HALF LIFE } \\ \text { (DAYS) }\end{gathered}$
MB-89
CONCEN (UCML)
CURIES
UNCERT(CURIES)
UNCERT(UCMM)
\% OF TOTAL
RE-188
CONCEN (UCML)
CURIES
UNCERT(CURIES)
UNCERT(UCAML)
\% OF TOTAL

SR-90
CONCEN (UCML)
CURIES
UNCERT(CURIES)
UNCERT(UCML)
$\%$ OF TOTAL
T.059E+04
TC-99M
CONCEN (UCML)
CURIES
UNCERT(CURIES)
UNCERT(UCAML)
\% OF TOTAL
XE-133
CONCEN (UCML)
CURIES
UNCERT(CURIES)
UNCERT(UCAML)
$\%$ OF TOTAL

JAMUARY FEBRUARY

$\begin{array}{llllll}2.750 E-09 & 1.398 E-09 & 2.008 E-09 & 1.548 E-09 & 3.018 E-09\end{array}$

$\begin{array}{llllll}2.429 E-01 & 7.235 E-02 & 9.883 E-02 & 9.233 E-02 & 1.747 E-01\end{array}$

3.400E-03 3.340E-03 5.070E-03 4.510E-03 5.500E-03

0.035

0.038

0.036

0.035

0.475

$6.803 E-13$

$3.349 E-05$

8.020E-06

\begin{abstract}
$9.648 E-16$
\begin{tabular}{lll}
$4.992 E-06$ & $4.605 E-07$ & $2.7514 E-15$ \\
\hline $.540 E-08$ & $3.170 E-08$ & $2.140 E-08$
\end{tabular}

$1.540 E-08 \quad 3.170 E-08 \quad 2.140 E-08$
\end{abstract}

5.469E-12 $\quad 1.019 E-11 \quad 8.966 E-12 \quad 6.392 E-12 \quad 1.427 E-12$ $\begin{array}{llllll}2.842 E-04 & 5.272 E-04 & 4.414 E-04 & 3.812 E-04 & 8.258 E-05\end{array}$ $\begin{array}{lllll}8.300 E-06 & 9.500 E-06 & 8.800 E-06 & 1.010 E-05 & 8.020 E-06\end{array}$

2.788E-08 $\quad 1.087 E-08 \quad 1.869 E-08 \quad 8.722 E-09$ $2.449 E+00 \quad 5.622 E-01 \quad 9.202 E-01 \quad 5.202 E-01$

$1.850 E-01$ 1.120E-01 1.480E-01 1.600E-01
0.359
0.296
0.336
0.195

XE-135 $3.792 E-01$

CONCEN (UCML)

CURIES

$\begin{array}{lllll}1.711 E-07 & 6.674 E-08 & 1.092 E-07 & 7.928 E-08 & 1.150 E-08\end{array}$

$8.890 E+00 \quad 3.453 E+00 \quad 5.376 E+00 \quad 4.728 E+00 \quad 6.658 E-01$

UNCERT( CURTES )

INCERT (UCML)

$\%$ OF TOTAL.

8. $900 E-02$

$\begin{array}{ll}4.728 E+00 & 6.658 E-01 \\ 5.300 E-02 & 2.260 E-02\end{array}$

2.205

2.820

1.963

1.773

1.810

$1.062 E-02$

CONCEN (UCML)

CORTES

$\begin{array}{llllll}7.962 E-08 & 2.681 E-08 & 5.322 E-08 & 3.697 E-08 & 7.495 E-09\end{array}$

$\begin{array}{lllll}4.138 E+00 & 1.387 E+00 & 2.620 E+00 & 2.20 .5 E+00 & 4.338 E-01\end{array}$

UNERT (CURIES )

UNCERT (UCAL)

\% OF TOTAL
$2.060 E-01$ 1.210E-01 1.520E-01 $1.310 E-015.3380 E-01$
1.026
0.731
0.957
0.827
1.179

$\begin{array}{lll} & \text { PAGE } 4 \text { RPT. } 120 \\ \text { TRA } 120 & \\ \text { ANUAL } & & \text { YEARLY } \\ \text { AVG CONCEN } & \text { YEARLY } & \text { RELEASE } \\ \text { TOT CURIES } & \text { RELEASE* CG** } & \% \text { OF CG }\end{array}$

2.078E-09 5.729E-16 9.00E-09

5.8IIE-0I

0.050

$1.298 E-13 \quad 3.302 E-20 \quad 6.00 E-09$

$3.349 E-0.5$

$2.796 E-15 \quad 7.707 E-22 \quad 9.00 E-12$ $7.818 E-07$

$6.140 E-12 \quad 1.692 E-18 \quad 1.00 E-07$ 1.717E-03

$1.235 E-08 \quad 3.403 E-15 \quad 5.00 E-07$ $3.452 E+00$

0.295

8.267E-08 2.279E-14 8.00E-08 $2.311 E+01$

1.975

$3.857 E-08 \quad 1.063 E-14 \quad 5.00 E-08$ $1.078 E+01$

0.921 
RADIOACTIVE WASTE MANAGEMENT INFORMATION SYSTEM

FOR JAN THROUGH JUN 1993

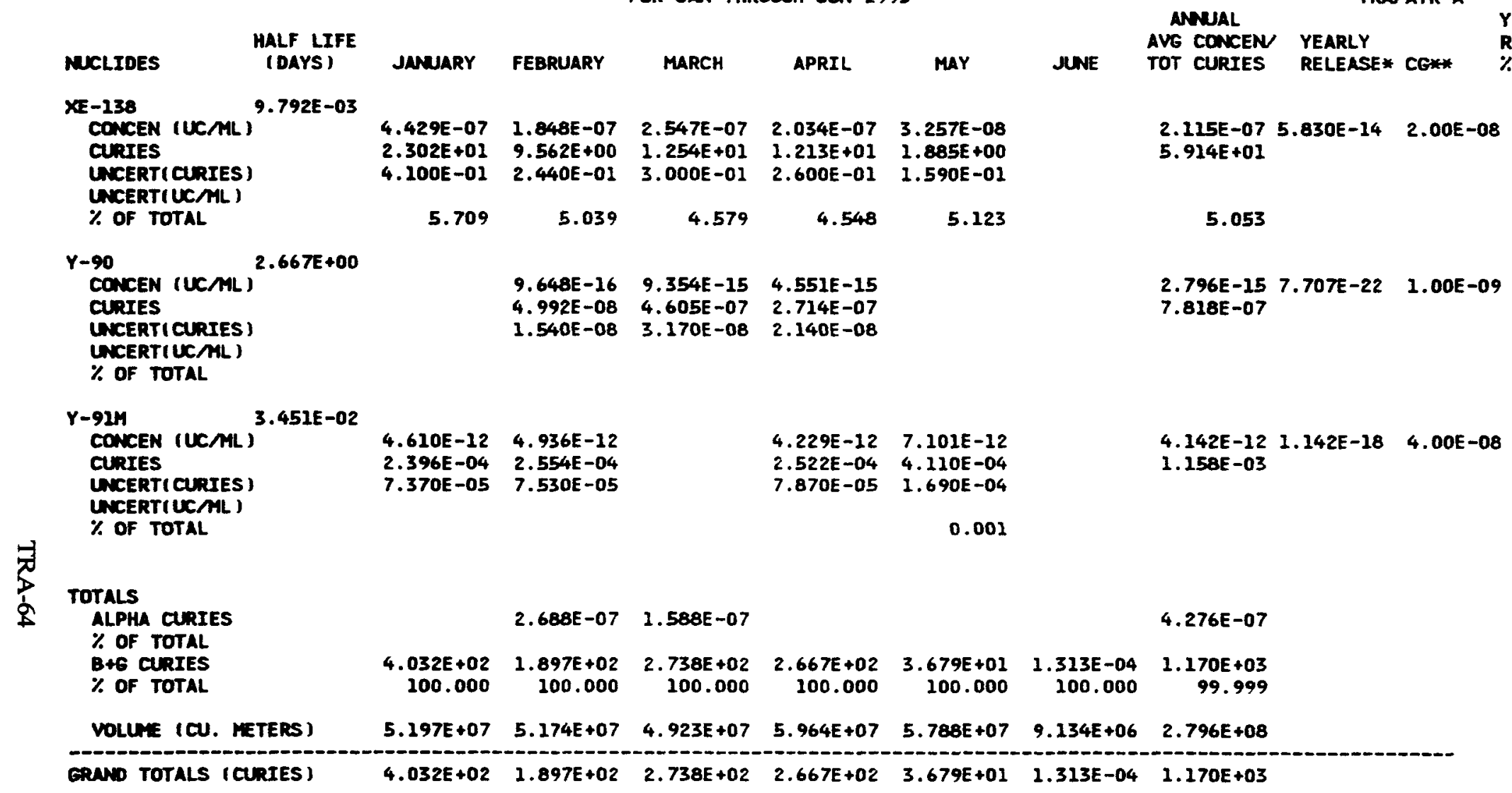

* This muber is derived from a dispersion factor equation. The dispersion factor is derived from the straight line gausian model and is provedad by the National Oceanic Atmospheric Adninistration (NOAA).

* NOTE: DCE value obtained from DOE ORDER 5400.5 deted February 8, 1990, Figures III-I and III-3 
RADIOACTIVE WASTE MANAGEMENT INFORMATION SYSTEM

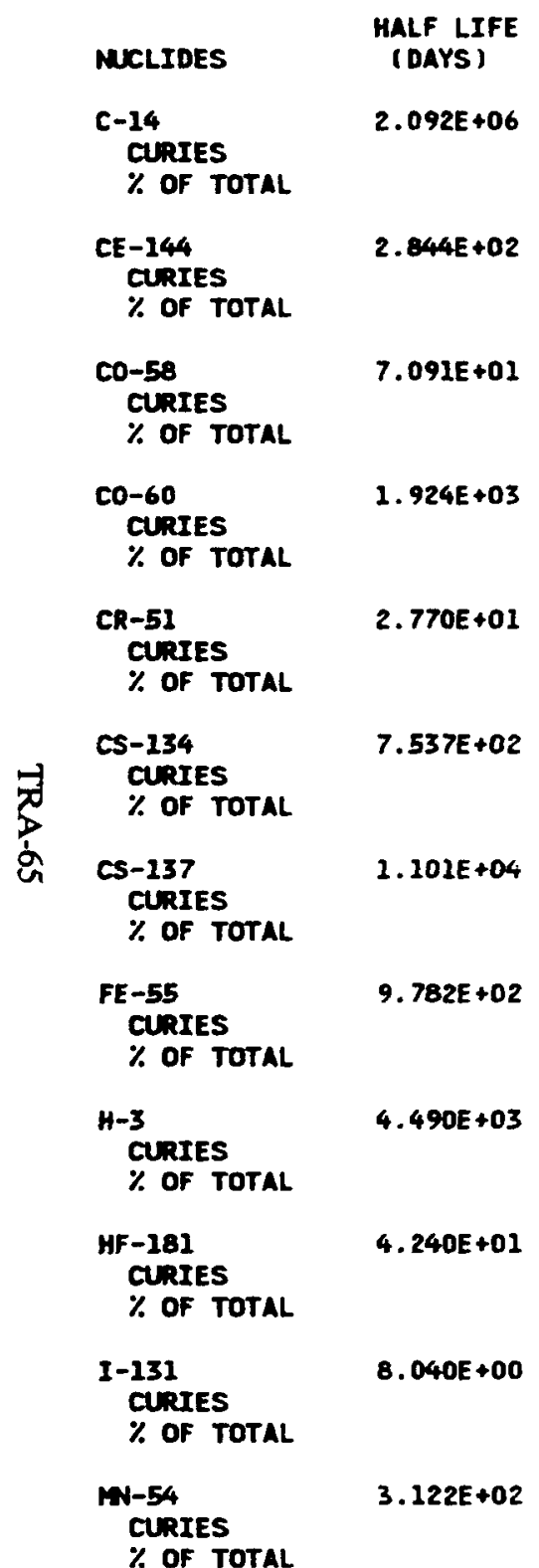

ATR DISPOSED SOLIO WASTE SUMMARY

FOR JAN THROUGH JN 1993

JANUARY FEBRUARY MARCH APRIL MAY TOTAL

$1.400 E-02$

0.001

$1.400 E-02$

$2.800 E-03 \quad 2.800 E-03$

$5.000 E+00$

0.424

3.000E-04 5.000E+00

0.003

$2.850 E+02$

24.150

$2.820 E+02 \quad 2.820 E+02 \quad 8.490 E+02$

0.283

0.284

0.424

$4.319 E+01 \quad 4.319 E+01$

$0.043 \quad 0.022$

$9.000 E-04 \quad 9.000 E-04$

$5.200 E-03 \quad 5.200 E-03$

$6.900 E+02$

58.468

$6.900 E+02$

0.345

1. 100E-04

$9.820 E+04$

$9.798 E+04 \quad 1.962 E+05$ 98.691 98.646

98.086

5. $900 E-03 \quad 5.900 E-03$

$6.400 E-03 \quad 6.400 E-03$

$8.300 E+01$

7.033

4.000E-04 8.300E+01

0.041 
TIONS OFFICE

U.S. DEPARTMENT OF ENERGY

RADIOACTIVE WASTE MANAGEMENT INFORMATION SYSTEM

ATR OISPOSED SOLID WASTE SUMMARY

MUCLIDES
M- 94
CURIES
$\%$ OF TOTAL

HALF LIFE

(DAYS)

$7.300 E+06$

M-95

CURIES

$\%$ OF TOTAL

NI -59

CURIES

$\%$ of TOTAL

NI -63

CURIES

$\%$ OF TOTAL

RU-103

CURIES

$\%$ of total

SB-124
CURIES
娄
\& OF TOTAL

$58-125$

$\%$ OF TOTA

SC-46

CURIES

\% DF TOTAL

SE-75
CURIES

$\%$ of total

SN-119M

CURIES

$2.930 E+02$

SR-90

CURIES

$\%$ OF TOTAL

TC-99

CURIES

$\%$ of total
$2.774 E+07$

$3.650 E+04$

$3.924 E+01$

$6.020 E+01$

$1.007 E+03$

$8.382 E+01$

$1.196 E+02$

$1.059 E+04$

$7.775 E+07$
FOR JAN THROUGH JUN 1993

JANUARY FEBRUARY MARCH APRIL

$9.000 E-07$

$4.360 E-02 \quad 4.360 E-02$

$1.120 E-0$

0.009

$1.120 E-01$

$1.500 E+01$

1.271

$5.000 E+00$

0.424

$$
\begin{array}{llr}
1.025 & 1.027 & 1.027
\end{array}
$$

1.700E-03 1.700E-03

5. DOOE-04 5.000E-04

4. D00E-04 5.000E+00

0.003

$1.000 E-03 \quad 1.000 E-03$

$9.000 E-03 \quad 9.000 E-03$

$1.500 E+01$

1.271

0.007

$2.500 E-09$

3.400E-03 3.400E -03

2.500E -05

$2.500 E-05$
RUN DATE: 05/12/94

PAGE 2 RPT. 122

TRAATR-S 

RADIOACTIVE WASTE MANAGEMENT INFORMATION SYSTEM

ATR DISPOSED SOLID WUSTE SUMLARY FOR JAN THROUGH JUN 1993

$\begin{array}{lc}\text { MUCLIOES } & \begin{array}{c}\text { HALF LIFE } \\ \text { (DAYS) }\end{array} \\ \begin{array}{l}\text { ZN-65 } \\ \text { CURIES }\end{array} & 2.438 E+02 \\ \% \text { OF TOTAL } & \\ \text { ZR-95 } & 6.403 E+01\end{array}$

CR-95

$\%$ Of TOTAL

$6.403 E+01$

\section{TOTALS}

ALPHA CURIES

$\%$ OF TOTAL

$B+G$ CURIES

$\%$ OF TOTAL

TOTAL GRAMS OF AM

TOTAL ERAMS OF PU

TOTAL GRANS OF PU-239+241

TOTAL ERNAS OF $U$

TOTAL ERAMS OF TH

TOTAL ERAMS OF NP

1
5 VOLUNE (CU. METERS)

GRAM TOTALS (CURIES)

MARCH

APRIL

maY
$8.200 E+01$

6.948
$7.640 E-01$

$4.080 E+02$

$1.180 E+03$

JNNE

ANMUAL

TOTAL

$1.600 E-03 \quad 8.200 E+01$

0.041

$7.500 E-03 \quad 7.500 E-03$

$1.180 E+03$

200.000

$$
\begin{array}{rrr}
9.950 E+04 & 9.933 E+04 & 2.000 E+05 \\
100.000 & 100.000 & 100.000
\end{array}
$$

PAGE

\section{RPT, 122}

$\begin{array}{lll}7.640 E-01 & 6.709 E+00 \quad 8.237 E+00\end{array}$

$2.109 E+02 \quad 8.459 E+03 \quad 9.078 E+03$

$9.950 E+04 \quad 9.933 E+04 \quad 2.000 E+05$ 
RADYOACTIVE WUSTE MUMAGEMENT INFORMATION SYSTEM

CHEM LAB ADD. AIRBORNE WASTE SUMMARY FOR JAN THROUGH NUN 1993

\section{MCLIDES \\ eross-ALPHA \\ CONCEN (UCML) \\ CURIES \\ UNCERTICURIES ) \\ UNCERTIUCML) \\ $\%$ OF TOTAL}

MALF LIFE
(DAYS)

eRoss-BETA

CONCEN (UCML)

CURIES

UNCERTI CURIES

UNCERT (UCAL)

$\%$ OF TOTAL

JANUARY FEBRUARY

MARCH

APRIL.

may

$-1.804 E-16-2.837 E-16$

$-1.590 E-09-2.500 E-09$

\begin{tabular}{l}
$4.210 E-09 \quad 2.480 E-09$ \\
\hline
\end{tabular}

1. 248E-15

$1.100 E-08$
$2.840 E-09$

$1.072 E-16$
$9.450 E-10$

$-22.682 \quad-35.014$

41.353

13.990

172.442

$122.682 \quad 135.014$

58.647

86.010

$-72.442$

JNE

$\begin{array}{lll}-16 & 2.450 E-16 & -3.030 E-16 \\ -10 & 2.090 E-09 & -2.670 E-09\end{array}$

$-40.826$

$\begin{array}{llllllll}9.758 E-16 & 1.094 E-15 & 1.770 E-15 & 6.593 E-16 & -1.029 E-16 & 1.045 E-15 & 9.123 E-16 & 1.942 E-22 \\ 8.600 E-09 & 9.640 E-09 & 1.560 E-08 & 5.810 E-09 & -8.780 E-10 & 9.210 E-09 & 4.798 E-08 & \\ 4.850 E-09 & 3.220 E-09 & 3.500 E-09 & 3.630 E-09 & 2.500 E-09 & 3.150 E-09 & \end{array}$

140.826

86.834

\section{TOTALS}

ALPHA CURIES

$\%$ Of total

B+G cuiries

$\%$ OF TOTAL

$-1.590 E-09-2.500 E-09$

$\begin{array}{rr}-22.682 & -35.014 \\ 8.600 E-09 & 9.640 E-09\end{array}$

1. $100 E-08$

41.353
$560 E-08$

$9.450 E-10$
13.990

2.090E-09-2.6

$810 E-09$

172.442

$122.682 \quad 135.014$

58.647

86.010

$-72.442$

$670 E-09$
-40.826

-40.826
$210 E-09$

140.826

7.275E-09

13.166

$98 E-08$

MNUAL
AVE CONCEN YEARLY YEARLY

PAGE 2 RPT. 116

TOT CURIES RELEASE* CG** \% OF CG

$1.383 E-16 \quad 2.944 E-23$

$.275 E-09$

13.166

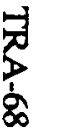

$\begin{array}{llllllll}\text { VOLUE (CU. METERS) } & 8.813 E+06 & 8.813 E+06 & 8.813 E+06 & 8.813 E+06 & 8.529 E+06 & 8.813 E+06 & 5.259 E+07\end{array}$

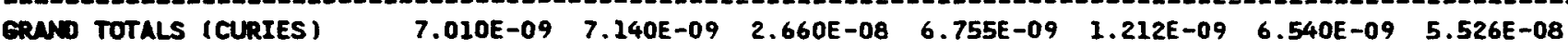

* This number is derived from a dispersion factor equetion. The dispersion factor is derived from the straight line sacssian model and is proveded by the Notional Oceanic Atmospheric Adninistration (NOAA).

* NOTE: DCG valu abtainad from DOE ORDER 5400.5 chted februry 8, 1990 , figures III-1 and III-3 
ETR AIRBORME WUSTE SUMAARY

FOR JAN THROUGH JN 1993

\section{MUCLIDES \\ eross-ALPHA CONCEN (UCAL) \\ CURIES \\ UNCERTI CURIES ) \\ UNCERT (UCAL) \\ $\%$ OF rotal}

MALF LIFE

\section{MLLF LIFE
(DAYS 3}

JANARY FEBRUARY

MARCH

APRIL

MaY

MNE

$-6.952 E-16 \quad 1.020 E-15 \quad 5.921 E-16$

-5.800E-09 8.510E-09 4.940E-09

4.220E-09 5.060E-09 5.270E-09

$-3.448$

2.374

1.615

enoss-aeta

CONCEN (UCAL)

CURIES

UNCERT (CURIES)

INCERT(ICML)

\% OF TOTAL

$\begin{array}{lll}2.086 E-14 & 4.195 E-14 & 3.608 E-14 \\ 1.740 E-07 & 3.500 E-07 & 3.010 E-07\end{array}$

9.400E-09 1.460E-08 1.310E-08

$\begin{array}{lll}103.448 & 97.626 \quad 98.385\end{array}$

-5.800E-09 8.510E-09 4.940E-09

$\begin{array}{rrr}-3.448 & 2.374 & 1.615\end{array}$

1.740E-07 3.500E-07 3.010E-07

$\begin{array}{lrr}103.448 & 97.626 & 98.385\end{array}$

ALPHA CURIES

$\%$ OF TOTAL

$\%$ of total

헝

VOLUKE (CU. METERS) $8.343 E+06 \quad 8.343 E+06 \quad 8.343 E+06$

ERND TOTALS (CURIES) $1.682 E-07$ 3.585E-07 3.059E-07

\begin{tabular}{lll} 
& PAGE I RPT. 110 \\
TRAETR-A & YNUAL & \multicolumn{1}{c}{ YEARLY } \\
AVG CONCEN & YEARLY & RELEASE \\
TOT CURIES & RELEASE* CE* & $\%$ OF CG
\end{tabular}

$3.056 E-16 \quad 4.310 E-23$

7.650E-09

0.919

$3.296 E-14 \quad 4.648 E-21$

8.250E-07

99.081

$7.650 E-09$ 0.919
$2505-07$

99.081

2.503E+07

8. 326E-07

* This mabar is carived from o dispersion factor equation. The dispersion factor is dorived from the straight line ocuseian modal and is proveded by the Netional Oceanic Atmospheric Adninistrotion (NOAA).

** NOTE: DCE value obtained from DOE OROER 5400.5 doted Februpry B, 1990, figures III-1 and III-3 
SCHED. NO. P62PMO09-H

IDAHO OPERATIONS OFFICE

U.S. DEPARTMENT OF ENERGY

RADIOACTIVE WASTE MANAGEMENT INFORMATION SYSTEM

ETR DISPOSED SOLID WASTE SUMARY

FOR JAN THROUGH JUN 1993

$\begin{array}{lc}\text { MUCLIDES } & \begin{array}{c}\text { HALF LIFE } \\ \text { (DAYS) }\end{array} \\ \begin{array}{c}\text { NH-241 } \\ \text { CURIES }\end{array} & 1.577 E+05 \\ \text { \% OF TOTAL } \\ \text { GRNAS }\end{array}$

\section{CH-242}

CURIES

$1.630 E+02$

$\%$ of total

CH-244

CURIES

$\%$ OF TOTAL

$6.610 E+03$

PU-238

CURIES

$\%$ of total

enars

PU-239

CURIES
$\%$ OF TOTAL

支

$8.800 E+06$

JANUARY FEBRUARY

MARCH

APRIL

MaY

7.540E-08

1.587

$2.192 E-08$

8. $000 E-10$

0.017

$1.360 E-08$

0.286

4.280E-06

90.109

2.503E-0.7

3. 800E -07

8.000

$6.119 E-06$

4. $750 E-06$

100.000

ALPHA CURIES

$\%$ OF TOTAL

$B+6$ CURIES

\% OF TOTAL

TOTAL GRAMS OF AM

TOTAL GRNAS OF PU

TOTAL GRars of PU-239+241

TOTAL ERANS OF U

TOTAL ERANS OF NP

VOLUNE (CU. METERS)

VEIEHT (KILOERWHS)

GRMD TOTALS (CURIES)

$2.192 E-08$

$6.369 E-06$

$6.129 E-06$

$3.856 \mathrm{E}+02$

4.750E-06
RUN DATE: 05/12/94

PAGE 1 RPT. 112 TRUETR-S

ANUAL
TOTAL

$7.540 E-08$

1.587

2. $192 E-08$

8.000E-10

0.017

$1.360 E-08$

0.286

4. 280E-06

90.109

$2.503 E-07$

$3.800 E-07$

8.000

$6.119 E-06$

4. $750 E-06$

100.000

$2.192 E-08$

$6.369 E-06$

6.119E-06

$3.620 E+00$

$3.856 E+02$

4. 750E-06 


\section{ITR AIRBORAE WUSTE SUMTARY}

MALF LIFE
MCLIDES
(DAYS)
CO-60
CONCEN (UCML)
CURIES
UNCERTICURIES)
UNCERT(UCML)
\% OF TOTAL

$$
\text { CS-137 }
$$

$1.101 E+04$

JANUARY FEBRUARY MARCH

APRIL

may

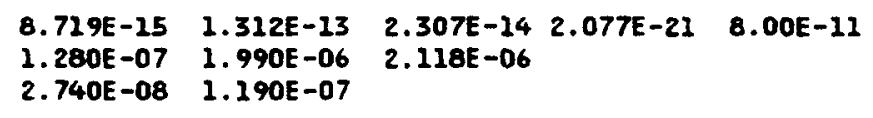

PAGE 1 RPT. 123 TRA TRA-A

YEARLY NWUAL AVG CONCEN YEARLY RELEASE \% OF CG
CURIES

UNCERT I CURIES )

$\%$ OF TOTAL

\section{GROSS-ALPHA \\ CONCEN (UCML) \\ CURIES \\ UNCERTICURIES I \\ UNCERT (UCAL) \\ $\%$ Of TOTAL \\ SROOSS-BETA \\ CONCEN (UCAN) \\ CURIES \\ UNCERT (CURIES) \\ UNCERT (UCAL) ) \\ \% OF TOTAL}

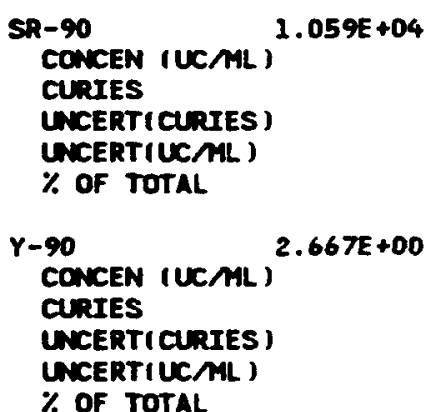

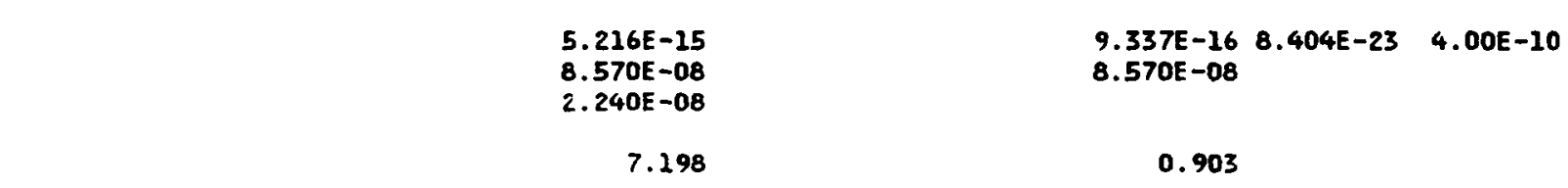

7.298

$9.337 E-16 \quad 8.404 E-23 \quad 4.00 E-10$ 8.570E-08

0.903

82.432

58.809

73.995 
IDAHO OPERATIONS OFFICE

U.S. DEPARTMENT OF ENERGY

RADIOACTIVE WASTE MANAGEMENT INFORMATION SYSTEM

MTR AIRBORNE HASTE SUMARY

FOR JAN THROUGH JUN 1993

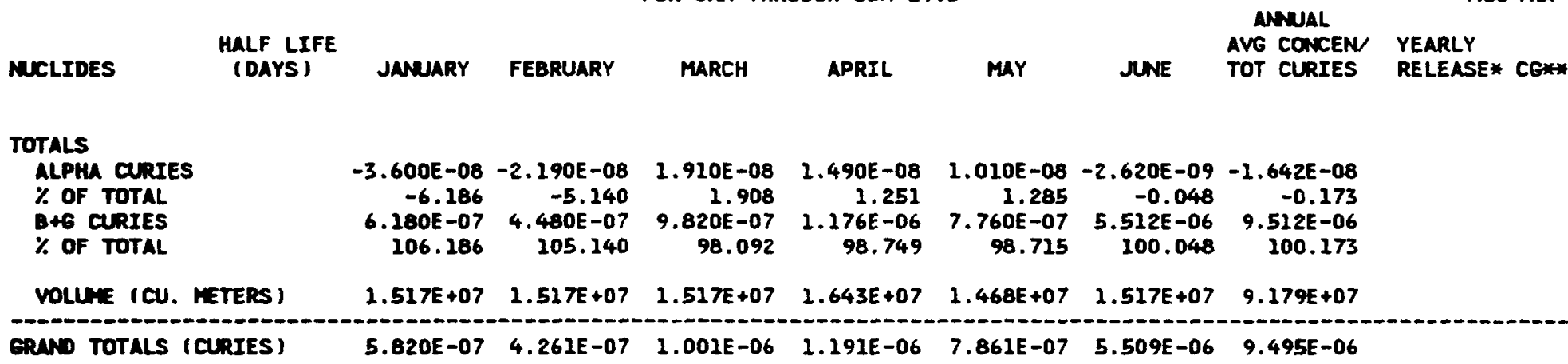

* This nubar is derived from a dispersion factor equation. The dispersion factor is derived from the straight 1 ine goussien model and is proveded by the Netional Dceanic Atmospheric Administration (NOAA).

** NOTE: DCG value obtained from DOE ORDER 5400.5 dated Fabmery 8, 1990, Figures III-1 and III-3
RUN DATE: 05/12/94

PAGE 2 RPT. 123

YEARLY RELEASE \% OF CG 
TRA HOT CELL AIRBORNE WUSTE SUMMARY FOR JAN THROUGH JUN 1993

\section{MCLIDES \\ eross-ALPHA \\ CONCEN (UCML) \\ CURIEs \\ UNCERT (CURIES ) \\ UNCERTIUCAL \\ $\%$ OF TOTAL}

\section{HALF LIFE}

EROSS-BETA

CONCEN (UCML)

CURIES

UNCERT (CURIES)

UNCERT(UCML)

$\%$ OF TOTAL

\section{Thart fegruary}

36.066 $1.873 E-14$
$7.560 E-06$ $.560 E-06$
$.720 E-09$

\section{4. $723 E-25$}

1. $970 E-08$

$7.710 E-09$

$1.801 E-14$

$1.882 E-14$

. $890 E-08 \quad 9.850 E-08$

$1.122 E-14 \quad 3.0$

402.041

112.106

88.005

$4.680 E-08$
$9.310 E-09$

38.066
63.934

3.047E-14

O50E-08 $9.000 E-09$
$7.510 E-09$

20.134

$9.879 E-0 B$

ANNAL

PAGE 1 RPT. 125 TRUTRA-A

AVG CONCEN

YEARLY

TOT CURIES RELEASE* CE** RELASE

\section{TOTALS}

ALPHA CURIES

$\%$ OF TOTAL

B+s curIEs

$-1.480 E-08-8.110 E-09$ $\begin{array}{r}-302.042,-12.106 \\ \hline, 970 E-08,720 E-08\end{array}$ $1.970 E-08 \quad 7.510 E-08$

\section{1. $070 E-08$} 11.996
$850 E-08$ $.850 E-08$
88.005

$8.559 E-15$
$3.570 E-0 B$

20.685

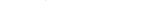

112.106
2.640E-08
36.066

.150E-08

$1.522 E-14$ 5.601E-22

$61.934 \quad 79.866$

79.315

\begin{tabular}{llllllll} 
VOLUNE ICU. METERS) & $4.171 E+06$ & $4.171 E+06$ & $4.171 E+06$ & $4.171 E+06$ & $4.037 E+06$ & $4.171 E+06$ & $2.489 E+07$ \\
\hline SRND TOTALS (CURIES) & $4.900 E-09$ & $6.699 E-08$ & $8.920 E-08$ & $7.320 E-08$ & $1.986 E-07$ & $4.470 E-08$ & $4.776 E-07$
\end{tabular}

* This nubar is derived from a dispersion factor equetion. The dispersion factor is derivod from the straight line goussian model and is proveded by the Nbtional Oceanic Atmospheric Administration (NOAA).

** NOTE: DCG velue obtainad from DOE ORDER 5400.5 dated February 8, 1990 , Figures III-I and III-3 
RADIOACTIVE MUSTE MANAGEMENT INFORMATION SYSTEM

TRA LIQUID MASTE SUMHARY

FOR JAN THROUGH JUN 1993

\begin{tabular}{|c|c|c|c|c|}
\hline MUCLIDES & $\begin{array}{l}\text { HALF LIFE } \\
\text { (DAYS ) }\end{array}$ & JANUAARY & FEBRUARY & MARCH \\
\hline $\begin{array}{l}\text { CE-141 } \\
\text { CONCEN (UCML) } \\
\text { CURIES } \\
\text { UNCERT (CURIES) } \\
\text { UNCERT (UCML) } \\
\text { \% OF TOTAL }\end{array}$ & $3.250 E+01$ & $\begin{array}{r}5.270 E-07 \\
2.799 E-03 \\
\\
1.500 E-08 \\
0.016\end{array}$ & $\begin{array}{r}2.710 E-07 \\
2.269 E-03 \\
1.400 E-08 \\
0.012\end{array}$ & \\
\hline $\begin{array}{l}\text { CE-144 } \\
\text { CONCEN (UCML) } \\
\text { CURIES } \\
\text { UNCERT(CURIES) } \\
\text { UNCERTIUCML) }\end{array}$ & $2.844 E+02$ & $\begin{array}{l}2.850 E-07 \\
1.514 E-03 \\
5.800 E-08\end{array}$ & $\begin{array}{l}3.310 E-07 \\
2.772 E-03 \\
8.600 E-08\end{array}$ & $\begin{array}{l}2.490 E-07 \\
1.732 E-03 \\
9.100 E-08\end{array}$ \\
\hline
\end{tabular}

PAGE 2 RPT. 105
TRA-L
ANWAL
TOT CONCEN
TOT CURIES CG *

\subsection{E-07 5.00E-05 \\ 0.24}

$5.068 E-03$

0.005

$\begin{array}{lll}1.431 E-07 & 7.00 E-06 & 2.04\end{array}$

$6.017 E-03$

0.006

5. $922 E-07 \quad 4.00 E-05$

1.48

CONCEN (UCML)

$7.091 E+01$

CURIES

UNCERTICURIES )

UNCERTIUCAL

$\%$ Of total

CO-60

$1.924 E+03$

CONCEN (UCAL)

CURIES

UNCERT (CURIES )

UNCERT(UCML)

$\%$ Of TOTAL

CR-51

CONCEN (UCAL)

CURIES

UNCERT(CURIES)

UNCERTIUCAML)

\% OF TOTAL

$2.770 E+01$

$7.537 E+02$

CS-134

CONCEN (UCAL)

CURIES

UNCERTI CURIES )

UNCERTIUCMLI

\% OF TOTAL

\section{CS-137}

1. 101E+04

CONCEN (UCML)

CURIES

UNCERT I CURIES )

UNCERTIUCALI

$\%$ of total

4


TRA LIQUID MUSTE SUMUAY

FOR JAN THROUGH JUN 1993

\begin{tabular}{|c|c|}
\hline MUCLIDES & $\begin{array}{l}\text { HALLF LIFE } \\
\text { (DAYS) }\end{array}$ \\
\hline $\begin{array}{l}\text { GROSS-ALPHA } \\
\text { CONCEN (UCML) } \\
\text { CURIES } \\
\text { UNCERT ( CURIES) } \\
\text { UNCERT (UCAML) } \\
\text { \% OF TOTAL }\end{array}$ & \\
\hline $\begin{array}{l}\text { CROSS-BETA } \\
\text { CONCEN (UCML) } \\
\text { CURIES } \\
\text { UNCERT(CURIES) } \\
\text { UNCERTIUCML) } \\
\text { \% OF TOTAL }\end{array}$ & \\
\hline $\begin{array}{l}\text { H-3 } \\
\text { CONCEN (UCML) } \\
\text { CURIES } \\
\text { UNCERT(CURIES) } \\
\text { UNCERT(UCAM) } \\
\text { \% OF TOTAL }\end{array}$ & $4.490 E+03$ \\
\hline $\begin{array}{l}\text { MA-24 } \\
\text { CONCEN (UCML) } \\
\text { CURIES } \\
\text { UNCERTI CURIES) } \\
\text { UNCERT(UCAL) } \\
\text { \% OF TOTAL }\end{array}$ & $6.237 E-01$ \\
\hline $\begin{array}{l}\text { SB-124 } \\
\text { CONCEN (UCML) } \\
\text { CURIES } \\
\text { UNCERT(CURIES) } \\
\text { UNCERT(UCAML) } \\
\text { \% OF TOTAL }\end{array}$ & $6.020 E+01$ \\
\hline $\begin{array}{l}\text { SR-90 } \\
\text { CONCEN (UCAL) } \\
\text { CURIES } \\
\text { UNCERT(CURIES) } \\
\text { UNCERT(UCML) } \\
\% \text { OF TOTAL }\end{array}$ & $1.059 E+04$ \\
\hline $\begin{array}{l}\text { TA-182 } \\
\text { CONCEN (UCAL) } \\
\text { CURIES } \\
\text { UNCERT(CURIES) } \\
\text { GCERT(UCAML) } \\
\text { \% OF TOTAL }\end{array}$ & $1.145 E+02$ \\
\hline
\end{tabular}

JNUARY FEBRUARY MARCH APRIL MAY JUNE TOT CURIES CG* $\%$ OF CG
$\begin{array}{ll}3.200 E-06 & 1.600 E-05 \\ 1.700 E-02 & 1.340 E-01\end{array}$
$1.600 E-05 \quad 1.700 E-05$
$1.700 E-05$
$1.182 E-01$
$\begin{array}{llll}5.500 E-06 & 2.000 E-05 & 9.400 E-05 & 2.472 E-05 \\ 4.266 E-02 & 1.501 E-01 & 5.775 E-01 & 1.039 E+00\end{array}$
5. 100E-07 3.000E-06
$3.100 E-06$
$9.000 E-07$
0.232
$3.000 E-06$
$1.500 E-05$
1.031

$\begin{array}{llllllll}3.180 E-03 & 2.110 E-03 & 5.000 E-03 & 2.350 E-03 & 1.210 E-03 & 1.280 E-04 & 2.317 E-03 & 2.00 E-03\end{array}$

$\begin{array}{lllllll}1.689 E+01 & 1.767 E+01 & 3.477 E+01 & 1.823 E+01 & 9.081 E+00 & 7.864 E-01 & 9.742 E+01\end{array}$

$\begin{array}{llllll}6.000 E-05 & 4.000 E-05 & 1.000 E-04 & 5.000 E-05 & 3.000 E-05 & 5.000 E-06\end{array}$

$1.060 E-06 \quad 2.040 E-06$

$5.630 E-03 \quad 1.708 E-02$

$3.000 E-08 \quad 1.400 E-07$

$\begin{array}{rr}0.033 & 1.400 E-07 \\ 0.094\end{array}$

\begin{tabular}{|c|c|c|c|c|c|c|}
\hline & & & $\begin{array}{r}2.510 E-07 \\
1.542 E-03 \\
4.600 E-08 \\
0.062\end{array}$ & $\begin{array}{l}3.668 E-08 \\
1.542 E-03\end{array}$ & $1.00 E-05$ & 0.37 \\
\hline $\begin{array}{l}7.900 E-07 \\
5.494 E-03\end{array}$ & $\begin{array}{l}2.890 E-06 \\
2.242 E-02\end{array}$ & $\begin{array}{l}6.800 E-06 \\
5.103 E-02\end{array}$ & & $\begin{array}{l}1.878 E-06 \\
7.895 E-02\end{array}$ & $1.00 E-06$ & $\begin{array}{r}187.76 \\
* \times \times * \times *\end{array}$ \\
\hline $\begin{array}{r}4.700 E-08 \\
0.016\end{array}$ & $\begin{array}{r}1.600 E-07 \\
0.122\end{array}$ & $\begin{array}{r}4.000 E-07 \\
0.543\end{array}$ & & 0.078 & & \\
\hline $\begin{array}{l}1.480 E-07 \\
1.029 E-03\end{array}$ & & & & $\begin{array}{l}2.448 E-08 \\
1.029 E-03\end{array}$ & $2.00 E-05$ & 0.12 \\
\hline $\begin{array}{r}9.600 E-08 \\
0.003\end{array}$ & & & & 0.001 & & \\
\hline
\end{tabular}

115.86

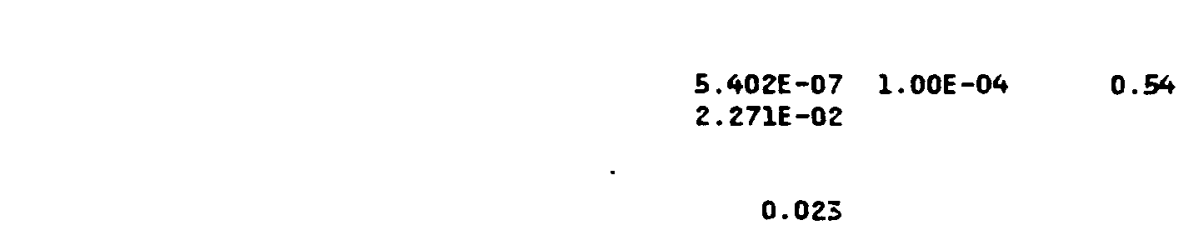

$\begin{array}{ccc}\begin{array}{c}5.402 E-07 \\ 2.271 E-02\end{array} & 1.00 E-04 & 0.54 \\ 0.025 & \end{array}$

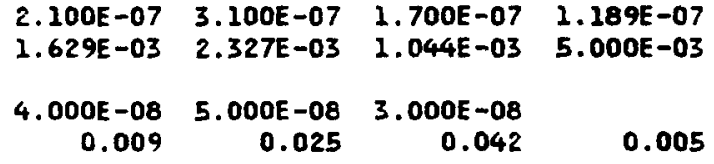


TRA LIQUID MASTE SUMYARY

FOR JAN THROUGH JUN 1993

$\begin{array}{lc}\text { MUCLIDES } & \begin{array}{c}\text { HALF LIFE } \\ \text { (DAYS) }\end{array} \\ \text { Y-90 } & 2.667 E+00 \\ \text { CONCEN (UCML) } & \\ \text { CURIES } & \\ \text { UNCERT(CURIES) } & \\ \text { UNCERT(LCML) } & \\ \text { \% OF TOTAL } & \end{array}$

JANUARY FEBRUARY

APRIL

0.122

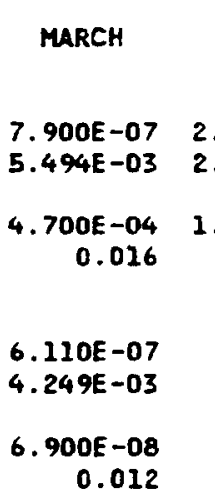
2.060E-07 5.390E-07 6.110E-07
$1.094 E-03 \quad 4.514 E-03 \quad 4.249 E-03$
$\begin{array}{rrr}4.000 E-08 & 6.600 E-08 & 6.900 E-08 \\ 0.006 & 0.025 & 0.012\end{array}$
0.025
.012

MaY

JNE

$\begin{array}{lrr}\text { PAGE } & 3 \text { RPT. } 105 \\ \text { TRA-L } \\ \text { AVUS CONCEN } \\ \text { TOT CURIES CG * }\end{array}$

$\begin{array}{ll}2.890 E-06 & 6.800 E-06 \\ 2.242 E-02 & 5.103 E-02\end{array}$

.600E-07 4.000E-07

0.543

$1.400 E-07$
$1.051 E-03$

1.140E-06 4.260E-07 9.00E-06

4.73

CURIES

UNCERTICURIES I

$\%$ of total

3. 400E-08

$1.800 E-07$

\section{$\begin{array}{lll}1.878 E-06 & 1.00 E-05 & 18.78\end{array}$}

$7.895 E-02$

0.078

TOTALS

ALPHA CURIES

$\%$ OF TOTAL

B+G CURIES

$\begin{array}{rrrrrrr} & & & 1.629 E-03 & 2.327 E-03 & 1.044 E-03 & 5.000 E-03 \\ 1.705 E+01 & 1.821 E+01 & 3.534 E+01 & 1.840 E+01 & 9.400 E+00 & 2.471 E+00 & 1.009 E+02 \\ 100.000 & 100.000 & 100.000 & 99.991 & 99.975 & 99.958 & 99.995\end{array}$

\section{volure (LITERS)}

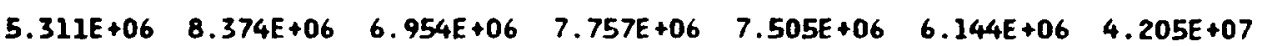

GRANO TOTALS (CURIES)

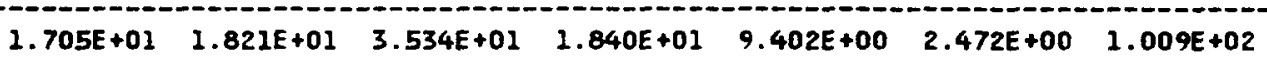

* NOTE: DCG value obtainad from DOE ORDER 5400.5 dated February8, 1990, Figures III-1 and III-3

* The CG limit is being approached or has been exceeded during the current month. Please verify the accuracy of the reported date. If the data is in error, please send correct data to haste Information. 


\section{Waste Experimental Reduction Facility 1993 Detail Data}

Waste Experimental Reduction Facility (WER) Bar Graphs

of Annual Data by Month WER-3

CY1993 Bar Graphs WER-5

WER Detail Data for Months July to December

Annual Average Concentration and Total Curies for January to December WER-7

Report 174-WER Airborne Waste Summary WER-9

Report 176-WER Disposed Solid Waste Summary WER-10

WER Detail Data for Months January to June

Annual Average Concentration and Total Curies for January to June

WER-13

Report 174-WER Airborne Waste Summary WER-15

Report 176-WER Disposed Solid Waste Summary WER-16

WER Facility Detail Data for Months July to December

Annual Average Concentration and Total Curies for January to December

WER-19

Report 180-WER No. Stack Airborne Waste Summary WER-21

Report 181-WER So. Stack Airborne Waste Summary WER-22

Report 182-W

WER 765 Stack Airborne Waste Summary

WER-23

Report 178-WER Incineration Operations, Disposed Solid Waste Summary

WER-24

WER Facility Detail Data for Months January to June

Annual Average Concentration and Total Curies for January to June

WER-27

Report 180-WER No. Stack Airborne Waste Summary WER-29

Report 181-WER So. Stack Airborne Waste Summary WER-30

Report 178-WER Incineration Operations, Disposed Solid Waste Summary

WER-31 



\section{Waste Experimental Reduction Facility (WER) Monthly Details - CY 1993}
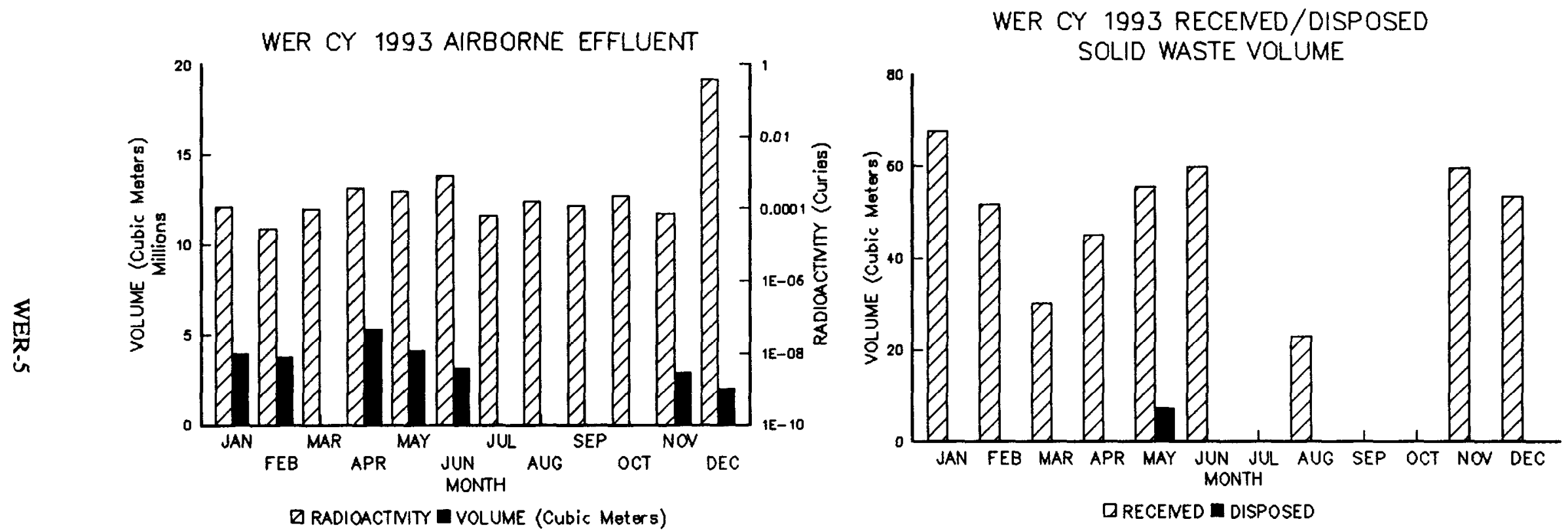

WER Detail Data for Months July to December Annual Average Concentration and Total Curies for January to December 


RAOIOACTIVE WASTE MANAGEMENT INFORMATION SYSTEM

NER AIRBORNE WASTE SUMMARY FOR JAN THROUEH DEC 1993

MUCLIDES
$\begin{aligned} & \text { HALF LIFE } \\ & \text { (DAYS) }\end{aligned}$
SR-90
CONCEN (UCAL)
CURIES
$\%$ OF TOTAL
Y-90
CONCEN (UCML)
CURIES
$\%$ OF TOTAL.

NULY

AUEUST SEPTEMBER

OCTOBER

MOVEMER

$-6.748 E-16-8.207 E-15-1.776 E-16$

$-8.350 E-09$

50.000

$.000 E-07$
50.000

$260 E-09$
50.000

$1.089 E-16$

$.280 E-09$
50.000

$2.520 E-10$
50.000

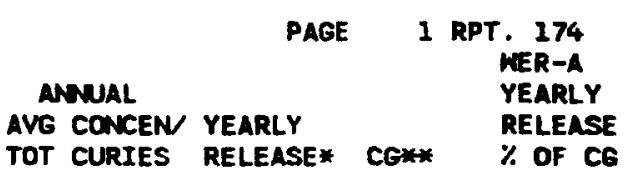

$-6.748 E-16-8.207 E-15-1.776 E-16$

$-8.350 E-09-1.000 E-07-2.260 E-09$
1.0
$1.089 E-16$
$1.280 E-09$
1.315E-17 -4.631E-16-2.336E-22
$2.520 E-20-7.162 E-08$

50.000

50.000

50.000

50.000

50.000

50.000

TOTALS

ALPHA CURIES

$\%$ OF TOTAL

B+G CURIES

\% OF TOTAL

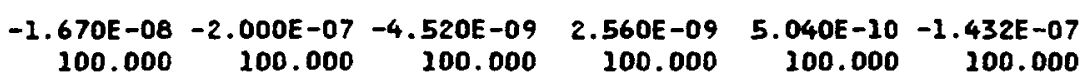

VOLUME (CU. METERS)

$1.161 E+07$

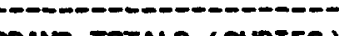

ERND TOTALS (CURIES)

$-1.670 \mathrm{E}-08-2.000 \mathrm{E}-07-4.520 \mathrm{E}-09 \quad 2.560 \mathrm{E}-09 \quad 5.040 \mathrm{E}-10-1.432 \mathrm{E}-07$

* This nuber is derived from a dispersion factor equetion. The dispersion factor is derived from the straight line Gauscian model and is provided by the National Oceanic Atmospheric Adninistration (NOAA).

** NOTE: DCG value obtained from DOE ORDER 5400.5 deted February 8, 1990, Figures III-1 and III-3 
SCHED. NO. P6IPMO09-G

TDAHO OPERATIONS OFFICE

U.S. DEPARTMENT OF ENEREY

RADIOACTIVE WASTE MANAGEMENT INFORMATION SYSTEM

WER DISPOSED SOLIO MASTE SUMMARY FOR JAN THROUSH DEC 1993

\begin{tabular}{|c|c|}
\hline MUCLIDES & $\begin{array}{l}\text { HALF LIFE } \\
\text { (DAYS) }\end{array}$ \\
\hline $\begin{array}{l}\text { AG-IOBM } \\
\text { CURIES } \\
\% \text { OF TOTAL }\end{array}$ & $4.745 E+04$ \\
\hline $\begin{array}{l}\text { AG-IIOM } \\
\text { CURIES } \\
\% \text { OF TOTAL }\end{array}$ & $2.498 E+02$ \\
\hline $\begin{array}{l}\text { AM- } 241 \\
\text { CURIES } \\
\% \text { OF TOTAL } \\
\text { GRAMS }\end{array}$ & $1.577 E+05$ \\
\hline $\begin{array}{l}\text { CM-244 } \\
\text { CURIES } \\
\% \text { OF TOTAL }\end{array}$ & $6.610 E+03$ \\
\hline $\begin{array}{l}\text { CO-60 } \\
\text { CURIES } \\
\% \text { OF TOTAL }\end{array}$ & $1.924 E+03$ \\
\hline $\begin{array}{l}\text { CS- } 134 \\
\text { CURIES } \\
\% \text { OF TOTAL }\end{array}$ & $7.537 E+02$ \\
\hline $\begin{array}{l}\text { CS- } 137 \\
\text { CURIES } \\
\% \text { OF TOTAL }\end{array}$ & $1.101 E+04$ \\
\hline $\begin{array}{l}\text { EU- } 152 \\
\text { CURIES } \\
\% \text { OF TOTAL }\end{array}$ & $4.891 E+03$ \\
\hline $\begin{array}{l}\text { EU- } 154 \\
\text { CURIES } \\
\% \text { OF TOTAL }\end{array}$ & $3.103 E+03$ \\
\hline $\begin{array}{l}\text { EU- } 155 \\
\text { CURIES } \\
\% \text { OF TOTAL }\end{array}$ & $1.727 E+03$ \\
\hline $\begin{array}{l}\text { N-54 } \\
\text { CURIES } \\
\% \text { OF TOTAL }\end{array}$ & $3.122 E+02$ \\
\hline $\begin{array}{l}\text { PU-238 } \\
\text { CURIES } \\
\% \text { OF TOTAL }\end{array}$ & $3.203 E+04$ \\
\hline
\end{tabular}

RUN DATE: 06/20/94

PAGE 1 RPT 176

anMéL

TOTAL

$2.880 E-07$

0.003

6.571E-06

0.070

$1.028 E-04$
1.095

$.989 E-05$

$1.975 E-05$

0.210

$4.108 E-03$

43.742

$6.305 E-05$

0.671

$3.395 E-03$

36.151

4.143E-05

0.441

$1.406 E-04$

1.497

4. $995 E-05$

0.532

$2.634 E-05$

0.280

4. $905 E-04$ 
MER DISPOSED SOLIO MASTE SUMARY

FOR JAN THROUGH DEC 1993

PAGE 2 RPT 176

MUCLIDES
GRANS
PU-239
CURIES
\% OF TOTAL
GRAMS

HALF LIFE (DAYS)

JULY

AUEUST

SEPTEYBER

OCTOBER NOVEMER DECEMBER

ANANAL

TOTAL

$2.868 E-05$

$8.800 E+06$

RU-106

CURIES

$3.726 E+02$

$\%$ OF TOTAL

SB-125

CURIES

$\%$ OF TOTAL

1. $007 E+03$

TH-230

CURIES
$\%$ OF TOTAL

\% OF TOTAL

$2.752 E+07$

CURIES

$\%$ OF TOTAL

GRars

5. 147E+12

$\mathrm{TH}-234$

CURIES

$\%$ OF TOTAL

$\%$ OF TOTAL

$2,410 E+01$

$-234$

CURIES

$\%$ OF TOTAL

GRANS

$8.943 E+07$

U-235

CURIES

$\%$ of TOtal

erams

$2.570 E+11$

U-238

CURIES

\% OF TOTAL

GRAMtS

$1.631 E+12$

$\mathrm{ZN}-65$

CURIES

$\%$ of total

9.300E-05 0.990

$1.498 E-03$

$1.315 E-04$ 1.400

1. 328E-04 1.415

3. 108E-05 0.331 1.509E-03

$1.973 E-05$
0.210 $1.810 E+02$

3.682E-07 0.004 1.587E-11

1. 783E-04 2.857E-02

1. 503E-06 0.016 6.961E-01

3. 277E-04 3.489
$.753 E+02$

3.101E-0.5 0.330 
SCHED. NO. P6IPMOO9-G

UDAHO OPERATIONS OFFICE

RADIOACTIVE MASTE MANAGEMENT INFOPMATION SYSTEM

MER DISPOSED SOLIO WASTE SUMARY FOR JAN THROUCH DEC 1993

HALF LIFE

(DAYS)

JULY

AUEUST

SEPTEMER

OCTOBER
RUN DATE: 06/20/94

PAGE

3 RPT 176

WER-S

\section{TOTALS}

ALPHA CURIES

\% OF TOTAL

B+G CURIES

$\%$ OF TOTAL

13.463

8. $127 E-03$

86.533

TOTAL GRAMS OF AM

TOTAL ERAMS OF AM

TOTAL GRAMS OF PU

TOTAL GRAYS OF PU-239+241

TOTAL GRAMS OF $U$

TOTAL GRAMS OF TH

TOTAL GRAMS OF NP

VOLUME (CU. METERS )

WEIGHT (KILOGRAMS)

$2.989 E-05$

0

$9.760 E+02$

$7.257 E+00$

$9.443 E+03$

GRAND TOTALS (CURIES )

9.391E-03 
WER Detail Data for Months January to June Annual Average Concentration and Total Curies for January to June 

MER AIRBORNE WASTE SUMARY FOR JAN THROUEH JUN 1993

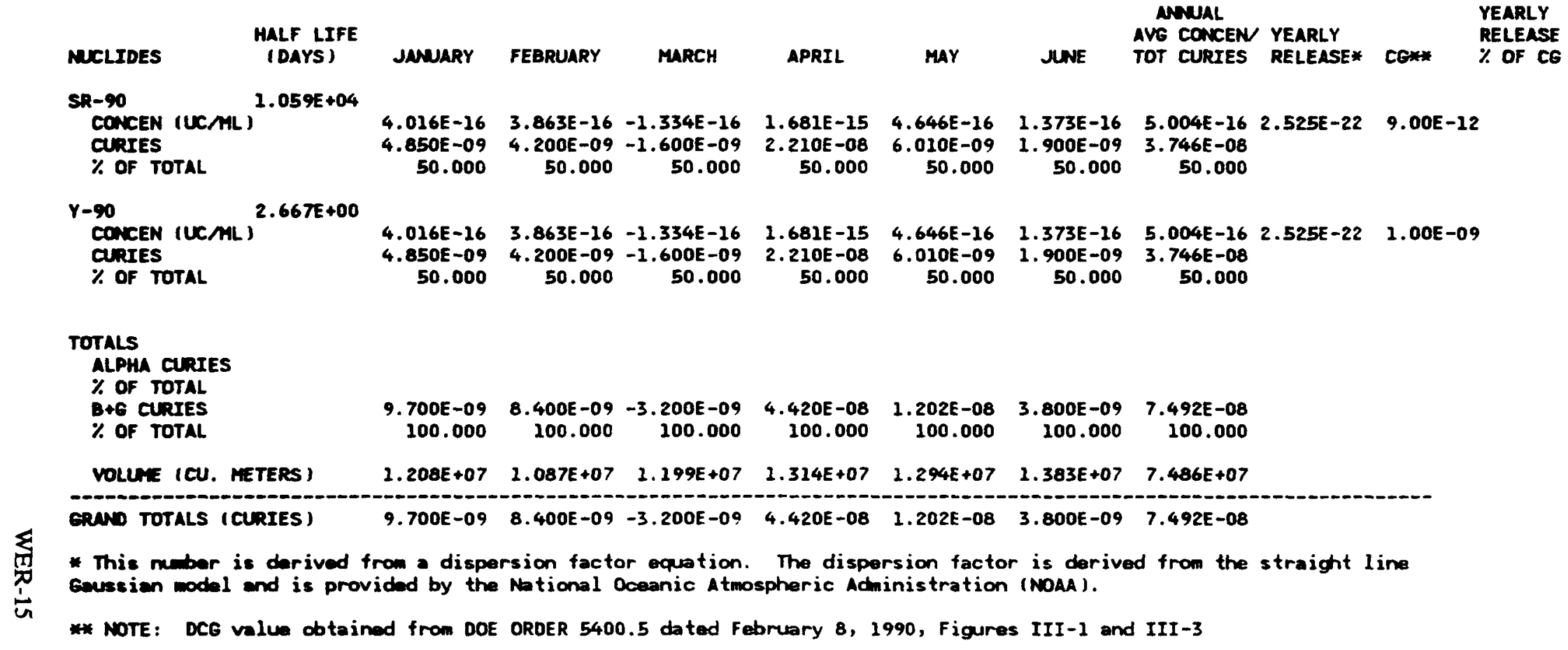



FOR JAN THROUGH JUN 2993

\begin{tabular}{|c|c|}
\hline MUCLIDES & $\begin{array}{l}\text { HALF LIFE } \\
\text { (DAYS) }\end{array}$ \\
\hline $\begin{array}{l}\text { AG-108M } \\
\text { CURIES } \\
\% \text { OF TOTAL }\end{array}$ & $4.745 E+04$ \\
\hline $\begin{array}{l}\text { AG-11OM } \\
\text { CURIES } \\
\% \text { OF TOTAL }\end{array}$ & $2.498 E+02$ \\
\hline $\begin{array}{l}\text { MM-241 } \\
\text { CURIES } \\
\text { \% OF TOTAL } \\
\text { ERNS }\end{array}$ & $1.577 E+05$ \\
\hline $\begin{array}{l}\text { CM-244 } \\
\text { CURIES } \\
\% \text { OF TOTAL }\end{array}$ & $6.610 E+03$ \\
\hline $\begin{array}{l}\text { CO-60 } \\
\text { CURIES } \\
\% \text { OF TOTAL }\end{array}$ & $1.924 E+03$ \\
\hline $\begin{array}{l}\text { CS-134 } \\
\text { CURIES } \\
\% \text { OF TOTAL }\end{array}$ & $7.537 E+02$ \\
\hline $\begin{array}{l}\text { CS-137 } \\
\text { CURIES } \\
\% \text { OF TOTAL }\end{array}$ & $1.101 E+04$ \\
\hline $\begin{array}{l}\text { EU-152 } \\
\text { CURIES } \\
\% \text { OF TOTAL }\end{array}$ & $4.891 E+03$ \\
\hline $\begin{array}{l}\text { EU-154 } \\
\text { CURIES } \\
\% \text { OF TOTAL }\end{array}$ & $3.103 E+03$ \\
\hline $\begin{array}{l}\text { EU-155 } \\
\text { CURIES } \\
\% \text { OF TOTAL }\end{array}$ & $1.727 E+03$ \\
\hline $\begin{array}{l}\text { MN-54 } \\
\text { CURIES } \\
\% \text { OF TOTAL }\end{array}$ & $3.122 E+02$ \\
\hline $\begin{array}{l}\text { PU-238 } \\
\text { CURIES }\end{array}$ & $3.203 E+04$ \\
\hline
\end{tabular}

SoLID WASTE sUrmarY
JANARY FEBRUARY MARCH

\begin{abstract}
APRIi
\end{abstract}
MaY

NNE

\section{$2.879 E-07$}

$$
0.003
$$

$6.571 E-06$
0.070

$$
0.070
$$

1. $028 E-04$

$$
\begin{array}{r}
1.095 \\
989 E-05
\end{array}
$$

2. $989 E-05$

$1.975 E-05$
0.210

$$
0.210
$$

4. $108 E-03$

$$
43.745
$$

6. 305E-05 0.671

$3.395 E-03$

36.253

4. 143E-0. 0.441

$1.406 E-04$
1.497

4. $995 E-05$

$$
\begin{array}{r}
0.532 \\
0.56
\end{array}
$$

2.634E-05

0.281

4. 905E-D4

5.223
PAGE 1 RPT 176

KER-S
ANUAL

TOTAL

$2.880 E-07$

$$
0.003
$$

6.571E-06 0.070

1. 028E-04

$$
1.095
$$

2. $989 E-05$

1. 975E-05 0.210

4. $108 E-03$ 43.742

6. 305E-05 0.671

$3.395 E-03$

36.151

4.143E-05 0.441

$1.406 E-04$ 1.497

4. 995E-05

$$
0.532
$$

2.634E-05 0.280

4. $905 E-04$ 
RADTOACTIVE WASTE MANAGEMENT INFORMATION SYSTEM

WER DISPOSED SOLID NASTE SUMMARY FOR JAN THROUGH JUN 1993

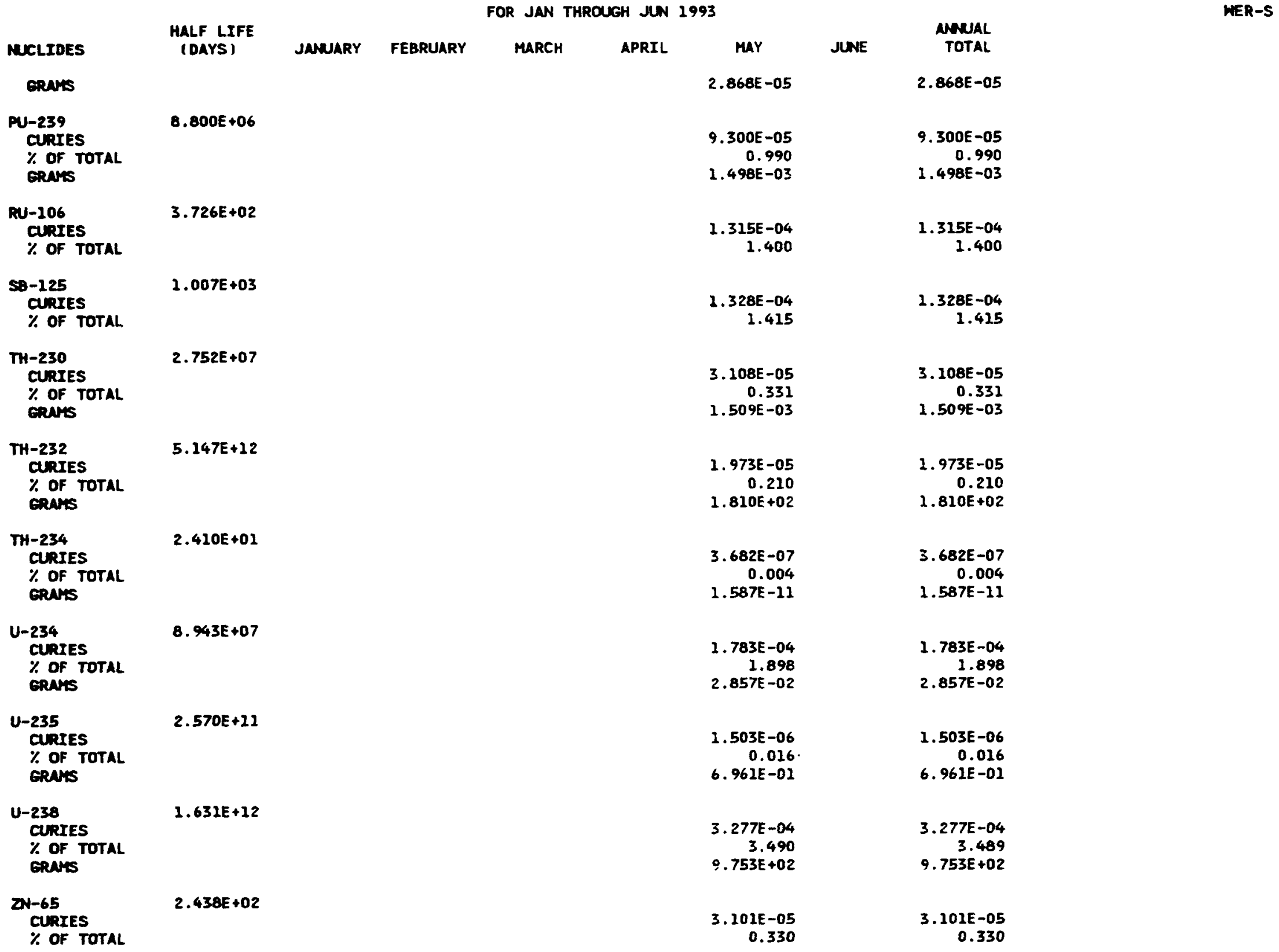


SCHED. NO. P61PM009-G

IDAHO OPERATIONS OFFICE

RADIOACTIVE WUSTE MANAGEMENT INFORMATION SYSTEM

MER DISPOSED SOLID MASTE SUMYARY FOR JAN THROUGH JUN 1993

MALF LIFE

(DAYS)

JANUARY

FEBRUARY

MARCH

APRIL

MAY

JUNE

\section{TOTALS}

ALPHA CURIES

$\%$ OF TOTAL

B+6 CURIES

$\%$ OF TOTAL

TOTAL GRAMS OF AM

TOTAL ERAMS OF AM

TOTAL ERATS OF PU

TOTAL ERANS OF PU-239+241

TOTAL ERANS OF $U$

TOTAL ERAMS OF TH

TOTAL ERANS OF NP

VOLUNE (CU. METERS)

MEIEAT (KILOERANS)

$1.264 E-03$

13.464

8.127E-03

86.539

RUN DATE: 05/12/94

GRIND TOTALS (CURIES)
2. $989 E-05$

0

976

181

$7.257 E+00$

$9.443 E+03$

$9.391 E-03$
PAGE

3 RPT 176

ANNuAL

TOTAL

$1.264 E-03$

13.463

8.127E-03

86.533

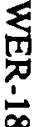

$2.989 E-05$

0

976

181

$7.257 E+00$

$9.443 E+03$

$9.391 E-03$ 
WER Facility Detail Data for Months July to December Annual Average Concentration and Total Curies for January to December 

RADIOACTIVE WASTE MANAGEMENT INFORMATION SYSTEM

WER NO. STACK AIRBORNE MASTE SUMYARY FOR JAN THROUGH DEC 1993

\begin{tabular}{|c|c|}
\hline XLIDES & $\begin{array}{l}\text { HALF LIFE } \\
\text { (DAYS ) }\end{array}$ \\
\hline $\begin{array}{l}\text { SR-90 } \\
\text { CONCEN (UCML) } \\
\text { CURIES } \\
\text { UNCERT(CURIES) } \\
\text { UNCERT(UCML) } \\
\% \text { OF TOTAL }\end{array}$ & $1.059 E+04$ \\
\hline $\begin{array}{l}\text { Y-90 } \\
\text { CONCEN (UCML) } \\
\text { CURIES } \\
\text { UNCERTI CURIES) } \\
\text { UNCERTILCML) } \\
\text { \% OF TOTAL }\end{array}$ & $2.667 E+00$ \\
\hline
\end{tabular}

rLY

Aueust

SEPTEMBER
$-8.940 E-16-1.124 E-14 \quad-2.309 E-16 \quad 1.563 E-16 \quad-3.313 E-17-7.837 E-16-3.954 E-22 \quad 9.00 E-12$
$-8.350 E-09-1.000 E-07-2.260 E-09 \quad 1.280 E-09-2.500 E-10-8.003 E-08$
$\begin{array}{rrrrr}1.350 E-09 & -1.000 E-07 & -2.260 E-09 & 1.280 E-09 & -2.500 E-10 \\ 1.250 E-08 & 4.000 E-07 & 1.810 E-09 & 8.530 E-10 & 5.000 E-10\end{array}$
50.000
50.000
50.000
50.000
50.000
50.000

$-8.940 E-16-1.124 E-14-2.309 E-16 \quad 1.563 E-16 \quad-3.313 E-17-7.837 E-16-3.954 E-22 \quad 1.00 E-09$ $-8.350 E-09-1.000 E-07-2.260 E-09 \quad 1.280 E-09-2.500 E-10-8.003 E-08$

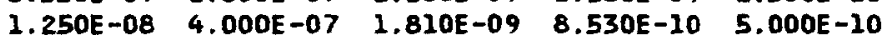
50.000
50.000
50.000
50.000
50.000
50.000

\section{TOTALS \\ ALPHA CURIES \\ $\%$ OF TOTAL \\ B+E CURIES}

VOLUAE (CU. METERS)

$-1.670 E-08-2.000 E-07-4.520 E-09$

$$
\begin{array}{rrrrrr}
.670 E-08 & -2.000 E-07 & -4.520 E-09 & 2.560 E-09 & -5.000 E-10 & -1.601 E-07 \\
100.000 & 100.000 & 100.000 & 100.000 & 100.000 & 100.000
\end{array}
$$

GRANo TOTALS (CURIES)

$\begin{array}{llllll}9.340 E+06 & 8.897 E+06 & 9.786 E+06 & 8.191 E+06 & 7.547 E+06 & 1.021 E+08\end{array}$

* This nuber is derived from a dispersion factor equation. The dispersion factor is derived from the straight line gaussian model and is proveded by the National Oceanic Atmospheric Administration (NOAA).

* NOTE: OCG value obtained from DOE ORDER 5400.5 dated Febmary 8, 1990 , Figures III-1 and III-3 
RADIOACTIVE WASTE MANAGEMENT INFORMATION SYSTEM

WER SO. STACK AIRBORNE MASTE SUMARY FOR JAN THROUGH DEC 1993

MCLIDES $\begin{gathered}\text { HALF LIFE } \\ \text { (DAYS) }\end{gathered}$
SR-90
CONCEN (UCML)
CURIES
UNCERT(CURIES)
UNCERT(UCML)
\% OF TOTAL
Y-90
CONCEN (UCML)
CURTES
UNCERT(CURIES)
UNCERTIUCML)
\% OF TOTAL

ULY AUEUST SEPTEMBER OCTOBER NOVEMBER DECEMBER

\begin{tabular}{|c|c|c|c|}
\hline & PA & WER ${ }^{1}$ & T. 181 \\
\hline $\begin{array}{l}\text { NWNUAL } \\
\text { VG CONCEN } \\
\text { OT CURIES }\end{array}$ & $\begin{array}{l}\text { YEARLY } \\
\text { RELEASE* }\end{array}$ & CGA* & $\begin{array}{l}\text { YEARLY } \\
\text { RELEASE } \\
\% \text { OF CG }\end{array}$ \\
\hline
\end{tabular}

1.617E-16 $8.159 E-23 \quad 9.00 E-12$ $7.910 E-09$

50.000

$1.617 E-16 \quad 8.159 E-23 \quad 1.00 E-09$

$7.910 E-09$

50.000

TOTALS

ALPHA CURIES

$\%$ OF TOTAL

B+G curIes

$\xi \%$ Of TOTAL

(IJ VOLUNE (CU. METERS)

$2.936 E+06$

$3.034 E+06$ GRNND TOTALS (CURIES)

* This muber is derived from a dispersion factor equation. The dispersion factor is derived from the straight line gaussian model and is provedad by the National Dceanic Atmospheric Administration (NOAA).

* NOTE: DCG value obtainod from DOE ORDER 5400.5 dated February 8, 1990 , Figures III-I and III-3 
WER 765 STACK AIRBORNE WASTE SUMMARY FOR JAN THROUGH DEC 1993

NUCLIDES
$\begin{aligned} & \text { HALF LIFE } \\ & \text { (DAYS) }\end{aligned}$
$\begin{aligned} & \text { SR-90 } \\ & \text { CONCEN (UCML) }\end{aligned}$
CURIES
UNCERT(CURIES)
UNCERT(UCML)
\% OF TOTAL
Y-90
CONCEN (UCML)
CURIES
UNCERT(CURTES)
UNCERT(UCML)
\% OF TOTAL

JULY

AUEUST

\begin{tabular}{|c|c|c|c|}
\hline & \multicolumn{3}{|c|}{ PAGE ${ }_{\text {WER }-A}^{1}$ RPT. 182} \\
\hline $\begin{array}{l}\text { ANNUAL } \\
\text { AVG CONCEN } \\
\text { TOT CURIES }\end{array}$ & $\begin{array}{l}\text { YEARLY } \\
\text { RELEASE: }\end{array}$ & cex* & $\begin{array}{l}\text { YEARLY } \\
\text { RELEASE } \\
\% \text { OF CG }\end{array}$ \\
\hline
\end{tabular}

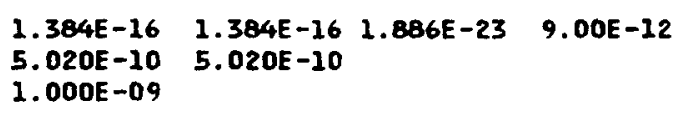

50.000

50.000
$1.384 E-16 \quad 1.384 E-16 \quad 1.886 E-23 \quad 1.00 E-09$ 5.020E-10 5.020E-10
1. $000 E-09$
50.000
50.000

\section{TOTALS}

ALPHA CURIES

$\%$ Of TOTAL

B+G CURIES

$\%$ OF TOTAL

VOLUME (CU. METERS)

$100.000 \quad 100.000$

GRAND TOTALS (CURIES)

$1.004 E-09 \quad 1.004 E-09$

* This nuber is derived from a dispersion factor equation. The dispersion factor is derived from the straight line gouscian model and is proveded by the National Oceanic Atmospheric Administration (NOAA).

** NOTE: DCG value obtained from DOE ORDER 5400.5 dated February 8, 1990, Figures III-1 and III-3 
RADIOACTIVE WUSTE MANAGEMENT INFORMATION SYSTEM

MER INCINERATION

OPERATIONS, DISPOSED SOLID WASTE SUMARY FOR JAN THROUEH DEC 1993

NUCLIDES

HALF LIFE

(DAYS)

JULY

$4.745 E+04$

AG-108M

CURIES

$\%$ OF TOTAL

AG-1IOM

CURIES

\% OF TOTAL

AM-241

CURIES

\% OF TOTAL

GRAMS

CM-244

CURIES

$\%$ OF TOTAL

CO-60

CURIES

$\%$ OF TOTAL

CS-134

CURIES

\% OF TOTAL

CS -137

CURIES

$\%$ OF TOTAL

EU-152

CURIES

$\%$ OF TOTAL

EU-154

CURIES

$\%$ OF TOTAL

EU-155

CURIES

$\%$ OF TOTAL

MN-54

CURIES

\% OF TOTAL

PU-238

CURIES

$\%$ OF TOTAL
$6.610 E+03$

$2.498 E+02$

$1.577 E+05$

$7.537 E+02$

$1.101 E+04$

$4.891 E+03$

$1.727 E+03$

$3.203 E+04$
PAGE 1 RPT. 178

WER-INC -S

AUCUST SEPTEMBER OCTOBER NOVEMBER DECEMBER T TOTAL

$2.880 E-07$ 0.003

$6.571 E-06$

$$
0.070
$$

1. 028E-04$$
\begin{array}{r}
1.095 \\
989 E-05
\end{array}
$$

1.975E-05 0.210

4. 108E-03

43.742

$6.305 E-05$

0.671

$3.395 E-03$

36.151

4.143E-05

0.441

$1.406 \mathrm{E}-04$ 1.497

4. $995 E-05$ 0.532

2.634E-05

$$
0.280
$$

4. 905E-04 5.223 
RADIOACTIVE WUSTE MUMAGEMENT INFORMATION SYSTEM

OPERATIONS, DISPOSED SOLID MUSTE SUMMARY FOR JAN THROUGH DEC 1993

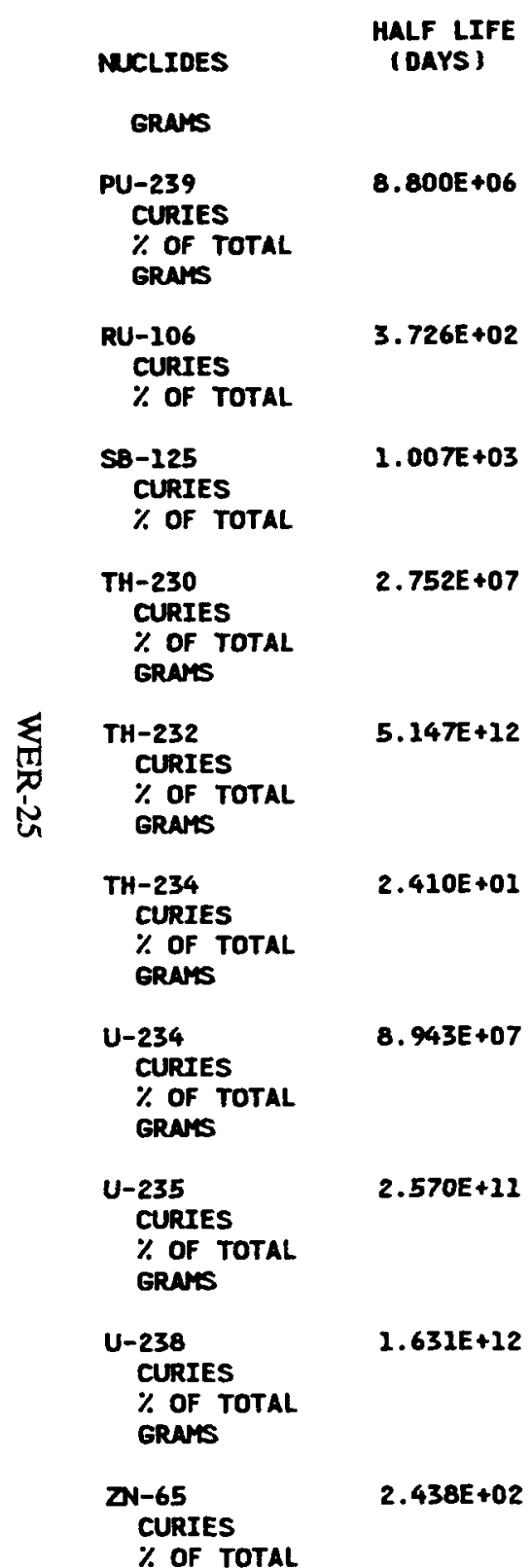

NLY AUGUST SEPTEMER OCTOBER NOVEFBER DECEMBER

NNUAL

TOTAL

2.868E-05

$9.300 E-05$

0.990

$1.498 E-03$

$1.315 E-04$

1.400

$1.328 E-04$

2.415

3. $108 E-05$

0.331

$1.509 E-03$

1. $973 E-05$

$1.810 E+02$

$3.682 E-07$

0.004

1.587E-11

1. $783 E-04$

1.898
$857 E-02$

1. 503E-06

0.016

6. 961E-01

3.277E-04

3.489
$753 E+02$

3.101E-05

0.330 
SCHED. NO. P61PMO99-H

IDAHO OPERATIONS OFFICE

RADIOACTIVE MASTE MANAGEMENT INFORMATION SYSTEM

\section{WER INCINERATION}

OPERATIONS, DISPOSED SOLID WASTE SUMMARY FOR JAN THROUEH DEC 1993

MUCLIDES HALF LIFE

(DAYS)

NUY

AUEUST

SEPTEMER

NOVEMER DECEMBER

RUN DATE: 06/27/94

PAGE

3 RPT. 178

MER-INC -S

TOTALS

ALPHA CURIES

$\%$ OF TOTAL

B+G CURIES

$\%$ OF TOTAL

TOTAL GRAMS OF NM

TOTAL ERAMS OF PU

TOTAL GRANS OF PU-239+24I

TOTAL GRAYS OF $U$

TOTAL GRAYS OF $U$

TOTAL GRANS OF TH
TOTAL GRAYS OF NP

VOLUME ICU. METERS )

WEIGHT (KILOGRAMS)

GRAND TOTALS (CURIES)

$1.264 E-03$
13.463

13.463
$127 E-03$

86.533

2. $989 E-05$

$1.526 E-03$

$1.498 E-03$

$9.760 E+02$

$1.810 E+02$

$7.257 E+00$

$9.443 E+03$

$9.391 E-03$

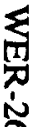


WER Facility Detail Data for Months January to June Annual Average Concentration and Total Curies for January to June 

RADIOACTIVE WASTE MUVAGEMENT INFOPRATION SYSTEM

\section{WER NO. STACK AIRBORNE MASTE SUMMARY} FOR JAN THROUGH JUN 1993

MALF LIFE
MCLIDES
(DAYS)
SR-90
CONCEN (UCML)
CURIES
UNCERT(CURIES )
UCERT(UCML)
\% OF TOTAL

$J$

$\begin{array}{lllll}5.363 E-16 & 5.359 E-16 & -1.786 E-16 & 2.186 E-15 \\ 4.850 E-09 & 4.200 E-09 & -1.600 E-09 & 2.210 E-08\end{array}$

$\begin{array}{rrrr}4.850 E-09 & 4.200 E-09 & -1.600 E-09 & 2.210 E-08 \\ 1.210 E-09 & 1.050 E-09 & 8.010 E-10 & 1.810 E-09\end{array}$

$\begin{array}{llll}50.000 & 50.000 & 50.000 & 50.000\end{array}$

MaY
JNE

\begin{tabular}{|c|c|c|c|}
\hline & \multicolumn{3}{|c|}{ PAGE $\begin{array}{c}1 \text { RPT } \\
\text { WER-A } \\
180\end{array}$} \\
\hline EN & $\begin{array}{l}\text { YEARLY } \\
\text { RELEASE }\end{array}$ & & $\begin{array}{l}\text { YEARLY } \\
\text { RELEASE } \\
\% \text { OF CE }\end{array}$ \\
\hline
\end{tabular}

$5.947 E-16 \quad 3.001 E-22 \quad 9.00 E-12$
$2.955 E-08$

50.000

5. $947 E-16 \quad 3.001 E-22 \quad 1.00 E-09$ $2.955 E-08$

50.000

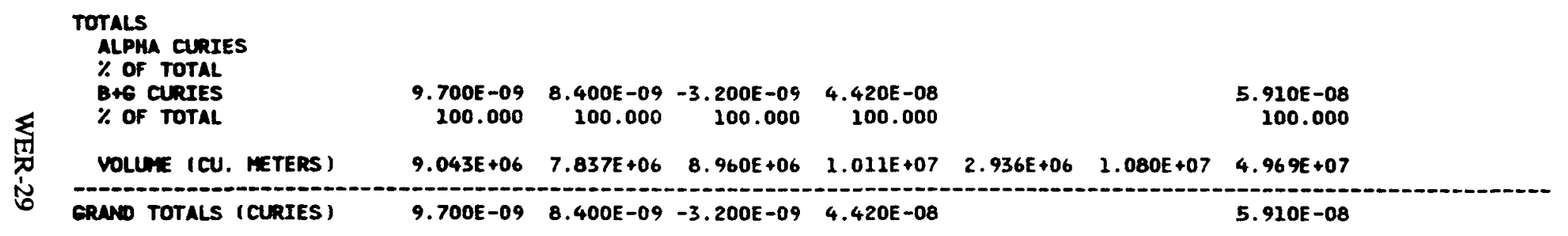

* This nuber is derived from a dispersion factor eqution. The dispersion factor is derived from the straight line gousien model and is proveded by the National Doeanic Atmospheric Administration (NOAA).

* NOTE: DCE value abtained from DOE ORDER 5400.5 dated Fabruary 8, 1990, Figures III-1 and III-3 


\section{RADIOACTIVE WUSTE MANAGEMENT INFORMATION SYSTEM}

ER SO. STACK AIRBORNE WASTE SUMMARY

FOR JAN THROUGH JUN 1993

MALF LIFE
(DAYS)
SR-90
CONCEN (UCML)
CURIES
UNCERT(CURIES)
UNCERT(UCAL)
\% OF TOTAL
Y-90
CONCEN (UCML
CURIES
UNCERT(CURIES)
UNCERT(UCML)
\% OF TOTAL

JMEARY FEBRUARY MARCH APRIL MAY TNE

\section{$\begin{array}{ll}6.010 E-16 & 6.262 E-16 \\ 6.010 E-09 & 1.900 E-09\end{array}$} $\begin{array}{ll}6.010 E-09 & 1.900 E-09 \\ 9.240 E-10 & 2.710 E-10\end{array}$

50.000

50.000

$6.010 E-16 \quad 6.262 E-16$

6.010E-09 $1.900 E-09$

$9.240 E-10 \quad 2.710 E-10$

50.000

50.000
PAGE

1 RPT. 181 WER $-A$

YEARLY RELEASE AVG CONCEN RELEASE* CG** $\%$ OF CG

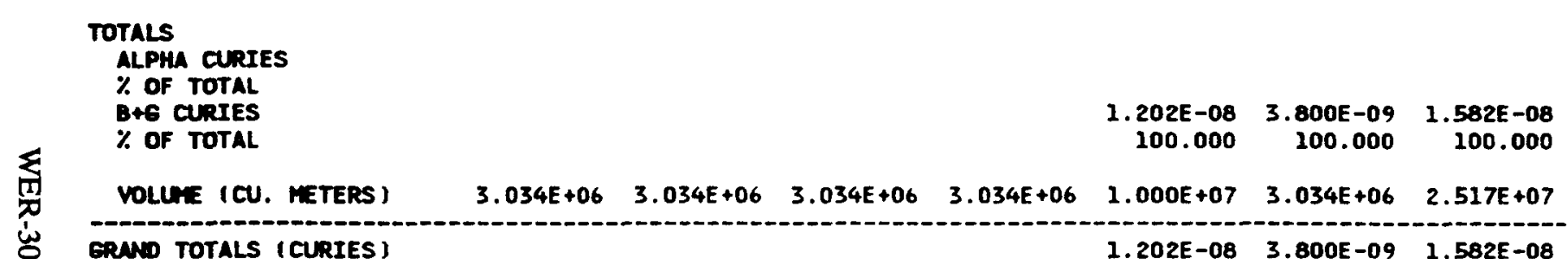

(n)

GRMD TOTALS (CURIES)

* This nuber is derived from a dispersion factor equation. The dispersion factor is derived from the straight line ouseien modal and is proveded by the National Oceanic Atmospheric Administration (NOAA).

** NOTE: DCG value abtainod from DOE ORDER 5400.5 dated February 8, 1990 , Figures III-I and III-3 
RADIOACTIVE WASTE MUNAGEMENT INFORMATION SYSTEM

\section{WER INCINERATION}

OPERATIONS, DISPOSED SOLID MASTE SUMARY FOR JAN THROUGH JUN 1993

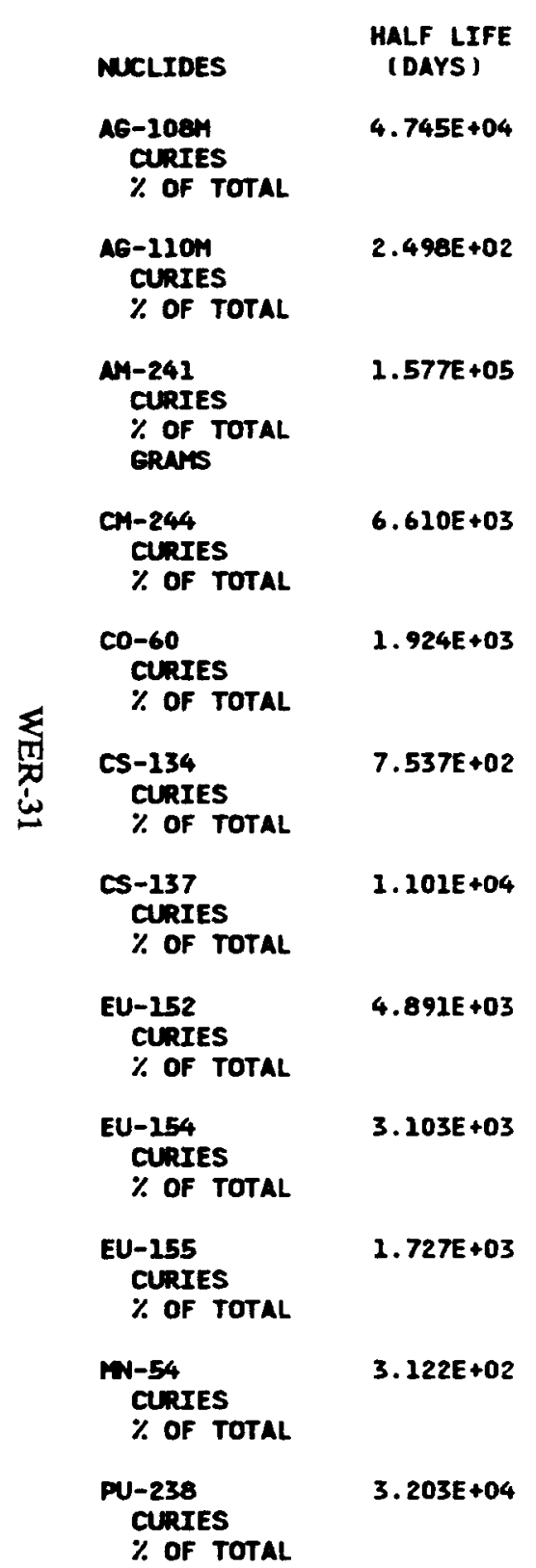

JANUARY FEBRUARY MARCH APRIL MAY JUNE

$2.879 E-07$
0.003

$6.571 E-06$
0.070

$2.880 E-07$

0.003

$6.571 E-06$

0.070

$1.028 E-04 \quad 1.028 E-04$

$1.095 \quad 1.095$

2. $989 E-05$

$2.989 E-05$
1. $975 E-05$
$1.975 E-05$

4. 108E-03

43.742
$6.305 E-05$
$6.305 E-05$

3. 395E-03

36.153

$3.395 E-03$ 36.151

$\begin{array}{r}4.243 E-05 \\ 0.441\end{array} \quad 4.143 E-05$

1.406E-04

1.497

4. $995 E-05 \quad 4.995 E-05$

$\begin{array}{ll}0.532 & 0.532\end{array}$

2.634E-05

0.280

4. $905 E-04 \quad 4.905 E-04$

PAGE 1 RPT. 178 WER-INC -S 


\section{WER INCINERATION}

OPERATIONS, DISPOSED SOLTO WASTE SUMARY FOR JAN THROUGH JUN 1993

$\begin{array}{lc}\text { MUCLIDES } & \begin{array}{c}\text { HALF LIFE } \\ \text { (DAYS) }\end{array} \\ \text { ERAMS } & \\ \text { PU-239 } & 8.800 E+06 \\ \text { CURIES } & \\ \text { \% OF TOTAL } & \\ \text { ERUNS } & \end{array}$

RU-206

$3.726 E+02$

$\%$ OF TOTAL

SB-125

CURIES

$\%$ OF TOTAL

1.007E+03

TH-230

CURIES

$\%$ OF TOTAL

eruis

䒝

TH-232

CURIES

\% OF TOTAL

grais

TH-234

CURIES

\% Of total

U-234

CLRIES

grans

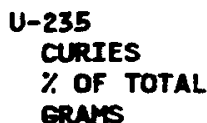

conars

U-238

CURIES

$\%$ OF TOTAL

corins

$1.631 \mathrm{E}+12$

2N-65

CURIES

$\%$ OF TOTAL

2.868E-05

$9.300 E-05$

0.990

$1.498 E-03$

$1.315 E-04$

1.400

$1.328 E-04$ 1.415

3.108E-05

$$
\begin{array}{r}
0.331 \\
509 E-03
\end{array}
$$

$1.973 E-05$

0.210

$1.810 E+02$

$3.682 E-07$

$$
\text { 0.004 }
$$

0.004
$1.587 E-11$

$1.783 E-04$
1.898

2.857E-02

1.503E-06

0.016

$6.961 E-01$

3. 277 E-04

3.490
$753 E+02$

$2.438 E+02$

3.101E-05

0.330
JANUARY FEBRUARY MARCH APRIL MAY JUNE

PAGE

2 RPT. 178

WER-INC -S

ANUAL

TOTAL

$2.868 E-05$

$9.300 E-05$

0.990

$1.498 \mathrm{E}-03$

1.315E-04

1.400

1. 328E-04 1.415

3.108E-05

$1.509 E-03$

1. 973E-05

$$
0.210
$$

$1.810 E+02$

3. 682E-07

0.004

1.587E-11

1.783E-04

1.898
$2.857 E-02$

$1.503 E-06$

0.016

$6.961 E-01$

3. 277E-04

$9.753 \mathrm{E}+02$

3.101E-05

0.330 
RADIOACTIVE WASTE MANAGEMENT INFORMATION SYSTEM

\section{WER INCINERATION}

OPERATIONS, OISPOSED SOLIO WASTE SUMARY

FOR JAN THROUGH HUN 1993

HALF LIFE
MUCLIDES JDAYS

\section{TOTALS}

ALPHA CURIES

$\%$ OF tOTAL

B+G CURIES

$\%$ of total

TOTAL ERANS OF AM

TOTAL ERANS OF PU

TOTAL ERMIS OF PU-239+241

TOTAL ERAMS OF $u$

TOTAL ERAMS OF $U$

TOTAL ERANS OF TH

TOTAL ERANS OF NP
VOLLAY ICU. METERS

WEIEHT (KILOGRANS)

GRAND TOTALS (CURIES)

1.264E -03

13.464

$8.127 E-03$

86.539

2. $989 E-05$

$1.526 E-03$

$1.498 E-03$

$9.760 E+02$

$1.810 E+02$

$7.257 E+00$ $9.443 E+03$

$9.391 E-03$
PAGE 3 RPT 178 NER-INC -S 

Engineered Release Points 

Table 1. Engineered Release Points to the Environment.

\begin{tabular}{|c|c|c|c|c|}
\hline $\begin{array}{l}\text { Type of } \\
\text { Discharge }\end{array}$ & Area/Location & $\begin{array}{l}\text { Type of Description } \\
\text { of Discharge Surface }\end{array}$ & $\begin{array}{l}\text { Air Emission } \\
\text { Inventory } \\
\text { Stack No. }\end{array}$ & $\begin{array}{c}\text { Active } \\
\text { (Yes/No) }\end{array}$ \\
\hline \multirow[t]{13}{*}{ Airborne } & ANL/EBR-II \& FCF & $\begin{array}{l}61-\mathrm{m} \text { glass-coated steel stack, } 30 \mathrm{~m}^{3} / \mathrm{s} \\
\text { discharge capacity, continuously moni- } \\
\text { tored. }\end{array}$ & ANL-764-001 & Yes \\
\hline & ANL/FASB & $\begin{array}{l}10-\mathrm{m} \text { stack, } 6.64 \mathrm{~m}^{3} / \mathrm{s} \text { discharge capacity, } \\
\text { continuously monitored. }\end{array}$ & ANL-787-001 & Yes \\
\hline & ANL/FMF & $\begin{array}{l}11.1-\mathrm{m} \text { stack, } 4.6 \mathrm{~m}^{3} / \mathrm{s} \text { discharge capac- } \\
\text { ity, continuously monitored. }\end{array}$ & ANL-704-008 & Yes \\
\hline & ANL/HFEF & $\begin{array}{l}28.6-\mathrm{m} \text { stack, } 21.7 \mathrm{~m}^{3} / \mathrm{s} \mathrm{discharge} \mathrm{capac-} \\
\text { ity, continuously monitored. }\end{array}$ & ANL-785-018 & Yes \\
\hline & ANL/L\&O & $\begin{array}{l}\text { Two separate stacks-one stack is } 15.2 \mathrm{~m} \\
\text { with } 10.9 \mathrm{~m}^{3} / \mathrm{s} \text { discharge capacity. The } \\
\text { other stack is } 11.3 \mathrm{~m} \text { with } 7.8 \mathrm{~m}^{3} / \mathrm{s} \text { dis- } \\
\text { charge capacity. Both stacks are continu- } \\
\text { ously monitored. }\end{array}$ & $\begin{array}{l}\text { ANL-752-004 } \\
\text { (MAIN) } \\
\text { ANL-752-005 } \\
\text { (NDA) }\end{array}$ & Yes \\
\hline & ANL/RLWTF & $\begin{array}{l}14.2-\mathrm{m} \mathrm{stack}, 1.7 \mathrm{~m}^{3} / \mathrm{s} \text { discharge capac- } \\
\text { ity, continuously monitored. }\end{array}$ & ANL-798-017 & Yes \\
\hline & ANL/SCMS & $\begin{array}{l}14.6-\mathrm{m} \text { stack, } 4.7 \mathrm{~m}^{3} / \mathrm{s} \text { discharge capac- } \\
\text { ity, continuously monitored. }\end{array}$ & ANL-793-001 & Yes \\
\hline & $\mathrm{ANL} / \mathrm{SPF}$ & $\begin{array}{l}6.9-\mathrm{m} \text { stack, } 2.1 \mathrm{~m}^{3} / \mathrm{s} \text { discharge capacity, } \\
\text { continuously monitored. This stack is } \\
\text { presently inactive. }\end{array}$ & ANL-799-010 & No \\
\hline & ANL/TREAT & $\begin{array}{l}42.7-\mathrm{m} \text { steel stack, } 2.8 \mathrm{~m}^{3} / \mathrm{s} \text { discharge } \\
\text { capacity, continuously monitored. }\end{array}$ & ANL-720-007 & Yes \\
\hline & ANL/ZPPR & $\begin{array}{l}22.9-\mathrm{m} \text { stack, } 2.3 \mathrm{~m}^{3} / \mathrm{s} \text { discharge capac- } \\
\text { ity, continuously monitored. }\end{array}$ & ANL-777-002 & Yes \\
\hline & ARA & $\begin{array}{l}9.1-\mathrm{m} \text { stack, } 1.4 \mathrm{~m}^{3} / \mathrm{s} \text { discharge capacity, } \\
\text { continuously monitored. These stacks } \\
\text { were capped in } 1989 . \text { D\&D removal } \\
\text { scheduled for } 1996 .\end{array}$ & ARA001 & No \\
\hline & CFA/West & $\begin{array}{l}\text { Two normal ventilation exhausts, respira- } \\
\text { tor maintenance } 0.6-\mathrm{m}^{3} / \mathrm{s} \text { flow, sampled } \\
\text { weekly when operating. Facility placed } \\
\text { on standby June } 1,1993 \text {. }\end{array}$ & $\begin{array}{l}\text { Drying Ovens } \\
\text { CFA-617-030 } \\
\text { CFA-617-031 }\end{array}$ & No \\
\hline & CFA/North & $\begin{array}{l}\text { Normal ventilation exhausts, laundry } \\
\text { dryer, } 6.1-\mathrm{m}^{3} / \mathrm{s} \text { flow, continuously } \\
\text { sampled when operating. }\end{array}$ & $\begin{array}{l}\text { CFA-617-010 } \\
\text { CFA-617-011 }\end{array}$ & Yes \\
\hline
\end{tabular}


Table 1. (continued)

\begin{tabular}{|c|c|c|c|c|}
\hline $\begin{array}{l}\text { Type of } \\
\text { Discharge }\end{array}$ & Area/Location & $\begin{array}{l}\text { Type of Description } \\
\text { of Discharge Surface }\end{array}$ & $\begin{array}{l}\text { Air Emission } \\
\text { Inventory } \\
\text { Stack No. }\end{array}$ & $\begin{array}{l}\text { Active } \\
\text { (Yes/No) }\end{array}$ \\
\hline & CPP/FAST & $\begin{array}{l}50-\mathrm{m} \text { stack, } 66.1 \mathrm{~m}^{3} / \mathrm{s} \text { discharge capac- } \\
\text { ity, continuously monitored and } \\
\text { sampled for particulates only. }\end{array}$ & CPP-767-001 & Yes \\
\hline & CPP/Main Stack & $\begin{array}{l}76.2-\mathrm{m} \text { stack, } 84.95 \mathrm{~m}^{3} / \mathrm{s} \text { discharge } \\
\text { capacity, continuously monitored. }\end{array}$ & CPP-708-001 & Yes \\
\hline & CPP/NWCF & $\begin{array}{l}22-\mathrm{m} \text { stack, } 51.9 \mathrm{~m}^{3} / \mathrm{s} \text { discharge capac- } \\
\text { ity, continuously monitored and } \\
\text { sampled. }\end{array}$ & CPP-659-033 & Yes \\
\hline & CPP/RAL Stack & $\begin{array}{l}14.8-\mathrm{m} \text { stack, } 8.5 \mathrm{~m}^{3} / \mathrm{s} \text { discharge capac- } \\
\text { ity, continuously monitored and } \\
\text { sampled. }\end{array}$ & CPP-684-001 & Yes \\
\hline & $\begin{array}{l}\text { CTF } \\
\text { (formerly LOF) }\end{array}$ & $\begin{array}{l}45.7-\mathrm{m} \text { stack, } 7.8 \mathrm{~m}^{3} / \mathrm{s} \text { discharge capac- } \\
\text { ity, continuously monitored. This facil- } \\
\text { ity is not functioning and has no future } \\
\text { plans to change status. }\end{array}$ & TAN-725-00I & No \\
\hline & NRF/AIW & $\begin{array}{l}17.7-\mathrm{m} \text { stack, } 22 \mathrm{~m}^{3} / \mathrm{s} \text { discharge capac- } \\
\text { ity, continuously monitored. }\end{array}$ & NRF-616-012 & Yes \\
\hline & NRF/AIW-RWDS & $\begin{array}{l}\text { Near-surface discharge, } 0.02 \mathrm{~m}^{3} / \mathrm{s} \mathrm{dis-} \\
\text { charge capacity, continuously moni- } \\
\text { tored. }\end{array}$ & NRF-628-006 & Yes \\
\hline & NRF/ECF & $\begin{array}{l}\text { Two stacks, one } 19.5-\mathrm{m} \text { stack, } 14.3 \mathrm{~m}^{3} / \mathrm{s} \\
\text { discharge capacity, one } 31-\mathrm{m} \text { stack } \\
23.13 \mathrm{~m}^{3} / \mathrm{s} \text { discharge capacity. Both } \\
\text { continuously monitored. }\end{array}$ & $\begin{array}{l}\text { NRF-618-103 } \\
\text { NRF-618-099 }\end{array}$ & Yes \\
\hline & NRF/Nonplant Areas & $\begin{array}{l}\text { 7.6-m stack, } 0.3 \mathrm{~m}^{3} / \mathrm{s} \text { discharge capac- } \\
\text { ity, continuously monitored. This stack } \\
\text { has been discommissioned and was } \\
\text { removed in the early } 1980 \mathrm{~s} \text {. }\end{array}$ & & No \\
\hline & NRF/SIW & $\begin{array}{l}\text { One } 45.1-\mathrm{m} \text { stack, } 22.96 \mathrm{~m}^{3} / \mathrm{s} \text { discharge } \\
\text { capacity, continuously monitored. One } \\
\text { filtered exhaust } 15.8-\mathrm{m}, 0.6 \mathrm{~m}^{3} / \mathrm{s} \mathrm{dis-} \\
\text { charge capacity, continuously moni- } \\
\text { tored. }\end{array}$ & NRF-601-019A & Yes \\
\hline & NRF/S5G & $\begin{array}{l}23.2-\mathrm{m} \text { stack, } 10.3 \mathrm{~m}^{3} / \mathrm{s} \text { discharge } \\
\text { capacity, continuously monitored. }\end{array}$ & NRF-633A-057 & Yes \\
\hline & PBF & $\begin{array}{l}\text { 24.4-m stack, } 2.8 \mathrm{~m}^{3} / \mathrm{s} \text { discharge capac- } \\
\text { ity, continuously monitored. }\end{array}$ & $\begin{array}{l}\text { PER620 } \\
\text { Vent } 016\end{array}$ & Yes \\
\hline & SMC/R\&D Process & $\begin{array}{l}17.7-\mathrm{m} \text { stack, } 23.6 \mathrm{~m}^{3} / \mathrm{s} \text { discharge } \\
\text { capacity, continuously monitored. }\end{array}$ & TAN-607-039 & Yes \\
\hline & SMC/MDF QC LAB & $\begin{array}{l}17.99-\mathrm{m} \text { stack, } 7.79 \mathrm{~m}^{3} / \mathrm{s} \text { discharge } \\
\text { capacity, continuously monitored. }\end{array}$ & TAN-607-119 & Yes \\
\hline
\end{tabular}




\begin{tabular}{|c|c|c|c|c|}
\hline $\begin{array}{c}\text { Type of } \\
\text { Discharge } \\
\end{array}$ & Area/Location & $\begin{array}{l}\text { Type of Description } \\
\text { of Discharge Surface }\end{array}$ & $\begin{array}{l}\text { Air Emission } \\
\text { Inventory } \\
\text { Stack No. }\end{array}$ & $\begin{array}{l}\text { Active } \\
\text { (Yes/No) }\end{array}$ \\
\hline & SMC/LINE $2 a$ & $\begin{array}{l}14.0-\mathrm{m} \text { stack, } 18.12 \mathrm{~m}^{3} / \mathrm{s} \text { discharge } \\
\text { capacity, continuously monitored. }\end{array}$ & TAN-629-013 & Yes \\
\hline & SMC/LINE $2 \mathrm{~b}$ & $\begin{array}{l}8.36-\mathrm{m} \text { stack, } 3.12 \mathrm{~m}^{3} / \mathrm{s} \text { discharge } \\
\text { capacity, continuously monitored. }\end{array}$ & TAN-629-014 & Yes \\
\hline & SMC/LINE 2B & $\begin{array}{l}\text { 8.36-m stack, } 3.12 \mathrm{~m}^{3} \text { discharge capac- } \\
\text { ity, continuously monitored. }\end{array}$ & TAN-629-012 & Yes \\
\hline & SMC/S. Process S6 & $\begin{array}{l}16.97-\mathrm{m} \text { stack, } 7.08 \mathrm{~m}^{3} / \mathrm{s} \text { discharge } \\
\text { capacity, continuously monitored. }\end{array}$ & TAN-679-027 & Yes \\
\hline & SMC/S. Process S7 & $\begin{array}{l}16.97-\mathrm{m} \text { stack, } 7.08 \mathrm{~m}^{3} / \mathrm{s} \text { discharge } \\
\text { capacity, continuously monitored. }\end{array}$ & TAN-679-026 & Yes \\
\hline & SMC/S. Process S8 & $\begin{array}{l}16.97-\mathrm{m} \mathrm{stack}, 7.08 \mathrm{~m}^{3} / \mathrm{s} \text { discharge } \\
\text { capacity, continuously monitored. }\end{array}$ & TAN-679-025 & Yes \\
\hline & SMC/S. Process S9 & $\begin{array}{l}16.97-\mathrm{m} \text { stack, } 7.08 \mathrm{~m}^{3} / \mathrm{s} \text { discharge } \\
\text { capacity, continuously monitored. }\end{array}$ & TAN-679-024 & Yes \\
\hline & SMC/S. Process S10 & $\begin{array}{l}16.97-\mathrm{m} \text { stack, } 7.08 \mathrm{~m}^{3} / \mathrm{s} \text { discharge } \\
\text { capacity, continuously monitored. }\end{array}$ & TAN-679-023 & Yes \\
\hline & SMC/S. Process S11 & $\begin{array}{l}16.97-\mathrm{m} \text { stack, } 7.08 \mathrm{~m}^{3} / \mathrm{s} \text { discharge } \\
\text { capacity, continuously monitored. }\end{array}$ & TAN-679-022 & Yes \\
\hline & SMC/Scrap Handling & $\begin{array}{l}16.97-\mathrm{m} \text { stack, } 7.93 \mathrm{~m}^{3} / \mathrm{s} \text { discharge } \\
\text { capacity, continuously monitored. }\end{array}$ & TAN-681-020 & Yes \\
\hline & SMC/Liquid Reclaim & $\begin{array}{l}16.97-\mathrm{m} \text { stack, } 7.93 \mathrm{~m}^{3} / \mathrm{s} \text { discharge } \\
\text { capacity, continuously monitored. }\end{array}$ & TAN-681-018 & Yes \\
\hline & SMC/PRF Stack S14 & $\begin{array}{l}33.54-\mathrm{m} \text { stack, } 0.94 \mathrm{~m}^{3} / \mathrm{s} \text { discharge } \\
\text { capacity, continuously monitored. }\end{array}$ & TAN-681-012 & Yes \\
\hline & TAN Decon & $\begin{array}{l}29.6-\mathrm{m} \mathrm{stack}, 24.9 \mathrm{~m}^{3} / \mathrm{s} \text { discharge } \\
\text { capacity, continuously monitored cur- } \\
\text { rently no emissions. Currently in pro- } \\
\text { cess of deactivation. }\end{array}$ & TAN-607-136 & No \\
\hline & TAN/TSF & $\begin{array}{l}51.4-\mathrm{m} \text { stack, } 7.9 \mathrm{~m}^{3} / \mathrm{s} \text { discharge capac- } \\
\text { ity, continuously monitored. }\end{array}$ & TAN-734-001 & Yes \\
\hline & TAN/PREPP & $\begin{array}{l}26.1-\mathrm{m}, 3 \mathrm{~m}^{3} / \mathrm{s} \text { discharge capacity, con- } \\
\text { tinuously monitored. Facility was never } \\
\text { activated-currently being scheduled } \\
\text { for decommissioning. }\end{array}$ & TAN-607-107 & No \\
\hline & TRA/ATR & $\begin{array}{l}76.2-\mathrm{m}, \text { stack, } 21.2 \mathrm{~m}^{3} / \mathrm{s} \text { discharge } \\
\text { capacity, continuously monitored. }\end{array}$ & TRA-770-001 & Yes \\
\hline & TRAVhem Lab & $\begin{array}{l}29.6-\mathrm{m} \text { stack, } 6.6 \mathrm{~m}^{3} / \mathrm{s} \text { discharge capac- } \\
\text { ity, continuously sampled. }\end{array}$ & TRA-604-035 & Yes \\
\hline
\end{tabular}


Table 1. (continued)

\begin{tabular}{|c|c|c|c|c|}
\hline $\begin{array}{l}\text { Type of } \\
\text { Discharge }\end{array}$ & Area/Location & $\begin{array}{c}\text { Type of Description } \\
\text { of Discharge Surface }\end{array}$ & $\begin{array}{l}\text { Air Emission } \\
\text { Inventory } \\
\text { Stack No. }\end{array}$ & $\begin{array}{c}\text { Active } \\
\text { (Yes/No) }\end{array}$ \\
\hline & TRAVETR & $\begin{array}{l}76.2-\mathrm{m} \mathrm{stack}, 9.4 \mathrm{~m}^{3} / \mathrm{s} \text { discharge capac- } \\
\text { ity, continuously monitored. As of } 3 / 88 \text {, } \\
\text { this stack is not monitored. No activity } \\
\text { is discharged. }\end{array}$ & TRA-753-001 & Yes \\
\hline & TRA/Hot Cells & $\begin{array}{l}15.2-\mathrm{m} \text { stack, } 1.6 \mathrm{~m}^{3} / \mathrm{s} \text { discharge capac- } \\
\text { ity, continuously sampled; three stacks } \\
\text { sampled as one. }\end{array}$ & TRA-632-030 & Yes \\
\hline & TRA/MTR & $\begin{array}{l}76.2-\mathrm{m} \text { stack, } 6 \mathrm{~m}^{3} / \mathrm{s} \text { discharge capacity, } \\
\text { continuously monitored. }\end{array}$ & TRA-710-001 & Yes \\
\hline & TRA/661 & $\begin{array}{l}8.43-\mathrm{m} \text { stack, } 3.3 \mathrm{~m}^{3} / \mathrm{s} \text { discharge capac- } \\
\text { ity, not monitored. }\end{array}$ & TRA-661-008 & Yes \\
\hline & WER/North & $\begin{array}{l}15-\mathrm{m} \text { stack, } 8 \mathrm{~m}^{3} / \mathrm{s} \text { discharge capacity, } \\
\text { continuously monitored. }\end{array}$ & $\begin{array}{l}\text { PER755 } \\
\text { Vent } 001\end{array}$ & Yes \\
\hline & WER/South & $\begin{array}{l}15-\mathrm{m} \text { stack, } 9.4 \mathrm{~m}^{3} / \mathrm{s} \text { discharge capacity, } \\
\text { continuously monitored. }\end{array}$ & $\begin{array}{l}\text { PER756 } \\
\text { Vent } 001\end{array}$ & Yes \\
\hline & WER/765 & $\begin{array}{l}15.5-\mathrm{m} \text { stack, } 4.7 \mathrm{~m}^{3} / \mathrm{s} \text { discharge capac- } \\
\text { ity, continuously monitored. }\end{array}$ & & \\
\hline & WMC/SWEPP & $\begin{array}{l}4.5 \text {-m stack, } 1.7 \mathrm{~m}^{3} / \mathrm{s} \text { discharge capac- } \\
\text { ity, continuously monitored during } \\
\text { active Drum Vent operation. }\end{array}$ & WMF-615-001 & Yes \\
\hline \multirow[t]{2}{*}{$\begin{array}{l}\text { Liquid } \\
\text { (injection well) }\end{array}$} & CPP & $\begin{array}{l}181 \mathrm{~m} \text { deep ( } 42.7 \mathrm{~m} \text { below water table), } \\
\text { constantly monitored by radiation } \\
\text { detector with a detection unit of } 2 \times \\
10^{-6} \mathrm{Ci} / \mathrm{mL} \text {, flow approximately } 170 \times \\
10^{6} \text { liters per month. (INEL discharges } \\
\text { to the injection well have been termi- } \\
\text { nated since } 1986 \text {. This well was closed } \\
\text { in November } 1989 .)\end{array}$ & & No \\
\hline & PBF & $\begin{array}{l}33.5 \mathrm{~m} \text { deep ( } 105 \mathrm{~m} \text { above water table), } \\
\text { constantly monitored, flow intermittent } \\
\text { with a maximum of } 750 \text { liters per min- } \\
\text { ute (no longer in use as of August } 1980 \text {; } \\
\text { in June } 1984 \text { this well was completely } \\
\text { filled with concrete). }\end{array}$ & & No \\
\hline $\begin{array}{l}\text { Liquid } \\
\text { (seepage } \\
\text { ponds, leach- } \\
\text { ing pits, cribs) }\end{array}$ & ANL/EBR-II \& L\&O & $\begin{array}{l}\text { Batch monitored pond approximately } \\
1.2 \times 10^{4} \mathrm{~m}^{2} \text { maximum depth of } 4 \mathrm{~m} \text {. }\end{array}$ & & Yes \\
\hline
\end{tabular}


Table 1. (continued)

\begin{tabular}{|c|c|c|c|c|}
\hline $\begin{array}{l}\text { Type of } \\
\text { Discharge }\end{array}$ & Area/Location & $\begin{array}{l}\text { Type of Description } \\
\text { of Discharge Surface }\end{array}$ & $\begin{array}{l}\text { Air Emission } \\
\text { Inventory } \\
\text { Stack No. }\end{array}$ & $\begin{array}{l}\text { Active } \\
\text { (Yes/No) }\end{array}$ \\
\hline & ARA & $\begin{array}{l}\text { Surface depression (approximately } \\
1 / 3 \text { acre), estimate flow } 1.05 \times 10^{7} \\
\text { liters per year, continuously monitored. } \\
\text { ARA completely shut down operations } \\
\text { in September } 1986 \text {, no further releases } \\
\text { are being made to this release point } \\
\text { except for drainage as a result of rain } \\
\text { water runoff. }\end{array}$ & & No \\
\hline & CFA & $\begin{array}{l}\text { Sewage plant tile drain field, } 610 \times \\
61 \mathrm{~m} \text {, average flow approximately } \\
15 \times 10^{6} \text { liters per month, continuously } \\
\text { monitored. }\end{array}$ & & Yes \\
\hline & CPP/Pond No. 1 & $\begin{array}{l}\text { Percolation pond, } 412 \mathrm{ft} \times 480 \mathrm{ft} \times \\
16 \mathrm{ft} \text {. CPP } 797 \text { is the discharge point } \\
\text { that is continuously monitored and } \\
\text { sampled. }\end{array}$ & & Yes \\
\hline & CPP/Pond No. 2 & $\begin{array}{l}\text { Percolation pond, } 498 \mathrm{ft} \times 498 \mathrm{ft} \times \\
12 \text { to } 14 \mathrm{ft} \text {. CPP } 797 \text { is the discharge } \\
\text { point that is continuously monitored } \\
\text { and sampled. }\end{array}$ & & Yes \\
\hline & $\begin{array}{l}\text { CTF } \\
\text { (formerly LOF) }\end{array}$ & $\begin{array}{l}\text { Continuously monitored pond approxi- } \\
\text { mately } 76 \times 152 \times \text { maximum } 5.5 \mathrm{~m} \\
\text { deep. }\end{array}$ & & Yes \\
\hline & NRF & $\begin{array}{l}\text { Continuously monitored leaching beds } \\
\text { handling } 95,000 \text { liters per month } \\
\text { (releases ended April 1979). }\end{array}$ & & No \\
\hline & TAN & $\begin{array}{l}\text { Continuously monitored pond approxi- } \\
\text { mately } 13 \text { hectares in size. }\end{array}$ & N/A & Yes \\
\hline & TRA & $\begin{array}{l}\text { Two ponds } 40 \times 73 \text { and one pond } 76 \times \\
122 \mathrm{~m} \text {. average flow approximately } \\
90 \times 10^{6} \text { liters per month, prior to } \\
1988 . \text { In } 1988 \text { average flow decreased } \\
\text { to } 4.7 \times 10^{6} \text { liters per month. Ponds are } \\
\text { continuously monitored. Removed from } \\
\text { service August } 1993 \text {. }\end{array}$ & N/A & No \\
\hline & TRA & $\begin{array}{l}\text { Evaporation pond. Two adjacent lined } \\
\text { ponds measuring } 70 \mathrm{~m} \times 140 \mathrm{~m} \text { each. } \\
\text { Double liner and leak detection system. } \\
\text { All particulate activity remains in the } \\
\text { lined pond } 3.0 \times 10^{6} \text { liters per month. } \\
\text { Continuously monitored. Zero release to } \\
\text { soil column. }\end{array}$ & & \\
\hline
\end{tabular}


Table 1. (continued)

\begin{tabular}{|c|c|c|c|c|}
\hline $\begin{array}{l}\text { Type of } \\
\text { Discharge }\end{array}$ & Area/Location & $\begin{array}{l}\text { Type of Description } \\
\text { of Discharge Surface }\end{array}$ & $\begin{array}{l}\text { Air Emission } \\
\text { Inventory } \\
\text { Stack No. }\end{array}$ & $\begin{array}{l}\text { Active } \\
\text { (Yes/No) }\end{array}$ \\
\hline \multirow[t]{6}{*}{ Solid } & All Facilities & $\begin{array}{l}\text { Disposal of radioactive solid waste } \\
\text { accomplished at the INEL Radioactive } \\
\text { Waste Management Complex (RWMC). }\end{array}$ & N/A & Yes \\
\hline & & $\begin{array}{l}\text { Storage of transuranic radioactive solid } \\
\text { waste accomplished at the INEL } \\
\text { RWMC. }\end{array}$ & $\mathrm{N} / \mathrm{A}$ & Yes \\
\hline & & $\begin{array}{l}\text { Reduction of applicable radioactive } \\
\text { solid waste volume accomplished at the } \\
\text { INEL WERF. }\end{array}$ & $\mathrm{N} / \mathrm{A}$ & Yes \\
\hline & & $\begin{array}{l}\text { Storage of calcinated solids accom- } \\
\text { plished at CPP. }\end{array}$ & $\mathrm{N} / \mathrm{A}$ & Yes \\
\hline & & $\begin{array}{l}\text { Storage of sodium-contaminated waste } \\
\text { generated by ANL accomplished at } \\
\text { ANL. }\end{array}$ & N/A & Yes \\
\hline & & $\begin{array}{l}\text { Storage of mixed waste accomplished at } \\
\text { Mixed Waste Storage Facility (MWSF). }\end{array}$ & N/A & Yes \\
\hline
\end{tabular}




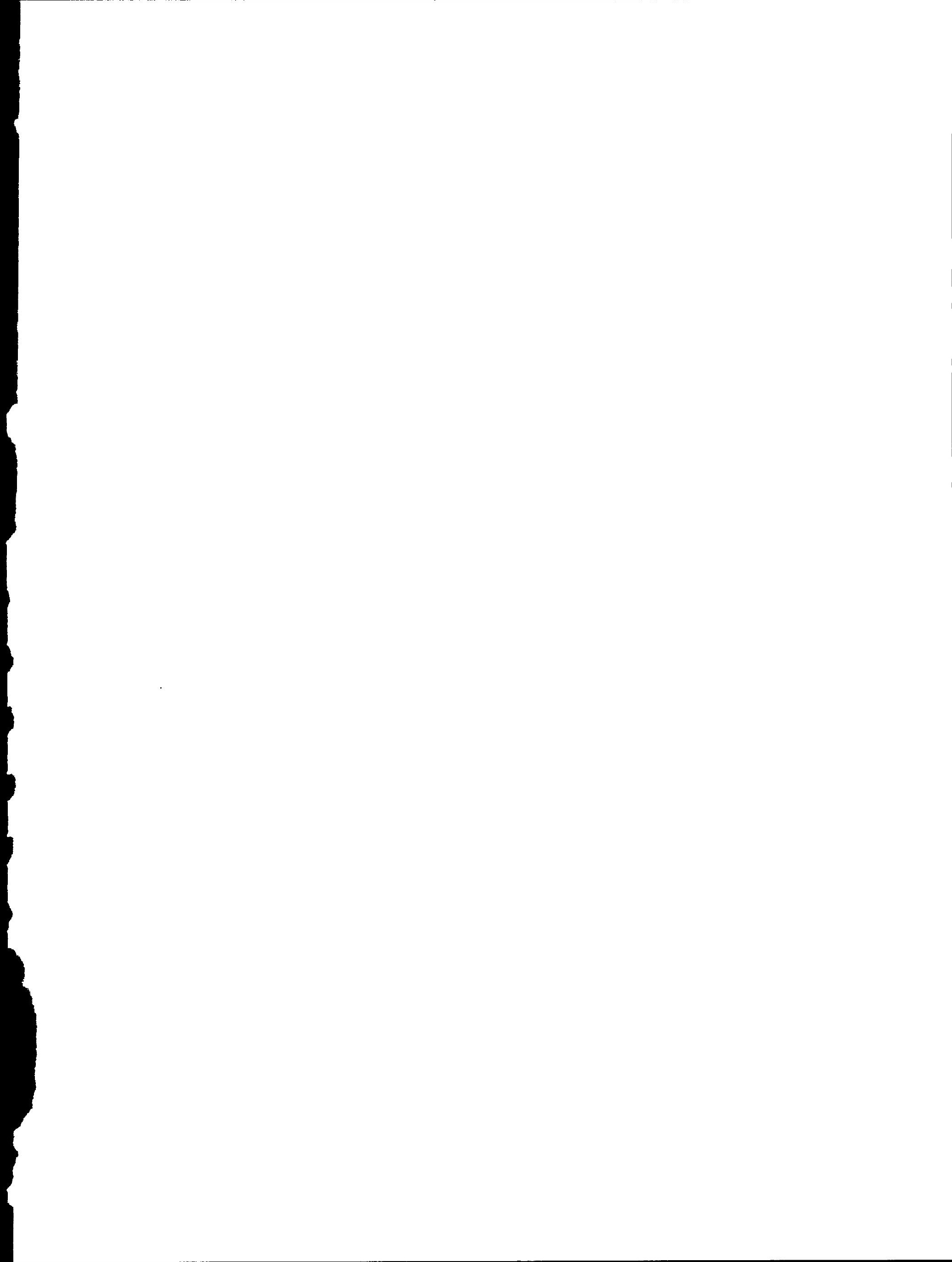


